
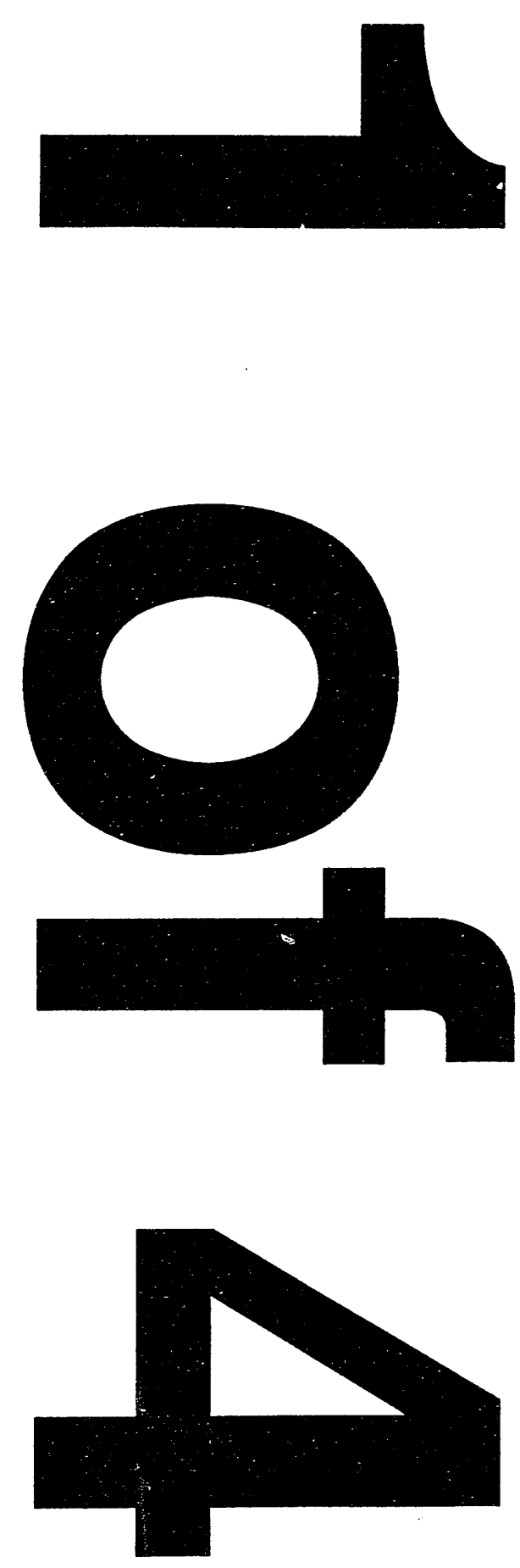


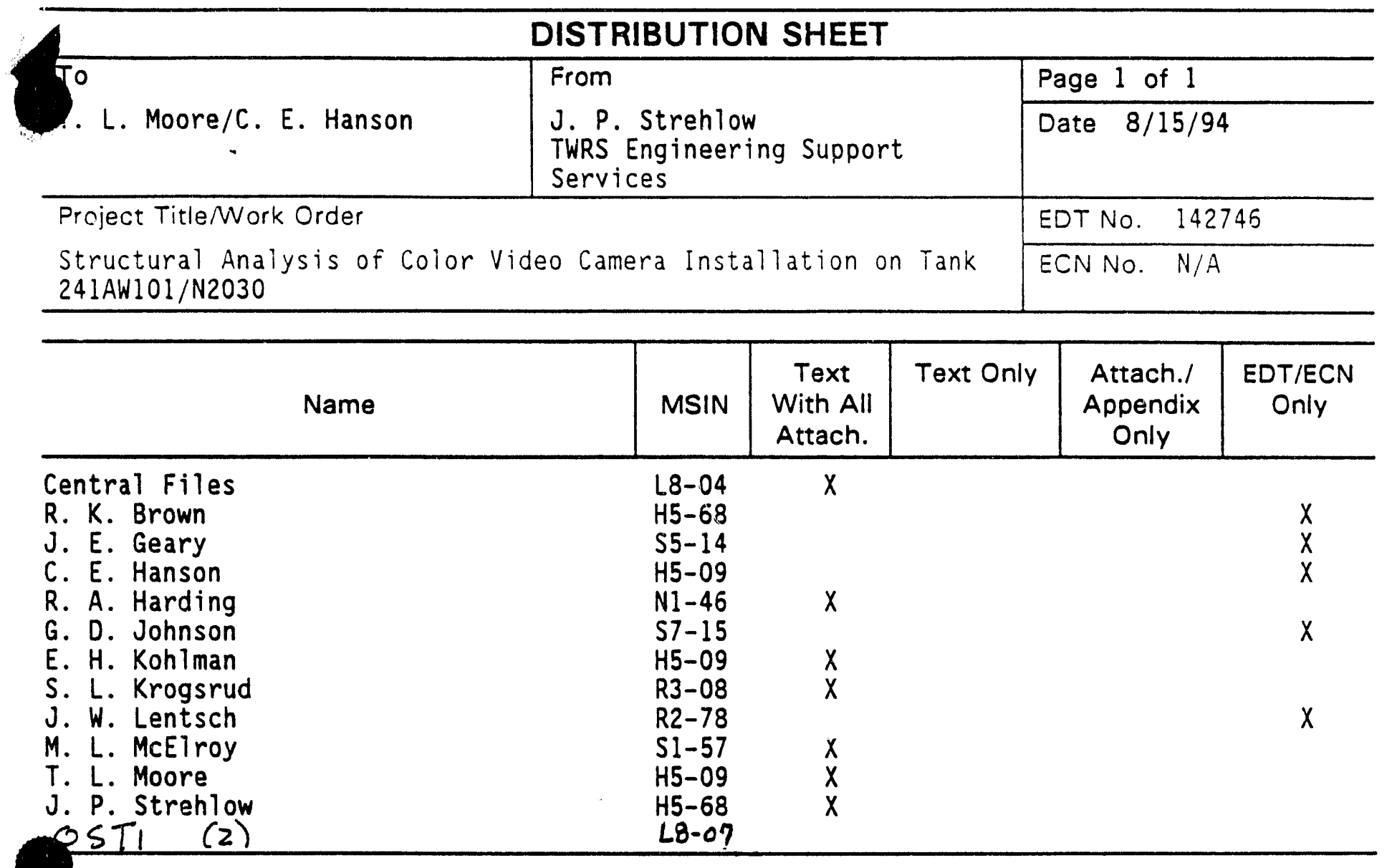




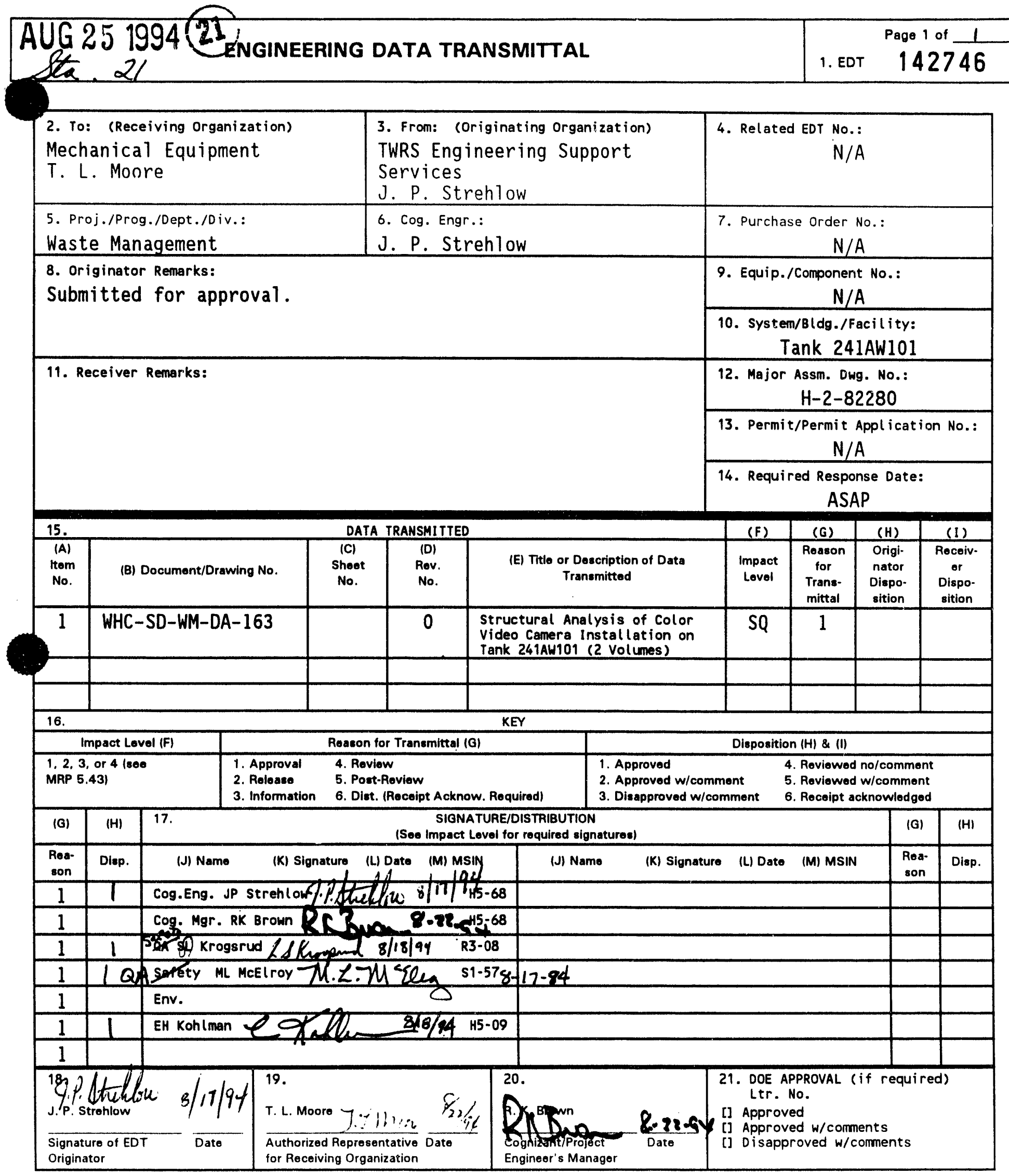


RELEASE AUTHORIZATION

Document Number: $\quad$ WHC-SD-WM-DA-163, REV. 0

Document Title: $\quad$ STRUCTURAL ANALYSIS OF COLOR VIDEO CAMERA

INSTALLATION ON TANK 241AW101 (2 VOLUMES)

Release Date: $\quad 8 / 24 / 94$

This document was reviewed following the procedures described in WHC-CM-3-4 and is:

APPROVED FOR PUBLIC RELEASE

***************

WHC Information Release Administration Specialist:

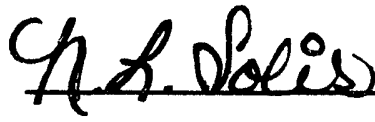

N.L. SOLIS

(Signature)

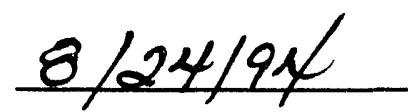

(Date) 
2. Title

Structural Analysis of Color Video Camera Installation on Tank 241AW101 (2 volumes)

5. Key Words

Video 241AW101

Camera Analysis

Color

Tank

Creston

3. Number

WHC-SD-WM-DA-163

6. Author

Nane: URSiJohn A. Blume?

Associates, Engineers/

J. P. Strehlow

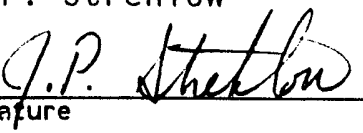

signafure

Organization/Charge Code

80220 / N2030

This supporting document provides the structural analysis of record for the color video camera assembly that will be installed in tank 24lAw101. This analysis includes both deadweight and seismic evaiuations. The camera assembly a purchased item, see CVI File 359426 for information on the camera.

8. PORPOSE AND USE OF $\angle D O C U M E N T$ - This document was prepared for use within the U.S. Dopantment of Energy and jts contractors. It is to be ysed only to perform, direct, for integrate work yuder U.S. Department of Energy contracts. This document is not agroved for public release until ceviewed.

PATENT STA.US - This document/copy, since it is transmitted in advance of pafent clearance, is made available in contidence solely for usf in performance of work under contracts with the U.S. Department of Energy This document is not to be pub ished nor $i$ ts contents otherwise /isseminated or used for ourposes other than spelified above befoce patent approval for such release or use has been secured, upon pequest, from the Patent Counsel, U.S. Department of Energy Field Office, Richland, WA.

10.

RELEASE STAMP

DISCLAIMER - This report was prepared as an accuunt of work sponsored by an agency of the United States Government. Neither the United States Government nor any agency thereof, nor any of their employees, nor any of their contractors, subcontractors or their employees, makes any warranty, express or implied, or assumes any legal liability or responsibility for the accuracy, completeness, or any third party's use or the results of such use of any information, apparatus, product, or process disclosed, or represents that its use would not infringe privately owned rights. Reference herein to any specific commercial product, process, or service by trade name, trademark, manufacturer, or otherwise, does not necessarily constitute or imply its endorsement, recommendation, or favoring by the United States Government or any agency thereof or its contractors or subcontractors. The views and opinions of authors expressed herein do not necessarily state or reflect those of the United States Government or any agency thereof.

9. Impact Level $\mathrm{SQ}$

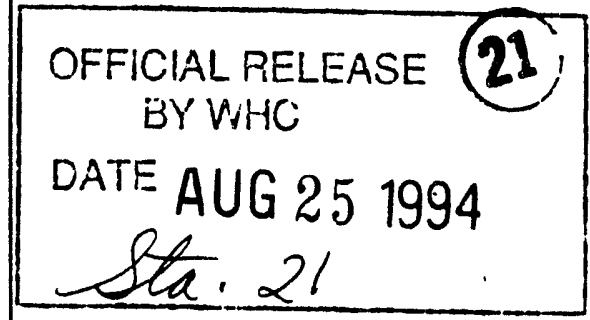


WHC-SD-WM-DA-163

Vol. I, Rev. 0

STRUCTURAL AI:ALYSIS OF COLOR VIDEO CAMERA

ON TANK 241AW101

$$
\text { Volume I of II }
$$

$\begin{array}{lll}\text { Volume I } & - & \text { Report } \\ \text { Volume II } & - & \text { Calculations }\end{array}$


WHC-SD-WM-DA-163

Vol. I, Rev. 0

\section{DESIGN VERIFICATION METHOD}

The need for design verification has been reviewed with the method selected as indicated below:

See Volume II

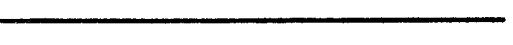

SD \# WHC-SD-WM-DA-163

EDT \# 142746

$D W G(S) \#$
Independent Review

Alternative Calculations

Qualification Testing

Formal Design Review

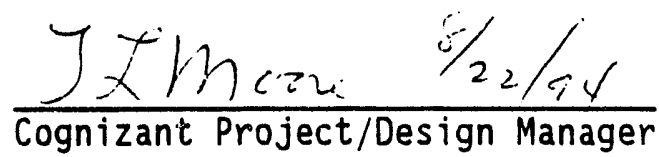


WHC-SD-WM-DA-163

Vol. I, Rev. 0

\section{TRADEMARKS}

The following trademarks are used in this report.

SAP9O is a trademark of Computers and Structures, Inc., 1995 University Avenue, Berkely, California.

SAPSTL is a trademark of Computers and Structures, Inc., 1995 University Avenue, Berkely, California. 
WHC-SD-WM-DA-163

Vol. I, Rev. 0

\title{
STRUCTURAL ANALYSIS OF CRESTON VIDEO CAMERA INSTALLATION ON TANK 241AW101
}

\section{VOLUME I of II - REPORT}

\author{
prepared for \\ Westinghouse Hanford Company \\ Richland, Washington
}

prepared by

URS/John A. Blume and Associates, Engineers

San Francisco, California

August 1994

\section{URS}

$I-1$ 
WHC-SD-WM-DA-163

Vol. I, Rev. 0

\section{TABLE OF CONTENTS}

COVER

$\begin{array}{lr}\text { TABLE OF CONTENTS } & 2\end{array}$

1.0 INTRODUCTION 3

1.1 Purpose and Scope 3

1.2 Structural Description of Creston Video Camera 3

1.3 Design Modifications 3

1.4 Design Codes, Standards and Specifications 4

1.5 As-Built Drawings 4

2.0 SEISMIC ANALYSIS 5

2.1 Seismic Load Criteria 5

2.2 Acceptance Criteria 5

2.3 Analysis Procedure and Stress Evaluation 5

$\begin{array}{lll}3.0 & \text { REFERENCES } & 7\end{array}$

$\begin{array}{ll}\text { TABLES } & 8\end{array}$

$\begin{array}{ll}\text { FIGURES } & 13\end{array}$

age

\section{(1)}

2

(1)

4
$\sqrt{20}$ 5 7 8 
WHC-SD-WM-DA-163

Vol. I, Rev. 0

\subsection{INTRODUCTION}

1.1 Purpose and Scope

A video camera is planned to be installed on the radioactive storage tank $241 \mathrm{AW} 10 \mathrm{l}$ at the DOE's Hanford Site in Richland, Washington. The camera will occupy the 20 inch port of the Multiport Flange riser which is to be installed on riser 5B of the tank 241AW101 $(3,5,10)$.

The objective of the project reported herein was to perform a seismic analysis and evaluation of the structural components of the camera for a postulated Design Basis Earthquake (DBE) per the reference Structural Design Specification (SDS) document (6). The detail of supporting engineering calculations is documented in URS/Blume Calculation \# 66481-01-CA-03 (1).

\subsection{Structural Description of Creston Video Camera}

The camera assembly consists of the camera unit itself and a steel frame, referred here as the camera mast structure, that holds the camera in position inside the tank 241AW101. The camera mast structure is secured to the 20 inch port of the Multiport Flange (MPF) riser by means of twenty $3 / 4$ inch A307 bolts. The camera mast structure is a four legged stainless steel structure made of four 1.25 in diameter pipes that are welded at top , bottom and intermediate locations to $1 / 4$ inch end plates. A discontinuous lacing using $1 \times 3 / 16$ flat steel ties the four corner pipes together. Figures 1-1, 1-2 and 1-3 show the upper mast, lower mast and the camera roll mechanism, respectively. The properties of the different components of the camera mast structure are summarized in Table 1-1. A set of preliminary shop drawings of the camera (2) were provided by WHC for this evaluation. A set of "as-built" drawings were also provided by WHC at a later date (11) for review (See also section 1.5).

\subsection{Design Modifications}

The original design of the camera mast, as shown in Figures $1-1$ and 1-2, include flat steel bars for diagonal lacing members. The single $1 \times 3 / 16$ flat steel diagonal members were found to be inadequate for compression and were replaced by a (tension only) cross bracing using the same size steel bars as shown in Figure 1-4. 
WHC-SD-WM-DA-163

Vol. I, Rev. 0

\subsection{Design Codes, Standards and Specifications}

The design of the Creston Video Camera was evaluated in accordance with the following Design codes, Standards and specifications.

1.2.1 "Structural Design Specification for Installation of Creston Video Camera on Tanks 241AW101, 241SY101 and 241AN107", Westinghouse Hanford Company, July 1994.

1.2.2 "Standard Architectural-Civil Design Criteria," Document No. SDC-4.1, Hanford Plant Standards, Rev. 11. Department of Energy, Richland Washington (9).

1.2.3 "General Design Criteria," United States Department of Energy, DOE Order No. 6430.1A.

1.2.4 "Design and Evaluation Guidelines for Department of Energy Facilities Subject to Natural Phenomenon Hazards," UCRL-15910, June 1990.

1.2.5 "Nuclear Facilities - Steel Safety Related Structures for Design, Fabrication and Erection," ANSI/AISC N690-1984.

1.2.6 "Seismic Analysis of Safety-Related Nuclear Structures and Commentary on Standard for Seismic Analysis of Safety Related Nuclear Structures," ASCE Standard ASCE 4-86.

\subsection{As-Built Drawings}

The as-built drawings (11) of the Creston camera mast were reviewed. Minor differences between the as-built and the preliminary drawings are summarized in reference (1). The differences had no impact on the results of seismic evaluation from using the preliminary set of drawings. 
WHC-SD-WM-DA-163

Vol. I, Rev. 0

\subsection{SEISMIC ANALYSIS}

\subsection{Seismic Load Criteria}

The camera mast structure supporting riser and its connection to the tank roof serve as a contain ment of radioactive material inside the tank and are classified as nonreactor safety class 2 structures (6). According to the specifications of reference 6, The MPF assembly and supporting 42 inch riser should be evaluated against application of normal loads and DBE loads as follows:

DL

DL + DBE

DL-DBE

Where

DL: Dead Loads

and

DBE: Design Basis Earthquake loads defined by a $5 \%$ damped ground surface acceleration response spectrum with a zero period acceleration (ZPA) of $0.12 \mathrm{~g}(6)$.

The reference (9) specifies that the MPF be subjected to DBE loads applied in three orthogonal direction simultaneously. The said reference also allows that the vertical component of the DBE ground motion be reduced by a factor of $2 / 3$. To account for potential amplification of the vertical ground motion by the tank roof, reference (6) specifies that the vertical motion not to be scaled by the $2 / 3$ factor.

\subsection{Acceptance Criteria}

The computed stresses in the Steel components of the MPF assembly were evaluated in accordance with the specifications of ANSI/AISC N690-84 (7). A summary of the applicable load combinations and corresponding stress allowables considered in this evaluation is tabulated in Table $2-1$. 
WHC-SD-WM-DA-163

Vol. I, Rev. 0

\subsection{Analysis Procedure and Stress Evaluation}

A finite element model of the camera mast structure, as shown in Figures 2-1 was developed using computer program SAP90 ( 8 ). To account for the flexibility of the suporting riser the stick model of the supporting Multiport Flang (MPF) structure and its supporting 42 inch nser, used for evaluation of MPF (10) in a separate study, was incorporated in the model of camera mast.

Frequency analyses of the camera model was first performed to extract the natural frequencies and mode shapes. Table 2-2 summarizes the properties of the first 20 modes of vibration for the camera models.

Response spectrum analysis of the model was then performed using the DBE free field acceleration response spectrum for $5 \%$ damping. The free field response spectrum was used because no tank roof response spectrum was available at the time of this evaluation. Reference (6) requires that the camera be subjected to the DBE spectra in three orthogonal directions simultaneously. To account for potential amplification of the verical ground motion by the roof of the 241AW101 tank, the vertical spectra was not reduced by $2 / 3$ factor.

The results from response spectra analyses were used to compute stresses in the components of the camera mast structure. Table 2-3 summarizes the computed stress ratio. As shown in Table 2-3, computed stress ratios for component of the camera are all within the specified limits with largest maximum stress ratios of 0.81 and 0.48 occurring at the weld connecting the lacing members to $1.25^{\prime \prime}$ SS pipes and welded connection between the light supports and the SS pipes, respectively.

The results from response spectrum analysis were also used to estimate the maximum lateral displacement of the camera mast structure to ensure that camera mast will not interact with any adjecent equipment. A plot of the deformed shape of the camera mast under DBE loads is shown on Figure 3-1. The maximum computed relative lateral displacement of 0.6 inches occures at the lowest nodal point of the mathematical model which represent the lowest point in the camera assembly structure. 


\subsection{REFERENCES}

1) "Seismic Analysis of Creston Camera Installation on Tanks 2+1AW 101 ", Volume II of II, URS/Blume Calculation no. 66481-01-CA-3, rev 0, August 1994..

2) Creston Video Camera Mast Preliminary Piece Part Drawings, Received from WHC on $5 / 28 / 1994$.

3) "Flanged Riser Installation, Tank 241-AW-101", WHC Drawings H-2-81102, SHT 1 of 1 , rev 0 .

4) (Not Used)

5) Selected 241AW Tank Farm drawings: H-2-70395, H-2-70396

6) "Structural Design Specification for Installation of Creston Video Camera on Tanks 241AW101, 241SY101 and 241AN107", Westinghouse Hanford Company, July 1994.

7) "Nuclear Facilities - Steel Safety Related Structures for Design, Fabrication and Erection," ANSI/ AISC N690-1984.

8) "SAP90 - A Series of Computer Programs for Finite Element Analysis of Structures," Computers and Structures, Inc., Berkeley, California.

9) "Standard Architectural-Civil Design Criteria," Document No. SDC-4.1, Hanford Plant Standards, Rev. 11. Department of Energy, Richland Washington.

10) "Structural Analysis of Multiport Flange Installation on Tanks 241AW101 and 241SY101", URS/Blume Report to Westinghouse Hanford Company, July 1994.

11) "As-Built" Creston Video Camera Mast Piece Part Drawings, Received from WHC on 8/1/1994. 
WHC-SD-WM-DA-163

Vol. I, Rev. 0

TABLE (1-1) Properties of Creston Camera Components for Tank 24 I AW 101

\begin{tabular}{|c|c|c|c|c|c|c|}
\hline $\begin{array}{c}\text { PART } \\
\text { NO. }\end{array}$ & DESCAIPTION & SIZE & THICK & MATERIAL & $\begin{array}{l}\text { YIELD } \\
\text { STRESS } \\
\text { Fy(kSI) }\end{array}$ & $\begin{array}{r}\text { TENSILE } \\
\text { STRENG } \\
\text { F(Iks) }\end{array}$ \\
\hline \multicolumn{7}{|c|}{ 42.1 UPPEA MAST ASSEMBLY } \\
\hline $42-2$ & SHIELDING PLATE & $22^{\circ} 0$ & $3^{*}$ & CARBON STEEL & 36 & 58 \\
\hline $42 \cdot 3$ & COVER PLATE & $22^{\circ} 0$ & $1 / 4^{*}$ & 300 SERIES SS & 25 & 70 \\
\hline 424 & MANIFOLD ASSEMBL & & & & & \\
\hline $42-5$ & MANIFOLD TUBE & 220 & $\mathbf{2}^{*}$ & 304 SS ASTM A312 & 35 & 85 \\
\hline $42-6$ & OIVIOING PLATE & $19.05 \times 4^{\circ}$ & $3 / 8^{*}$ & 300 SERIES SS & 25 & 70 \\
\hline 42.7 & fLANGE PLATE & $27.5 \circ 0$ & $3 / 4^{\circ}$ & 30.4 SS ASTM A24O & 25 & 70 \\
\hline 428 & 1 1/4 IPS PIPE & 107.9" & $0.14^{\circ}$ & $\mathrm{SCH} 40304 \mathrm{SS}$ & 30 & 75 \\
\hline $42-0$ & \multicolumn{6}{|l|}{ PIPE WELDING DETAIL } \\
\hline $42 \cdot 10$ & \multicolumn{6}{|l|}{ CROSS BRACE DETAIL } \\
\hline $42-11$ & \multicolumn{2}{|l|}{ FLANGE PLT GASKET } & $1 / 8^{\bullet}$ & GARLOCK 3000 & N/A & N/A \\
\hline $42-12$ & \multicolumn{2}{|l|}{ MATING PLT GASKET } & $1 / 8^{\bullet}$ & GAALOCK 3000 & N/A & N/A \\
\hline 40.3 & MATING PLATE & $13^{\circ} \times 13^{\circ}$ & $1 / 2^{\circ}$ & 300 SERIES SS & 25 & 70 \\
\hline $40-5$ & CROSS BRACE & $1{ }^{\circ} \times 15^{\circ}$ & $3 / 16^{\circ}$ & 300 SEAIES SS FLA & 25 & 70 \\
\hline \multicolumn{7}{|c|}{ 40-1 LOWER MAST ASSEMBLY } \\
\hline $40-2$ & BOTTOM PLATE & $13^{\circ} \times 13^{\circ}$ & $1 / 2^{*}$ & 300 SEAIES SS & 25 & 70 \\
\hline $40-3$ & MATING PLATE & $13^{\circ} \times 13^{\circ}$ & $1 / 2^{*}$ & 300 SERIES SS & 25 & 70 \\
\hline 404 & UGHT SUPPORT & $15.6^{\circ} \times 1^{\circ}$ & $3 / 8^{\bullet}$ & 300 SERIES SS FLA & 25 & 70 \\
\hline $40-5$ & CAOSS BRACE & $1{ }^{\circ} \times 15^{\circ}$ & $1 / 4^{\circ}$ & 300 SERIES SS FLA & 25 & 70 \\
\hline $40-6$ & \multicolumn{6}{|l|}{ GAS TUBE DETAIL } \\
\hline $40-7$ & \multicolumn{6}{|l|}{ LGHT FITING DETAIL } \\
\hline $40-8$ & \multicolumn{6}{|l|}{ CROSS BRACE DETAIL } \\
\hline $40-9$ & \multicolumn{6}{|l|}{ PIPE WELDING DETAIL } \\
\hline $40-10$ & 1/2NPT NIPPLE MO & ס $84^{\circ}$ & $1 / 2^{\circ}$ & \multicolumn{2}{|c|}{$1 / 2$ NPT $\times 21 / 2$ SS NIPPLE } & N/A \\
\hline $40-11$ & $11 / 4$ IPS PIPE & $86.0^{\circ}$ & $0.14^{\circ}$ & $\mathrm{SCH} 40304 \mathrm{SS}$ & 30 & 73 \\
\hline $40-12$ & 3/8 IPS GAS PIPE & $\begin{array}{l}675^{\circ} 0 \\
\times 9.34^{\circ}\end{array}$ & $0.15^{\circ}$ & $3 / 8$ IPS SCH $40304 \mathrm{~S}$ & $\begin{array}{r}\text { SS PIPE } \\
25\end{array}$ & 75 \\
\hline $40-13$ & FITTING & & $4^{\circ}$ & 300 SERIES SS & 25 & 75 \\
\hline $40-14$ & FITTING GASKET & & $1 / 8^{\bullet}$ & GARLOCK $3000 \mathrm{GA}$ & N/A & $N / A$ \\
\hline
\end{tabular}


WHC-SD-WM-DA-163

Vol. I. Rev. 0

TABLE (2-1) - Load Combinations and allowable stress limits

\begin{tabular}{llll}
$\begin{array}{l}\text { Structure } \\
\text { Category }\end{array}$ & $\begin{array}{l}\text { Load } \\
\text { Category }\end{array}$ & Load Combinations & $\begin{array}{l}\text { Stress Limit } \\
\text { Coefficient }(*)\end{array}$ \\
\hline \multirow{2}{*}{ Steel } & Normal & DL & 1.0 \\
& Extreme & DL + DBE & 1.6 \\
\hline
\end{tabular}

Where

$\mathrm{DL} \quad=\quad$ Dead Loads

$\mathrm{DBE}=$ Design Basis Earthquake load

(*) The shear stress allowables for steel and bolts are increased by 1.4 .

All allowable stresses should not exceed $0.7 * \mathrm{Fu}$ where:

$\mathrm{Fu}=$ Ultimate tensile strength. 


\section{- TABLE (2-2) - Summary of Frequency Analysis}

\begin{tabular}{|c|c|c|c|}
\hline & $\begin{array}{c}\text { MODE } \\
\text { NUMBER }\end{array}$ & $\begin{array}{r}\text { FREQUENCY } \\
(\mathrm{HZ})\end{array}$ & $\begin{array}{r}\text { PERIOD } \\
\text { (SEC) }\end{array}$ \\
\hline & 1 & 3.182 & 0.31423 \\
\hline & 2 & 3.200 & 0.31247 \\
\hline & 3 & 10.475 & 0.09547 \\
\hline & 4 & 11.881 & 0.08417 \\
\hline & 5 & 14.510 & 0.06892 \\
\hline & 6 & 27.528 & 0.03633 \\
\hline & 7 & 27.548 & 0.03630 \\
\hline & 8 & 32.057 & 0.03119 \\
\hline & 9 & 38.304 & 0.02611 \\
\hline & 10 & 38.786 & 0.02578 \\
\hline & 11 & 39.243 & 0.02548 \\
\hline & 12 & 45.454 & 0.02200 \\
\hline & 13 & 50.228 & 0.01991 \\
\hline & is 14 & 57.144 & 0.01750 \\
\hline & 15 & 72.505 & 0.01379 \\
\hline & 16 & 78.109 & 0.01280 \\
\hline & 17 & 112.010 & 0.00893 \\
\hline & 18 & 150.223 & 0.00666 \\
\hline & * 19 & 186.701 & 0.00536 \\
\hline & 20 & 200.561 & 0.00499 \\
\hline
\end{tabular}

\begin{tabular}{|c|c|c|c|c|c|c|}
\hline \multicolumn{7}{|c|}{ PAATICIPATING MASS - (percent) } \\
\hline MODE & X-DIR & Y-DIR & Z-DIR & X-sum & Y-SUM & Z-SUM \\
\hline 1 & 0.000 & 3.466 & 0.000 & 0.000 & 3.466 & 0.000 \\
\hline 2 & 3.474 & 0.000 & 0.000 & 3.474 & 3.466 & 0000 \\
\hline 3 & 0.262 & 0.243 & 0.000 & 3.737 & 3.710 & 0.000 \\
\hline 4 & 0.369 & 0.415 & 0.001 & 4.105 & 4.125 & 0.001 \\
\hline 5 & 0.185 & 0.159 & 0.000 & 4.290 & 4.284 & 0.001 \\
\hline 6 & 79.650 & 1.494 & 0.013 & 83.940 & 5.779 & 0.014 \\
\hline 7 & 1.485 & 79.807 & 0.001 & 85.426 & 85.586 & 0.015 \\
\hline 8 & 0.014 & 0.013 & 0.000 & 85.440 & 85.599 & 0015 \\
\hline 9 & 0.070 & 0.158 & 0.000 & 85.510 & 85.757 & 0015 \\
\hline 10 & 0.127 & 0.037 & 0.000 & 85.637 & 85.793 & 0.015 \\
\hline 11 & 0.026 & 0.021 & 0.000 & 85.663 & 85.814 & 0.015 \\
\hline 12 & 0.006 & 0.010 & 0.001 & 85.669 & 85.824 & 0.016 \\
\hline 13 & 0.006 & 0.025 & 0.086 & 85.674 & 85.848 & 0103 \\
\hline 14 & 0.004 & 0.000 & 0.027 & 85.678 & 85.848 & 0.130 \\
\hline 15 & 0.000 & 0.009 & 0.791 & 85.678 & 85.857 & 0921 \\
\hline 16 & 0.002 & 0.000 & 0.194 & 85.680 & 85.857 & 1.115 \\
\hline 17 & 0.010 & 0.000 & 6.948 & 85.690 & 85.857 & 8.063 \\
\hline 18 & 0.284 & 0.005 & 77.174 & 85.974 & 85.862 & 85237 \\
\hline 19 & 3.711 & 2.898 & 2.609 & 89.685 & 88.760 & 87846 \\
\hline 20 & 3.448 & 4.470 & 0.816 & 93.133 & 93.230 & 88662 \\
\hline
\end{tabular}


WHC-SD-WM-DA-163

Vol. I. Rev. 0

TABLE (2-3) - Summary of Computed Stress Ratios

\begin{tabular}{|c|c|c|c|c|}
\hline \multirow{2}{*}{ PART } & \multirow[b]{3}{*}{ DESCAIPTION } & \multirow{3}{*}{$\begin{array}{l}\text { MAX } \\
\text { STAESS } \\
\text { KSII }\end{array}$} & \multicolumn{2}{|c|}{ ALLOWABLE } \\
\hline & & & STRESS & STAESS \\
\hline & & & KSII & AATIC \\
\hline \multicolumn{5}{|c|}{ 42.: UPDER MAST ASSEMBLY } \\
\hline \multirow[t]{2}{*}{42.2} & SHIELDING PLATE & 0.0549 & 20.16 & 00027 \\
\hline & BOLT w/Manifdd Tube & 0.922 & 33.8 & 0.0274 \\
\hline $42-3$ & COVEA PLATE & & & \\
\hline $42-4$ & MANIFOLD ASSEMBLY & & & \\
\hline \multirow[t]{2}{*}{$42-5$} & MANIFOLD TUEE & 0.80 & 14 & 0.0036 \\
\hline & BOLT w/ Shielding Plate & 0.974 & 10.5 & 0.0928 \\
\hline 126 & DIVIDING PLATE & & & \\
\hline \multirow[t]{3}{*}{42.7} & flange plate & 2.24 & 14 & 0.2100 \\
\hline & BOLT w/ $20^{\circ}$ Piser & $.851 k$ & $30.4 k$ & 0.0280 \\
\hline & WELD w/ SS Pipe & 0.758 & 2.625 & 0.2888 \\
\hline $42-6$ & $11 / 4$ IPS PIPE & & & 0.2200 \\
\hline 42.9 & PIPE WELDING DETAIL & & & \\
\hline $42-10$ & CROSS BRACE DETAIL & & & \\
\hline $42-11$ & fLANGE PLT GASKet & & & \\
\hline 42.12 & MATING PLT GASKET & & & \\
\hline \multirow[t]{2}{*}{$40-3$} & mating plate & 2.0 & 14 & 0.1857 \\
\hline & WELD w/ SS Pipes & 0.312 & 2.625 & 0.1180 \\
\hline \multirow[t]{2}{*}{$40-5$} & CAOSS BRACE & & & 0.3560 \\
\hline & WELD w/ SS Pipe & 1968 & 5.25 & 0.3749 \\
\hline \multicolumn{5}{|c|}{ LO-1 LOWEA MAST ASSEMELY } \\
\hline \multirow[t]{2}{*}{$42-2$} & BOTTOM PLATE & 0.0157 & 14 & 0.0011 \\
\hline & WELO w/ SS PIPE & 0.541 & 2.625 & 0.2061 \\
\hline $40-3$ & MATING PLATE & \multicolumn{2}{|c|}{ (SEE ABOVE) } & \\
\hline \multirow[t]{2}{*}{$40-4$} & LUGT SUPPOAT & & & 0.1670 \\
\hline & WEL W/ SS PIpe & 0.75 & 3.5 & 0.2143 \\
\hline $40-5$ & CROSS BRACE & \multicolumn{2}{|c|}{ (SEE ABOVE) } & \\
\hline $40-8$ & GAS TUBE DETAIL & & & \\
\hline $40-7$ & UGHT FITTING DETAIL & & & \\
\hline 400 & CAOSS BRACE DETAIL & & & \\
\hline 400 & PIPE WELDING DETAIL. & & & \\
\hline $40-10$ & 1/2NPT NIPPLE MOOS & & & \\
\hline $40-11$ & $11 / 4$ IPS PIPE & & & 0.1450 \\
\hline 40.12 & 3/8 IPS GAS PIPE & & & 0.0870 \\
\hline & WELD w/ SS Pipes & 0.140 & 3.5 & 0.0 .426 \\
\hline $40-13$ & FITTING & & . & \\
\hline $40-14$ & FITTNG GASKET & & & \\
\hline
\end{tabular}


WHC-SD-WM-DA-163

Vol. I. Rev. 0

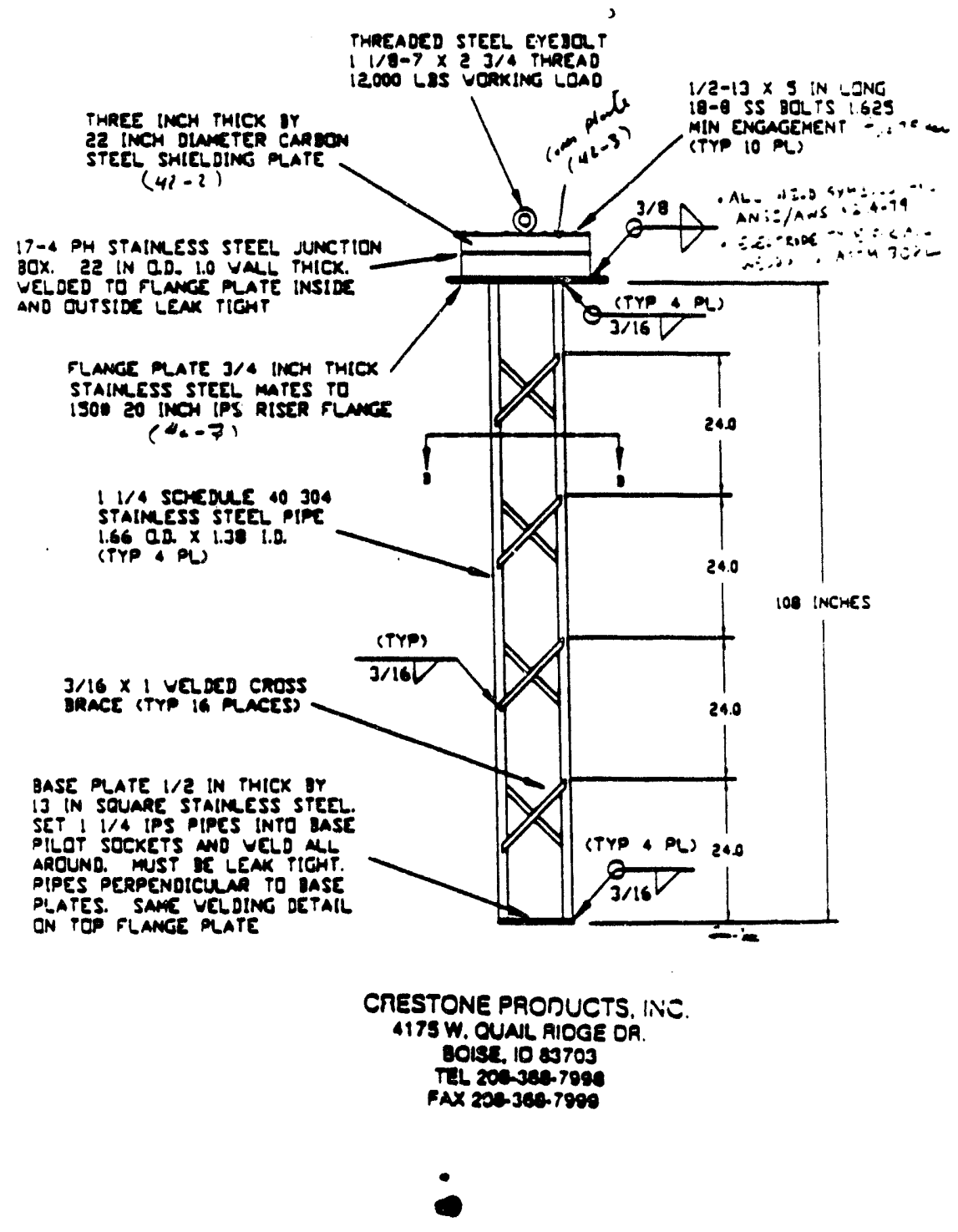

Figure (1-1) - Structure of Upper Mast Assembly 
WHC-SD-WM-DA-163

Vol. I. Rev. 0

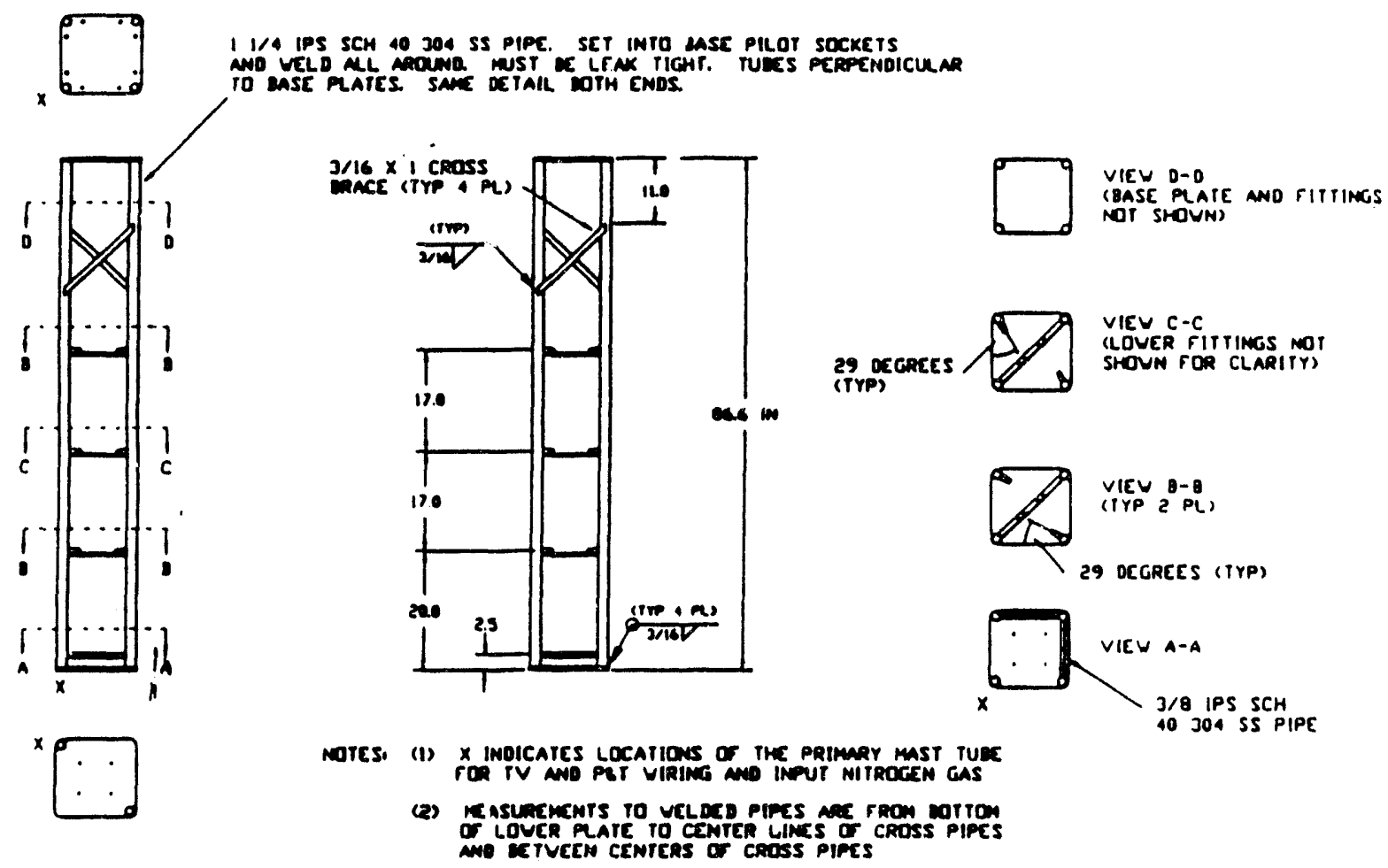

Figure (1-2) - Structure of Lower Mast Assembly 
WHC-SD-WM-DA-163

Vol. I, Rev. 0.

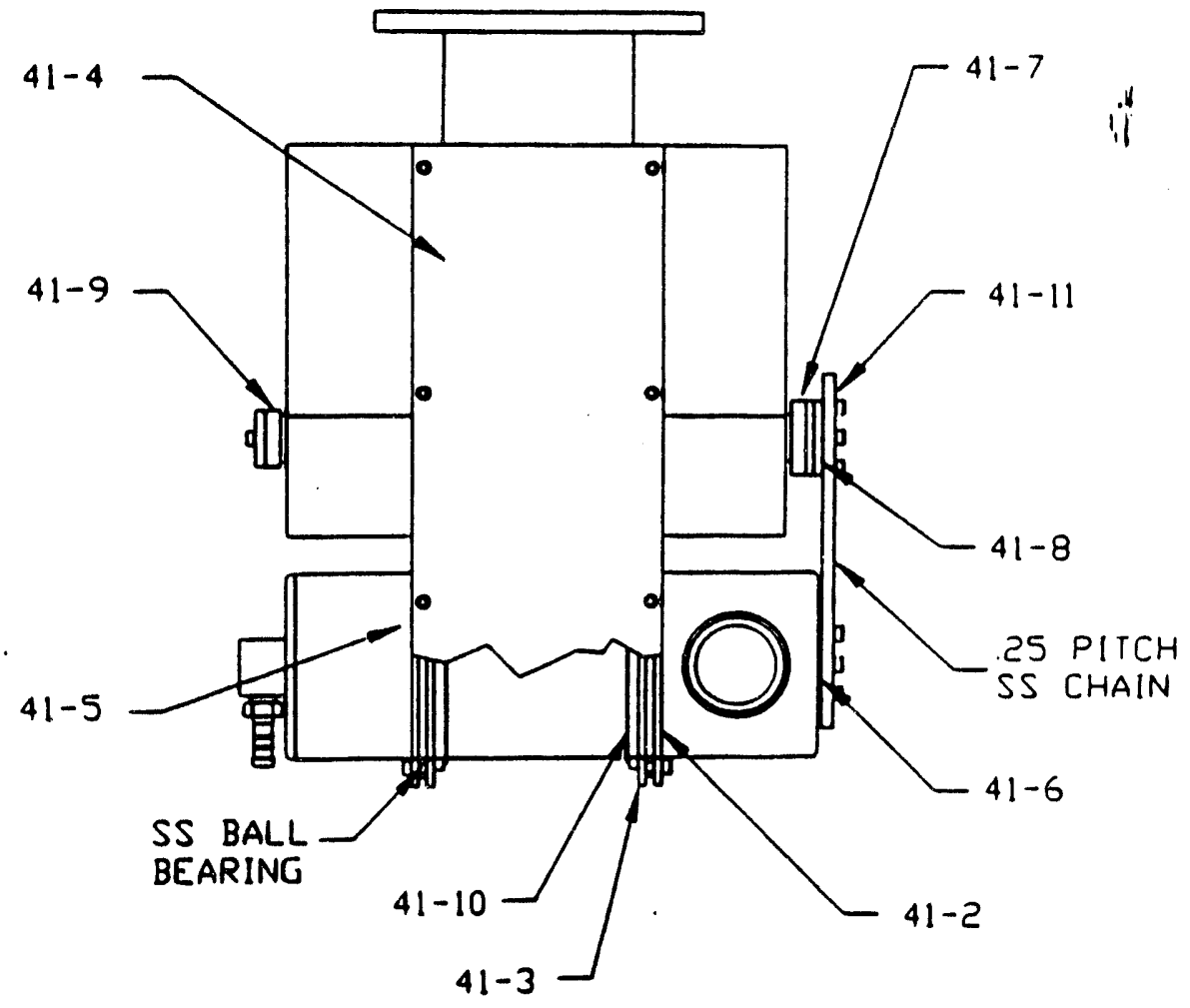

Figure (1-3) - Camera Roll Assembly 
WHC-SD-WM-DA-163

Vol. I, Rev. 0
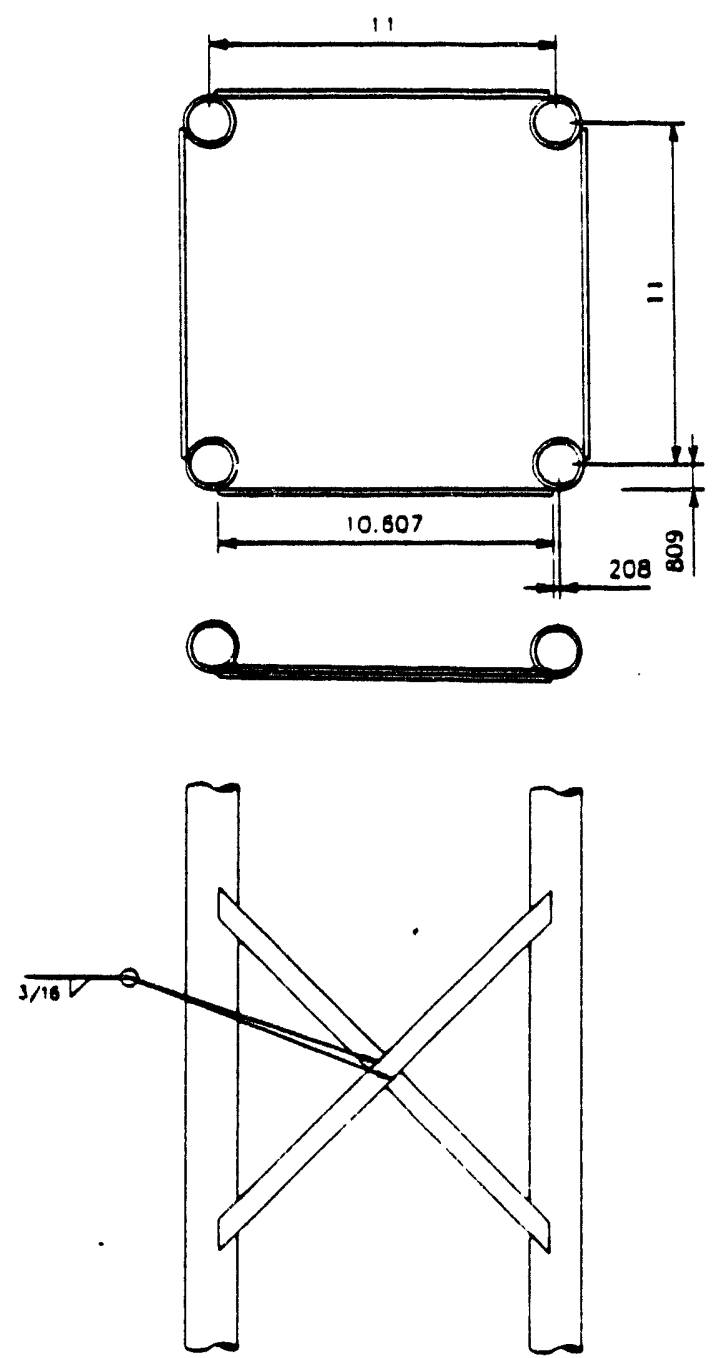

Figure (1-4) - Modification to Lacing of the Camera Mast 
WHC-SD-WM-DA-163

Vol. I, Rev. 0

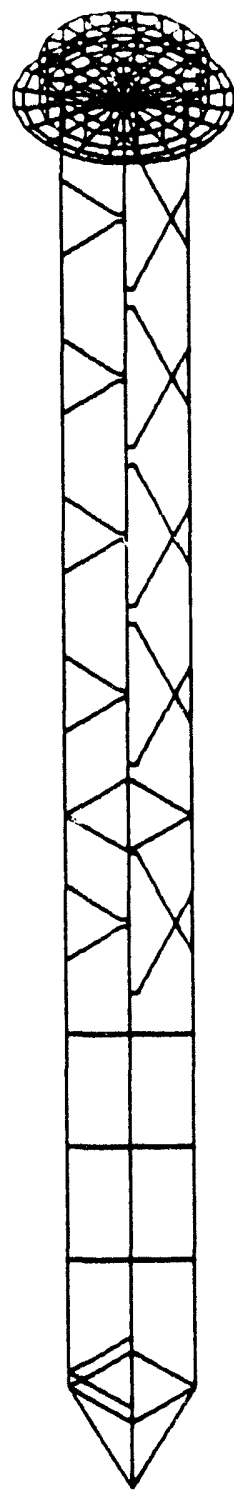

Figure (2-1) - Finite Element Model of Camera Mast Structure 
WHC-SD-WM-DA. 163

Voi. I, Rev. 0

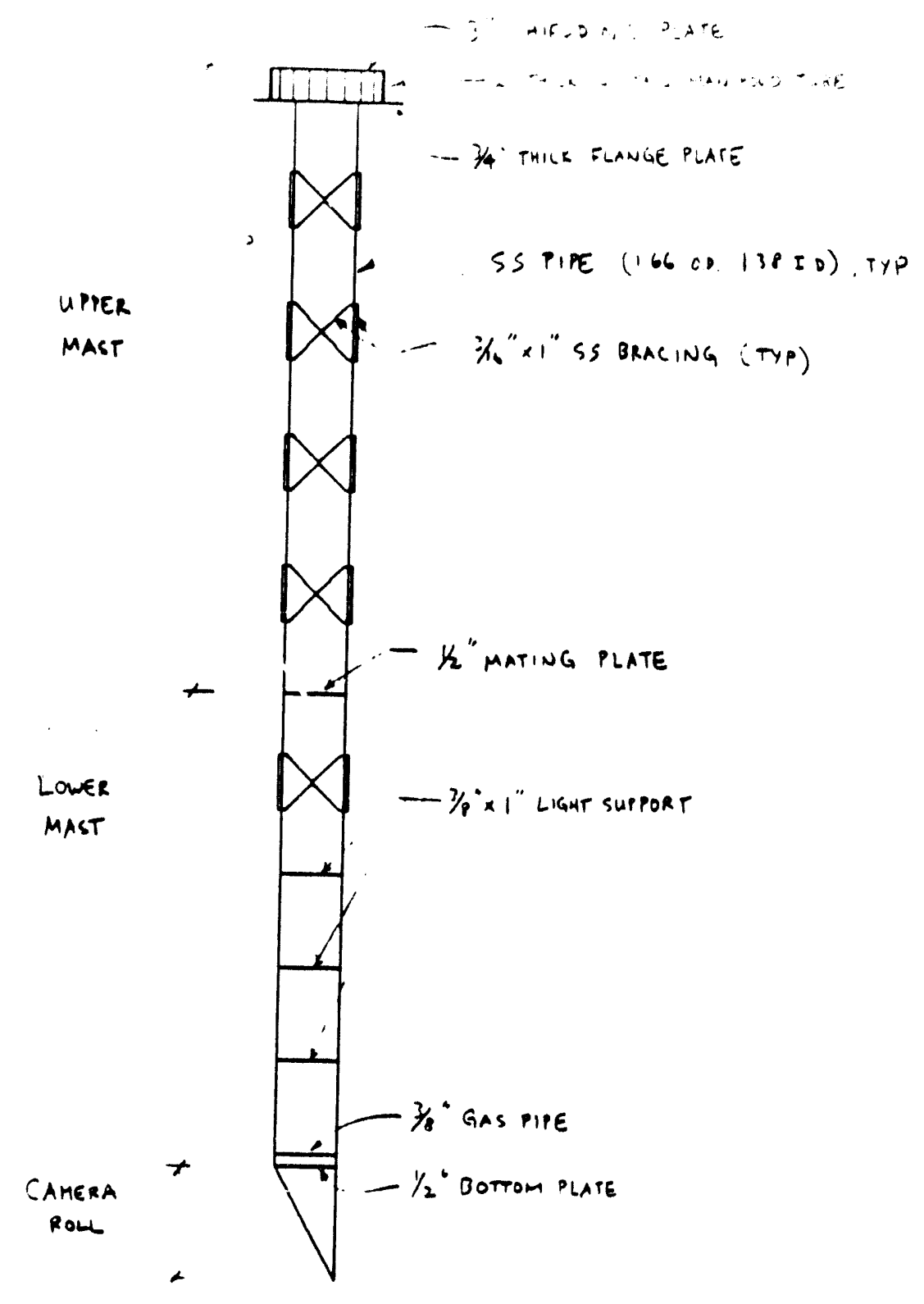

Figure (2-2) - Components of the Finite Elemengt Model of Camera 


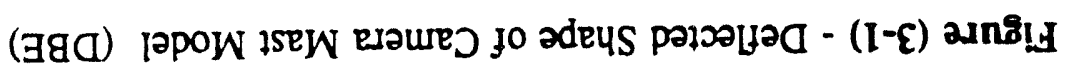

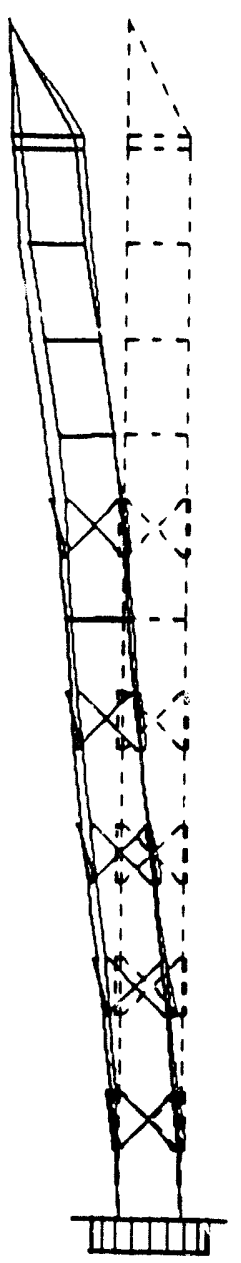


WHC-SD-WM-DA-163

Vol. I, Rev. 0

ATTACHMENT 1

STRUCTURAL DESIGN SPECIFICATICN

FOR

VIDEO CAMERAS IN TANKS

241AW101, 241SY101, AND 241AN107

I Att $1-i$ 
WHC-SD-WM-DA-163

Vol. I, Rev. 0

. STRUCTURAL DESIGN SPECIFICATION

FOR

VIDEO CAMERAS IN TANKS

241AW101, 241SY101, AND 241AN107

July, 1994

Prepared by J.P. Strehlow

I Att $1-i j$ 
WHC-SD-WM-DA-163

Vol. I, Rev. 0

CONTENTS

1.0 INTRODUCTION . . . . . . . . . . . . . . . . . . . . . . . .

1.1 Scope

1.2 Jurisdictional Boundary

1.3 Definitions

2.0 GENERAL REQUIREMENTS ...................... 3

2.1 Analysis Requirements . . . . . . . . . . . . . . . 3

2.2 Structural Report .................. . . . 4

2.3 Conflicting Requirements . . . . . . . . . . . . . . . 5

3.0 DESIGN-BASIS LOADS . . . . . . . . . . . . . . . . . . . 6

3.1 Dead Load $(D)$. . . . . . . . . . . . . . . . . . . . . . . . 6

3.2 Design-Basis Earthquake Load . . . . . . . . . . . . . . . . . 6

3.3 Design-Basis Wind Load $(W)$. . . . . . . . . . . . . . . . . 6

3.4 Design-Basis Ashfall Load (A) . . . . . . . . . . . . . . . 6

3.5 Design-Basis Snow Load $(S N)$. . . . . . . . . . . . . . . . 6

4.0 BEYOND-DESIGN-BASIS LOADS . • . . . .

4.1 Hydrogen Burn Load (H2) (Tank 241SY101 only) . . . . . . . . . 7

5.0 TRANSPORTATION AND NORMAL INSTALLATION LOADS . . . . . . . . . . 8

6.0 LOAD COMBINATIONS. . . . . . . . . . . . . . . . . . . . . . . 9

6.1 Design-Basis Load Combination for the Video Camera Assembly . 9

6.2 Modal and Directional Combination . . . . . . . . . . . . . . 9

6.3 Beyond-Design-Basis Load Combination . . . . . . . . . . . . . 9

6.4 Transportation Load Combination . . . . . . . . . . . . . . . 9 9

6.5 Normal Installation Load Combination . . . . . . . . . . . . . 9

7.0 EVALUATION AND ACCEPTANCE CRITERIA . . . . . . . . . . . . . 10

7.1 Desian-Basis Acceptance Criteria . . . . . . . . . . . . . . . 10

7.2 Beyond-Design-Basis Acceptance Criteria . . . . . . . . . 10

8.0 REFERENCES ............................ 11 
WHC-SD-WM-DA-163

Vol. I, Rev. 0

\section{STRUCTURAL DESIGN SPECIFICATION \\ FOR \\ VIDEO CAMERAS IN TANKS \\ 241AW101, 241SY101, AND 241AN107}

\subsection{INTRODUCTION}

This document establishes the requirements and guidelines for performing the structural evaluation of the video cameras that will be installed on tanks 241AW101, 241SY101, and 241AN1J7. The video camera on tank 241AW101 will be installed in a multiport flange (MPF) assembly (H-2818144) while the video camera on tank 241 SY101 will be installed in the multi-port riser (MPR) assembly (H-2-821101). The video camera on tank 241AN107 will be installed directly on the existing 12-in. riser 7A. This document addresses scope of the analysis and the loading and the load cases that are to be analyzed and establishes the acceptance criteria for evaluating the results of the structural analysis.

\subsection{Scope}

The scope of this ciocument includes the following:

- Identification of the design requirements applicable to the video camera design from the Hanford Plant Standard, SDC-4.1 (Reference 8.2), U.S. Department of Energy Order No. 6430.1A (Reference 8.3) and UCRL-15910 (Reference 8.4), documents

- Definition of the various design-basis, beyond-design-basis, transportation, and normal installation loads to be considered in the structural evaluation of the video cameras and the loads that the video cameras will have on their supporting structure

- Definition of the loads and load combinations

- Provision of acceptance criteria to serve as the basis for qualification of the video cameras under design-basis and beyonddesign-basis loads. 
WHC-SD-WM-DA-163

Vol. I, Rev. 0

\subsection{Jurisdictional Boundary}

This structural design specification covers the entire video camera assembly, including the bolts that connect it to either the MPF. MPR, or existing tank riser. Evaluation of the effect that the reaction forcus, and moments from the AW 101 and SY101 video camera assemblies will have on their supporting structures, the MPF or the MPR, are not within the scope of the camera structural analysis. However, these loads shall be reported so that an evaluation of their effect can be performed and incorporated in the structural analys is for these support structures. The effect that the AN107 camera wi11 have on it's support structure, the existing tank riser $7 \mathrm{~A}$, is within the scope of the camera structural analysis.

\subsection{Definitions}

Shall Consider: Requires a referable assessment with discussion of

Shall:

Must: rationale when an alternate method is selected.

Should: Denotes a requirement. Denotes a requirement. Denotes a recommendation. 
WHC-SD-WM-DA-163

Vol. I, Rev. 0

\subsection{GENERAL REQUIREMENTS}

\subsection{Analys is Requirements}

SDC-4.1 (Reference 8.2) provides the criteria for establishing the design loads for facilities. These criteria are based on four safety classifications (Safety Class 1 through 4 ) for the structure, system and component. The safety classes can be correlated respectively to the High-Hazard, Moderate-Hazard, Low-Hazard/Important and General-Use usage categories defined in the 1990 version of UCRL-15910 (Reference 8.4) for non-reactor facilities. In the $1992 \mathrm{draft}$ version of UCRL-15910, these descriptive classifications have been modified, in reverse order, to Performance Categories 1 through 4 . Safety Class 1 structures, systems, and components are those that perform a function required for nuclear criticality safety or whose failure might result in a significant release of radioactive, hazardous, or toxic materials as defined by DOE Order $6430.1 \mathrm{~A}$, Division 13 , as it relates to non-reactor nuclear facilities.

In addition to normal loads, non-reactor Safety Class 1 structures, systems and components are to withstand the effects of extreme environmental loads including design-basis earthquake (DBE), designbasis wind (DBW), design-basis flood, volcanic eruptions and other abnormal loads considered on a case by case basis. Non-reactor Safety Class 2,3 and 4 structures, systems, and components are those that are not Safety Class 1; these are specified respectively as onsite safety-related, occupational safety-related and non-safety related items.

Tanks 241AW101, 241SY101, and 241AN107 are non-reactor Safety Class 1 structures. The risers and the supporting structures for the video cameras, the MPF, and MPR, are non-reactor Safety Class 2 structures. The video cameras that will be installed in these tanks will also be considered non-reactor Safety Class 2 components for the purpose of these structural analyses because they transfer reaction loads to Safety Class 2 structures and because their connection flange will provide containment of radioactive and toxic materials under normal operating conditions.

Section 0111-99.0.4 of Reference 8.3 states that "the adequacy of systems, components, and structures to withstand a seismic event shall be verified by a dynamic analysis, except where it can be demonstrated that the use of a simplified approach, such as a static load method, component testing, or a combination of testing and analysis provides assurance of adequate seismic design. The ratio of vertical-tohorizontal atceleration shall be two-thirds, unless site-specific data justify the use of a different ratio." Therefore, the MPF together with the tank and the other supporting structures shall be evaluated by dynamic analysis for DBE loads. A response spectrum method of structural analysis is an acceptable method of analysis for this purpose. Section 0111-99.0.8 of Reference 8.3 states that "Safety class structures and structural members shall be designed to resist the appropriate load combinations provided in UCRL-15910." Therefore, the 
WHC-SD-WM-DA-163

Vol. I, Rev. 0

load combinations provided in Section 6.0 of this document shall be based on UCRL-15910.

Section 0111-99.0.8 of Reference 8.3 al so states that "Safety class steel structures shall meet the design, fabrication. and erection requirements of ANSI/AISC $N 590$ for new construction or original cor equivalent) codes for existing construction providing the margin of safety of the overall facility is maintained." For the video camera assemblies, the structural evaluation shall be based on ANSI/AISC N69084 (Reference 8.6 ) requirements.

Section $0111-2.4 .1$ of Reference 8.3 states that "The structural frame and exterior components of all buildings, signs, tanks, towers, and other exposed structures shall be designed to resist pressures due to wind assumed to act from any direction. Partial wind loading shall be considered if it produces a more severe effect." Therefore, wind load shall be included as one of the design-basis loads in the design and analysis of the video camera assembly. The wind load calculations shall be in accordance with procedures in ASCE 7-88 (Reference 8.7) with the basic wind speed being obtained from SDC-4.1. In addition to the DBW load, the design-basis ashfall and snow loads shall be included in the design and analysis of the MPF assembly.

Additional requirements that shall be factored into the structural evaluation of the video camera assembly.

- The video camera assembly installed in tank $2415 Y 101$ shall not become a missile if there is a hydrogen burn inside the tank (tank 241SY101 only).

- All structural members designed to the requirements of this Structural Design Specification must be fabricated from a material that can be characterized in terms of the material designations found in the ASME Boiler and Pressure Vessel Code, Section VIII (Reference 8.8), ANSI/AISC $N 690$ or a similar nationally recognized, widely accepted industry design standard. Where possible lightweight, high-strength material shall be selected to minimize loads applied to the tank dome.

The transnational and rotational stiffness in the orthognnal directions at support point for the video camera assembly shall be considered in the structural analyses of the video camera assemblies. For the AW101 and SY101 cameras these stiffnesses can be obtained from the MPF or MPR structural analysis report. These stiffnesses shall be used in the camera analysis if they cause an increase in either the camera stresses or the reaction forces and moments transmitted to the support structure.

\subsection{Structural Report}

A11 engineering reports documenting the satisfaction of this specification and the acceptance criteria shall be prepared in accordance with the requirements of WHC Standard Engineering Practices, EP-1.12 (Reference 8.9). All calculations supporting the engineering 
WHC-SD-WM-DA-163

Vol. I, Rev. 0

report shall be prepared in accordance with the requirements of WHC Standard Engineering Practices, EP-1.11 (Reference 8.10). Changes to engineering reports shall be performed in accordance with the requirements of WHC Standard Engineering Practices, EP-2.2

(Reference 8.11 ). The design verification of engineering reports and the supporting document; shall be performed in accordance $31^{\text {th }}$ the requirements of WHC Standard Engineering Practices, EP-4.1

(Reference 8.12). All engineering reports submitted to WHC Engineering shall be subjected to independent review. Submittal to WHC shall include, in hard-copy form, all engineering reports, supporting documents, and computer output associated with the supporting documents. The submittal shall also include input run-streams of all computer models either on a 5.25- or a 3.5-in. floppy diskette.

The reactions (forces and moments) that the video camera assembly imposes on its support structure, either the MPF or the MPR, shall be reported for both the normal and the extreme load combinations.

The maximum lateral deflection of the camera for either of the load combinations shall be reported. This lateral deflection shall be determined at an elevation corresponding to the bottom of the $42-i n$. riser. This information will be used to ensure that sufficient clearance is maintained between the camera and the riser.

\subsection{Conflicting Requirements}

If requirements imposed by this Structural Design Specification conflict with requirements listed in another specification applicable to the MPF, The conflict shall be brought to the attention of the WHC Engineering Department for resolution. 
WHC-SD-WM-DA-163

Vol. $\cdot$ I, ReV. 0

\subsection{DESIGN-BASIS LOADS}

3.1 Dead Load (D)

Dead load for the video camera assembly shall include the total wion of all individual components that form the MPF assembly.

\subsection{Design-Basis Earthquake Load}

The DBE load shall be included as a design-basis extreme load. The response spectra curves provided in SDC 4.1 (Reference 8.2 ) shall be used to determine the DBE loads. The DBE response spectra curves for a $0.12 \mathrm{~g}$ zero-period acceleration shall be used to determine seismic loads on the video camera assembly. The required damping for the video camera assembly shall be $5 \%$, as specified in SDC 4.1 .

To account for possible amplification of the tank dome in the vertical direction, the full horizontal response spectra shall be applied as the vertical response spectra for components attached to the tank dome.

\subsection{Design-Basis Wind Load (W)}

The video cameras installed over the 42-in. risers on tanks 241 AW101 and 241SY101 and on the existing 12-in. riser on tank 241AN107 are exposed to environmental loads such as the DBW. However, in each case the video camera assembly does not extend above its support structure far enough to be exposed to significant wind loads. Therefore, the wind load on the projected surface of the video camera assembly can be neglected.

\subsection{Design-Basis Ashfall Load (A)}

The design-basis ashfall load defined in SDC 4.1 will have a negligible effect on the video camera assembly. Therefore, this load can be neglected.

\subsection{Design-Basis Snow Load (SN)}

The design-basis snow load defined in SDC 4.1 will have a negligible effect on the video camera assembly. Therefore, it can be neglected. 
WHC-SD-WM-DA-163

Vol. I, Rev. 0

\subsection{BEYOND-DESIGN-BASIS LOADS}

\subsection{Hydrogen Burn Load (H2) (Tank 241SY101 only)}

The waste within tank $2415 Y 101$ generates hydrogen and nitrous oxide gases. The concentration of these gases inside the tank can rain flammability levels during episodic releases of these gases from the waste during a waste rollover. This situation creates the possibility of a hydrogen burn. This event is referred to as the maximum expected burp (MEB) burn. Several burn events have been evaluated including a 1.25 MEB and a best-estimate MEB. For this analysis the transient pressure loadings and the tank dome displacement/acceleration time histories that correspond to the 1.25 MEB (Reference 8.15 ) shall be applied. 
WHC-SD-WM-DA-163

Vol. I, Rev. 0

\subsection{TRANSPORTATION AND NORMAL INSTALLATION LOADS}

The party who installs the video camera assembiy shall evaluate it for various transportation loads. The installer shall be responsible for providing adequate support to the assembly during transportation t? prevent physical damage or operational impairment. Road shipment. shock and vibration forces in accordance with Federal Highway Administration. DOT 49 Code of Federal Regulations (CFR) 393.100, are to be assumed during transportation (Reference 8.16).

The party who installs the video camera assembly shall be responsible for providing adequate rigging and support for the assembly, to assure that it is not damaged during normal installation. Installation procedures shall be provided to WHC cognizant engineer for review and comment in accordance with the schedule established by Project

Management. The installation procedure shall contain a listing of all equipment to be used. The estimated total weight of all equipment and personnel to be located above the tank at any one time during the installation shall be included in the procedure. The estimated total weight shall be reported to Project Management.

Lifting lugs or components on the video camera assembly shati be evaluated for a deadweight load with a dynamic amplification load factor of 2.0 applied to the load. Stresses within the lifting lugs and their attachment welds shall satisfy the requirements of ANSI N690-1984

(Reference 8.6) for a normal loads. If commercial components are used, the total lifting load shall be less that the maximum allowable working load published by the manufacture. 
WHC-SD-WM-DA-163

Vol. I, Rev. 0

\subsection{LOAD COMBINATIONS.}

\subsection{Design-Basis Load Combination for the Video Camera Assembly}

The following load combinations shall be used in evaluating the vides camera assembly.

\begin{tabular}{|c|c|c|}
\hline Category & Load Combination & $\begin{array}{c}\text { Stress } \\
\text { Limit* } \\
\text { Coefficient }\end{array}$ \\
\hline Normal & $n$ & 1.0 \\
\hline Extreme & $D+[$ DBE] & 1.6 \\
\hline
\end{tabular}

Where $D=$ dead load

$D B E=$ design-basis earthquake load

* For items subject to the requirements of ANSI N690-1984.

\subsection{Modal and Directional Combination}

A three-directional response spectrum analysis with DBE loading shall be performed to obtain the structural response of the video camera assembly to the earthquake loading. The Grouping Method as stated in the U.S. Nuclear Regulatory Commission's (NRC) Guide 1.92 (Reference 8.17) or the complete quadratic combination (CQC) method shall be used to perform the modal combination. The responses for each of the three directions of seismic data shall be combined by the SRSS method. The missing mass effect shall be accounted for in the total response of the video camera assembly if the number of modes included in the analys is are not sufficient to insure that $90 \%$ or more of the total system response has been achieved:

\subsection{Beyond-Desian-Basis Load Combination}

The stresses in the video camera assembly and its connection bolts that are caused by the beyond-design-basis load need not be combined with those stresses in the video camera assembly resulting from design-basis loads.

\subsection{Transportation Load Combination}

The stresses in the video camera assembly resulting from transportation loads need not be combined with any other design-basis normal loads.

\subsection{Normal Installation Load Combination}

The stresses in the video camera assembly during normal installation need not be combined with any other design-basis normal loads 
WHC-SD-WM-DA-163

Vol. I, Rev. 0

\subsection{EVALUATION AND ACCEPTANCE CRITERIA}

\subsection{Desigri-Basis Acceptance Criteria}

The stresses in the video camera assembly and its connection bot a developed by the external loadings imposed on the assembly. Therofore. these stresses are of a primary nature and they are not self-limiting. The primary stresses and shall satisfy the requirements of ANSI N690-1984 (Reference 8.6).

Stress-limit coefficients shall be applied to the stress allowable as shown in References 8.6 and 8.19. In accordance with Reference 8.6, the stress allowable after applying the stress 1 imit coefficient shall not exceed 0.7 times $F_{u}$ in axial tension nor $0.7 F_{y}$ times the ratio $Z / S$ for tension plus bending. Here, $F_{u}$ is the ultimate stress of the material, $Z$ is the plastic section modulus of the member, and $S$ is the elastic section modulus of the member. Alternatively, for the extreme load case, the computed stress can reach the member plastic capacity of the member, which is computed as the shape factor times the material yield stress of the member.

Stress limit coefficients in shear for extreme and abnormal load conditions shall be limited to 1.4 .

\subsection{Beyond-Desion-Basis Acceptance Criteria}

The acceptance criterion applicable to the hydrogen burn is that the video camera assembly shall not become a missile. 
WHC-SD-WM-DA-163

Vol. I, Rev. 0

\subsection{REFERENCES}

8.1 (Not used)

8.2 Standard Architectural-Civil Design Criteria. SOC-4.1. Rey. !. U.S. Department of Energy. Richland Jperation. iff:ca. $\because:$ : : Washington.

8.3 General Design Criteria, DOE Order 6430.1A, U.S. Department of Energy, Washington, D.C.

8.4 Robert P. Kennedy, et al., Design and Evaluation Guidelines for Department of Energy Facilities Subjected to Natural Phenomena Hazards, UCRL-15910, June 1990.

8.5 "Code Requirements for Nuclear Safety Related Concrete Structures (ACI 349-90)," American Concrete Institute, Detroit, Michigan.

8.6 "Nuclear Facilities - Steel Safety Related Structures for Design Fabrication and Erection," ANSI/AISC N690-1984, CITY, STATE, 1984.

8.7 "Minimum Design Loads for Buildings and Other Structures," ASCE Standard, ASCE 7-88 (Formerly ANSI A58.1), American Society of Civil Engineers, CITY, STATE, 1990.

8.8 "Boiler and Pressure Vessel Code," Section VIII, Division 2, Appendix 5, American Society of Mechanical Engineers, New York, New York, 1992.

8.9 "Preparation of Design Analysis Reports, ASME Design Reports, and ASME Load Capacity Data Sheets, "EP-1.11, Rev. 3, Standard Engineering Practices, WHC-CM-6-1, Westinghouse Hanford Company, Richl and, Washington.

8.10 "Supporting Documents," Standard Engineering Practices, EP-1.12, Rev. 7, WHC-CM-6-1, EP-1.12, Westinghouse Hanford Company, Richl and, Washington.

8.11 "Engineering Document Change Control," EP-2.2, Rev. 6, Standard Engineering Practices, WHC-CM-6-1, Westinghouse Hanford Company, Richland, Washington.

8.12 "Design Verification Requirements," EP-4.1, Rev. 4, Standard Engineering Practices, WHC-CM-6-1, Westinghouse Hanford Company, Richland, Washington.

8.13 N. M. Newmark, and W. J. Hall, "Development of Criteria for Seismic Review of Selected Nuclear Power Plants," U.S. Nuclear Regulatory Commission Regulatory Report, NUREG/CR-0098, Washington, D.C., 1978. 
WHC-SD-WM-DA-163

Vol. I, Rev. 0

8.14 J. N. Edwards, "A Safety Assessment for Proposed Pump Mixing Operations to Mitigate Episodic Gas Releases in Tank SY-101," LA-UR-92-3196, Rev. 8, 1994, Los Alamos National Laboratory, Los Alamos. New Mexico.

3.15 E. A. Rodriguez, "Structural Analy"is of HLid Tank Si.. in Subjected to Burn Pressure Transient with Spark Ignition at Dome Apex," Calc. Note PSA-CN-WT-SA-ST-062, Rev. 0, 1994, LoS Alamos National Laboratory, Los Alamos, New Mexico.

8.16 Federal Highway Administration, DOT 49, Code of Federal Regulations 393.100 , as amended.

8.17 "Combining Modal Responses and Spatial Components in Seismic Response Analysis," U.S. Nuclear Regulatory Commission Regulatory Guide 1.92, Washington, D.C., February 1976.

8.18 "Standard Architectural-Civil Design Criteria - Design and Installation of Expansion Anchors," Hanford Plant Standard SDC-4.2, Rev. 0, U.S. Department of Energy, Richland Operations Office, Richl and, Washington.

8.19 Manual of Steel Construction, American Institute of Steel Construction, 9th Edition, CITY, STATE. 
WHC-SD-WM-DA-163

Vol. II, Rev. 0

STRUCTURAL ANALYSIS OF COLOR VIDEO CAMERA INSTALLATION ON TANK 241AW101

Volume II of II

Volume I - Report

Volume II - Calculations 
WHC-SD-WM-DA-163

Vol. II, Rev. 0

\section{INDEPENDENT REVIEW}

Document Reviewed Structural Analysis of color Video Camera Installition Tank 241AW101

Author URS/John A. Blume Report No. WHC-SD-WM-DA-163 EDT No. 142746 and Associates, Engineering Rev. 0

The subject document has been reviewed by the undersigned. The reviewer reviewed and verified the following items as applicable [EP.4.1].

- Engineering Specification

- Design Input

- Basic Assumption

- Approach/Design Methodology

- Related Information

- Conclusion/Result Interpretation

ADVENT Engineering Services. Inc. 2. BALICA

Reviewer

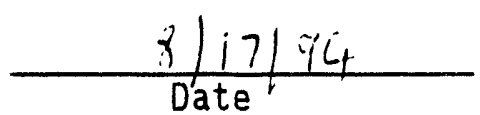

See Attachment 1 for review comment resolutions. 


\title{
STRUCTURAL ANALYSIS OF CRESTON VIDEO CAMERA INSTALLATION ON TANK 241AW101
}

\section{VOLUME II - CALCULATIONS}

\author{
prepared for \\ Westinghouse Hanford Company \\ Richland, Washington
}

prepared by

URS/John A. Blume and Associates, Engineers

San Francisco, California

August 1994

\section{URS}


WHC-SD-WM-DA-163

Vol. II, Rev. 0

URS Consultants, Inc. CALCULATION COVER SHEET

Cient: Westinghuuse Hanford Cumpany Projec Viame: WHC MPF E: illiadun

Project/Calculation Number: 43-66481-01-CA-3

Title: "Seismic Analysis of Creston Video Camera Installation on Tank241AW 101"

Total number of pages (including cover sheet):

Total number of computer runs:

Prepared by: Reter Lum Date: August 17, 1994_

Checked by: Kery MaCoy, Rabieh Khoury Date:_August 17, 1994

Description and Purpose:

Stated Within

Design bases/references/assumptions:

Stated Within

Remarks/conclusions:

Stated Within

Calculation Approved by:

Bahram Mosaddad Auggust 17, 1994 Project Manager/Date

Revision No.: Description of Revision:

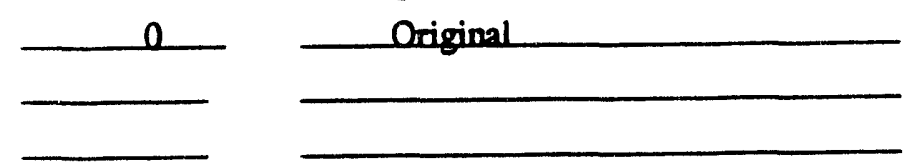

Approved by:

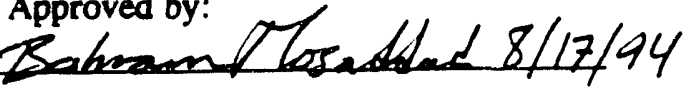

Project Manager/Date

QAM $5.608 / 15 / 93$ 
URS CONSULTANTS WHC-SD-WM-DA-163

Vol. II, Rev. 0

Job \#:66481-01 Job: WHC/MPF CAMERA ASSEMBLY ANALYSIS

Sheet No.

By Pue Date $6 / 8: 94$

Client: WHC Subject: SAP9O ANALYSIS DOCUMENTATION

Chk'd Y/m Date F/1:2!',

OUTLINE OF DOCUMENTATION SAP9O/SAPSTL ANALISIS PACKAGE

1. Criteria

1.1 Project Description

1.2 Analysis Criteria

1.3 Analysis Approach

1.4 Quality Objectives

2. Computer Model

2.1 General Discussion

2.2 SAP9O input parameters

2.3 SAP90 Model sketches

2.4 SAPSTI input parameters

3. Loads

3.1 General Discussion

3.2 Camera Assembly Dead Load

3.3 Response Spectra

4. SAP90/SAPSTL Input Data

4.1 General Discussion

4.2 Input data files for SAP9O

4.3 Input data files for SAPSTL

5. SAP90/SAPSTL Output files

5.1 General Discussion

5.2 Output files for SAP9O

5.3 Output files for SAPSTL

5.4 Selective graphical output

6. Check Element Stresses

6.1 General Discussion

6.2 Check Shell Element Stresses

6.3 Check Beam Element Stresses

7. Check Bolt and Weld Connections

7.1 General Discussion

7.2 Check Bolt Connection

7.3 Check Weld Connection

8. Check Displacements

8.1 General Discussion

8.2 Summary of Displacements

9. Summary

9.1 Summary of Stress Ratios

9.2 Overall Analysis Conservatism 


\section{URS CONSULTANTS}

WHC-SD-WM-DA-163

Vol. II, Rev. 0

Job \#:66481-01 Job: WHC/MPF CAMERA ASSEMBLY ANALYSIS

Sheet No. $\mu$

Client: WHC Subject: SAP9O ANALYSIS DOCUMENTATION

By $P$ - Date $3 / 10,{ }^{\text {ad }}$

Chk'd RIKDate $8 / 16 / 914$

Attachment A Preliminary Camera Assembly Drawings Erom

Crestone Products, Inic.

Attachment B (not used)

Attachment C Allowable Stresses

Attachment D Letter from wHC to URS/Blume dated May 25, 1994 which contains the information for the multiport flange and video camera analysis

Attachment E List of "As-Built" Camera Assembly Drawings from Crestone Products, Inc dated 6/18/94, received by URS/Blume on $8 / 1 / 94$, and evaluation of changes between preliminary and "As-Built" drawings. 
URS CONSULTANTS

Job \#:66481-01 Job: WHC/MPF CAMERA ASSEMBLY ANALYSIS

Client: WHC Subject: SAP90 ANALYSIS DOCUMENTATION
WHC-SD-WM-DA-163

Vol. II, Rev. 0 


\section{URS CONSULTANTS}

WHC-SD-WM-DA-163

Vol. II, Rev. 0

Shert No. 1.2

Job \#:66481-01 Job: WHC/MPF CAMERA ASSEMBLY ANALYSIS

By Ifm Date $9 / 1$ i?

Client: WHC Subject: SAP90 ANALYSIS DOCUMENTATION

Chk'd RIKDate Pjí, 1 -

Load combinations and applicable stress limit coefficients are:

\begin{tabular}{|c|c|c|c|}
\hline \multicolumn{3}{|c|}{$\begin{array}{l}\text { Load } \\
\text { Combination }\end{array}$} & $\begin{array}{l}\text { Stress limit } \\
\text { Coefficients }\end{array}$ \\
\hline Normal & Dead Load (DL) & $D L$ & 1.0 \\
\hline Extreme & $D L+$ Seismic (EQ) & $\begin{array}{l}D L+E Q \\
D L-E Q\end{array}$ & $\begin{array}{l}1.6 \\
1.6\end{array}$ \\
\hline & For shear & $\begin{array}{l}D L+E Q \\
D L-E Q\end{array}$ & $\begin{array}{l}1.4 \\
1.4\end{array}$ \\
\hline
\end{tabular}

The camera assembly is categorized as "Safety class 2 " structure.

1.3 Analysis Approach

The general-purpose elastic structural computer software, SAP90, is used for analysis (refer to SAP90 version 5.4, computer porgram vrification, $7 / 27 / 93$, URS Consultants). The SAPSTL, post-processor of SAP90, is used for checking the stress ratios of beam elements.

The stresses of the steel plates, which were modelled as shell elements (both bending and membrane), are checked against the allowable stresses. All the connecting elements such as bolts and welding are also checked.

The maximum displacements of the camera assembly are checked for the minimum clearance to avoid any interference with other surrounding elements.

\subsection{Quality Objectives}

- Check SAP90/SAPSTL models for assumptions, boundary conditions, loads and other input parameters for accuracy.

- Review of the methodology for checking the analysis against allowable stresses and displacements. 
URS CONSULTANTS $\begin{aligned} & \text { WHC-SD-WM-DA-163 } \\ & \text { VoI.II, Rev. } 0\end{aligned}$

Job \#:66481-01 Job: WHC/MPF CAMERA ASSEMBLY ANALYSIS

Client: WHC Subject: SAP90 ANALYSIS DOCUMENTATION
Sheet No. 20

By Prue Date 6/?:14 Cak'd_s Date $=$,

\section{SECTION 2: COMPUTER MOCEL}

\section{TABLE OF CONTENTS}

SECTION CONTENTS

2.1

2.2

$2 \cdot 3$

2.4
General Discussion

SAP90 input parameters

SAP90 Model sketches

SAPSTL input parameters
PAGE NO,

$211-21+$

22.1

$231-2313$

24.1 


\section{URS CONSULTANTS WHC-SD-WM-DA-163}

Job \#:66481-01 Job: WHC/MPF CAMERA ASSEMBLY ANALYSIS

Client: WHC Subject: SAP90 ANALYSIS DOCUMENTATION
Sheet No. 211

By Pru Date ${ }^{-1}=3 / a+$

Chk'd R if Date $8 / 3 / 94$

2.: General Discussion

Modelling

The computer model is created using the SAP9O computer software which has the basic assumptions of 3-Dimensional elastic analysis.

Two analytical models were created as follows:

Model Description

Model A Camera Assembly unit only

Model B Camera Assembly plus MPF riser stick Model
Purpose

Obtain Maximum Element stresses

Obtain Maximum Displacements of Camera

Model A:

Restraints: The center point of the 20" riser was modelled as fixed in 6 degrees of freedom (DOF), with weightless stiff beams which span to the bolt holes of the flange plate. The flange plate shared the same node numbers as the bolt holes in the model. This approach allows the flange plate to move when it is subjected to loads, while the bolt holes remain stationary.

Elements: The plates were modelled as shell elements (both bending and membrane) with nodes located at the centerline of the plate. Therefore, the "rigid zone" between the thicknesses of the connecting plates are ignored.

The pipes and the braces were modelled as beam elements. The braces were welded on the pipes. Rigid links were used to span from the center of the pipes to the braces. The stiffness properties of the rigid links were obtained by an iterative process such that they were extremely rigid yet avoiding numerical instability in the model. 
URS CONSULTANTS WHC-SD-WM-DA-163

Vol. II, Rev. 0

Job \#:66481-01 Job: WHC/MPF CAMERA ASSEMBLY ANALYSIS

Client: WHC Subject: SAP9O ANALYSIS DOCUMENTATION

Sheet No. 212

By Pre Date $5 / 5 / 97$

Chk'd RikDate $? / 5 / 9+$

Description

of Parts:

Using the drawings and the list of parts $5:=m$

Attachment $A$, the description ot pat:j tid

their connections which include SAP9o node

and element numbers, size, thickness,

material, yield and tensile strengths are

tabulated in Table 2-1.

Model B:

Restraints: Model B was fixed in 6 DOF at the roof of the tank and shares the same node with the 20 " riser.

Elements :

The same elements were used from Model A with additional beam elements from the MPF riser stick model. 
TABLE 2-1: DESCRIPTION OF PARTS

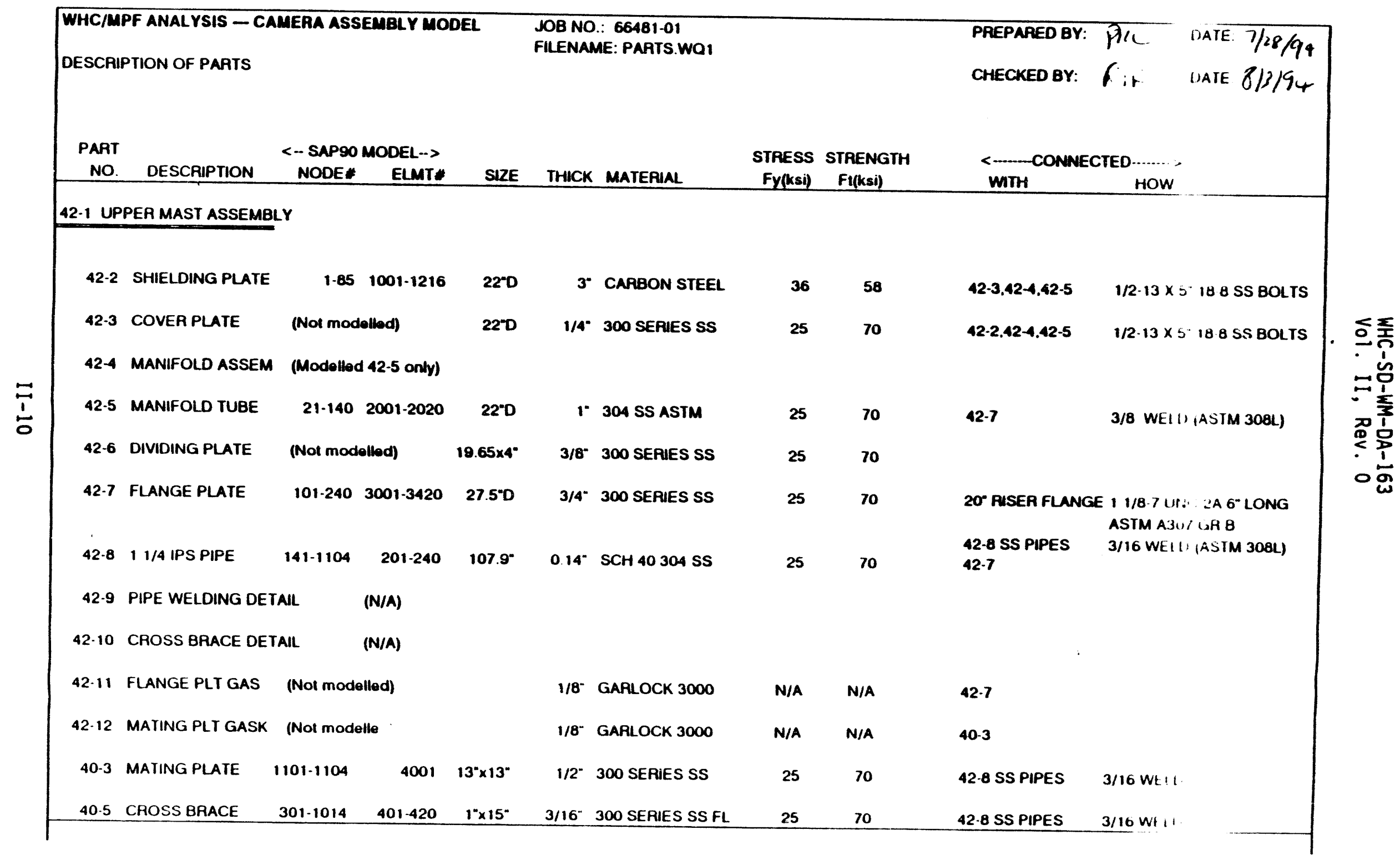


TABLE 2-1: DESCRIPTION OF PARTS (CONTINUED)

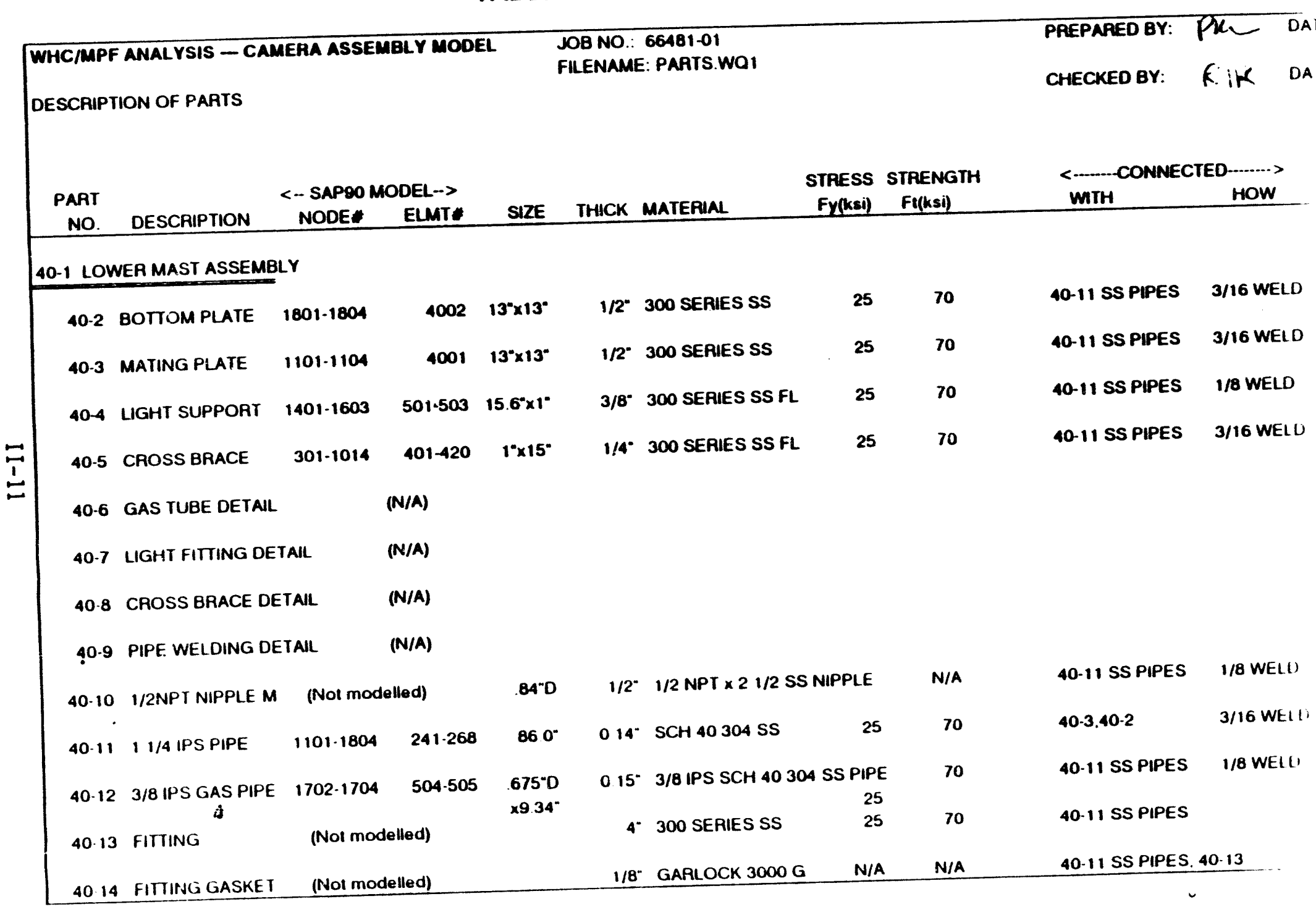


URS CONSULTANTS WHC-SD-WM-DA-163

Vol. II, Rev. 0

Job \#:66481-0ْ Job: WHC/MPF CAMERA ASSEMBLY ANALYSIS

Sheet No. $\geq 21$

Clieat: WHC Subject: SAP9O ANALYSIS DOCUMENTATION

By Due Date $3 / 5 / 94$

Chk d RIK Date

2.2 SAP9o Input Parameters

Model A: Nodes \& Elements

Using the drawings from Attachment A, the SAP9o Model A was created with 271 nodes with the following elements:

Beam Elements:

20 Weightless stiff beams connect from bolts to Master Node

68 Pipes

20 Braces

40 Short stiff beams span from center of pipes to braces

3 Light support beams

2 3/8" Gas pipes

4 Stiff beams connect from bottom plate to camera

157 Total beam elements

Shell Elements:

60 elements for 3 " thick Shielding plate

20 elements for 2" thick, 4" high manifold tube

100 elements for 3/4" thick Flange plate

1 element for 1/2" thick mating plate

1 element for $1 / 2$ " thick bottom plate

$-\cdots--$

182 Total shell elements

Material Properties:

$\begin{array}{ll}\text { Es }= & 29,000 \mathrm{ksi} \\ \text { Steel Density } & =0.49 \mathrm{kcf}\end{array}$

steel Density $=0.49 \mathrm{kCf}$

Since the entire camera assembly is given as $1,2271 \mathrm{bs}$, adjustment factors were applied to the steel density of selective components to account for the unmodelled parts of the manifold assembly. See section 3 for details.

Model Bi Nodes \& Elements:

Model $B$ was created by using the same nodes and elements of Model $A$, and additionally 11 nodes and 20 beam elements. 
URS CONSULTANTS WHC-SD-WM-DA-163

Vol. II, Rev. 0

Sheet No. 2.3 .1

By PICL Date

Job \#:66481-01 Job: WHC/MPF CAMERA ASSEMBLY ANALYSIS

Chk'd is Date $\overline{3}, 3$;

Client: WHC Subject: SAP90 ANALYSIS DOCUMENTATION

Che'

2.3 SAP9O Model Sketches

Selective sketches of the SAP9O Model A and Model B are given in this section. 
42-! UPPER MAST ASSEMBLY WITH SHIELDING PLATE

$$
\text { tital bot: }: 227 l_{2} \quad(5 / 26 / 94)
$$

THREADED STEEL EYEBOLT

$1 \angle 8-7 \times 23 / 4$ THREAD

12,000 LBS WORKING LQAD

THREE INCH THICK BY 22 INCH DIAMETER CARBON STEEL SHIELDING PLATE $(42-2)$

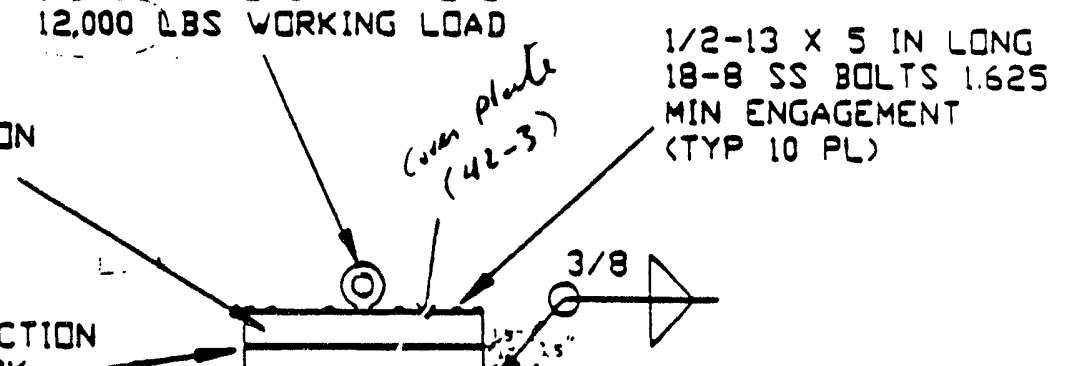

17-4 PH STAINLESS STEEL JUNCTION BOX. 22 IN Q.D. 1.0 WALL THICK. WELDED TO FLANGE PLATE INSIDE AND QUTSIDE LEAK TICHT

FLANCE PLATE $3 / 4$ INCH THICK STAINLESS STEEL MATES TQ ISO 20 INCH IPS RISER FLANGE. $(4<-7)$

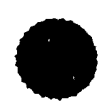

$11 / 4$ SCHEDULE 40304 STAINLESS STEEL PIPE 1.66 0.D. $\times$ 1.38 I.D. . (TYP 4 PL) .

BASE PLATE I/2 IN THICK BY 13 IN SOUARE STAINLESS STEEL. SET $11 / 4$ IPS PIPES INTO BASE PILUT SOCKETS AND WELD ALL AROUND. MUST BE LEAK TIEHT. PIPES PERPENDICULAR TO BASE . PLATES. SAME WELDING DETAIL ON TOP FLANCE PLATE

\section{3/16 $\times 1$ WELDED CROSS} BRACE (TYP IS PLACES)
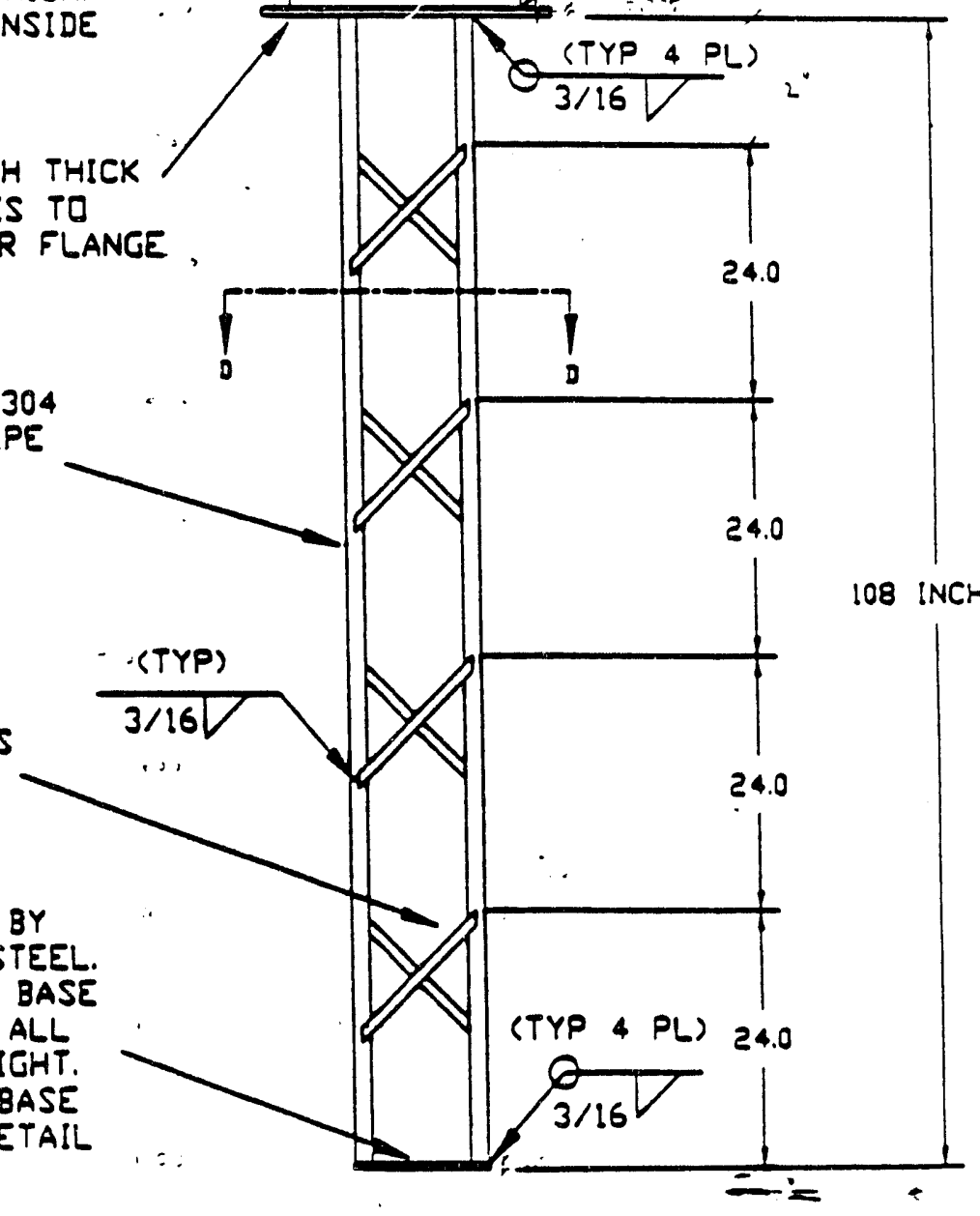

(TYP \& PL) 24.0

CRESTONE PRODUCTS, INE

$4175 \mathrm{~W}$. QUAIL RIDGE DR.

BOISE. 1083703

TEL 208.368.7998

FAX 208.368.7999 


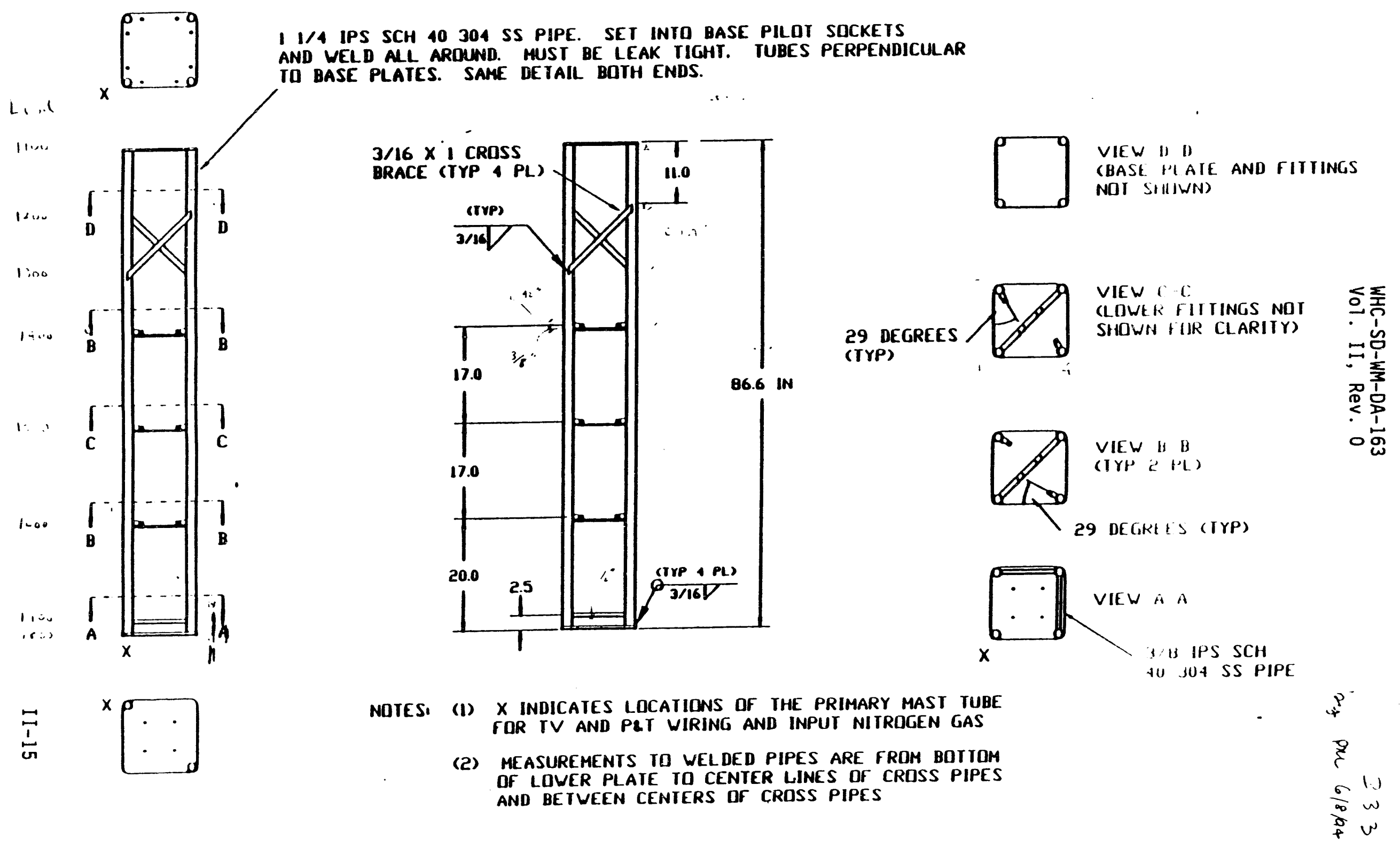




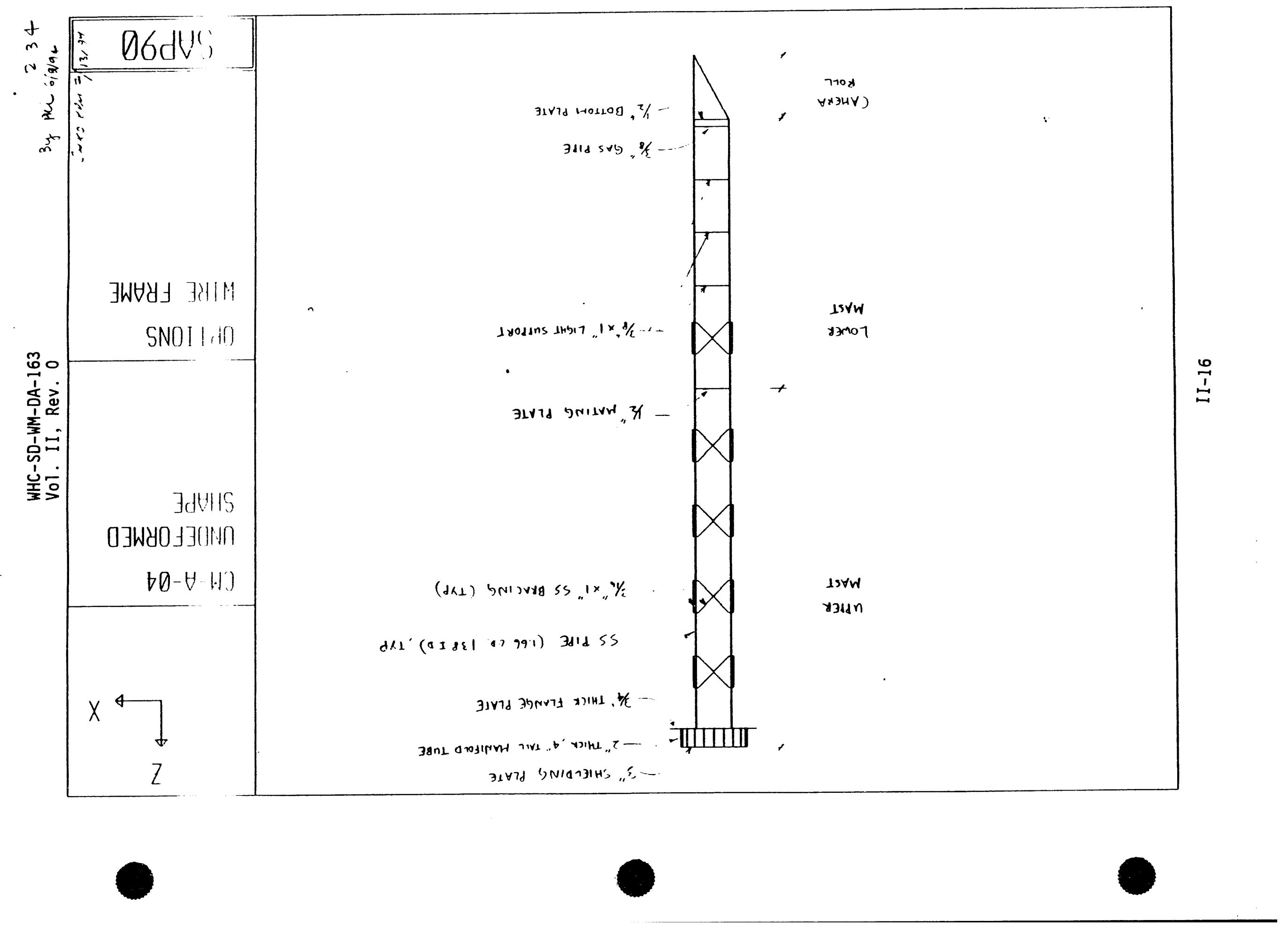


WHC-SD-WM-DA-163

Vol. II, Rev. 0

an ou bisiat $=35$

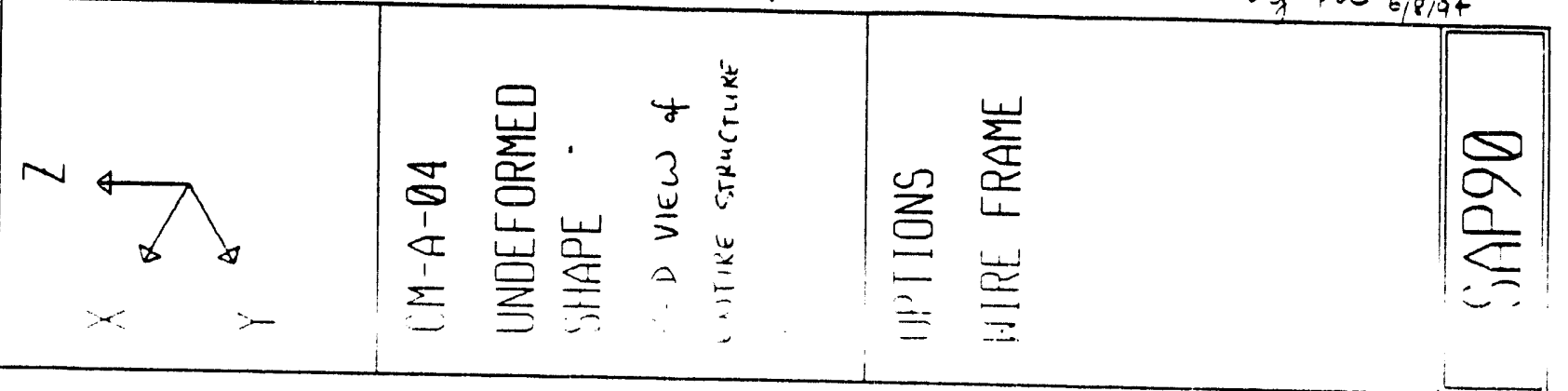
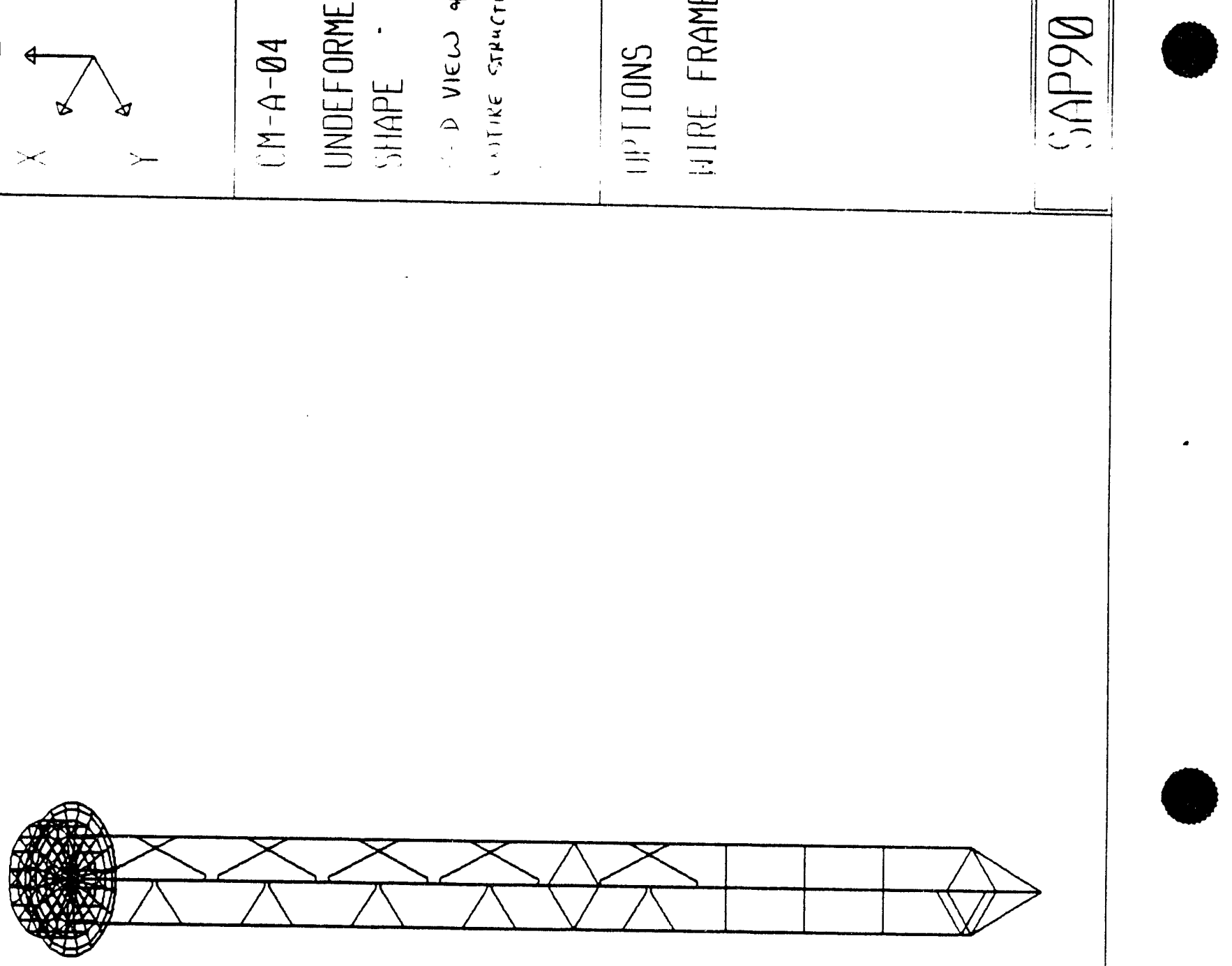

$=$ 

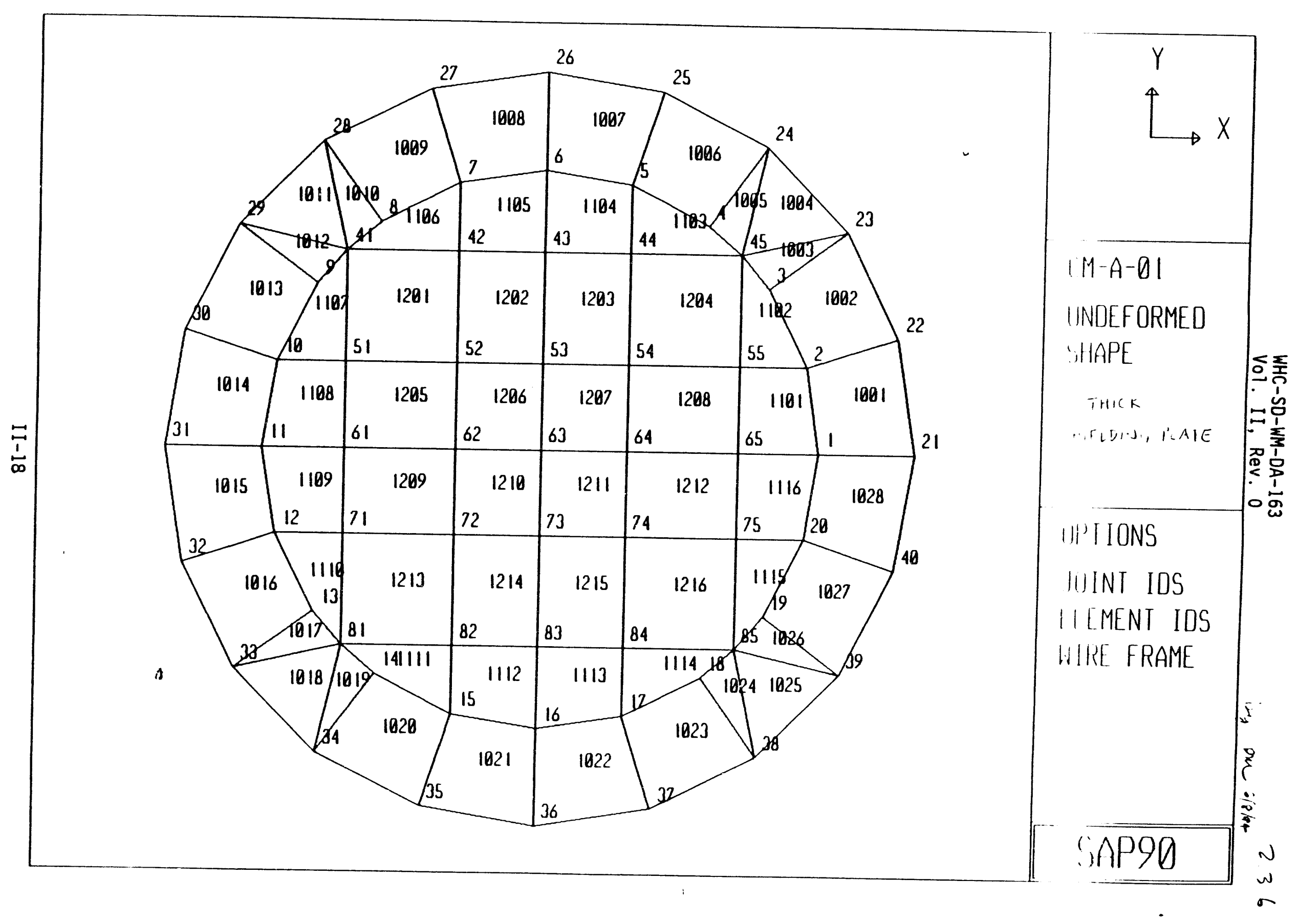


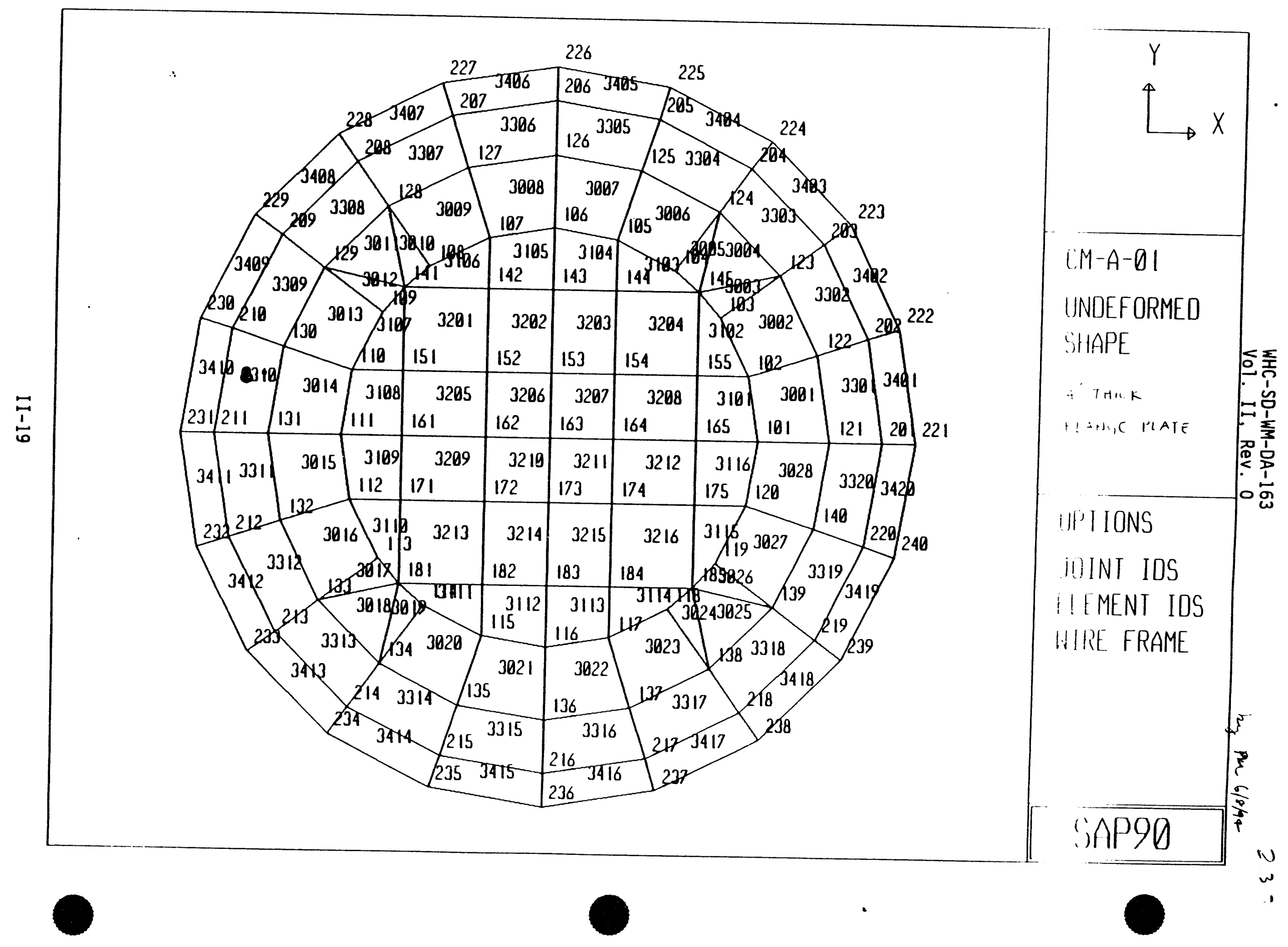


WHC-SD-WM-DA-163

2.38

Vol. II, Rev. 0

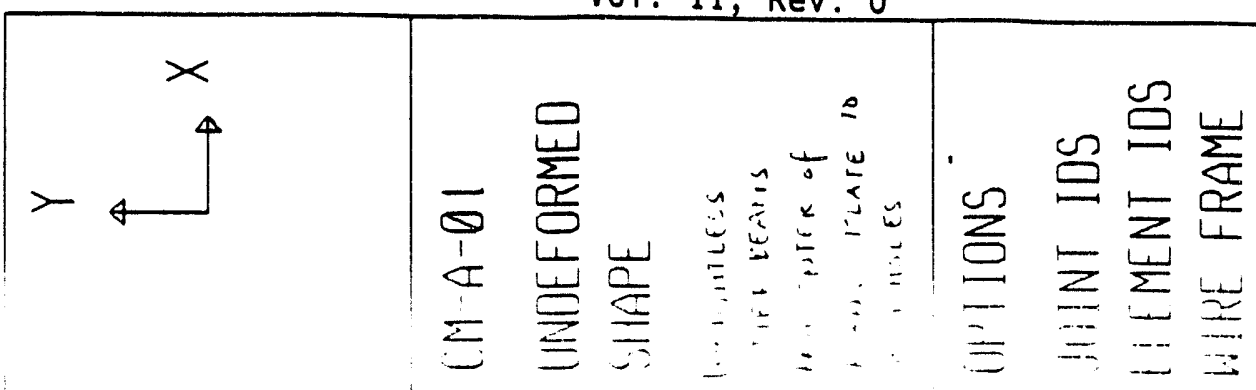

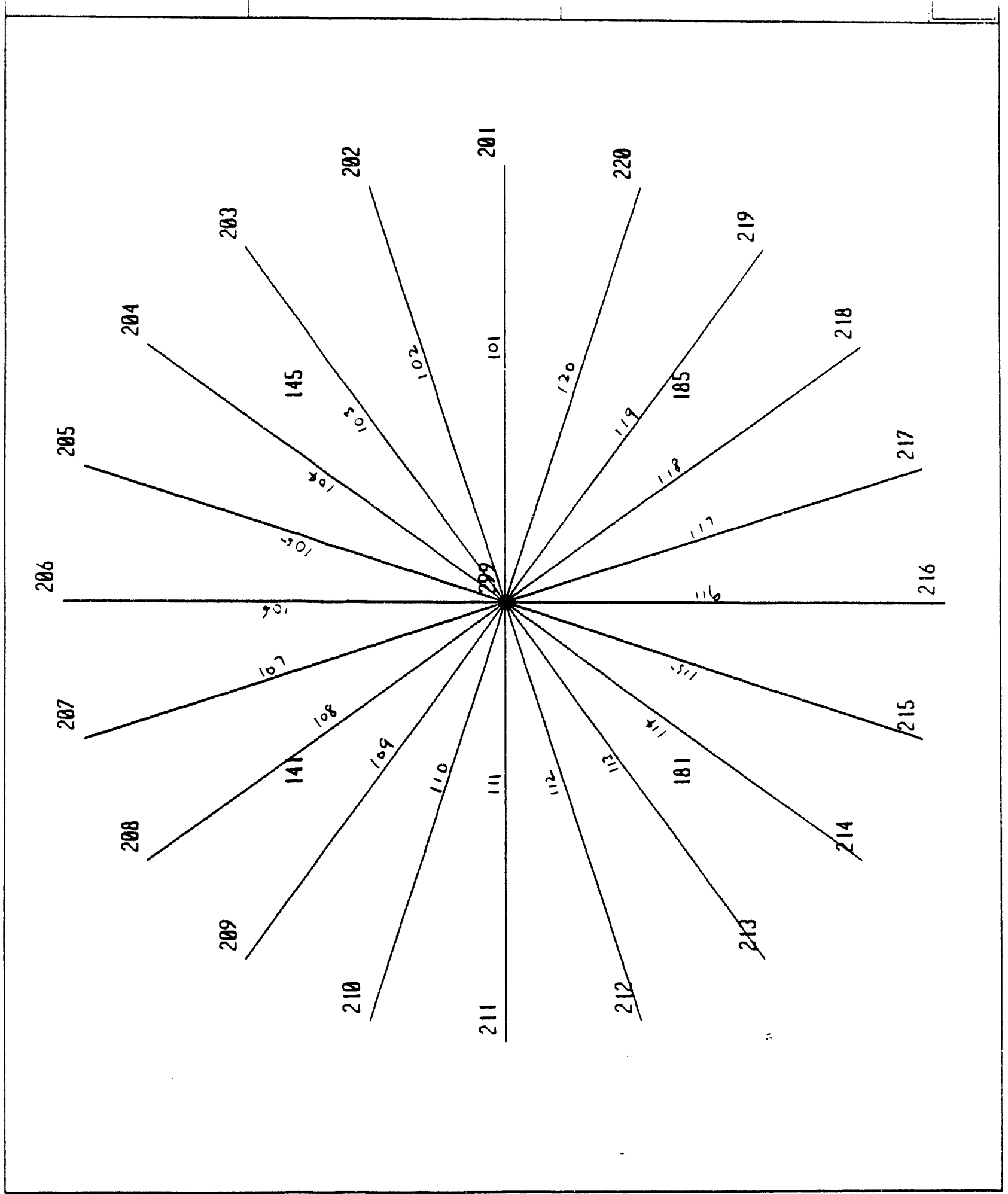




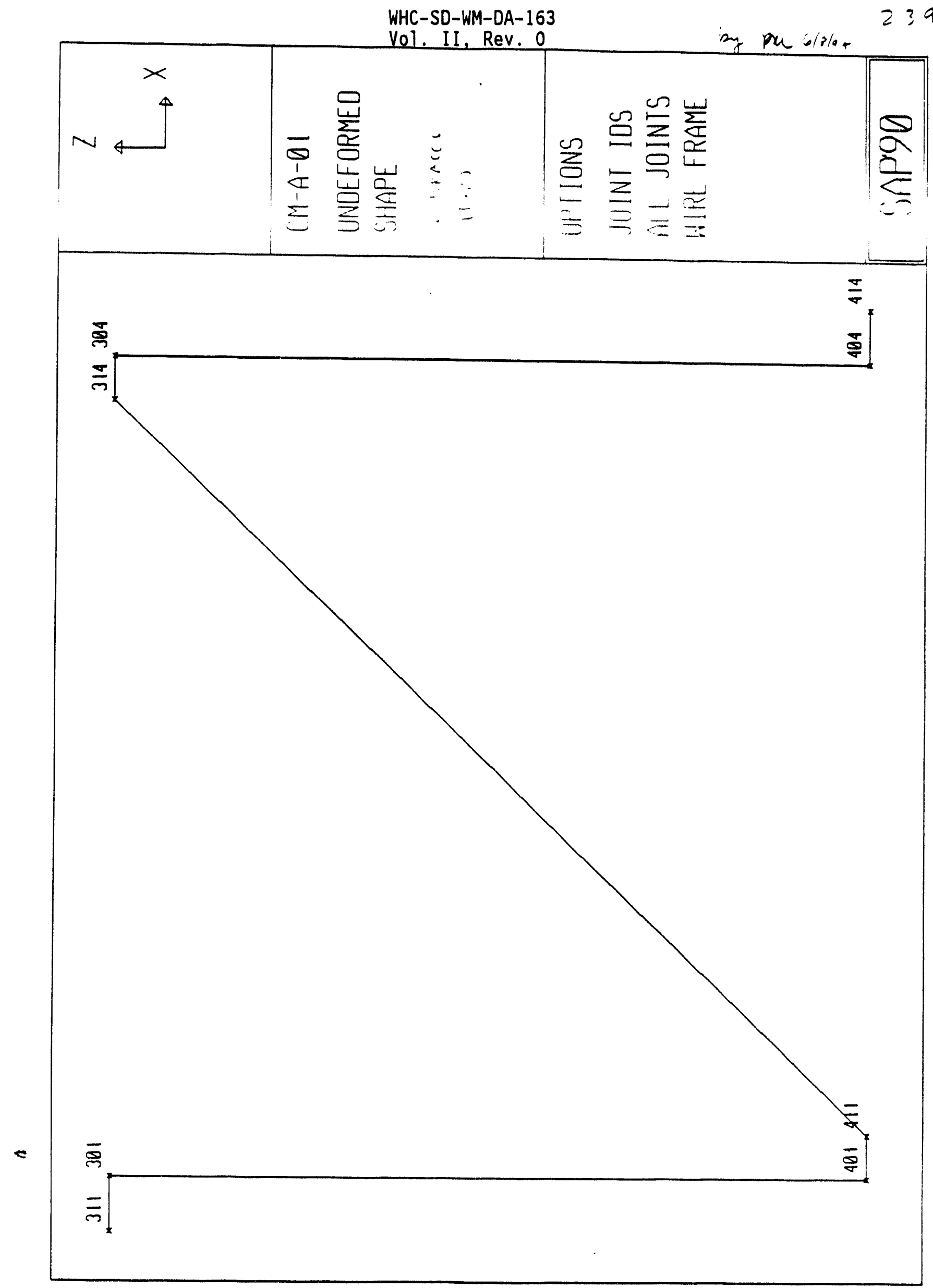

II-2] 
WHC-SD-WM-DA-163

$=310$

Vol. II, Rev. 0

ra pur aisiat
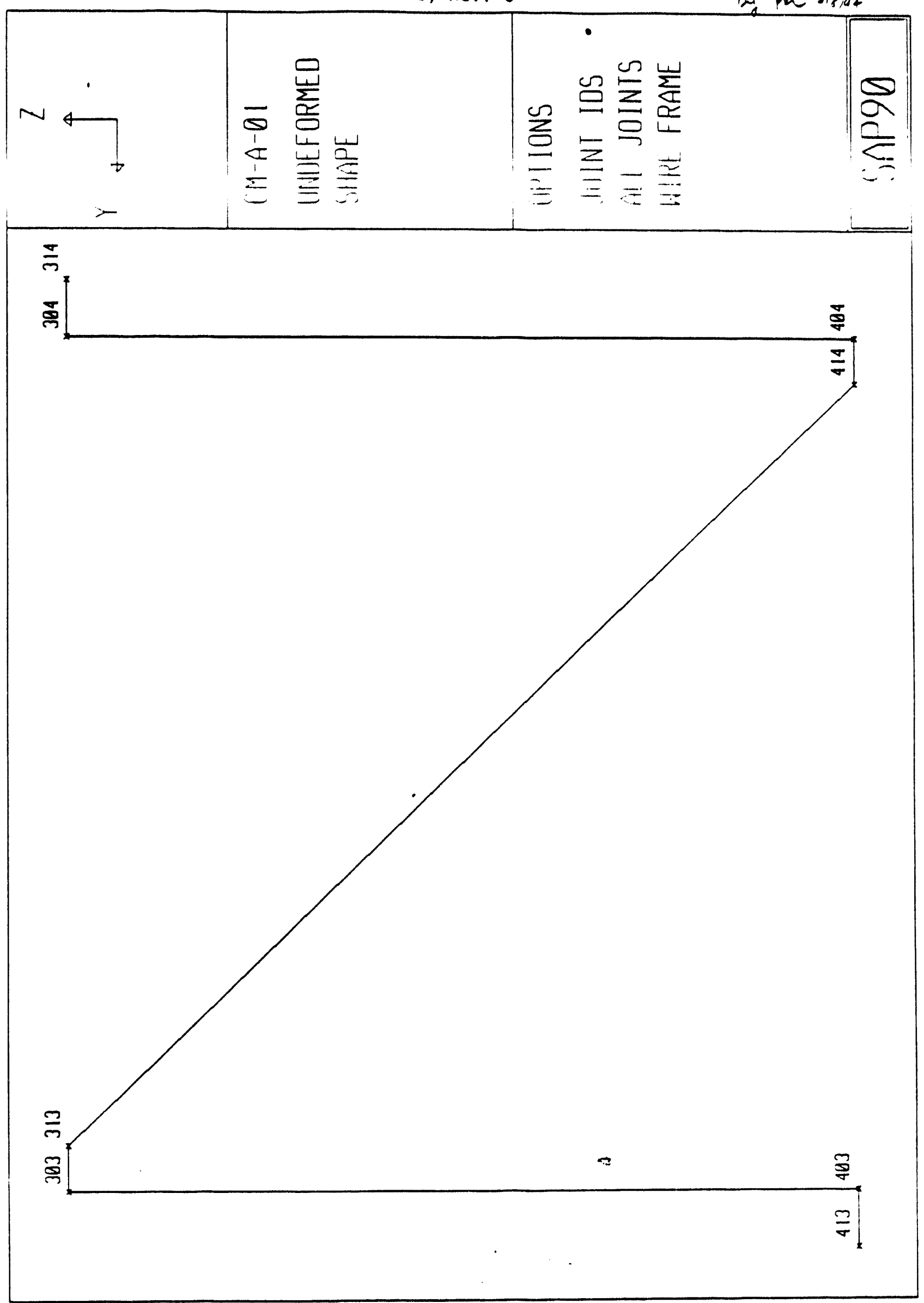


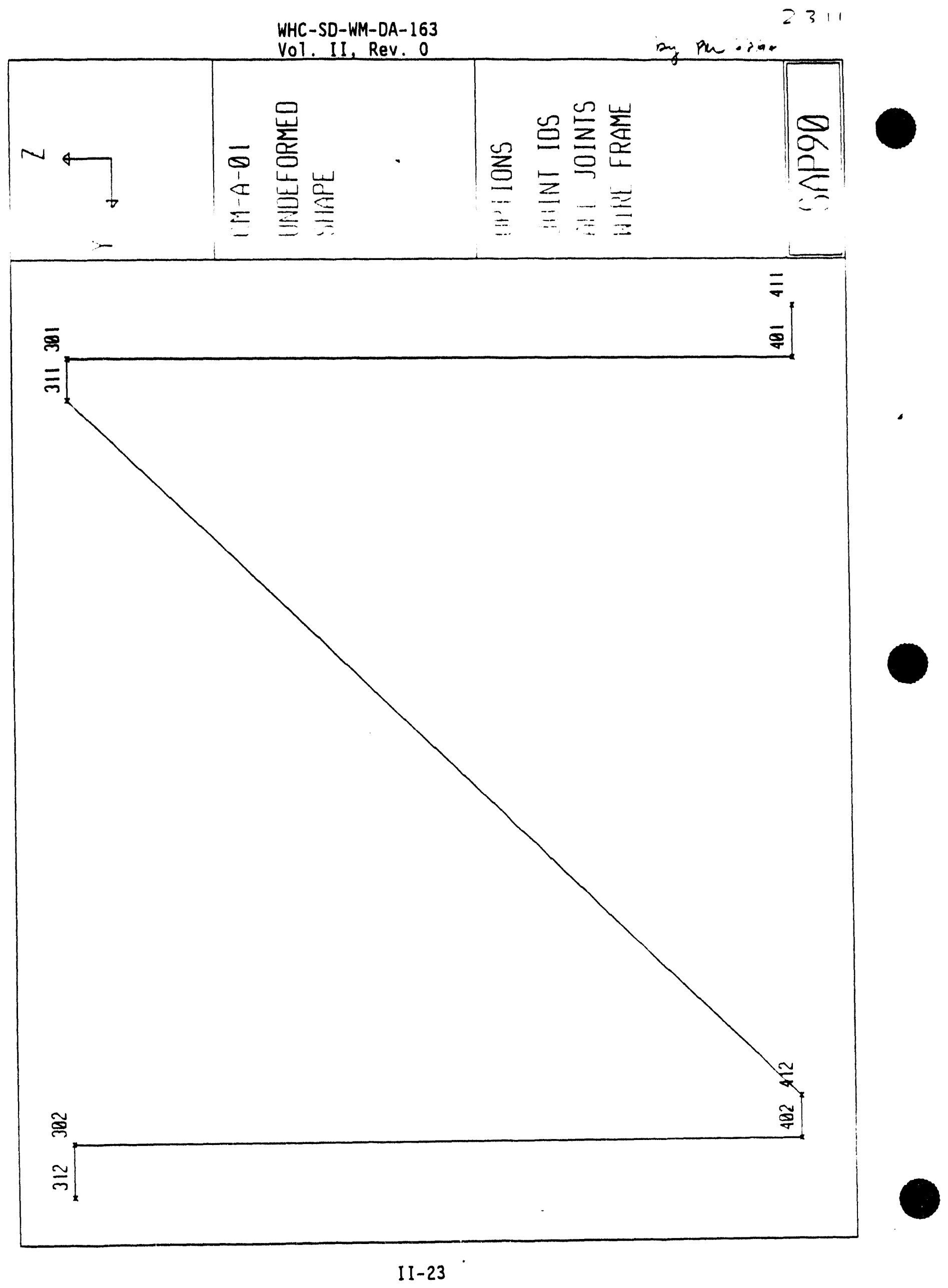


WHC-SD-WM-DA-163

23.12
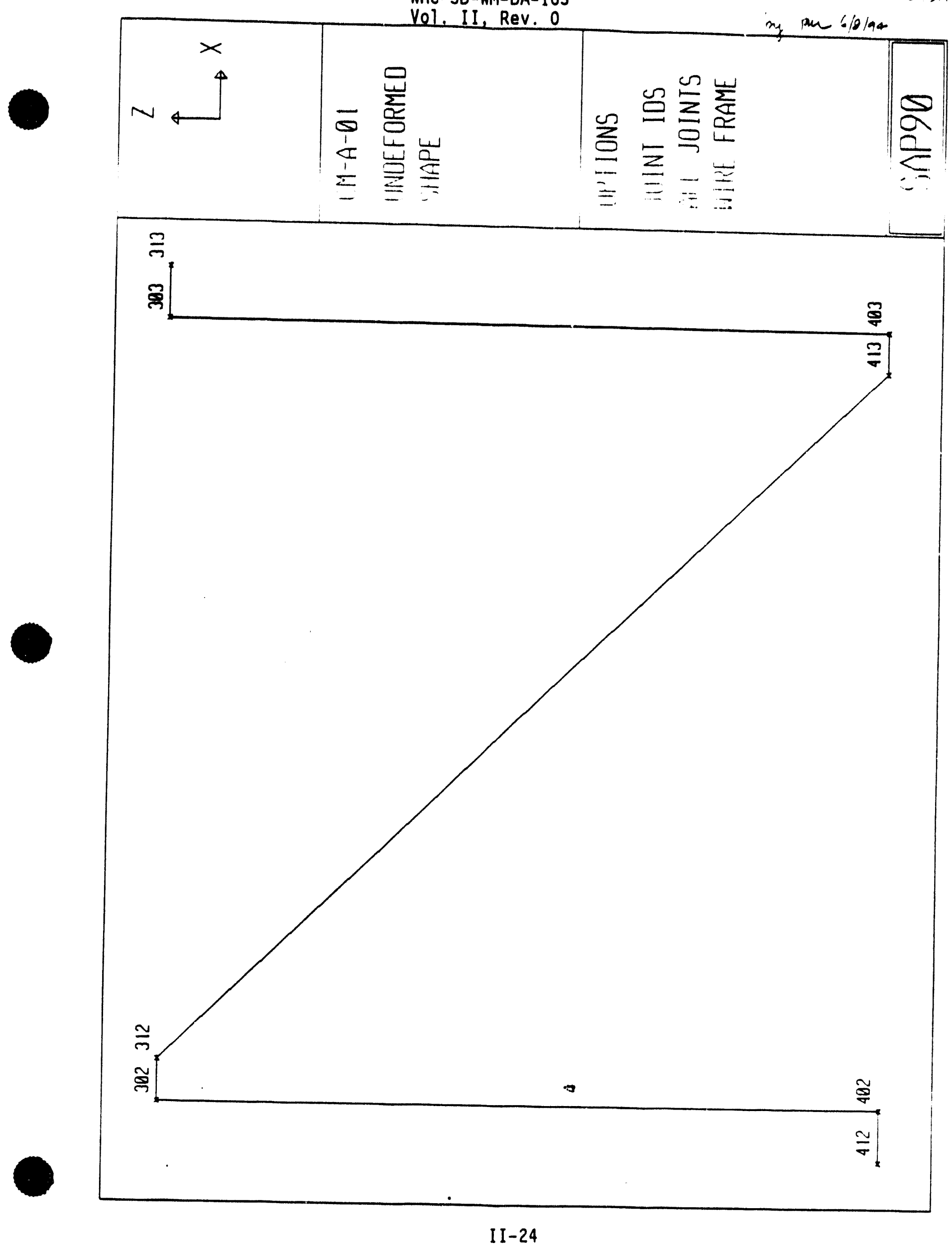


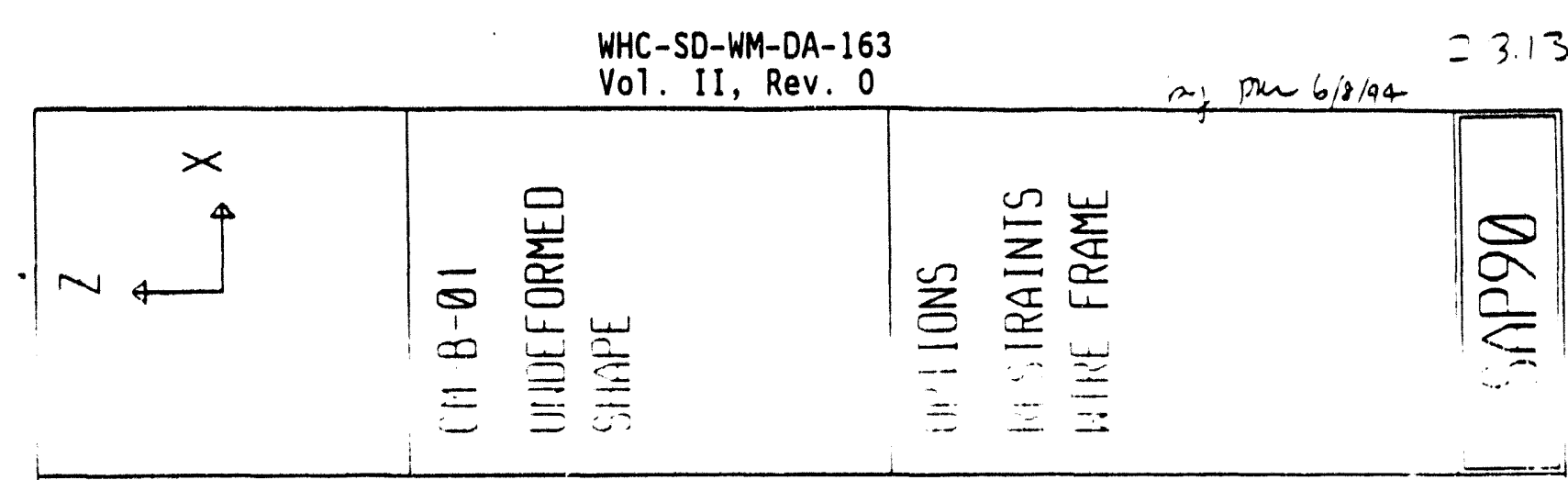

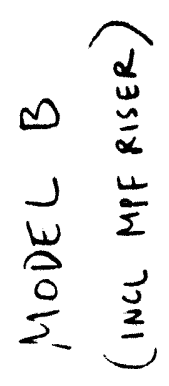

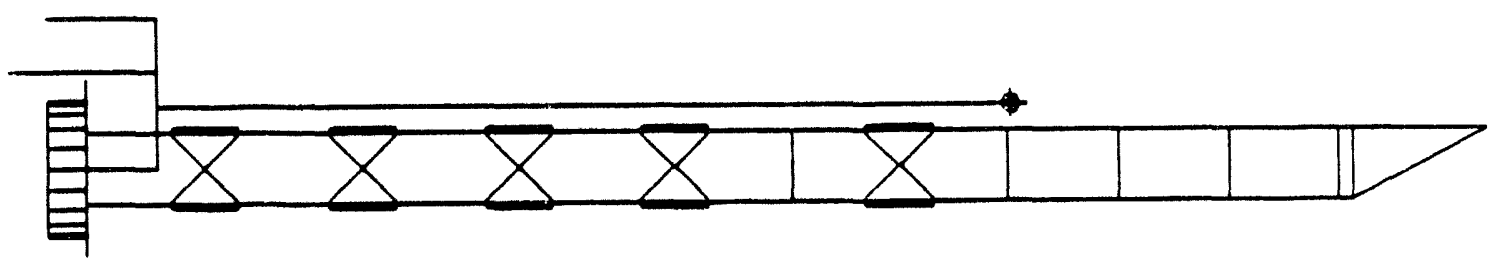




\section{URS CONSULTANTS WHC-SO-WM-DA-163}

Vol. II, Rev. 0

Sheet No. 261

Job *:66481-01 Job: WHC/MPF CAMERA ASSEMBLY ANALYSIS

By PuL Date $8 / 5 / 97$

Client: WHC Subject: SAP9O ANALYSIS DOCUMENTATION

Chk'd RIKDate $8 / 5 / 4$

2.4 SAPSTL Input parameters

The purpose of using the SAPSTL post-processor was to check

the stress ratios of the pipes and the braces.

The material property parameter, $M N=N$, was used for beam elements other than pipes and braces (such as weightless stiff beams). This parameter suppressed the unnecessary output.

"K" factor was set to be:

$K=2$ for cross braces
$K=1$ for all others

cm was globally set to be 0.85 for all elements.

The following allowable stresses were computed and input to SAPSTL to override the default built-in allowable formulas:

Fb33 Major bending stress

Fb22 Minor bending stress

Fac Compressive axial stress

Ft Tension axial stress

Fv Shear stress 
URS CONSULTANTS $\begin{aligned} & \text { WHC-SD-WM-DA-163 } \\ & \text { Vol. II, Rev. } 0\end{aligned}$

Job \#:66481-01 Job: WHC/MPF CAMERA ASSEMBLY ANALYSIS

Sheet No. 3.0

Client: WHC

Subject:

SAP9O ANALYSIS DOCUMENTATION

By Du Date $6 / 8 / a 4$

Chk'd:/,- Date $7 /:]^{04}$

\section{SECTION 3: LOADS}

\section{TABLE OF CONTENTS}

\section{SECTION CONTENTS}

3.1

3.2

3.3
General Discussion

Camera Assembly Dead Loads

Response Spectra
PAGE NO.

3.1 .1

$321-325$

$3.3 .1-3.3 .9$ 
URS CONSULTANTS WHC-SD-WM-DA-163

Vol. II, Rev. 0

Job \#:66481-01 Job: WHC/MPF CAMERA ASSEMBLY ANALYSIS

Client: WHC Subject: SAP9O ANALYSIS DOCUMENTATION

Sheet No. 3.1 .1

By Pu Date $3 / 5 / 94$

Chk'd RIKDate $3 / 5 / 94$

3.1 General Discussion

The Dead load (DL) of the camera assembly was given by wHC

as 1,227 lbs (see Attachment D).

The Response Spectrum (RS) for the Non-reactor Safety Class $2,2 \%$ of critical damping was used. This spectrum was applied in 3 directions:

$$
\begin{aligned}
& 100 \% \text { in X-dir (horizontal) } \\
& 100 \% \text { in } Y \text {-dir (horizontal) } \\
& 1008 \text { in } \mathrm{Z} \text {-dir (Vertical) }
\end{aligned}
$$

Note that the scale factor of $2 / 3$ was not used to scale the vertical spectrum per specification of the SDC 4.1 Rev. 12 ("Design Loads for Facilities", Non-reactor Safety Class 2), to account for potential amplification of vertical ground motion by the roof of the tank. This is considered as a conservative assumption.

Four load cases were performed:

$$
\begin{aligned}
& \text { 1. } 1.0 * \text { DL } \\
& \text { 2. } 1.0 * \text { RS } \\
& 3.01 .0 * D L+1.0 * \text { RS } \\
& \text { 4. } 1.0 \star D L-1.0 \text { RS }
\end{aligned}
$$




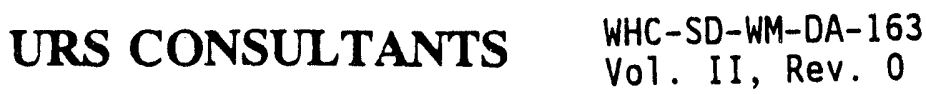

Job \#:66481-01 Job: WHC/MPF CAMERA ASSEMBLY ANALYSIS

Sheet No. 321

Client: WHC Subject: SAP9O ANALYSIS DOCUMENTATTON

By Mu Date 6/8/a4

Chk'd I// Date $2, \cdots, 11$

3.2 Camera Assembly Dead Loads

Summary of Camera's Weights

The weights of the camera assembly elements in SAP9o are :

Beam elements 168.2 lbs

Shell elements 931.8 lbs

Camera $\quad 128.0 \mathrm{lbs}$

Total $1,228.01 \mathrm{bs}$

(close enough for a total of 1,227 lbs)

\section{Check SAPgo Element Self-Weight}

Hand calculations of weights were performed for each unit and compared to the SAP9O self-weights as computed by the software. Due to modelling and other unaccounted parts, there were some discrepancies. Weight adjustments were made to remedy the differences. The hand calculations, explanations of discrepancies of weights, and weight adjustments are given in the following pages. 
URS Consultants Vol. II, Rev. 0

URS/John A. Blume \& Associates, Engineers

Job no. 6481-01 Job WHC/MPF ANALYSIS

Client

Subject

CHECK WEIGHTS
Sheet No.

Calc. No.

Rev. No.

By Piu Date $\frac{\dot{b} / 2 / x 4}{7 /, \ldots 11}$

\section{BHEL_E_LUENT SELF-WEIGHTS}

$$
\text { WT of 3" SHIELDING PLATE: } \begin{aligned}
D & =22^{\prime \prime} \quad A=\frac{\pi D^{2}}{4}=380 \mathrm{~m}^{2} \quad t=3^{\prime \prime} \\
\text { WT } & =3^{\prime \prime} \times 380 \mathrm{~m}^{\prime} \times \frac{49^{k}}{1728 \mathrm{~m}^{3}} \\
& =0.323^{k} \\
\text { MASS } & =\frac{0.323^{k}}{386.4}=0.0008366
\end{aligned}
$$

WT. of MANIFOLD TUBE

$$
O D=22^{\prime \prime} I D=20^{\prime \prime} \quad h=4^{\prime \prime}
$$

$$
\begin{aligned}
& A=\frac{\pi}{4}\left(22^{2}-20^{2}\right)=65.97 \mathrm{~m}^{2} \\
& W T=4^{\prime \prime} \times 65.97 \times \frac{49}{1728}=0.0748^{\mathrm{k}} \\
& \text { MASS }=\frac{0.0748^{\mathrm{k}}}{386.4}=0.0001937
\end{aligned}
$$

lut of flange plate

$$
D=27.5^{\prime \prime} \quad A=\frac{\pi \partial^{2}}{4}=594 \mathrm{in}^{2} \quad t=0.75^{\prime \prime}
$$

$$
\text { WT }=0.75 \times 594 \times \frac{.49}{1728}=0.1263^{k}
$$

WT of mating plate.

$$
\begin{aligned}
& A=13^{\prime \prime} \times 13^{\prime \prime}=169 \quad t=0.5^{\prime \prime} \\
& W T=0.5 \times 169 \times \frac{.49}{1728}=0.024^{k}
\end{aligned}
$$


URS Consultants Vol. II, Rev. O URS/John A. Blume \& Associates, Engineers

Job no. 66481.01 Job WHC/UPF ANALYSIS

Client

Subject

CHECK WEIGHTS
Sheet No.

$3=3$

Calc. No

Rev. No.

By Pue Date $\frac{6 / 2 / 9+}{7 j, ? / 9+}$

\section{ZEAM ELEMENTS DELF LUEIGHTS}

WT of PIPES : $\quad 0 . D=1.66^{\prime \prime} I D=1.38^{\prime \prime} \quad A=\frac{\pi}{4}\left(1.66^{2}-138^{\circ}\right)=0.6655 \mathrm{~m}^{2}$ LENGTH $=108^{\prime \prime}+86.6^{\prime \prime}=194.6^{\prime \prime}$

$W T=0.6685 \times 194.6 \times \frac{.49}{1728}=00369$

4 PIPES $=4 \times 0.0369=0.1476^{k}$

CRoSS BRACES: $L=14^{\prime \prime} \quad t=3 / 16 \quad h=1^{\prime \prime} \quad A=3 / 6 \mathrm{~m}^{2}$

$W T=\frac{3}{16} \times 14 \times \frac{.49}{1728}=0.000744^{\mathrm{K}} /$ BRACE

20 BRACES $=20 \times 0.000744^{k}=0.0149$

LIGHT SUPPORT: $L=13.9^{\prime \prime} \quad t=3 / 8^{\prime \prime} \quad h=1^{\prime \prime}$

$W T=\frac{3}{8} \times 13.9 \times \frac{49}{1728}=0.001478 /$ support

3 SUPPORTS $=3 \times 0.001478=0.0044^{K}$

3/8"GASpIPES O.D. $=0.675^{\prime \prime}$ I $D=0.375^{\prime \prime} \quad A=\frac{\pi}{4}\left(0.675^{2}-0.375^{\circ}\right)=0.247 \mathrm{in}^{2}$ LENGTH $=9.34^{\prime \prime}$

$W T=0.247 \times 9.34 \times \frac{49}{1728}=0.000655^{k} /$ PIPE

2 PIPES $=2 \times 0.0000655^{k}=0.00131^{k}$ 
URS Consultants Vol. II, Rev. 0

URS/John A. Blume \& Associates, Engineers

Job no. $e_{0}+3 \mid-01$ Job WHC/MPF ANALYSIS

Client

Subject

SHECK WEIGHTS
Sheer No. $32 \div$

Calc. No.

Rev. No

By PuL Date b/2/94

Chkd re Date 31,1

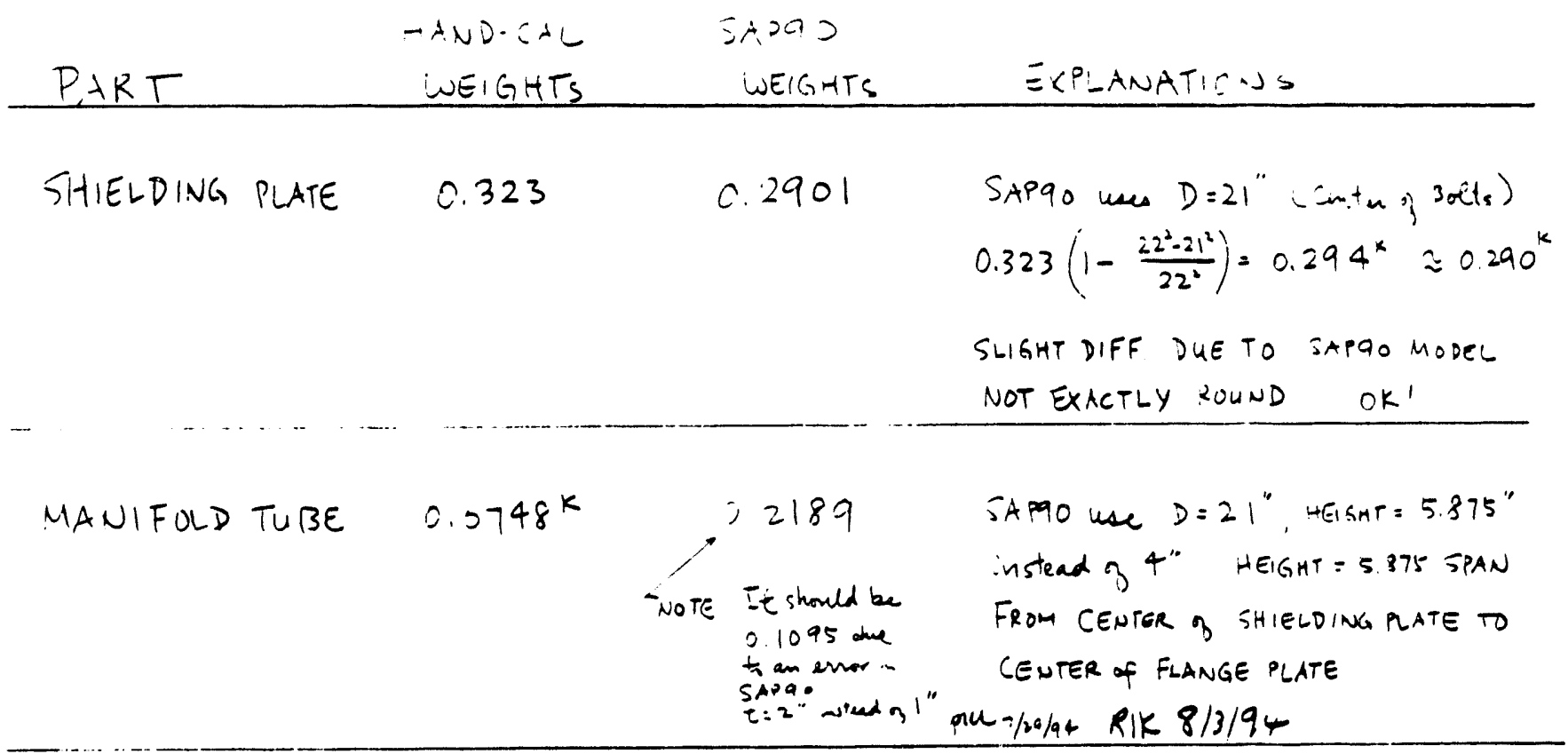

Flange plate $\quad 0.1263^{k} \quad 0.1243^{k}$

SLISHT DIFF DUE TO SAPO. MODEL NoT EXACTLY ROUND, OK!

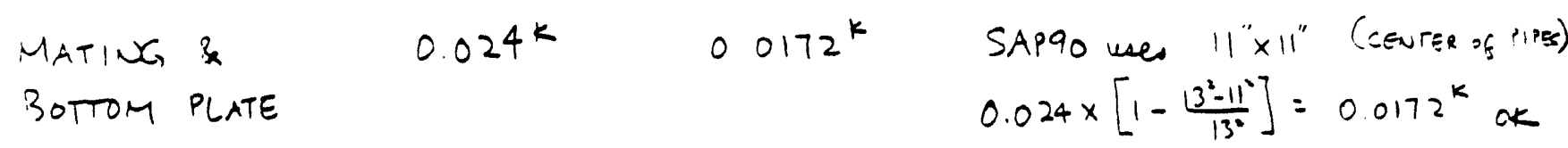

$\begin{array}{lll}\text { PIPES } 0.1476^{K} & 0.1578^{k} & \frac{01579-0.1476}{0.1476} \approx 7 \% \text { INCREASE of WT } \\ & \text { DUE TO SAP90 NODE TS CENTER of } \\ & \text { PLATES. OK }\end{array}$

\begin{tabular}{|c|c|c|c|}
\hline LROSS BRACES & $0.0149^{k}$ & $\begin{array}{l}0.0150^{K} \\
\therefore\end{array}$ & OK \\
\hline LIGHT SUPPORT & $0.0044^{k}$ & $0.005^{k}$ & SARPOD nODEL TO CENTER of PIS RK \\
\hline $3 / 8$ GAS PIPES & $0.0013^{k}$ & $0.0015^{k}$ & . \\
\hline
\end{tabular}

$\bar{\Sigma}=0739^{k} \quad$ II -32




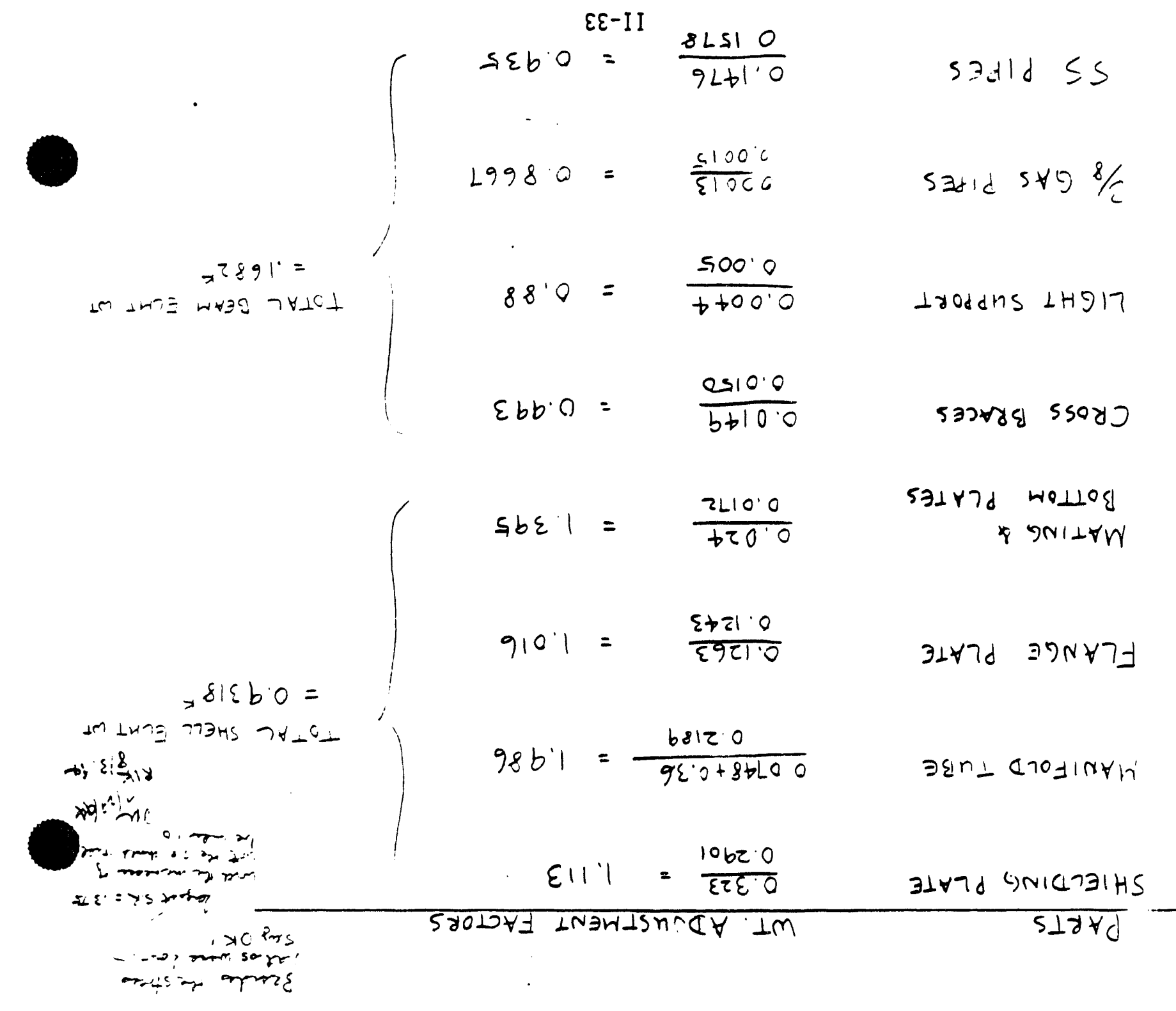




\section{URS CONSULTANTS WHC-SD-WM-DA-163}

Vol. II, Rev. 0

Job \#:66481-01 Job: WHC/MPF CAMERA ASSEMBLY ANALYSIS

Sheet No. 331

Client: WHC Subject: SAP9O ANALYSIS DOCUMENTATION

By Pue Date $\dot{8} / 5 / 94$

Chr'dRiLDate $8 / 5 / 94$

3.3 Response Spectra Analys is

The response spectrum was obtained from SDC 4.1 Rev. 12, Figure 5 (log-log scale) with tabulated design spectra in Table 2 of the same document. A spreadsheet (SA.WQ1) was created to interpolate from $\log -\log$ scale to linear-Iinear scale.

The interpolated spectrum and its plots are given in this section.

During the eignvalue extraction computations, the participating mass in the vertical direction was less than $90 \%$ for 20 mode cut-off. The main reasons were:

1. The masses of the shielding plate, manifold tube and the flange plate are high ( $76 \%$ of the total mass of the entire structure).

2. The flange plate is fixed in all directions at the center of the plate.

3. The stiffness of the plates and tube are comparatively much higher than the 195" pipes hanging down from the flange plate.

To alleviate the mass participation problem, the shell elements of the shielding plate, manifold tube and the flange plate were treated as massless. Their masses were applied as equivalent static loads, load condition 2 (LC2), as follows:

$F(x)=$ Mass $* 2 P A$
$F(y)=$ Mass $* 2 P A$
$F(z)=$ Mass $* 2 P A$

where $\mathrm{ZPA}=$ Zero Period Acceleration of $0.12 \mathrm{~g}$ for Class 2 structure

The load combinations are:

$\begin{array}{lll}\text { 1. } & D L & \text { Dead load only } \\ \text { 2. } & L C 2+S A & \text { Spectrum only } \\ \text { 3. } & D L+L C 2+S A & \text { Dead load + Spectrum } \\ \text { 4. } & D L-L C 2-S A & \text { Dead Load - Spectrum }\end{array}$


WHC/MPF ANALYSIS ... CAMERA ASSEMBLY MODEL

NON-REACTOR SAFETY CLASS 2

JOB NO. 66458-01

FILENAME: SA.WO1

2\% DAMPED DESIGN SPECTRUM

SOURCE: SDC 4.1 REVISION 12. DESIGN LOADS FOR FACIUTIES

FIGURE 3 \& TABLE 2, SCALED BY 0.6 FOR CLASS 2

NOTE: DESIGN SPECTRUM IS GIVEN IN LOG-LOG SCALE

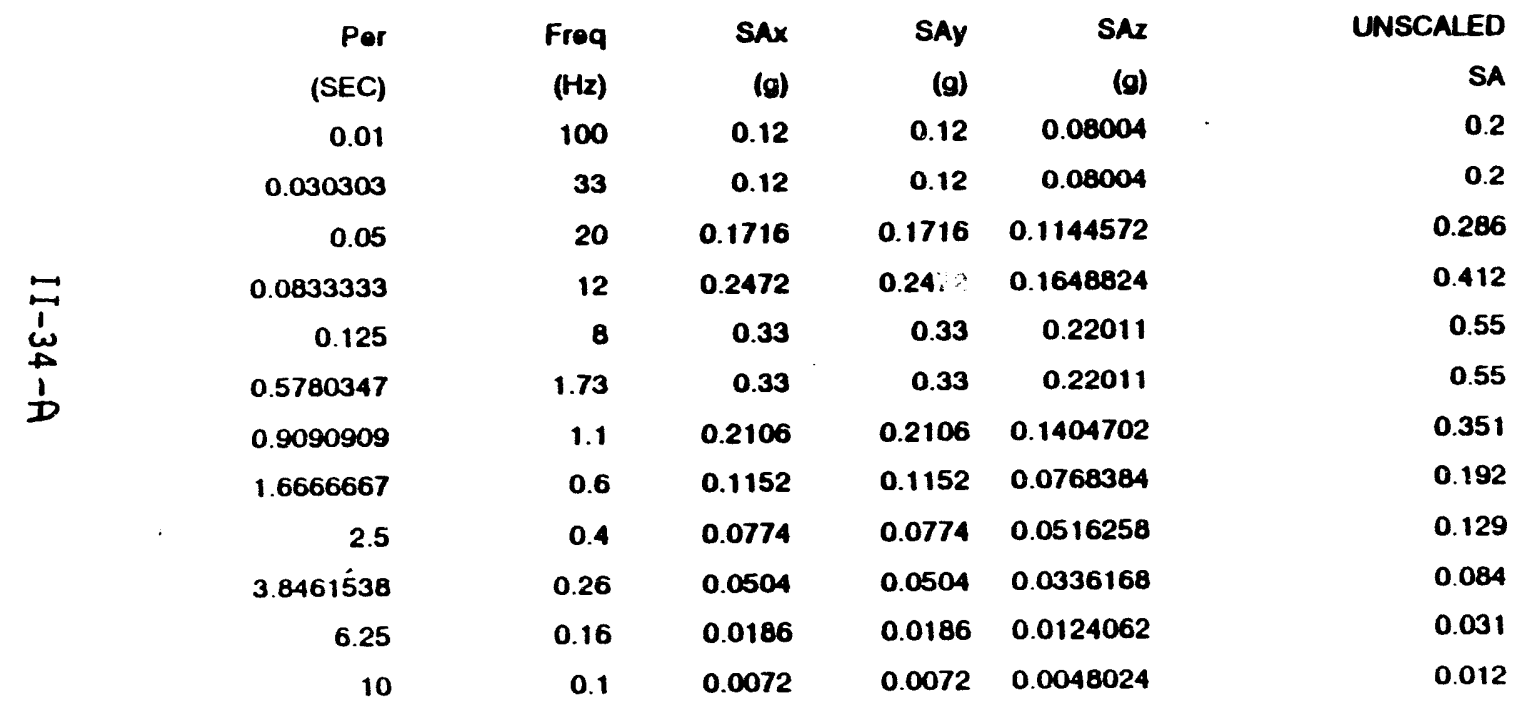

Prepared by: PKC Dato 6/8/94

Checked by: 4 Date $=\ldots, 4$ 
WHC/MPF ANALYSIS -- CAMERA ASSEMBLY MODEL

NON-REACTOR SAFETY CLASS 2

$2 \%$ DAMPED DESIGN SPECTAUM
JOB NO. 66458-01

FILENAME: SA.WQ1
Prepared by: PL Dato $: / 8 / 94$

Checked by: K/-3, Date Sir/,

CONVERT LOG-LOG DESIGN SPECTRUM TO NATURAL-NATURAL FORMAT

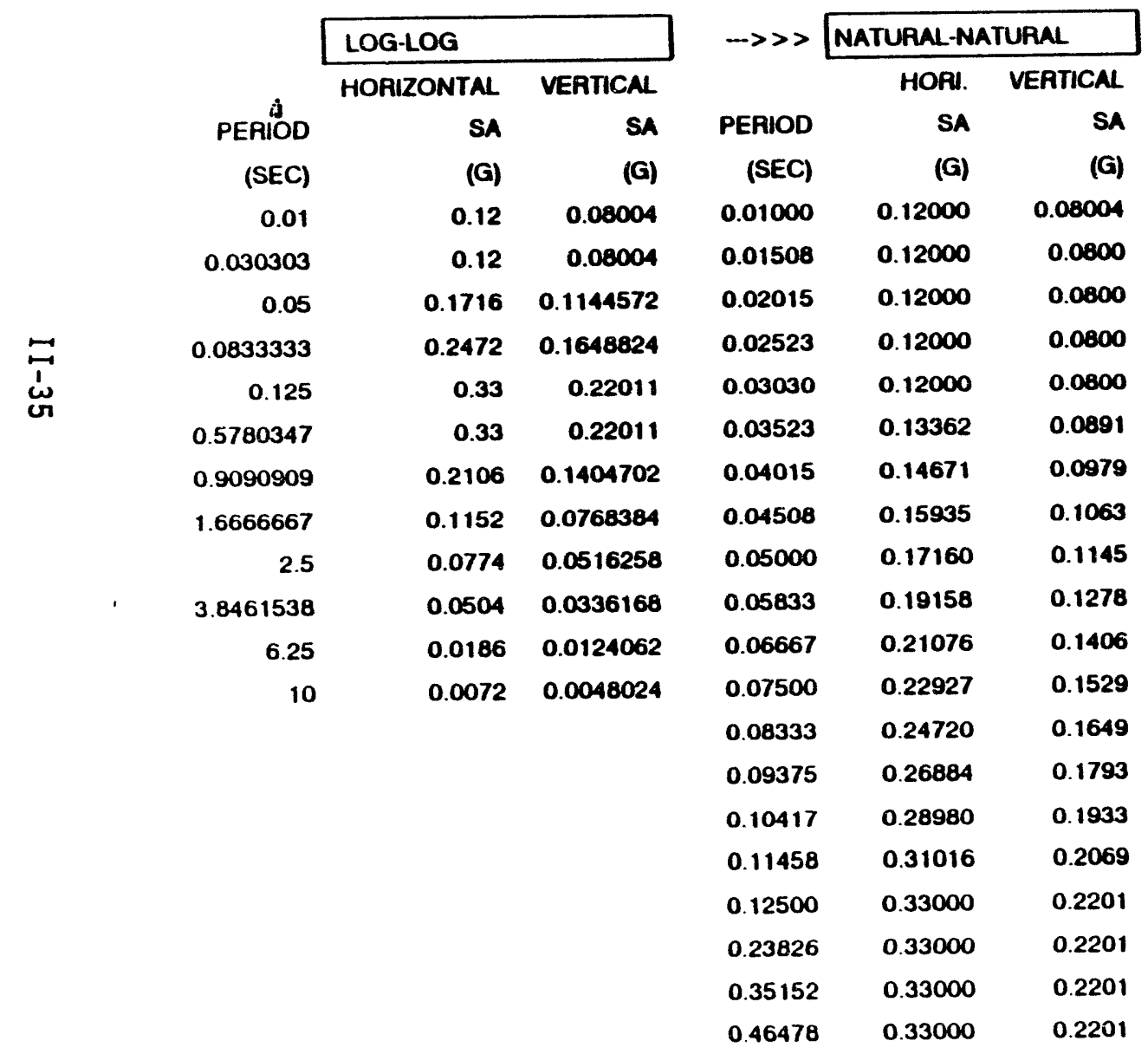


WHC/MPF ANALYSIS -.- CAMERA ASSEMBLY MODEL

NON-REACIOR SAFETY CLASS 2

$2 \%$ DAMPED DESIGN SPECTAUM
JOB NO. 66458-01

FILENAME: SA.WO1
Prepared by: PKL Daic $8 / 94$

Chocked by:
Dato $x$ is ty

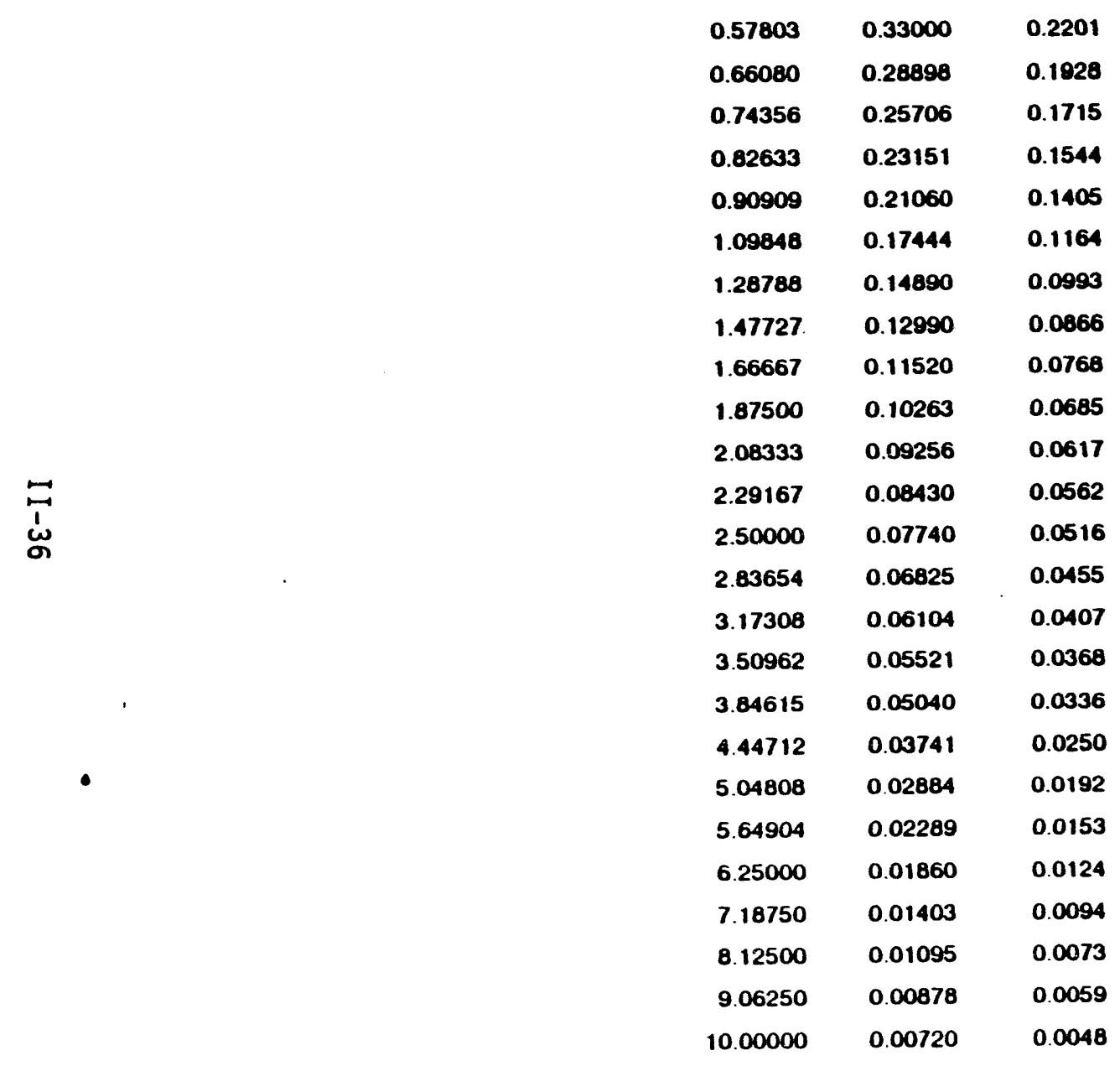


WHC-SD-WM-DA-163

Vol. II, Rev. 0

SHeit $\because 335$

ing Pul 6/8/a4

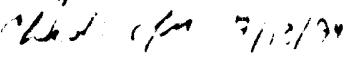

ININIERPOLATED DIRETLY FRM TARE:

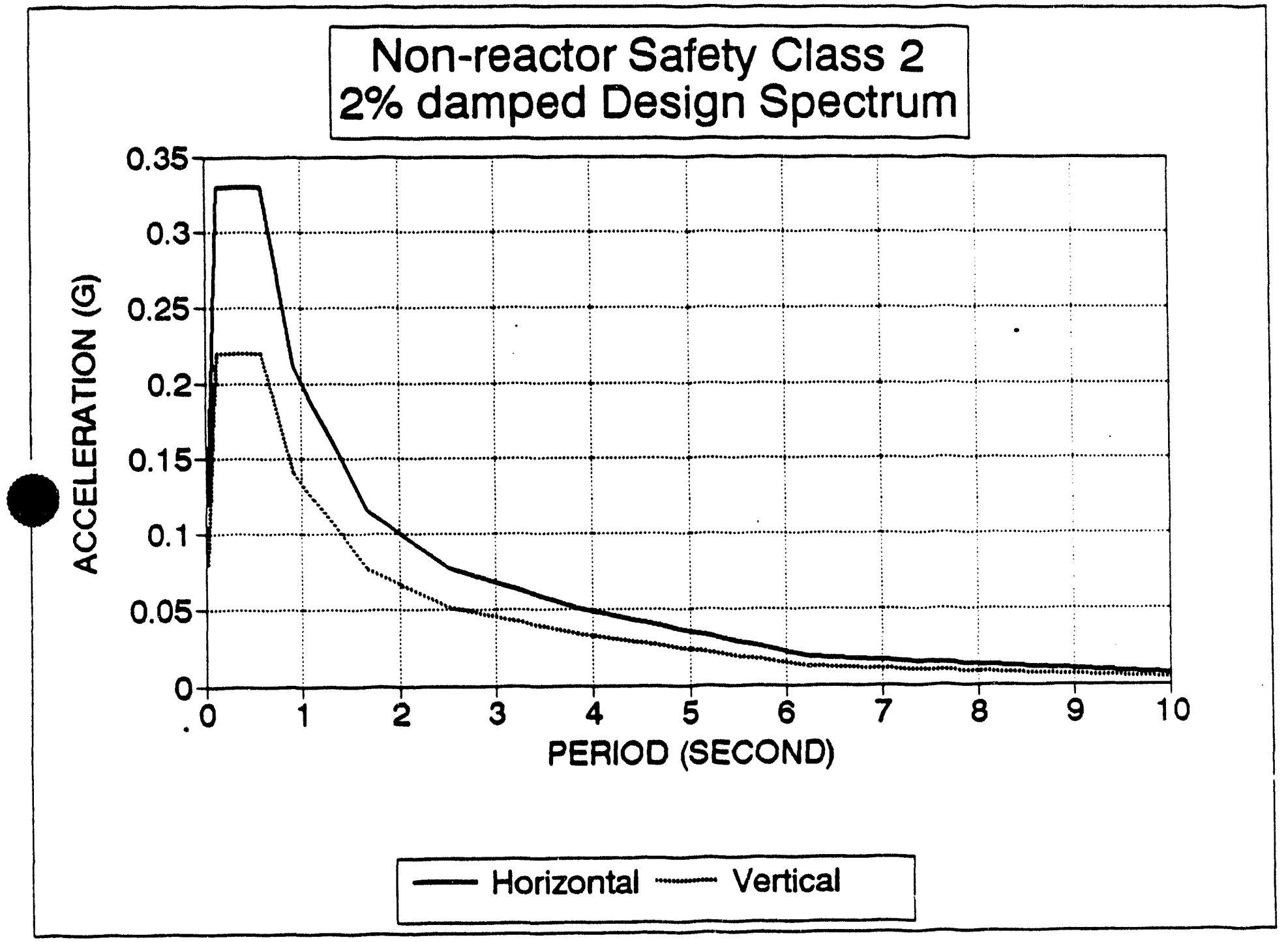


WHC-SD-WM-DA-163

Vol. II. Rev. 0

Sitest ve 236

mate re/at

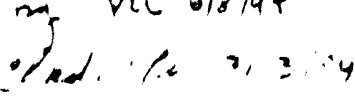

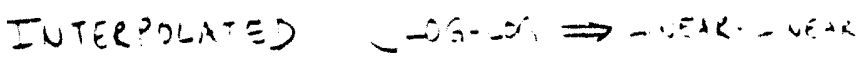

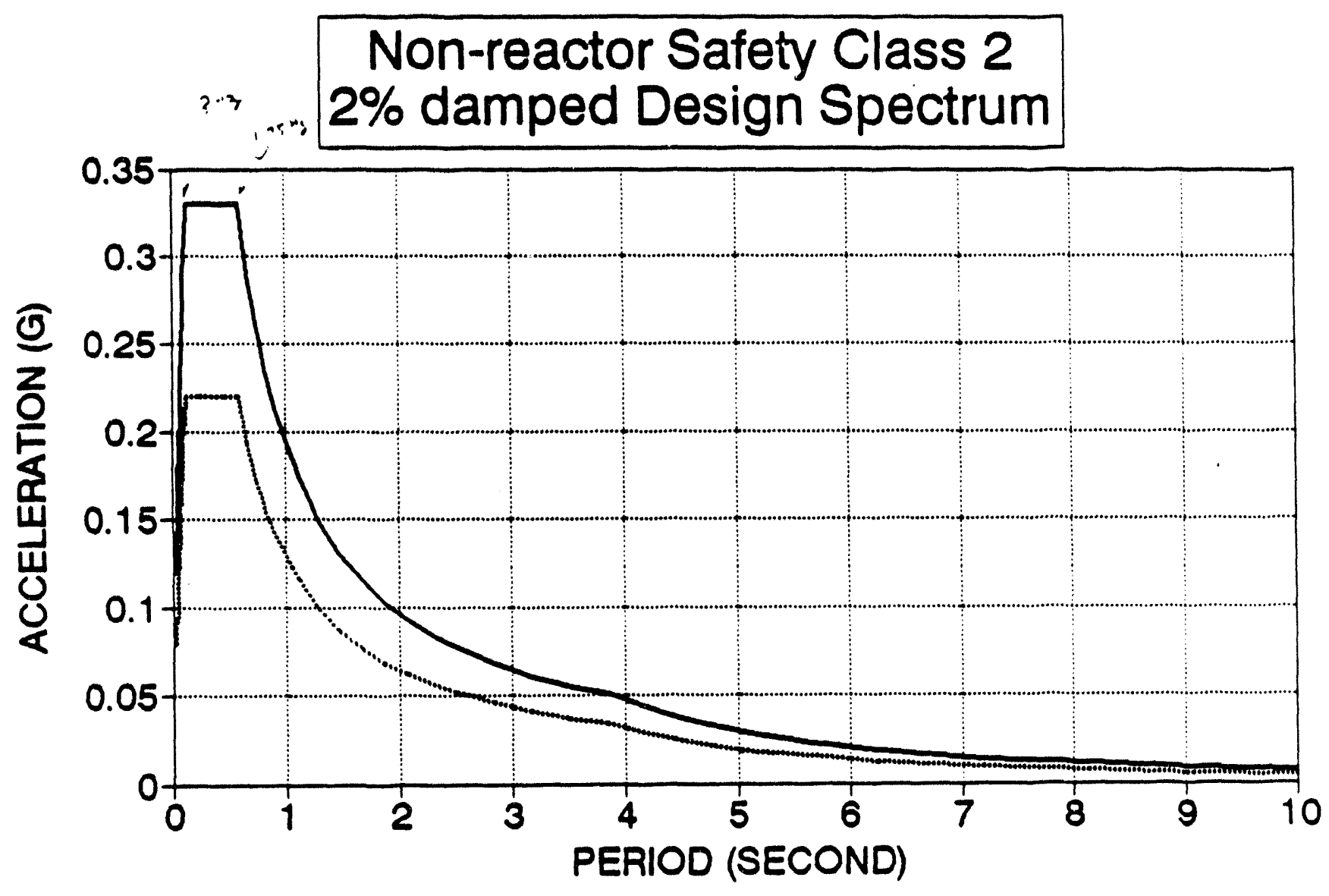

Horizontal - Vertical 
WHC-SD-WM-DA-163

Vol. II. Rev. 0

SOC 4.1 Revision 12

Page 32

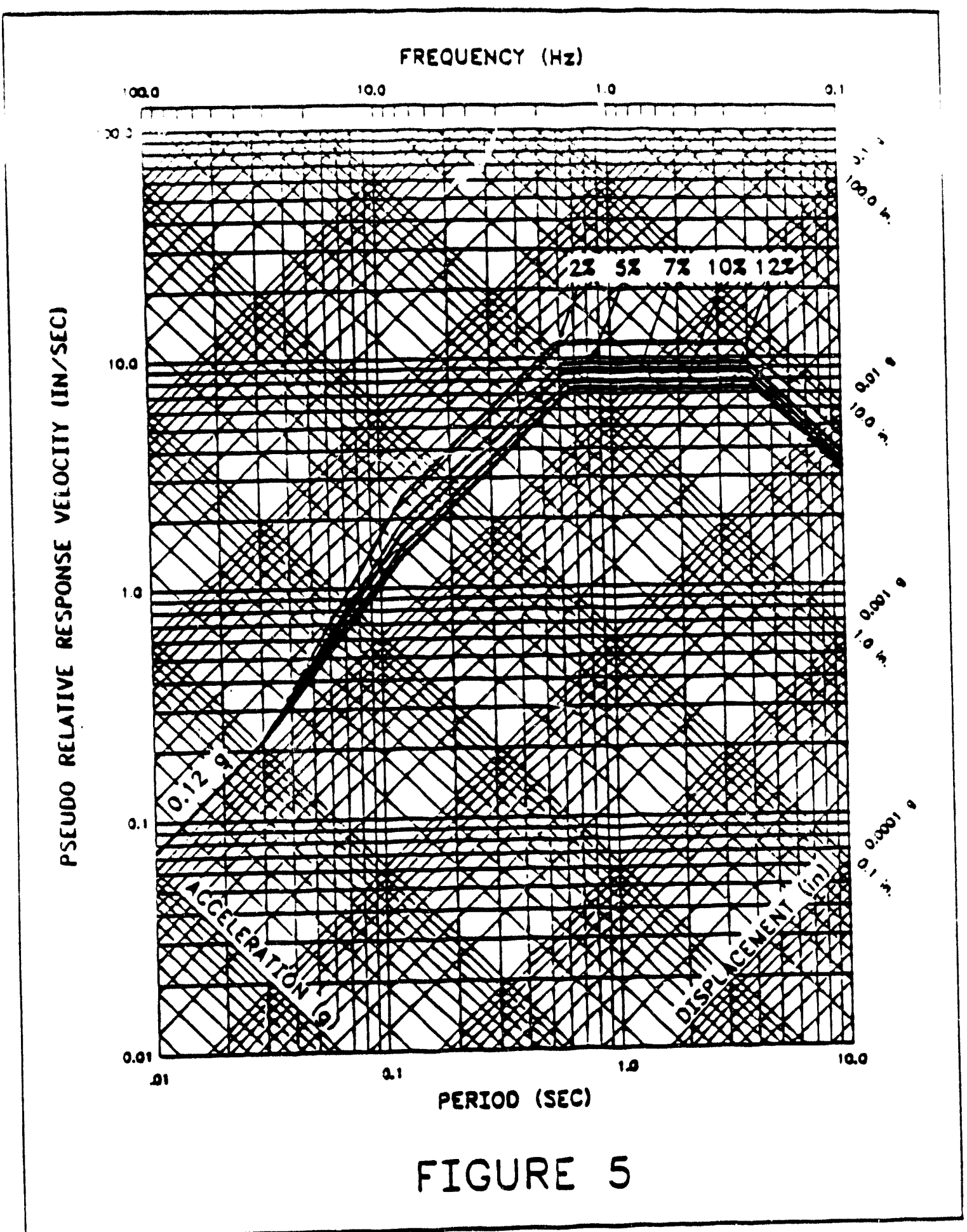

Figure 5. Response Spectra $0.12 \mathrm{~g}$, Non-reactor Safety Class 2

(2\%, 5\%, $7 \%, 10 \%$ and $12 \%$ Critical Damping) 
TABLE 2. $0.2 \mathrm{~g}$ Response-Spectra Control Points (Corresponds to Figure 3 ).

\begin{tabular}{|c|c|c|c|c|c|c|c|c|c|c|}
\hline \multirow{2}{*}{$\begin{array}{r}\text { Control } \\
\text { Point } \\
\text { Sec }\end{array}$} & \multicolumn{2}{|c|}{ 2x Damping } & \multicolumn{2}{|c|}{$5 \%$ Damping } & \multicolumn{2}{|c|}{$7 x$ Damping } & \multicolumn{2}{|c|}{$10 \%$ Damp Ing } & \multicolumn{2}{|c|}{ 12\% Damping } \\
\hline & $f(i) z)$ & $g$ & $f(1 / z)$ & $g$ & $f\left(H_{2}\right)$ & $g$ & $f(\mathrm{~Hz})$ & $g$ & $f(H z)$ & $g$ \\
\hline E $\quad 10$ & 0.10 & 0.012 & 0.10 & 0.0102 & 0.10 & 0.0095 & 0.10 & 0.0088 & 0.10 & 0.0083 \\
\hline 625 & 0.16 & 0.031 & 0.16 & 0.026 & 0.16 & 0.024 & 0.16 & 0.023 & 0.16 & 0.021 \\
\hline D 3.25 & 0.26 & 0.084 & 0.25 & 0.065 & 0.25 & 0.059 & 0.24 & 0.052 & 0.24 & 0.048 \\
\hline 25 & 0.40 & 0.129 & 0.40 & 0.104 & 0.40 & 0.095 & 0.40 & 0.087 & 0.40 & 0.080 \\
\hline 167 & 0.60 & 0.192 & 0.60 & 0.155 & 0.60 & 0.142 & 0.60 & 0.130 & 0.60 & 0.120 \\
\hline 04.1 & 1.10 & 0.351 & 1.10 & 0.283 & 1.10 & 0.261 & 1.10 & 0.237 & 1.10 & 0.220 \\
\hline C $5 \pi$ & 1.73 & 0.550 & 1.64 & 0.420 & 1.60 & 0.380 & 1.53 & 0.330 & 1.50 & 0.300 \\
\hline B .125 & 8.00 & 0.550 & 8.00 & 0.420 & 8.00 & 0.380 & 8.00 & 0.330 & 8.00 & 0.300 \\
\hline .083 & 12.00 & 0.412 & 12.00 & 0.340 & 12.00 & 0.316 & 12.00 & 0.286 & 12.00 & 0.261 \\
\hline .05 & 20.00 & 0.286 & 20.00 & 0.260 & 20.00 & 0.251 & 20.00 & 0.239 & 20.00 & 0.231 \\
\hline A .03 & 33.00 & 0.200 & 33.00 & 0.200 & 33.00 & 0.200 & 33.00 & 0.200 & 33.00 & 0.200 \\
\hline$A^{\prime} \quad .01$ & 100.0 & 0.200 & 100.0 & 0.200 & 100.0 & 0.200 & 100.0 & 0.200 & 100.0 & 0.200 \\
\hline
\end{tabular}

The response spectra accelerations for the Safety Class $10.20 \mathrm{~g}$. DBE shall be

- scaled by 0.60 to obtain the response spectra accelerations for the Safety Class 2 and 3 $0.12 \mathrm{~g} \mathrm{DBE}$; and

- scaled by 0.45 to obtain the response spectra accelerations for the Safety Class $40.09 \mathrm{~g} 0 \mathrm{BE}$. 
URS Consultants Vol. II. Rev. 0

URS/John A. Blume \& Associates, Engineers

job no. $66481-01$ job WHC/MPF tnALysis

Client

SUbject MASSES AS STATIC OOADS
Sheet No. 339

Calc. No.

Rev. No

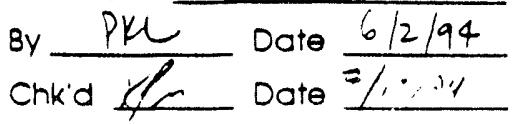

\section{AP?LY WASSES of SMIELDUG PLATE \& UANIFOLD TUBE AS TAE -AAUS}

- llass of shielding plate: $\frac{0.323^{k}}{386.4}=0.0008366$

$Z P A=0.12 \mathrm{~g} \quad, \quad F_{x}=F_{y}=F_{z}=0.12(0.0008366)(386.4)=003876^{\mathrm{K}}$

APPLY@ CENTER of 4 PIPES (NODES 41,45,81,85) $=\frac{0.03876}{4}=0.00969^{k}$

- MASS of MANIFOLI TUBE: $\frac{0.36+0.0748^{k}}{g}=\frac{0.4348}{g}$

$Z P A=0.12 \mathrm{~g} \quad F_{x}=F_{y}=F_{z}=0.12 \mathrm{~g}\left(\frac{0.4348}{\mathrm{~g}}\right)=0.0522^{\mathrm{K}}$

APPLY@NODES 121 thm 140 (20 NODEs EQuALYY), F= $\frac{0.0522}{20}=0.00261^{\mathrm{K}}$

- Mass of flange plate. $\frac{0.1263^{K}}{g}$

$Z P A=0.12 \mathrm{~g} \quad F_{x}=F_{y}=F_{z}=0.12 \mathrm{~g}\left(\frac{0.1263}{\mathrm{~g}}\right)=0.0152^{k}$

APPLY@ CENTER of 4 PIPES (NODES 141, 45, 181,185) $=\frac{0.0152}{4}=00038^{1-}$ 
URS CONSULTANTS SD-WM-DA-163
Vol. II. Rev. 0

Job \#:66481-01 Job: WHC/MPF CAMERA ASSEMBLY ANALYSIS

Client: WHC Subject: SAP90 ANALYSIS DOCUMENTATION
Sheet No. 40

By piu Date $\% / \xi \%$

Chk'd $\underline{S m}$ Date $=3,24$

SECTION 4: SAPQO/SAPSTI INPUT DATA

\section{TABLE OF CONTENTS}

\section{SECTION CONTENTS}

4.1

4.2

$4 \cdot 3$
General Discussion

Input data files for SAP9o

Input data file for SAPSTL
PAGE NO.

4.1 .1

$\Delta=1-\Delta 217$

$431-4.37$ 
URS CONSULTANTS WHC-SD-WM-DA-163

Vol. II. Rev. 0

Sheet No. 41.1

Job \#:66481-01 Job: WHC/MPF CAMERA ASSEMBLY ANALYSIS

By Phe Date $8 / 5 / 94$

Client: WHC Subject: SAP90 ANALYSIS DOCUMENTATION

Chk'd RiKDate?/S/9

\subsection{General Discussion}

File Naming Convention:

The SAP9O or SAPSTL input data files are named using the following file naming convention, for example:

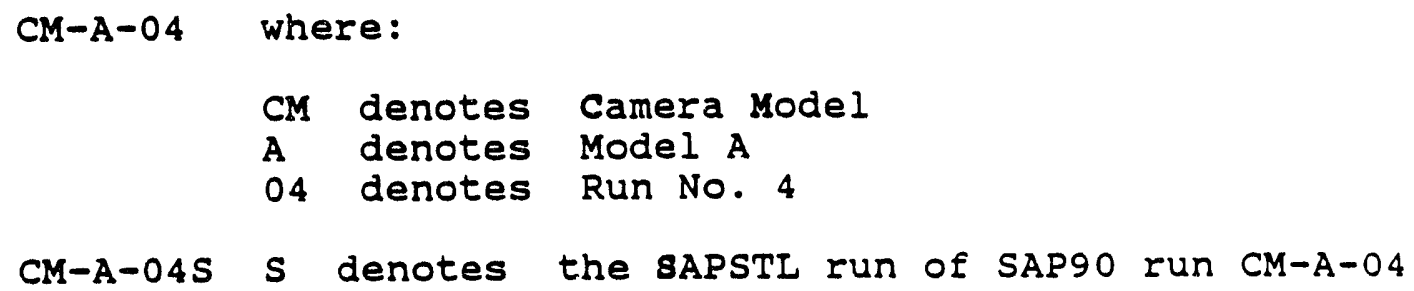

At the beginning of each input data file, comment lines are provided for documentation purposes. Also, the input parameters for each section are commented for easy reference. See Page 4.2.2 for sample input data file.

\section{History of Computer Runs:}

There were several intermediate SAP9o runs before the model was finalized. The final runs were:

Model A: Camera assembly unit only, with restrains in all 6 directions at the center of Flange plate

$$
\begin{aligned}
& \text { Run Ids: CM-A-04 SAP9O file } \\
& \text { CM-A-04S SAPSTL file for } C M-A-04
\end{aligned}
$$

Model B: Camera assembly unit is combined with the MPF riser model with the node at 20 "riser share the same node with at the center of Flange plate from the camera assembly unit model.

Run Ids: CM-B-01 SAP90 file

\section{PC Diskettes}

For the intermediate SAP9o runs, only the input data files are saved on diskettes. For the $C M-A-04$ and $C M-B-01$ runs, both input and output files are saved for future references. 
URS CONSULTANTS WHC-SD-WM-DA-163

Vol. II. Rev. 0

Sheet No. 42.1

Job \#:66481-01 Job: WHC/MPF CAMERA ASSEMBLY ANALYSIS

By PRCDate-12:94

Clieat: WHC

Subject:

SAP90 ANALYSIS DOCUMENTATION

Chk'd Lim Date = ?

4.2 Listing of Input Data File

The echoprint of the input data files, $C M-A-04$ and $C M-B-01$ are given in this section. 
06/02/1994 17:12

Filenam: $\mathrm{CH}-\mathrm{A}-04$

Page 1

MHC/MPF CAMERA ASSEMBLY MOOEL JOBH: 66458-01 RUN: CH-A-04

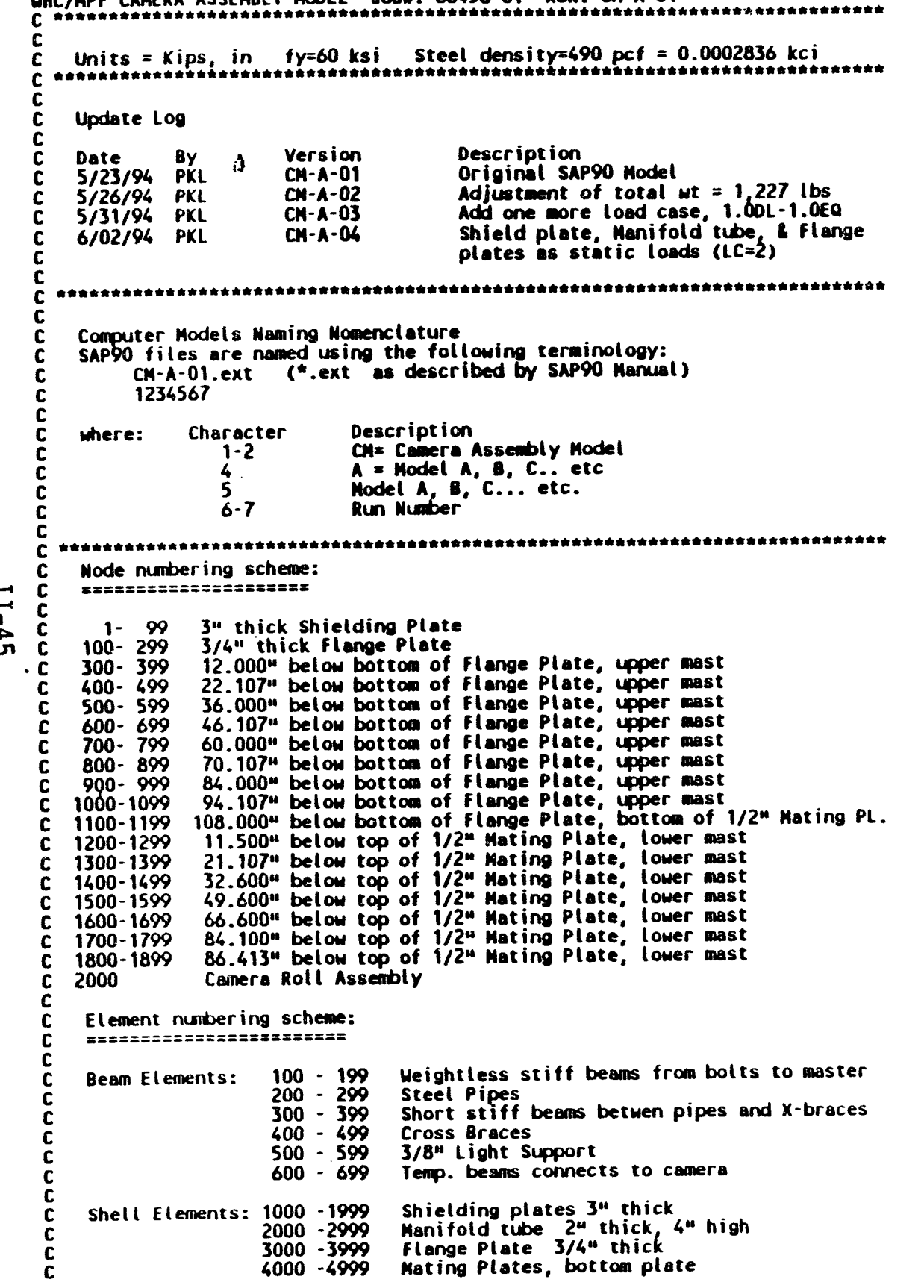

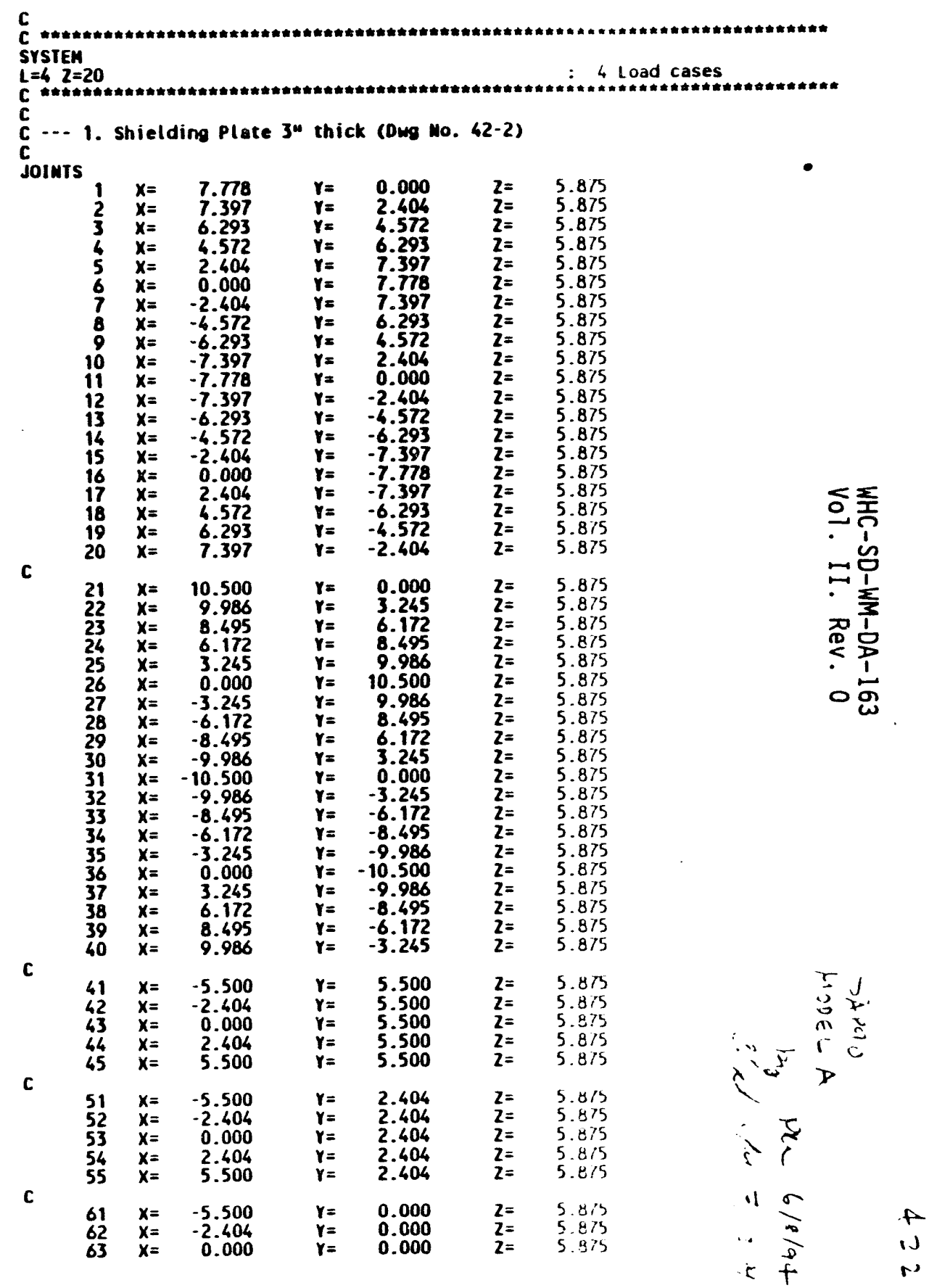


WHC-SD-WM-DA-163

Vol. II. Rev. 0

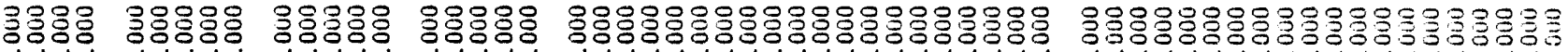

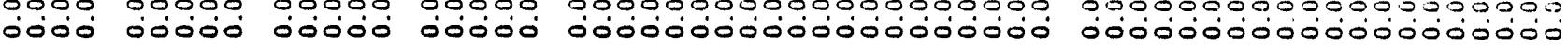

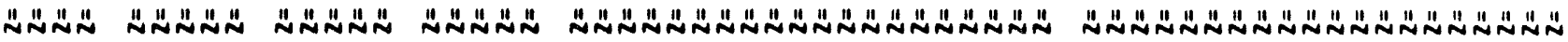

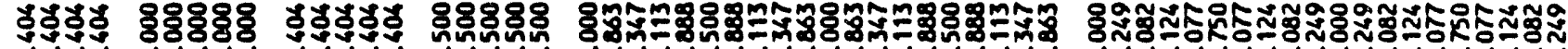

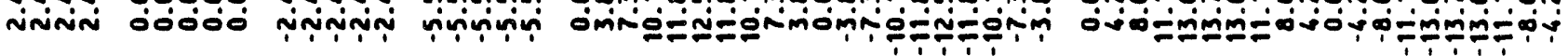

는

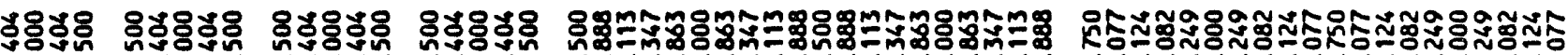

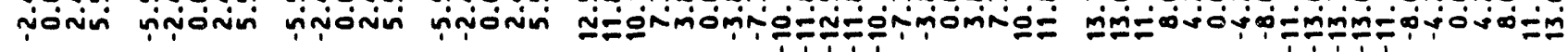

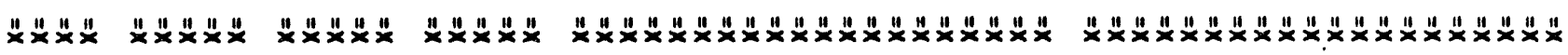

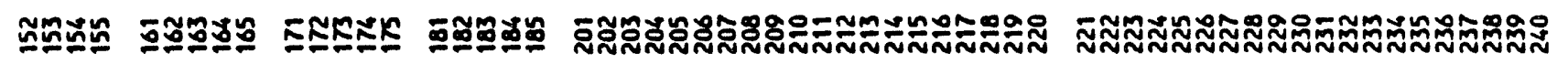
西

$$
\text { 西 }
$$

$\checkmark$

$\omega$

$\omega$

$\omega$

$u$

u

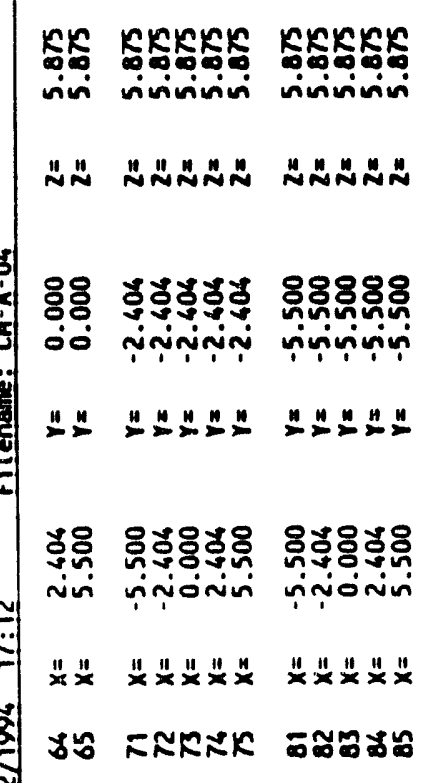

$u$

טuט

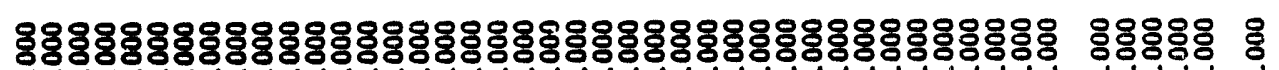

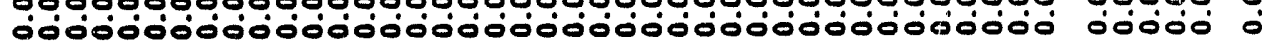

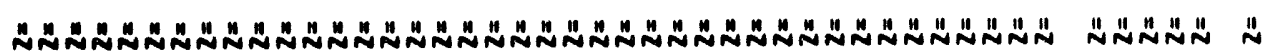

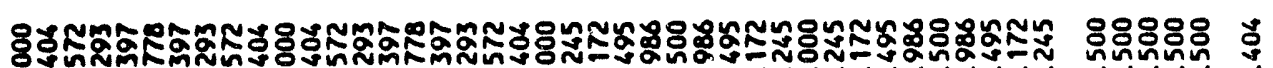

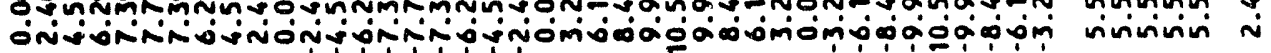

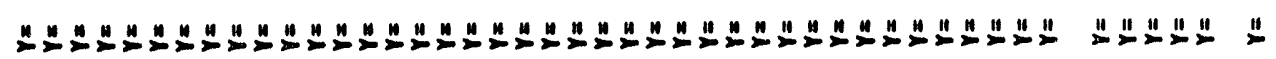

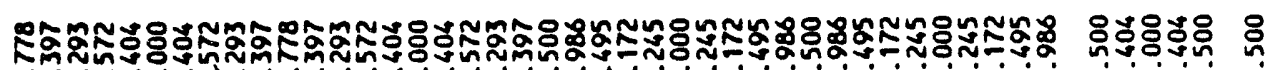

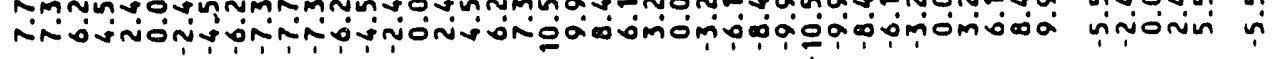

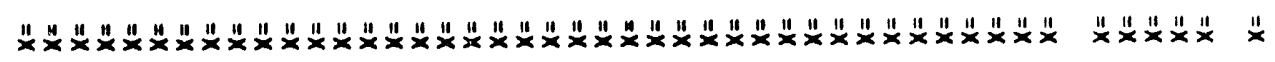

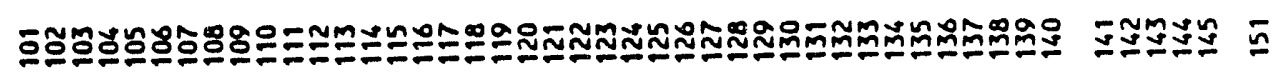
II -46 
WHC-SD-WM-DA-163

Vol. II. Rev. 0

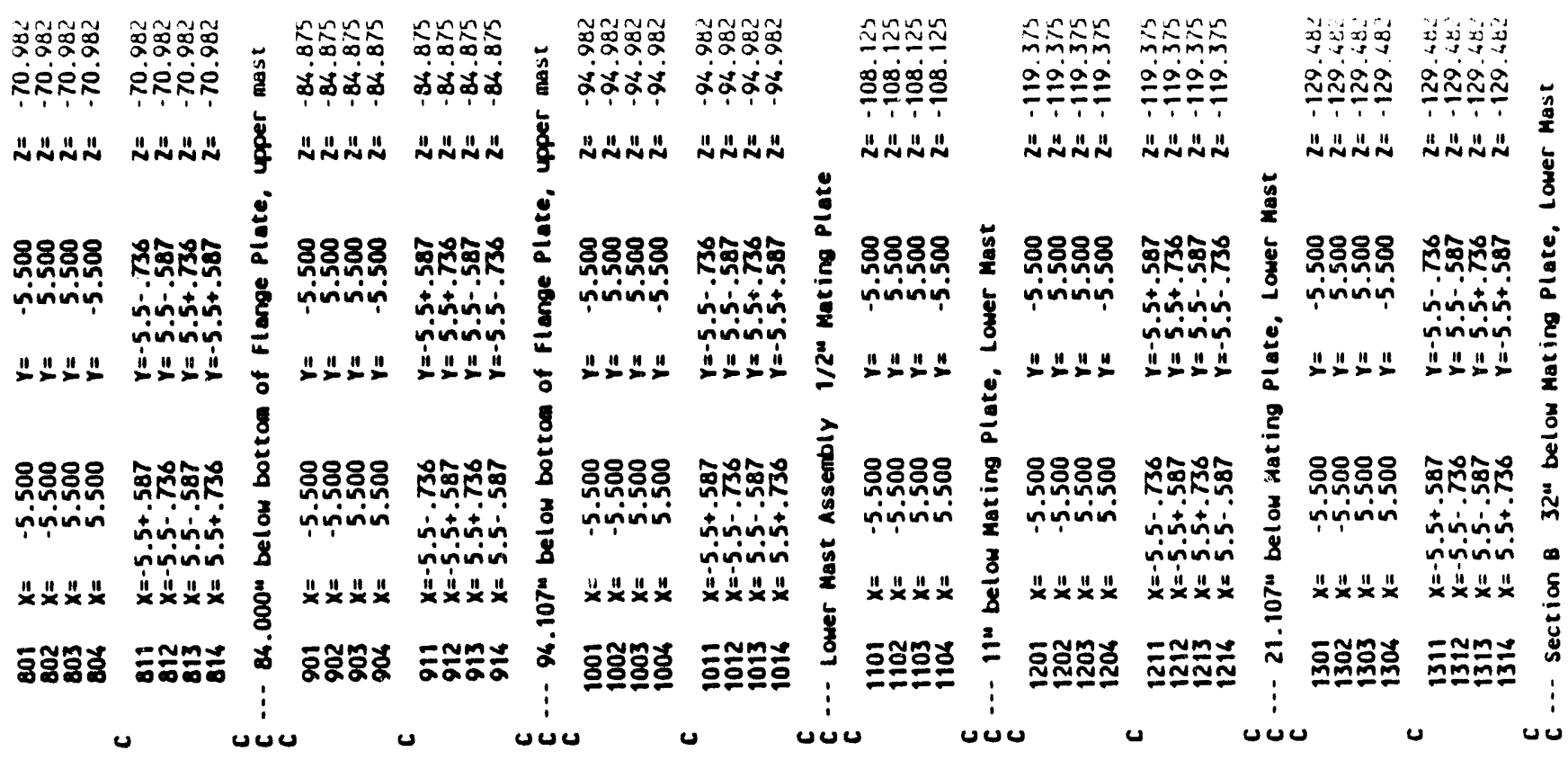

영

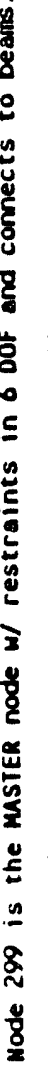

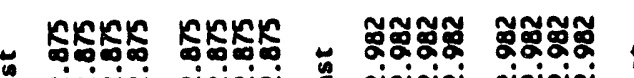

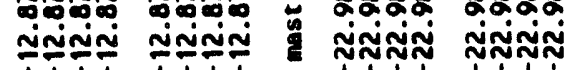

ckck sim

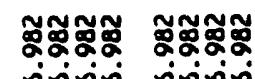

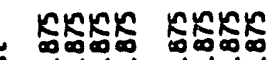

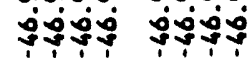

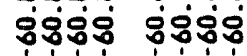

药

0
0

-1
0

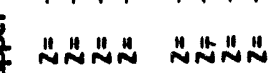

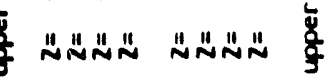

:

goso

8888 คำ

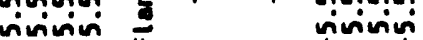

rivining

\section{\%}

जinninin

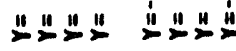

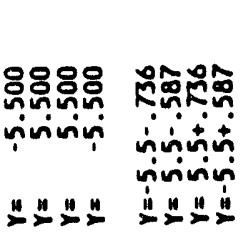

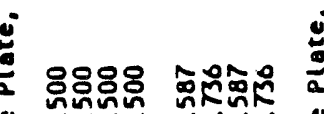

vivivin ininining

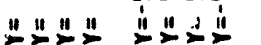

눈

$\geq 2 x+2 \pm 2 \div$

要

政

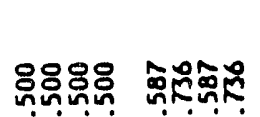
ninivini $\overline{8}$

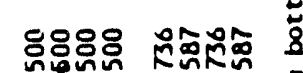

rivinum inininin $\frac{3}{8}$

vinivin

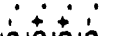
ninivin 8

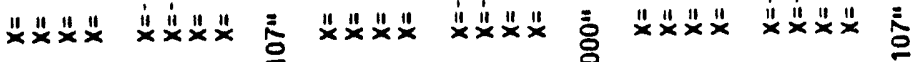

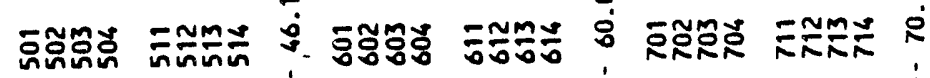

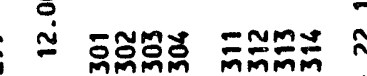

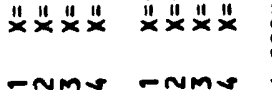

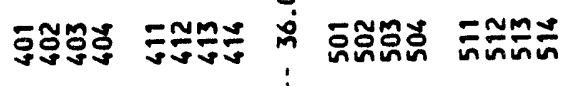


$06 / 02 / 1994 \quad 17: 12$

Filename: $\mathrm{CM}-\mathrm{A}-\mathrm{O}$

Page 7

$\begin{array}{llrlrl}1401 & X= & -5.500 & y= & -5.500 & z=-141.083 \\ 1402 & x= & -5.500 & Y= & 5.500 & Z=-141.083 \\ 1403 & X= & 5.500 & \gamma= & 5.500 & z=-141.083 \\ 1404 & X= & 5.500 & Y= & -5.500 & 2=-141.083\end{array}$

C ... Section C 49" below Mating Plate, Lower Mast

$\begin{array}{rrrrrr}1501 & x= & -5.500 & y= & -5.500 & z=-158.083 \\ 1502 & x= & -5.500 & y= & 5.500 & z=-158.083 \\ 1503 & x= & 5.500 & y= & 5.500 & 2=-158.083 \\ 1504 & x= & 5.500 & y= & -5.500 & z=-158.083\end{array}$

C -.. Section D 115 w below Mating Plate, Lower Mast

$\begin{array}{llrlrl}1601 & x= & -5.500 & \gamma= & -5.500 & z=-175.083 \\ 1602 & x= & -5.500 & \gamma= & 5.500 & z=-175.083 \\ 1603 & x= & 5.500 & \gamma= & 5.500 & z=-175.083 \\ 1604 & x= & 5.500 & \gamma= & -5.500 & z=-175.083\end{array}$

C -.. Section A Upper portion, oh.1* below Mating Plate, Lower Mast$$
\begin{array}{rrrrrr}
1701 & x= & -5.500 & \gamma= & -5.500 & z=-191.975 \\
1772 & x= & -5.500 & \gamma= & 5.500 & 2=-191.975 \\
1703 & x= & 5.500 & y= & 5.500 & z=-191.975 \\
1704 & x= & 5.500 & \gamma= & -5.500 & z=-191.975
\end{array}
$$

C --- Section A Bottom plate, 96.6 " below Mating flate, Lower Mast

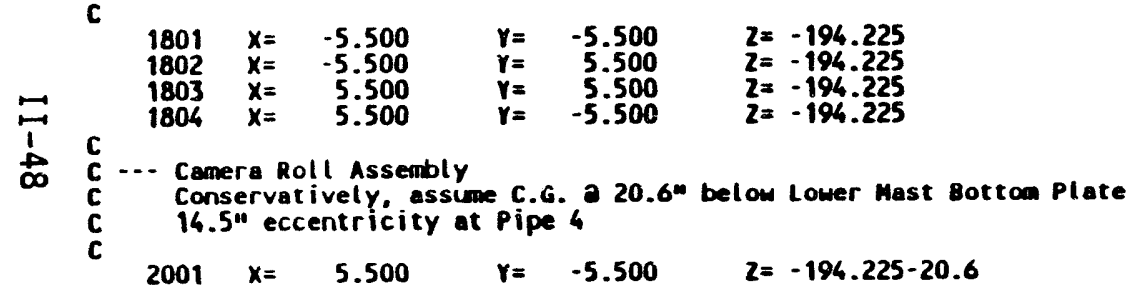

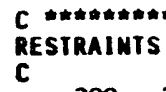

$2992991 R=1,1,1,1,1,1$

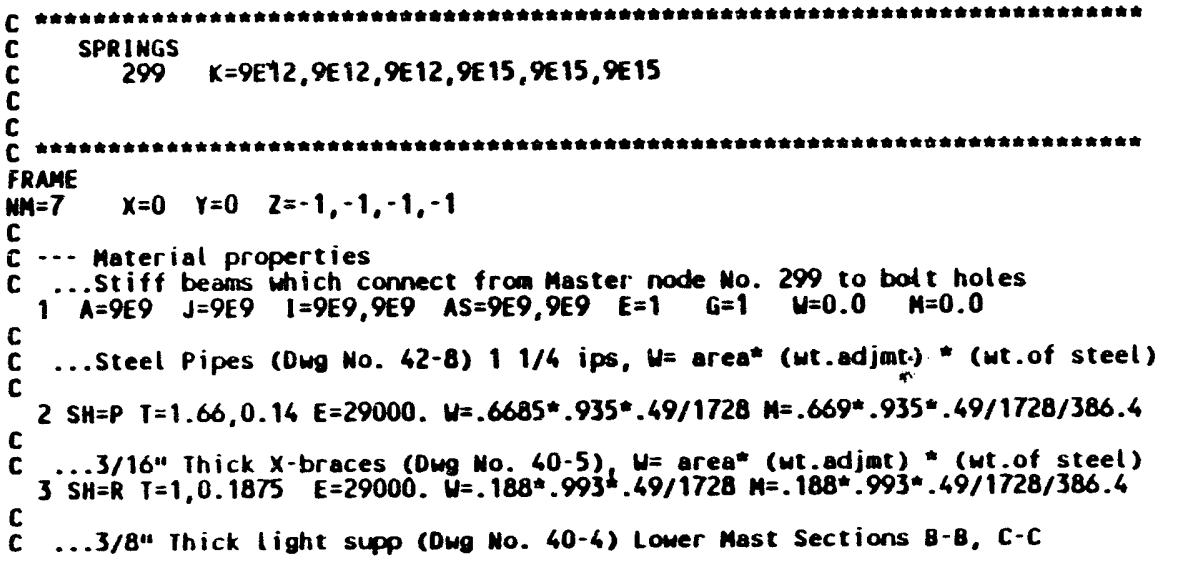

06/02/1994 17:12 Fil enase: CM-A-04

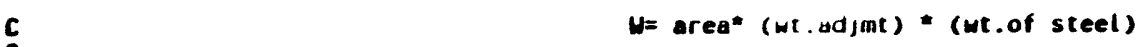

${ }^{4} S_{S H}=R \quad I=1,0.375 \quad E=29000 . \quad W=.375^{*} .88^{*} .49 / 1728 \quad M=.375 * .88^{*} .49 / 1728 / 386.4$

C ...short link from center of pipes to braces

C $A=9 E 4 \quad J=9 E 6$ I=9E6,9E6 AS=9E6,9E6 E=1. $G=1 . \quad N=0.0 \quad M=0.0$

C ...Gas Pipes (Dwg Mo. 40-12) Lower Mast Sect ion A-A

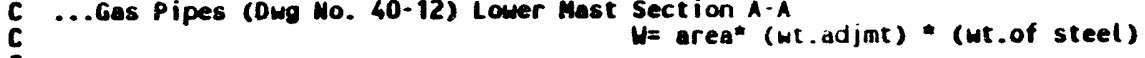

6 SH=P $T=0.675,0.15 \quad E=29000 . W=.247^{*} .867^{*} .49 / 1728 M=.247^{*} .867^{*} .49 / 1728 / 386.4$

C...stiff beans wich connect from lower ast bottom plate to C.G. of camera

C 7 A=9E5 J=9E7 I=9E7, 9E7 AS=9E5, gES E=1 G=1 $\quad W=0.0 \quad M=0.0$

C -.. Weightless stiff beans connect from bolt holes tu Master node 299

\begin{tabular}{|c|c|c|c|c|}
\hline $\begin{array}{ll}101 & 299 \\
102 & 299 \\
103 & 299 \\
104 & 299 \\
105 & 299 \\
106 & 299 \\
107 & 299 \\
108 & 299 \\
109 & 299 \\
110 & 299 \\
111 & 299 \\
112 & 299 \\
113 & 299 \\
114 & 299 \\
1115 & 299 \\
1116 & 299 \\
117 & 299 \\
118 & 299 \\
119 & 299\end{array}$ & 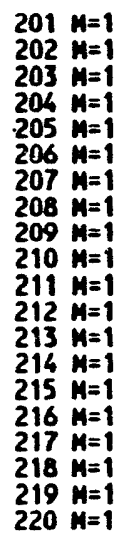 & 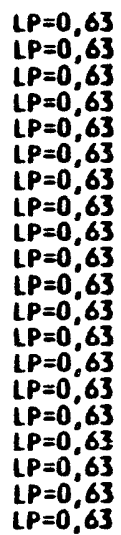 & $\begin{array}{l}\vdots \\
\vdots \\
\vdots \\
\vdots \\
\vdots \\
\vdots\end{array}$ & 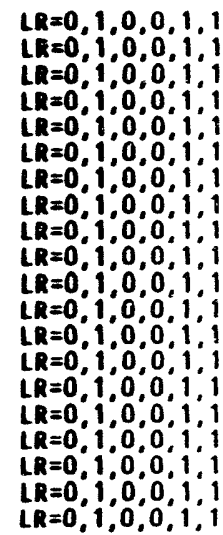 \\
\hline
\end{tabular}

C -... steel Pipes

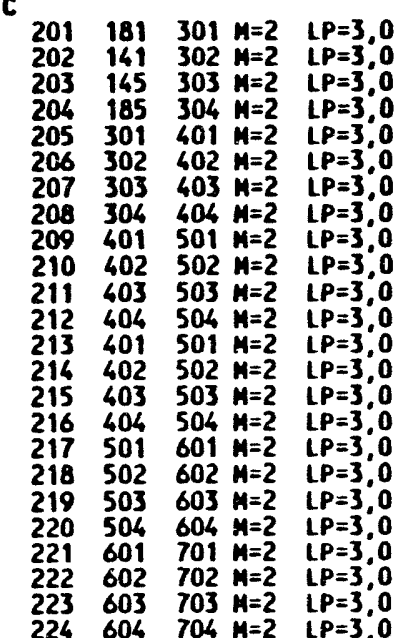


WHC-SD-WM-DA-163

Vol. II. Rev. 0

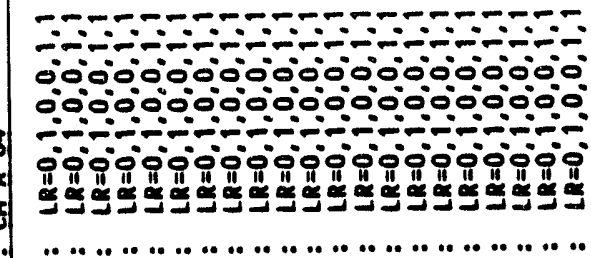

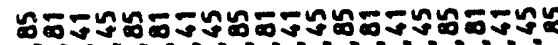

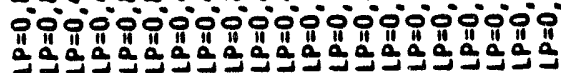

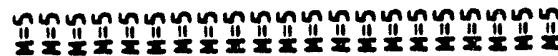

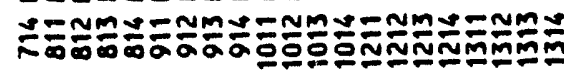

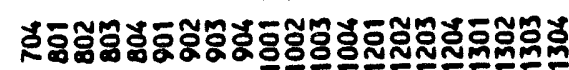

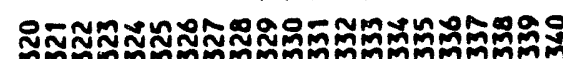

ニำ 00000000000000 อง

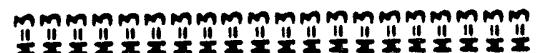

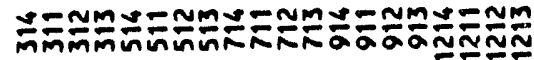

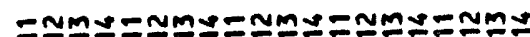

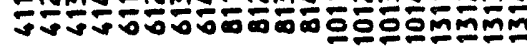
бำํำ uU

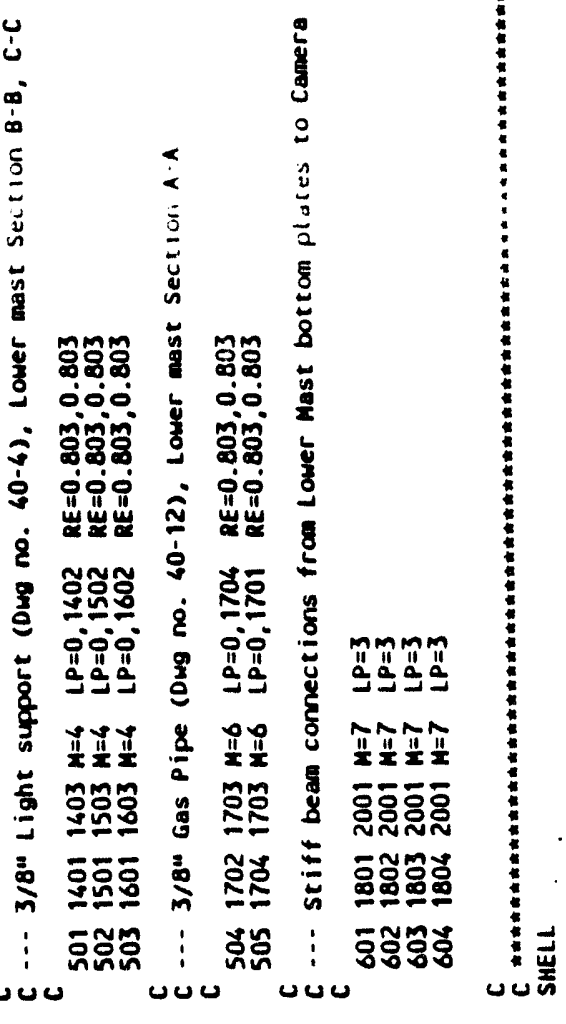

00000000000000000000000000000000000000000000000

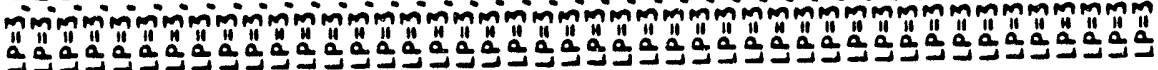

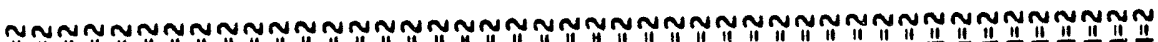

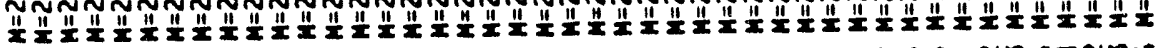

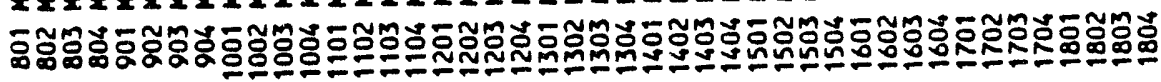

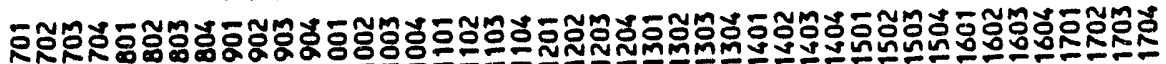
ะ

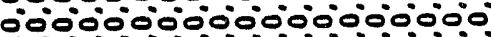
o

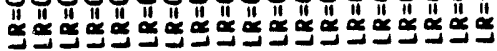

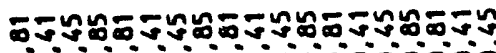
o0 $0^{\circ} 0^{\circ} 0^{\circ} 0^{\circ} 0^{\circ} 0^{\circ} 0^{\circ} 0^{\circ} 0^{\circ}$ 药

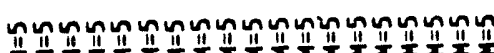

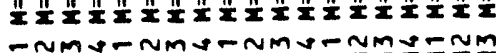

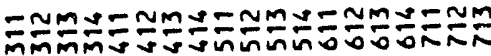

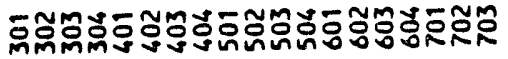
ธำ טu' 


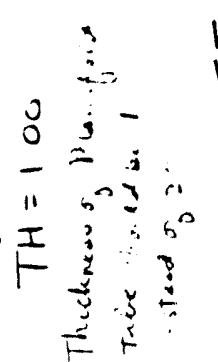

WHC-SD-WM-DA-163

Vol. II. Rev. 0

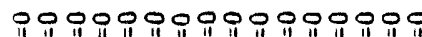
åa

0000000000000000

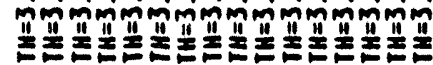

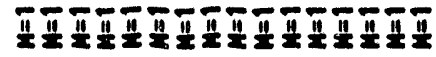

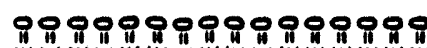

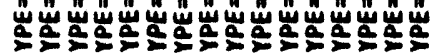

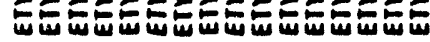

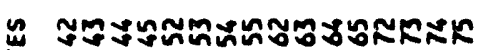

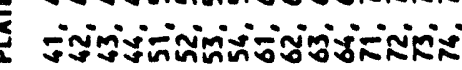

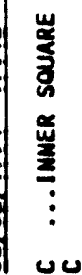

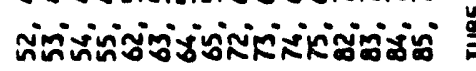

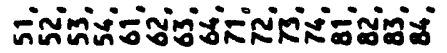

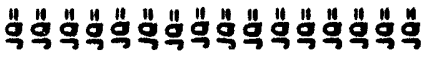

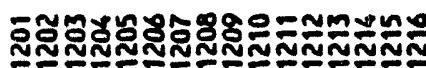

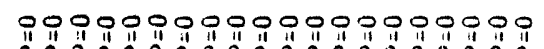

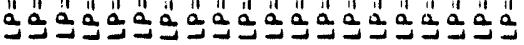

† 88888888888888888888

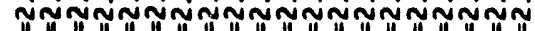

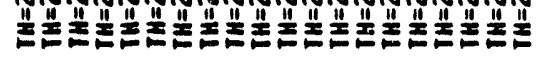

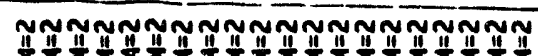

ż

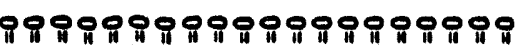

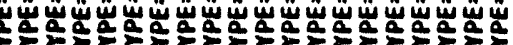

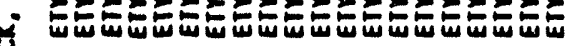

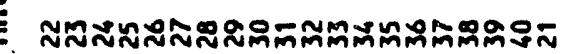

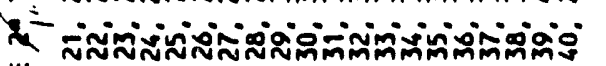

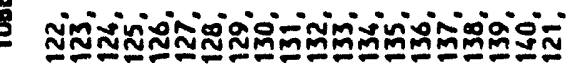

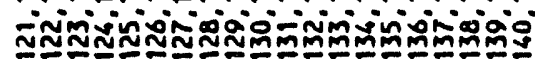

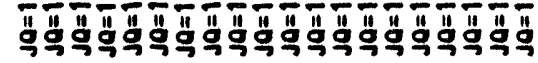

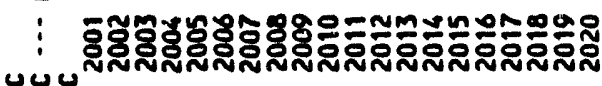

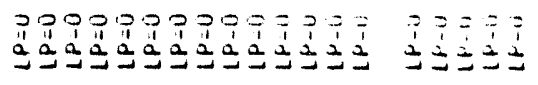

KKKKKKKKKKNKKK KNKKK

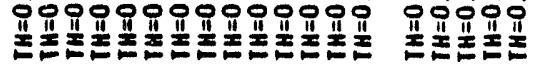

minmmmmmmmmm

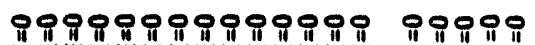

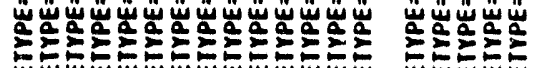

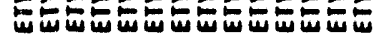

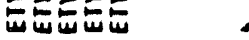

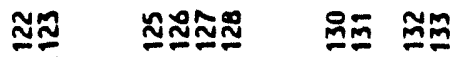

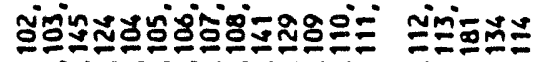

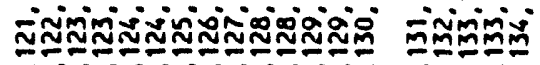

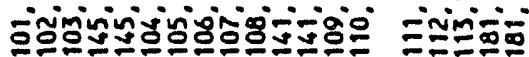

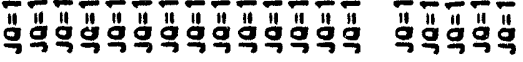

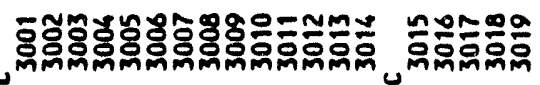

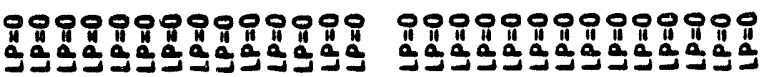

00000000000000 minning

00000000000000 minnminmminmmingm

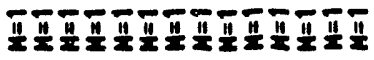

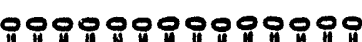

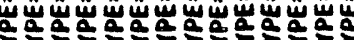

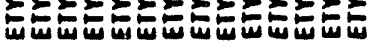

กผ กัก๊ิ

ㅇำ ำก

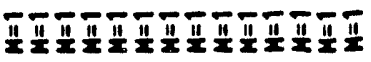
Nimis

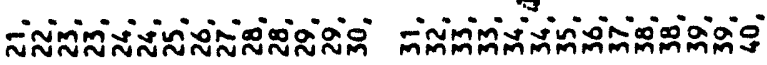

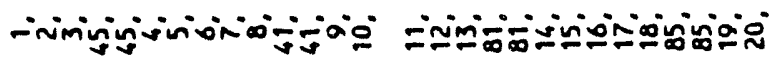

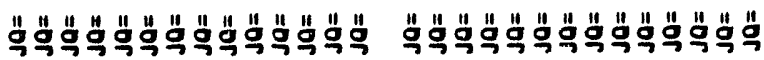

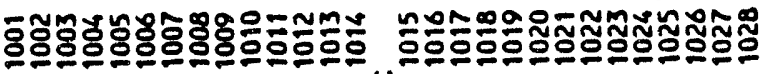

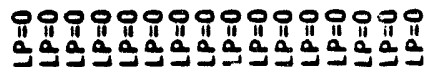

0000000000000000

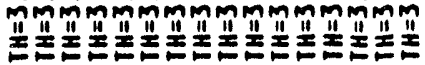

峞

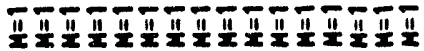

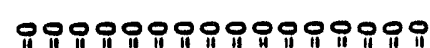

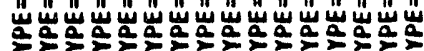

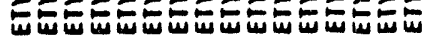

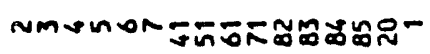
ทีง - invن ติنง و

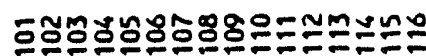


06/02/1994 17:12 Fil ename: $\mathrm{CH}-\mathrm{A}-06$

Page 13

$3020 \quad J 0=114,134,115,135$ ETYPE $=0 \quad M=3 \quad I M=0.75 \quad L P=0$

$3021 \quad J 0=115,135,116,136 \quad$ EIYPE $=0 \quad M=3 \quad T H=0.75 \quad$ LP $=0$

$3023 \quad J 0=117 ; 137 ; 118,138$ EIYPE=0 $M=3 \quad M=0.75 \quad L P=0$

$3024 \quad J 0=118^{\prime}, 138,185$ ETYPE $=0 \quad M=3 \quad$ I $M=0.75 \quad L P=0$

$3025 \quad J 0=185,138,139 \quad$ EIYPE $=0 \quad M=3 \quad$ I $H=0.75 \quad L P=0$

$3026 \quad J 0=185,139,119 \quad$ ETYPE $=0 \quad M=3 \quad T H=0.75 \quad L P=0$

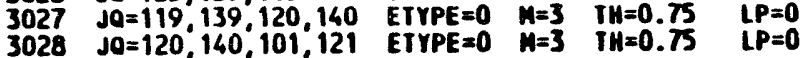

C ... CIRCULAR S SQUARE BOUMDARY PLATES

$3101 \quad J Q=165,101,155,102$ ETYPE $=0 \quad M=3 \quad T H=0.75 \quad L P=0$

$102 \quad J 0=155,102,145,103$ ETYPE $=0 \quad M=3 \quad$ I $M=0.75 \quad$ LP=0

$103 \quad J 0=144,145,105,104$ ETYPE $=0 \quad M=3 \quad I M=0.75 \quad L P=0$

EIYPE $=0 \quad M=3 \quad T H=0.75 \quad L P=0$

$3106 \quad J Q=141,142,108,107$ EIYPE=0 $M=3 \quad T H=0.75 \quad L P=0$

$3107 \quad J Q=110,151,109,141$ ETYPE $=0 \quad M=3 \quad$ TH=0.75 $\quad L P=0$

J108 Ja=111,161,110,151 EIYPE=0 $M=3 \quad T H=0.75 \quad$ LP=0

$3109 \quad J Q=112,171,111,161$ ETYPE $=0 \quad M=3 \quad T H=0.75 \quad L P=0$

$3110 \quad J a=113,181,112,171$ EIYPE $=0$ Mas $\quad M=0.75 \quad L P=0$

$3111 \quad J 0=114,115,181,182 \quad$ EIPPE $=0 \quad M=3 \quad T H=0.75 \quad L P=0$

作

3114 JQ=117, 118, 184, 185 ETYPE=0 $\quad M=3 \quad T H=0.75 \quad I P=0$

$3115 \quad M=185,119,175,120$ ETYPE $=0 \quad M=3 \quad I H=0.75 \quad L P=0$

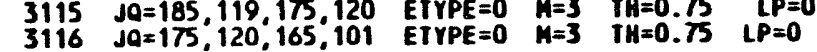

C ... IMMER square plates

$3201 \quad J Q=151,152,141,142$ ETYPE $=0 \quad M=3 \quad I H=0.75 \quad L P=0$

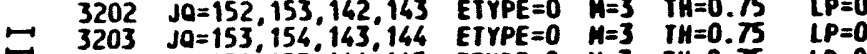

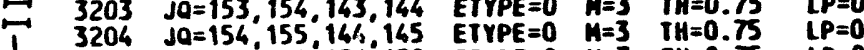

G 3205 JO=161, 162, 15i,152 ETYPE $=0 \quad M=3 \quad$ IH $=0.75 \quad$ LP=0

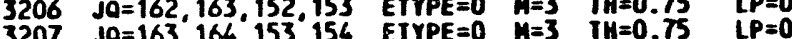

3208 J0164, 165, 156, 155 EIYPE=0 $M=3 \quad T H=0.75 \quad$ LP=0

$3209 \quad J 0=171,172,161,162$ ETYPE $=0 \quad M=3 \quad T H=0.75 \quad L P=0$

$3210 \quad J Q=172,173,162,163$ ETYPE $=0 \quad M=3 \quad$ TH=0.75 LP=0

$3211 \quad J Q=173,174,163,164$ EIYPE $=0 \quad H=3 \quad T H=0.75 \quad L P=0$

$3212 \quad J Q=174,175,164,165$ ETYPE $=0 \quad H=3 \quad T H=0.75 \quad L P=0$

$3213 J 0=181,182,171,172 \quad$ EIYPE $=0 \quad K=3 \quad T M=0.75 \quad L P=0$

$3214 \mathrm{JO}=182,183,12,173 \quad$ ETrPE $=0 \quad M=3 \quad T M=0.75 \quad L P=0$

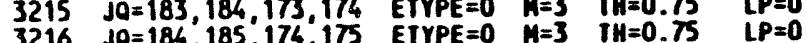

C... 2ND TO THE OUIER RIMG (BOLT LOCATIONS)

$3301 \quad J 0=121,201,122,202$ ETYPE $=0 \quad M=3 \quad T H=0.75 \quad L P=0$

$3302 J 0=122,202,123,203$ EIYPE $=0 \quad M=3 \quad M=0.75$

$3303 \quad J O=123,203,124,204$ ETYPE $=0 \quad M=3 \quad T H=0.75 \quad L P=0$

$3304 \mathrm{~J} Q=124,204,125,205$ EIYPE $=0 \quad H=3 \quad$ TH=0.75 LP L

$3305 \quad J Q=125,205,126,206 \quad$ ETYPE $=0 \quad M=3 \quad$ I $H=0.75 \quad L P=0$

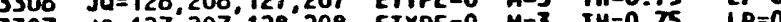

J

N $10=129,209,130,210$ ETYPE $=0 \quad M=3 \quad T H=0.75 \quad L P=0$

$3310 \mathrm{~J} O=130,210,131,211$ ETYPE $=0 \quad M=3 \quad$ IH=0.75 $\quad$ LP=0

$3311 \quad 0=131,211,132,212 \quad$ ETYPE $=0 \quad M=3 \quad$ TH=0.75 LP=0

$3312 \quad J Q=132,212,133,213$ ETYPE $=0 \quad M=3 \quad I H=0.75 \quad L P=0$

$3313 \quad J 0=133,213,134,214 \quad$ ETYPE $=0 \quad M=3 \quad H H=0.75 \quad L P=0$

$3314 \quad J 0=134,214,135,215 \quad$ ETYPE=0 $\quad M=3 \quad M \quad M=0.75 \quad L P=0$

06/02/1994 17:12 Fillename: $\mathrm{CH}-\mathrm{A}-\mathrm{OC}$

$3316 \quad J 0=136,216,137,217$ ETYPE $=0 \quad M=3 \quad T M=0.75 \quad L P=0$

$3317 \quad J Q=137,217,138,218$ EIYPE $=0 \quad M=3 \quad I H=0.75 \quad L P=0$

$3318 \quad J Q=138,218,139,219 \quad$ EIYPE $=0 \quad M=3 \quad T M=0.75 \quad$ LP $\quad M=0$

$3320 \quad J Q=140,220,121,201$ EIYPE $=0 \quad M=3 \quad I M=0.75 \quad$ IP=0

C ... OUTER RIMG

C $3401 \quad J Q=201,221,202,222$ ETYPE $=0 \quad M=3 \quad T M=0.75 \quad L P=0$

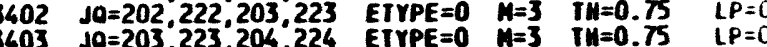

$3406 \quad J 0=204,224,205,225$ ETYPE $=0 \quad M=3 \quad$ TM=0.75 $\quad L P=0$

$3405 \quad J 0=205,225,206,226$ ETYPE $=0 \quad M=3 \quad T H=0.75 \quad L P=0$

$3406 \quad J Q=206,226,207,227$ ETYPE $=0 \quad K=3 \quad T H=0.75 \quad$ I $P=0$

$3607 \quad J O=207,227,208,228 \quad$ EIYPE $=0 \quad M=3 \quad I M=0.75 \quad L P=0$

$3408 J a=208,228,209,229$ EIrPE $=0 \quad M=3 \quad$ I $M=0.75 \quad L P=0$

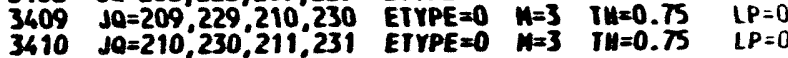

C $3411 \quad 0=211,231, \quad M=3 \quad I M=0.75 \quad L P=0$

$3411 \mathrm{JQ}=211,231,212,232$ ETrPE=0 $\quad M=3 \quad T H=0.75 \quad L P=0$

3413

$3416 \quad 10=214,234,215,235$ ETYPE $=0 \quad M=3 \quad$ TM=0.75 $\quad L P=0$

$3415 \quad J 0=215,235,216,236$ EIYPE $=0 \quad M=3 \quad T M=0.75 \quad$ LP=0

$3416 \quad J Q=216,236,217,237$ ETYPE $=0 \quad M=3 \quad T H=0.75 \quad$ LP=0

$3417 \quad J 0=217,237,218,238$ EIYPE $=0 \quad M=3 \quad$ IM=0.75 $\quad$ LP=0

$3418 \quad J Q=218,238,219.239$ ETrPE $=0 \quad M=3 \quad I M=0.75 \quad L P=0$

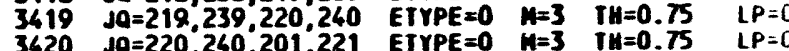

C... Mating Plate Upper/lower Mast, 1/2" thick

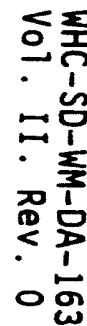

c ... Bottom Plote, 1/2" thick

$4002+10=1801,1804,1802,1803$ ETYPE $=0 \quad H=5 \quad$ IH $H=0.5 \quad$ t $F=0$

Lonos

tonos

C ... Dead Load of Camere

$2001 \quad L=1 \quad f=0,0,-.128$

C -.. Masses of Shield plate applied at center of 4 ss pipes

$41 L=2 \quad F=.00969, .00969, .00969$

$45 \quad L=2 \quad F=.00969, .00969, .00969$

$81 \quad L=2 \quad F=.00969, .00969, .00969$

$85 \quad L=2 \quad F=.0096$

C -.. Masses of Manifold Tube applied at Modes $121 \mathrm{thr}$, 140 equally

$121 \quad L=2 \quad F=.00261, .00261, .00261$

$122 \quad L=2 \quad F=.00261, .00261 \ldots .00261$

$124 \quad L=2 \quad F=.00261, .00261, .0026$

$125 \quad l=2 \quad F=.00261, .00261, .00261$

$126 \quad L=2 \quad F=.00261, .00261, .0026$

$127 \quad L=2 \quad F=.00261, .00261, .0026$

$28 \quad l=2 \quad f=.00261, .00261, .00261$

$130 \quad l=2 \quad F=00261,00261.00261$

$131 \quad l=2 \quad F=.00261, .00261, .00261$ 
06/02/1994 17:1 Fil eneme: $\mathrm{CM}-\mathrm{A}-\mathrm{OC}$

$133 L=2 \quad F=.00261, .00261, .0026$

$134 \mathrm{~L}=2 \quad F=.00261, .00261, .0026$

$35 \quad L=2 \quad F=.00261, .00261, .0026$

$137 \quad L=2 \quad f=.00261, .00261, .0026$

$38 \quad L=2 \quad F=.00261,00261, .0026$

t $=2 \quad F=.00261, .00261, .0026$

C -.. Masses of Flange plate applied at center of the 6 ss pipes

$141 \mathrm{~L}=2 \quad f=.0038, .0038, .0038$

$145 \quad L=2 \quad F=.0038, .0038, .0038$

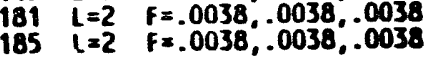

\section{CMSSES}

WO01 $H=128 / 386.4, .128 / 386.4, .128 / 386.4$

SPEC

$$
S=386.4 \quad A=0.0 \quad D=0.02
$$

C

Mon-reactor Safety Class 2 2X damped Design Spectrun from Figure 3 and C Table 2, scaled by 0.6, SDC 4.1 Revision 12, Design Laeds for Facilities

c

$\begin{array}{llll}3.84615 & 0.05040 & 0.05040 & 0.05040\end{array}$

4.447120 .037410 .037410 .03741

$\begin{array}{llll}5.64904 & 0.02289 & 0.02289 & 0.02289\end{array}$

$\begin{array}{llll}6.25000 & 0.01860 & 0.01860 & 0.01860\end{array}$

$\begin{array}{llll}7.18750 & 0.01403 & 0.01403 & 0.01403\end{array}$

8.125000 .010950 .010950 .01095

$\begin{array}{rrrr}9.06250 & 0.00878 & 0.00878 & 0.00878 \\ 10.00000 & 0.00720 & 0.00720 & 0.00720\end{array}$

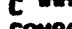

$C=1, \quad: D L$

$C=0.1 \quad D=1.0$ : Masses of Plates + Spectre

$\begin{array}{ll}C=1,1 & D=1.0\end{array}: D L$ + (Masses of Plotes) + Spectra

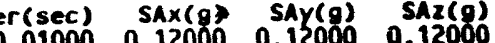

0.015080 .12000 0.12000 0.12000

$0.020150 .12000 \quad 0.12000 \quad 0.12000$

$0.02523 \quad 0.12000 \quad 0.12000 \quad 0.12000$

$\begin{array}{llll}0.03030 & 0.12000 & 0.12000 & 0.12000 \\ 0.03523 & 0.13362 & 0.13362 & 0.13362\end{array}$

$\begin{array}{llll}0.03523 & 0.13362 & 0.13362 & 0.13562 \\ 0.04015 & 0.14671 & 0.14671 & 0.14671\end{array}$

$0.04508 \quad 0.15935 \quad 0.159350 .15935$

0.05000 0.1760 0.171600 .17160

$\begin{array}{lllll}0.05833 & 0.191076 & 0.21076 & 0.21076\end{array}$

$\begin{array}{lllll}0.07500 & 0.22927 & 0.22927 & 0.22927\end{array}$

$\begin{array}{lllll}0.08333 & 0.24720 & 0.24720 & 0.24720\end{array}$

0.093750 .268840 .268840 .26884

$\begin{array}{lllll}0.10417 & 0.28980 & 0.28980 & 0.28980 \\ 0.11458 & 0.31016 & 0.31016 & 0.31016\end{array}$

$\begin{array}{llll}0.11458 & 0.31016 & 0.31016 & 0.31016 \\ 0.12500 & 0.33000 & 0.33000 & 0.33000\end{array}$

$\begin{array}{lllll}0.23826 & 0.33000 & 0.33000 & 0.33000\end{array}$

$\begin{array}{llll}0.35152 & 0.33000 & 0.33000 & 0.33000\end{array}$

$\begin{array}{llll}0.46478 & 0.33000 & 0.33000 & 0.33000\end{array}$

0.578030 .33000 0.33000 0.33000

$\begin{array}{llllll}0.74356 & 0.25706 & 0.25706 & 0.25706\end{array}$

0.826330 .231510 .231510 .23151

$\begin{array}{lllll}0.90909 & 0.21060 & 0.21060 & 0.21060\end{array}$

1.098480 .174440 .174440 .17446

$\begin{array}{lllll}1.28788 & 0.14890 & 0.14890 & 0.14890\end{array}$

$\begin{array}{llll}1.47727 & 0.12990 & 0.12990 & 0.12990\end{array}$

1.060670 .115200 .115200 .11520

1.083330 .092560 .002560 .09256

2.063330 .092560 .092560 .09256

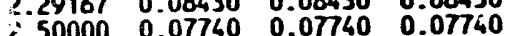

$\begin{array}{lllll}2.83654 & 0.06825 & 0.06825 & 0.06825\end{array}$

$\begin{array}{llll}3.17308 & 0.06104 & 0.06104 & 0.06104 \\ 3.50962 & 0.05529 & 0.05521 & 0.05521\end{array}$ 


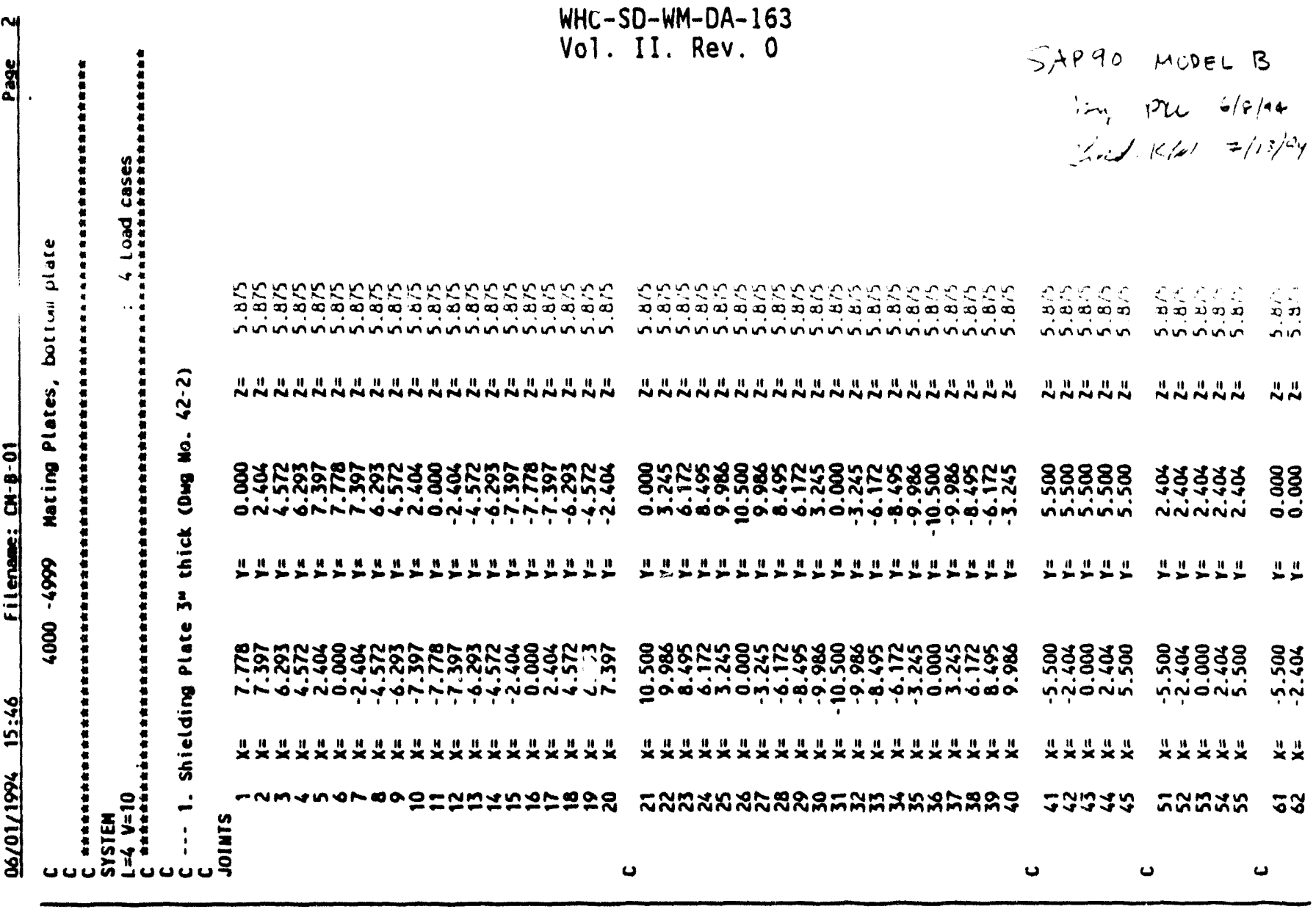

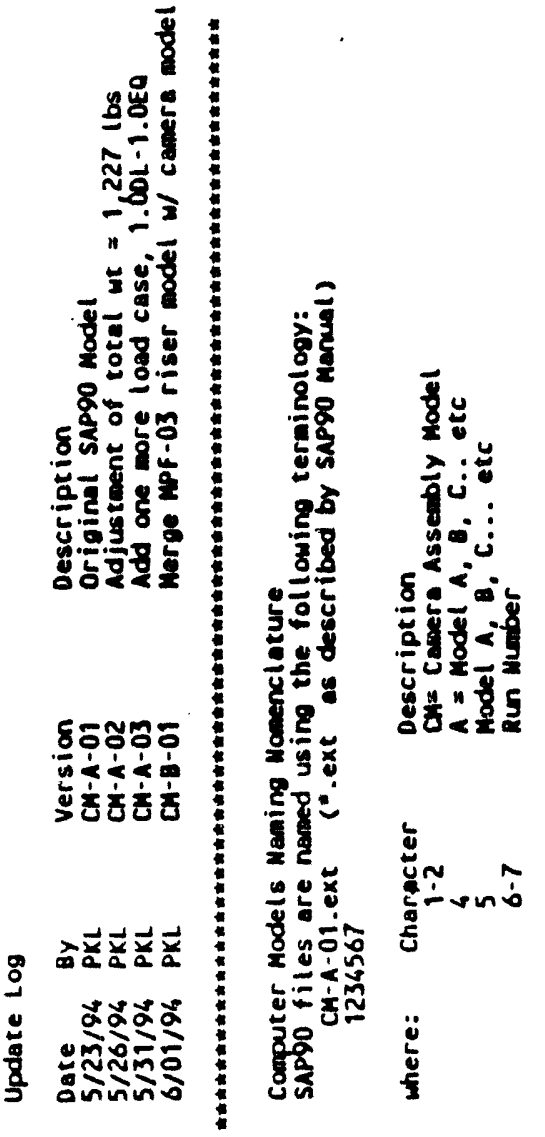

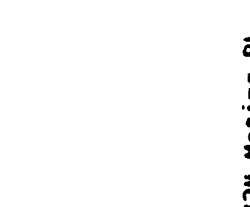

范

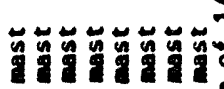

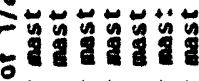

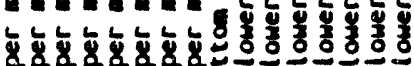

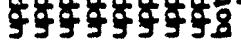
isiogio

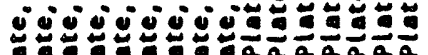

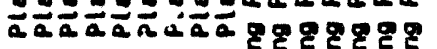

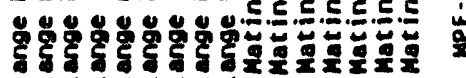

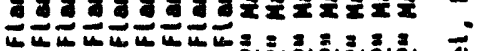

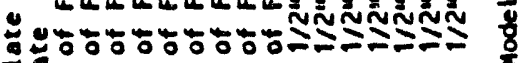

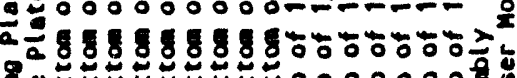

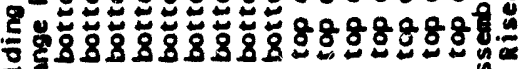

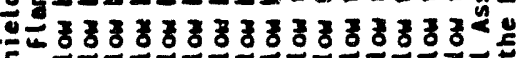

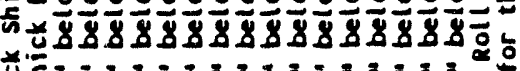

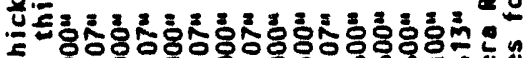

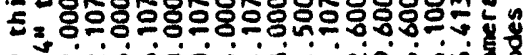

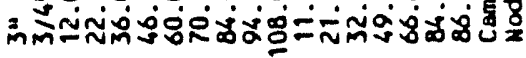

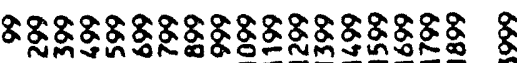

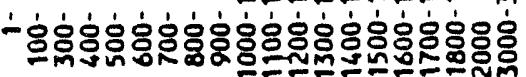

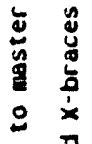

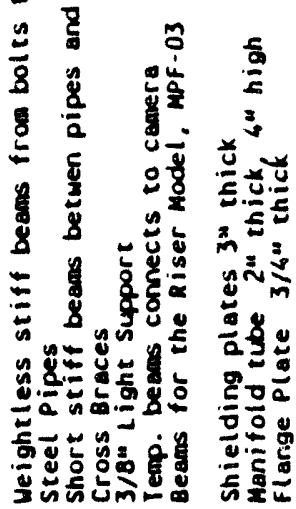

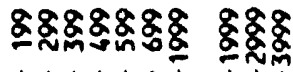

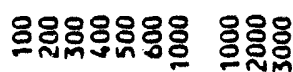

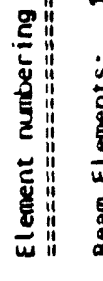



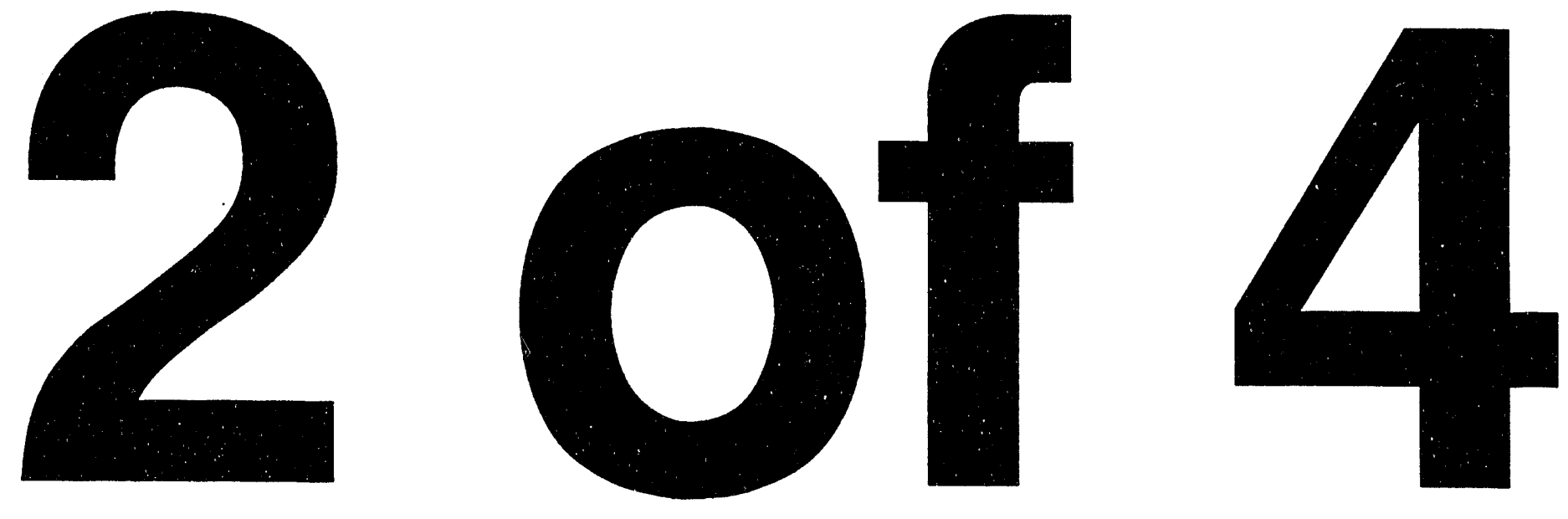


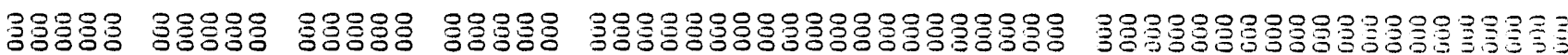

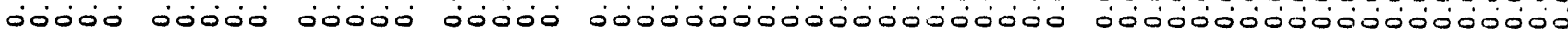

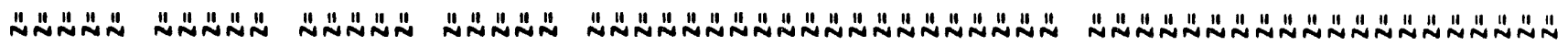

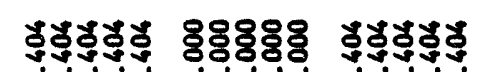

inininini

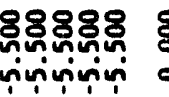

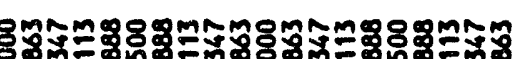

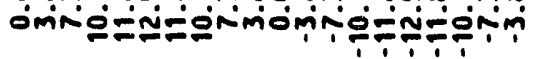

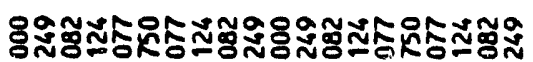

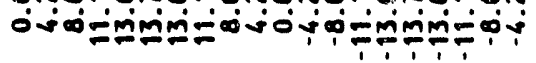

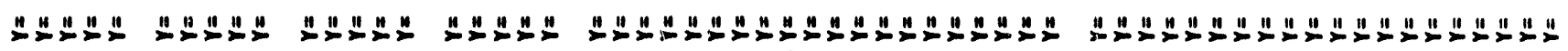

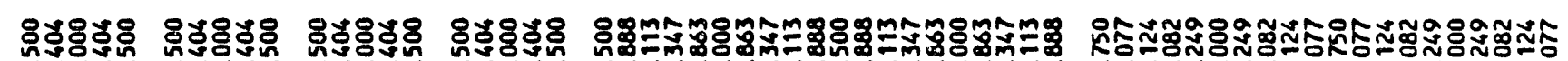

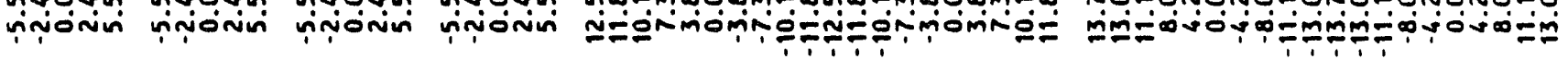

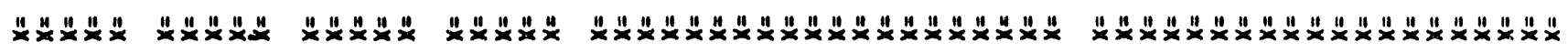

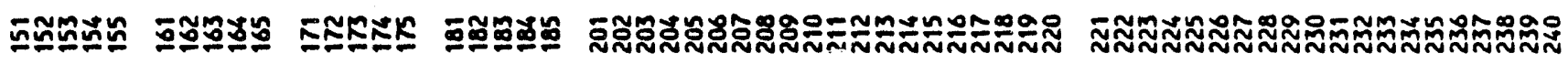

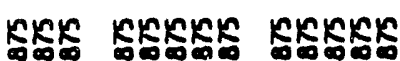

viniv vinivivin vivinivin

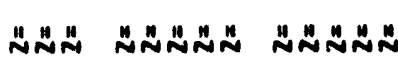

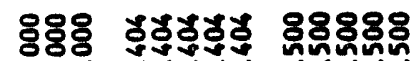

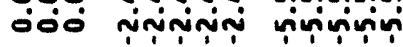

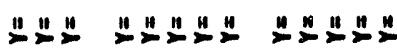

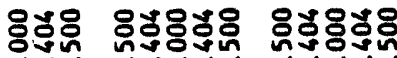

onin vinónin ninónin

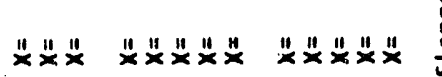

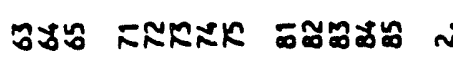

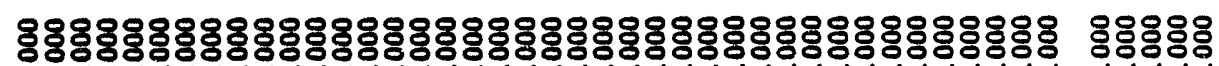

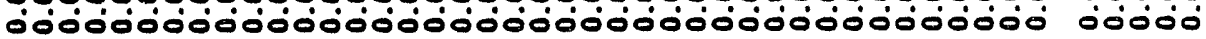

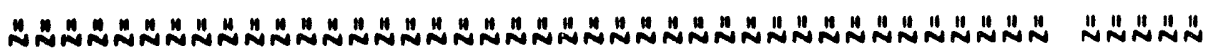

8ิชำ

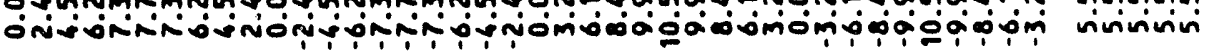

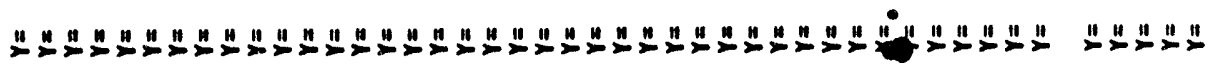
คำ

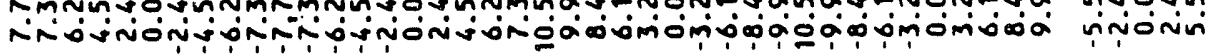

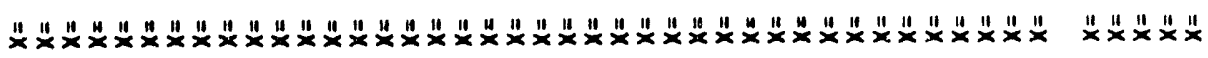

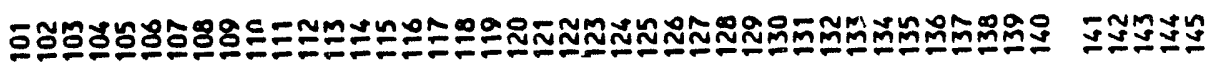
II -54 . 
Mode 163 connect to the plate;
C Mode 299 is the muSIER node w/ restraints in 6 DOf and connects to beans.

c $299 x=0.000 \quad y=0.000 \quad z=0.000$

C -.. 12.000" below bottom of flange Plate, upper mast

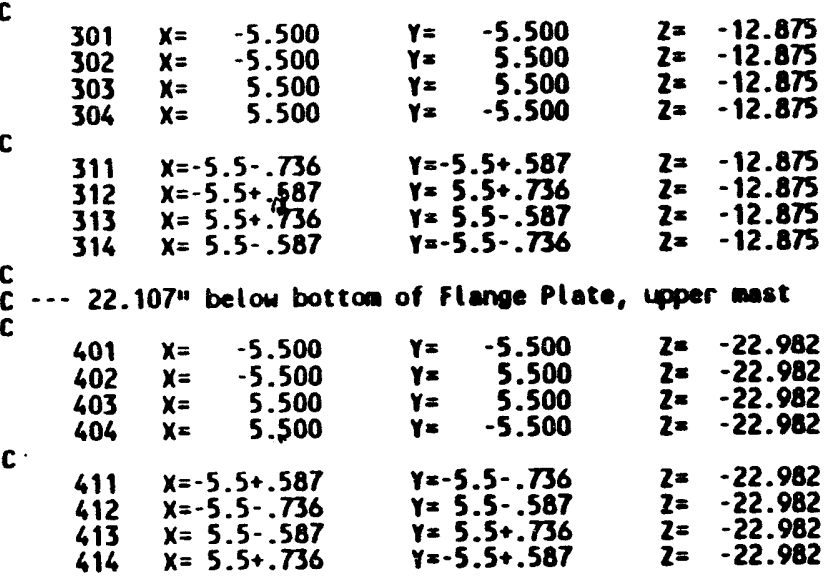

$\exists \quad$ C ... 36.000" below bottom of Flange plate, upper mast

\begin{tabular}{|c|c|c|c|c|c|c|c|}
\hline C & $\begin{array}{l}501 \\
502 \\
503 \\
504\end{array}$ & $\begin{array}{l}x= \\
x= \\
x= \\
x=\end{array}$ & $\begin{array}{r}-5.500 \\
-5.500 \\
5.500 \\
5.500\end{array}$ & $\begin{array}{l}y= \\
y= \\
y= \\
y=\end{array}$ & $\begin{array}{r}-5.500 \\
5.500 \\
5.500 \\
-5.500\end{array}$ & $\begin{array}{l}2= \\
2= \\
2= \\
2=\end{array}$ & $\begin{array}{l}-36.875 \\
-36.875 \\
-36.875 \\
-36.875\end{array}$ \\
\hline & $\begin{array}{l}511 \\
512 \\
513 \\
514\end{array}$ & $\begin{array}{l}x=- \\
x=- \\
x= \\
x=\end{array}$ & $\begin{array}{l}.736 \\
.587 \\
.736 \\
-.587\end{array}$ & $\begin{array}{l}y=- \\
y= \\
y= \\
y=-\end{array}$ & $\begin{array}{r}.5+.587 \\
.5+.736 \\
.5-.587 \\
.5-.736\end{array}$ & $\begin{array}{l}2= \\
z= \\
z= \\
2=\end{array}$ & $\begin{array}{l}-36.875 \\
-36.875 \\
-36.875 \\
-36.875\end{array}$ \\
\hline
\end{tabular}

C ... '46.107" below bottom of Flange Plate, upper mast

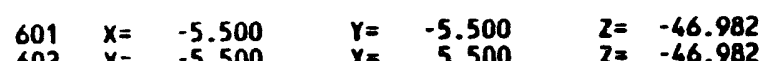

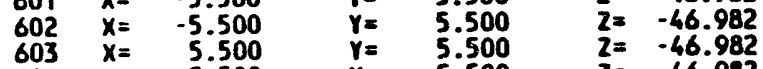

$\begin{array}{llll}604 & X=5.500 & & \\ 611 & X=-5.5+.587 & Y=-5.5-.736 & Z=-46.982 \\ 612 & X=-5.5-.736 & Y=5.5-.587 & Z=-46.982\end{array}$

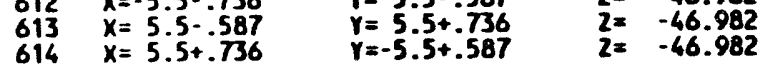

C -.- 60.000" below bottion of flenge Plate, upper mast
$\begin{array}{ll}701 & x=-5.500 \\ 702 & x= \\ x & -5.500\end{array}$
$Y=\quad-5.500$
$Y=5.500$
$z=-60.875$
$\begin{array}{lll}703 & x= & 5.500 \\ 704 & x= & 5.500\end{array}$
$Z=-60.875$
$Z=-60.875$
$Z=-60.875$
$\begin{array}{llll}711 & x=-5.5-.736 & y=-5.5+.587 & Z=-60.875 \\ 712 & x=-5.5+.587 & y=5.5+.736 & z=-60.875\end{array}$
$\begin{array}{llll}713 & x=5.55 .736 & y=5.5-.587 & z=-60.875 \\ 716 & x=5.5-.587 & y=-5.5-.736 & Z=-60.875\end{array}$

c

c ... 70.107" below botton of flange Plate, upper mast

\begin{tabular}{|lrr} 
& 801 \\
& 802 \\
& 803 \\
& 8 \\
& 8 \\
& 8 \\
& 8
\end{tabular}

$r=-5.500 \quad Z=-70.982$ $r=5.500 \quad z=-70.982$ $\begin{array}{rlrl}\gamma= & 5.500 & Z= & -70.982 \\ \gamma=-5.500 & Z= & -70.982\end{array}$ $\gamma=-5.5-.736 \quad z=-70.982$ $\begin{array}{ll}Y=5.5-.587 & z=-70.982 \\ Y=5.5+.736 & z=-70.982\end{array}$

C ... 84.006" below bottom of Flange Plate, upper mast

$$
\text { c }
$$
$901 x=-5.500$
$902 x=-5.500$
$\begin{array}{ll}903 & x=5.500 \\ 904 & x=5.500\end{array}$
$Y=-5.500$
$Y=\quad 5.500$
$\begin{array}{lr}Y= & 5.500 \\ Y= & 5.500 \\ Y= & -5.500\end{array}$
$Z=-84.875$
$911 \quad x=-5.5-.736$

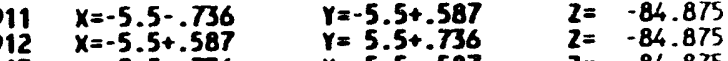
$913 \quad x=5.5+.736 \quad y=5.55 .587 \quad z=-84.875$

C

C ... 94.107" below bottom of flange Plate, upper mast

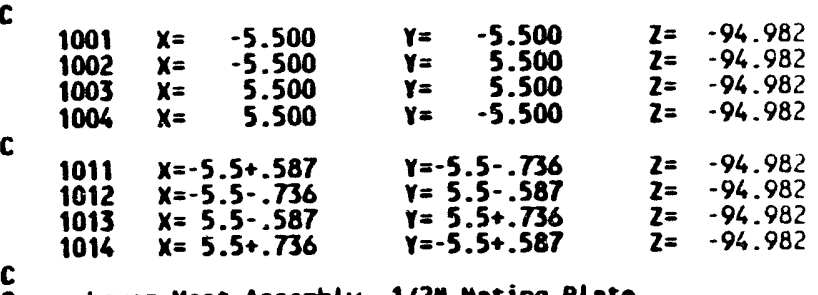
C -.- Lower Mast Asseably 1/2" Mating Plate

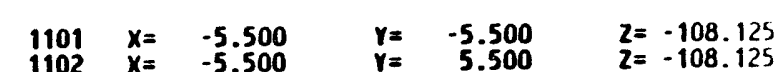

$\begin{array}{llrlrl}1102 & X= & -5.500 & Y= & 5.500 & Z=-108.125 \\ 1103 & X= & 5.500 & Y= & 5.500 & Z=-108.125 \\ 1104 & X= & 5.500 & Y= & -5.500 & Z=-108.125\end{array}$

c -.. 11" below Mating Plate, Lower Mast

c

\begin{tabular}{|c|c|c|c|}
\hline $\begin{array}{l}1201 \\
1202 \\
1203 \\
1206\end{array}$ & $\begin{array}{lr}x= & -5.500 \\
x= & -5.500 \\
x= & 5.500 \\
x= & 5.500\end{array}$ & $\begin{array}{lr}Y= & -5.500 \\
Y= & 5.500 \\
Y= & 5.500 \\
Y= & -5.500\end{array}$ & $\begin{array}{l}z=-119.375 \\
z=-119.375 \\
z=-119.375 \\
z=-119.375\end{array}$ \\
\hline $\begin{array}{l}1211 \\
1212 \\
1213 \\
1214\end{array}$ & $\begin{array}{l}x=-5.5-.736 \\
x=-5.5+.587 \\
x=5.5+.736 \\
x=5.5-.587\end{array}$ & $\begin{array}{l}Y=-5.5+.587 \\
Y=5.5+.736 \\
Y=5.5-.587 \\
Y=-5.5-.736\end{array}$ & $\begin{array}{l}z=-119.375 \\
z=-119.375 \\
z=-119.375 \\
z=-119.375\end{array}$ \\
\hline
\end{tabular}

C -.. 21.107" below Mating Plate, Lower Mast

\begin{tabular}{|c|c|c|c|}
\hline $\begin{array}{l}1301 \\
1302 \\
1303 \\
1304\end{array}$ & $\begin{array}{lr}x= & -5.500 \\
x= & -5.500 \\
x= & 5.500 \\
x= & 5.500\end{array}$ & $\begin{array}{lr}Y= & -5.500 \\
Y= & 5.500 \\
Y= & 5.500 \\
Y= & -5.500\end{array}$ & $\begin{array}{l}Z=-129.482 \\
Z=-129.482 \\
Z=-129.482 \\
Z=-129.48 z\end{array}$ \\
\hline $\begin{array}{l}1311 \\
1312 \\
1313 \\
1314\end{array}$ & $\begin{array}{l}x=-5.5+.587 \\
x=-5.5-.736 \\
x=5.5-.587 \\
x=5.5+.736\end{array}$ & $\begin{array}{l}\gamma=-5.5-.736 \\
\gamma=5.5-.587 \\
\gamma=5.5+.756 \\
\gamma=-5.5+.587\end{array}$ & $\begin{array}{l}Z=-129.48 z \\
Z=-129.482 \\
Z=-129.48 z \\
Z=-129.48 Z\end{array}$ \\
\hline
\end{tabular}


06/01/1994 15:46 Filename: CM-B-01

Page 7

c -.. Section B 32" below Mating Plate, Lower Mast

$\begin{array}{rlrrrr}1401 & X= & -5.500 & \gamma= & -5.500 & z=-141.083 \\ 1402 & X= & -5.500 & \gamma= & 5.500 & z=-141.083 \\ 1403 & X= & 5.500 & \gamma= & 5.500 & z=-141.083 \\ 1404 & X= & 5.500 & \gamma= & -5.500 & Z=-141.083\end{array}$

C ... Section C 49" below Mating Plate, Lower Mast

$\begin{array}{llrlrl}1501 & x= & -5.500 & \gamma= & -5.500 & z=-158.083 \\ 1502 & x= & -5.500 & \gamma= & 5.500 & 2=-158.083 \\ 1503 & x= & 5.500 & \gamma= & 5.500 & 2=-158.083 \\ 1504 & x= & 5.500 & \gamma= & -5.500 & 2=-158.003\end{array}$

C -.- Section D 115" below Mating Plate, Lower Mast

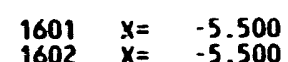
$Y=-5.500$
$Y=5.500$
$2=-175.083$
$1603 x=$
$1604 x=5.500$
$\gamma=5.500$
$\gamma=-5.500$
$2=-175.083$

C -.. Section A Upper portion, 84.1" below Mating Plate, Lower Mast

$\begin{array}{rlrlrl}1701 & x= & -5.500 & y= & -5.500 & z=-191.975 \\ 1702 & x= & -5.7 .10 & y= & 5.500 & z=-191.975 \\ 1703 & x= & 5.500 & y= & 5.500 & z=-191.975 \\ 1704 & x= & 5.500 & y= & -5.500 & z=-191.975\end{array}$

C ... Section A Botton plate, 96.6" below Mating Plate, Lower Mast

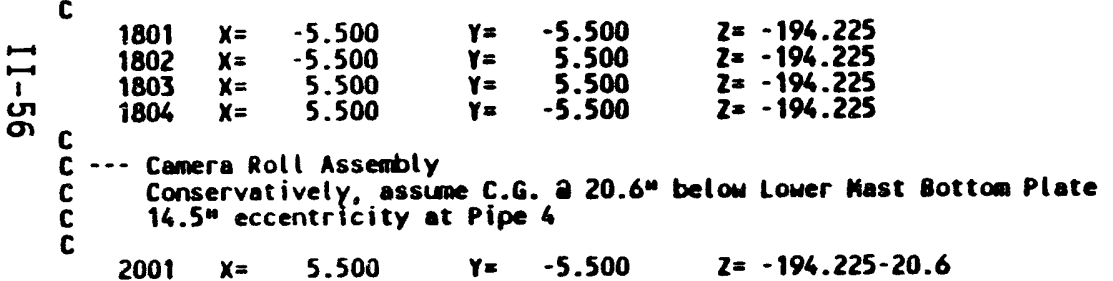

C -..' 'Modes for the Riser Model

\begin{tabular}{|c|c|c|c|c|}
\hline $\begin{array}{l}3002 \\
3000 \\
3004 \\
3005 \\
3006 \\
3007 \\
3008 \\
3009 \\
3010\end{array}$ & $\begin{array}{l}x=0 \\
x=23.25 \\
x=15 . \\
x=15 . \\
x=0 \\
x=23.25 \\
x=15 . \\
x=15 \\
x=9.5 \\
x=10.07645\end{array}$ & $\begin{array}{l}Y=0 . \\
Y=0 . \\
Y=-14.75 \\
Y=14.75 \\
Y=0 . \\
Y=0 . \\
Y=-14.75 \\
Y=14.75 \\
\gamma=0 . \\
Y=0 .\end{array}$ & $\begin{array}{l}z=0 . \\
2=6 . \\
2=12 . \\
z=12 . \\
z=-10.7 \\
2=-10.7 \\
2=-10.7 \\
z=-10.7 \\
z=-10.7 \\
z=-10.7\end{array}$ & 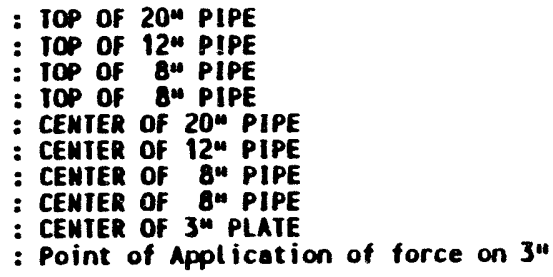 \\
\hline$\Delta$ & $x=9.5$ & $\gamma=0$. & $z=-24$. & : BOTIOA OF MPE, CENTER OF $3 / 4^{4 \mu}$ PLATE \\
\hline 3021 & $x=9.5$ & $r=0$. & $Z=-141.5$ & $\begin{array}{l}G=3011,3021,1 \\
\text { : DOME OF COMCRETE IAMK }\end{array}$ \\
\hline
\end{tabular}

c

RESTRAINIS

RESTRAINS $2992991 R=1,1,1,1,1,1$
C $3021 \quad R=1,1,1,1,1,1 \quad$ : TAMK DOME AT RADIUS 20 FEET
06/01/1994 15:46 Filename: CA-8-01 Page 8

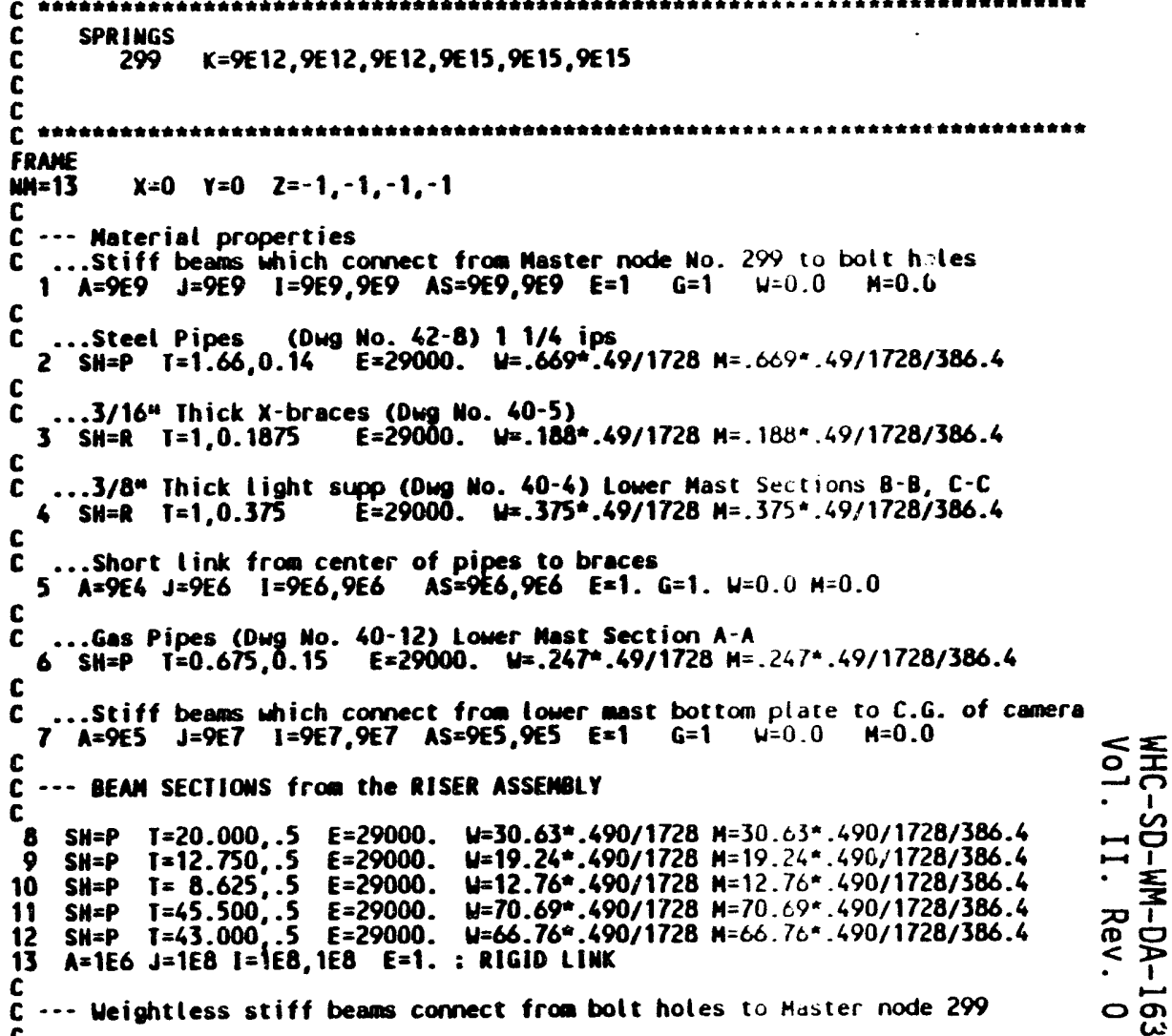

$101299201 \quad \mu=1 \quad L P=0.63$

: $L R=0,1,0,0,1,1$

$102299202 \quad H=1 \quad L P=0.63 \quad: L R=0,1,0,0,1,1$

$103299203 \quad K=1 \quad L P=0,63 \quad \vdots \quad L R=0,1,0,0,1,1$

$104299 \quad 204 \quad M=1 \quad L P=0.63$

$106299 \quad 206 \quad M=1 \quad L P=0.63$

$107299 \quad 207 \quad M=1 \quad L P=0,63$

$106299 \quad 208 \quad M=1 \quad L P=0.63$

$\begin{array}{lll}109 & 299 & 209 \\ 110 & 299 & 210 \\ M=1 & L P=0,63 \\ 1 & L P=0.63\end{array}$

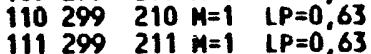

$\begin{array}{lllll}111 & 299 & 211 & M=1 & L P=0.63 \\ 112 & 299 & 212 & M=1 & L P=0.63\end{array}$

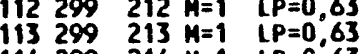

$114299214 \quad H=1 \quad L P=0,63$

$115299215 \quad M=1 \quad L P=0.63$

$116299 \quad 216 \quad M=1 \quad L P=0.63$

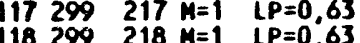

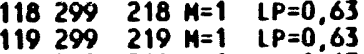

$120299 \quad 220 \quad M=1 \quad L P=0,63$

$L R=0,1,0,0,1,1$

- $\angle R=0,1,0,0,1,1$

$=\quad 2=0,1,0,0,1,1$

- $\quad L R=0,1,0,0,1,1$

- $\quad L R=0,1,0,0,1,1$

- $\quad R=0.1,0,0.1 .1$

- $R=0.1,0.1$

LR=0.100.1

- LR=0,1,0,0,1

- LR=0,1,0,0,1,1

- $\quad \angle R=0,1,0,0,1,1$

L $\quad L=0,1,0,0,1,1$

$L_{R=0,1,0,0,1,1}$

$\vdots \quad L R=0,1,0,0,1,1$ 


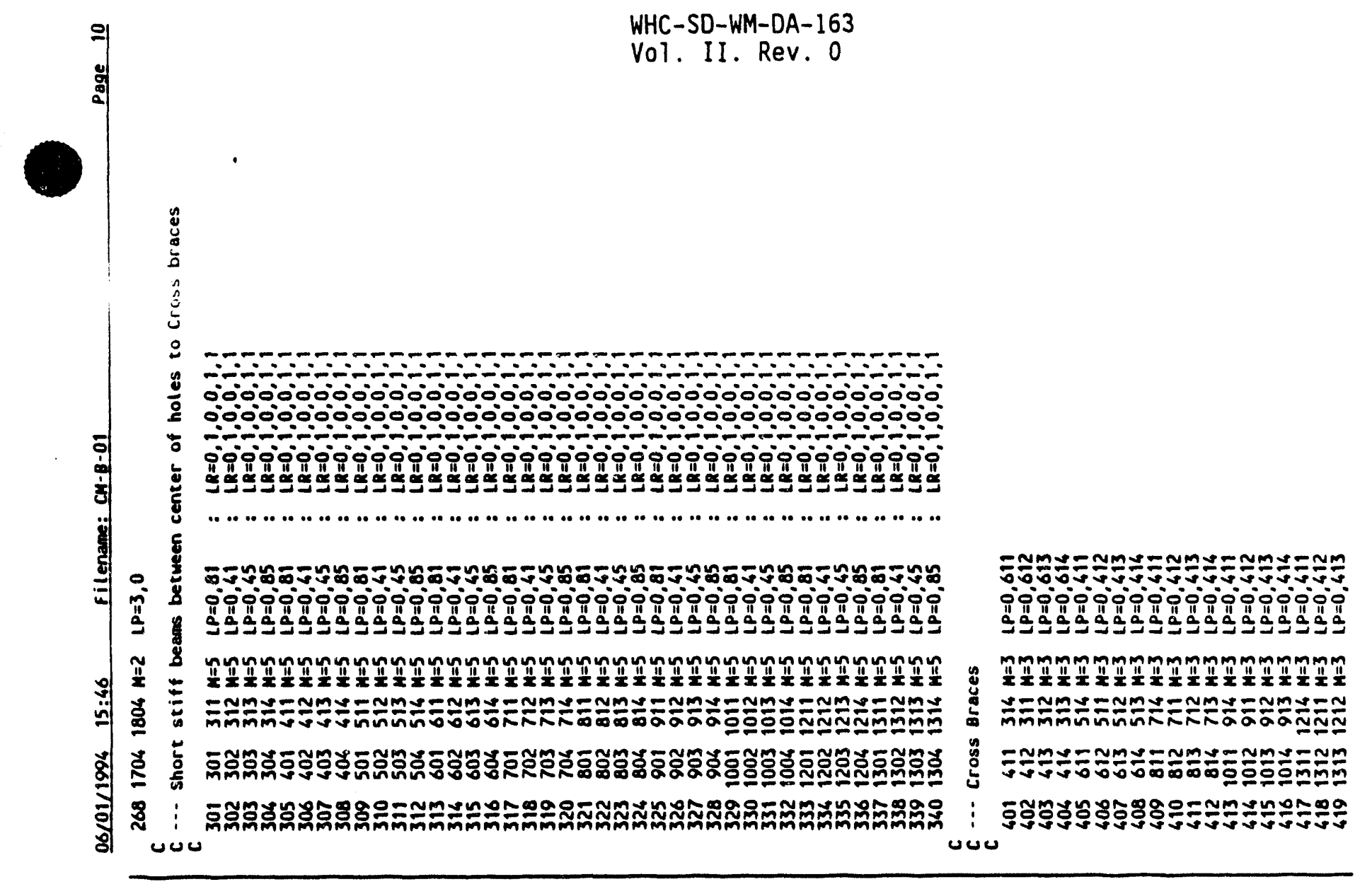

0

000000000000000000000000000000000000000000000000000000000000000000.

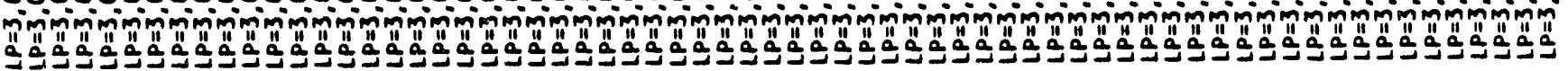

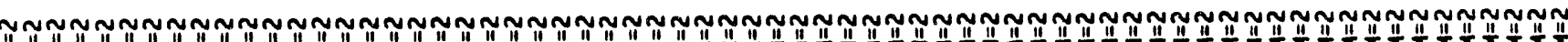

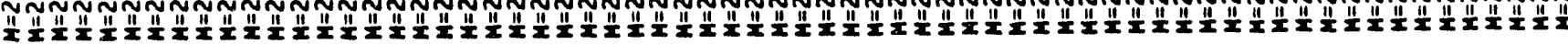

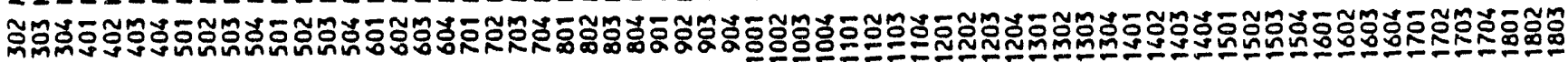

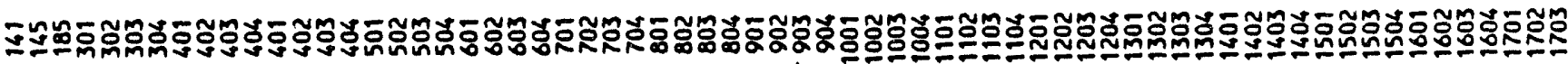

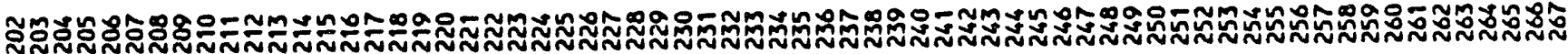


89-II

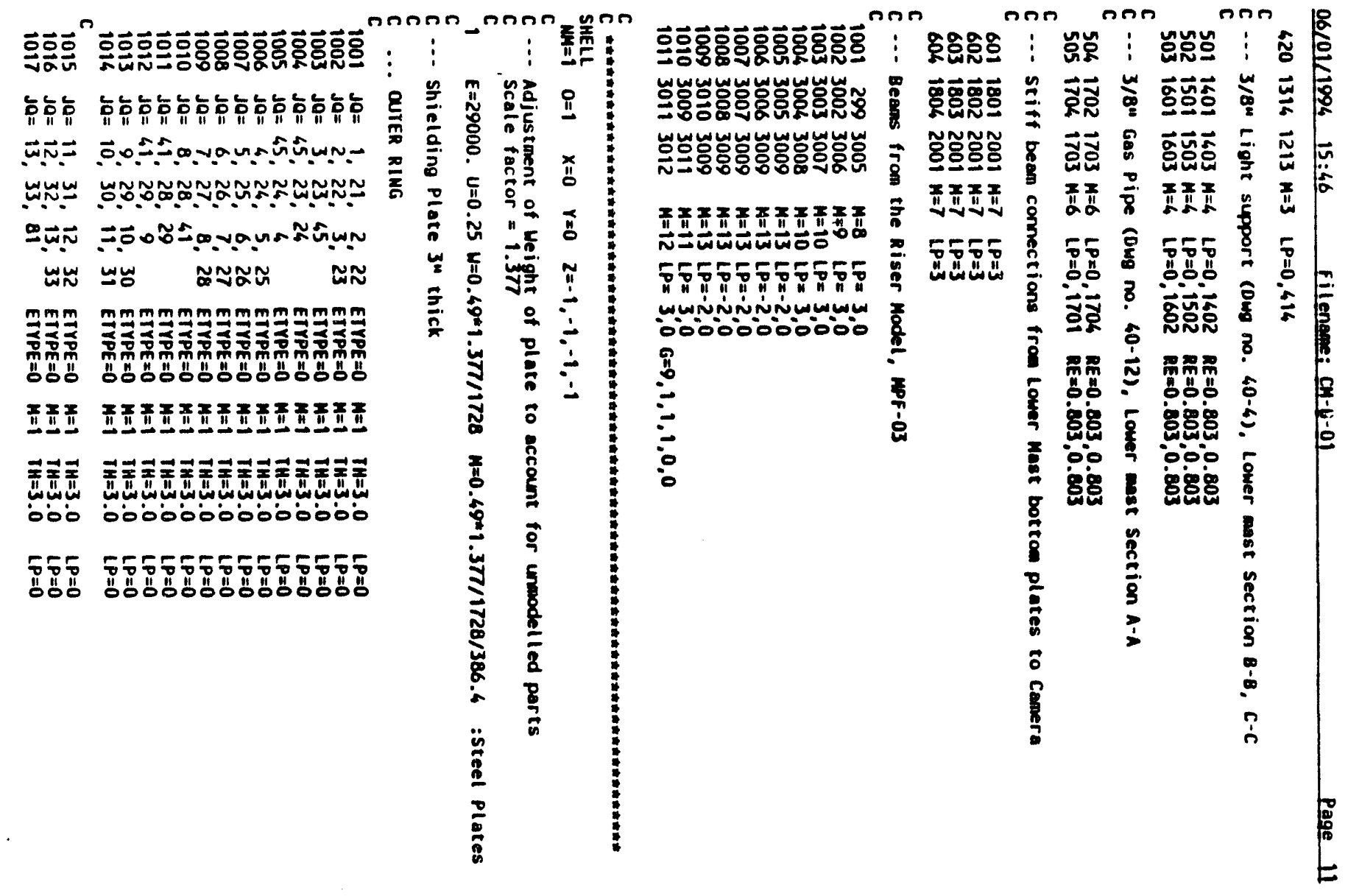

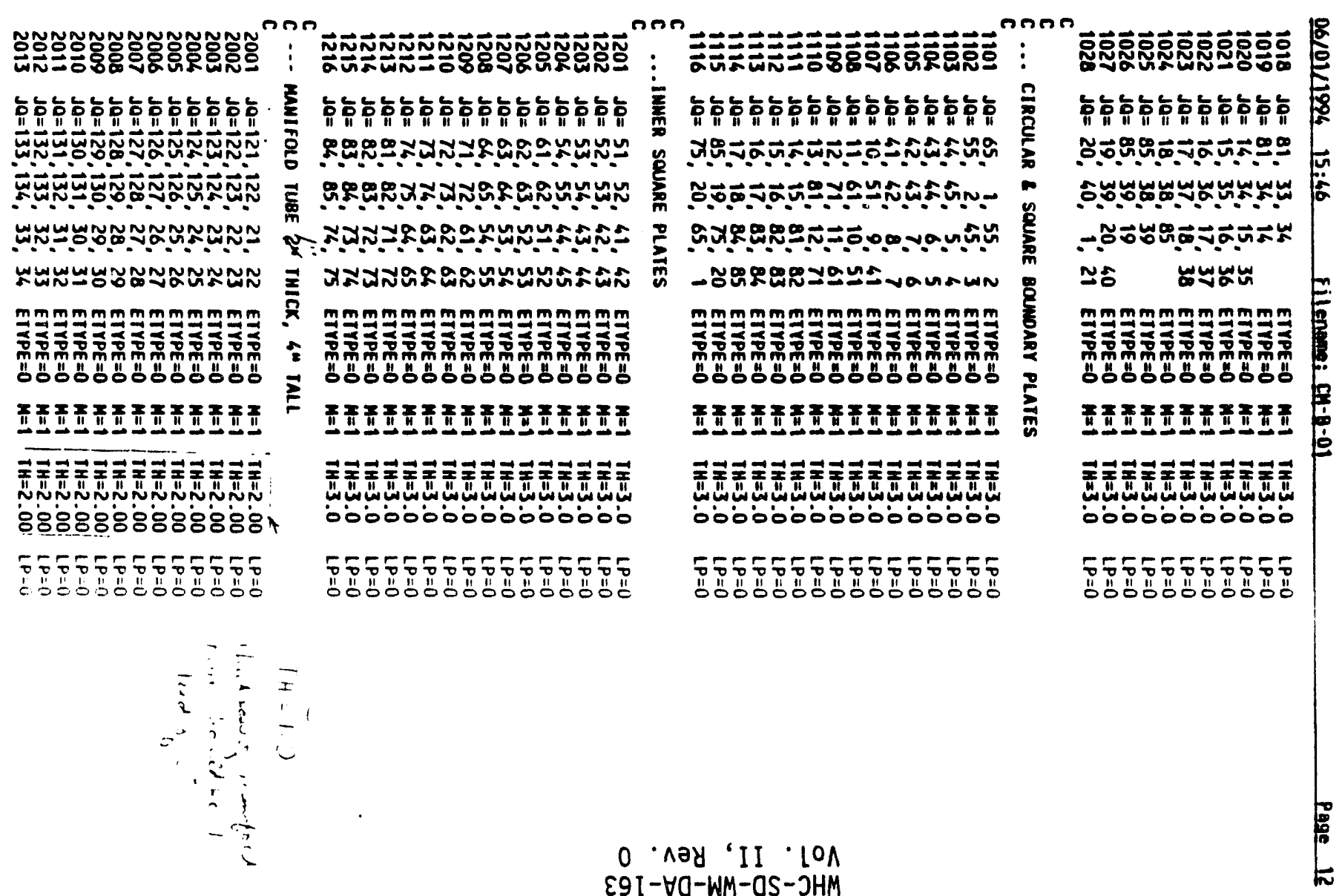




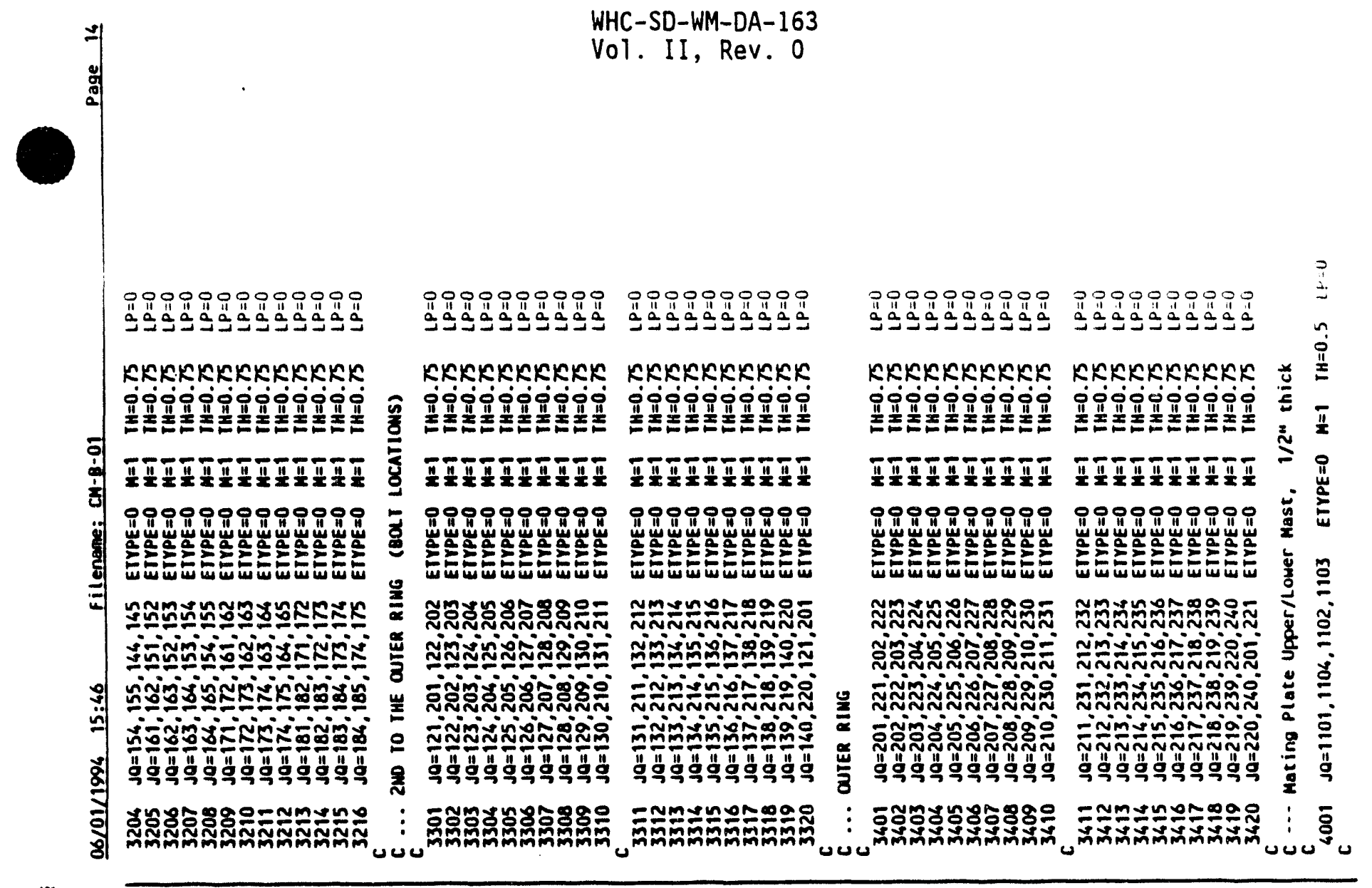

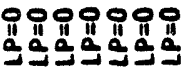

8888888

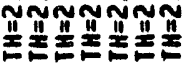

$\frac{\pi}{2} \frac{\pi}{2} \frac{\pi}{2} \frac{\pi}{2} \frac{\pi}{2} \frac{\pi}{2}$

유압윰애매유

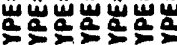

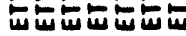

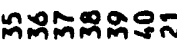

जिinipinimin:

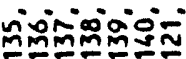

vivisinisio

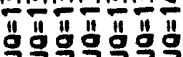

ว้ำํํํํํํํํํํำ

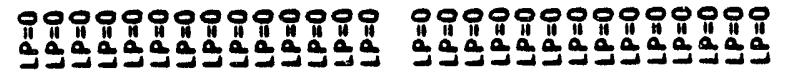

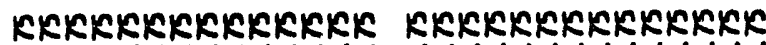

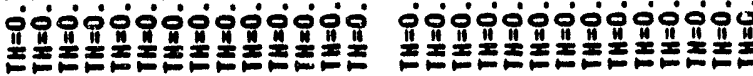

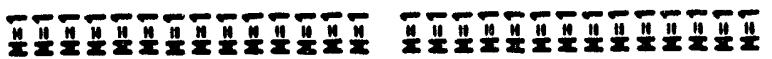

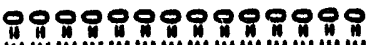

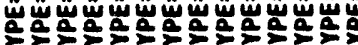

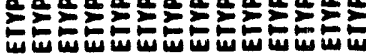

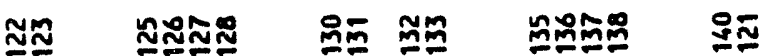

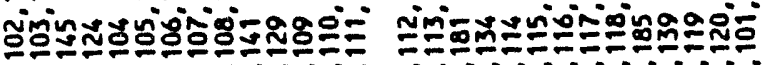

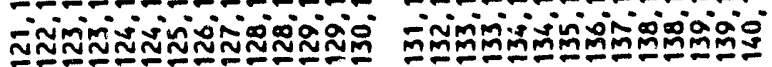

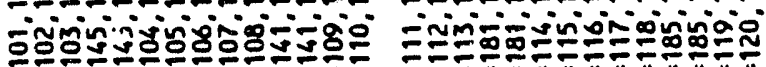

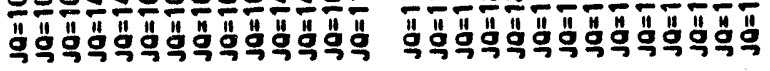

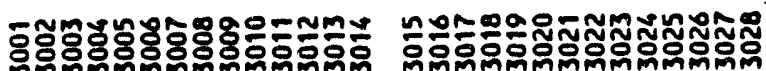

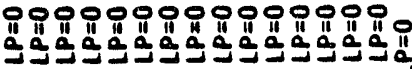

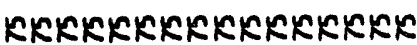

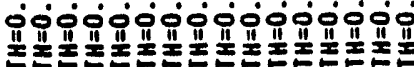

茨

전 $\frac{7 \pi}{2} \frac{\pi}{2} \frac{\pi}{2} \frac{\pi}{2} \frac{\pi}{2} \frac{\pi}{2} \frac{\pi}{2} \frac{\pi}{2} \frac{\pi}{2} \frac{\pi}{2} \frac{\pi}{2} \frac{\pi}{2} \frac{\pi}{2} \frac{\pi}{2}$

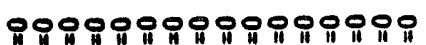

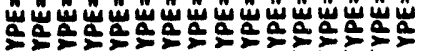

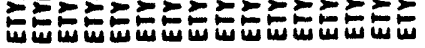

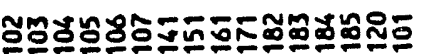

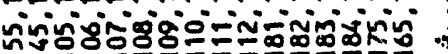

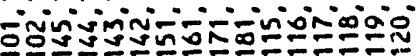
nิن

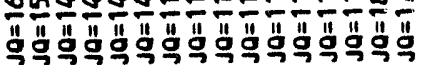

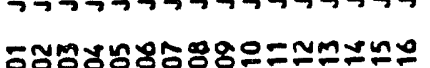

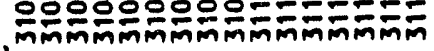

뮬ํํำ

nnn

iㅗ뉸픈

표 $\frac{\pi}{2} \frac{\pi}{2}$

애윰요

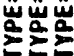

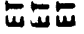

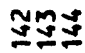

इing

inis:

กิตูบ

ining

"ำ

อะกิ

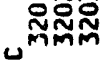


C -. Bottom Plate, 1/2" thick

$4002 J 0=1801,1804,1802,1803$ ETYPE $=0 \quad H=1 \quad I H=0.5 \quad$ LP=0

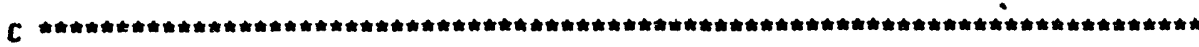

LOADS

$2001 \quad L=1 \quad F=0,0,-.128$

C - $\therefore$ Loads from the Riser Model, MPf-03

$299 \quad F=0,0,-.441$

$3002 F=0,0,-.247$

$3003 \quad F=0,0,-.11762$

(3003 $F=0,0,-11762$

$3010 \quad F=0,0 ;-2.538$

$l=1$

$\begin{array}{ll}3010 \quad F=0,0,-2.538 & l=1 \\ 3011 \quad F=0,0,-.29676) & L=1: 244.3 \mathrm{lbs}+45.66 \mathrm{lbs}+6.8 \mathrm{lbs}\end{array}$

$l=1$

\section{MASSES}

$2001 M=.128 / 386.4, .128 / 386.4, .128 / 386.4$

C -.. Masses from the Riser Model, MPF-03

C $M=.441 / 386.4, .441 / 386.4, .441 / 386.6$

$3002 M=.267 / 386.4, \quad 247 / 386.4, \quad .247 / 386.6$

$3003 \quad M=.11762 / 386.4, .11762 / 386.4 . .11762 / 386.4$

$3004 M=.11762 / 386.4, .11762 / 386.4, .11762 / 386.6$

$3009 \quad M=.0191 / 386.4, .0191 / 386.4, .0191 / 386.4$

$3010 \quad H=2.538 / 386.4,2.538 / 386.4,2.538 / 386$.

$\begin{array}{rlll}0.90909 & 0.21060 & 0.21060 & 0.21060 \\ 1.09848 & 0.17444 & 0.17444 & 0.17444 \\ 1.28788 & 0.14890 & 0.14890 & 0.14890 \\ 1.47727 & 0.12990 & 0.12990 & 0.12990 \\ 1.66667 & 0.11520 & 0.11520 & 0.11520 \\ 1.87500 & 0.10263 & 0.10263 & 0.10263 \\ 2.08333 & 0.09256 & 0.09256 & 0.09256 \\ 2.29167 & 0.08430 & 0.08430 & 0.08430 \\ 2.50000 & 0.07740 & 0.07740 & 0.07740 \\ 2.50006 & 0.0685 \\ 2.83654 & 0.06825 & 0.06825 & 0.06825 \\ 3.17308 & 0.06104 & 0.06104 & 0.06104 \\ 3.50962 & 0.05521 & 0.05521 & 0.05521 \\ 3.84615 & 0.05040 & 0.05040 & 0.05040 \\ 4.44712 & 0.03741 & 0.03741 & 0.03741 \\ 5.04808 & 0.02884 & 0.02884 & 0.02884 \\ 5.64904 & 0.02289 & 0.02289 & 0.02289 \\ 6.25000 & 0.01860 & 0.01860 & 0.01860 \\ 7.18750 & 0.01403 & 0.01403 & 0.01403 \\ 8.12500 & 0.01095 & 0.01095 & 0.01095 \\ 9.06250 & 0.00878 & 0.00878 & 0.00878 \\ 10.00000 & 0.00720 & 0.00720 & 0.00720\end{array}$

congo

$1 \mathrm{C=1.0}$

$2 \quad C=1.0 \quad D=1.0$

$\begin{array}{lll}C=1.0 & D=1.0 \\ C=1.0 & D=-1.0\end{array}$

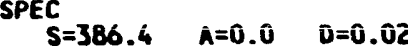

C Non-reactor Safety Class $22 x$ damped Design Spectrum from figure 3 and C Table 2, scaled by 0.6, SDC 4.1 Revision 13. Design Loads for Facilities

Convervatively, Sa is applied $100 x$ in all 3 directions ( 2 hori., 1 vert)

Peri(sec) SAX(g) SAYY(g) SAz(g)

$0.01000 \quad 0.12000 \quad 0.12000 \quad 0.12000$

$0.01508 \quad 0.12000 \quad 0.12000 \quad 0.12000$

$\begin{array}{llll}0.02015 & 0.12000 & 0.12000 & 0.12000 \\ 0.02523 & 0.12000 & 0.12000 & 0.12000\end{array}$

$0.03030 \quad 0.12000 \quad 0.12000 \quad 0.12000$

$0.03523 \quad 0.13362 \quad 0.13362 \quad 0.13362$

0.04015 0.146?1 0.146710 .14671

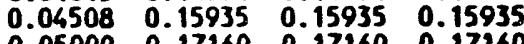

$\begin{array}{llll}0.05000 & 0.17160 & 0.17160 & 0.17160\end{array}$

$\begin{array}{llll}0.05833 & 0.19158 & 0.19158 & 0.19158\end{array}$

$0.060670 .21076 \quad 0.21076 \quad 0.21076$

$0.08333 \quad 0.24720 \quad 0.24720 \quad 0.24720$

0.093750 .268840 .268840 .26884

$\begin{array}{lllll}0.09375 & 0.26894 & 0.26824 & 0.26884\end{array}$

$\begin{array}{llll}0.11658 & 0.31016 & 0.31016 & 0.31016\end{array}$

$\begin{array}{llll}0.12500 & 0.33000 & 0.33000 & 0.33000\end{array}$

$\begin{array}{lllll}0.23826 & 0.33000 & 0.33000 & 0.33000\end{array}$

$\begin{array}{llll}0.35152 & 0.33000 & 0.33000 & 0.33000\end{array}$

$\begin{array}{llll}0.46478 & 0.33000 & 0.33000 & 0.33000\end{array}$

$\begin{array}{lllll}0.57803 & 0.33000 & 0.33000 & 0.33000\end{array}$

$\begin{array}{llll}0.66080 & 0.28898 & 0.28898 & 0.28898\end{array}$

$\begin{array}{llll}0.74356 & 0.25706 & 0.25706 & 0.25706 \\ 0.82633 & 0.23151 & 0.23151 & 0.23151\end{array}$ 


\section{URS CONSULTANTS}

WHC-SD-WM-DA-163

Vol. II, Rev. 0

Job \#:66481-01 Job: WHC/MPF CAMERA ASSEMBLY ANALYSIS

Client: WHC
Subject: SAP9O ANALYSIS DOCUMENTATION
Sheet No.

By $\because-$ Date

Chk'dRIKDate $8 / 16 / 94$

4.3 Input data file for SAPSTL

Since stainless steel is used, the SAPSTL built-in formulas for ordinary structural steel are not applicable. Hand calculations are given in this section to provide the input parameters for the allowable stresses to SAPSTL for combined stress evaluations. (refer to ANSI/AISC N690-1984, section Q1.5.9, "Austentic Stainless steel").

The echoprint of the input data file, $C M-A-04 S$, is also given in this section. 
URS Consultants URS/John A. Blum \& Associates, Engineers job no. 66458 -01 Job WHC/MPF ANALYSIS Client

Subject SECTION PROPERTIES
WHC-SD-WM-DA-163

Vol. II, Rev. 0

Sheet No.

432

Calk. No.

Rev. No.

BY PK U Date ol.194

Chk'd $\leq /, n$ Date $z, 1 ?: 1$

STEEL PIPES

$$
\begin{aligned}
& E=29,000 \mathrm{ki} \\
& f_{y}=30 \mathrm{ki}
\end{aligned}
$$

PIPE: COMPACT SECTION

$$
K=2 \text { (LACINGS ARE StagGered) }
$$

Allowable:

$$
\begin{aligned}
F_{b} & =1.6\left(0.66 f_{y}\right)=31.68 \mathrm{ka} \\
F_{b m i} & =1.6\left(0.75 f_{y}\right)=36 \mathrm{ksi} \\
F_{a c} & =\left(\frac{f_{y}}{2.15}-\frac{\left(\frac{f_{y}}{2.15}-6\right)}{120} \frac{\mathrm{kl}}{r}\right) \times 1.6 \quad \frac{\mathrm{kl}}{r}=\frac{.24)}{.54}=88.39=120 \\
& =\left[13.95-0.0663\left(\frac{\mathrm{kl}}{r}\right)\right] \times 1.6=12.89 \\
F_{a t} & =1.6\left(0.6 f_{y}\right)=28.8 \mathrm{ksi} \\
F_{v} & =1.4\left(0.4 f_{y}\right)=16.8 \mathrm{ksi}
\end{aligned}
$$

LACINGS

$$
f_{y}=25 \mathrm{ks}
$$

$$
b=1^{\prime \prime} \quad t=3 / 16 " \frac{b}{t}=533<\frac{52.5}{\sqrt{f_{y}}}=10.5 \text { COMPACT SECT. }
$$

Allowable.

$$
\begin{aligned}
& F_{b}=1.6\left(0.66 f_{y}\right)=26.4 \mathrm{ks} \\
& F_{b . .}=1.6(0.75 \mathrm{fy})=30 \mathrm{ki} \\
& F_{a c}=\left[12-\frac{1}{20}\left(\frac{k l}{r}\right)\right] \times 1.6 \\
& \frac{K l}{r}=\frac{14096}{0054}=261>200 \mathrm{~N} \cdot G .
\end{aligned}
$$

use 1/4" THICKNESS INSTEAD of $3 / 16$ "

$$
\begin{aligned}
& \text { Fir } t=1 / 4^{\prime \prime} \quad \frac{K l}{r}=\frac{14.096}{.072}=195.8 \\
& F_{a c}=\left[12-\frac{1}{20}(195.8)\right] \times 1.6=3.53 \mathrm{~km} \\
& F_{t}=1.6\left(0.45 f_{y}\right)=18 \mathrm{Kni} \text { (Assize pindenuestiou) } \\
& F_{v}=1.6\left(0.4 f_{y}\right)=16 \mathrm{ks}
\end{aligned}
$$

II -62 
URS Consultants

WHC-SD-WM-DA-163

URS/John A. Blame \& Associates, Engineers

Sheet No.

43.3

Job no. $66758^{\circ}-01$ Job JHC/MPF ANALYSIS

Call. No.

Client

Rev. No.

subject

SECTION PROPERTIES

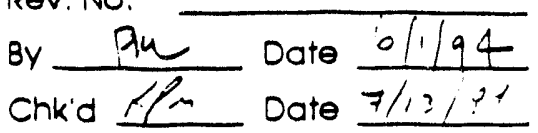

$\because \quad \therefore-5.0002$

$$
\begin{aligned}
& f_{y}=25 \mathrm{kar} \quad 0^{\prime \prime} \quad t=3 / 3 " \quad \frac{c}{i}=2.67<\frac{52.5}{\sqrt{f_{y}}}=3.5 \text { CMmpet seer } \\
& \text { Allowable. } \quad F_{b}=1.6\left(0.66 \mathrm{fy}_{y}\right)=26.4 \mathrm{kmi} \\
& F_{b m m}=1.6\left(0.75 f_{y}\right)=30 \mathrm{kic} \\
& \frac{k l}{r}=\frac{1(15.56)}{0.108}=144>120 \\
& F_{a c}=\left[12-\frac{1}{20}\left(\frac{k l}{r}\right)\right] \times 1.6=7.67 \mathrm{ksi} \\
& F_{a t}=1.6\left(0.45 f_{y}\right)=18 \mathrm{ksi} \\
& F_{v}=1.6\left(0.4 f_{y}\right)=16 \mathrm{ksi}
\end{aligned}
$$

$3 / 8$ " GAS PIPES

$$
\begin{aligned}
& f_{y}=25 \mathrm{~km} \quad \text { PIPE } \rightarrow \text { COMPACT } \\
& \text { ALLOWABLE: } \quad F_{b}=1.6\left(0.66 f_{y}\right)=26.4 \mathrm{~km} \\
& F_{b \text { max }}=1.6\left(0.15 f_{y}\right)=30 \mathrm{~km} \\
& \frac{k \ell}{r}=-\frac{11}{0.193}=57<120 \\
& F_{a c}=\left[\frac{f_{y}}{215}-\frac{\left(\frac{f_{y}}{21.5}-6\right)}{120}\left(\frac{k_{y}}{r}\right)\right] \times 1.6=14.33 \mathrm{ken} \\
& F_{a t}=1.6\left(0.45 f_{y}\right)=18 \mathrm{ks} \\
& F_{v}=1.6\left(0.4 f_{y}\right)=16 \mathrm{~km}
\end{aligned}
$$

II -63 
URS Consultants

WHC-SD-WM-DA-163

URS/John A. Blame \& Associates, Engineers

Job no. $06458-01$ Job WHC/MPF ANALYSIS

Client

Subject

Sheet No. 13.4

Call. No.

Rev. No.

By PK e Date 6/2/94

Coked $\mathrm{H} / \mathrm{m}$ Date $=3$ i:

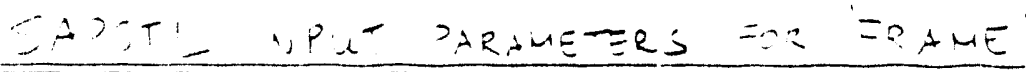

SteEL PIPES: $\quad K=2, \quad C=0.85$

$(K=2 \quad$ - ACUGS ARE StAGgERED $)$

LACINGS: $K=1, C=0.85$

(w ese $1 / 4$ "THICK)

LIGHT SUPPORT: $\quad K=1, \quad C=0.85$

GAS PIPES $\quad K=1, \quad c=0.85$

II -64 
MHC/MPF CAMERA ASSEMBLY ANALYSIS JOBN: 66458-01 RUM ID: CM-A-04S C

Units $=x i p s$ in $f y=60 \mathrm{ksi}$ steel density $=490 \mathrm{pcf}=0.0002836 \mathrm{kci}$

C

C Update Log

$\begin{array}{llll}\text { Date } & B Y & \text { Version } & \text { Description } \\ 5 / 26 / 94 & P K L & C H-A-01 S & \text { SAPSIL for SAP90 run } C M-A-01 \\ 5 / 27 / 94 & \text { PKL } & \text { CH-A-02S } & \text { SAPSIL for SAP90 run CA-A-02 } \\ 5 / 31 / 94 & \text { PKL } & C H-A-03 S & \text { SAPSIL for SAP90 rUn CH-A-03 }\end{array}$

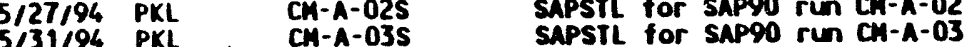

6/02/94 PKL + CH-A-03S SAPSIL for SAP9O rUn CH-A-04

\section{Computer Models Maming Manenclature}

wo following termingl ogy

(*.ext as described by SAP9O Manial)

CN-A-015.
12345678

\section{where: Character Description}

\section{$C M=$ Camera Assembly Model}

$4 \quad A=$ Model $A, B$. C.. etc

6.7 Run Number

s denotes SAPSIL file

c

COMTROL

$\operatorname{Cow}_{i x=2}$

$c$.

:DATACHECK =1, MORMLL=0, STRESS FILE=2

сомво

$\begin{array}{ll}1 & C=1 \\ 2 & 0=1\end{array}$

$\begin{array}{lll}3 & C=1,1 & D=1 \\ 4 & C=1,-1 & D=-1\end{array}$

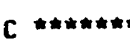

SECTIONS

C ... Material properties

C...Stiff beams which connect from Master node No. 299 to bolt holes

$1 \quad M N=M \quad A=9 E 9 \quad J=9 E 9 \quad I=9 E 9,9 E 9 \quad A S=9 E 9$, 9E9 $\quad E=1 \quad G=1 \quad W=0.0 \quad M=0.0$

C ...steel Pipes is (Dwg No. 42-8) $11 / 4$ ips

2 MN=S SH=P $T=1.66,0.14 \quad E=29000 . \quad F Y=30 \quad F=31.68,36,12.89,28.8,16.8$

C ...3/16" Thick $x$-braces (Dwg No. 40-5) "*REVISED to use 1/4" thick"***

C $3 \quad M N=S \quad S H=R \quad T=1,0.25 \quad E=29000$. $F Y=25 \quad F=26.4,30,3.53,18,16$

C ...3/8" Thick light supp (Dwg No. 40-4) Lower Mast Sections B-B, C-C

C $M H=S \quad S H=R \quad i=1,0.375 \quad E=29000 . \quad F Y=25 \quad F=26.4,30,7.67,18,16$

C $M H=S \quad S H=R \quad T=1,0.375 \quad E=29000$. $\quad F Y=25$

C …Short link from center of pipes to braces

C ...Gas Pipes (Dng No. 40-12) Lower Mast Section A-A

C $\quad M N=S \quad S H=P \quad I=0.675,0.15 \quad E=29000 . \quad F Y=25 \quad F=26.4,30,14.33,18,16$
C ...stiff beans which connect from lower mast bottuli plate to C.G. of camera C 7 MN=N $A=9 E 5$ J=9E7 I=9E7,9E7 AS=9E5,9E5 E=1 $G=1 \quad M=0.0 \quad M=0.0$ FRAME

c

C ...steel Pipes (Dug No. 42-8) $11 / 4$ ips

$201268, I=1 \quad n=2 \quad K=2,2 \quad C=0.85,0.85$

C ...3/16" Thick $x$-braces (Dwg No. 40-5) "\#REVISEO to use 1/4" thick"\#\#

$401420, T=1 \quad K=3 \quad K=1,1 \quad C=0.85,0.85$

C ...3/8" Thick light supp (Dwg Mo. 40-4) Lower Mast Sections B-B, C-C

$501503, T=1 \quad H=4 \quad K=1,1 \quad C=0.85,0.85$

C ...Ges Pipes (Dug Mo. 40-12) Laver Mast Section $A-A$

$504505 \quad 1 \quad I=1 \quad M=6 \quad K=1,1 \quad C=0.85,0.85$

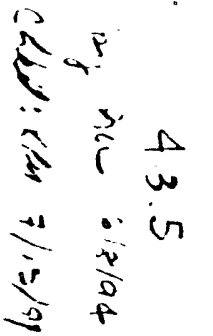




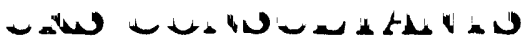

Vol. II, Rev. 0

Sheet No. 5.0

Job \#:66481-01 Job: WHC/MPF CAMERA ASSEMBLY ANALYSIS

By Plu Date 6/8/94

Client: WHC Subject: SAP9O ANALYSIS DOCUMENTATION

Chk'd I/m Date $\#$ ? il

SECTION 5: SAP9O/SAPSTL OUTPUT FILES

TABLE OF CONTENTS

$\begin{array}{lll}\text { SECTION } & \text { CONTENTS } & \frac{\text { PAGE NO. }}{5.1} \\ 5.1 & \text { General Discussion } & 5.1 \\ 5.2 & \text { Output files for SAP90 } & 5.2 .1 \\ 5.3 & \text { Output file for SAPSTL } & 5.3 .1-5.314 \\ 5.4 & \text { Selective graphical output } & 54.1-5460\end{array}$


UKS CONSULTANTS

WHC-SD-WM-DA-163

Vol. II, Rev. 0

Sheet No. 5.1 .1

Job \#:66481-01 Job: WHC/MPF CAMERA ASSEMBLY ANALYSIS

By Pu Date 8/5/94

Client: WHC Subject: SAP9O ANALYSIS DOCUMENTATION

Chk'd R/KDate 3/5/94

5.1 General Discussion

\section{SAP90 Output}

The extensions of the SAP9o output filename are described below:

- SAP Tabulated Input Data Echo

-EQN Optimized Equation Numbers

-RIT Frequencies \& other Model Parameters

-SPC Spectral Analysis Modal Factors

. SOL Joint Displacements and Reactions

.F3F FRAME element forces

- F4F SHELI element stresses

-FEF Element Joint forces

\section{SAPLOT Graphical Results}

Using the SAPLOT graphics post-processor, SAP9O output such as mode shapes, shell element stresses are presented graphically.

\section{SAPSTL OUTPUT}

The extensions of the SAPSTL output filename are described below:

.STL Echo of input data; $K$-factor values, controlling load combinations and stress ratios.

.SHR not used in this analysis

The SAPSTL output file, CM-A-04S.STL, is included in section 5.3 .

\section{PC diskettes}

The final runs of SAP90 / SAPSTL output files are saved on PC diskettes and they are provided with this documentation. 


\section{URS CONSULTANTS}

WHC-SD-WM-DA-163

Vol. II, Rev. 0

Job \#:66481-01 Job: WHC/MPF CAMERA ASSEMBLY ANALYSIS

Client: WHC Subject: SAP90 ANALYSIS DOCUMENTATION

Sheet No. 521

By Der Date $8 / 5 / 94$

Chk'dRIK Date $R / S / 94$

\subsection{SAP9O Output}

Since all of the output files are saved on PC diskettes, oniy selected output is printed for stress and displacement checking purposes in sections 6 through 7 . 
URS CONSULTANTS WHC-SD-WM-DA-163

Vol. II, Rev. 0

Sheet No. 53.1

Job \#:66481-01 Job: WHC/MPF CAMERA ASSEMBLY ANALYSIS

By inu Date 6/8/94

Client: WHC Subject: SAP9O ANALYSIS DOCUMENTATION

Chk'd Date

5.3 SAPSTL Output

The SAPSTL output file, CM-A-04S.STL, is included in this section.

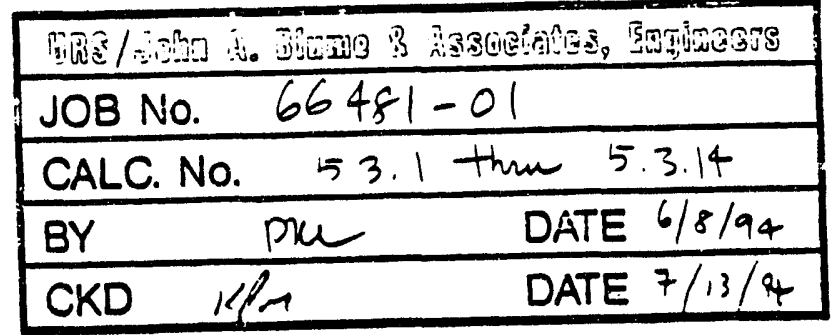


07/13/1994 10:37 Fil ename: $\mathrm{CM}-\mathrm{A}-04 \mathrm{~S}$

Page 1

UHC/MPF CAMERA ASSEMBLY ANALYSIS JOBX: $66458-01$ RUM ID: CH-A-OCS

C Units $=$ Kips, in fy=60 ksi steel density $=490 \mathrm{pcf}=0.0002836 \mathrm{kci}$

\section{Update $\log$}

$\begin{array}{ll}\text { Date } & \text { BY } \\ 5 / 26 / 94 & \text { PKL } \\ 5 / 27 / 94 & \text { PKL }\end{array}$

$\begin{array}{ll}5 / 27 / 94 & \text { PKL } \\ 5 / 31 / 94 & \text { PKL }\end{array}$

6/02/94 PKL

Version
CM-A-0is

CA-A-02S

CM-A-03S

CH-A-03S

Description

SAPSTL for SAP9O run CA- $A-01$

SAPSIL for SAP9O rUn CA-A-OD

SAPSTL for SAP9O rUn CA-A-03
SAPSTL for SAP9O run $C A-A-04$

c.

Computer Models Maming Monenclature

SAP90 files are named using the foll louing terninol ogy:

$C M-A$-01S.ext (".ext as described by SAP9O Manual) 12345678

where: Character Description

where: Charter $\quad C H=$ Camera Assembly Model

C $\quad 4 \quad A=$ Model A, B. C.. etc

$\begin{array}{lll}C & 5 & \text { Model Af B, C... etc. } \\ c & 6-7 & \text { Run Number }\end{array}$

年

Control

:DATACHECK=1, MORMAL=0, STRESS FILE=2

† conso

I. COABO 1

3 . $\begin{array}{ll}D=1,1 & D=1\end{array}$

$\begin{array}{lll}3 & C=1,1 & D=1 \\ 4 & C=1,-1 & D=-1\end{array}$

SECTIOAS

C -. Material properties

C...Stiff beams which connect from Master node Mo. 299 to bolt holes

C $1 \quad M N=N \quad A=9 E 9$ J=9E9 I=9E9,9E9 AS=9E9,9E9 E=1 G=1 $N=0.0 \quad H=0.0$

C ...steel Pipes (Dwg No. 42-8) $11 / 4$ ips

$2 \quad M M=S \quad S H=P \quad T=1.66,0.14 \quad E=29000$. $F Y=30 \quad F=31.68,36,12.89,28.8,16.8$

C ...3/16" Thick $x$-braces (Dwg Mo. 40-5) "\#REVISED to use 1/4" thick"*\#*

C 3 NH=S SH=R $I=1,0.25 \quad E=29000 . \quad F Y=25 \quad F=26.4,30,3.53,18,16$

C ...3/8" Thick light supp (Dug No. 40-4) Lower Mast Sections B-B, C-C

$4 \quad M M=S \quad S H=R \quad I=1,0.375 \quad E=29000 . \quad F Y=25 \quad F=26.4,30,7.67,18,16$

C ...Short link from center of pipes to braces

C $M H=N \quad A=9 E 4 \quad J=9 E 6 \quad I=9 E 6,9 E 6 \quad A S=9 E 6,9 E 6 \quad E=1 . \quad G=1 . \quad N=0.0 \quad M=0.0$

C ...Gas Pipes (Dwg No. 40-12) Lower Mast Section A-A

C $M H=S$ SH=P $I=0.675,0.15 \quad E=29000 . \quad F Y=25 \quad F=26.4,30,14.33,18,16$
07/13/1994 10:37 Filename: CM-A-045 Page 2

C ...stiff beans which connect from lower mast botton plate to C.G. of camera

7 MN $A=9 E 5 \quad J=9 E 7$ I=9E7,9E7 AS=9E5,9E5 E=1 $G=1 \quad H=0.0 \quad M=0.0$

Frane

rano

C ...steel Pipes (Dwg Mo. 42-8) $11 / 4$ ips

$201268 \quad I \quad T=1 \quad M=2 \quad K=2,2 \quad i=0.85,0.85$

C ...3/16" Thick $x$-braces (Dwg Mo. 40-5) "*\#REVISED to use 1/4" thick"*\#

$401420 \quad I \quad J=1 \quad M=3 \quad K=1,1 \quad C=0.85,0.85$

C ...3/8" Thick light supp (Dug Mo. 40-4) Lower Mast Sections B-B, C-C

$501503, I=1 \quad M=4 \quad K=1,1 \quad C=0.85,0.85$

C ...Gas Pipes (Dug Mo. 40-12) Lower Mast Section $A-A$

$504505, T=1 \quad M=6 \quad K=1,1 \quad C=0.85,0.85$ 
pansesas sjyb!s ilr

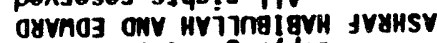
$2661-9861$ (J) 346 ! JNdoj

17. Sd molsabn

06dVS \&04 Хכэнว SS3y1S 73315

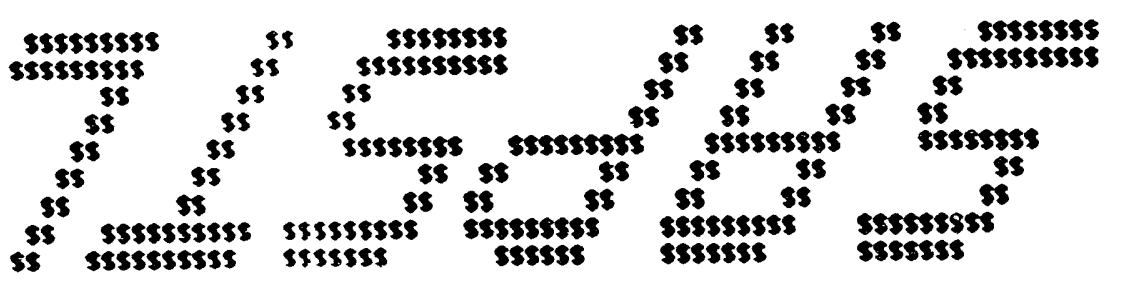

58 s5 55 sकss5sss

\$5\$5555

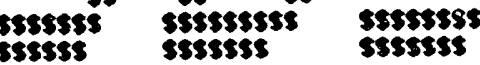


SAP9O FILE:CM-A-OL/SAPSTL FILE:CM-A-0LS.STI UHC/MPF CAMERA ASSEMBLY AMALYSIS JOB\#: 66458-01 RUM ID: CH-A-04S CONTROL DAIA

EXECUTION MODE ........... 2

COOE IYPE $\ldots \ldots \ldots$

1 (AISC. 1989 ASD)

PRINT SUPPRESSION FLAG - ......

0

OVERRIDE FLAG FOR UNITY K-FACTORS - -

IYPE OF UNITS .....................

INTERACTION STRESS RATIO CUTOFF - -

SHEAR STRESS RATIO CUTOFF - ...

0.0005
SAC/MPF CAMERA ASSEMBIY AMALYFILE:CM-A-0L/SAPSIL_FILE:CM-A-04S.SIL

LIVELOAD

Lonp
1
2
3
4 
SAP90 FILE:CM-A-OL/SAPSIL FILE:CH-A-04S.STI WHC/MPF CAMERA ASSEMBLY AMALYSIS JOQH: 66458-01 RUA ID: CH=A-04S

$\begin{array}{rrrr}\text { LOAD CONDIT ION } & \text { CO A B } \\ \text { COMBIMATION } & \text { CONDITION } & \text { FACTOR } \\ 1 & 1 & 1.000 \\ 2 & 0 & 1.000 \\ 3 & 1 & 1.000 \\ & 2 & 1.000 \\ 4 & 0 & 1.000 \\ & 1 & 1.000 \\ & 2 & -1.000 \\ & & -1.000\end{array}$

$\frac{1}{\omega}$
SAP90 FILE:CM-A-04/SAPSIL_FILE:CM-A-0LS.STL

SECIIOA PROPERTY DATA

PROP SECTION

ID TYPE

$\begin{array}{ll}1 & G \\ 2 & p \\ 3 & R \\ 4 & R \\ 5 & G \\ 6 & P \\ 7 & G\end{array}$

DEPTM FLAMGE FLANGE

MIDIH

$\begin{array}{ll}\text { (in) } & \text { (in) } \\ 0.000 & 0.000\end{array}$

(in)

(in)

FLAMGE FLANGE

$0.140 \quad 0.140$

$1.000 \quad 0.250$

$1.000 \quad 0.375$

$0.000 \quad 0.000$

$0.675-0.675$

$.150 \quad 0.150$ 
SAP9O FILE:CH-A-0L/SAPSIL FILE:CM-A-0LS.SIL UMC/MPF CAMERA ASSEMBLY AMALYSIS JOB*: B6458-01 RUM ID: CH=A-04S

SECIION PROPERTY DATA

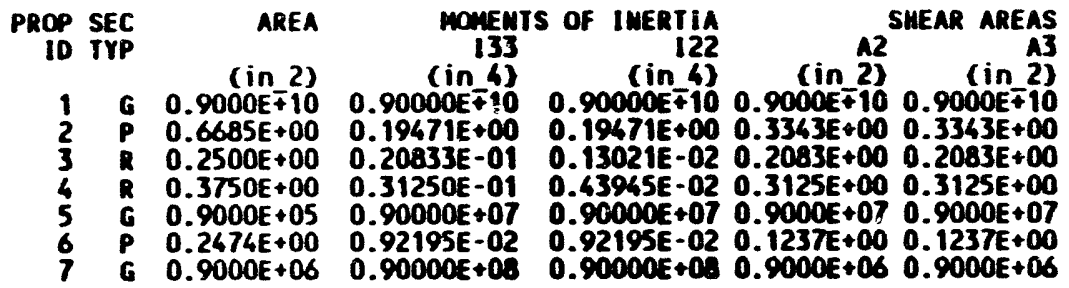

$\stackrel{1}{1}$

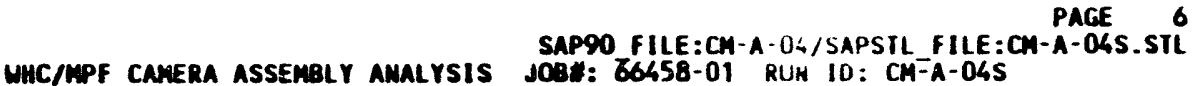
SECIION PROPERIY DAIA

PROP SEC SECTION MOOUL!

ID IYP in

322

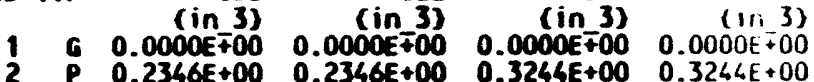

$\begin{array}{llll}0.4167 E-01 & 0.1042 E-01 & 0.6250 E-01 & 0.1563 E-01\end{array}$

R $0.6250 E-01 \quad 0.2344 E-01 \quad 0.9375 E-01 \quad 0.3516 E-01$

G $0.0000 E+00$ 0.0000E+00 $0.0006 E+000.0000 E+00$

$\begin{array}{llllll}6 & \text { P } & 0.2732 E-01 & 0.2732 E-01 & 0.4267 E-01 & 0.4267 E-01 \\ 7 & G & 0.0000 E+00 & 0.0000 E+00 & 0.0000 E+00 & 0.0000 E+00\end{array}$
RADII OF GYRATIO R33 R22

(in)

0.00

0.540

0.289

0.289

0.000

0.193 
SAPQO FILE:CH-A-0G/SAPSIL FILE:CM-A-0LSS STL HHC/MPF CAMERA ASSEMBLY AMALYSIS J08*: 66458-01 RUA 10: CH-A-04S

AATERIAL PROPERTY DATA

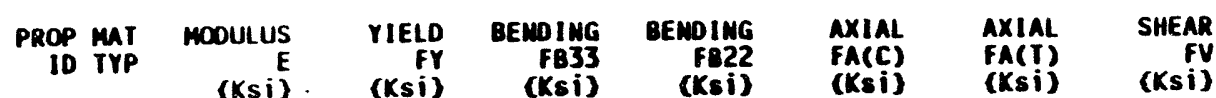

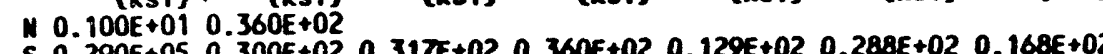
S $0.290 E+050.300 E+020.317 E+020.360 E+020.129 E+02 \quad 0.280 E+02 \quad 0.160 E+02$ S $0.290 E+05 \quad 0.250 E+02 \quad 0.264 E+02 \quad 0.300 E+02 \quad 0.767 E+01 \quad 0.180 E+02 \quad 0.160 E+02$

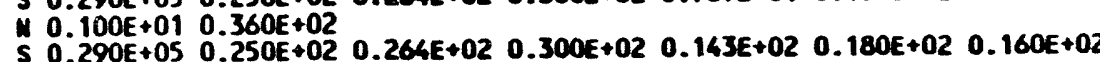

N $0.100 E+010.360 E+02$
SAP9O FILE:CM-A-04/SAPSIL FILE:CA
HHCMPPF CAMERA ASSEMBLY AMALYSIS J08*: $6658-01$ RUN 10: CH=A-04S

PAGE B

\section{FRAME ELEMENT DESIGN OATA}

$\begin{array}{rrrrr}\text { ELI } & \text { ELI } & \text { PROP } & \text { FACIOR } & \text { FACTOO } \\ 10 & \text { IYPE } & \text { ID } & \text { K33 } & \text { K22 } \\ 101 & 1 & 1 & 1.000 & 1.000 \\ 102 & 1 & 1 & 1.000 & 1.000 \\ 103 & 1 & 1 & 1.000 & 1.000 \\ 104 & 1 & 1 & 1.000 & 1.000 \\ 105 & 1 & 1 & 1.000 & 1.000 \\ 106 & 1 & 1 & 1.000 & 1.000 \\ 107 & 1 & 1 & 1.000 & 1.000 \\ 108 & 1 & 1 & 1.000 & 1.000 \\ 109 & 1 & 1 & 1.000 & 1.000 \\ 110 & 1 & 1 & 1.000 & 1.000 \\ 111 & 1 & 1 & 1.000 & 1.000 \\ 112 & 1 & 1 & 1.000 & 1.000 \\ 113 & 1 & 1 & 1.000 & 1.000 \\ 114 & 1 & 1 & 1.000 & 1.000 \\ 115 & 1 & 1 & 1.000 & 1.000 \\ 116 & 1 & 1 & 1.000 & 1.000 \\ 117 & 1 & 1 & 1.000 & 1.000 \\ 118 & 1 & 1 & 1.000 & 1.000 \\ 119 & 1 & 1 & 1.000 & 1.000 \\ 120 & 1 & 1 & 1.000 & 1.000 \\ 201 & 1 & 2 & 2.000 & 2.000 \\ 202 & 1 & 2 & 2.000 & 2.000 \\ 203 & 1 & 2 & 2.000 & 2.000 \\ 204 & 1 & 2 & 2.000 & 2.000 \\ 205 & 1 & 2 & 2.000 & 2.000 \\ 206 & 1 & 2 & 2.000 & 2.000 \\ 207 & 1 & 2 & 2.000 & 2.000 \\ 208 & 1 & 2 & 2.000 & 2.000 \\ 209 & 1 & 2 & 2.000 & 2.000 \\ 210 & 1 & 2 & 2.000 & 2.000 \\ 211 & 1 & 2 & 2.000 & 2.000 \\ 212 & 1 & 2 & 2.000 & 2.000 \\ 213 & 1 & 2 & 2.000 & 2.000 \\ 214 & 1 & 2 & 2.000 & 2.000 \\ 215 & 1 & 2 & 2.000 & 2.000 \\ 216 & 1 & 2 & 2.000 & 2.000 \\ 217 & 1 & 2 & 2.000 & 2.000 \\ 218 & 1 & 2 & 2.000 & 2.000 \\ 219 & 1 & 2 & 2.000 & 2.000 \\ 220 & 1 & 2 & 2.000 & 2.000 \\ 221 & 1 & 2 & 2.000 & 2.000 \\ 222 & 1 & 2 & 2.000 & 2.000 \\ 223 & 1 & 2 & 2.000 & 2.000 \\ 224 & 1 & 2 & 2.000 & 2.000 \\ 225 & 1 & 2 & 2.000 & 2.000 \\ 226 & 1 & 2 & 2.000 & 2.000 \\ 227 & 1 & 2 & 2.000 & 2.000 \\ 228 & 1 & 2 & 2.000 & 2.000 \\ 229 & 1 & 2 & 2.000 & 2.000 \\ 230 & 1 & 2 & 2.000 & 2.000 \\ 231 & 1 & 2 & 2.000 & 2.000 \\ & & & & \end{array}$

factor faciur coeff COEFf coeff factod FOR L33 FOR L22 CM33 CM22 CB FOR LL

$\begin{array}{llllll}\mathbf{1 . 0 0 0} & 1.000 & 0.00 & 0.00 & 0.00 & 1.00 \\ 1.000 & 1.000 & 0.00 & 0.00 & 0.00 & 1.00\end{array}$ 1.000 1.000

1.000

1.000

1.000

1.000

1.000
1.000

1.000

1.000

1.000

1.000
1.000

1.000

1.000

1.000

1.000

1.000

1.000

1.000
1.000

1.000

1.000

1.000

1.000

1.000

1.000

1.000

1.000
1.000

1.000
1.000

1.000
1.000

1.000

1.000

1.000

1.000

1.000
1.000

1.000

1.000

1.000

1.000

1.000

1.000

1.000

1.000
1.000

1.000
1.000
1.000

$\begin{array}{lllll}1.000 & 0.00 & 0.00 & 0.00 & 1.00\end{array}$

$\begin{array}{lllll}1.000 & 0.00 & 0.00 & 0.00 & 1.00\end{array}$

$1.000 \quad 0.00 \quad 0.00 \quad 0.00 \quad 1.00$

$\begin{array}{lllll}000 & 0.00 & 0.00 & 0.00 & 1.00\end{array}$

$\begin{array}{llllll}1.000 & 0.00 & 0.00 & 0.00 & 1.00\end{array}$

$\begin{array}{lllll}0.000 & 0.00 & 0.00 & 0.00 & 1.00\end{array}$

$\begin{array}{lllll}000 & 0.00 & 0.00 & 0.00 & 1.00\end{array}$

$\begin{array}{llllll}000 & 0.00 & 0.00 & 0.00 & 1.00\end{array}$

$\begin{array}{lllll}1.000 & 0.00 & 0.00 & 0.00 & 1.00\end{array}$

$\begin{array}{lllll}1.0000 & 0.00 & 0.00 & 0.00 & 1.00\end{array}$

$1.000 \quad 0.00 \quad 0.00 \quad 0.00 \quad 1.00$

$1.000 \quad 0.00 \quad 0.00 \quad 0.00$

$\begin{array}{llll}000 & 0.00 & 0.00 & 0.00\end{array}$

$\begin{array}{llll}.000 & 0.00 & 0.00 & 0.00\end{array}$

$\begin{array}{llllll}1.000 & 0.00 & 0.00 & 0.00 & 1.00 \\ 1.000 & 0.00 & 0.00 & 0.00 \quad 1.00\end{array}$

$1.000 \quad 0.00 \quad 0.00 \quad 0.00$

$1.000 \quad 0.85 \quad 0.85 \quad 0.00$

1.000 .850 .850 .00

$\begin{array}{llll}1.000 & 0.85 & 0.85 & 0.00\end{array}$

$\begin{array}{llll}1.000 & 0.85 & 0.85 & 0.00 \\ 1.000 & 0.85 & 0.85 & 0.00\end{array}$

$\begin{array}{llll}.000 & 0.85 & 0.85 & 0.00\end{array}$

$\begin{array}{llll}000 & 0.85 & 0.85 & 0.00\end{array}$

$\begin{array}{llll}1.000 & 0.85 & 0.85 & 0.00\end{array}$

$\begin{array}{llll}1.000 & 0.85 & 0.85 & 0.00\end{array}$

$\begin{array}{llll}0100 & 0.85 & 0.85 & 0.00\end{array}$

$\begin{array}{llll}\text { Quo } & 0.85 & 0.85 & 0.00\end{array}$

$\begin{array}{llll}1.000 & 0.85 & 0.85 & 0.00\end{array}$

$\begin{array}{llll}.000 & 0.85 & 0.85 & 0.00\end{array}$

$\begin{array}{lllll}1.0(1 i) & 0.85 & 0.85 & 0.00 & 1.00\end{array}$

$\begin{array}{lllll}1 . i j w 0 & 0.85 & 0.85 & 0.00 & 1.00\end{array}$

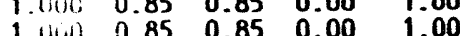

$1.1400 .85 \quad 0.85 \quad 0.00 \quad 1.00$

$\begin{array}{llll}8.85 & 0.85 & 0.00 & 1.00\end{array}$

$1.0000 .850 .850 .00 \quad 1.00$

$1.0000 .85 \quad 0.85 \quad 0.00 \quad 1.00$

$1.0400 .85 \quad 0.85 \quad 0.00 \quad 1.00$

$\begin{array}{lllll}1.5 i v i & 0.85 & 0.85 & 0.00 & 1.00\end{array}$

$\begin{array}{llllll}1.400 & 0.85 & 0.85 & 0.00 & 1.00\end{array}$

$\begin{array}{lllll}1.006 & 0.85 & 0.85 & 0.00 & 1.00\end{array}$

$\begin{array}{lllll}1.304 & 0.85 & 0.85 & 0.00 & 1.00 \\ 1.404 & 0.85 & 0.85 & 0.00 & 1.00\end{array}$ 
SAP90 FILE:CH-A-OL/SAPSTL FILE:CM-A-04S.SIL

FRAME ELEMENT DESIGM DATA

\begin{tabular}{|c|c|c|c|c|c|c|c|c|c|c|}
\hline $\begin{array}{l}\text { ELI } \\
10 \\
232 \\
233 \\
234 \\
235 \\
236 \\
237 \\
238 \\
239 \\
240 \\
241 \\
242 \\
243 \\
244 \\
245 \\
246 \\
247 \\
248 \\
249 \\
250 \\
251 \\
252 \\
253 \\
254 \\
255 \\
256 \\
257 \\
258 \\
259 \\
260 \\
261 \\
262 \\
263 \\
264 \\
265 \\
266 \\
267 \\
268 \\
301 \\
302 \\
303\end{array}$ & $\begin{array}{r}\text { ELI } \\
\text { IYPE } \\
\mathbf{1} \\
\mathbf{1} \\
\mathbf{1} \\
\mathbf{1} \\
\mathbf{1} \\
\mathbf{1} \\
\mathbf{1} \\
\mathbf{1} \\
\mathbf{1} \\
\mathbf{1} \\
\mathbf{1} \\
\mathbf{1} \\
\mathbf{1} \\
\mathbf{1} \\
\mathbf{1} \\
\mathbf{1} \\
\mathbf{1} \\
\mathbf{1} \\
\mathbf{1} \\
\mathbf{1} \\
\mathbf{1} \\
\mathbf{1} \\
\mathbf{1} \\
\mathbf{1} \\
\mathbf{1} \\
\mathbf{1} \\
\mathbf{1} \\
\mathbf{1} \\
\mathbf{1} \\
\mathbf{1} \\
\mathbf{1} \\
\mathbf{1} \\
\mathbf{1} \\
\mathbf{1} \\
\mathbf{1} \\
\mathbf{1} \\
\mathbf{1} \\
\mathbf{1} \\
\mathbf{1} \\
\mathbf{1} \\
\mathbf{1}\end{array}$ & $\begin{array}{r}P R O P \\
10 \\
2 \\
2 \\
2 \\
2 \\
2 \\
2 \\
2 \\
2 \\
2 \\
2 \\
2 \\
2 \\
2 \\
2 \\
2 \\
2 \\
2 \\
2 \\
2 \\
2 \\
2 \\
2 \\
2 \\
2 \\
2 \\
2 \\
2 \\
2 \\
2 \\
2 \\
2 \\
2 \\
2 \\
2 \\
2 \\
2 \\
2 \\
2 \\
2 \\
5 \\
5 \\
5 \\
5 \\
5 \\
5 \\
5 \\
5 \\
5 \\
5 \\
5 \\
5\end{array}$ & $\begin{array}{r}\text { FACTOR } \\
\text { K33 } \\
2.000 \\
2.000 \\
2.000 \\
2.000 \\
2.000 \\
2.000 \\
2.000 \\
2.000 \\
2.000 \\
2.000 \\
2.000 \\
2.000 \\
2.000 \\
2.000 \\
2.000 \\
2.000 \\
2.000 \\
2.000 \\
2.000 \\
2.000 \\
2.000 \\
2.000 \\
2.000 \\
2.000 \\
2.000 \\
2.000 \\
2.000 \\
2.000 \\
2.000 \\
2.000 \\
2.000 \\
2.000 \\
2.000 \\
2.000 \\
2.000 \\
2.000 \\
2.000 \\
1.000 \\
1.000 \\
1.000 \\
1.000 \\
1.000 \\
1.000 \\
1.000 \\
1.000 \\
1.000 \\
1.000 \\
1.000 \\
1.000\end{array}$ & $\begin{array}{r}\text { FACTO0 } \\
\times 22 \\
2.000 \\
2.000 \\
2.000 \\
2.000 \\
2.000 \\
2.000 \\
2.000 \\
2.000 \\
2.000 \\
2.000 \\
2.000 \\
2.000 \\
2.000 \\
2.000 \\
2.000 \\
2.000 \\
2.000 \\
2.000 \\
2.000 \\
2.000 \\
2.000 \\
2.000 \\
2.000 \\
2.000 \\
2.000 \\
2.000 \\
2.000 \\
2.000 \\
2.000 \\
2.000 \\
2.000 \\
2.000 \\
2.000 \\
2.000 \\
2.000 \\
2.000 \\
2.000 \\
1.000 \\
1.000 \\
1.000 \\
1.000 \\
1.000 \\
1.000 \\
1.000 \\
1.000 \\
1.000\end{array}$ & $\begin{array}{r}\text { FACTOR } \\
\text { FOQ } 133 \\
1.000 \\
1.000 \\
1.000 \\
1.000 \\
1.000 \\
1.000 \\
1.000 \\
1.000 \\
1.000 \\
1.000 \\
1.000 \\
1.000 \\
1.000 \\
1.000 \\
1.000 \\
1.000 \\
1.000 \\
1.000 \\
1.000 \\
1.000 \\
1.000 \\
1.000 \\
1.000 \\
1.000 \\
1.000 \\
1.000 \\
1.000 \\
1.000 \\
1.000 \\
1.000 \\
1.000 \\
1.000 \\
1.000 \\
1.000 \\
1.000 \\
1.000\end{array}$ & $\begin{array}{r}\text { FACIOR } \\
\text { FOR } 122 \\
1.000 \\
1.000 \\
1.000 \\
1.000 \\
1.000 \\
1.000 \\
1.000 \\
1.000 \\
1.000 \\
1.000 \\
1.000 \\
1.000 \\
1.000 \\
1.000 \\
1.000 \\
1.000 \\
1.000 \\
1.000 \\
1.000 \\
1.000 \\
1.000 \\
1.000 \\
1.000 \\
1.000 \\
1.000 \\
1.000 \\
1.000 \\
1.000 \\
1.000 \\
1.000 \\
1.000 \\
1.000 \\
1.000 \\
1.000 \\
1.000 \\
1.000\end{array}$ & $\begin{array}{l}\text { COEFF } \\
\text { Cn33 } \\
0.85 \\
0.85 \\
0.85 \\
0.85 \\
0.85 \\
0.85 \\
0.85 \\
0.85 \\
0.85 \\
0.85 \\
0.85 \\
0.85 \\
0.85 \\
0.85 \\
0.85 \\
0.85 \\
0.85 \\
0.85 \\
0.85 \\
0.85 \\
0.85 \\
0.85 \\
0.85 \\
0.85 \\
0.85 \\
0.85 \\
0.85 \\
0.85 \\
0.85 \\
0.85 \\
0.85 \\
0.85 \\
0.85 \\
0.85 \\
0.85 \\
0.85 \\
0.85 \\
0.00 \\
0.00 \\
0.00 \\
0.00 \\
0.00 \\
0.00 \\
0.00 \\
0.00 \\
0.00 \\
0.00 \\
0.00 \\
0.00 \\
0.00 \\
0.00\end{array}$ & $\begin{array}{l}\text { COEFF } \\
\text { CW22 } \\
0.85 \\
0.85 \\
0.85 \\
0.85 \\
0.85 \\
0.85 \\
0.85 \\
0.85 \\
0.85 \\
0.85 \\
0.85 \\
0.85 \\
0.85 \\
0.85 \\
0.85 \\
0.85 \\
0.85 \\
0.85 \\
0.85 \\
0.85 \\
0.85 \\
0.85 \\
0.85 \\
0.85 \\
0.85 \\
0.85 \\
0.85 \\
0.85 \\
0.85 \\
0.85 \\
0.85 \\
0.85 \\
0.85 \\
0.85 \\
0.85 \\
0.85 \\
0.85 \\
0.00 \\
0.00 \\
0.00 \\
0.00 \\
0.00 \\
0.00 \\
0.00 \\
0.00 \\
0.00 \\
0.00 \\
0.00 \\
0.00 \\
0.00 \\
0.00\end{array}$ & $\begin{array}{r}\text { COEFF } \\
\text { CB } \\
0.00 \\
0.00 \\
0.00 \\
0.00 \\
0.00 \\
0.00 \\
0.00 \\
0.00 \\
0.00 \\
0.00 \\
0.00 \\
0.00 \\
0.00 \\
0.00 \\
0.00 \\
0.00 \\
0.00 \\
0.00 \\
0.00 \\
0.00 \\
0.00 \\
0.00 \\
0.00 \\
0.00 \\
0.00 \\
0.00 \\
0.00 \\
0.00 \\
0.00 \\
0.00 \\
0.00 \\
0.00 \\
0.00 \\
0.00 \\
0.00 \\
0.00 \\
0.00 \\
0.00 \\
0.00 \\
0.00 \\
0.00 \\
0.00\end{array}$ & $\begin{array}{lr}\text { F FACTOR } \\
\text { B } \\
\text { FOR } & \text { LL } \\
0 & 1.00 \\
0 & 1.00 \\
0 & 1.00 \\
0 & 1.00 \\
0 & 1.00 \\
0 & 1.00 \\
0 & 1.00 \\
0 & 1.00 \\
0 & 1.00 \\
0 & 1.00 \\
0 & 1.00 \\
0 & 1.00 \\
0 & 1.00 \\
0 & 1.00 \\
0 & 1.00\end{array}$ \\
\hline
\end{tabular}

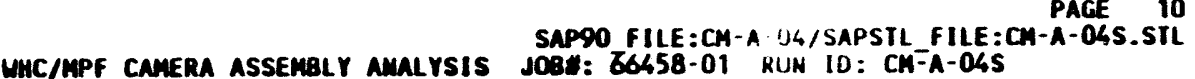

FRAME ELEMENT DESIGN DATA

\begin{tabular}{|c|c|c|c|}
\hline $\begin{array}{l}\text { ELI } \\
\text { ID } \\
315 \\
316 \\
317 \\
318 \\
319 \\
330 \\
321 \\
322 \\
323 \\
324 \\
325 \\
326 \\
327 \\
328 \\
329 \\
330 \\
331 \\
332 \\
333 \\
334 \\
335 \\
336 \\
337 \\
338 \\
339 \\
340 \\
401 \\
402 \\
403 \\
404 \\
405 \\
406 \\
407 \\
408 \\
409 \\
410 \\
411 \\
412\end{array}$ & $\begin{array}{r}\text { ELI } \\
\text { IYPE } \\
\mathbf{1} \\
\mathbf{1} \\
\mathbf{1} \\
\mathbf{1} \\
\mathbf{1} \\
\mathbf{1} \\
\mathbf{1} \\
\mathbf{1} \\
\mathbf{1} \\
\mathbf{1} \\
\mathbf{1} \\
\mathbf{1} \\
\mathbf{1} \\
\mathbf{1} \\
\mathbf{1} \\
\mathbf{1} \\
\mathbf{1} \\
\mathbf{1} \\
\mathbf{1} \\
\mathbf{1} \\
\mathbf{1} \\
\mathbf{1} \\
\mathbf{1} \\
\mathbf{1} \\
\mathbf{1} \\
\mathbf{1} \\
\mathbf{1} \\
\mathbf{1} \\
\mathbf{1} \\
\mathbf{1} \\
\mathbf{1} \\
\mathbf{1} \\
\mathbf{1} \\
\mathbf{1} \\
\mathbf{1} \\
\mathbf{1} \\
\mathbf{1} \\
\mathbf{1} \\
\mathbf{1} \\
\mathbf{1} \\
\mathbf{1} \\
\mathbf{1} \\
\mathbf{1} \\
\mathbf{1} \\
\mathbf{1}\end{array}$ & $\begin{array}{r}\text { PROP } \\
10 \\
5 \\
5 \\
5 \\
5 \\
5 \\
5 \\
5 \\
5 \\
5 \\
5 \\
5 \\
5 \\
5 \\
5 \\
5 \\
5 \\
5 \\
5 \\
5 \\
5 \\
5 \\
5 \\
5 \\
5 \\
5 \\
5 \\
3 \\
3 \\
3 \\
3 \\
3 \\
3 \\
3 \\
3 \\
3 \\
3 \\
3 \\
3 \\
3 \\
3 \\
3 \\
3 \\
3 \\
3 \\
3 \\
3\end{array}$ & $\begin{array}{r}\text { FACTOR } \\
1.33 \\
1.000 \\
1.000 \\
1.000 \\
1.000 \\
1.000 \\
1.000 \\
1.000 \\
1.000 \\
1.000 \\
1.000 \\
1.000 \\
1.000 \\
1.000 \\
1.000 \\
1.000 \\
1.000 \\
1.000 \\
1.000 \\
1.000 \\
1.000 \\
1.000 \\
1.000 \\
1.000 \\
1.000 \\
1.000 \\
1.000 \\
1.000 \\
1.000 \\
1.000 \\
1.000 \\
1.000 \\
1.000 \\
1.000 \\
1.000 \\
1.000 \\
1.000 \\
1.000 \\
1.000 \\
1.000 \\
1.000 \\
1.000 \\
1.000 \\
1.000 \\
1.000 \\
1.000 \\
1.000 \\
1.000 \\
1.000 \\
1.000 \\
1.000 \\
1.000\end{array}$ \\
\hline
\end{tabular}

FACTOR FACIUK COEFF COEFF COEFF FACIOR OR L33 FOR 122 CH33 CM22 CB FOR LL $\begin{array}{llllll}1.000 & 1.000 & 0.00 & 0.00 & 0.00 & 1.00 \\ 1.000 & 1.000 & 0.00 & 0.00 & 0.00 & 1.00\end{array}$ $\begin{array}{llllll}1.000 & 1.000 & 0.00 & 0.00 & 0.00 & 1.00 \\ 1.000 & 1.000 & 0.00 & 0.00 & 0.00 & 1.00\end{array}$ $\begin{array}{llllll}1.000 & 1.000 & 0.00 & 0.00 & 0.00 & 1.00\end{array}$ $\begin{array}{llllll}1.000 & 1.000 & 0.00 & 0.00 & 0.00 & 1.00\end{array}$ $\begin{array}{llllll}1.000 & 1.000 & 0.00 & 0.00 & 0.00 & 1.00\end{array}$ $\begin{array}{lllll}1.000 & 0.00 & 0.00 & 0.00 & 1.00\end{array}$ $\begin{array}{llllll}1.000 & 1.000 & 0.00 & 0.00 & 0.00 & 1.00 \\ 1.000 & 1.000 & 0.00 & 0.00 & 0.00 & 1.00\end{array}$ $\begin{array}{llllll}1.000 & 1.000 & 0.00 & 0.00 & 0.00 & 1.00 \\ 1.000 & 1.000 & 0.00 & 0.00 & 0.00 & 1.00\end{array}$ $\begin{array}{llllll}1.000 & 1.000 & 0.00 & 0.00 & 0.00 & 1.00 \\ 1.000 & 1.000 & 0.00 & 0.00 & 0.00 & 1.00\end{array}$ $\begin{array}{llllll}1.000 & 1.000 & 0.00 & 0.00 & 0.00 & 1.00 \\ 1.000 & 1.000 & 0.00 & 0.00 & 0.00 & 1.00\end{array}$ $\begin{array}{llllll}1.000 & 1.000 & 0.00 & 0.00 & 0.00 & 1.00\end{array}$ $\begin{array}{llllll}1.000 & 1.000 & 0.00 & 0.00 & 0.00 & 1.00 \\ 1.000 & 1.000 & 0.00 & 0.00 & 0.00 & 1.00\end{array}$ $\begin{array}{llllll}1.000 & 1.000 & 0.00 & 0.00 & 0.00 & 1.00<\end{array}$

1.000

1.000

1.000

1.000

1.000
1.000

1.000
1.000

1.000

1.000

1.000

1.000
1.000

1.000
1.000

1.000
1.000

1.0000

1.000

1.000

1.000

1.000
1.000

1.000

1.000

1.000

1.000

1.000

1.000

1.000

1.000
1.000

1.000
1.000

1.000
1.000

1.000
1.000 $\begin{array}{lllll}1.000 & 0.00 & 0.00 & 0.00 & 1.000 \\ 1.00 & \end{array}$

$1.000 \quad 0.00 \quad 0.00 \quad 6.00 \quad 1.00-$

$\begin{array}{lllll}1.000 & 0.00 & 0.00 & 0.00 & 1.00 \\ 1.000 & 0.00 & 0.00 & 0.00 & 1.00 .\end{array}$

$1.000 \quad 0.00 \quad 0.00 \quad 0.00$

1.0000 .000 .000 .00

$\begin{array}{lllll}1.000 & 0.00 & 0.00 & 0.00 & 1.00 \\ 1.000 & 0.00 & 0.00 & 0.00 & 1.00\end{array}$

$\begin{array}{lllll}1.000 & 0.00 & 0.00 & 0.00 & 1.00<\end{array}$

$\begin{array}{lllll}1.000 & 0.85 & 0.85 & 0.00 & 1.00 \\ 1.000 & 0.85 & 0.85 & 0.00 & 1.000 \%\end{array}$

$\begin{array}{lllll}1.900 & 0.85 & 0.85 & 0.00 & 1.00\end{array}$

$\begin{array}{lllll}1.000 & 0.85 & 0.85 & 0.00 & 1.00\end{array}$

$\begin{array}{lllll}1.000 & 0.85 & 0.85 & 0.00 & 1.00\end{array}$

$\begin{array}{lllll}1.000 & 0.85 & 0.85 & 0.00 & 1.00 \\ 1.000 & 0.85 & 0.85 & 0.00 & 1.00\end{array}$

$\begin{array}{llllll}1.000 & 0.85 & 0.85 & 0.00 & 1.00\end{array}$

$\begin{array}{lllll}1.000 & 0.85 & 0.85 & 0.00 & 1.00\end{array}$

$\begin{array}{lllll}1.000 & 0.85 & 0.85 & 0.00 & 1.00\end{array}$

$\begin{array}{lllll}1.000 & 0.85 & 0.85 & 0.00 & 1.00\end{array}$

$\begin{array}{lllll}1.300 & 0.85 & 0.85 & 0.00 & 1.00\end{array}$

$\begin{array}{lllll}1.000 & 0.85 & 0.85 & 0.00 & 1.00\end{array}$

$1.0000 .850 .850 .00 \quad 1.00$

$\begin{array}{llllll}1.000 & 0.85 & 0.85 & 0.00 & 1.00\end{array}$

$\begin{array}{lllll}1.000 & 0.85 & 0.85 & 0.00 & 1.00\end{array}$

$1.4000 .85 \quad 0.85 \quad 0.00 \quad 1.00$

$\begin{array}{llllll}1.400 & 0.85 & 0.85 & 0.00 & 1.00\end{array}$

$\begin{array}{lllll}1.1 .040 & 0.85 & 0.85 & 0.00 & 1.00\end{array}$

$\begin{array}{lllll}1.6100 & 0.85 & 0.85 & 0.00 & 1.00\end{array}$

$\begin{array}{lllll}1.660 & 0.85 & 0.85 & 0.00 & 1.00 \\ 1.3110 & 0.85 & 0.85 & 0.00 & 1.00\end{array}$ $\begin{array}{llllll}1.000 & 1.000 & 0.00 & 0.00 & 0.00 & 1.00 \\ 1.000 & 1.000 & 0.00 & 0.00 & 0.00 & 1.00\end{array}$

$\begin{array}{llllll}1.400 & 0.85 & 0.85 & 0.00 & 1.00\end{array}$ 
SAP9O FILE:CA-A-04/SAPSIL_FILC:CM-A-04S.SIL UHC/MPF CAMERA ASSEMBLY AMALYSIS JOB: 66458-01 RUN ID: CH= $A$-04S

FRAME ELEMENT DESIGN DATA

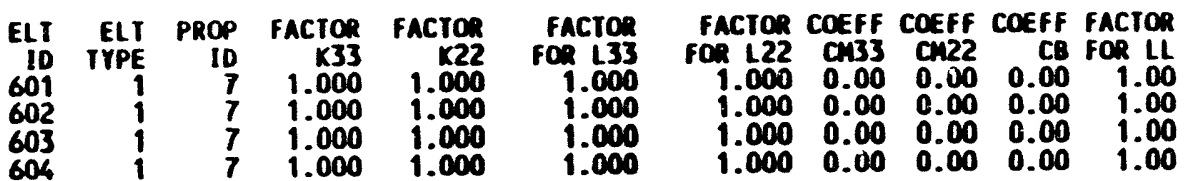

$\vec{v}$
SAP90 FILE:CM-A-OL/SAPSIL FILE:
UHCIMPF CAMERA ASSEMBLY AMALYSIS J08: $66458-01$ KUN ID: CH-A-04S

PAGE 12

SAP9O FRAME ELEMENT DATA

ELI JOINT JOIMT ELEMENT
ID END-I END-J

$\begin{array}{llll}101 & 299 & 201 & (\mathrm{in}) \\ 12.500\end{array}$

$\begin{array}{llll}102 & 299 & 201 & 12.500 \\ 103 & 209 & 203 & 12.500\end{array}$

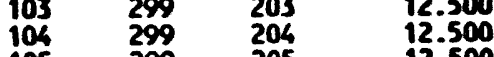

$\begin{array}{llll}105 & 299 & 205 & 12.500 \\ 106 & 299 & 206 & 12.500\end{array}$

$107 \quad 299 \quad 207 \quad 12.500$

$108 \quad 299 \quad 208 \quad 12.500$

$\begin{array}{llll}109 & 299 & 209 & 12.500\end{array}$

$110 \quad 299 \quad 211 \quad 12.500$

$\begin{array}{llll}111 & 299 & 211 & 12.500\end{array}$

$\begin{array}{llll}113 & 299 & 213 & 12.500 \\ 114 & 299 & 214 & 12.500\end{array}$

$\begin{array}{llll}115 & 299 & 215 & 12.500 \\ 116 & 299 & 216 & 12.500\end{array}$

$\begin{array}{llll}116 & 299 & 216 & 12.500 \\ 117 & 299 & 217 & 12.500\end{array}$

$\begin{array}{llll}118 & 299 & 218 & 12.500\end{array}$

$\begin{array}{llll}119 & 299 & 219 & 12.500\end{array}$

12.500

12.875

12.875

12.875

12.875
10.107

10.107

10.107

10.107

13.893

13.893

13.893

13.893

13.893

13.893

10.107

10.107

10.107

10.107

13.893

13.893

13.893

13.893
10.107

10.107

10.107

10.107

10.107

13.893 
SAPGO FILE:CH-A-04/SAPSTL FILE:CH-A-0LS.SIL UMCIMPF CAMERA ASSEMBLY AMALYSIS JOQU: 66458-01 RUM 1D: CH=A-04S

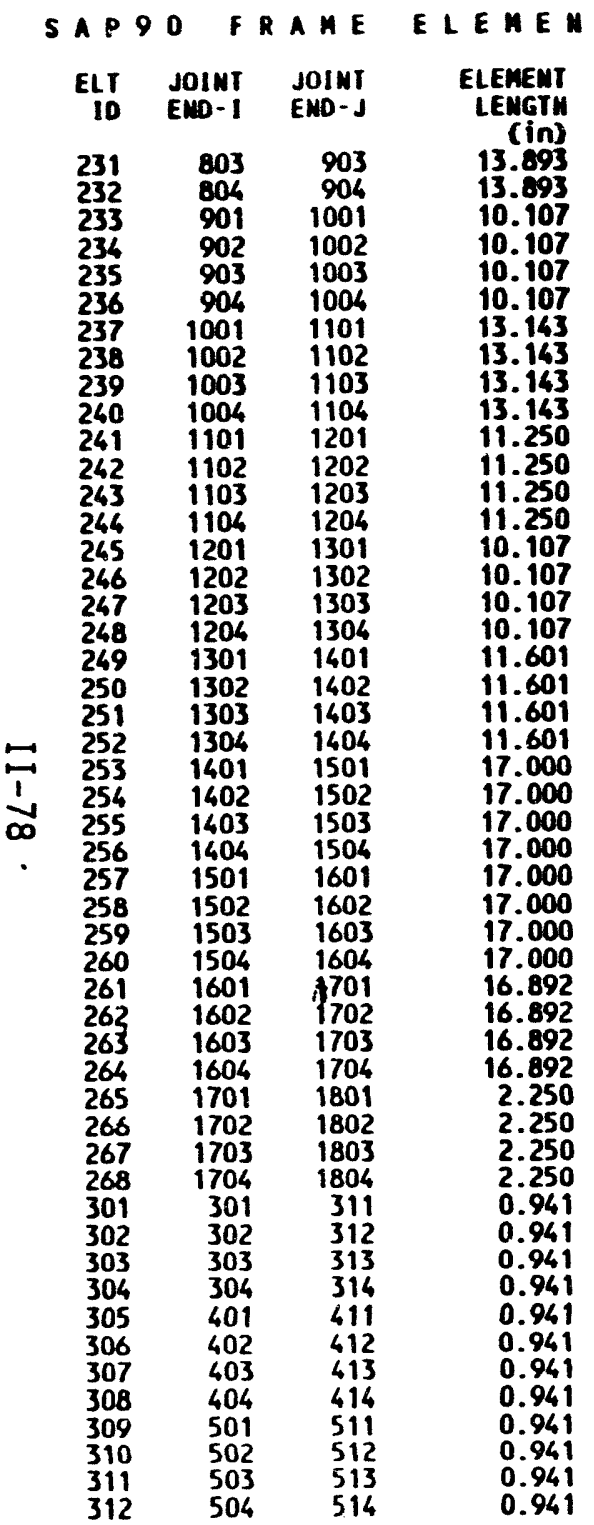

SAPSO FILE:CM-A US/SAPSIL_FILE:CM-A-0LS.SIL UMC/MPF CAMERA ASSEMBLY AMALYSIS JOBH: 86458-01 KUN ID: CH-A-04S SAPGO FRAME ELEMENT DAIA

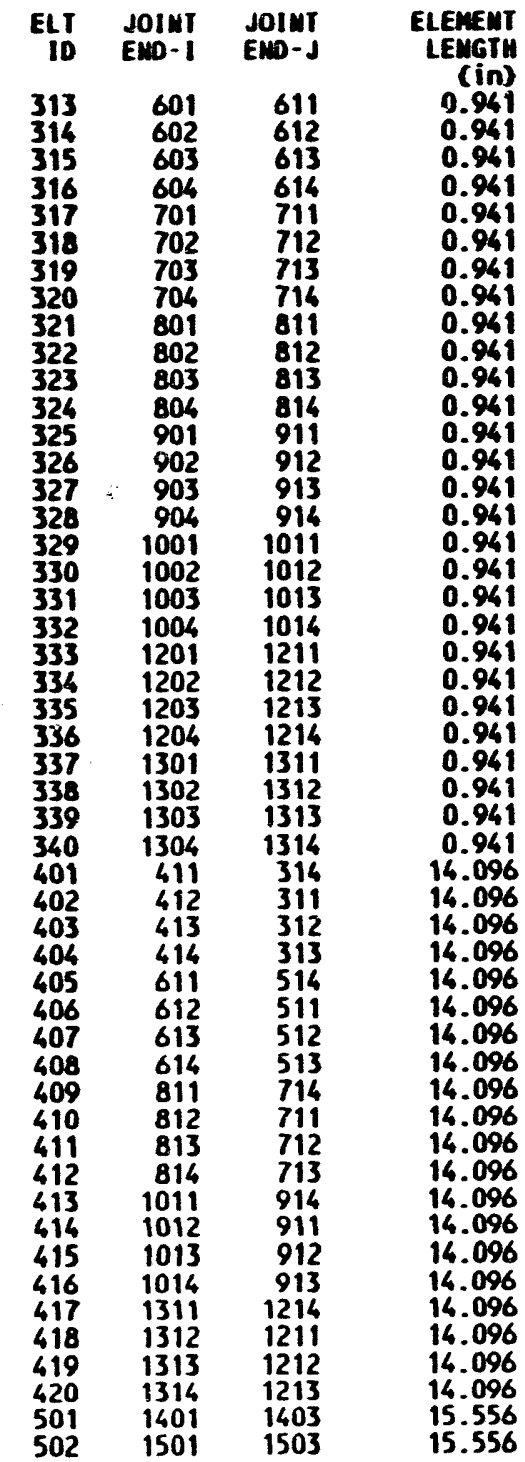


SAP90 FILE:CM-A-04/SAPSIL FILE:CM-A-04S.SIL
PAGE SAP9o fRAME ELEnENT DATA

\begin{tabular}{|c|c|c|c|}
\hline $\begin{array}{l}\text { ELI } \\
\text { ID }\end{array}$ & $\begin{array}{l}\text { JOINT } \\
\text { END-I }\end{array}$ & $\begin{array}{l}\text { JOINI } \\
\text { END-J }\end{array}$ & $\begin{array}{l}\text { ELEMENT } \\
\text { LEMGTH }\end{array}$ \\
\hline $\begin{array}{l}503 \\
504 \\
505 \\
601 \\
602 \\
603 \\
604\end{array}$ & $\begin{array}{l}1601 \\
1702 \\
1704 \\
1801 \\
1802 \\
1803 \\
1804\end{array}$ & $\begin{array}{l}1603 \\
1703 \\
1703 \\
2001 \\
2001 \\
2001 \\
2001\end{array}$ & $\begin{array}{l}15.556 \\
11.000 \\
11.000 \\
23.353 \\
25.814 \\
23.353 \\
20.600\end{array}$ \\
\hline
\end{tabular}

$\stackrel{1}{\frac{1}{6}}$
SAP9OFILE:CM-A-UL/SAPSIL FILE:CM-A-04S.SIL AISC SPECIFICATIONS, ASO 1989 AXIAL LOAD \& BIAXIAi MONENT IMTERACTION STRESS CHECA

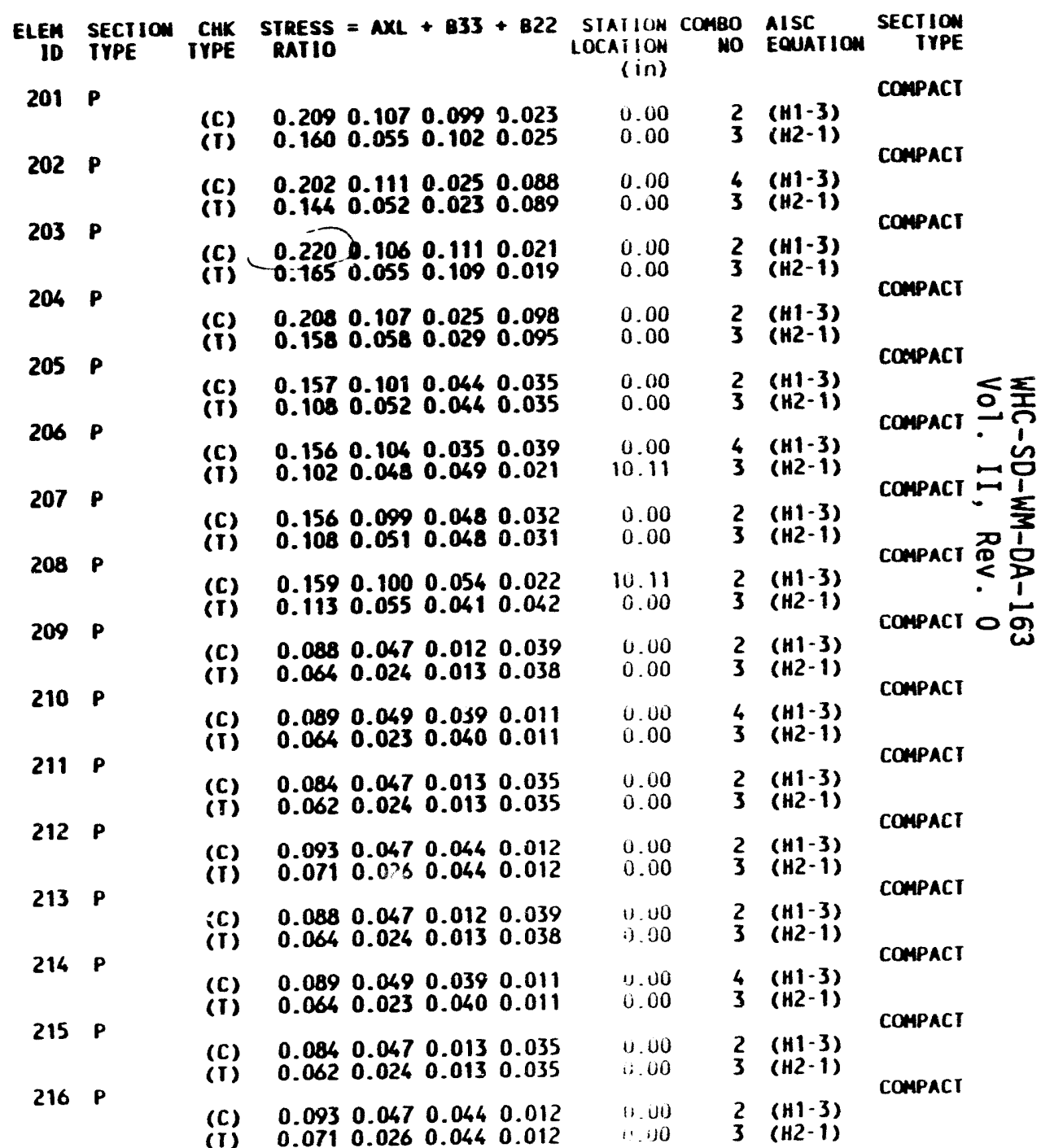


MHC/MPF CAMERA ASSEMBLY AMALYSIS SAP90 FILE:CM-A-04/SAPSIL FILE:CM-A-04S.SIL AISC SPECIFICATIONS, ASO 1969 AXIAL LOAD \& GIAXIAL MONEMT IMTERACTION STRESS CHECK

\begin{tabular}{|c|c|c|c|c|c|c|c|}
\hline $\begin{array}{l}\text { ELEN } \\
\text { ID }\end{array}$ & $\begin{array}{l}\text { SECIIOW } \\
\text { TYPE }\end{array}$ & $\underset{\text { TYPE }}{\text { Clik }}$ & $\begin{array}{l}\text { SIRESS } \\
\text { RATIO }\end{array}$ & $\begin{array}{r}\text { Station } \\
\text { LOCATION } \\
\text { cin) }\end{array}$ & $\begin{array}{r}\text { COnso } \\
\text { MO }\end{array}$ & $\begin{array}{l}\text { AISC } \\
\text { EOUATION }\end{array}$ & SECTIO \\
\hline 217 & $P$ & (C) & $\begin{array}{llll}0.138 & 0.090 & 0.039 & 0.028 \\
0.095 & 0.046 & 0.040 & 0.028\end{array}$ & $\begin{array}{l}0.00 \\
0.00\end{array}$ & $\frac{2}{3}$ & $\begin{array}{l}(H 1-3) \\
(H 2-1)\end{array}$ & \\
\hline 218 & $\mathbf{P}$ & (C) & $\begin{array}{llll}0.138 & 0.093 & 0.028 & 0.035 \\
0.089 & 0.043 & 0.029 & 0.035\end{array}$ & $\begin{array}{l}0.00 \\
0.00\end{array}$ & 4 & $\begin{array}{l}(H 1-3) \\
(H 2-1)\end{array}$ & \\
\hline 218 & $p$ & (C) & $\begin{array}{llll}0.139 & 0.088 & 0.044 & 0.026 \\
0.097 & 0.046 & 0.044 & 0.026\end{array}$ & $\begin{array}{l}0.00 \\
0.00\end{array}$ & 2 & $\begin{array}{l}(H 1-3) \\
(H 2-1)\end{array}$ & \\
\hline $2<0$ & $p$ & (C) & $\begin{array}{llll}0.140 & 0.090 & 0.033 & 0.038 \\
0.039 & 0.050 & 0.032 & 0.038\end{array}$ & $\begin{array}{l}0.00 \\
0.00\end{array}$ & 3 & $\begin{array}{l}(H 1-3) \\
(H 2-1)\end{array}$ & \\
\hline ckt & $r$ & (C) & $\begin{array}{llll}0.124 & 0.084 & 0.013 & 0.038 \\
0.083 & 0.044 & 0.014 & 0.037\end{array}$ & $\begin{array}{l}0.00 \\
0.00\end{array}$ & $\frac{2}{3}$ & $\begin{array}{l}(\mathrm{H} 1-3) \\
(\mathrm{H} 2-1)\end{array}$ & \\
\hline CC: & $r$ & (C) & $\begin{array}{llll}0.128 & 0.088 & 0.038 & 0.012 \\
0.081 & 0.040 & 0.039 & 0.011\end{array}$ & $\begin{array}{l}0.00 \\
0.00\end{array}$ & 4 & $\begin{array}{l}\left(\begin{array}{c}H 1-3) \\
(H 2-1)\end{array}\right. \\
(H 2-1)\end{array}$ & \\
\hline$<2$ & $r$ & $\begin{array}{l}\text { (C) } \\
\text { (T) }\end{array}$ & $\begin{array}{llll}0.121 & 0.084 & 0.015 & 0.034 \\
0.080 & 0.043 & 0.015 & 0.034\end{array}$ & $\begin{array}{l}0.00 \\
0.00\end{array}$ & $\frac{2}{3}$ & $\begin{array}{l}(\mathrm{H} 1-3) \\
(\mathrm{H} 2-1)\end{array}$ & \\
\hline 224 & $\mathbf{P}$ & (C) & $\begin{array}{llll}0.129 & 0.084 & 0.043 & 0.013 \\
0.092 & 0.047 & 0.043 & 0.013\end{array}$ & $\begin{array}{l}0.00 \\
0.00\end{array}$ & $\frac{2}{3}$ & $\begin{array}{l}(H 1-3) \\
(H 2-1)\end{array}$ & \\
\hline 225 & $\mathbf{p}$ & $\begin{array}{l}\text { (C) } \\
\text { (I) }\end{array}$ & $\begin{array}{llll}0.113 & 0.080 & 0.013 & 0.030 \\
0.074 & 0.041 & 0.014 & 0.030\end{array}$ & $\begin{array}{l}10.11 \\
10.11\end{array}$ & 2 & $\begin{array}{l}(H 1-3) \\
(H 2-1)\end{array}$ & \\
\hline 226 & $p$ & $\begin{array}{l}\text { (C) } \\
\text { (I) }\end{array}$ & $\begin{array}{llll}0.115 & 0.083 & 0.030 & 0.011 \\
0.070 & 0.038 & 0.030 & 0.011\end{array}$ & $\begin{array}{l}10.11 \\
10.11\end{array}$ & $\begin{array}{l}4 \\
3\end{array}$ & $\begin{array}{l}\left(\begin{array}{c}H 1-3) \\
(H 2-1)\end{array}\right) \\
(H 2\end{array}$ & \\
\hline $2<4$ & $p$ & (C) & $\begin{array}{llll}0.108 & 0.078 & 0.015 & 0.026 \\
0.071 & 0.041 & 0.015 & 0.026\end{array}$ & $\begin{array}{l}10.11 \\
10.11\end{array}$ & 2 & $\begin{array}{l}(H 1-3) \\
(H 2-1)\end{array}$ & \\
\hline$<<0$ & $r$ & $\begin{array}{l}\text { (C) } \\
\text { (I) }\end{array}$ & $\begin{array}{llll}0.116 & 0.080 & 0.034 & 0.013 \\
0.081 & 0.044 & 0.034 & 0.013\end{array}$ & $\begin{array}{l}10.11 \\
10.11\end{array}$ & 2 & $\begin{array}{l}\left(\begin{array}{l}H 1-3) \\
(H 2-1)\end{array}\right. \\
(12-1)\end{array}$ & \\
\hline 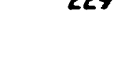 & $r$ & $\begin{array}{l}\text { (C) } \\
\text { (I) }\end{array}$ & $\begin{array}{llll}0.138 & 0.075 & 0.063 & 0.009 \\
0.102 & 0.039 & 0.063 & 0.009\end{array}$ & $\begin{array}{l}13.89 \\
13.89\end{array}$ & $\frac{2}{3}$ & $\begin{array}{l}(H 1-3) \\
(H 2-1)\end{array}$ & \\
\hline $\cos$ & $p$ & (C) & $\begin{array}{llll}0.134 & 0.078 & 0.009 & 0.055 \\
0.091 & 0.035 & 0.009 & 0.054\end{array}$ & $\begin{array}{l}13.89 \\
13.89\end{array}$ & $\begin{array}{l}4 \\
3\end{array}$ & $\begin{array}{l}(H 1-3) \\
(H 2-1)\end{array}$ & \\
\hline 251 & $\mathbf{P}$ & $\begin{array}{l}\text { (C) } \\
\text { (I) }\end{array}$ & $\begin{array}{llll}0.146 & 0.074 & 0.071 & 0.009 \\
0.111 & 0.038 & 0.072 & 0.008\end{array}$ & $\begin{array}{l}13.89 \\
13.89\end{array}$ & $\frac{2}{3}$ & $\begin{array}{l}\left(\begin{array}{c}H 1-3) \\
(H 2-1)\end{array}\right. \\
(H 2)\end{array}$ & \\
\hline 23 & $p$ & (C) & $\begin{array}{llll}0.138 & 0.075 & 0.011 & 0.063 \\
0.106 & 0.042 & 0.011 & 0.063\end{array}$ & $\begin{array}{l}13.89 \\
13.89\end{array}$ & $\frac{2}{3}$ & $\begin{array}{l}(H 1-3) \\
(H 2-1)\end{array}$ & \\
\hline
\end{tabular}

PAP90 FILE:CM-A-06/SAPSIL_FILE:CM-A-06S.SIL 18
FIL WHCNAPF CAMERA ASSEMBLY AMALYSIS JOBH: 66458-01 RUN 10: CM=A-04S

AISC SPECIFICATIONS, ASD 1989 AXIAL LOND \& BIAXIAi MOMENT IMTERACTION STRESS CHECA

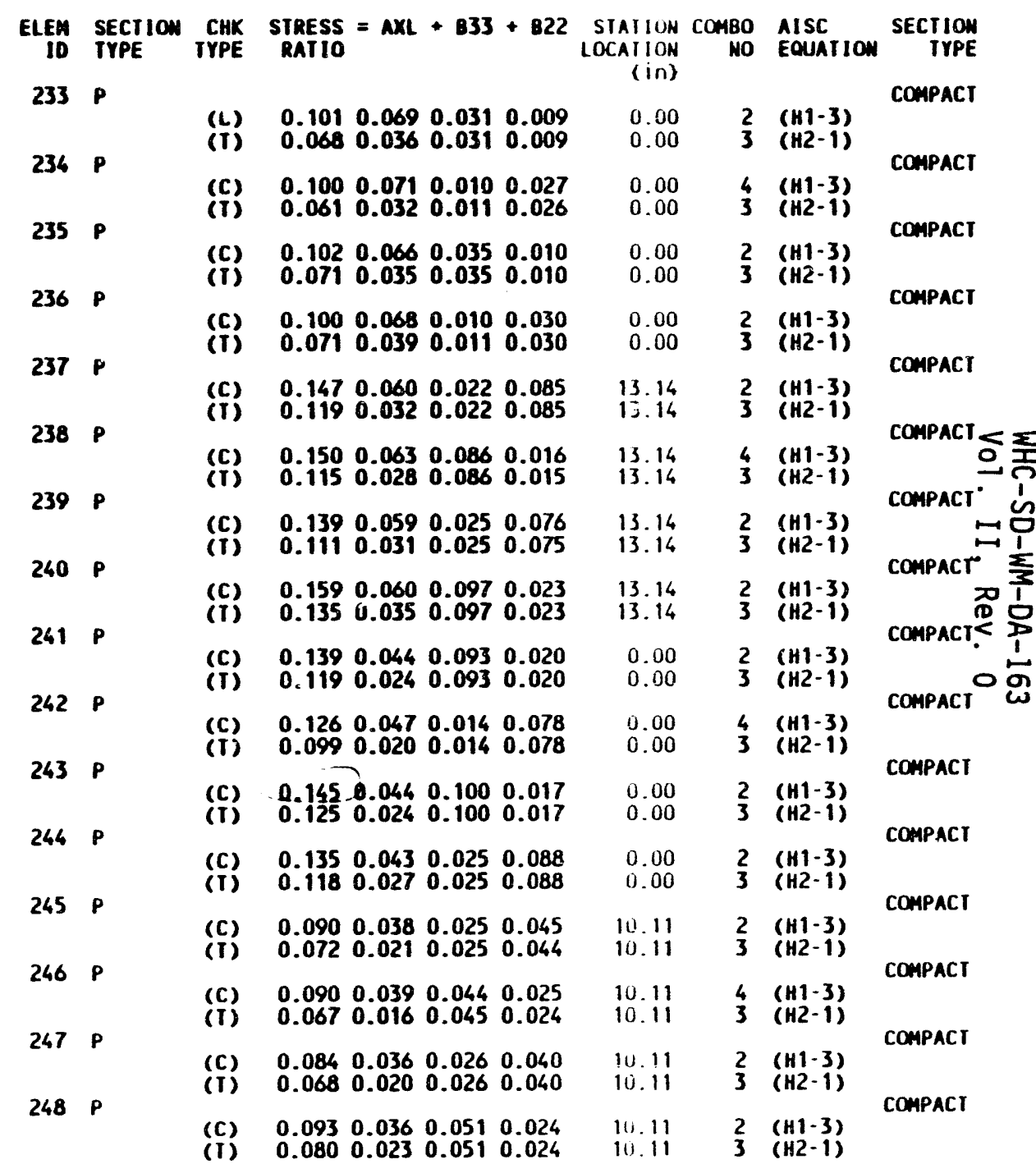


SAP90 FILE:CH-A-04/SAPSIL FILE:CM-A-04S.SIL UHC/MPF CAMERA ASSEMBLY AMALYSIS JOB*: 66458-01 RUW ID: CH-A-04S

AISC SPECIFICATIONS, ASD 1989

AXIAL LOAD \& BIAXIAL MOMENT INTERACTION STRESS CHECK

\begin{tabular}{|c|c|c|c|c|c|c|c|}
\hline $\begin{array}{r}\text { ELEM } \\
\text { ID }\end{array}$ & $\begin{array}{l}\text { SECTION } \\
\text { IYPE }\end{array}$ & $\begin{array}{l}\text { CHK } \\
\text { TYPE }\end{array}$ & $\underset{\text { RATIO }}{\text { STRESS }}=A X L+833+822$ & $\begin{array}{c}\text { stayion } \\
\text { Locaigon } \\
\text { (in) }\end{array}$ & $\begin{array}{r}\text { COMBO } \\
\text { NO }\end{array}$ & $\begin{array}{l}\text { AISC } \\
\text { EOUATION }\end{array}$ & $\begin{array}{l}\text { SECTIOW } \\
\text { IYPE }\end{array}$ \\
\hline 249 & P & (C) & $\begin{array}{llll}0.111 & 0.029 & 0.026 & 0.079 \\
0.099 & 0.016 & 0.026 & 0.078\end{array}$ & $\begin{array}{l}0.00 \\
0.00\end{array}$ & 2 & $\begin{array}{l}(\mathrm{H1}-3) \\
(\mathrm{H2}-1)\end{array}$ & \\
\hline 250 & $\mathbf{P}$ & (C) & $\begin{array}{llll}0.112 & 0.030 & 0.079 & 0.023 \\
0.096 & 0.012 & 0.079 & 0.022\end{array}$ & $\begin{array}{l}0.00 \\
0.00\end{array}$ & 4 & 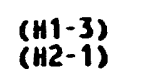 & \\
\hline 251 & $\mathbf{P}$ & (C) & $\begin{array}{llll}0.105 & 0.030 & 0.028 & 0.070 \\
0.092 & 0.017 & 0.028 & 0.070\end{array}$ & $\begin{array}{l}0.00 \\
0.00\end{array}$ & $\frac{2}{3}$ & $\begin{array}{l}(\mathrm{H1}-3) \\
(\mathrm{H2}-1)\end{array}$ & \\
\hline 252 & $\mathbf{P}$ & (C) & $\begin{array}{llll}0.119 & 0.027 & 0.089 & 0.023 \\
0.111 & 0.019 & 0.089 & 0.023\end{array}$ & $\begin{array}{l}0.00 \\
0.00\end{array}$ & $\frac{2}{3}$ & $\begin{array}{l}(H 1-3) \\
(H 2-1)\end{array}$ & \\
\hline & $D$ & (C) & $\begin{array}{llll}0.073 & 0.027 & 0.011 & 0.045 \\
0.062 & 0.015 & 0.011 & 0.045\end{array}$ & $\begin{array}{l}0.00 \\
0.00\end{array}$ & $\frac{2}{3}$ & $\begin{array}{l}(\mathrm{H} 1-3) \\
(\mathrm{HL}-1)\end{array}$ & \\
\hline 28 & 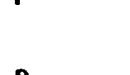 & (C) & $\begin{array}{llll}0.077 & 0.030 & 0.046 & 0.008 \\
0.059 & 0.012 & 0.067 & 0.008\end{array}$ & $\begin{array}{l}0.00 \\
0.00\end{array}$ & $\frac{4}{3}$ & $\begin{array}{l}(H 1-3) \\
(H 2-1)\end{array}$ & \\
\hline 2כ) & $p$ & (C) & $\begin{array}{llll}0.076 & 0.028 & 0.014 & 0.046 \\
0.063 & 0.016 & 0.014 & 0.045\end{array}$ & $\begin{array}{l}0.00 \\
0.00\end{array}$ & $\frac{2}{3}$ & $\begin{array}{l}(\mathrm{H} 1-3) \\
(\mathrm{H} 2-1)\end{array}$ & \\
\hline $2 J 0$ & & (C) & $\begin{array}{llll}0.080 & 0.027 & 0.053 & 0.007 \\
0.072 & 0.018 & 0.053 & 0.007\end{array}$ & $\begin{array}{l}0.60 \\
0.00\end{array}$ & $\frac{2}{3}$ & $\begin{array}{l}(\mathrm{H1}-3) \\
(\mathrm{H2}-1)\end{array}$ & \\
\hline & & $\begin{array}{l}\text { (C) } \\
\text { (I) }\end{array}$ & $\begin{array}{llll}0.084 & 0.025 & 0.038 & 0.045 \\
0.073 & 0.014 & 0.038 & 0.044\end{array}$ & $\begin{array}{l}17.00 \\
17.00\end{array}$ & $\begin{array}{l}2 \\
3\end{array}$ & $\begin{array}{l}(H 1-3) \\
(H 2-1)\end{array}$ & \\
\hline 258 & $\mathbf{P}$ & $\begin{array}{l}\text { (C) } \\
\text { (I })\end{array}$ & $\begin{array}{llll}0.085 & 0.030 & 0.045 & 0.031 \\
0.067 & 0.012 & 0.045 & 0.031\end{array}$ & $\begin{array}{l}17.00 \\
17.00\end{array}$ & $\begin{array}{l}4 \\
2\end{array}$ & $\begin{array}{l}(H 1-3) \\
(H 2-1)\end{array}$ & \\
\hline 259 & $\mathbf{P}$ & $\begin{array}{l}\text { (C) } \\
\text { (I) }\end{array}$ & $\begin{array}{llll}0.085 & 0.026 & 0.041 & 0.042 \\
0.073 & 0.014 & 0.041 & 0.042\end{array}$ & $\begin{array}{l}17.00 \\
17.00\end{array}$ & $\begin{array}{l}2 \\
3\end{array}$ & $\begin{array}{l}\left(\begin{array}{l}H 1-3 \\
(H 2-1)\end{array}\right) \\
(H 2)\end{array}$ & \\
\hline 260 & $p$ & $\begin{array}{l}\text { (C) } \\
\text { (I) }\end{array}$ & $\begin{array}{llll}0.088 & 0.027 & 0.051 & 0.034 \\
0.079 & 0.018 & 0.051 & 0.034\end{array}$ & $\begin{array}{l}17.00 \\
17.00\end{array}$ & $\frac{2}{3}$ & $\begin{array}{l}(\mathrm{H} 1-3) \\
(\mathrm{H} 2-1)\end{array}$ & \\
\hline 201 & $r$ & $\begin{array}{l}\text { (C) } \\
\text { (!) }\end{array}$ & $\begin{array}{llll}0.125 & 0.024 & 0.055 & 0.085 \\
0.114 & 0.013 & 0.056 & 0.085\end{array}$ & $\begin{array}{l}16.99 \\
16.89\end{array}$ & $\frac{2}{3}$ & $\begin{array}{l}(\mathrm{H} 1-3) \\
(\mathrm{H} 2-1)\end{array}$ & \\
\hline 20 & $\boldsymbol{r}$ & (c) & $\begin{array}{llll}0.130 & 0.030 & 0.087 & 0.048 \\
0.111 & 0.012 & 0.087 & 0.048\end{array}$ & $\begin{array}{l}16.89 \\
16.89\end{array}$ & $\begin{array}{l}4 \\
2\end{array}$ & $\begin{array}{l}(H 1-3) \\
(H 2-1)\end{array}$ & \\
\hline 63 & & (C) & $\begin{array}{llll}0.127 & 0.025 & 0.065 & 0.079 \\
0.116 & 0.013 & 0.066 & 0.079\end{array}$ & $\begin{array}{l}16.89 \\
16.89\end{array}$ & $\frac{2}{3}$ & $\begin{array}{l}(H 1-3) \\
(H 2-1)\end{array}$ & \\
\hline 204 & $r$ & (C) & $\begin{array}{llll}0.138 & 0.027 & 0.099 & 0.051 \\
0.129 & 0.018 & 0.099 & 0.051\end{array}$ & $\begin{array}{l}16.89 \\
16.89\end{array}$ & $\frac{2}{3}$ & $\begin{array}{l}\left(\begin{array}{l}H 1-3) \\
(H 2-1)\end{array}\right.\end{array}$ & \\
\hline
\end{tabular}

SAP90 FILE:CM-A-OLL/SAPSIL FILE:CM-A-0LS SIL SAP90 FILE:CM-A-04/SAPSTL FILE:CM-A-04S.SIL
UHC/MPF CAMERA ASSEMBLY AMALYSIS JOB\%: $66458-01$ AISC SPECIFICATIONS, ASD 1989 AXIAL LOAD \& BIAXIAL MOHENT INTERACTION SIRESS CHECK

ELEM SECIION CHK SIRESS $=A X L+B 33+822$ SIAIION COMBO AISC 10 TYPE IYPE RATIO SECIION $265 P$

$266 P$

(C) $0.1320 .0240 .058 \quad 0.091$ (in)

$267 P$

2.25

(T) $\quad 0.1180 .0120 .0920 .053$ 2.25
2.25

$268 P$

(C) 0.1230 .0230 .0710 .070

(C) 0.1310 .0260 .0910 .051

2.25
2.25

$401 R$

$\begin{array}{lllll}\text { (C) } & 0.310 & 0.100 & 0.192 & 0.019 \\ \text { (I) } 0.231 & 0.020 & 0.192 & 0.018\end{array}$

$402 R$

(C) $0.276 \quad 0.088 \quad 0.1690 .019$

$403 R$

$404 R$

$405 R$

$406 R$

$407 R$

$408 R$

$409 R$

$410 R$

$411 R$

$412 R$
(C) $0.2750 .090 \quad 0.1710 .015$

(T) 0.2050 .0180 .1720 .015

(C) 0.3090 .1010 .1930 .015

(I) 0.2290 .0200 .1940 .015

(C) $0.376 \quad 0.048 \quad 0.304 \quad 0.024$

(C) 0.3380 .0430 .2700 .025

$\begin{array}{lllll}\text { (C) } & 0.338 & 0.043 & 0.270 & 0.025 \\ \text { (I) } & 0.303 & 0.008 & 0.270 & 0.025\end{array}$

(C) $\quad 0.335 \quad 0.043 \quad 0.2710 .02$

(I) $0.301 \quad 0.008 \quad 0.271 \quad 0.021$

(C) $0.373 \quad 0.048 \quad 0.305 \quad 0.021$

(I) 0.3520 .0090 .3200 .023

(r) 0.3150 .0080 .2830 .024

(C) $\begin{array}{lllll} & 0.352 & 0.041 & 0.284 & 0.027 \\ \text { (I) } & 0.319 & 0.008 & 0.284 & 0.027\end{array}$

(C) 0.3920 .0460 .3210 .026

(C) 0.3920 .0460 .3210 .026
(I) $0.335 \quad 0.009 \quad 0.305 \quad 0.02$

(C) $0.3890 .045 \quad 0.3200 .023$

(C) $0.3480 .041 \quad 0.283 \quad 0.024$
0.00
0.00

14.10

14.10

14. 10

14.

16.10
14.10

14.10

14. 10

0.00

U. 00
0.00

0.00

0.00

$U .00$
0.00

16. 10

14.10

14.10

14.10
14.10

1.. 10

1.. 10
(H1-3)

( $\mathrm{H2}-1)$

2 (H1-3)

2 (H1

COMPACT

COMPACt

COMfACt

COMPACT

2 (H1 - 3$)$
3 (H2-1)

2 (HI-3)

3 (H2-1)

4 (H1-3)

2 (HI-3)

H2-1)

$2\left(\begin{array}{c}\mathrm{H} 1-3) \\ (\mathrm{H} 2-1)\end{array}\right.$

2 (H1-3)

$2(\mathrm{H2}-1)$

2 (H1-3)

$2(\mathrm{H} 2-1)$

$2(\mathrm{H} 1-3)$

$2 \quad(\mathrm{H1}-3)$

$4(H 1-3)$

4 (H1-3)

4 (H2-1)

4 ( ${ }_{4}(\mathrm{HL}-3)$

4 (H1-3)
COAPAC

Compact $\frac{5}{2}$

COMPACT

COMPACT 总

COAPACT O

COMPACT

COMPACT

COMPACt

COMPACT

COMPACT

COMPACT

COMPACT 
SAP90 FILE:CH-A-04/SAPSTLLFILE:CA
WHC/MPF CAMERA ASSEMBLY AMALYSIS JOBH: 66458-01 RUM ID: CH-A-04S

AISC SPECIFICATIONS, ASD 1989
AXIAL LOAD E BIAXIAL MOMEWI IMTERACTION STRESS CMECK

\begin{tabular}{|c|c|c|c|c|c|c|c|}
\hline ELEM & $\begin{array}{l}\text { SECTION } \\
\text { TYPE }\end{array}$ & $\begin{array}{c}\text { CHK } \\
\text { IYPE }\end{array}$ & $\underset{\text { RATIO }}{\operatorname{STRESS}}=A \times L+833+B 22$ & $\begin{array}{l}\text { STation } \\
\text { LOCATion } \\
\text { (in) }\end{array}$ & $\begin{array}{r}\cos 80 \\
\text { MO }\end{array}$ & $\begin{array}{l}\text { AISC } \\
\text { EOUATION }\end{array}$ & $\begin{array}{l}\text { SECTION } \\
\text { IYPE }\end{array}$ \\
\hline 413 & R & $\begin{array}{l}\text { (C) } \\
\text { (I) }\end{array}$ & $\begin{array}{llll}0.362 & 0.119 & 0.226 & 0.017 \\
0.267 & 0.023 & 0.226 & 0.017\end{array}$ & $\begin{array}{l}0.00 \\
0.00\end{array}$ & $\frac{2}{3}$ & $\begin{array}{l}(\mathrm{H1}-3) \\
(\mathrm{H2}-1)\end{array}$ & СОМРАCT \\
\hline 414 & $\boldsymbol{k}$ & (C) & $\begin{array}{llll}0.320 & 0.104 & 0.198 & 0.018 \\
0.237 & 0.020 & 0.198 & 0.018\end{array}$ & $\begin{array}{l}0.00 \\
0.00\end{array}$ & $\frac{2}{2}$ & $\begin{array}{l}(\mathrm{H1}-3) \\
(\mathrm{HL}-1)\end{array}$ & COMPACT \\
\hline 415 & $R$ & $\begin{array}{l}\text { (C) } \\
\text { (I) }\end{array}$ & $\begin{array}{llll}0.317 & 0.102 & 0.197 & 0.018 \\
0.235 & 0.020 & 0.197 & 0.018\end{array}$ & $\begin{array}{l}0.00 \\
0.00\end{array}$ & $\frac{2}{2}$ & $\begin{array}{l}(\mathrm{H} 1-3) \\
(\mathrm{HL}-1)\end{array}$ & COMPA \\
\hline 410 & $R$ & (C) & $\begin{array}{llll}0.361 & 0.118 & 0.226 & 0.017 \\
0.266 & 0.023 & 0.226 & 0.017\end{array}$ & $\begin{array}{l}0.00 \\
0.00\end{array}$ & $\frac{2}{2}$ & $\begin{array}{l}(H T-3) \\
(H 2-1)\end{array}$ & COAPACI \\
\hline 417 & $R$ & (C) & $\begin{array}{llll}0.380 & 0.130 & 0.229 & 0.021 \\
0.276 & 0.026 & 0.229 & 0.021\end{array}$ & $\begin{array}{l}14.10 \\
14.10\end{array}$ & 4 & $\begin{array}{l}(n 1-3) \\
(H 2-1)\end{array}$ & COAPAC \\
\hline 618 & R & (c) & $\begin{array}{llll}0.332 & 0.106 & 0.207 & 0.019 \\
0.247 & 0.021 & 0.207 & 0.019\end{array}$ & $\begin{array}{l}14.10 \\
14.10\end{array}$ & $\frac{2}{2}$ & $\begin{array}{l}(H 1-3) \\
(H 2-1)\end{array}$ & Contra \\
\hline 419 & $\mathrm{x}$ & (C) & $\begin{array}{llll}0.327 & 0.107 & 0.202 & 0.018 \\
0.241 & 0.021 & 0.202 & 0.018\end{array}$ & $\begin{array}{l}14.10 \\
14.10\end{array}$ & $\begin{array}{l}4 \\
4\end{array}$ & $\begin{array}{l}(H 1-3) \\
(H 2-1)\end{array}$ & atra \\
\hline 420 & K & (c) & $\begin{array}{llll}0.371 & 0.121 & 0.230 & 0.020 \\
0.274 & 0.024 & 0.230 & 0.020\end{array}$ & $\begin{array}{l}14.10 \\
14.10\end{array}$ & 4 & $\begin{array}{l}(\mathrm{H1}-3) \\
(\mathrm{H} 2-1)\end{array}$ & 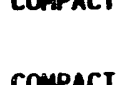 \\
\hline sol & $\boldsymbol{k}$ & (c) & 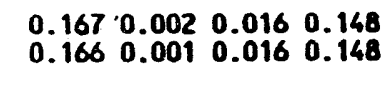 & $\begin{array}{l}14.75 \\
14.75\end{array}$ & $\hat{4}$ & $\begin{array}{l}(H 1-3) \\
(H 2-1)\end{array}$ & Cunt at \\
\hline Juc & $n$ & (C) & $\begin{array}{llll}0.164 & 0.001 & 0.004 & 0.160 \\
0.164 & 0.000 & 0.004 & 0.160\end{array}$ & $\begin{array}{l}0.80 \\
0.80\end{array}$ & 4 & $\begin{array}{l}(\mathrm{H} 1-3) \\
(\mathrm{H2}-1)\end{array}$ & CO \\
\hline 503 & R & (C) & $\begin{array}{llll}0.132 & 0.000 & 0.014 & 0.118 \\
0.132 & 0.000 & 0.014 & 0.118\end{array}$ & $\begin{array}{l}0.80 \\
0.80\end{array}$ & 4 & $\begin{array}{l}(\mathrm{H} 1-3) \\
(\mathrm{H} 2-1)\end{array}$ & COAPACT \\
\hline 504 & $\mathbf{P}$ & $\begin{array}{l}\text { (C) } \\
(I)\end{array}$ & $\begin{array}{llll}0.062 & 0.009 & 0.009 & 0.052 \\
0.061 & 0.007 & 0.009 & 0.052\end{array}$ & $\begin{array}{l}10.20 \\
10.20\end{array}$ & $\begin{array}{l}3 \\
3\end{array}$ & $\begin{array}{l}(\mathrm{H} 1-3) \\
(\mathrm{H} 2-1)\end{array}$ & CONAPA \\
\hline 505 & $\mathbf{P}$ & $\begin{array}{l}\text { (C) } \\
\text { (I) }\end{array}$ & $\begin{array}{llll}0.067 & 0.008 & 0.010 & 0.058 \\
0.065 & 0.006 & 0.010 & 0.058\end{array}$ & $\begin{array}{l}0.80 \\
0.80\end{array}$ & 2 & $\begin{array}{l}(\mathrm{H} 1-3) \\
(\mathrm{H} 2-1)\end{array}$ & \\
\hline
\end{tabular}




\section{URS CONSURTANTS WHC-SU-WIY-UA-16S \\ Vol. II, Rev. 0}

Job \#:66481-01 Job: WHC/MPF CAMERA ASSEMBLY ANALYSIS

Client: WHC Subject: SAP9O ANALYSIS DOCUMENTATION
Sheet No. $\$ 4.1$

By pue Date $0 / 3 / 9+$

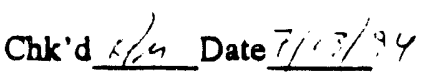

5.4 Selective Graphical Output

The following are given in this section:

- Mode shapes \& Frequencies, including:

CM-A-04.RIT for Model A (Camera assembly only)
CM-B-01.RIT for Model B (Camera + MPF risers)

- Shell Element stresses for

- 3" thick Shielding plate

- 2" thick 4" high Manifold tube

- 3/4" thick flange plate

- 1/2" Mating plate

- $\quad 1 / 2 "$ Bottom plate

Each set of plots includes:

SMXT Maximum principal stresses at top of plate SMXB Maximum principal stresses at bottom of plate SVM Maximum shear stresses

for both load case 3 (DL + LC2 + RS) and for load case 4 (DL - LC2 - RS) as described in section 3.

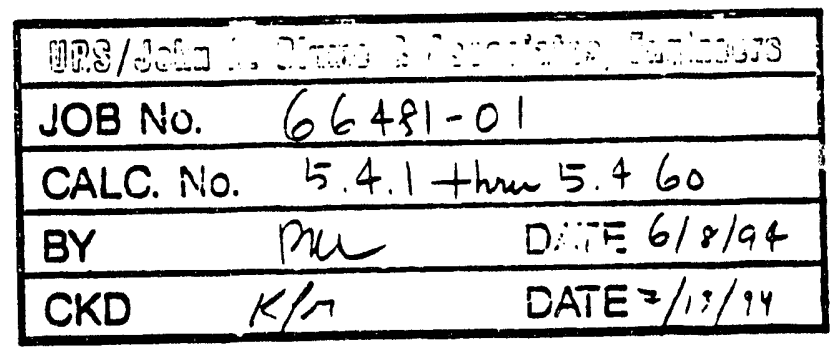




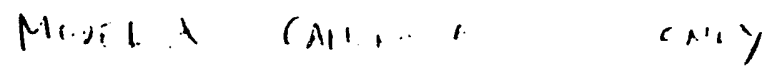

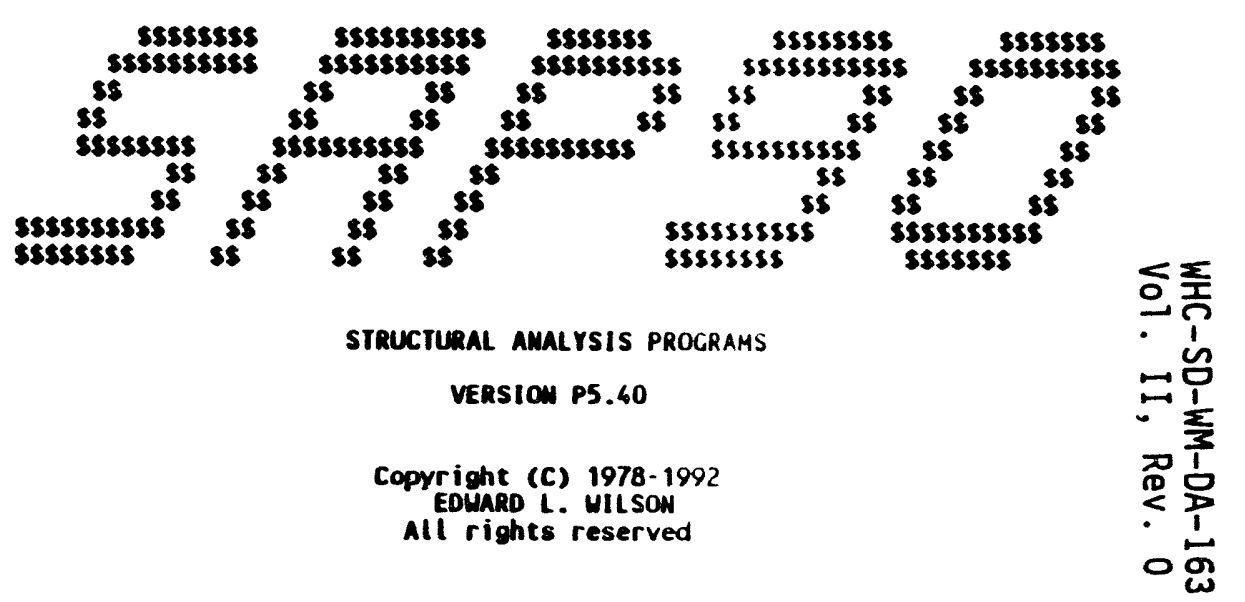




\section{PROGRA:SAP9O/FILE:CM-BGE} MHC MPF CAMERA ASSEMBLY MODEL JOBN: 66458-01 RUW: CH-A-06

II 2 S Y S IE

MUMBER OF EQUATIOWS

MUMBER OF MASSES

MUMBER OF DIRECTION VECTORS

$=1650$

$=\quad 20$

Ð

\section{PAGE 2
PROLRAK:SAP90/FILE:CA-B-04.RII} MHC/MPF CAMERA ASSEMBLY MODEL JOBH: $66458-01$ RUN: CM-A-04

EIGENVALUES A D FREQUENCIES

\begin{tabular}{|c|c|c|c|c|c|}
\hline $\begin{array}{r}\text { MODE } \\
\text { MUMBER } \\
1 \\
2 \\
3 \\
4 \\
5 \\
6 \\
7 \\
8 \\
9 \\
10 \\
11 \\
12 \\
13 \\
14 \\
15 \\
16 \\
17 \\
18 \\
19 \\
20\end{array}$ & $\begin{array}{r}\text { EIGENVALUE } \\
(R A D / S E C)=402 \\
0.407498 E+03 \\
0.411552 E+03 \\
0.449698 E+04 \\
0.578889 E+04 \\
0.864802 E+04 \\
0.424371 E+05 \\
0.579778 E+05 \\
0.611514 E+05 \\
0.628768 E+05 \\
0.859494 E+05 \\
0.105259 E+06 \\
0.127686 E+06 \\
0.212823 E+06 \\
0.222035 E+06 \\
0.257731 E+06 \\
0.321171 E+06 \\
0.423339 E+06 \\
0.601809 E+06 \\
0.756031 E+06 \\
0.105368 E+07\end{array}$ & 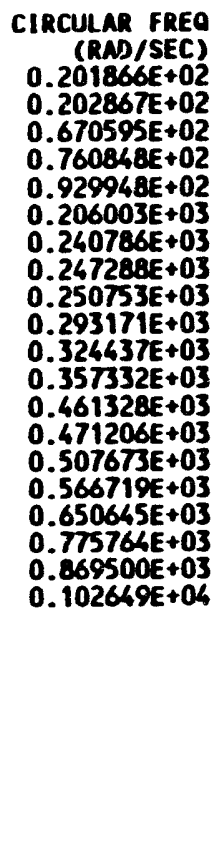 & $\begin{array}{r}\text { FREQUENCY } \\
\text { (CrCLES/SEC) } \\
3.212794 \\
3.228734 \\
10.672851 \\
12.109266 \\
14.800574 \\
32.786347 \\
38.322254 \\
39.357134 \\
39.908501 \\
46.659657 \\
51.635676 \\
56.871182 \\
73.422577 \\
74.994830 \\
80.790591 \\
90.196163 \\
103.553415 \\
123.466621 \\
138.385264 \\
163.370948\end{array}$ & $\begin{array}{l}\text { PER100 } \\
\text { (SEC) } \\
0.311256 \\
0.309719 \\
0.093696 \\
0.082581 \\
0.067565 \\
0.030501 \\
0.026094 \\
0.025408 \\
0.025057 \\
0.021432 \\
0.019366 \\
0.017584 \\
0.013620 \\
0.013334 \\
0.012376 \\
0.011087 \\
0.009657 \\
0.008099 \\
0.007226 \\
0.006121\end{array}$ & 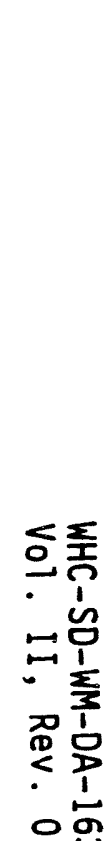 \\
\hline
\end{tabular}


MHC/MPF CAMERA ASSEMBLY MOOEL JOB: :6658-01 RUA: CH-A-04 Base FORCE REACTIOM FACTORS

MODE PERIOD

( $10.3110 .122 E-02 \quad 0.262 E-01-0.150 E-03 \quad 0.483 E+01-0.219 E+00 \quad 0.918 E-01$ $2 \quad 0.310 \quad 0.263 E-01-0.131 E-02 \quad 0.152 E-03-0.225 E+00-0.484 E+010.834 E-01$ $0.083-0.690 E-02 \quad 0.739 E-02 \quad 0.427 E-03 \quad 0.150 E+00 \quad 0.907 E+00 \quad 0.141 E-02$ $5 \quad 0.0680 .412 E-02 \quad 0.376 E-02-0.585 E-04-0.335 E-01 \quad 0.141 E-01 \quad 0.104 E+00$ $6 \quad 0.031-0.820 E-03-0.781 E-03-0.943 E-04-0.296 E-01$ 0.299E-01 $0.605 E-01$ 0.026-0.332E-03-0.354E-05-0.45E-05-0.705E-02 O. TOE-02 0.200E-02 0.025-0.347E-02 0.530E-02-0.604E-04 0.737E-01 0.335E-02 0.240E-02 9 0.025 0.5 110.0210 .034 12 0.018 0.936E-03-0.102 12 0.014-0.036E-03 $0.441 E-03-0.7025-02$ 0.599-01 $0.046-010.54$

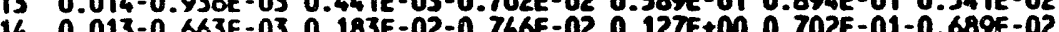
15 0.012 0.214E-02 0.528E-04 0.182E-02 0.100E-01-0 101E+00-0.527E-02 16 0. 011 0.125E-02 0.273E-02 $0.146 E-02$ 0.772E-01-0.543E-01 0.638E-02 $170.010-0.308 E-02$ 0.172E-02 0.907E-02-0.351E-02 0.230E-01-0.376E-02 $180.0080 .250 E-02 \quad 0.283 E-02 \quad 0.486 E-02 \quad 0.644 E-02-0.883 E-02-0.754 E-03$ 19 0.007-0.135E-02 0.224E-02-0.176E-01 0.989E-02 0.241E-02-0.237E-03 20 0.006-0.155E-02 0.126E-02 0.150E-01 0.434E-01 0.256E-01 0.461E-02
UHCAMPF CAMERA ASSEMBLY MOOEL JOB*: 66458-01 RUN: CM-A-04

\begin{abstract}
PARIICIPATING MASS-(percent)
\end{abstract}

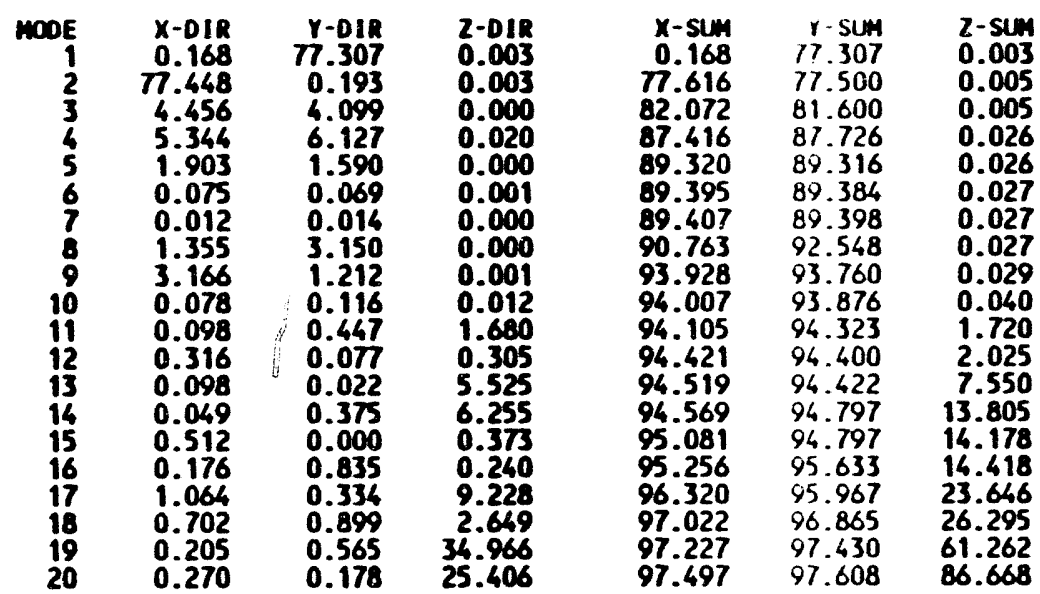


WHC-SD-WM-DA-163

$5+5$

Vol. II, Rev. 0
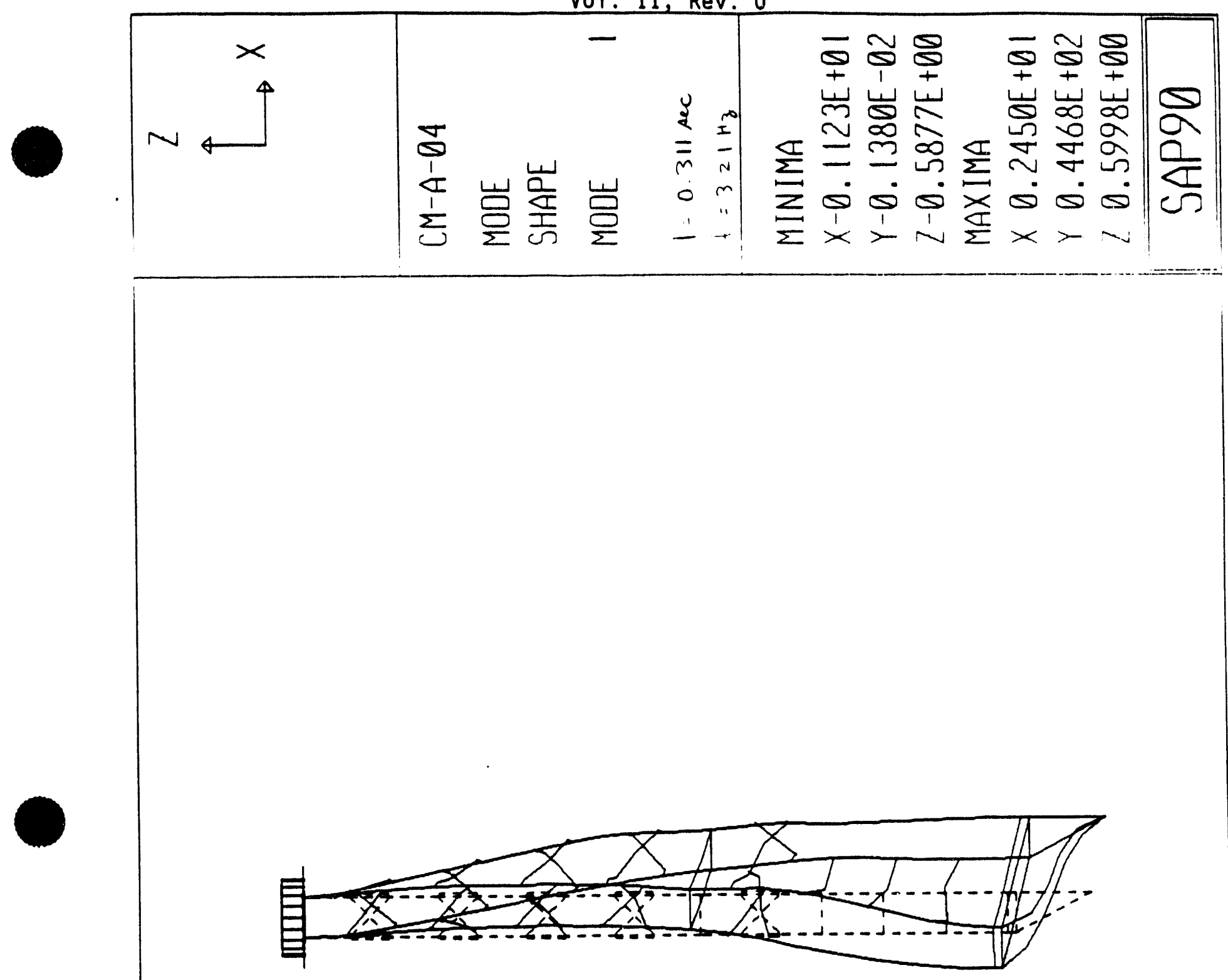


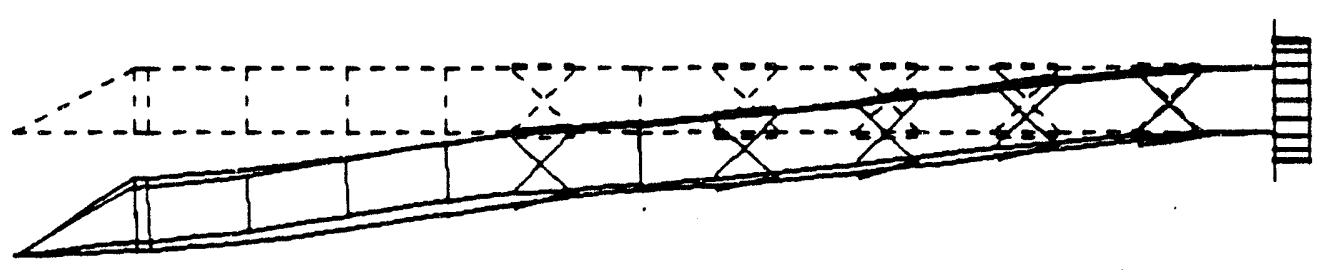

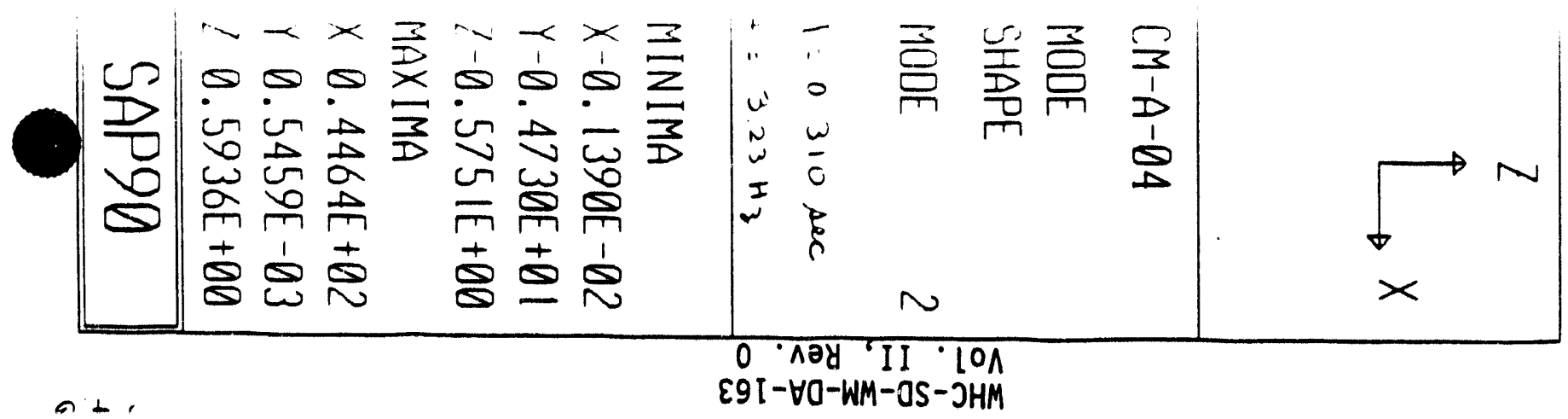


Vol. II, Rev. 0

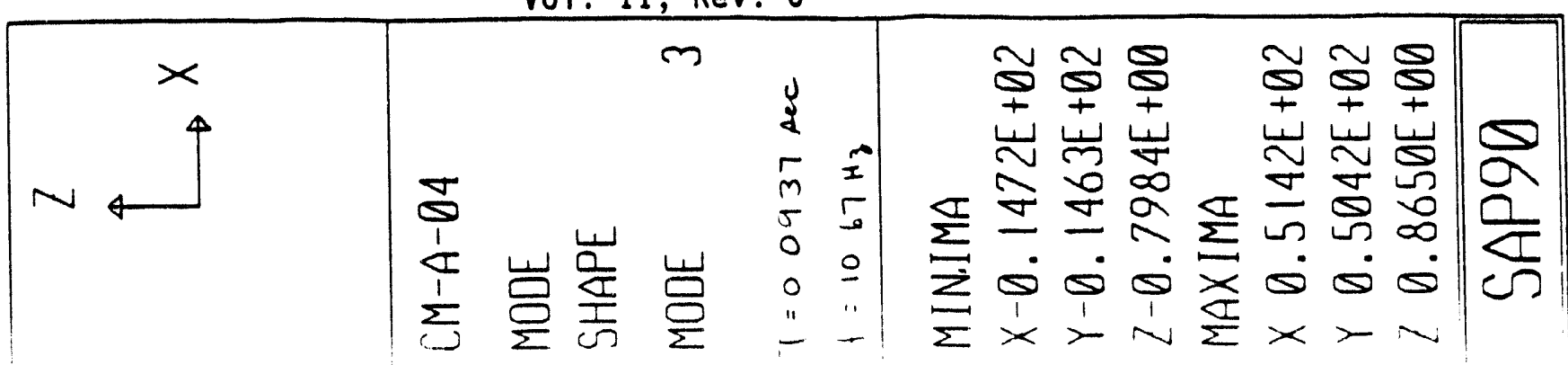

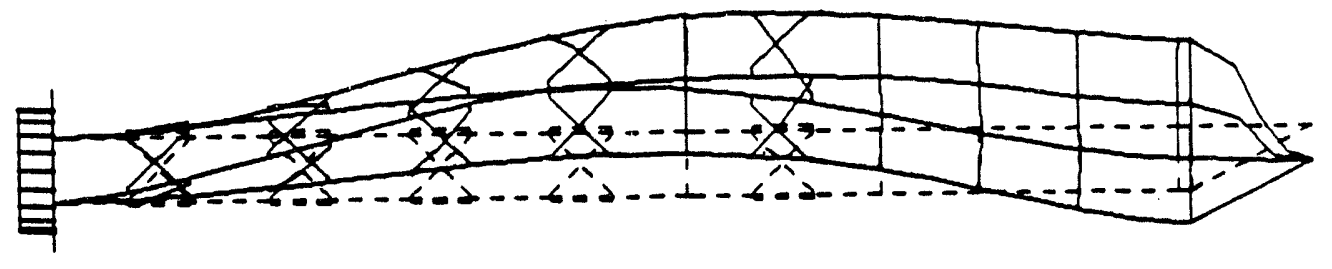


Vol. II, Rev. 0

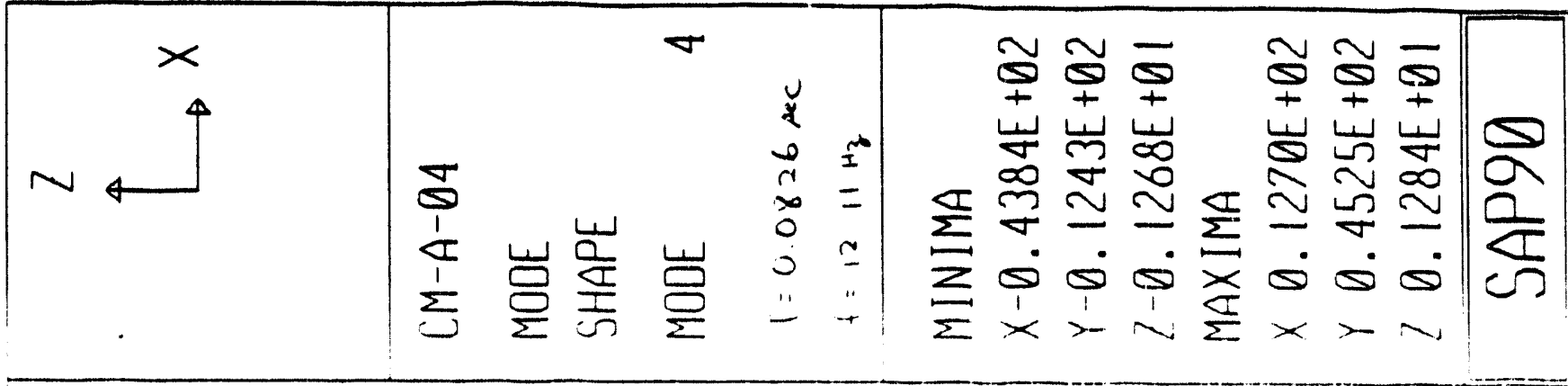

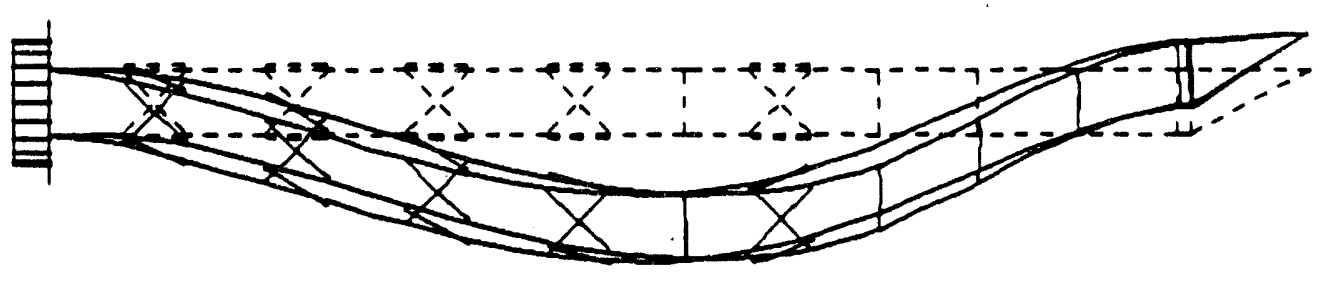


WHC-SD-WM-DA-163

Vol. II, Rev. 0

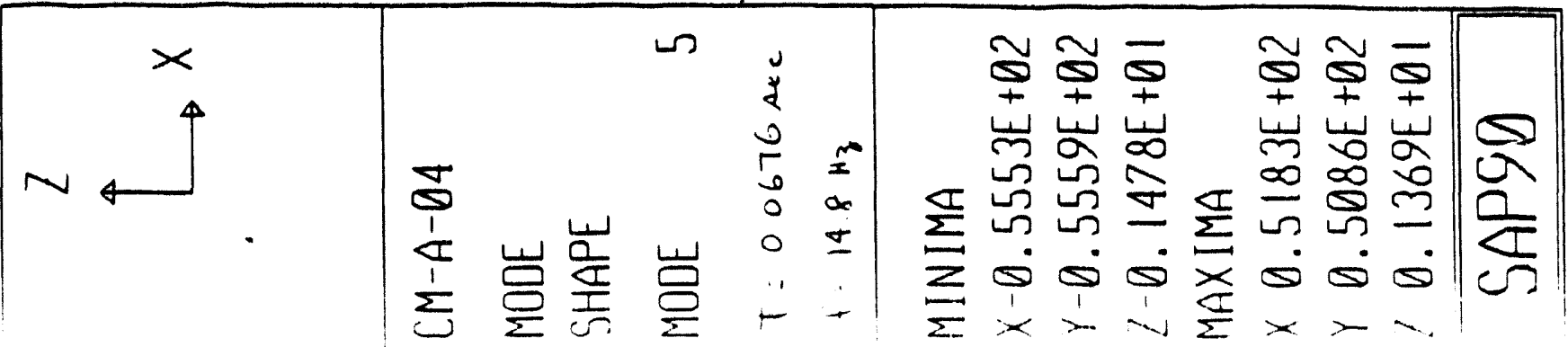

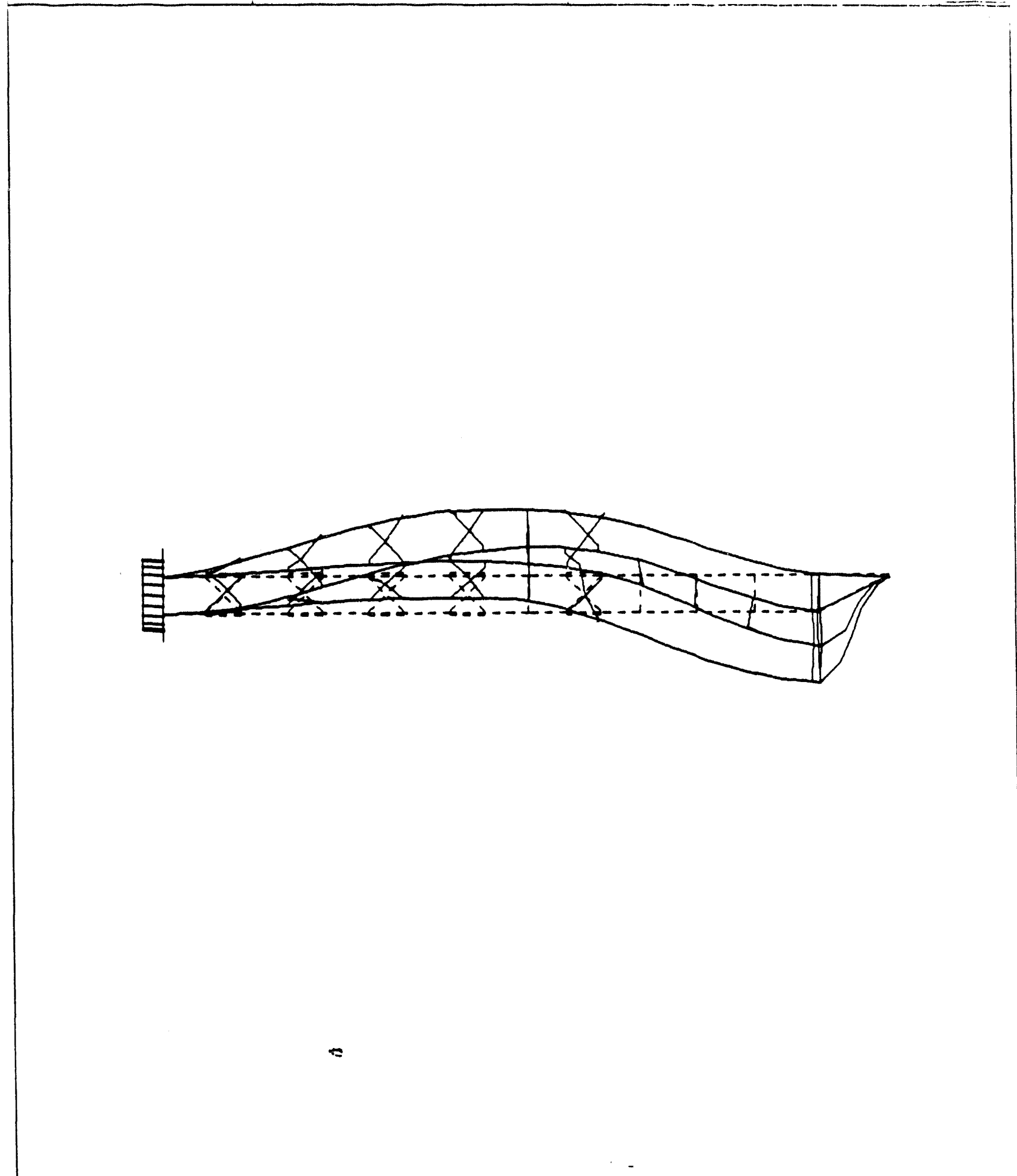



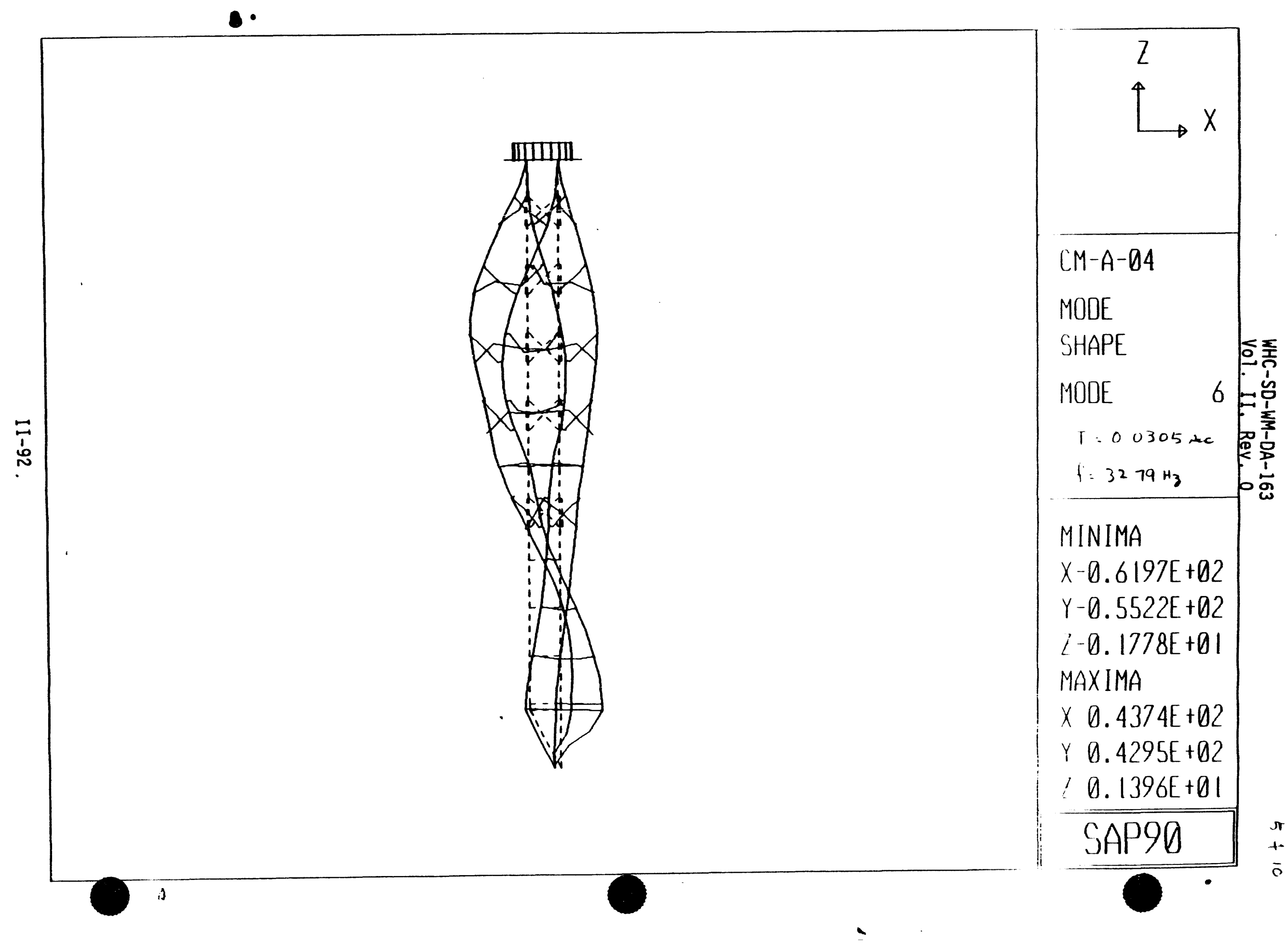
Vol. II, Rev. 0

\begin{tabular}{|c|c|c|c|c|c|c|c|c|}
\hline $\begin{array}{r}x \\
4\end{array}$ & $\sum_{\substack{1\\
}}^{\frac{1}{1}}$ & 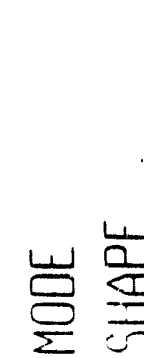 & N & \begin{tabular}{l}
0 \\
\multirow{0}{*}{} \\
0 \\
0 \\
0 \\
0 \\
-1
\end{tabular} & 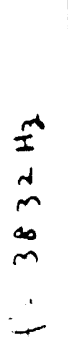 & 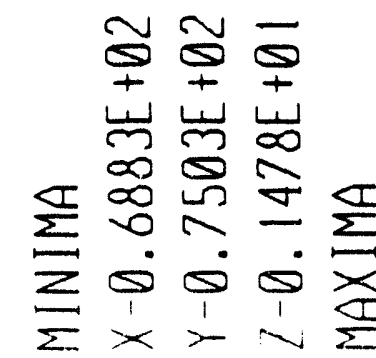 & 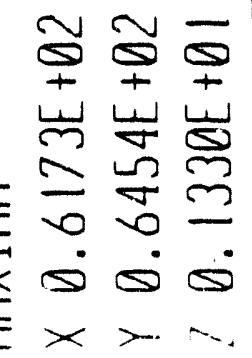 & $\frac{\sqrt[\sigma]{\sigma}}{\frac{\sigma}{\sigma}}$ \\
\hline
\end{tabular}

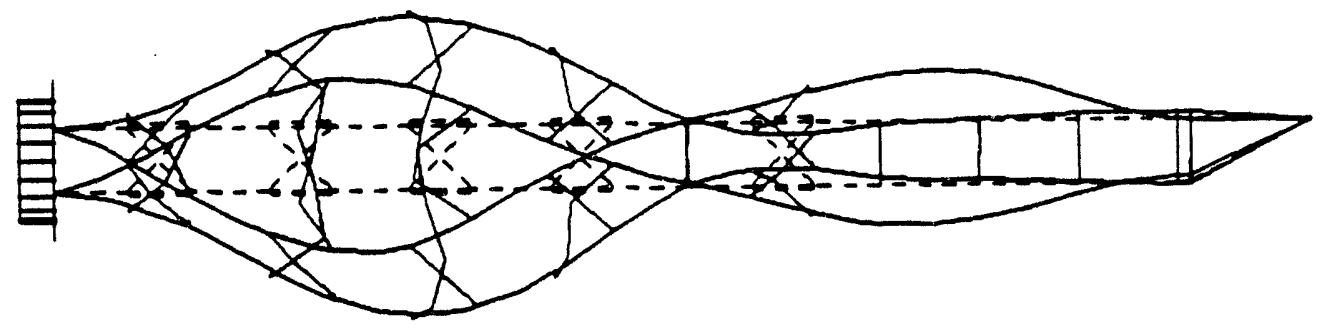


WHC-SD-WM-DA-163

Vol. II, Rev. 0

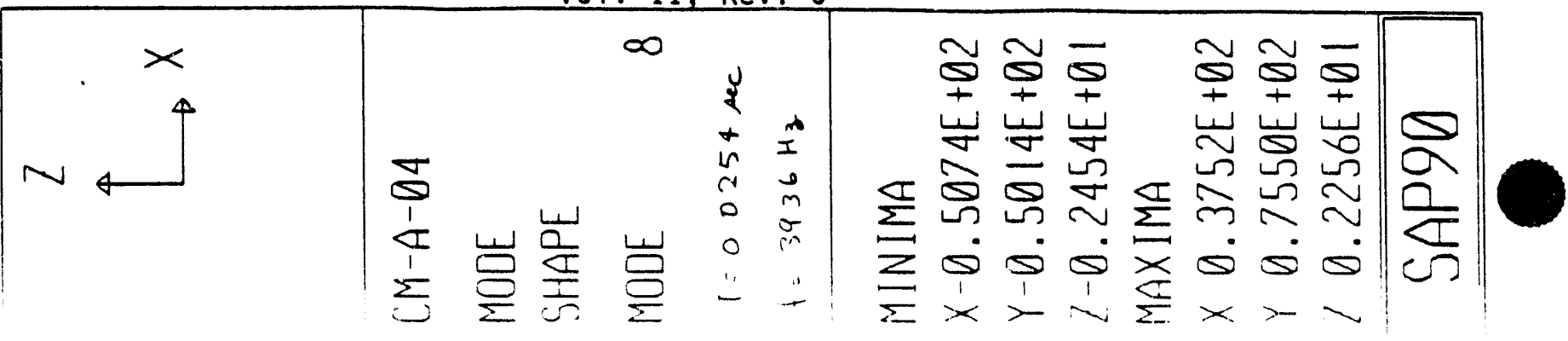

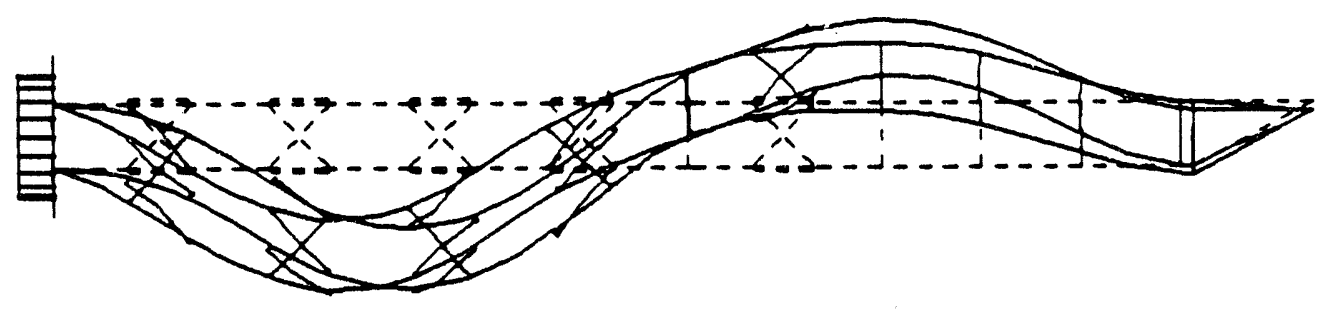


WHC-SD-WM-DA-163

Vol. II, Rev. 0

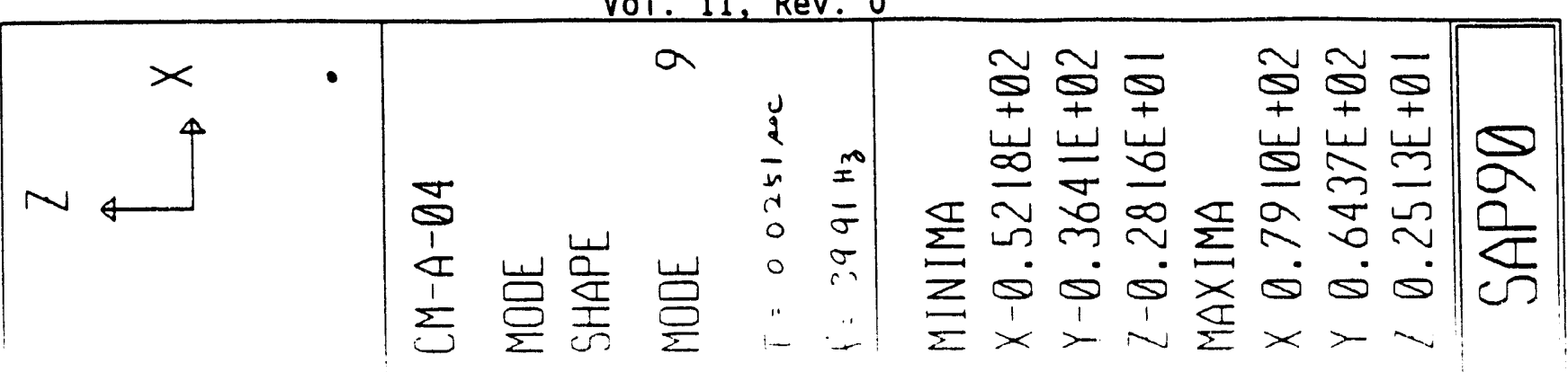

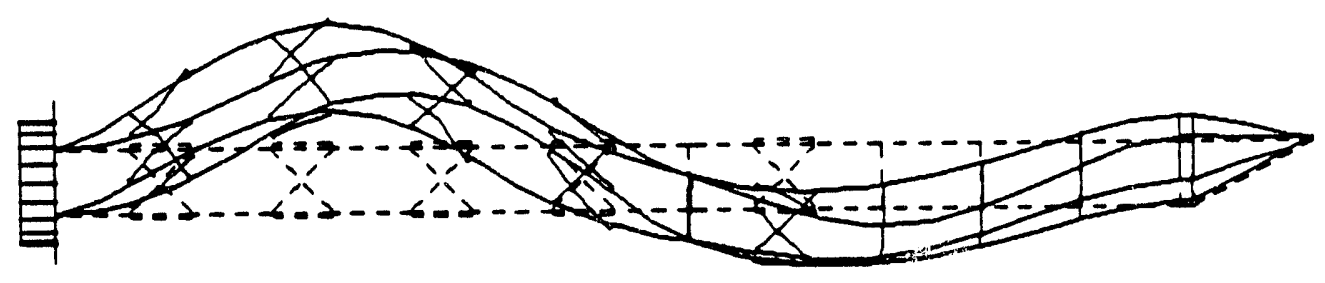


WHC-SD-WM-DA-163

Vol. II, Rev. 0

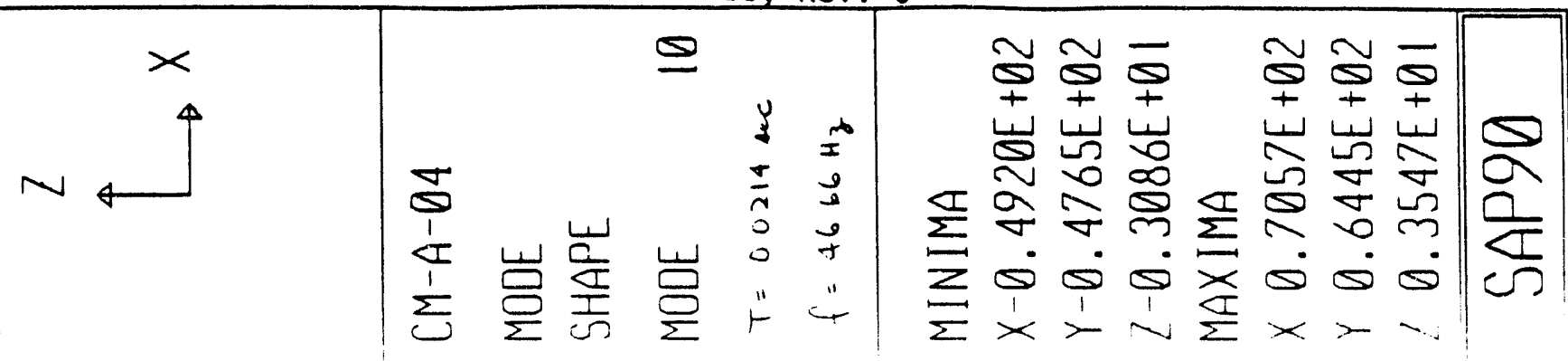

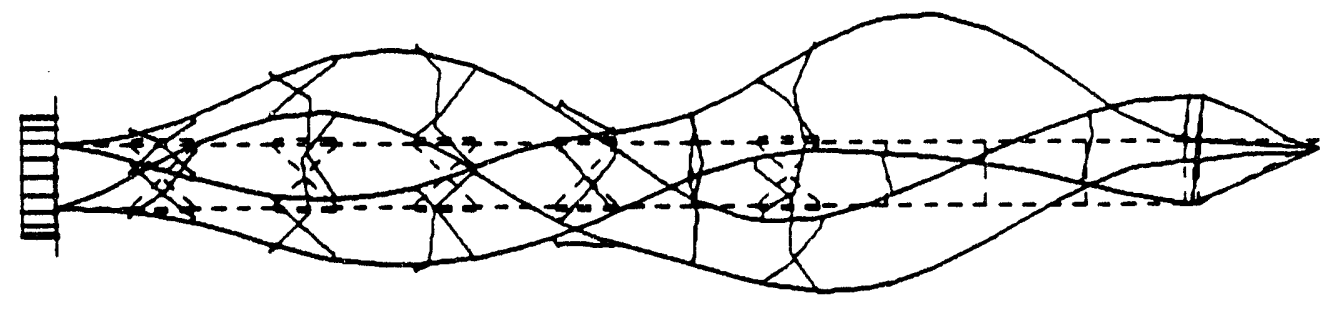


Vol. II, Rev. 0

\begin{tabular}{|c|c|c|c|c|c|c|c|c|c|c|c|c|c|c|}
\hline$N_{4}^{x}$ & $\sum_{\substack{1\\
}}^{J}$ & 岂 $\frac{\omega}{\frac{1}{n}}$ & $\underset{巳}{巳}$ & 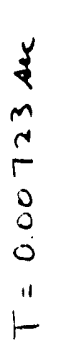 & $\begin{array}{l}\infty \\
I \\
+ \\
\infty \\
m \\
\cdots \\
-\end{array}$ & $\begin{array}{l}\sum_{\sum}^{I} \\
\sum \\
\sum\end{array}$ & 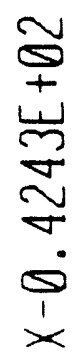 & 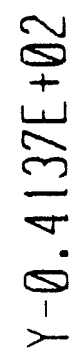 & 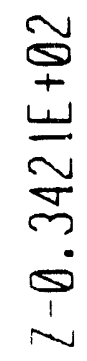 & $\sum_{\frac{\Sigma}{\Sigma}}^{\frac{\sigma}{\Sigma}}$ & 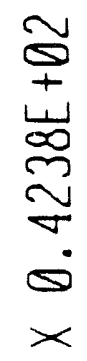 & 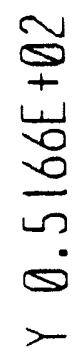 & 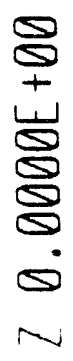 & $\frac{1}{20}$ \\
\hline
\end{tabular}

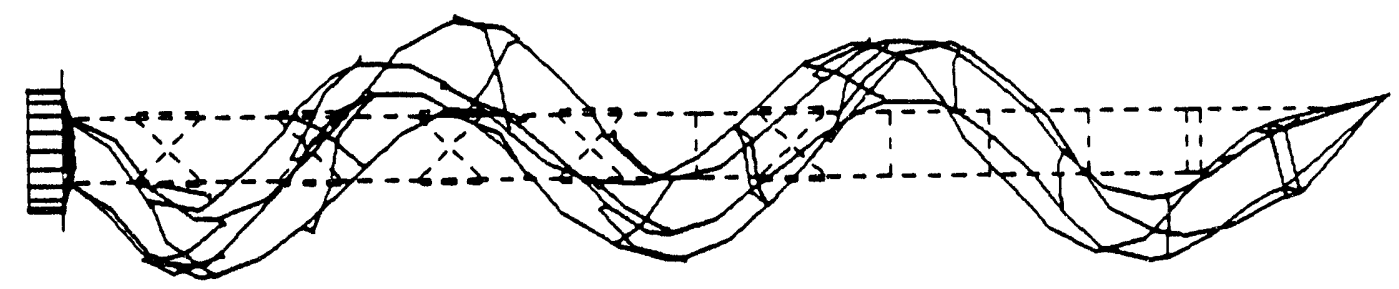


Vol. II, Rev. 0

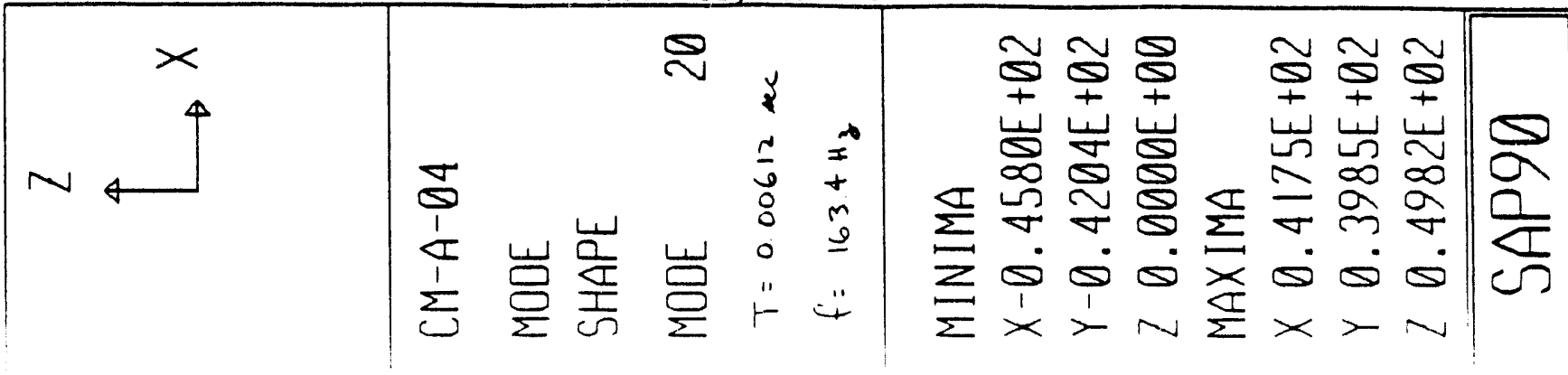

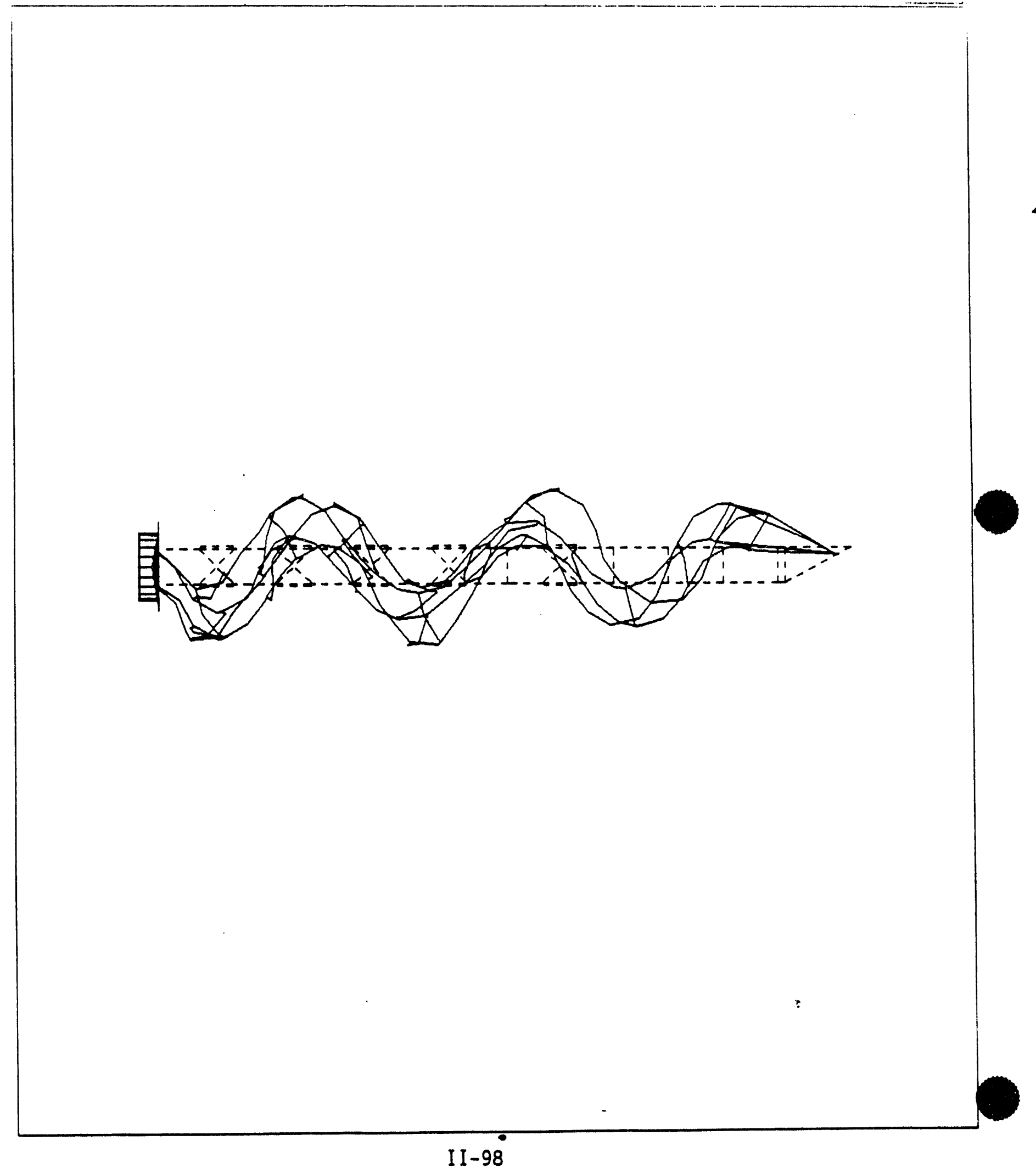



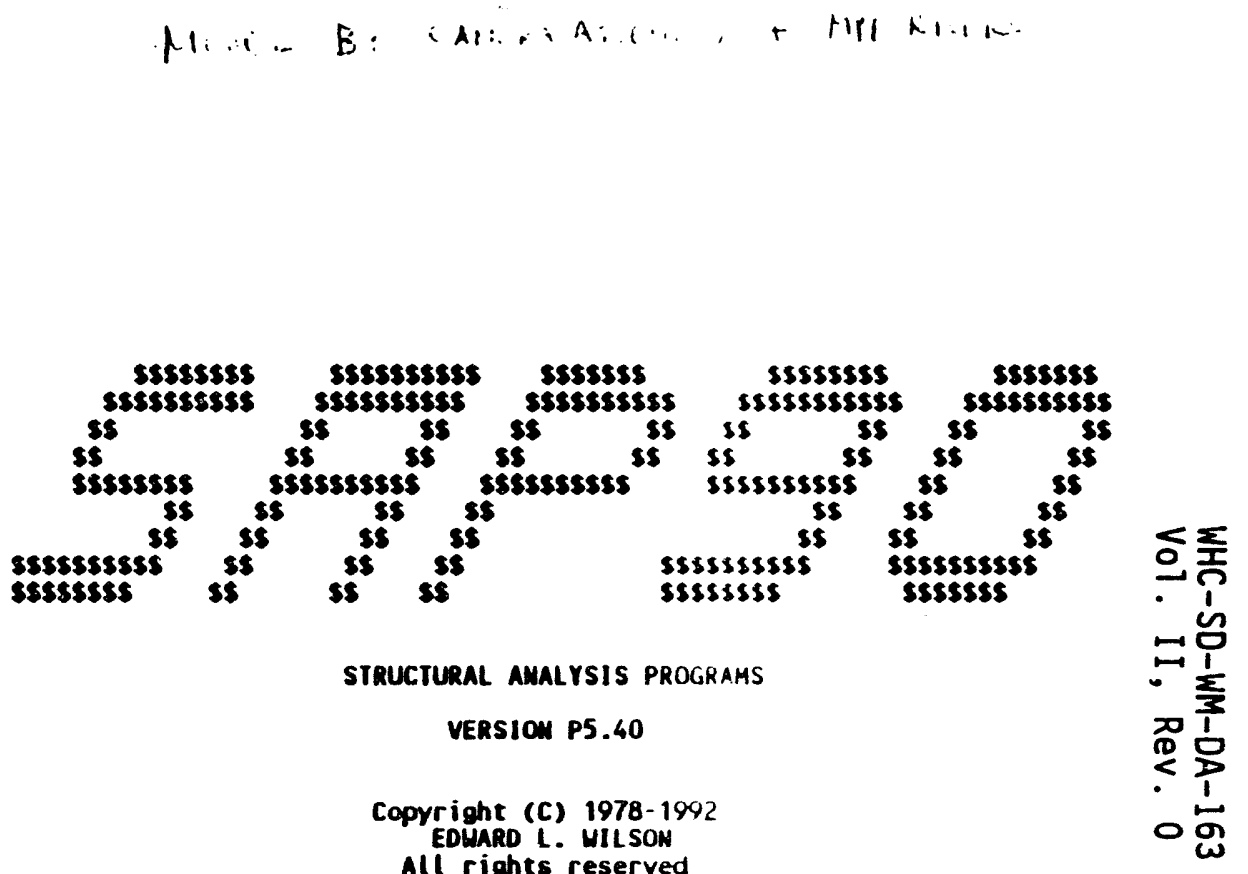
RIIZ SYSIEM PARANEIERS

MUMBER OF EQUATIIONS

MUNER OF MASSE

MUABER OF DIRECTION VECTORS

꿍
$=1770$
$=\quad 885$
$=\quad 20$

$=3$
MHC/MPF CAMERA ASSEMBLY MODEL JOB*: 66458-01 RUH: CM-B-01

EIGENVALUES A D FREOUENCIES

MODE EIGEMVALUE CIRCULAR FREQ FREQUENCY (RAD/SEC)"\#2 $0.399807 E+03$ $0.404339 E+03$ $0.433175+04$ 0.831171E+04 O.831171E+04 .

$0.057125+05$

$0.579230 E+05$

$0.5938996+05$

$0.607981 E+05$ $0.815664 \mathrm{E}+05$

$0.995993 E+05$

$0.128916 E+06$

$0.207535 E+06$

$0.24005 \%$ E +06

$0.495304 E+06$

$0.890902 E+06$

$0.137611 E+07$

$0.158802 E+07$

(RNO/SEC)

(CYCLES/SEC)

$0.199952 E+02$

$0.201082 E+02$

$0.658162 E+02$

3.200315

(SEC)

10.4749710 .095466

11.8814470 .084165

$27.528432-0.036326$

$27.548363 \quad 0.036300$

$32.057469-0.031194$

$38.304137 \quad 0.026107$

$0.263700 E+03$

$0.246573 E+03$

$\begin{array}{lll}0.285598 E+03 & 45.454396 & 0.022000\end{array}$

$\begin{array}{lll}0.315594 E+03 & 50.228270 & 0.019909\end{array}$

0.359049E+03 57.1444E8 0.017500

$\begin{array}{lll}0.455560 E+03 & 72.504645 & 0.013792\end{array}$

$0.490774 E+03 \quad 78.1090 C$ C 0.012803

0.703776 to3 $\quad 112.00978-0.008928$

$0.943876 E+03 \quad 150.2225420 .00665$

$0.117308 E+04$ (1) 
PROGRAM:SAP9O/FILE:CM-B-01.RIT UHC/MPF CAMERA ASSEMBLY MODEL JOBN: 66458-01 RUM: CH-8-01

\section{BASE FORCE REACTION FACIORS}

MOOE PERICD DIRECIIION DIRECTION DIRECIION MOMENT MOMEMT MOMENT * (sec) DIRECTION DIRECTION DIRECTION MONENT MONENT MOMENT $0.3120 .264 E-01-0.108 E-03$ 0.133E-03-0.283E-02-0.487E+01 $0.870 E-01$ $0.095 \quad 0.724 E-02 \quad 0.698 E-02 \quad 0.303 E-06 \quad 0.205 E+00-0.230 E+00-0.137 E+00$ $0.084-0.859 E-02$ 0.911E-02 0.400E-03 0. $0.0690 .608 E-02$ 0.565E-02-0.401E-04-0. $0.036-0.126 E+00$ 0.1 $0.0360 .172 E-01$ 0.126E+00 0. 0.03 0.026 0.303E-02 0.270E-02 0 313E-03 0.178E-01-0.47LE-01 0.391E-01 11 0.025 0.228E-02 $0.205 E-02-0.559 E-05$ 0.631E-01-0.280E-01 0.212E-01 12 0.022 0 107E-02 0.139E-02-0.461E-03 0.565E-01-0.440E-01 0.524E-01 13 0.020-0.107E-02 $0.222 E-02-0.416 E-02 \quad 0.115 E+00 \quad 0.939 E-01 \quad 0.348 E-01$ 14 0.017-0.839E-03-0.160E-03-0.233E-02 0.135E-01 0.526E-01 $0.120 E-02$ $150.0140 .911 E-04-0.132 E-020.126 E-01-0.149 E+00-0.146 E+00-0.176 E-02$ $160.013 \quad 0.608 E-03 \quad 0.136 E-04-0.623 E-02-0.289 E-02-0.804 E-02 \quad 0.508 E-03$ $170.009 \quad 0.144 E-02 \quad 0.827 E-04-0.373 E-01-0.451 E-02 \quad 0.201 E+00 \quad 0.135 E-01$ $180.0070 .754 E-02 \quad 0.951 E-03-0.124 E+00 \quad 0.113 E+00 \quad 0.339 E+000.568 E-02$ $190.0050 .272 E-010.241 E-010.220 E-010.270 E+01-0.328 E+010.308 E+00$ $200.0050 .263 E-01-0.299 E-010.128 E-01-0.335 E+01-0.307 E+01-0.384 E+00$

$\stackrel{\Xi}{\circ}$
PARIICIPATING MAS S - (percent)

$\begin{array}{rr}\text { MOE } & X-D 1 R \\ 1 & 0.000 \\ 2 & 3.474 \\ 3 & 0.262 \\ 4 & 0.369 \\ 5 & 0.185 \\ 6 & 79.650 \\ 7 & 1.485 \\ 8 & 0.014 \\ 9 & 0.070 \\ 10 & 0.127 \\ 11 & 0.026 \\ 12 & 0.006 \\ 13 & 0.006 \\ 14 & 0.004 \\ 15 & 0.000 \\ 16 & 0.002 \\ 17 & 0.010 \\ 18 & 0.284 \\ 19 & 3.711 \\ 20 & 3.448\end{array}$

$2.01 R$
0.000
0.000
0.000
0.001
0.000
0.013
0.001
0.000
0.000
0.000
0.000
0.001
0.086
0.027
0.791
0.194
6.948
7.174
2.609
0.816 2-sum

0.000

0.000

0.001

0.001

0.014

0.015

0.015

0.015

0.015

0.015

0.016

0.103

0.130

0.921

1.115
8.063

8.063
85.237

87.846 
Vol. II, Rev. 0

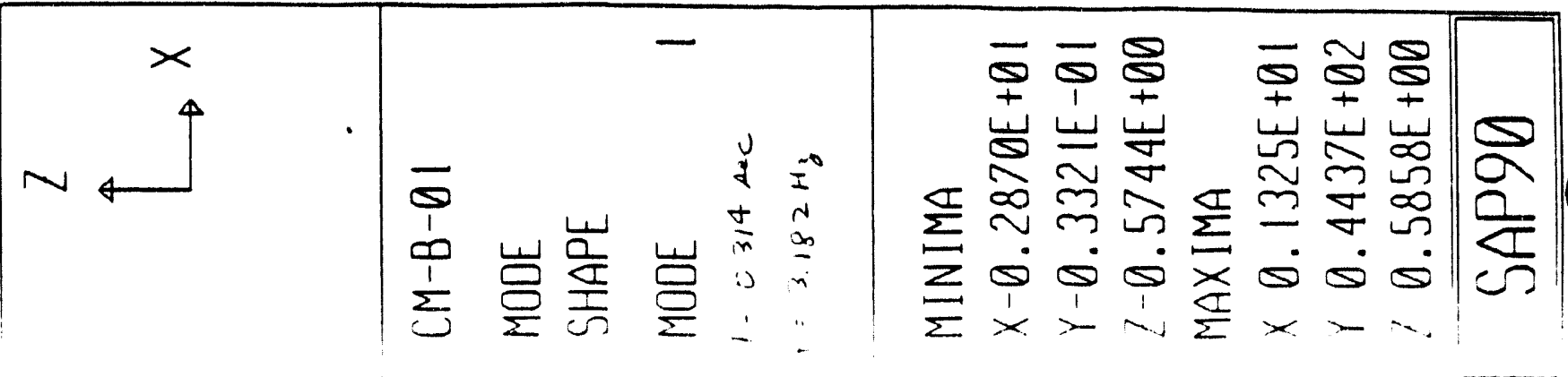

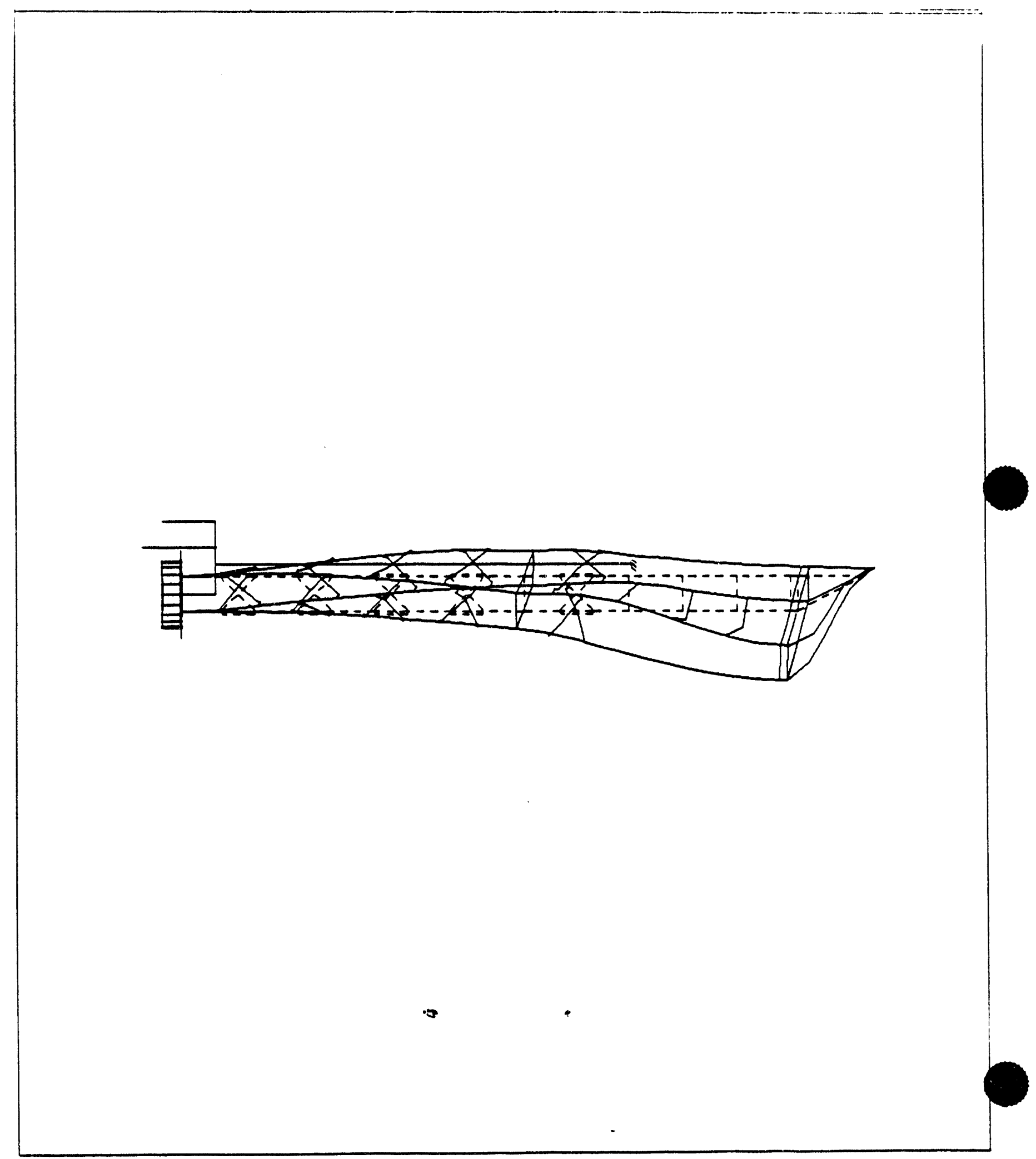


WHC-SD-WM-DA-163

5421

Vol. II, Rev. 0

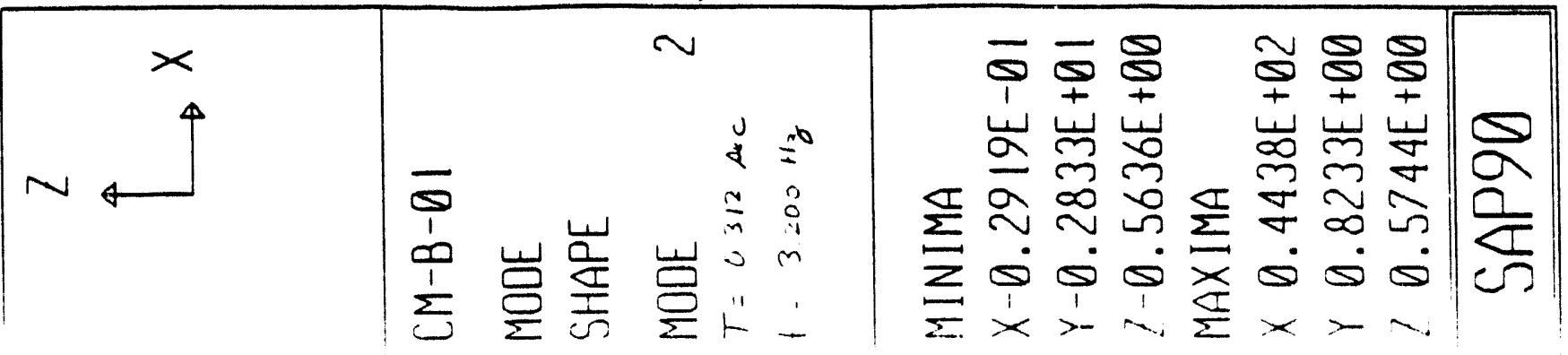

- 
WHL-SU-WM-UA-IOS

Vol. II, Rev. 0

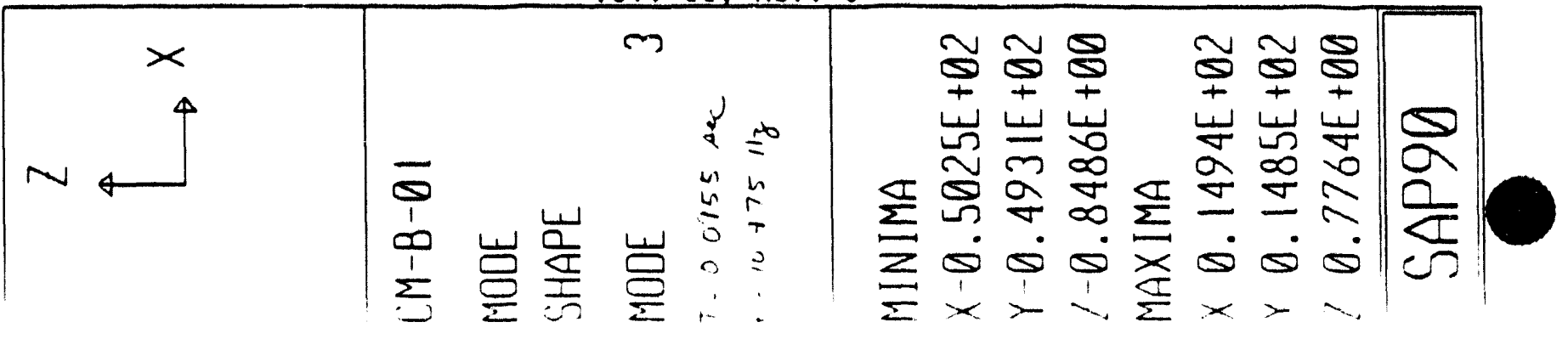

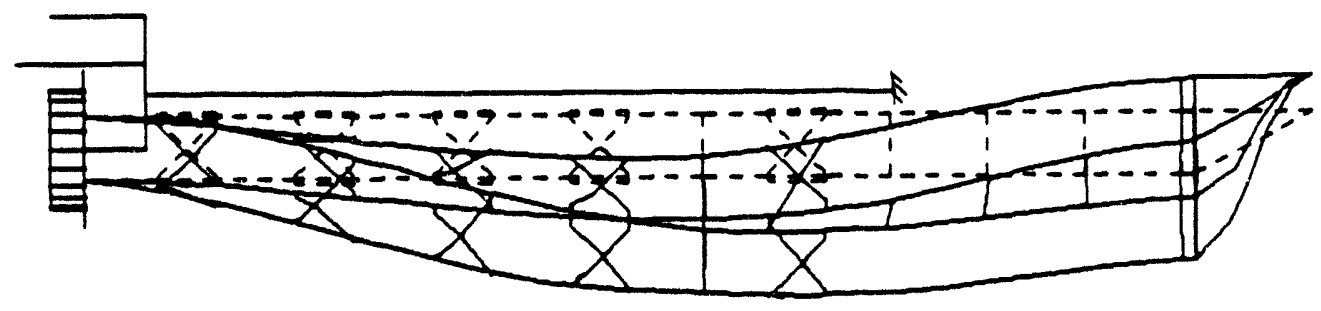


Vol. II, Rev. 0

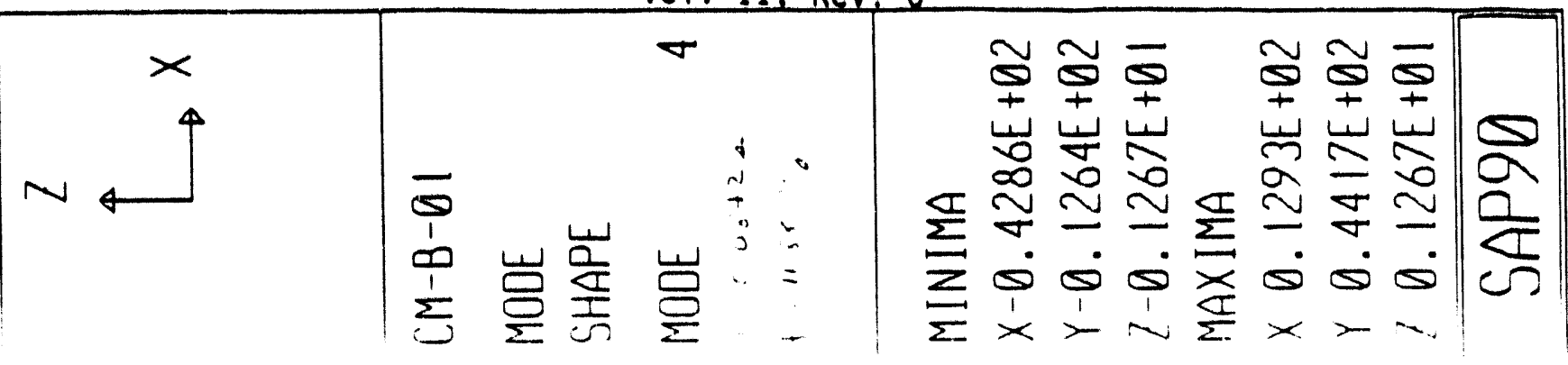

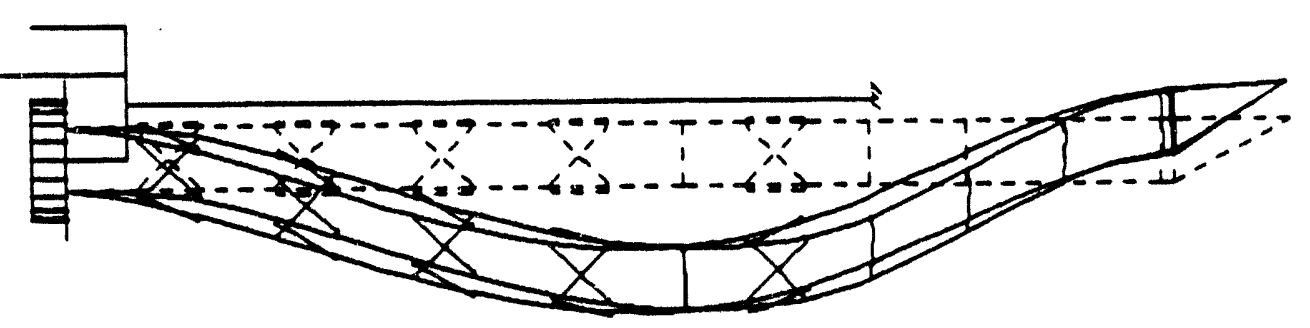


WHC-SD-WM-DA-163

Vol. II, Rev. 0

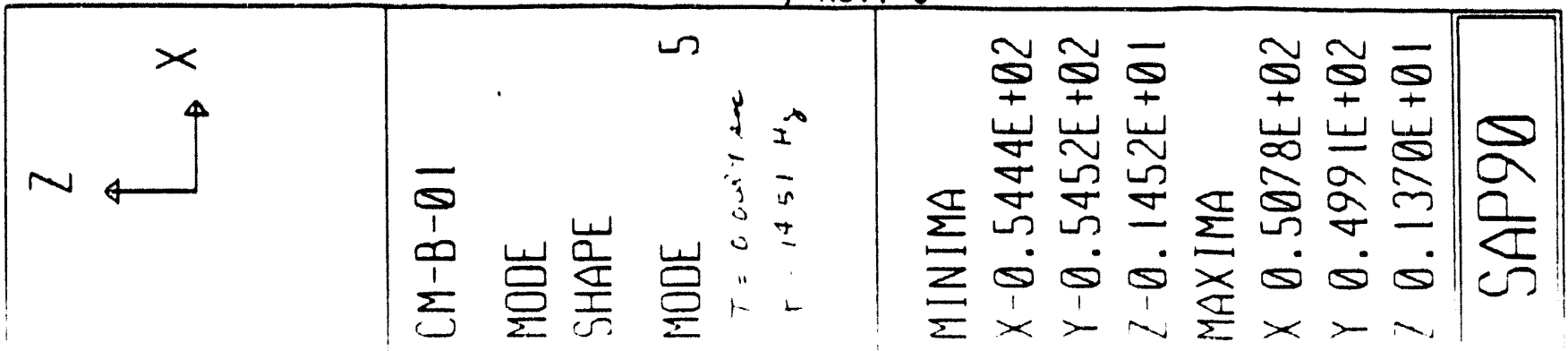

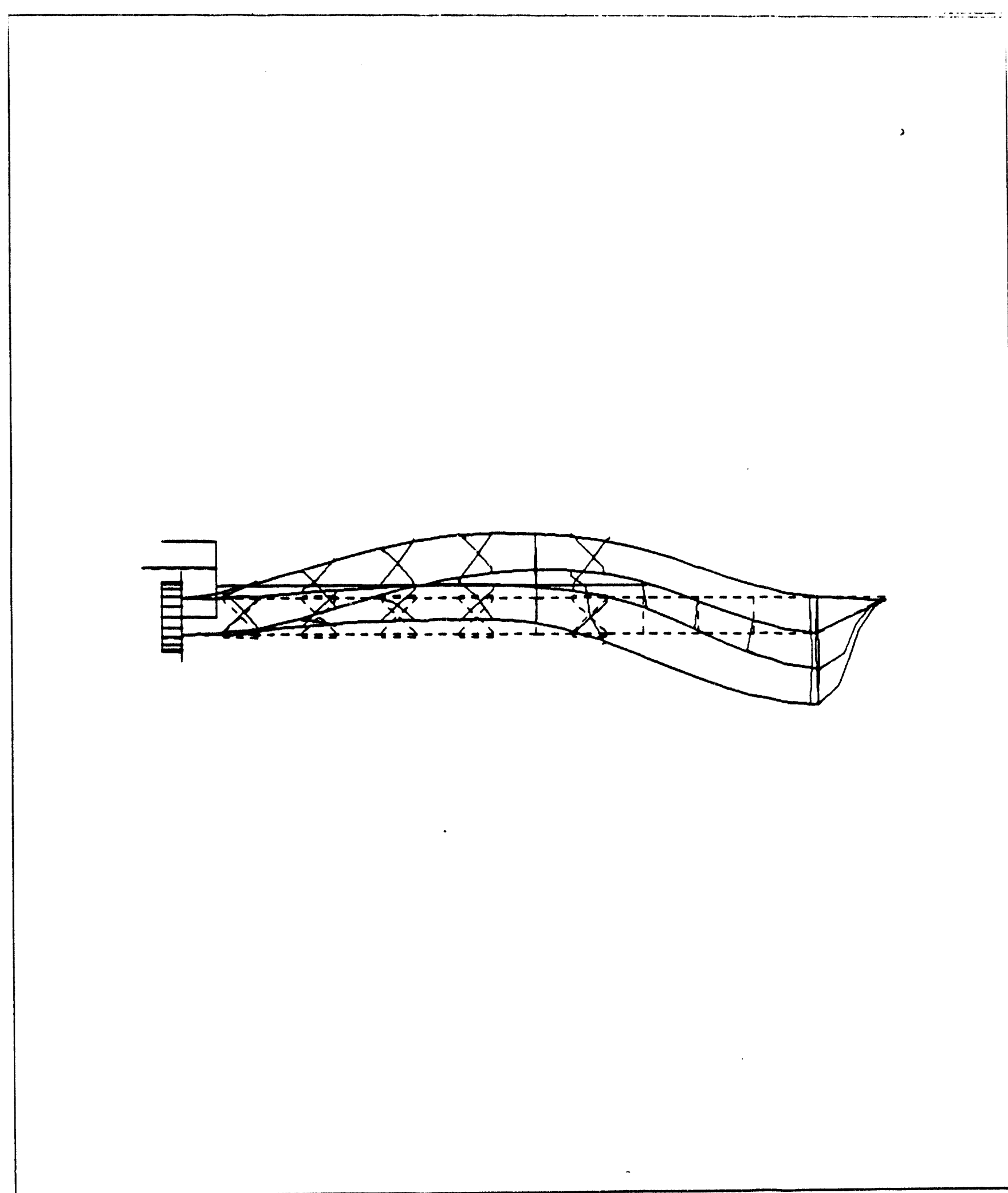


WHC-SD-WM-DA-163

Vol. II, ReV. 0

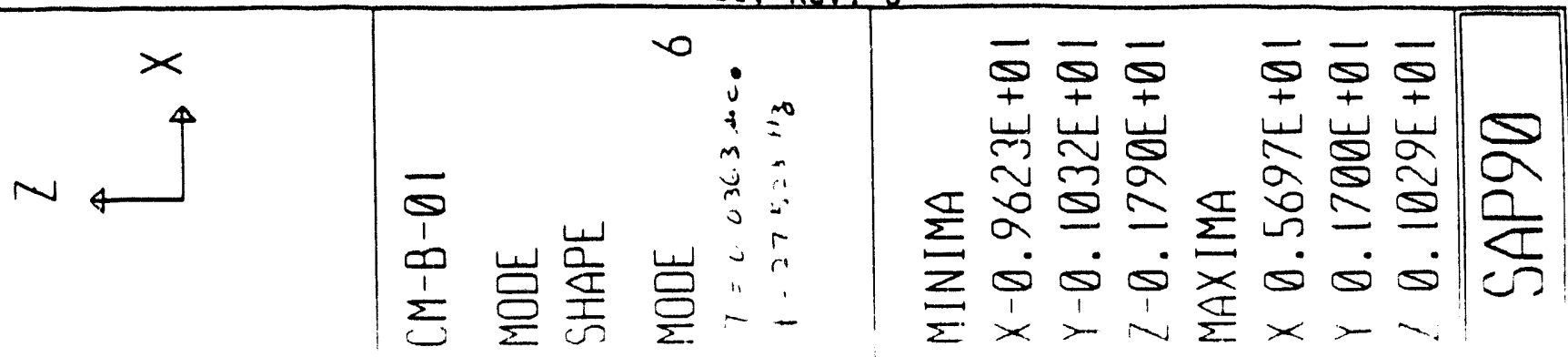

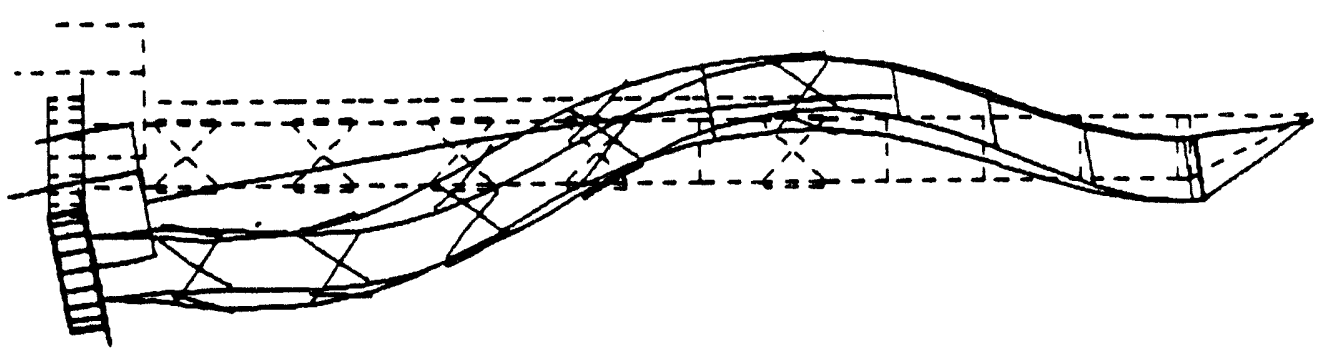


$80 I^{-1}$

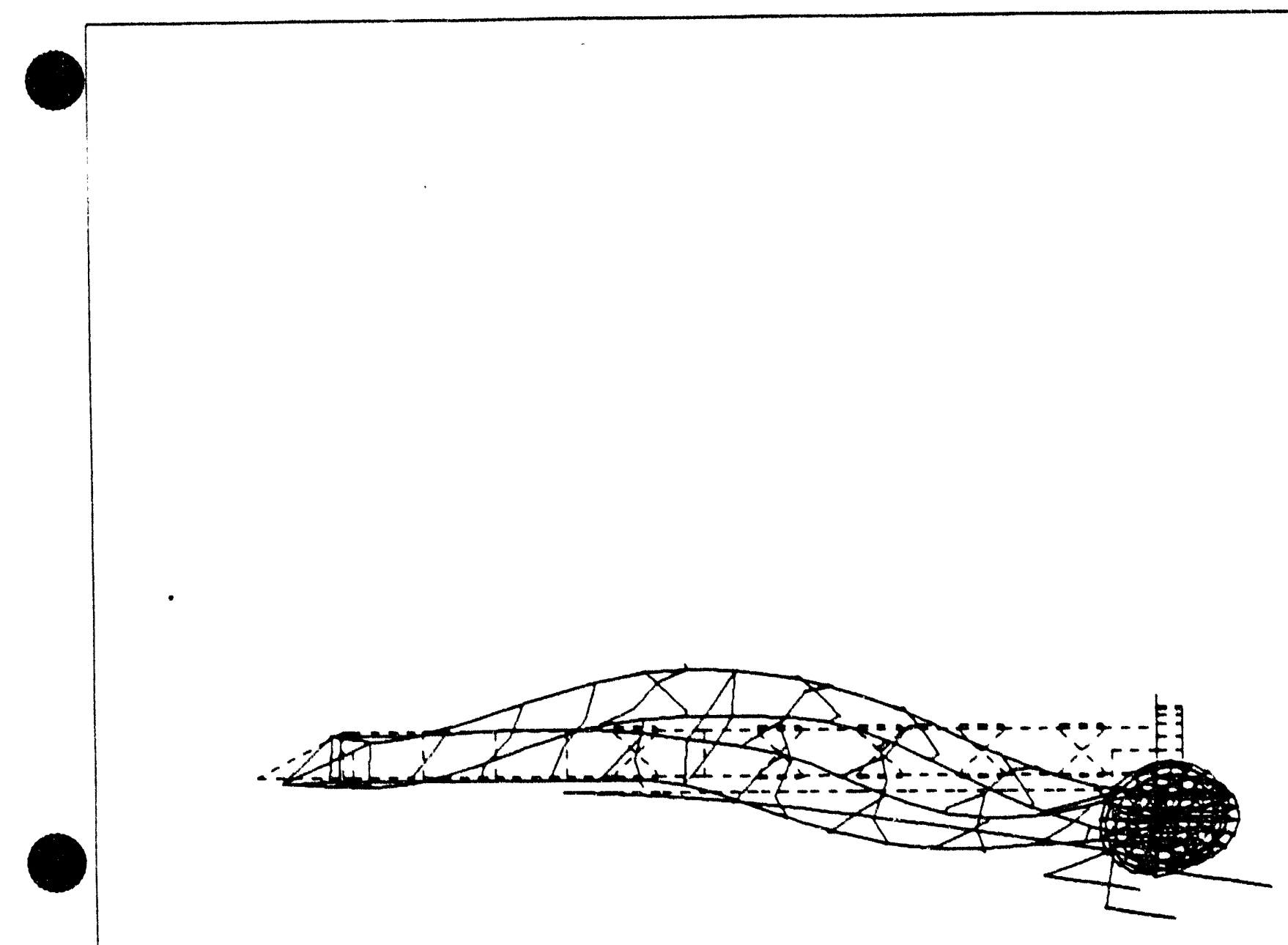

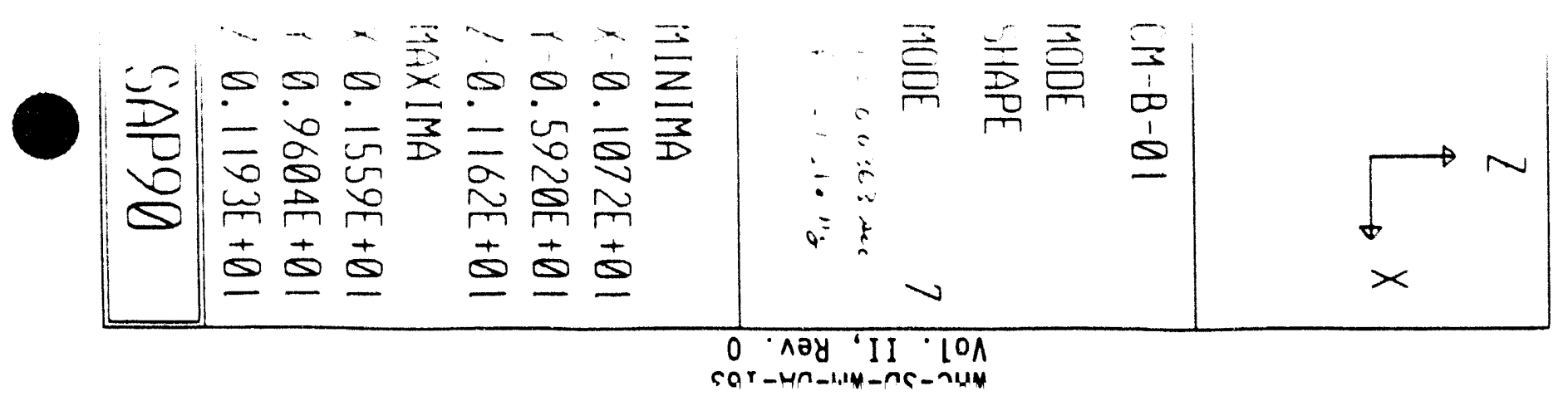


WHC-SD-WM-DA-163

Vol. II Rev 0

\begin{tabular}{|c|c|c|c|c|c|c|c|c|c|c|c|c|c|c|}
\hline${ }_{4}^{x}$ & $\begin{array}{l}\bar{Q} \\
\infty \\
\sum_{0}^{1}\end{array}$ & $\sum_{\sum}^{\frac{\omega}{\alpha}} \frac{\frac{\omega}{I}}{\sigma}$ & $\begin{array}{l}\infty \\
巳 \\
巳\end{array}$ & $\begin{array}{l}j \\
j \\
j \\
\bar{m} \\
0 \\
0 \\
11 \\
1\end{array}$ & $\begin{array}{l}\hat{x} \\
\hat{3} \\
\vec{b} \\
\therefore \\
\therefore\end{array}$ & $\frac{I}{\sum} \underset{\Sigma}{\sum}$ & 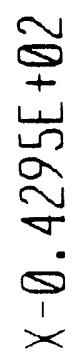 & 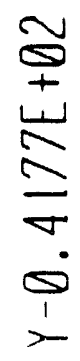 & 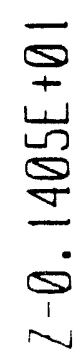 & $\frac{\pi}{\sum} \frac{\pi}{\sum}$ & $\begin{aligned} & \underset{+}{+} \\
= & \frac{\infty}{0} \\
= & \infty \\
= & \infty\end{aligned}$ & 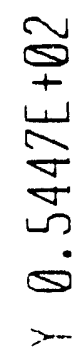 & 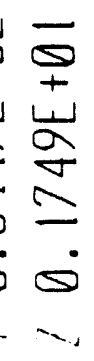 & $\frac{1}{\Omega}$ \\
\hline
\end{tabular}

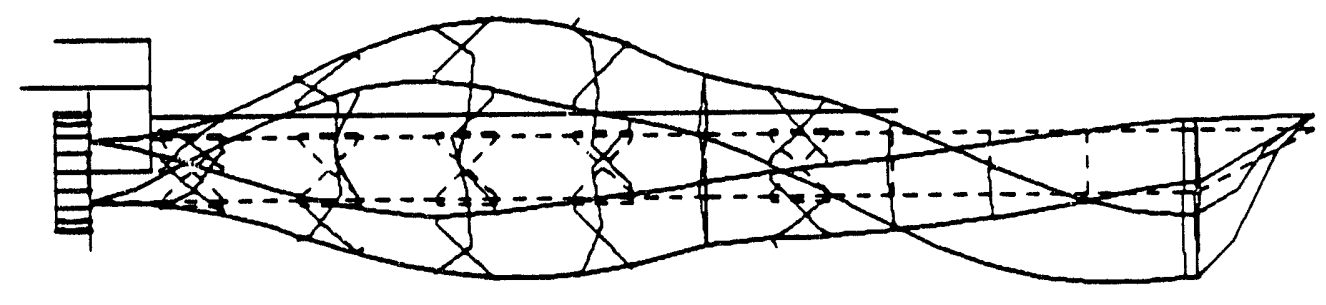

$=$ 
Vol. II, Rev. 0

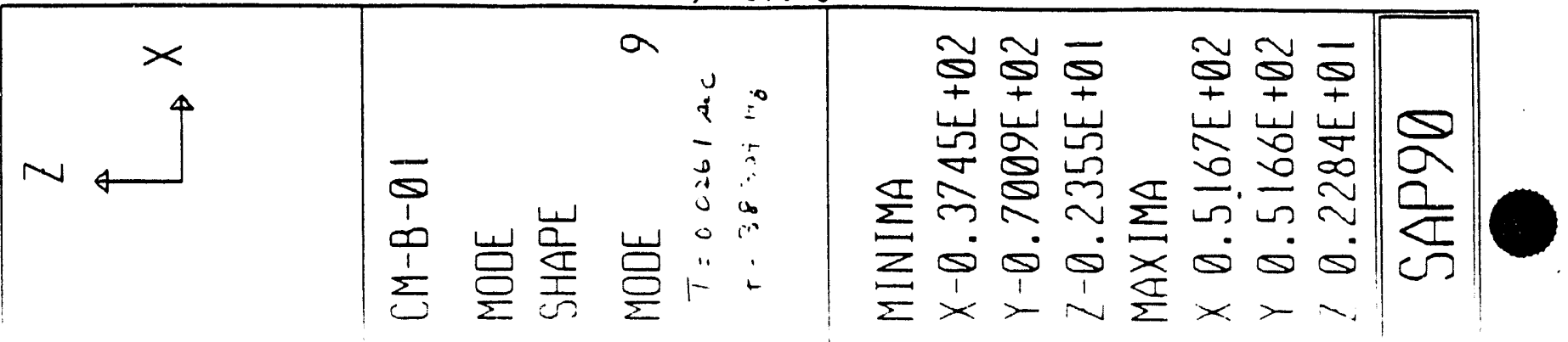

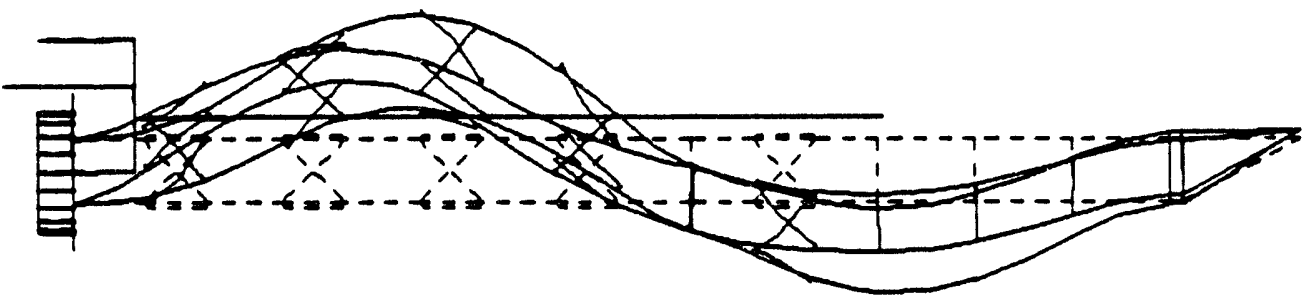


WHC-SD-WM-DA-163

Vol. II, Rev. 0

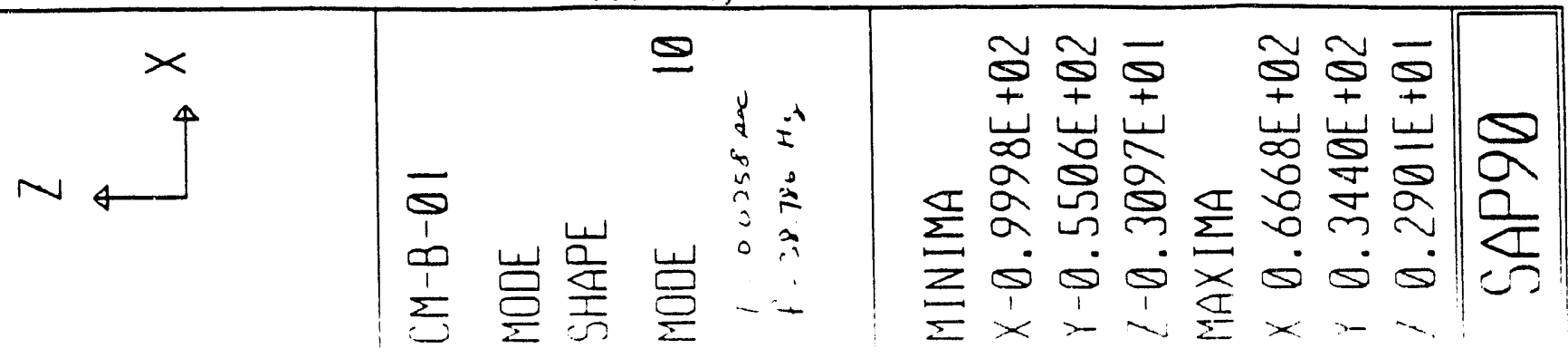

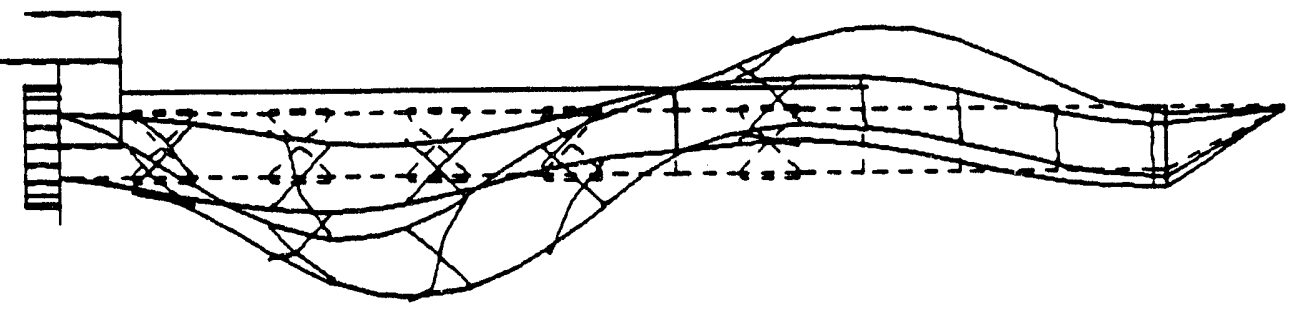




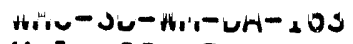

Vol. II, Rev. 0

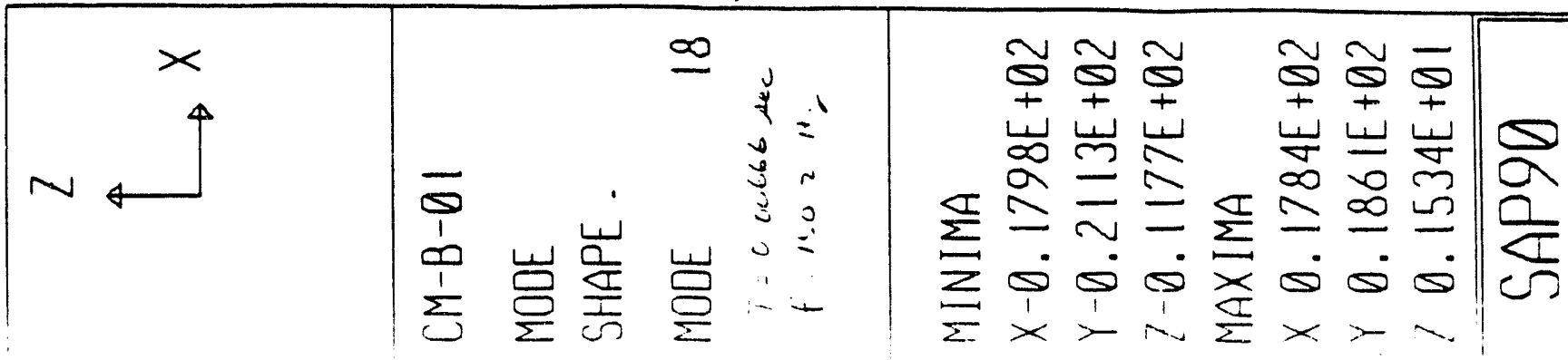

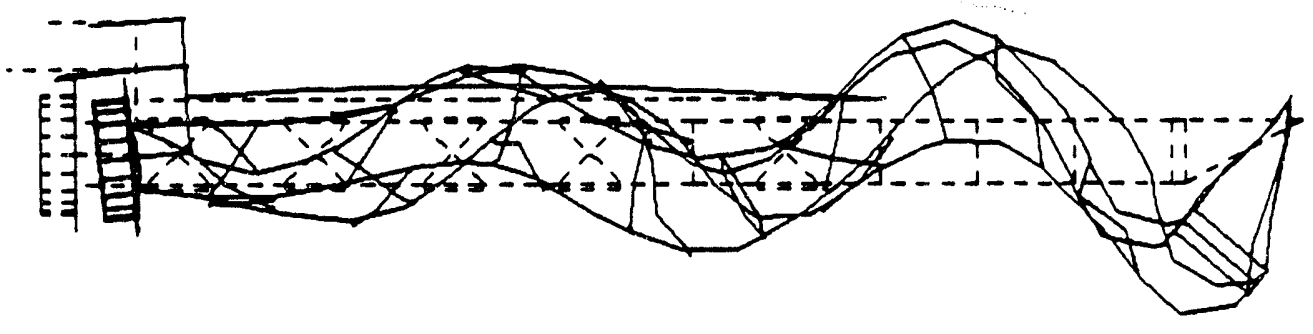




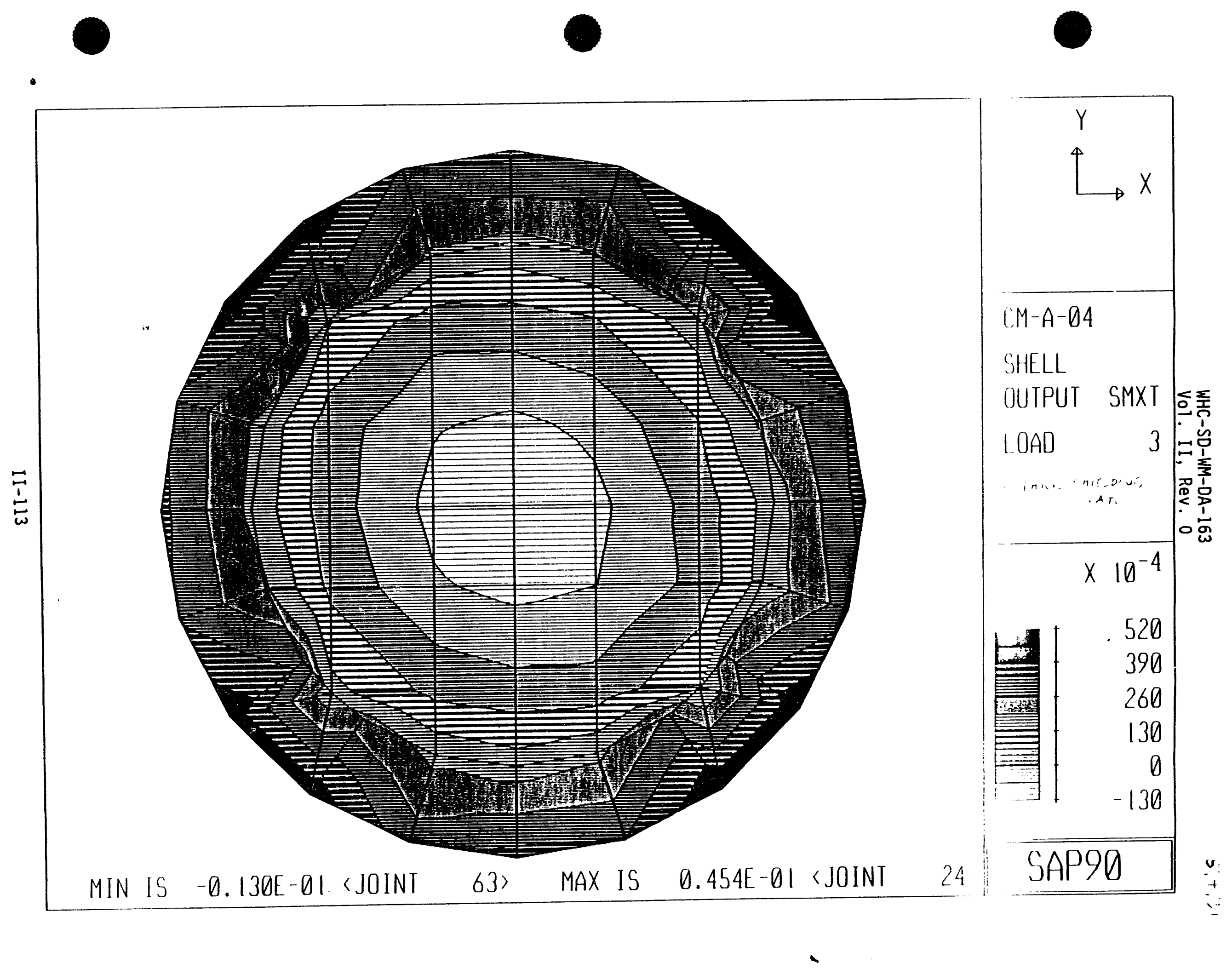


tII-II

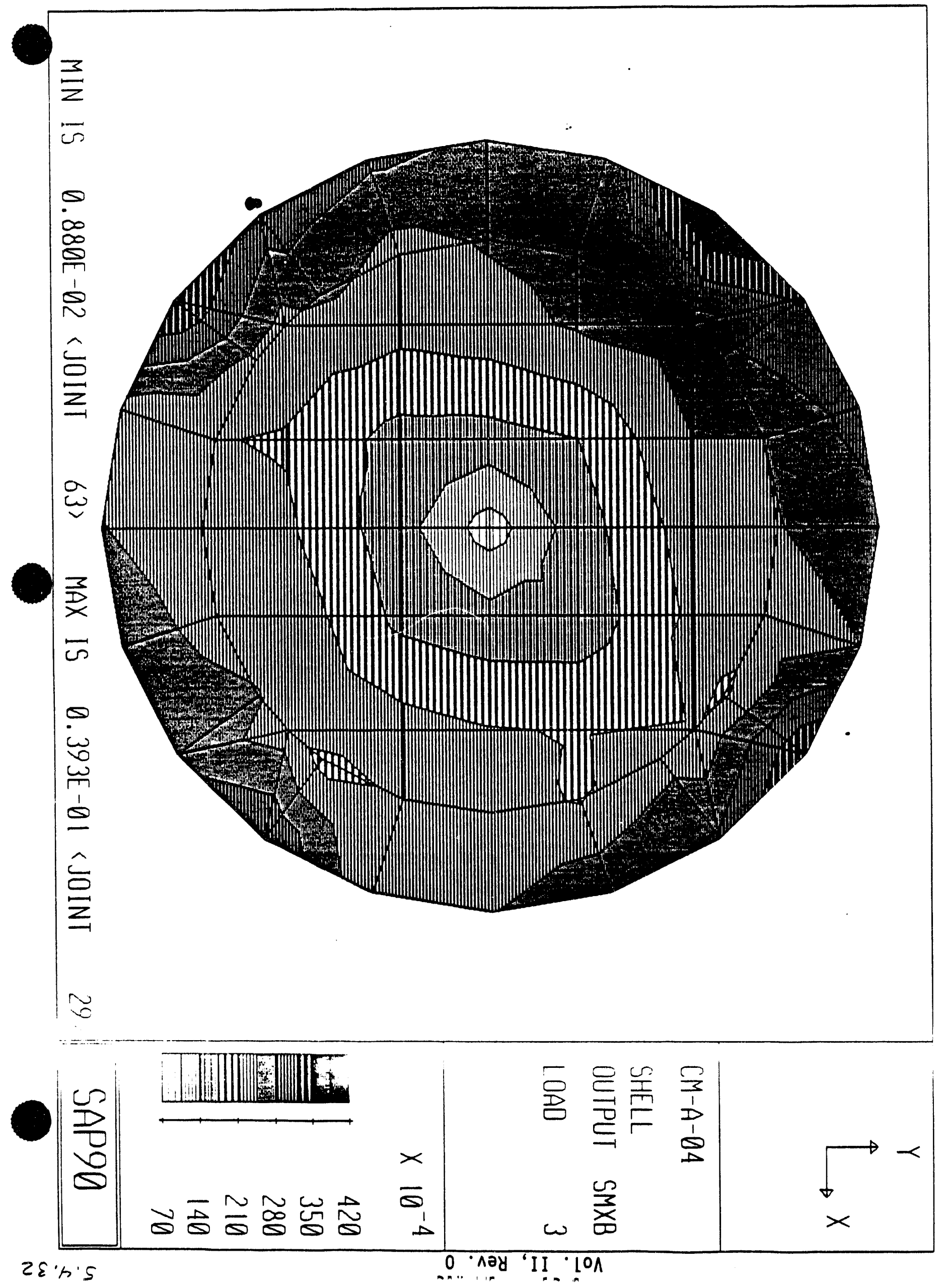




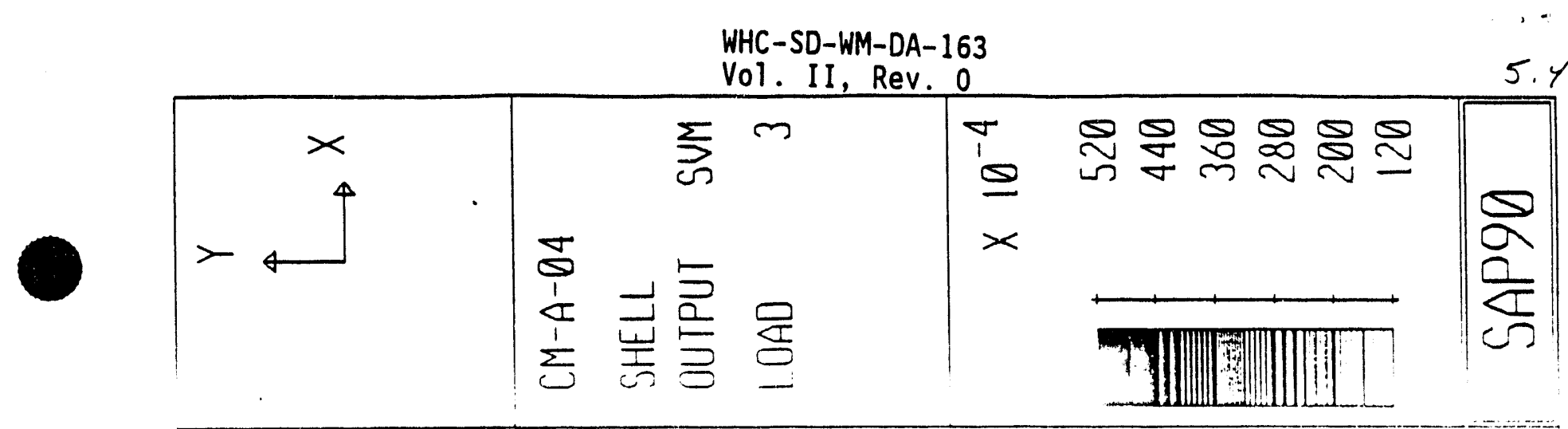

5.4 .33

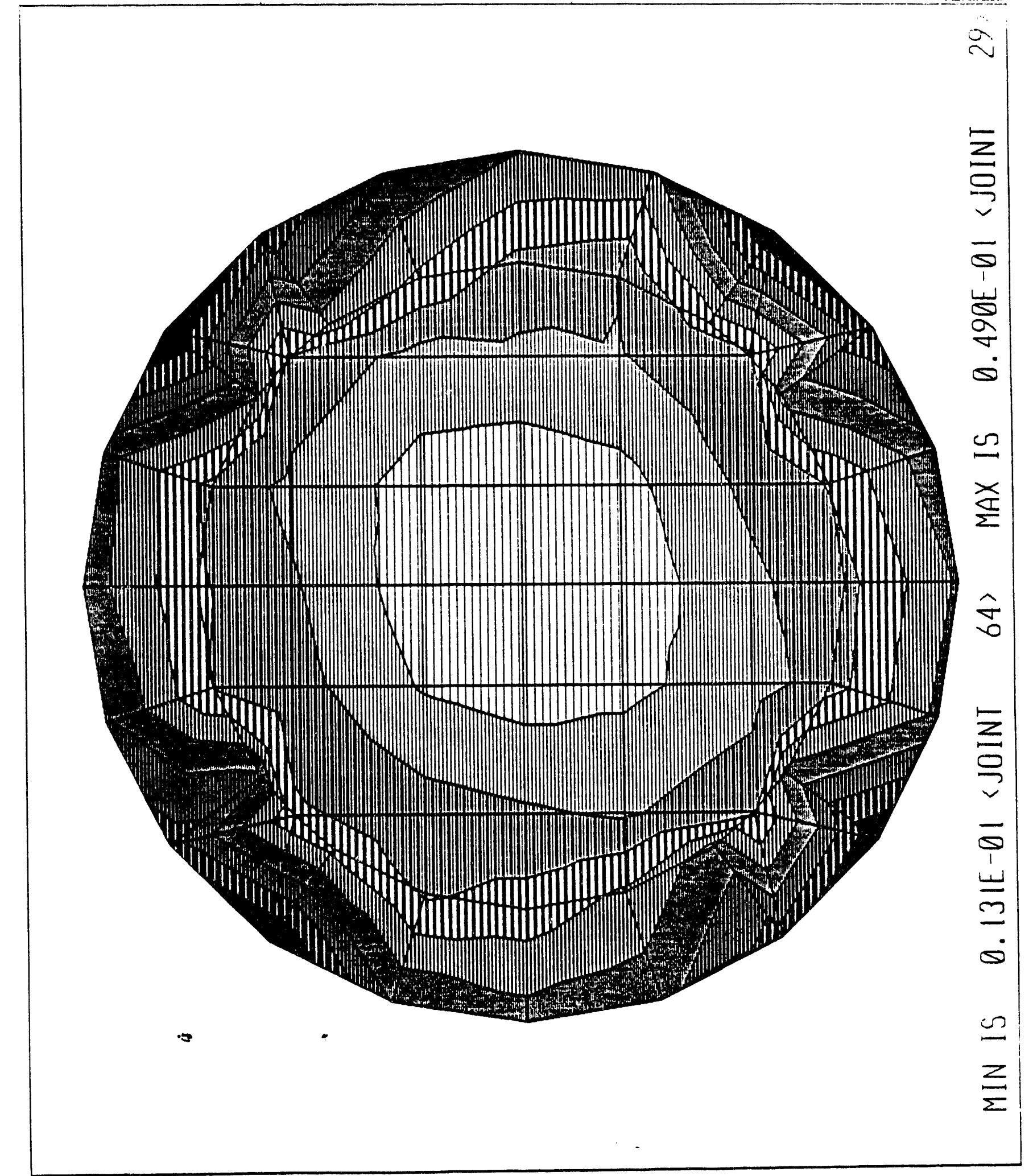




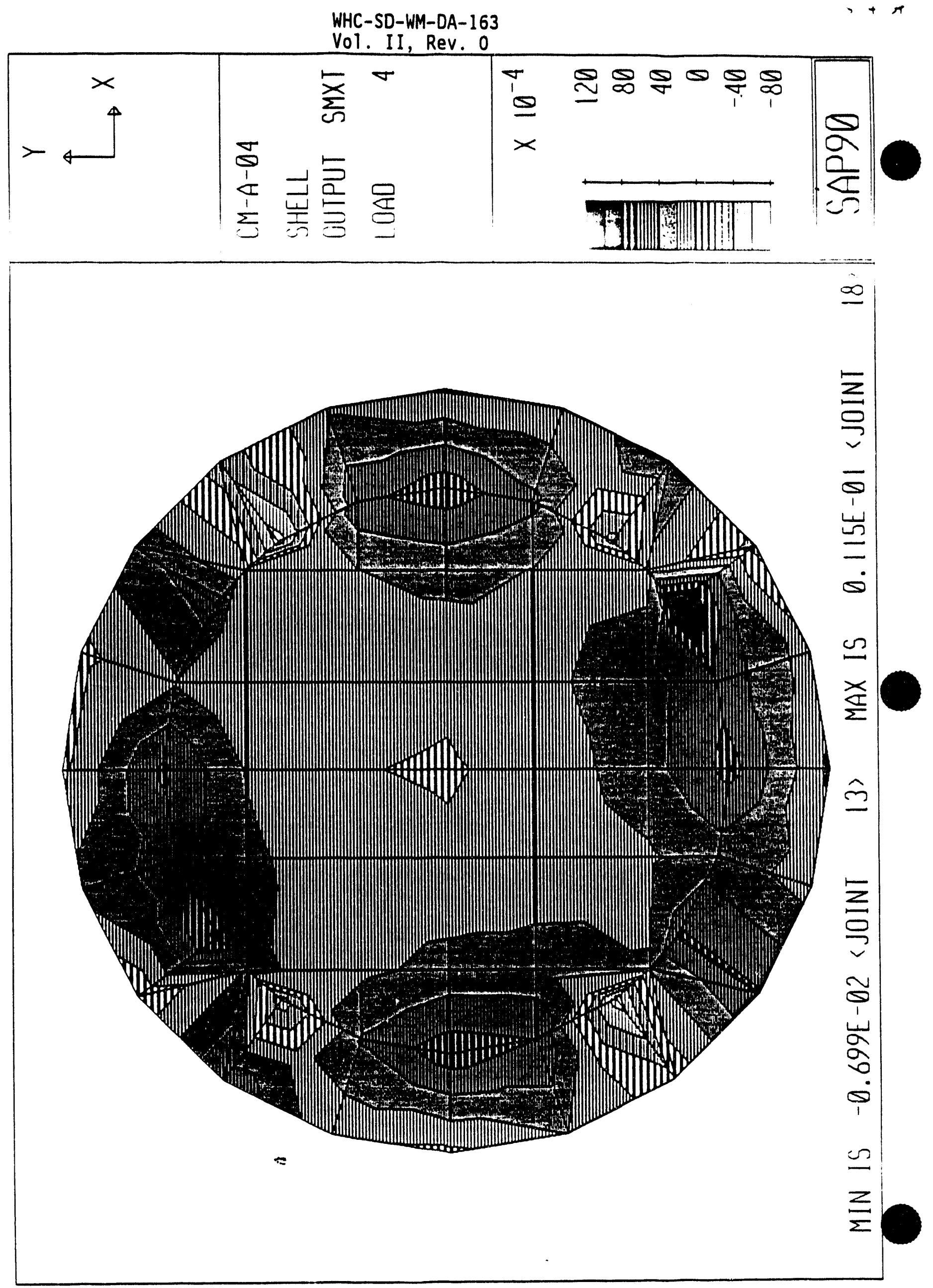




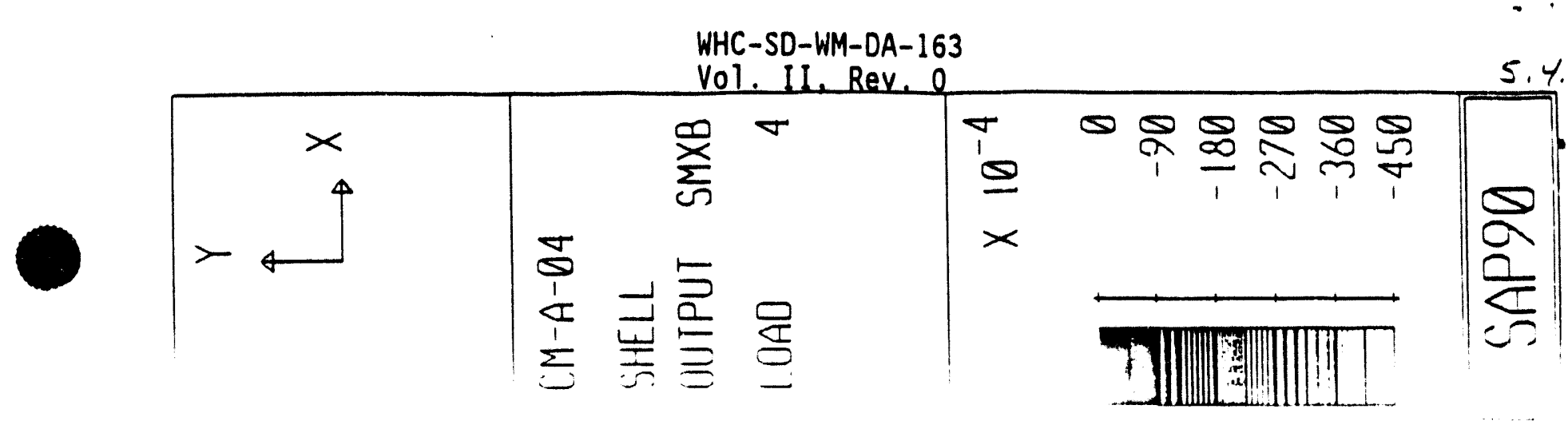

5.4 .35 
WHC-SD-WM-DA-163

Vol. II. ReV. 0
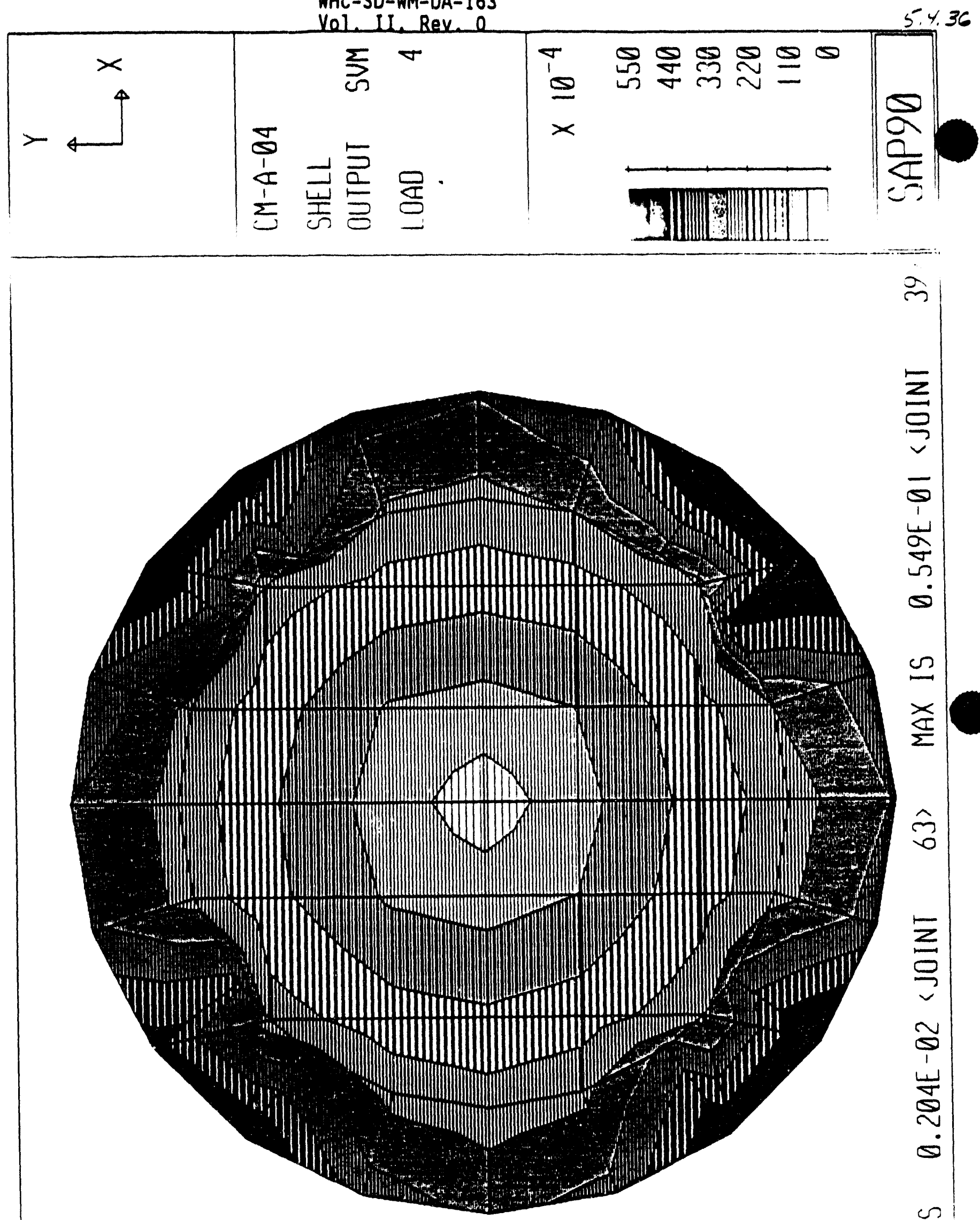

जे

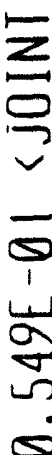

$\frac{1}{2}$
$\frac{x}{2}$
$\hat{n}$

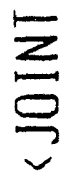

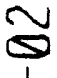

嵌

on

를 


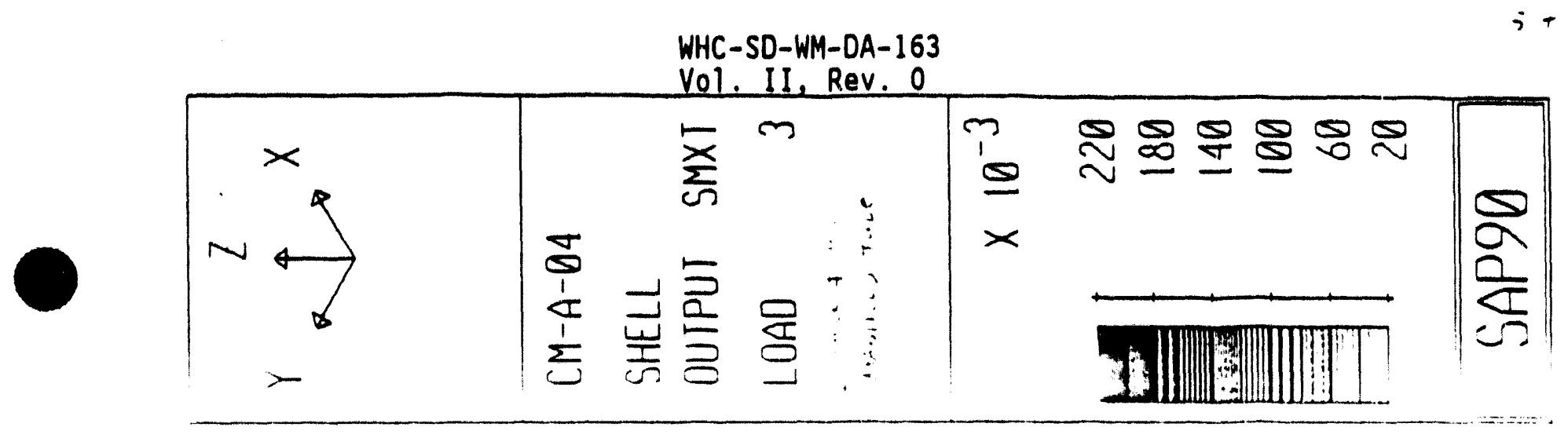

i $+i$

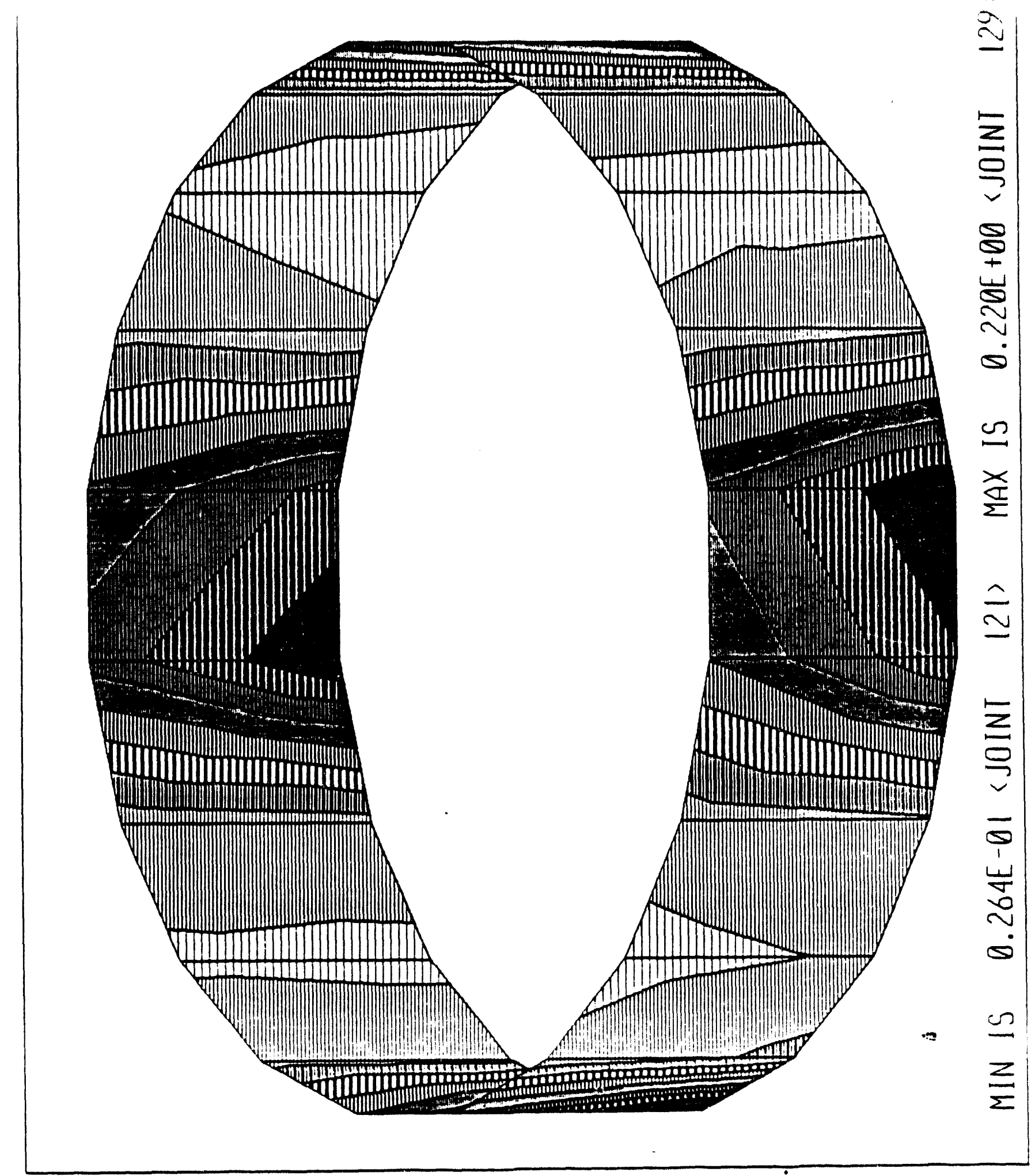

II -119 


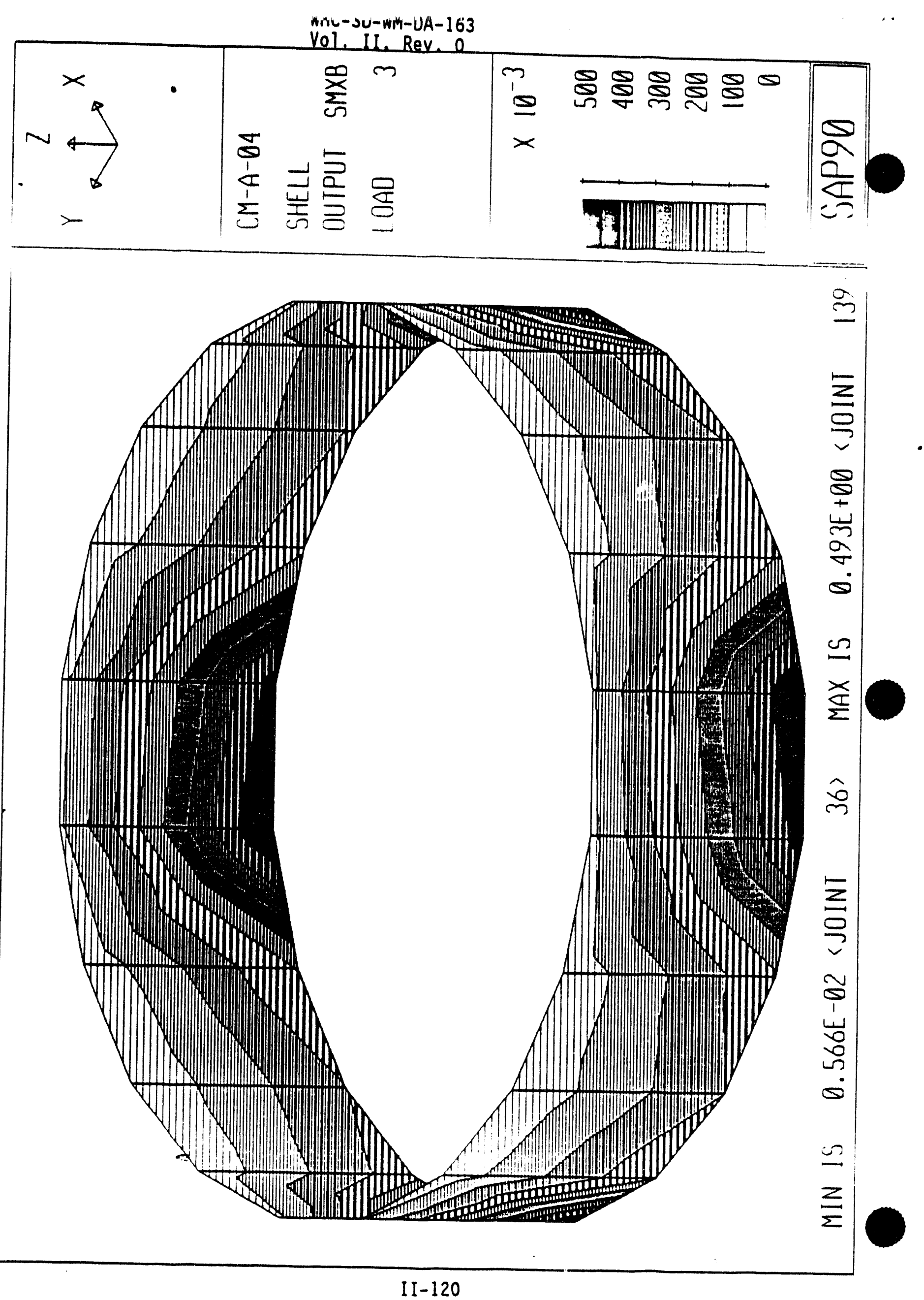



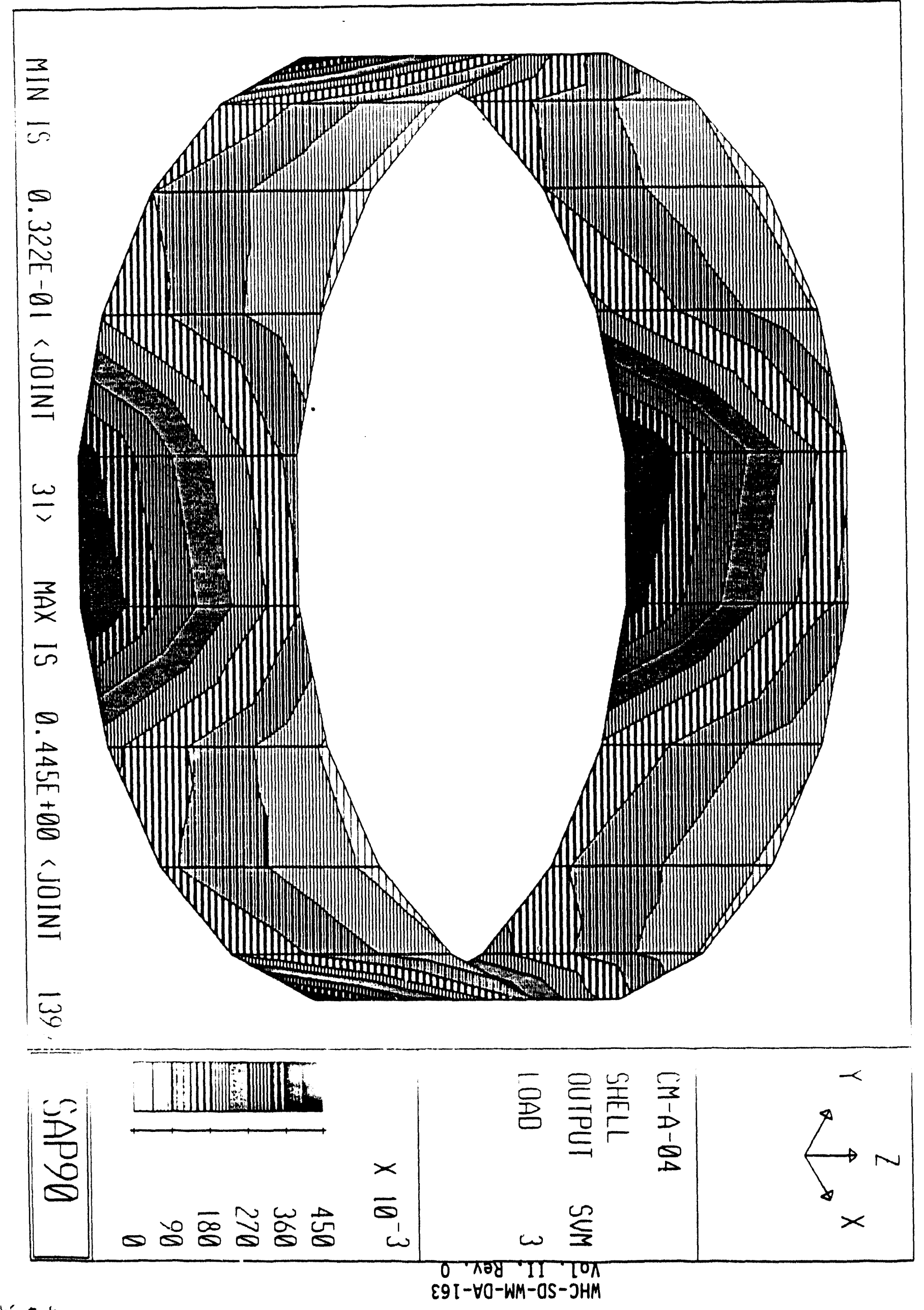
WHC-SD-WM-DA-163

Vol. II, Rev. 0

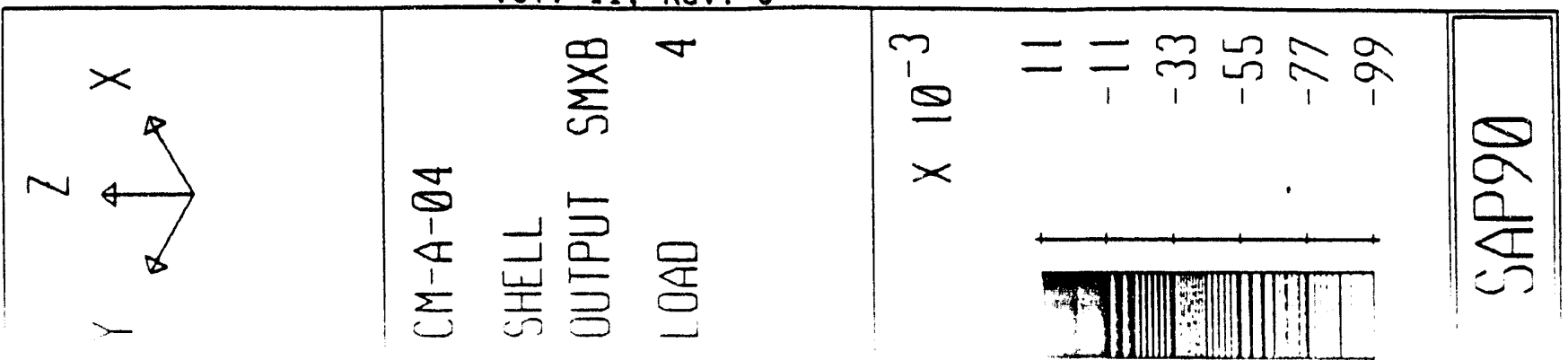

5.4 .41

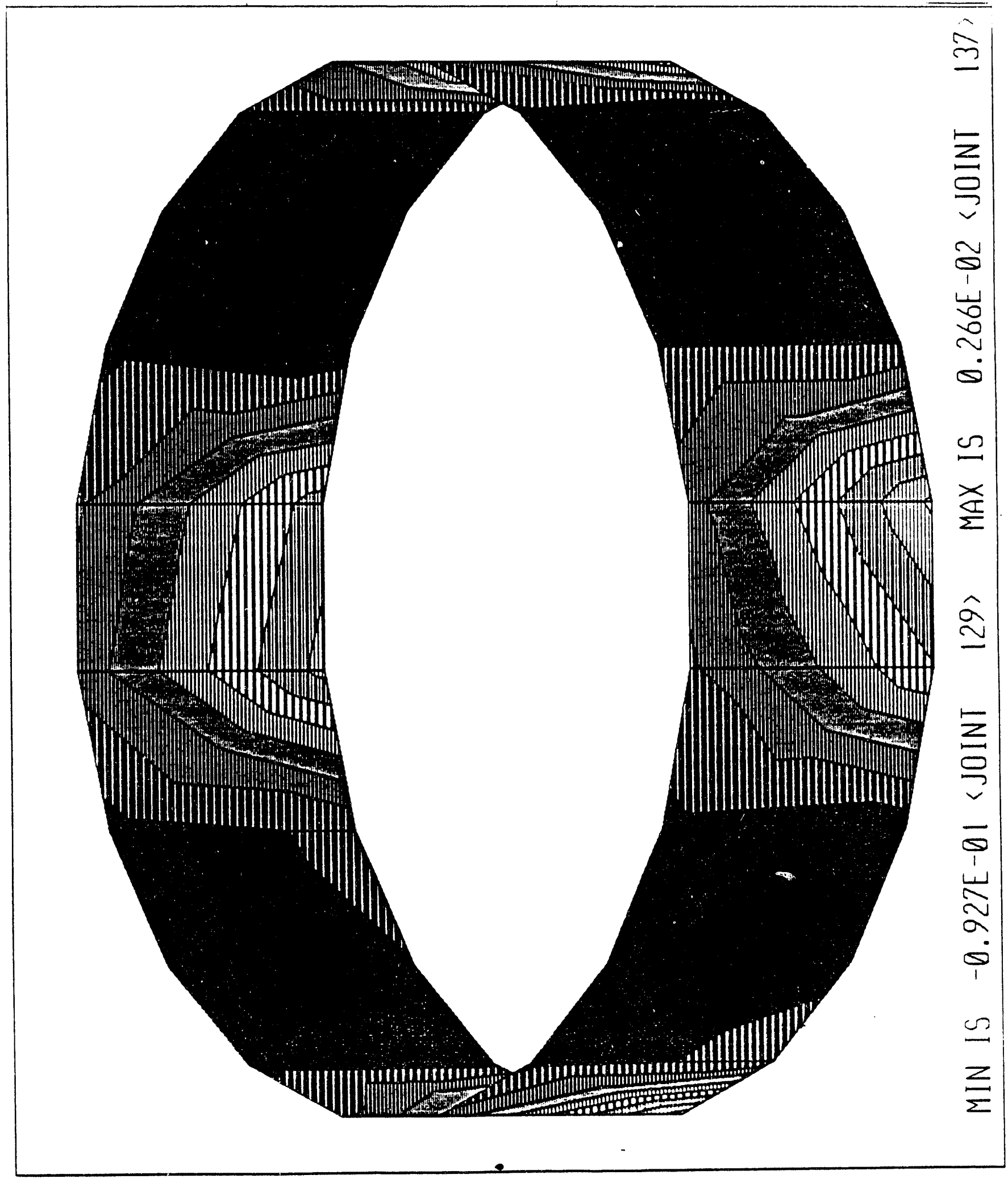




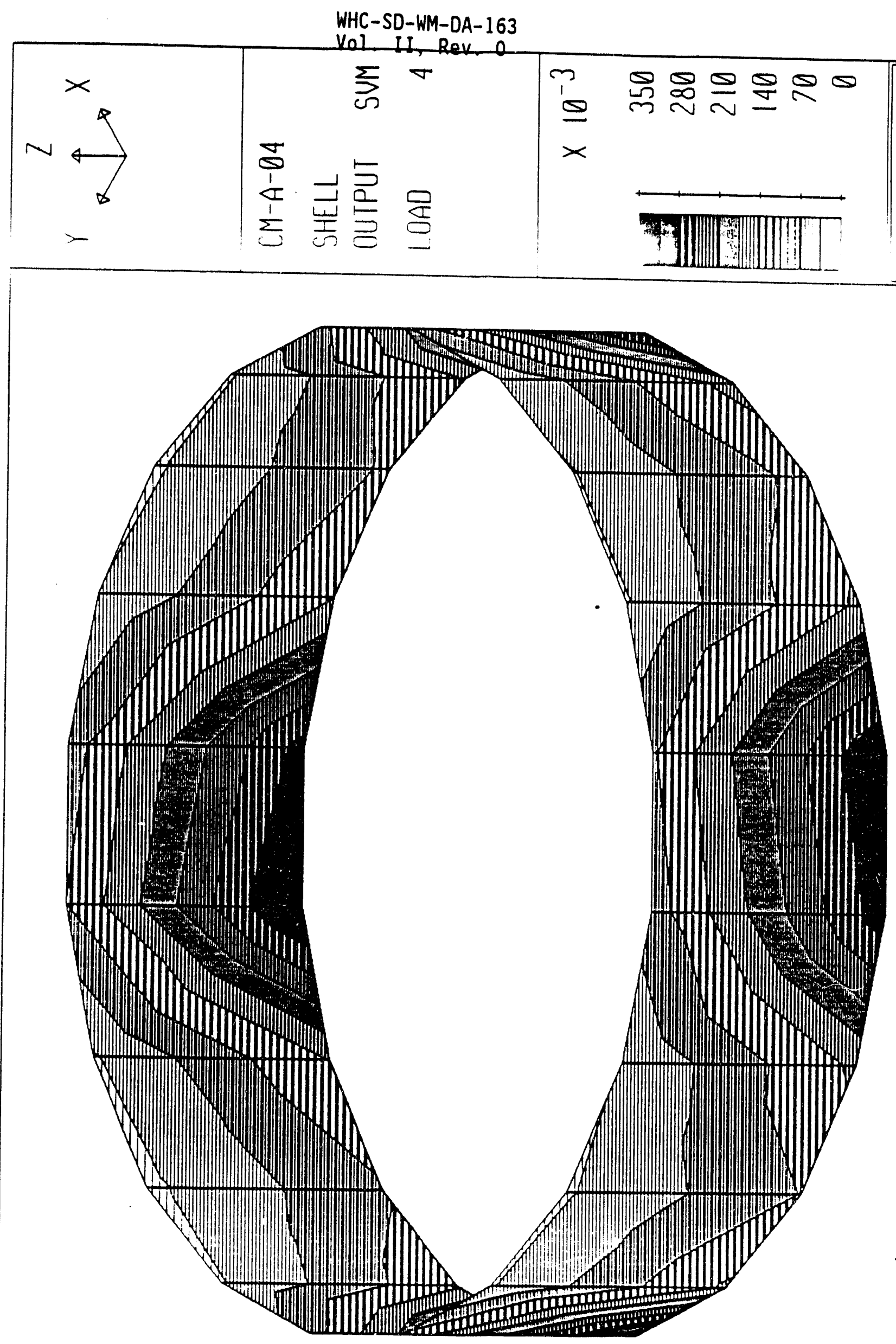




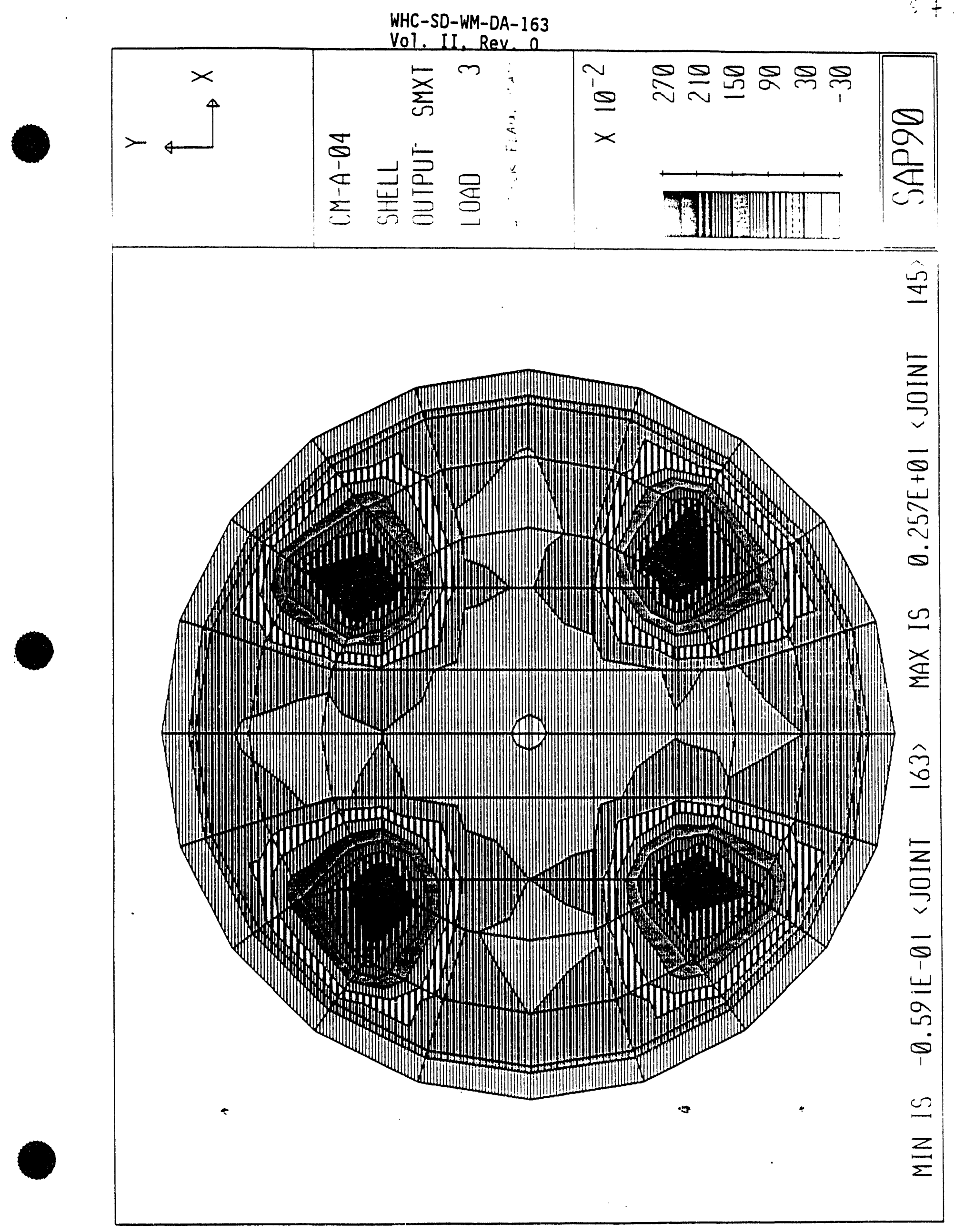




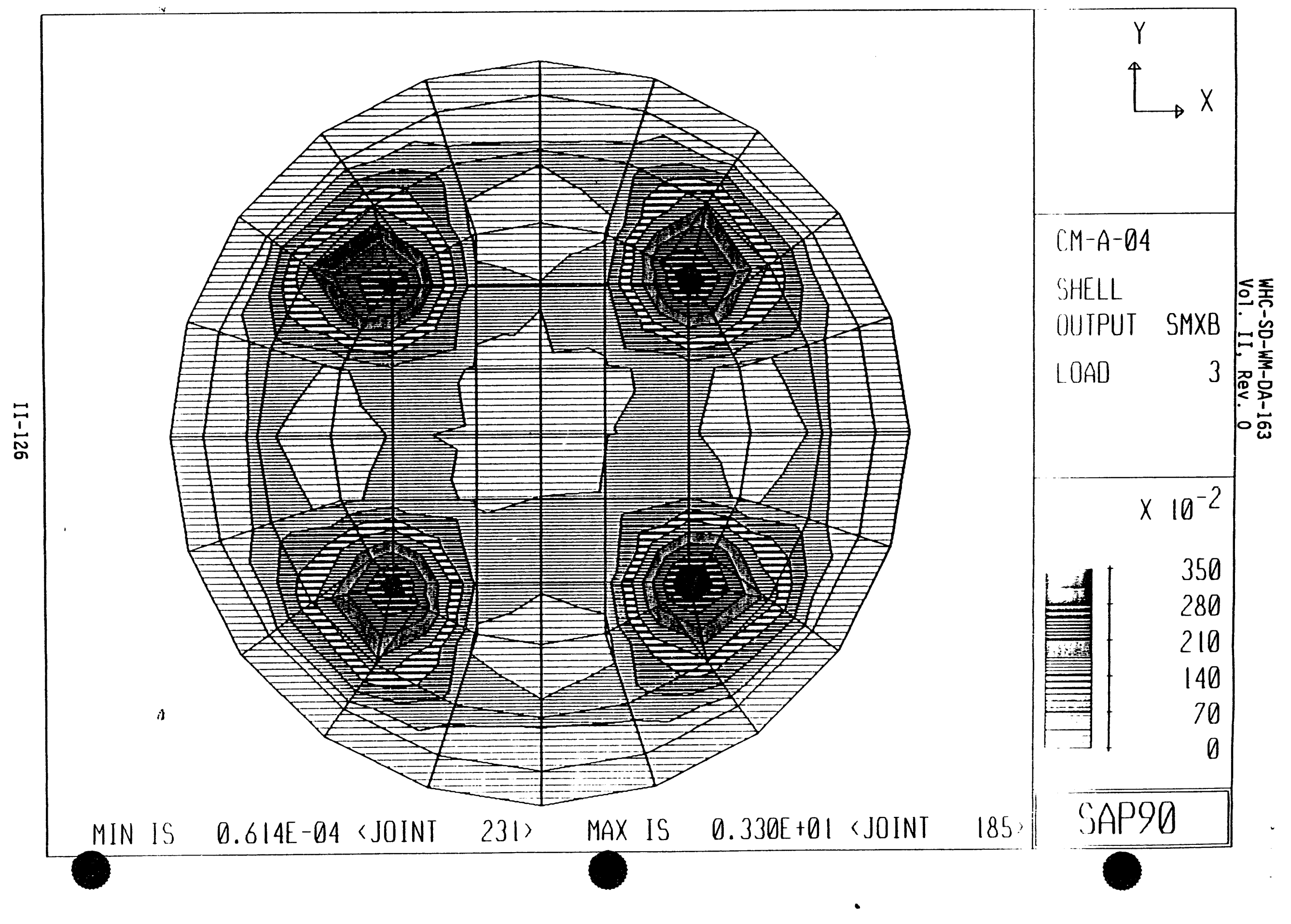




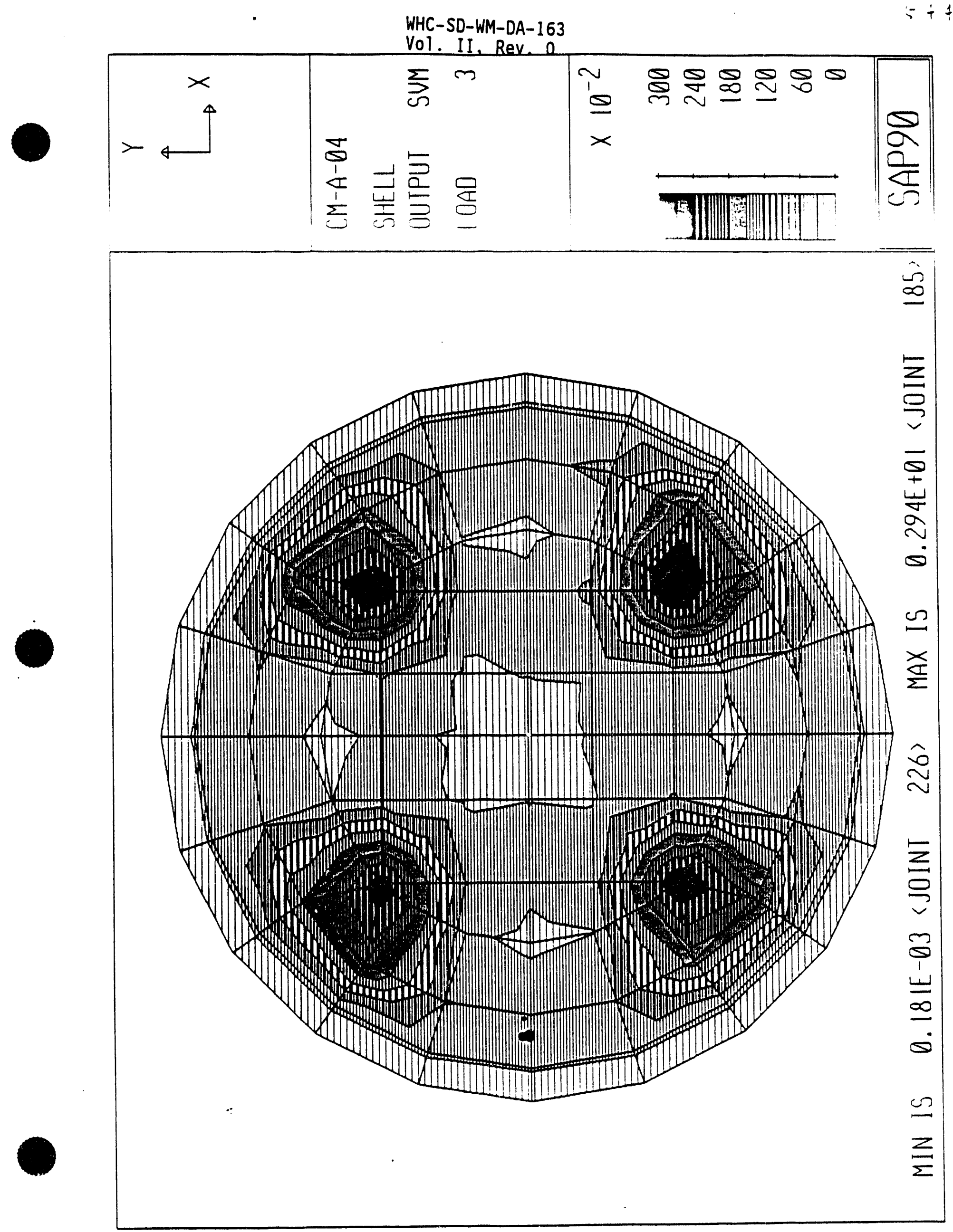



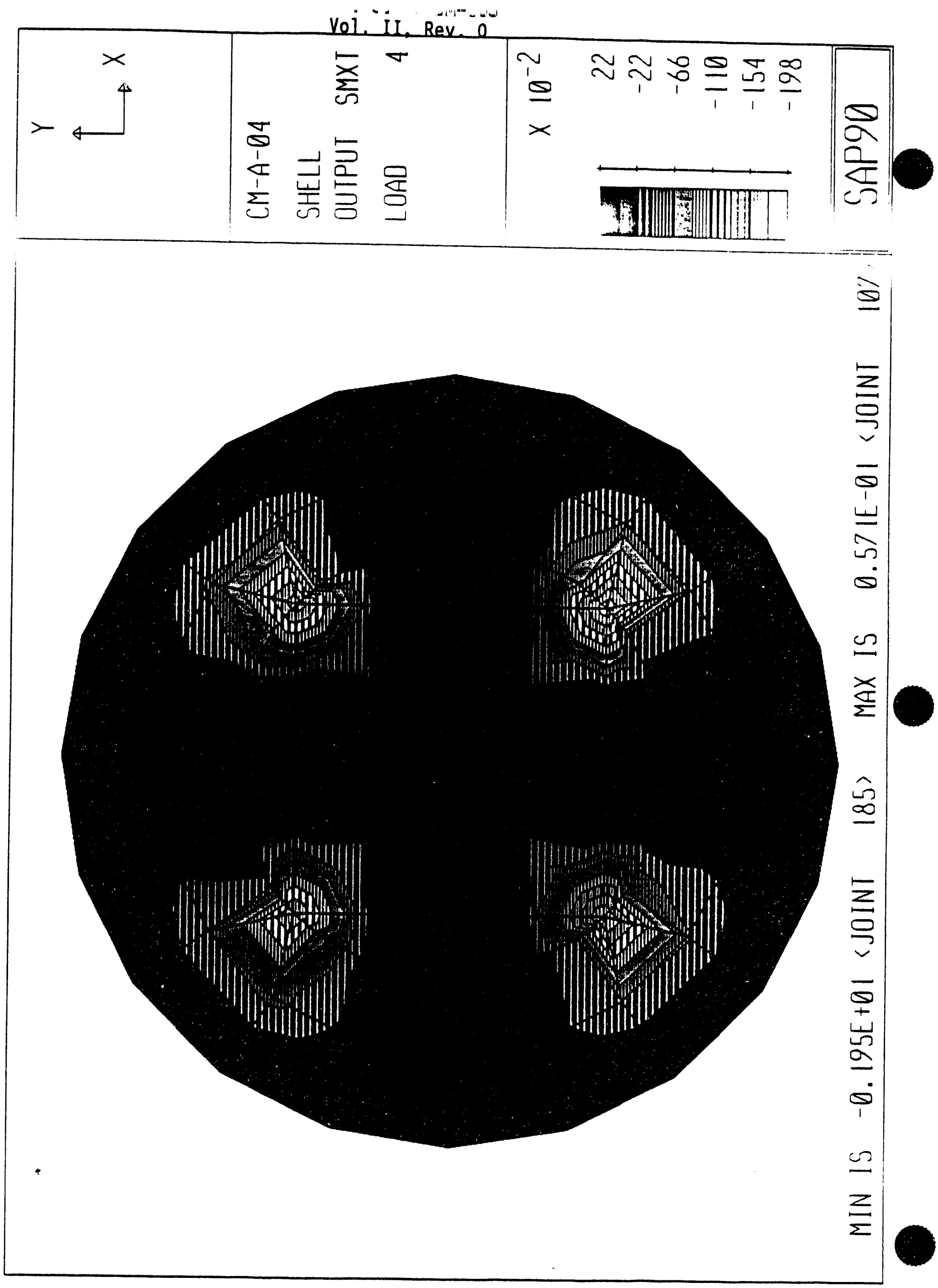


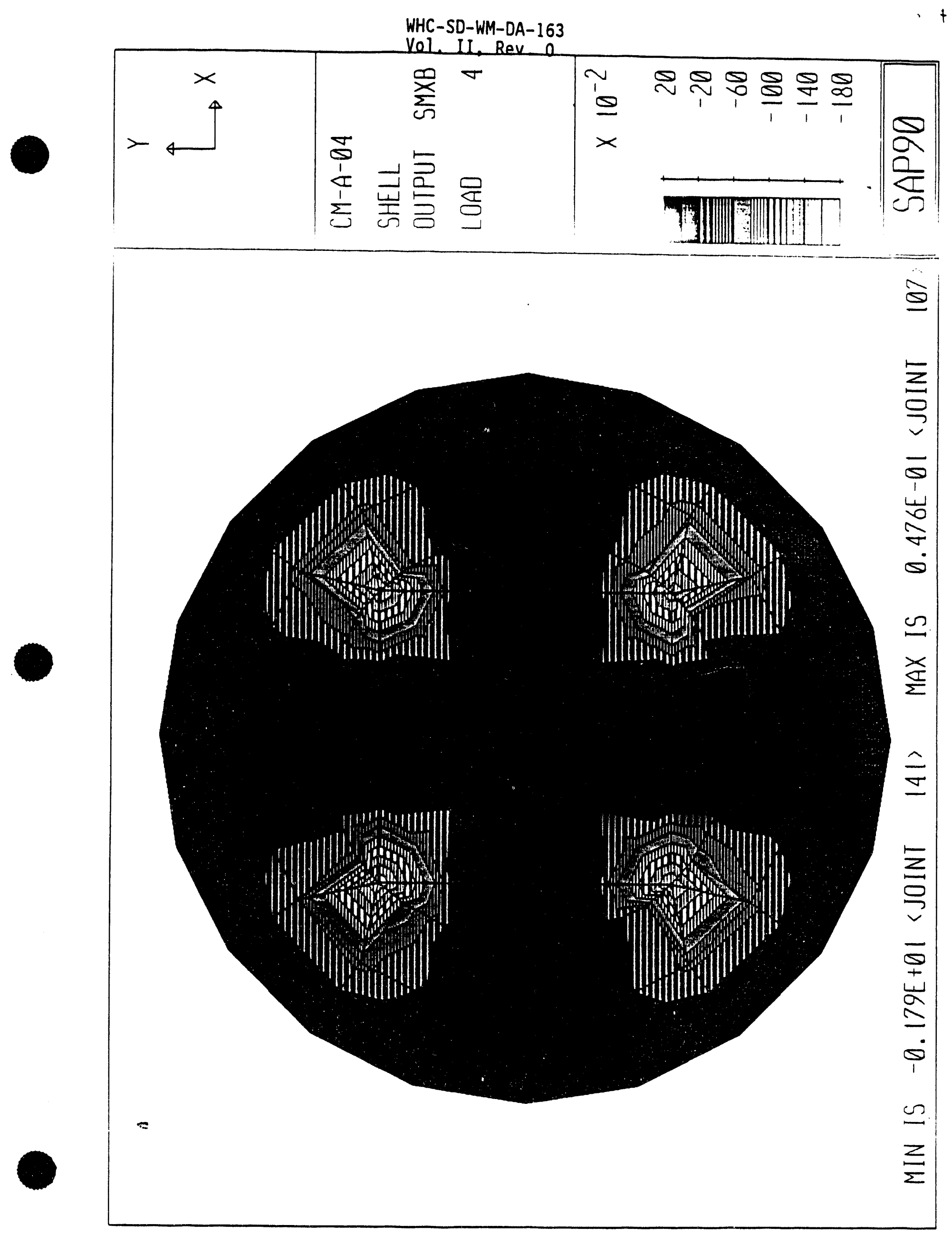


WHC-SD-WM-DA-163

$\mathrm{Vol}$. II ReV. 0
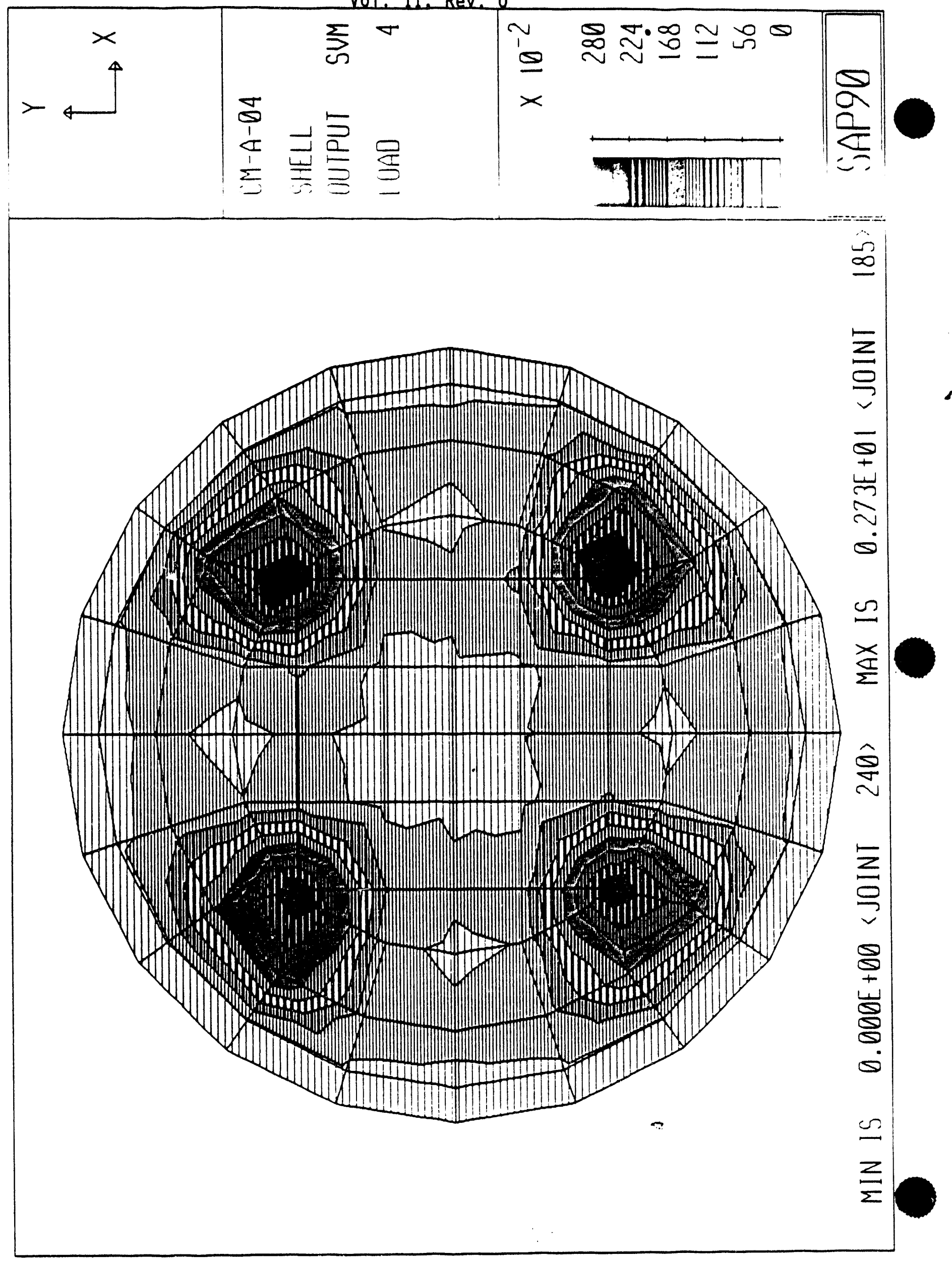

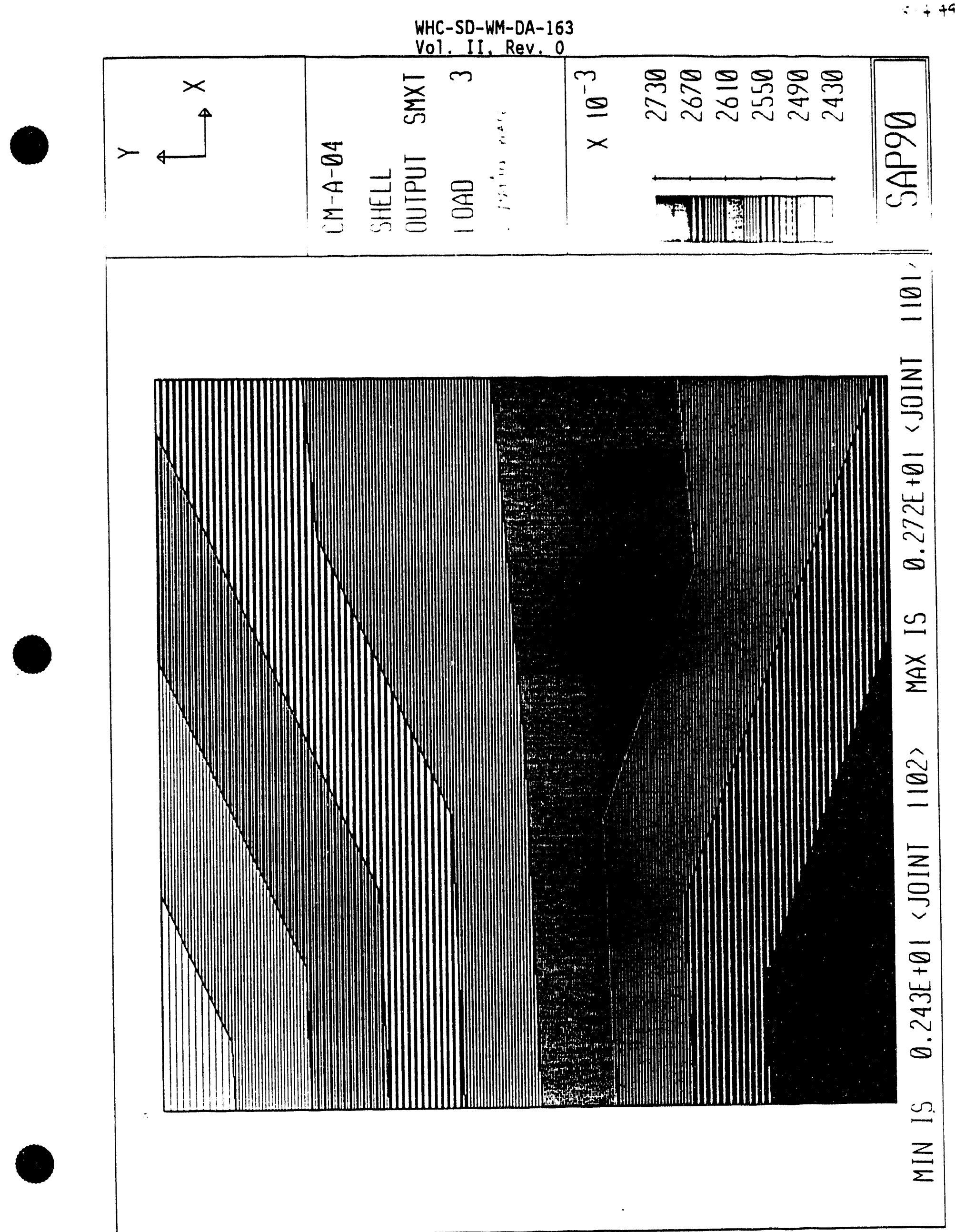


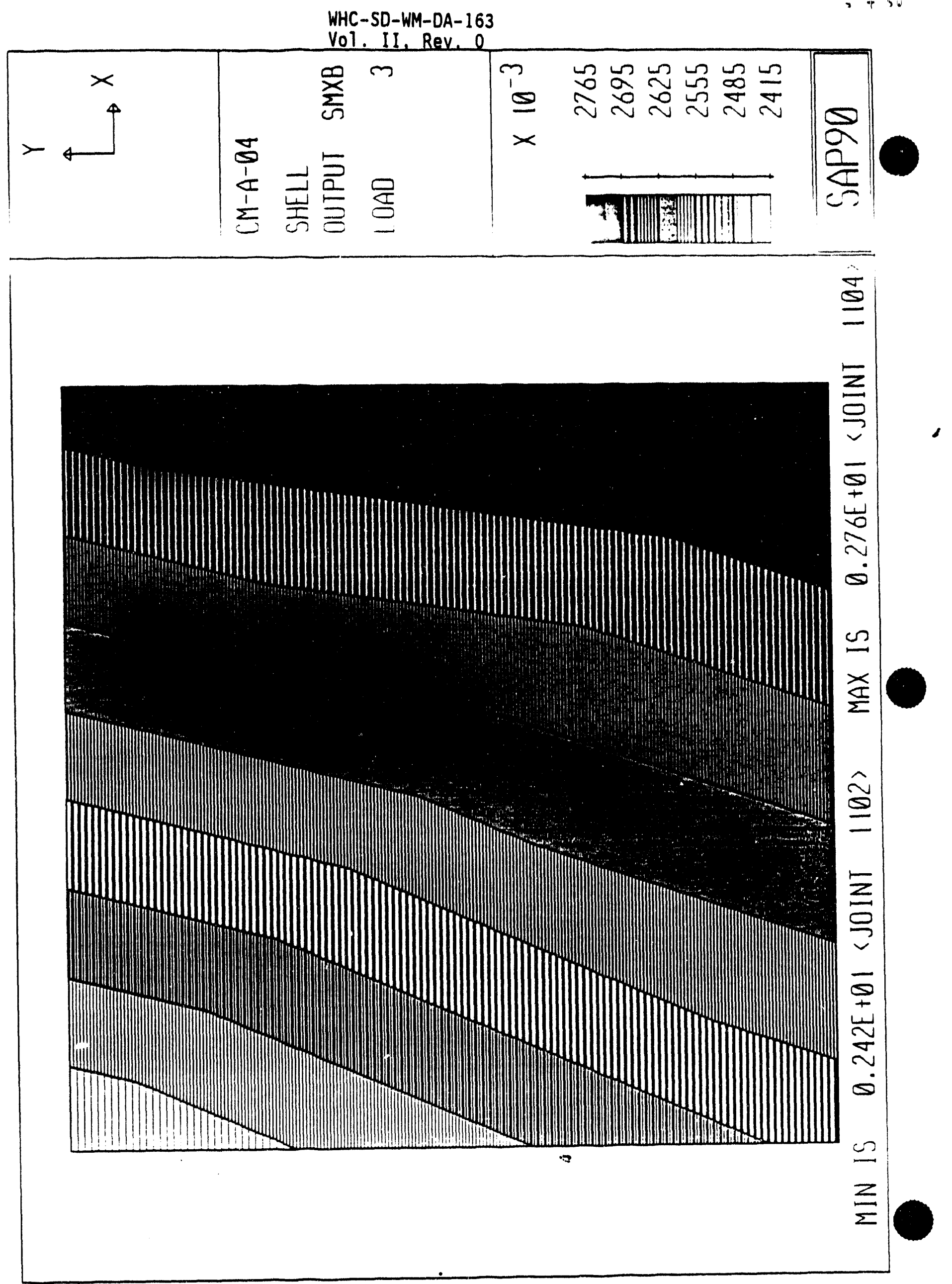




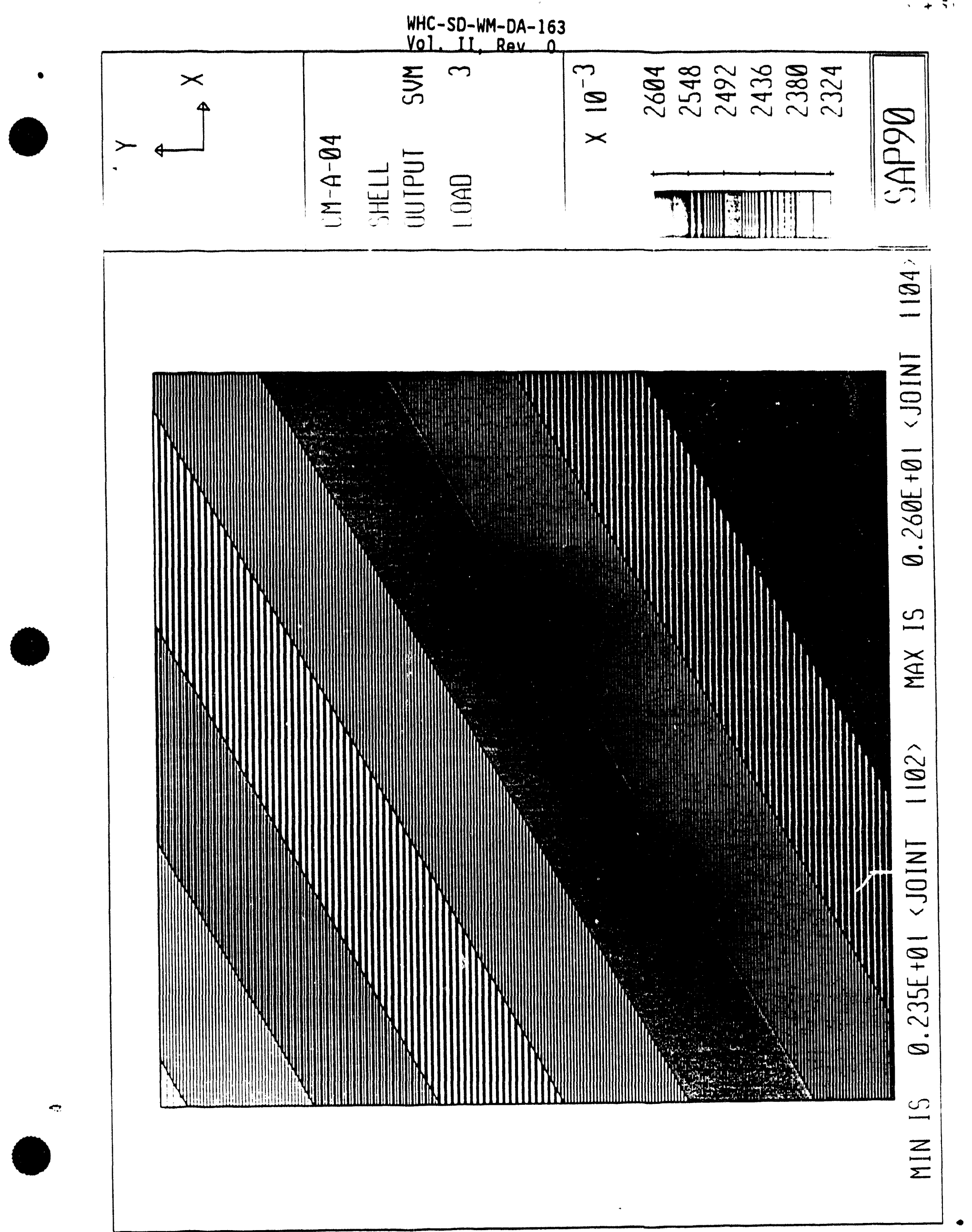




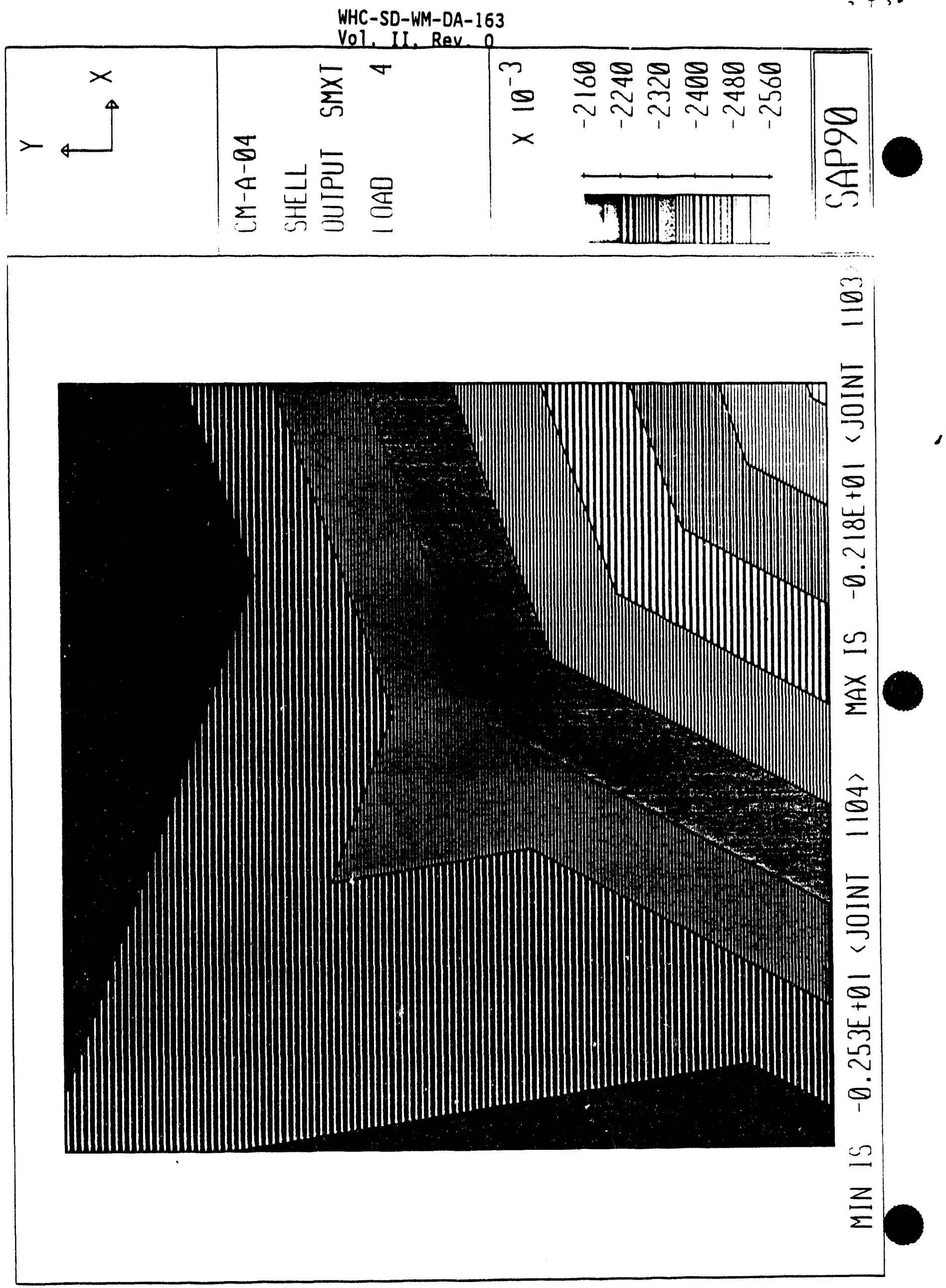




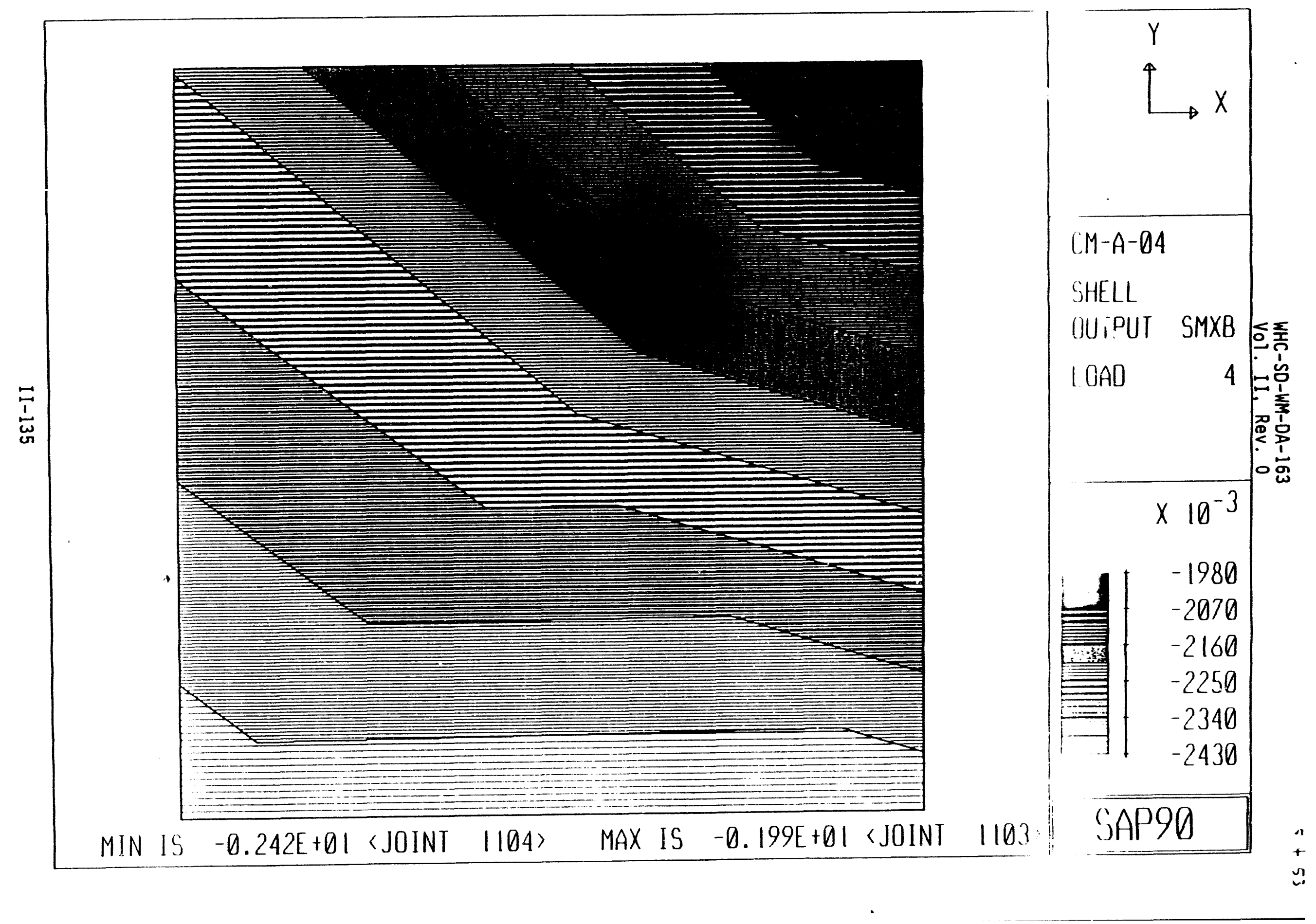



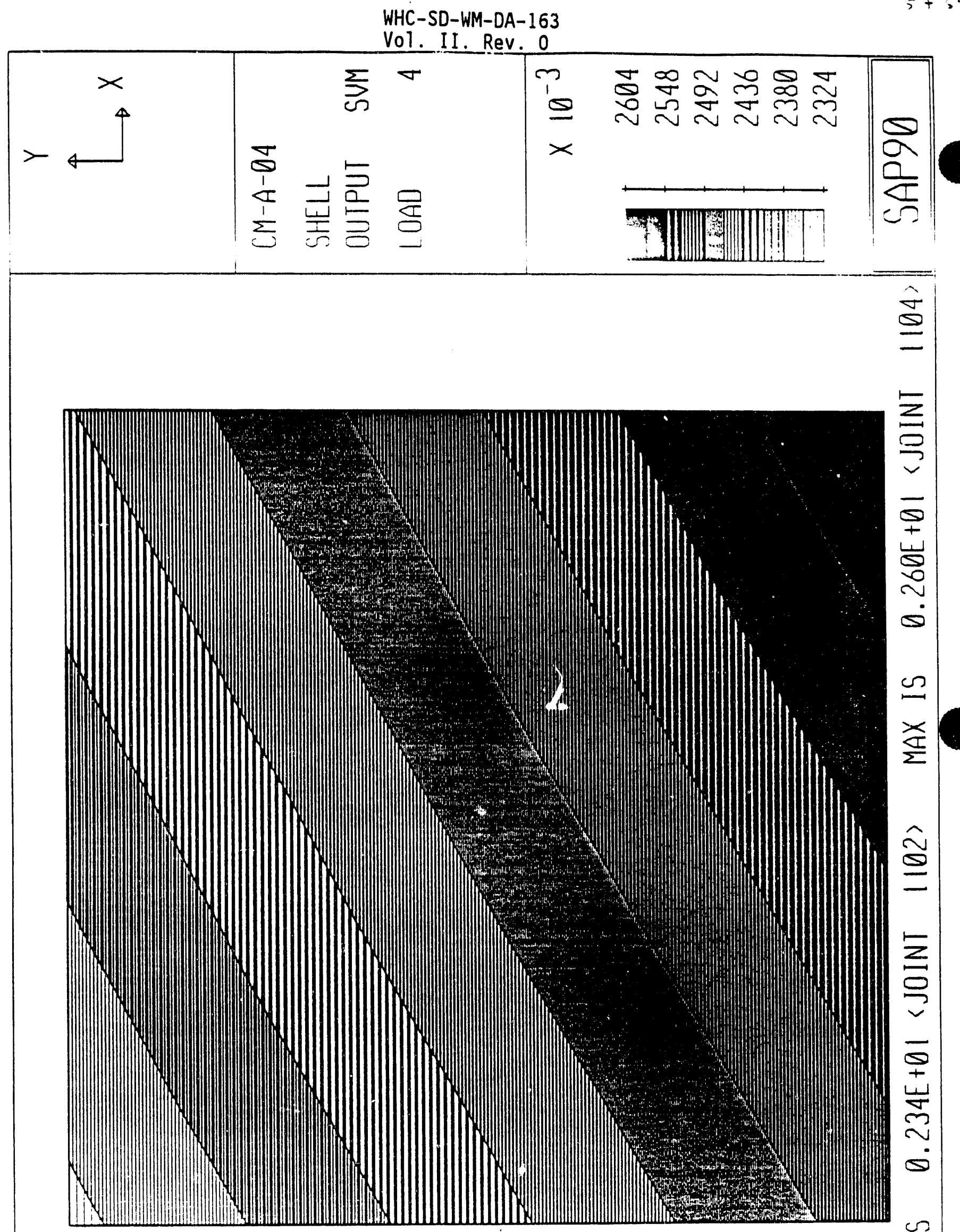

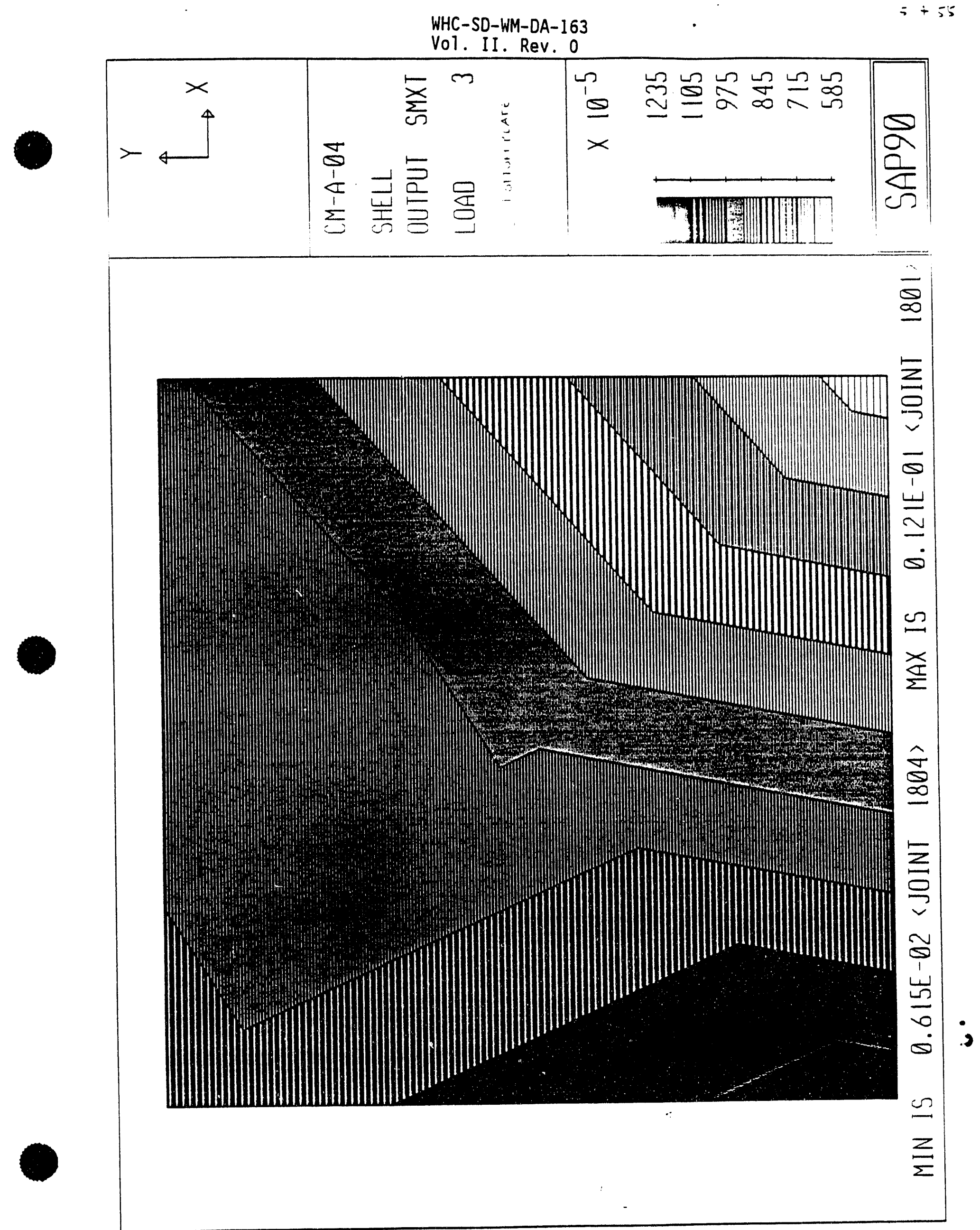

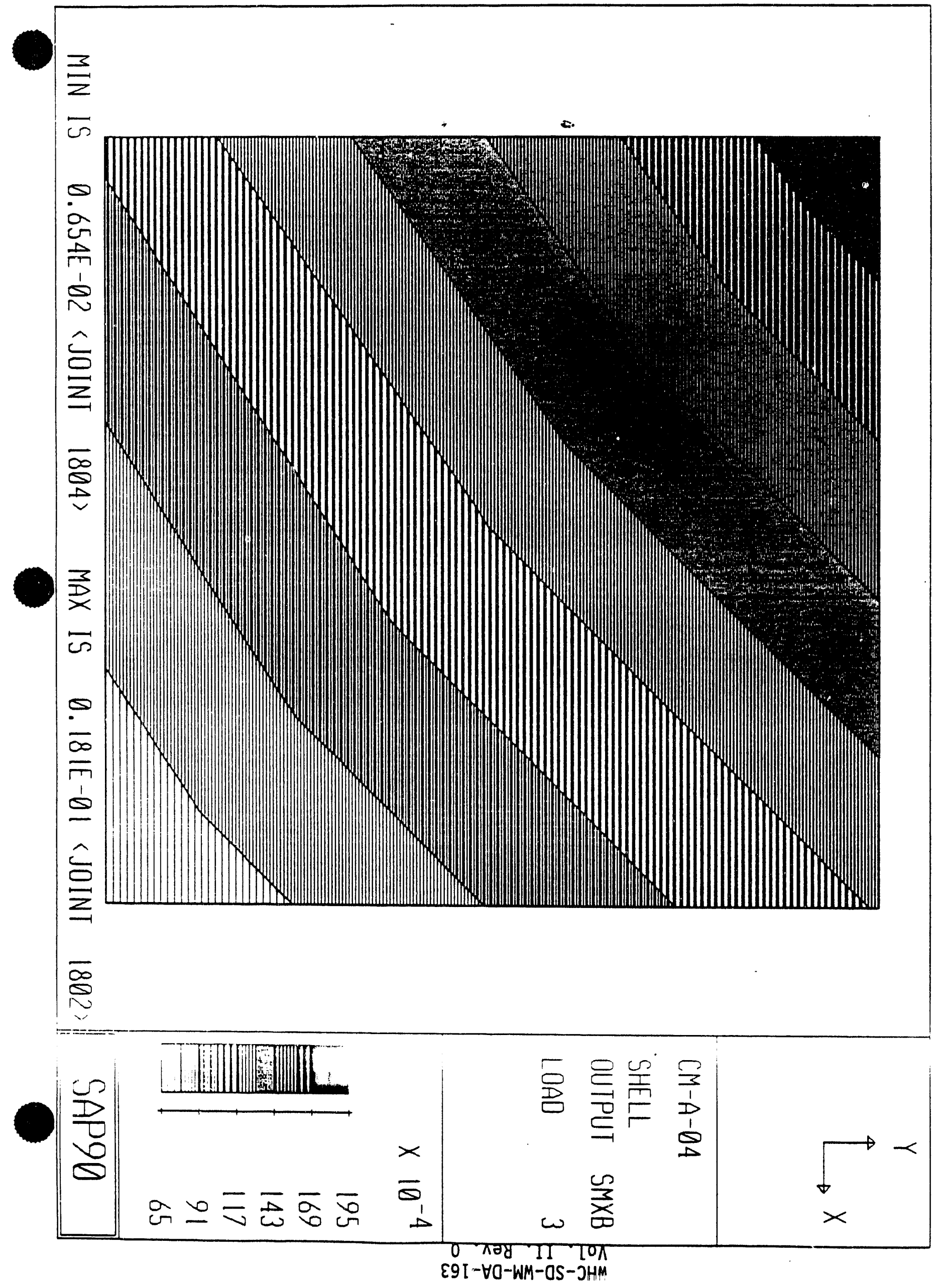

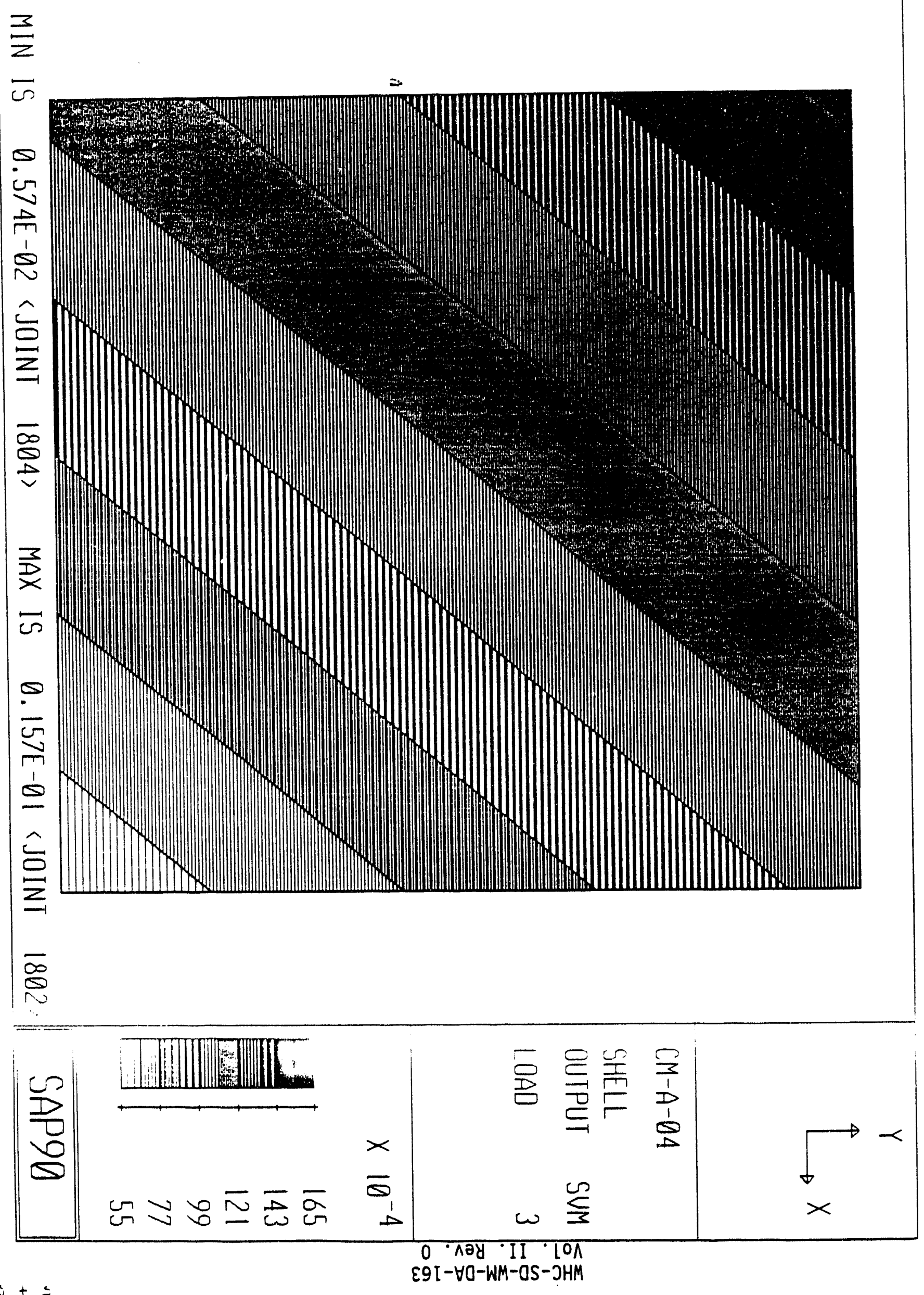


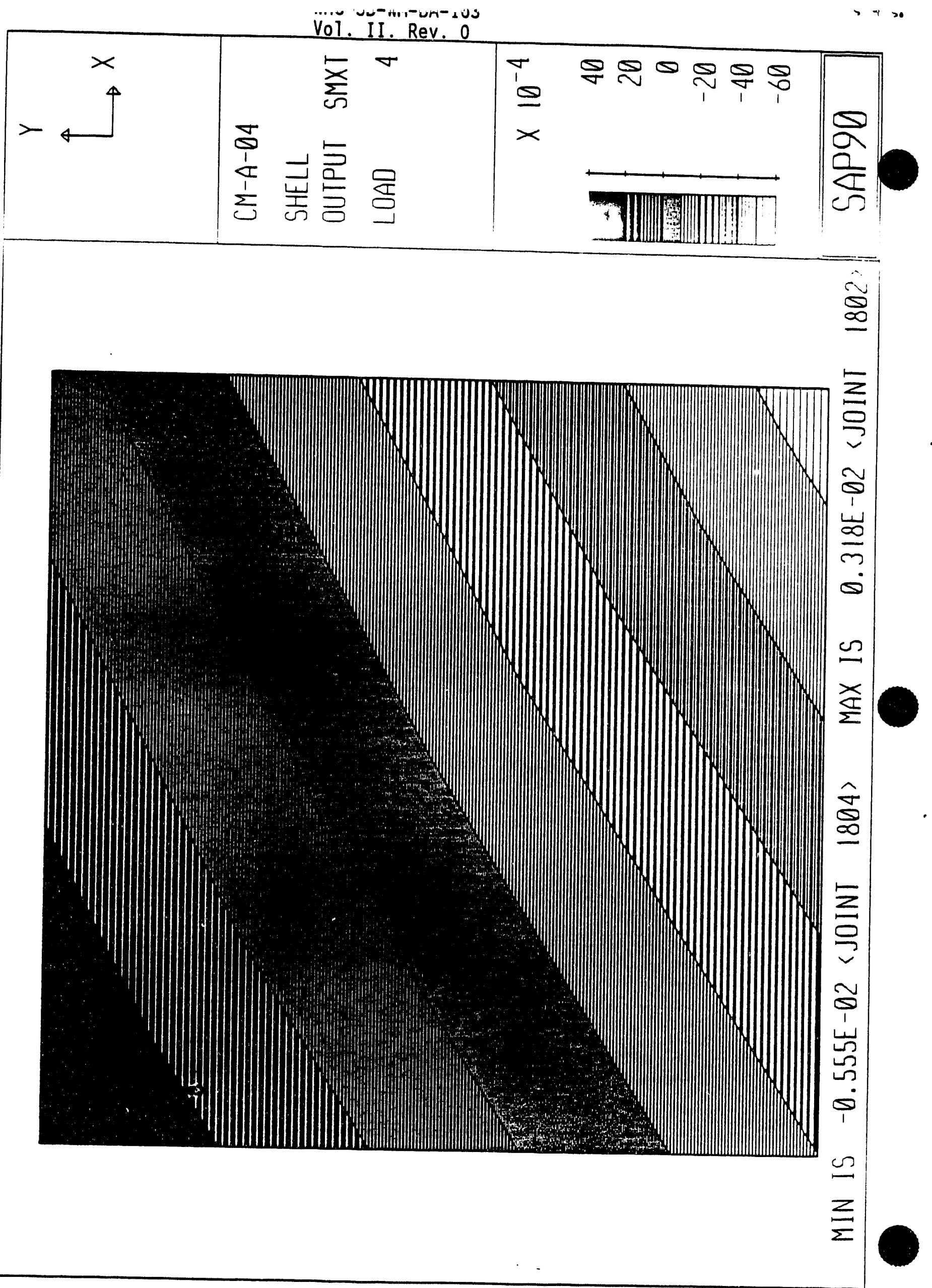




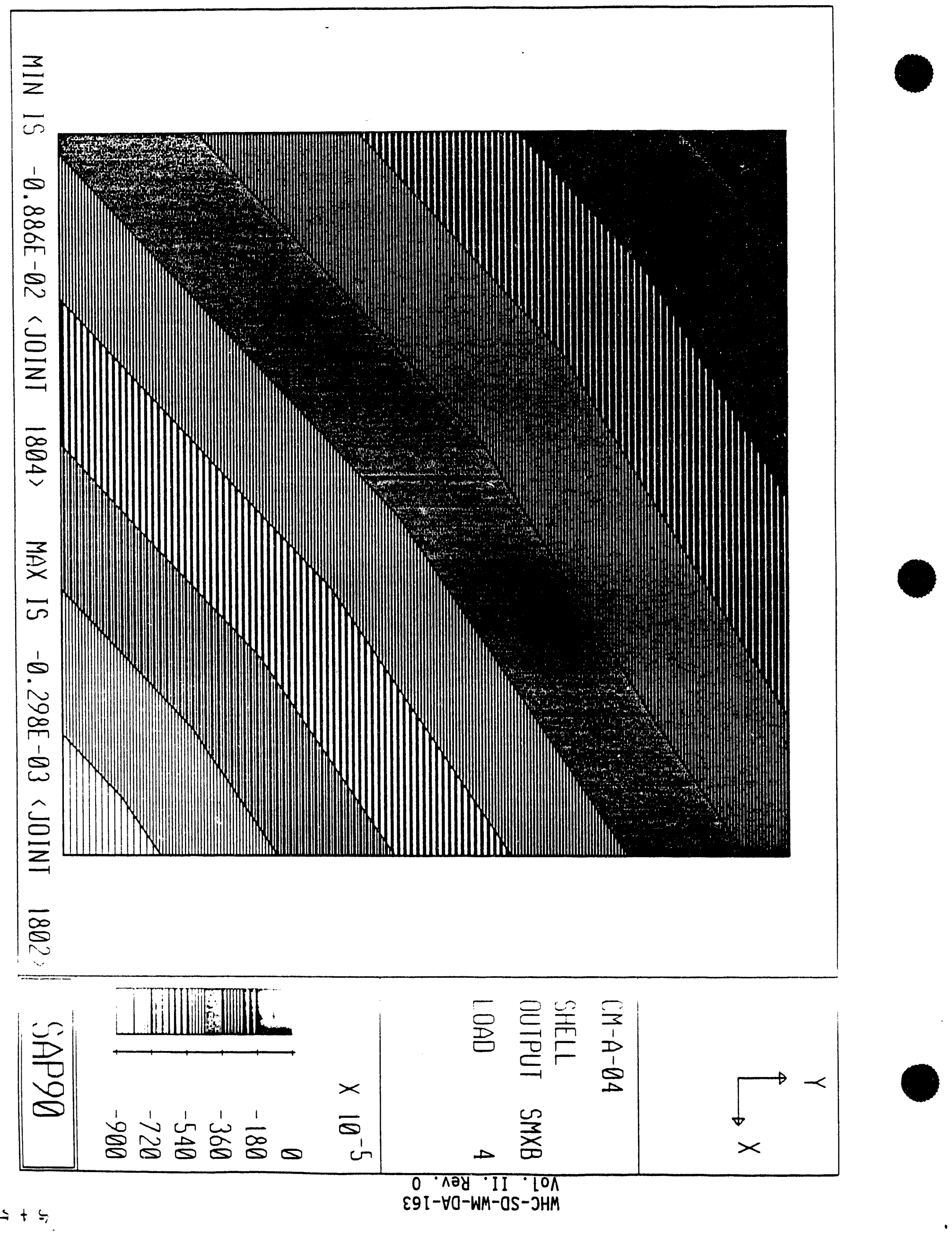



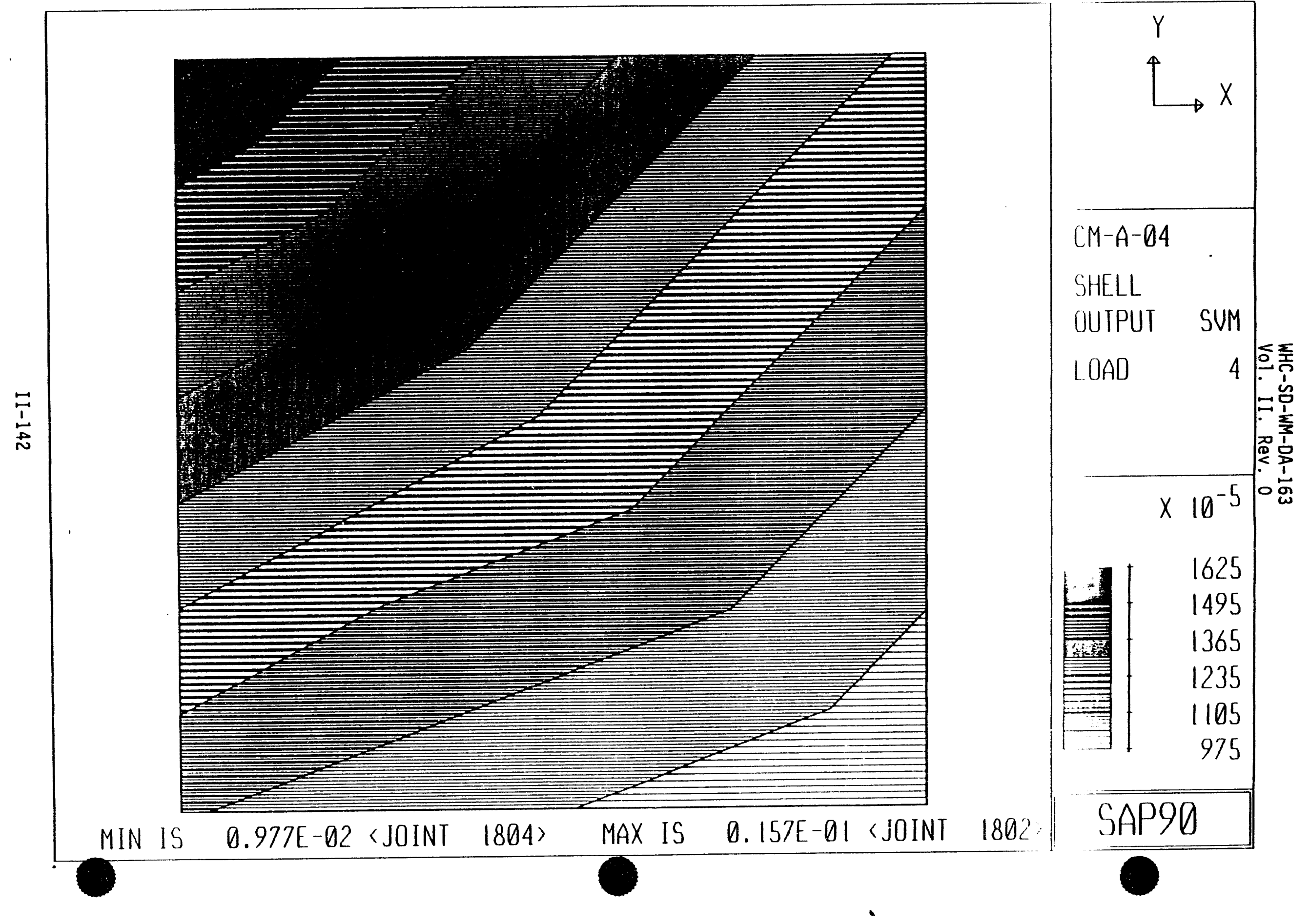
WHC-SD-WM-DA-163
URS CONSULTANTS II. Rev. 0

Job \#:66481-01 Job: WHC/MPF CAMERA ASSEMBLY ANALYSIS

Client: WHC Subject: SAP9O ANALYSIS DOCUMENTATION
Sheet No. 6.0

By Mu Date b/r/a4

Chk'd_/,.. Date $7 /: ? / 1$,

\section{CHECK ELEMENT STRESSES}

TABLE OF CONTENTS

SEECTION CONTENTS

6.1

General Discussion

6.2

6.3
Check Shell Element Stresses

Check Beam Element Stresses
PAGE NO.

61.1

$6.2 .1-6.27$

$6.3 .1-6.3 .2$ 
Job \#:66481-01 Job: WHC/MPF CAMERA ASSEMBLY ANALYSIS

Sheet No. $\frac{1}{0.11}$

Client: WHC Subject: SAP9O ANALYSIS DOCUMENTATION

By $P x \in$ Date $7 / 2 s / 14$

Chk'd RiKDate 8, is:

\subsection{General Discussion}

SAP9O Model A (SAP9O Run ID CM-A-04) resules are evailiazed because the element stresses are higher when the flange plate is fixed at the center of plate than when the flange plate is free to displace (Model B).

Using the given material from the drawings (Attachment $A$ ) and the allowable stresses (Attachment $C$ ) were computed. The maximum element stresses were compared to the allowable stresses and the stress ratios were computed for the shell elements. For the beam elements, the stress ratios from SAPSTL were obtained and analyzed.

The checking of the bolts and welding connections are given in Section 7 . 
URS CONSULTANTS WHC-SD-WM-DA-163

Vol. II. Rev. 0

Sheet No. 6.21

Job \#:66481-01 Job: WHC/MPF CAMERA ASSEMBLY ANALYSIS

By Pru Date $=18 / 94$

Client: WHC Subject: SAP9O ANALYSIS DOCUMENTATION

Chk'd + Date ${ }_{1, \ldots},+$

6.2 Check Shell Element Stresses

Hand calculations of the following are given in this section:

- 3" thick Shielding Plate

- 2" thick Manifold Tube

- 3/4" thick Flange Plate

- 1/2" thick Mating \& Bottom Plates 
URS Consultants

WHC-SD-WM-DA-163

URS/John A. Blame \& Associates, Engineers

Job no. $66458-01$ Job WHC/MOF ANALYSIS

Client

Subject $\frac{\text { SHIELDING PLATE }}{\text { PART NO. }+2.2)}$
Sheet No. $\quad 6.22$

Cali. No.

Rev. No.

By PK u Date $\frac{5,31 / 34}{2 / 3 / 1}$

Chk'd Elm Date $2 / \% / 1$

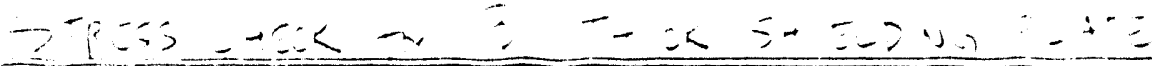

WAT: CARBON STEEL

$f_{y}=36 \mathrm{ksi} \quad f_{u}=58 \mathrm{ku}$

ALLOWABLE STRESSES:

$$
\begin{aligned}
\text { BENDING } & =1.6\left(0.6 \mathrm{fy}_{y}\right) \\
& =34.56 \mathrm{kai}<0.7 \mathrm{f}_{4}=40.6 \mathrm{~km} \text { ok } \\
\text { SHEAR } & =1.4\left(0.4 \mathrm{fy}_{y}\right) \\
& =20.16 \mathrm{kii}
\end{aligned}
$$

where 1.6 = stress Limit Coff. jor extreme case (See ANSI/AisC N690-1994, TABLE Q 1 5.7.1)

SAtP90 RESULTS

From sAPLOT of ELEMENT STRESSES, MAX. STRESSES ARE:

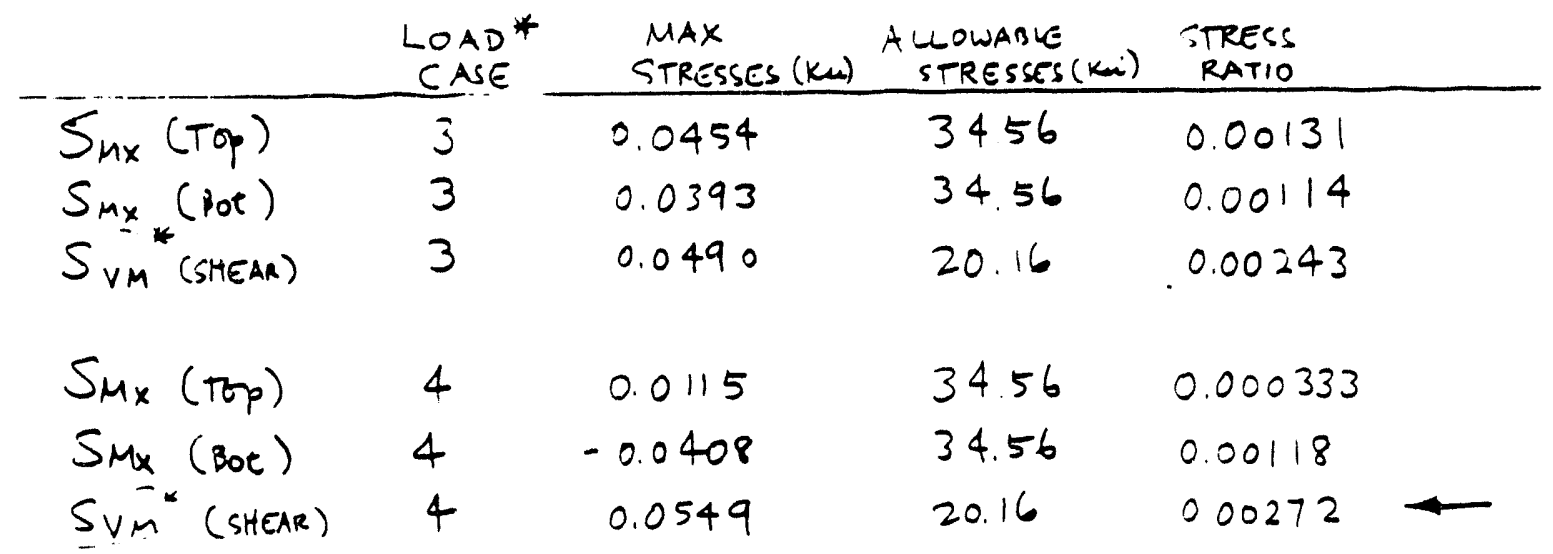

Land case $3 \cdot D L+E Q$

L. $D L-E Q$

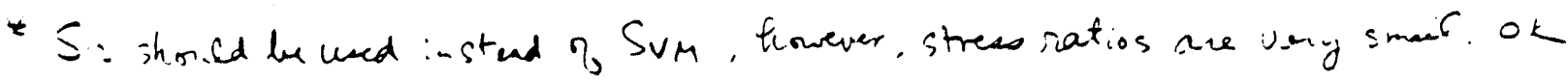
II -146 


\section{URS Consultants WHC-SD-WM-DA-163 URS/John A. Blume \& Associates, Engineers} job no. $66458-01$ job WHC/MPF ANALYSIS

Client Subject $\frac{\text { MANIFOLD TUBE }}{\text { (PART NO. 42.5) }}$

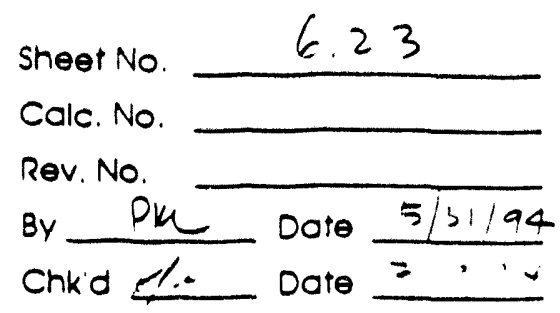

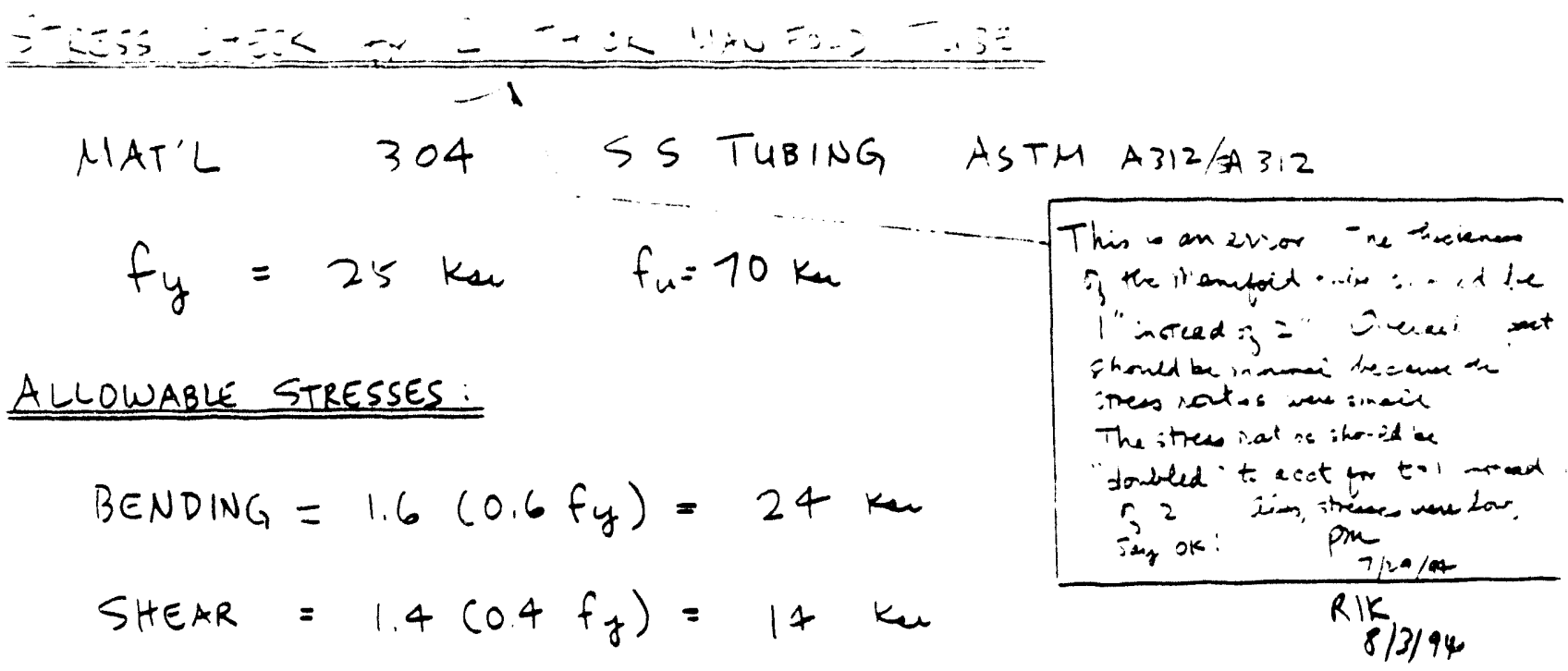

\section{SAP 90 RESULTS:}

From saplot of ELEMENT STRESSES, MAX. ARE

\begin{tabular}{|c|c|c|c|c|c|}
\hline & $\begin{array}{l}\text { LOAD } \\
\text { CASE }\end{array}$ & $\begin{array}{l}\text { MAX } \\
\text { STRESSES (Kai) }\end{array}$ & $\begin{array}{c}\text { AUDWABLE } \\
\text { STRESSES } \\
(K L)\end{array}$ & $\begin{array}{l}\text { STRESS } \\
\text { RATIO }\end{array}$ & \\
\hline$S_{M x}($ top) & 3 & 0.22 & 24 & 200917 & \\
\hline $\operatorname{Smx}(B \circ t)$ & 3 & 0.493 & 24 & 0.2054 & \\
\hline$S V_{M}^{*}(S H E \lambda R)$ & 3 & $\frac{0.445}{2.45 x}$ & $1+$ & 0.03178 & $\begin{array}{l}\text { o } 03173 \times 2 \\
=00636\end{array}$ \\
\hline$S_{M x}(t o p)$ & 4 & -0.0504 & 27 & 0.2015 & \\
\hline $\operatorname{Smx}(B \circ t)$ & 4 & -0.0927 & 27 & 000276 & \\
\hline SVM* (SHEAR) & 4 & 0.346 & 14 & 00.765 & \\
\hline
\end{tabular}

\footnotetext{
5

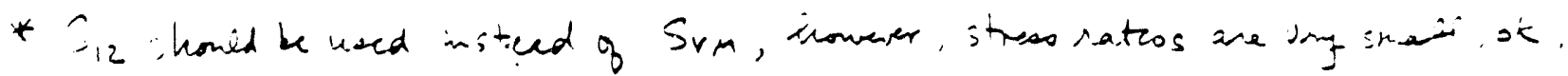


URS Consultants Vol. II. Rev. 0 URS/John A. Blame \& Associates, Engineers Joono. $\$ 6458.01$ Job WHC/MPF ANALYSIS Client

Subject $\frac{\text { FLANGE PLATE }}{\text { (PAR TAO 42-T) }}$

Sheet No.

$6.2 \div$

Call. No.

Rev. No.

By PKL Date $5 / 31 / 94$

Coked KP, Date $2 /, 3, \cdot y$

Pis pix

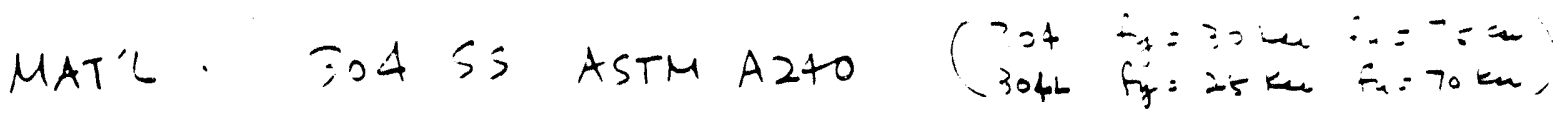
$f_{y}=25 \mathrm{ku} \quad f_{u}=70 \mathrm{ku}$

ALLOWABLE STRESSES

$$
\begin{aligned}
\text { BENDING } & =1.6\left(0.6 f_{y}\right) \\
& =24 \mathrm{ksi}<0.7 f_{u}=49 \mathrm{ksi}=K \\
\text { SHEAR } & =1.4\left(0.4 \mathrm{f}_{y}\right) \\
& =14 \mathrm{ksi}
\end{aligned}
$$

SAP OO RESULTS.

From satplot of element stresses, max. are

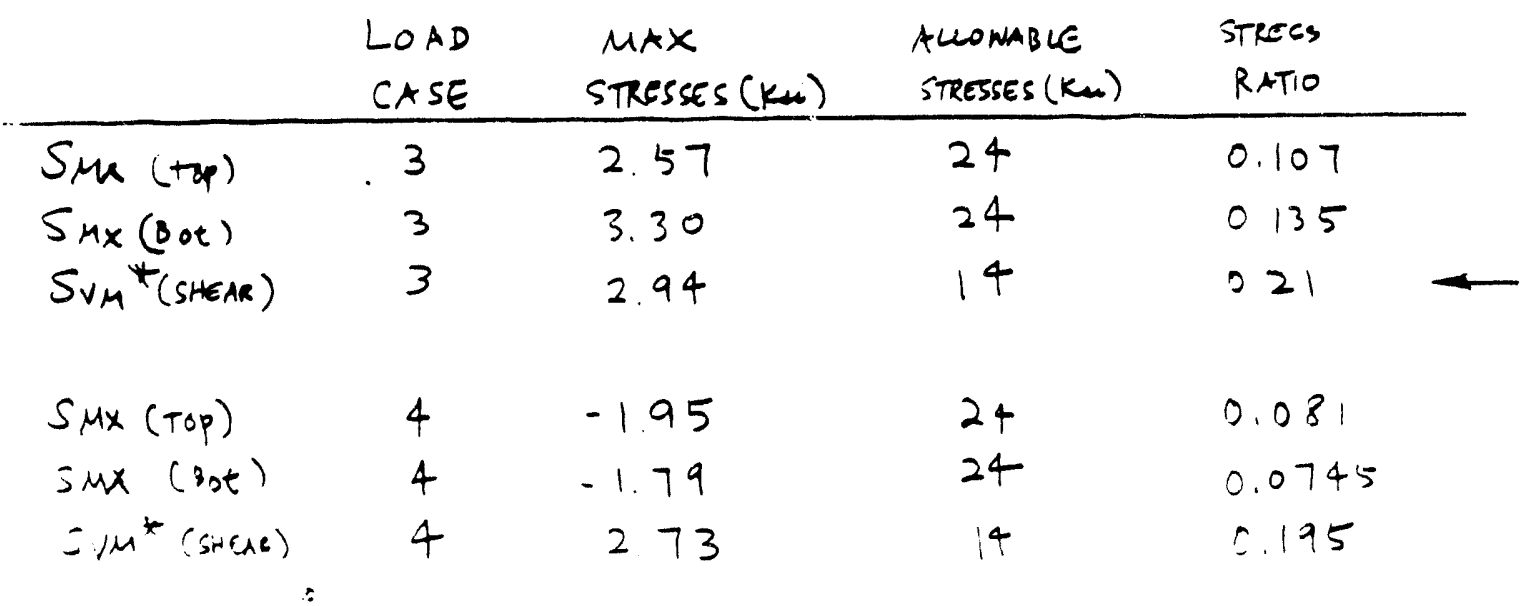

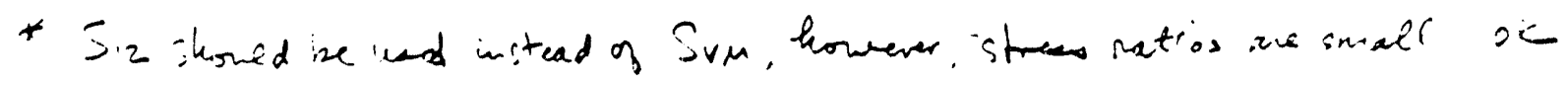
II -148 
$\rightarrow 01 \gg 9020.0=\frac{\nabla 1}{b 020}$ : 0114x ssards

$\max \neq 1=(s \tau x+0)+1=\left(h_{j}+0\right)+1$. ss3rds 3า8षM0า7t

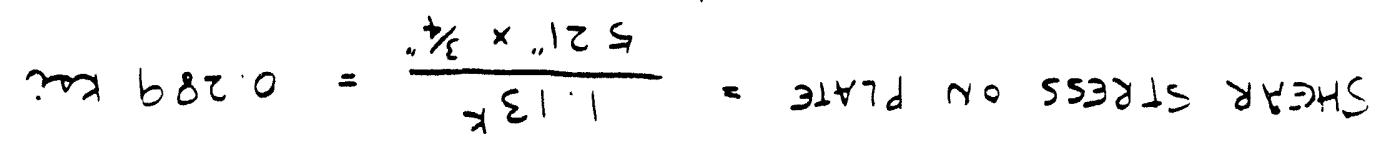

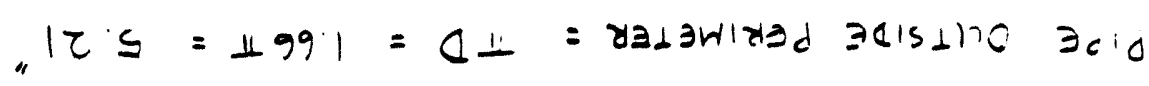
$.991=00$ Dic

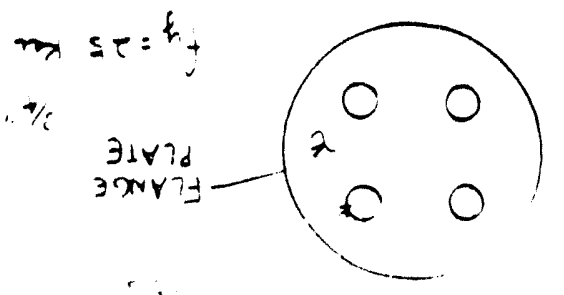

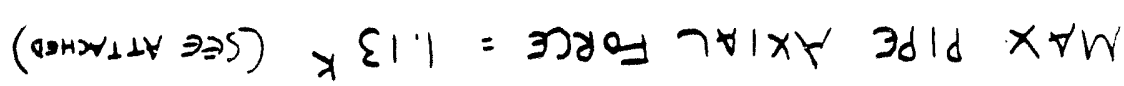

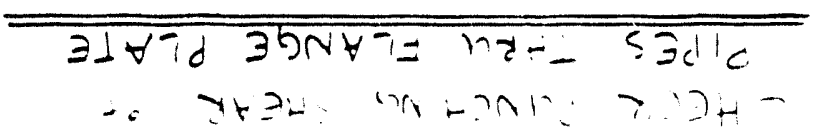

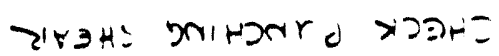

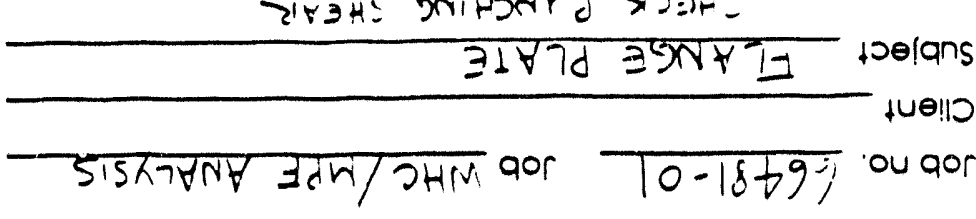
sdeoulbug 'seje!ooss 8 ounig $\nabla$ uyor/syn $0 \cdot \wedge$ - II ' $10 \wedge$ $\varepsilon 9[-\forall O-W M-O S-J H M$ 

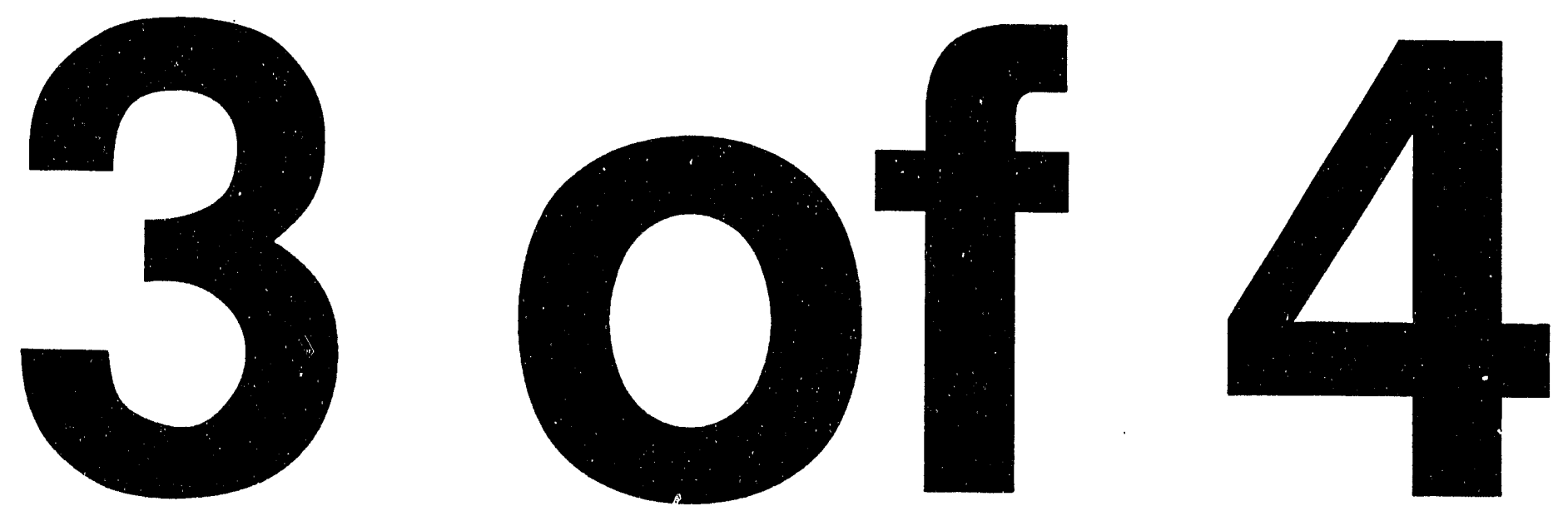


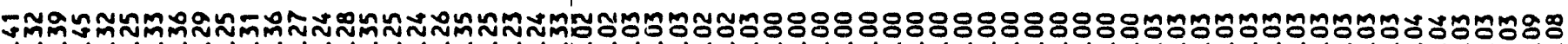
000000000000000000000000000000000000000000000000000000000000000000

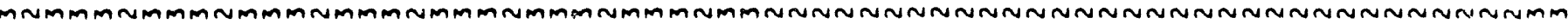

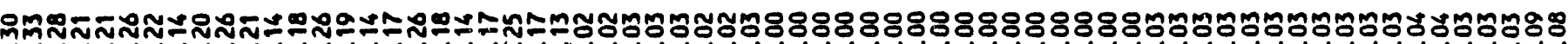

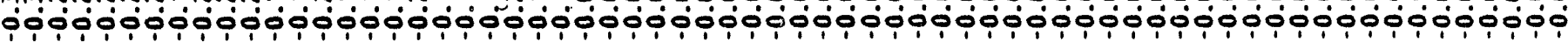

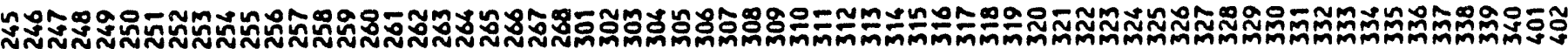

$$
\begin{gathered}
\text { sald } \\
\text { is }
\end{gathered}
$$

\section{$\frac{x}{2}$}

$\checkmark$

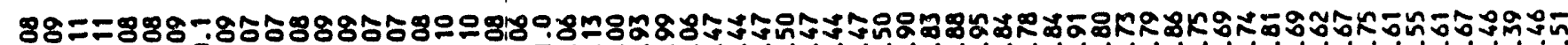

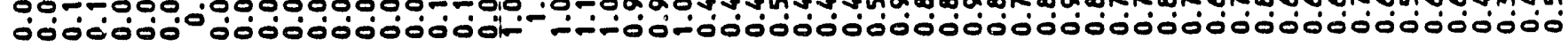

mmmmmmmmmmmmmmmmmmmminmmmmmmmmmmmmmmmmmmmmmmmmmmmmmmmmmmmmmmmm

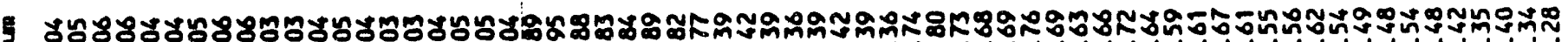

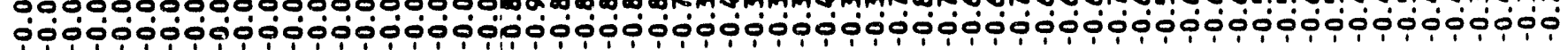

\section{0}

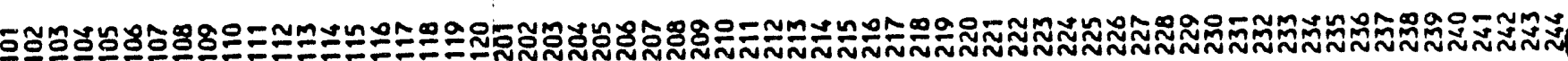

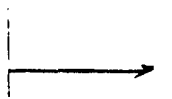


URS Consultants

$$
\text { WHC-SD-WM-DA-163 }
$$$$
\text { Vo 1. II. Rev. } 0
$$

URS/John A. Blame \& Associates, Engineers

Job no. $66.58-01$ Job WHC/MPF ANALYsis

Client

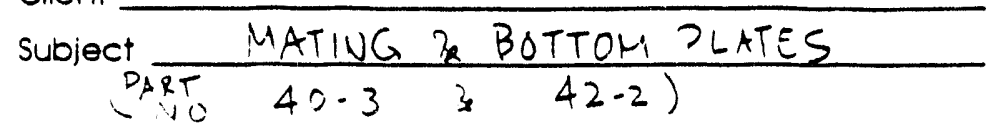

Sheet No.

$\leq 27$

Talc. No.

Rev. No.

By Pu e Date $5 / 31 / 94$

Chad $1 / f$ r Date $7 / 1: 1: 1$

$$
\begin{aligned}
& \text { MAT'L : } 300 \text { SERIES SS } \\
& f_{y}=25 \mathrm{ksi} \quad f_{u}=70 \mathrm{ksi}
\end{aligned}
$$

ALLOWABLE STRESSES:

$$
\begin{aligned}
\text { BENDING } & =1.6\left(0.6 \mathrm{fy}_{y}\right) \\
& =24 \mathrm{ksi}<0.7 \mathrm{f}_{4}=49 \mathrm{~km} \text { oK } \\
\text { SHEAR } & =1.4(0.4 \mathrm{fy}) \\
& =14 \mathrm{kn}<0.3 \mathrm{fu}=21 \mathrm{~km} \%
\end{aligned}
$$

SAP OO RESULTS:

From SAPLOT of ELEMENT STRESSES, MAX ARE:

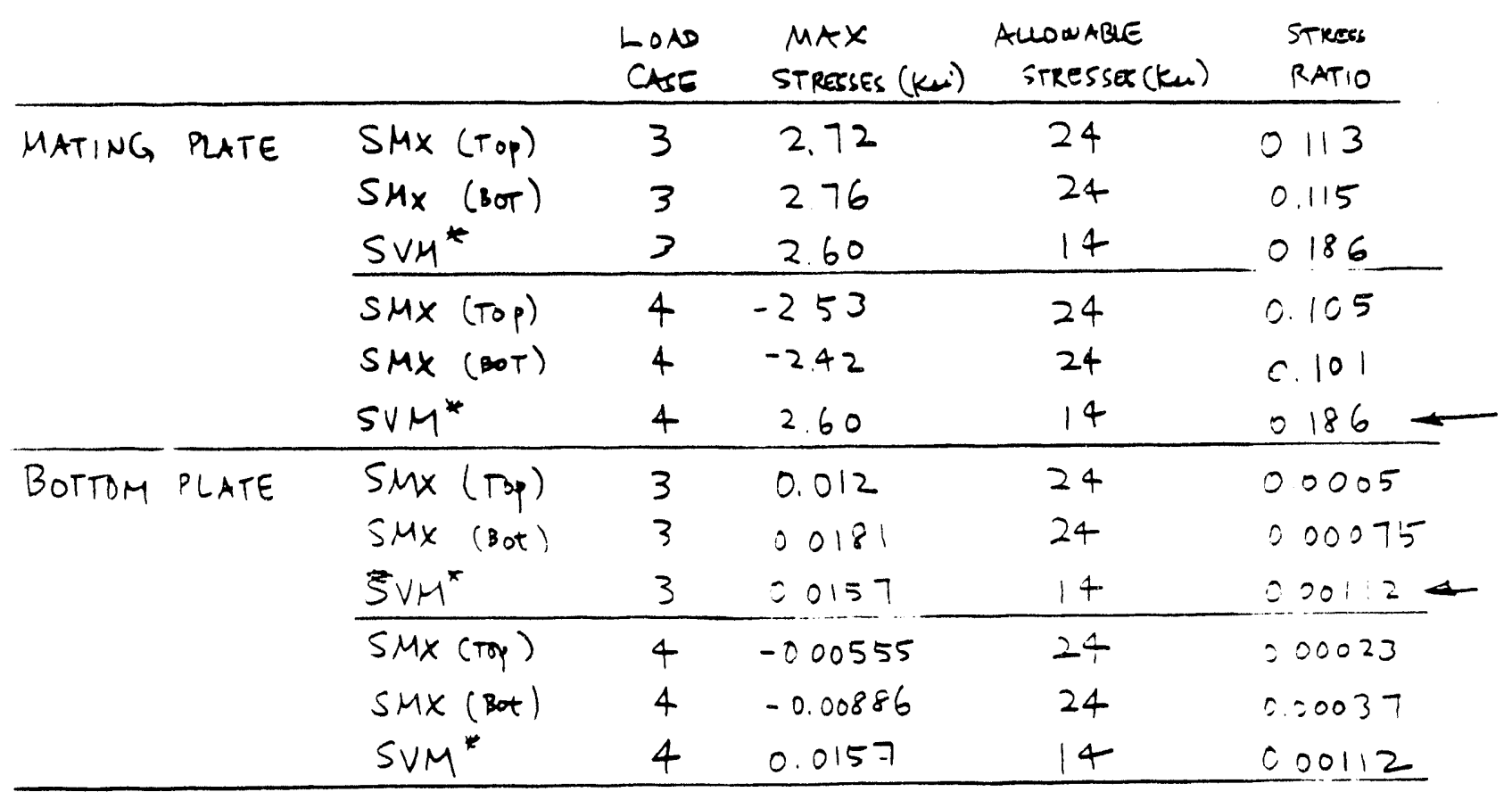
* $S_{12}$ should be wed, however stress ratios we un y small EK 
URS CONSULTANTS WHC-SD-WM-DA-163

Vol. II. Rev. 0

Job \#:66481-01 Job: WHC/MPF CAMERA ASSEMBLY ANALYSIS

Sheet No.

63

Client: WHC Subject: SAP9O ANALYSIS DOCUMENTATION

By Pre Date E/8/94

Chk'd K/m Date $3 / 13 / 6 \%$

6.3 Check Beam Element Stresses

SAPSTL analyzed the following beam elements:

- 1.66"O.D. 1.38"I.D. Stainless Steel Pipes

- 3/16" Braces (Note that 1/4" was actually used because the KI/R for $3 / 16^{\prime \prime}$ exceeds 200 .

- 3/8" Light Support

- 3/8" Gas Support

Summary of the enveloped stress ratios are given in the following page. 
WHC-SD-WM-DA-163

URS Consultants Vol. II. Rev. 0

URS/John A. Blume \& Associates, Engineers

Job no. 6488101 Job WHC/MPF ANALYsis

Client

Subject

Sheet No.

Calc. No.

Rev. No.

By Pue Date $6 / 3 / 94$

Chk'd Lin Date $23+4$

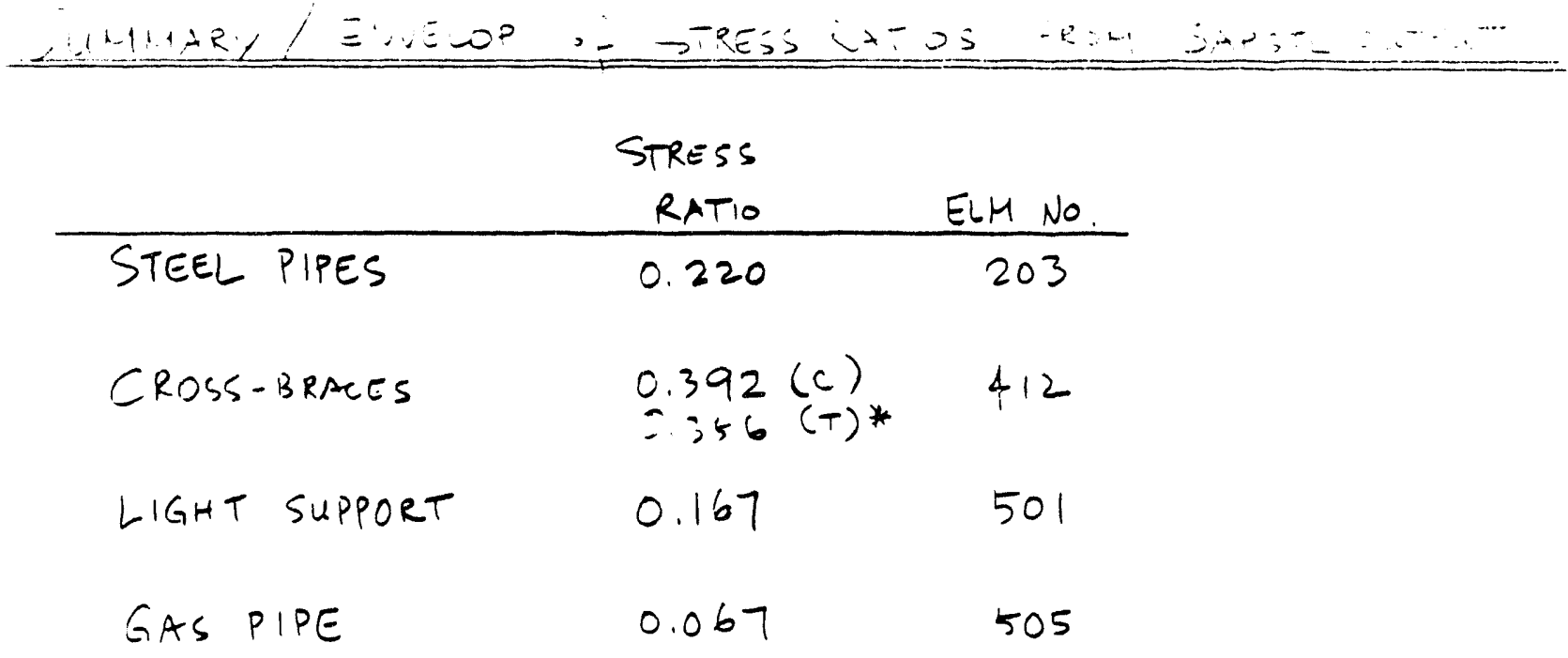

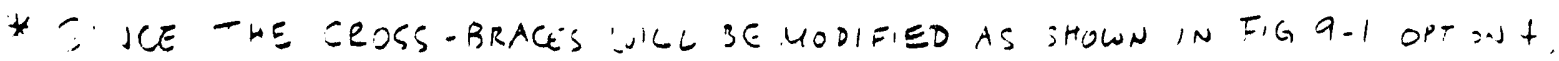

IISE STRCSS RATIO FOK TENSION, 0.356 


\section{URS CONSULTANTS}

Job \#:66481-01 Job: 'WHC/MPF CAMERA ASSEMBLY ANALYSIS

Client: WHC Subject: SAP9O ANALYSIS DOCUMENTATION

WHC-SD-WM-DA-163

Vol. II. Rev. 0
Sheet No. 7.0

By_ple Date'b/r/a4

Chk'd Kf- Date $=3 / 04$

\section{CHECK BOLT AND WELD CONNECTIONS}

\section{TABLE OF CONTENTS}

$\begin{array}{lll}\text { SECTION } & \text { CONTENTS } & \text { PAGE NO. } \\ 7.1 & \text { General Discussion } & 7.1 .1 \\ 7.2 & \text { Check Bolt Connections } & 7.2 .1-7.28 \\ 7.3 & \text { Check Weld Connections } & 7.3 .1-7.3 .18\end{array}$

Cheng 
$\begin{array}{ll}\text { URS CONSULTANTS } & \text { WHC-SD-WM-DA-163 } \\ \text { Vol. II, ReV. } 0\end{array}$

Job \#:66481-01 Job: WHC/MPF CAMERA ASSEMBLY ANALYSIS

Sheet No. 7.11

Client: WHC Subject: SAP90 ANALYSIS DOCUMENTATION

By Pre Date 7/:8/a4

Chk'd R!KDate $8^{\prime}: ?-$

7.1 General Discussion

The following bolts are checked:

Connecting $3 "$ Shielding Plate and 1" Manifold Tube

Bolts :

$1 / 2-13 \times 5 " 18-8$ sS

Minimum tensile strength $=70 \mathrm{ksi}$

Loads :

Lift Load (904 lbs) (------ Control

Dead load plus or minus response spectra

Connecting 3/4" Flange Plate and 20" Riser

Bolts :

1 1/8-7 UNC-2A 6" long A307 GR B

Allowable tensile stress $=20 \mathrm{ksi}$

Allowable single shear stress $=10 \mathrm{ksi}$

Loads :

60 psi Hydrogen burn pressure <----- Control Dead load plus or minus response spectra

The allowable stresses from Attachment $C$ for the bolts were compared with the bolt stresses. Stress ratios were computed and evaluated.

The following weld connections were checked:

Fillet
$3 / 8^{\prime \prime}$
$3 / 16^{\prime \prime}$
$3 / 16^{\prime \prime}$
$3 / 16^{\prime \prime}$
$3 / 16^{\prime \prime}$
$1 / 8^{\prime \prime}$
$1 / 8^{\prime \prime}$

Part/Part No.
Flange Plate $(42-7)$
Flange Plate $(42-7)$
Mating Plate $(40-3)$
Bottom Plate $(42-2)$
Cross Braces $(40-5)$
Light support $(40-4)$
3/8" Gas Pipe $(40-12)$

Part/Part No. Manifold Tube $(42-5)$

SS Pipe $(42-8)$

SS Pipe $(42-8)$

SS Pipe $(42-8)$

SS Pipe $(42-8)$

SS Pipe $(42-8)$

$1 / 8^{\prime \prime}$

SS Pipe $(42-8)$

The allowable stresses from Attachment $C$ for the welds ( $F W$ ) were compared with the weld stresses ( $f w$ ). Stress ratios were computed and evaluated. 
Job \#:66481-01 Job: WHC/MPF CAMERA ASSEMBLY ANALYSIS By Pur Date $1 / 8 / 94$

Client: WHC Subject: SAP9O ANALYSIS DOCUMENTATION

Chk'd K/A Date $7 / Y^{\prime}: 1$

7.2 Check Bolt Connections

Hand calculations for checking bolt connections are provided in this section. 
URS Consultants WHC-SD-WM-DA-163

URS/John A. Blame \& Associates, Engineers

Job no. 66453.01 Job WHC/ MPF ANALYSIS

Client

SUbject CHECK BOLTS
Sheet No.

72.2

Coals. No.

Rev. No.

By PK ᄃ Date $\frac{016 / 94}{9 / 12 / 14}$

Chi V/. Date $7 / / 3 / 14$

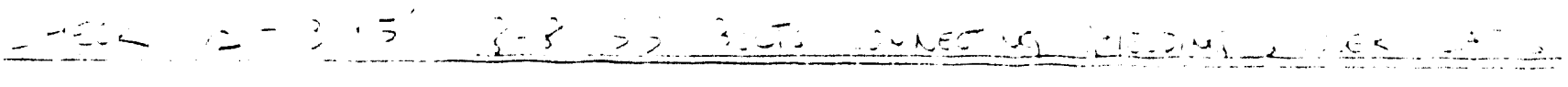

$. M A T^{\prime} L \cdot 18-8$ SS

$F_{+}=70 \mathrm{~km} \quad$ area $f \operatorname{bot}\left(\%_{2}^{\prime \prime} p\right)=0.196 \mathrm{~m}{ }^{2}$

ALLOWABLE STRESSES

$$
\begin{aligned}
F_{t} & =16\left(0.3 \times f_{t}\right) \\
& =33.6 \mathrm{ker}
\end{aligned}
$$

-AFT LOAD

$$
\begin{aligned}
& \text {-jat- WT of CAMERA ASSEMBLY }=1227 \mathrm{k} \\
& \text { SHEELDUG PLATE oNLY }=\frac{-323 \mathrm{ld}}{904 \mathrm{lb}} \\
& \text { tor } 10 \text { locus or } 704 \% \text { coste } \\
& \text { TRANSPDRTATIOU LOAD FACTOR }=2 \quad 2 \times 90.4=180.8 \mathrm{lb} / \text { Bolt } \\
& \text { Best stress }=\frac{01808^{k}}{0196}=0.922 \mathrm{ksi} \\
& \text { Sires rate }=\frac{0.722}{33.6}=0.0275
\end{aligned}
$$

II -157 
WHC-SD-WM-DA-163

URS Consultants Vol. II, Rev. 0 URS/John A. Blume \& Associates, Engineers

Job no. 16658.0 Job WHC/MPF ANALYSIS

Client

Subject

EHECK BOLTS
Sheet No.

$7=3$

Calc. No.

Rev. No.

By $\frac{p u}{P_{\mu}}$ Date $\frac{5 / 31 / 94}{7 / 2}$

$$
\begin{aligned}
& \text { MAT'D A } 307 G R B, \quad D=1 \frac{1}{8}{ }^{\prime \prime} \quad A_{B \alpha T}=0994 \mathrm{~m}^{2} \\
& F_{t}=19^{\mathrm{K}} / \text { BOLT (THREADED ASTENERS) } \\
& F_{V}-9.9</ \text { BOLT }
\end{aligned}
$$

\section{AllOWABLE TENSION \& SHEAR FORCES}

$$
\begin{aligned}
& F_{t}=16(19 \mathrm{~K} / \text { bect })=30.4^{\mathrm{k}} / \text { bolt } \\
& F_{v}=1.4(9.9 \mathrm{~K} / \text { bot })=13.86^{\circ} / \text { bolt }
\end{aligned}
$$

STD TO RESHITS

TENSION

ElMT loAd force allowade

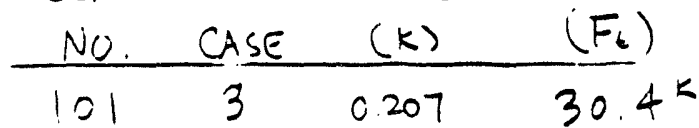

1023

1033

$104 \quad 3 \quad 0.212$

105330.204

$106 \quad 3 \quad 0.195$

$107 \quad 3 \quad 0.196$

$\begin{array}{lll}108 & 3 & 0.202\end{array}$

$1093 \quad 0.205$

11040.200

11140195

$112+0.200$

$112+2.209$

$114 \quad 4 \quad 0215$

$115+0211$

$\begin{array}{lll}116 \quad 1 & 0207\end{array}$

11740211

$118+0220$

$\begin{array}{ll}119 & 4 \\ 120 & 4\end{array}$
0.223 (MAX)

0.216
sTRESS

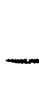

RATIO (K)

0.107

0105

0.100

0.087

0.088

0.091

3.089

0072

0.080

0.089

$\therefore 101$

0108

max)

$2207 \div 9$

3101

0.096

0.099

0.102

0.098

0.00734

0.091

0.088

SHEAR

WOWABLE

RATIO

$13.86^{k}$

-

I $I-158$ 
URS Consultants WHC-SD-WM-DA-163

URS/John A. Glume \& Associates, Vol. II, Rev.

Job no. $.06+58.01$ Job WHC/MPF ANALYSIS

Sheet No. - $2+$

Client

Call. No.

Rev. No.

By Dice Date 6/194

Subject CHECK BOLTS

Chk'd KL Date $71,: 11$

20 Bolts @ Flange plate

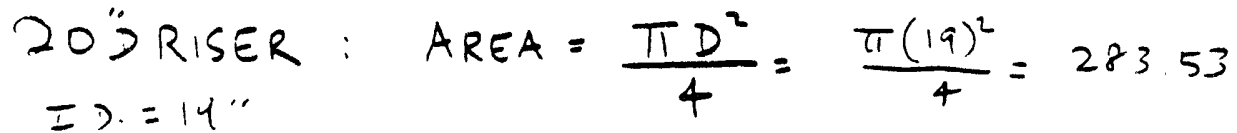

$$
\begin{aligned}
& F=28353(60 \text { pst })=17,011 \quad G=17.011 \mathrm{kms}
\end{aligned}
$$

ForCE PER BOLT $=\frac{17.011}{20}=0851^{\mathrm{k}}$

$$
\begin{aligned}
& F_{t}=3,0.4^{k} / \text { bott } \\
& \frac{D}{C}=\frac{5351^{k}}{30+k / 80 L T}=0028<1 \quad \text { (OK for tyduogen bum ) }
\end{aligned}
$$

II -159 

UHC/MPF CAMERA ASSEMBLY MODEL J08E: 66458-01 RUN: CH-A-03

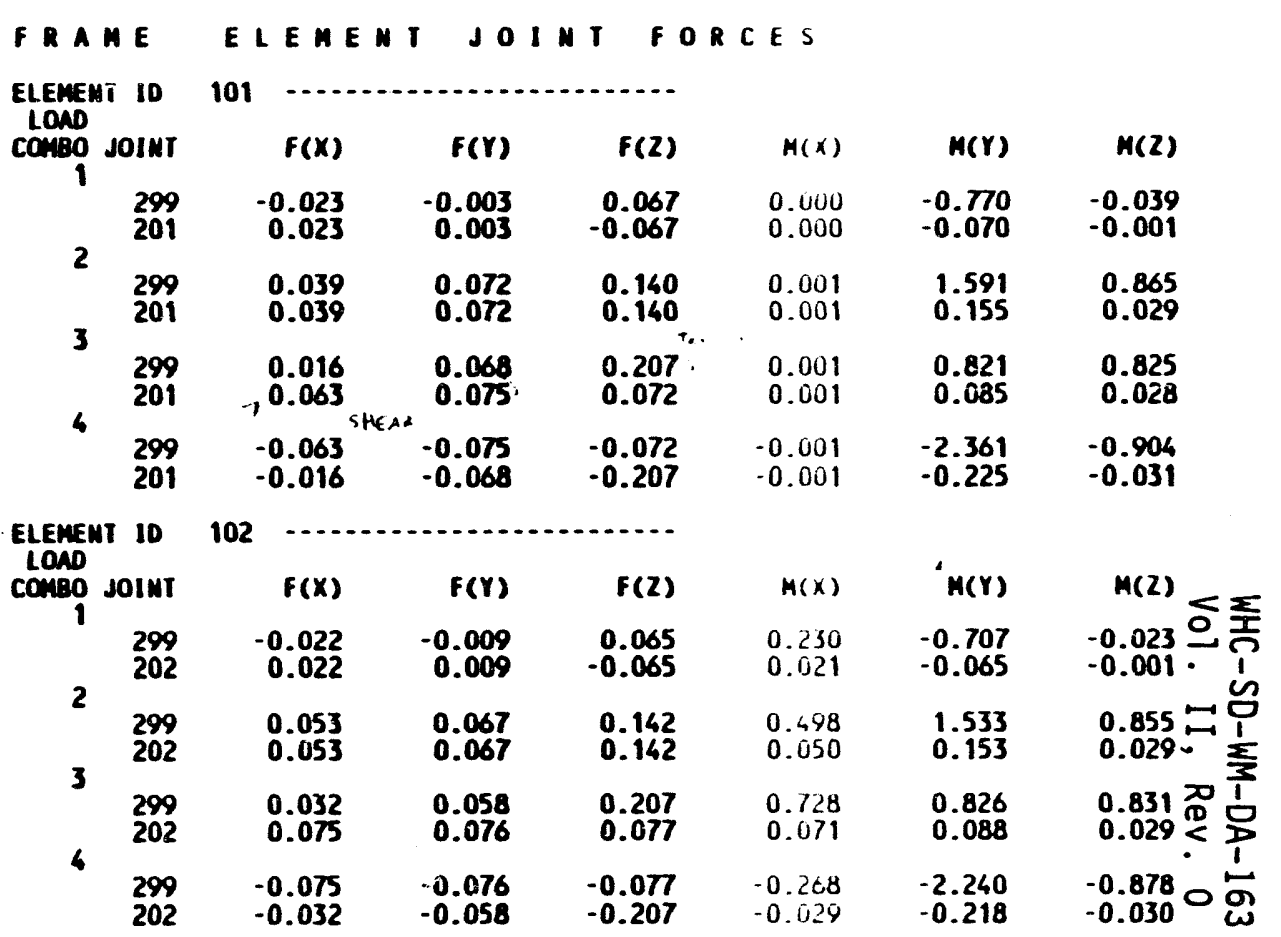

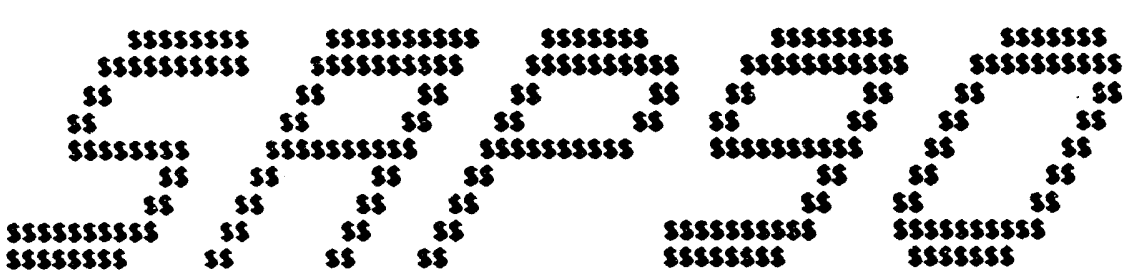

STRUCTURAL AMALYSIS PROGRAMS

Copyright (C) 1978-1992

VERSION P5.40

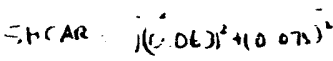
$=0.098^{K}$ All rights reserved

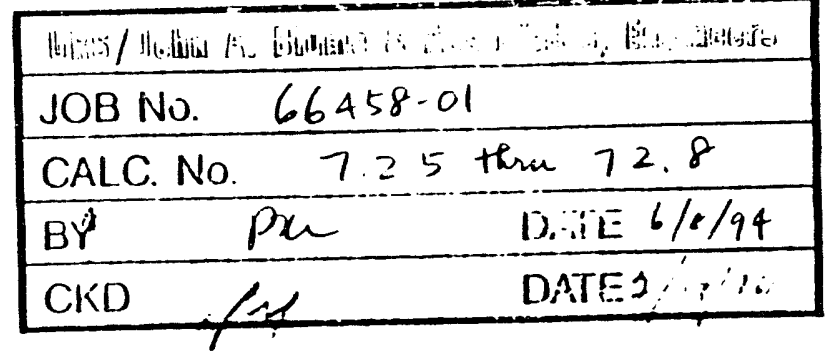

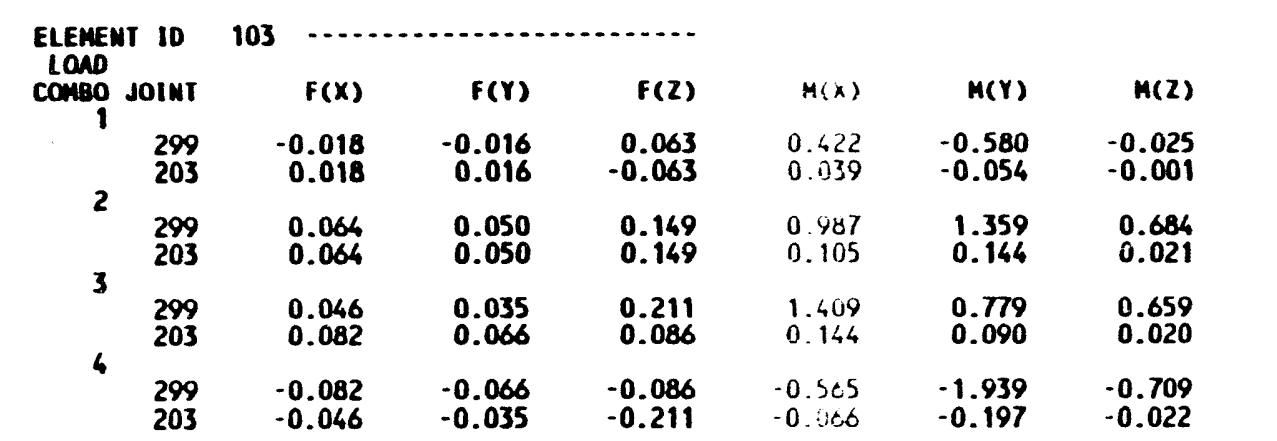


PROGRAM: SAPQO/FILE:CM-A-03.FEF WHC/MP "MEQR ASSEMBLY MODEL JOBW: 66458-01 RUM: CM-A-03

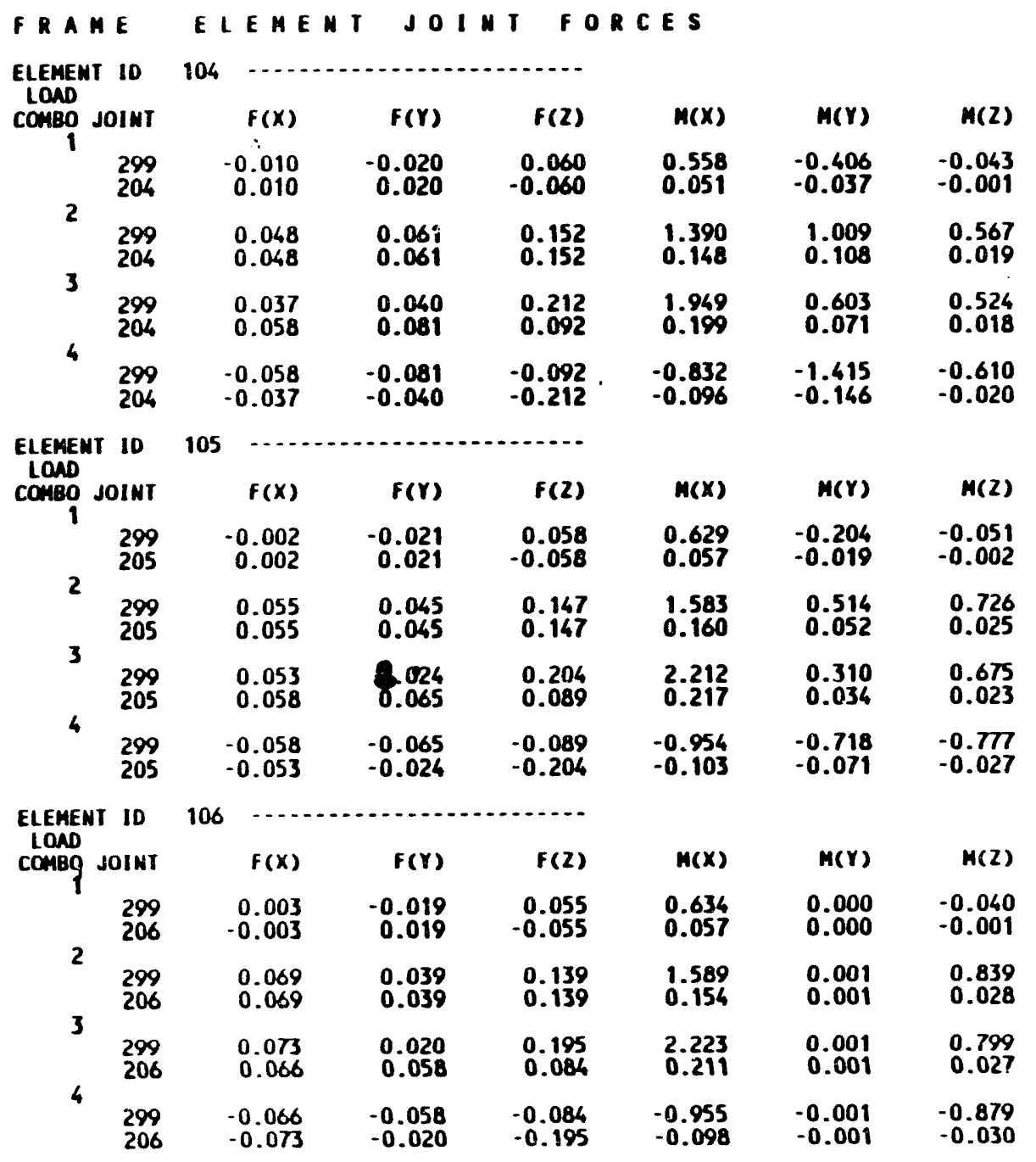

UHCJMPF CAMERA ASSEMBLY MODEL J034: 66458-01 PUOGRAM:SAP90/FILE:CH-A-03.FEF

FRAME ELEMENT JOINT FORCES

\begin{tabular}{|c|c|c|c|c|c|c|c|}
\hline conso & JOINT & $f(x)$ & $f(Y)$ & $f(z)$ & $H(x)$ & $M(Y)$ & $M(z)$ \\
\hline & $\begin{array}{l}299 \\
207\end{array}$ & $\begin{array}{r}0.008 \\
-0.008\end{array}$ & $\begin{array}{r}-0.017 \\
0.017\end{array}$ & $\begin{array}{r}0.053 \\
-0.053\end{array}$ & $\begin{array}{l}0.582 \\
0.052\end{array}$ & $\begin{array}{l}0.189 \\
0.017\end{array}$ & $\begin{array}{l}-0.022 \\
-0.001\end{array}$ \\
\hline & $\begin{array}{l}299 \\
207\end{array}$ & $\begin{array}{l}0.064 \\
0.064\end{array}$ & $\begin{array}{l}0.054 \\
0.054\end{array}$ & $\begin{array}{l}0.142 \\
0.142\end{array}$ & $\begin{array}{l}1.557 \\
0.154\end{array}$ & $\begin{array}{l}0.499 \\
0.050\end{array}$ & $\begin{array}{l}0.830 \\
0.029\end{array}$ \\
\hline & $\begin{array}{l}299 \\
207\end{array}$ & $\begin{array}{l}0.072 \\
0.057\end{array}$ & $\begin{array}{l}0.037 \\
0.071\end{array}$ & $\begin{array}{l}0.196 \\
0.089\end{array}$ & $\begin{array}{l}2.120 \\
0.200\end{array}$ & $\begin{array}{l}0.689 \\
0.067\end{array}$ & $\begin{array}{l}0.808 \\
0.028\end{array}$ \\
\hline & $\begin{array}{l}299 \\
207\end{array}$ & $\begin{array}{l}-0.057 \\
-0.072\end{array}$ & $\begin{array}{l}-0.071 \\
-0.037\end{array}$ & $\begin{array}{l}-0.089 \\
-0.196\end{array}$ & $\begin{array}{l}-0.955 \\
-0.102\end{array}$ & $\begin{array}{l}-0.310 \\
-0.033\end{array}$ & $\begin{array}{l}-0.852 \\
-0.028\end{array}$ \\
\hline
\end{tabular}

\begin{tabular}{|c|c|c|c|c|c|c|c|}
\hline conso & JOIMT & $f(x)$ & $f(Y)$ & $f(z)$ & $M(x)$ & $M(Y)$ & $M(Z)$ \\
\hline & $\begin{array}{l}299 \\
208\end{array}$ & $\begin{array}{r}0.012 \\
-0.012\end{array}$ & $\begin{array}{r}-0.015 \\
0.015\end{array}$ & $\begin{array}{r}0.052 \\
-0.052\end{array}$ & $\begin{array}{l}0.486 \\
0.044\end{array}$ & $\begin{array}{l}0.353 \\
0.032\end{array}$ & $\begin{array}{r}-0.006 \\
0.000\end{array}$ \\
\hline 2 & $\begin{array}{l}299 \\
208\end{array}$ & $\begin{array}{l}0.047 \\
0.047\end{array}$ & $\begin{array}{l}0.066 \\
0.066\end{array}$ & $\begin{array}{l}0.149 \\
0.149\end{array}$ & $\begin{array}{l}1.305 \\
0.145\end{array}$ & $\begin{array}{l}0.991 \\
0.106\end{array}$ & $\begin{array}{l}0.643 \\
0.022\end{array}$ \\
\hline & $\begin{array}{l}299 \\
208\end{array}$ & $\begin{array}{l}0.059 \\
0.036\end{array}$ & $\begin{array}{l}0.051 \\
0.081\end{array}$ & $\begin{array}{l}0.202 \\
0.097\end{array}$ & $\begin{array}{l}1.851 \\
0.188\end{array}$ & $\begin{array}{l}1.344 \\
0.137\end{array}$ & $\begin{array}{l}0.637 \\
0.022\end{array}$ \\
\hline 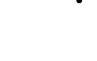 & $\begin{array}{l}299 \\
208\end{array}$ & $\begin{array}{l}-0.036 \\
-0.059\end{array}$ & $\begin{array}{l}-0.081 \\
-0.051\end{array}$ & $\begin{array}{l}-0.097 \\
-0.202\end{array}$ & $\begin{array}{l}-0.878 \\
-0.101\end{array}$ & $\begin{array}{l}-0.638 \\
-0.074\end{array}$ & $\begin{array}{l}-0.650 \\
-0.022\end{array}$ \\
\hline
\end{tabular}

\begin{tabular}{|c|c|c|c|c|c|c|c|}
\hline $\begin{array}{l}\text { lond } \\
\text { conso }\end{array}$ & JOINT & $f(x)$ & $f(r)$ & $F(z)$ & $M(x)$ & $M(Y)$ & $M(Z)$ \\
\hline & $\begin{array}{l}299 \\
209\end{array}$ & $\begin{array}{r}0.015 \\
-0.015\end{array}$ & $\begin{array}{r}-0.011 \\
0.011\end{array}$ & $\begin{array}{r}0.052 \\
-0.052\end{array}$ & $\begin{array}{l}0.353 \\
0.032\end{array}$ & $\begin{array}{l}0.486 \\
0.044\end{array}$ & $\begin{array}{l}0.006 \\
0.000\end{array}$ \\
\hline 2 & $\begin{array}{l}299 \\
209\end{array}$ & $\begin{array}{l}0.061 \\
0.061\end{array}$ & $\begin{array}{l}0.045 \\
0.045\end{array}$ & $\begin{array}{l}0.153 \\
0.153\end{array}$ & $\begin{array}{l}1.015 \\
0.109\end{array}$ & $\begin{array}{l}1.396 \\
0.148\end{array}$ & $\begin{array}{l}0.547 \\
0.020\end{array}$ \\
\hline 3 & $\begin{array}{l}299 \\
209\end{array}$ & $\begin{array}{l}0.076 \\
0.046\end{array}$ & $\begin{array}{l}0.033 \\
0.056\end{array}$ & $\begin{array}{l}0.205 \\
0.100\end{array}$ & $\begin{array}{l}1.50 \% \\
0.160\end{array}$ & $\begin{array}{l}1.883 \\
0.192\end{array}$ & $\begin{array}{l}0.553 \\
0.020\end{array}$ \\
\hline 4 & $\begin{array}{l}299 \\
209\end{array}$ & $\begin{array}{l}-0.046 \\
-0.076\end{array}$ & $\begin{array}{l}-0.056 \\
-0.033\end{array}$ & $\begin{array}{l}-0.100 \\
-0.205\end{array}$ & $\begin{array}{l}-0.600 \\
-0.077\end{array}$ & $\begin{array}{l}-0.910 \\
-0.105\end{array}$ & $\begin{array}{l}-0.541 \\
-0.019\end{array}$ \\
\hline
\end{tabular}


HHCIMPF CAMERA ASSEMBLY MODEL JOB: $66458-01$ PROGRAM:SAP90/FILE:CH-A-03.FEF

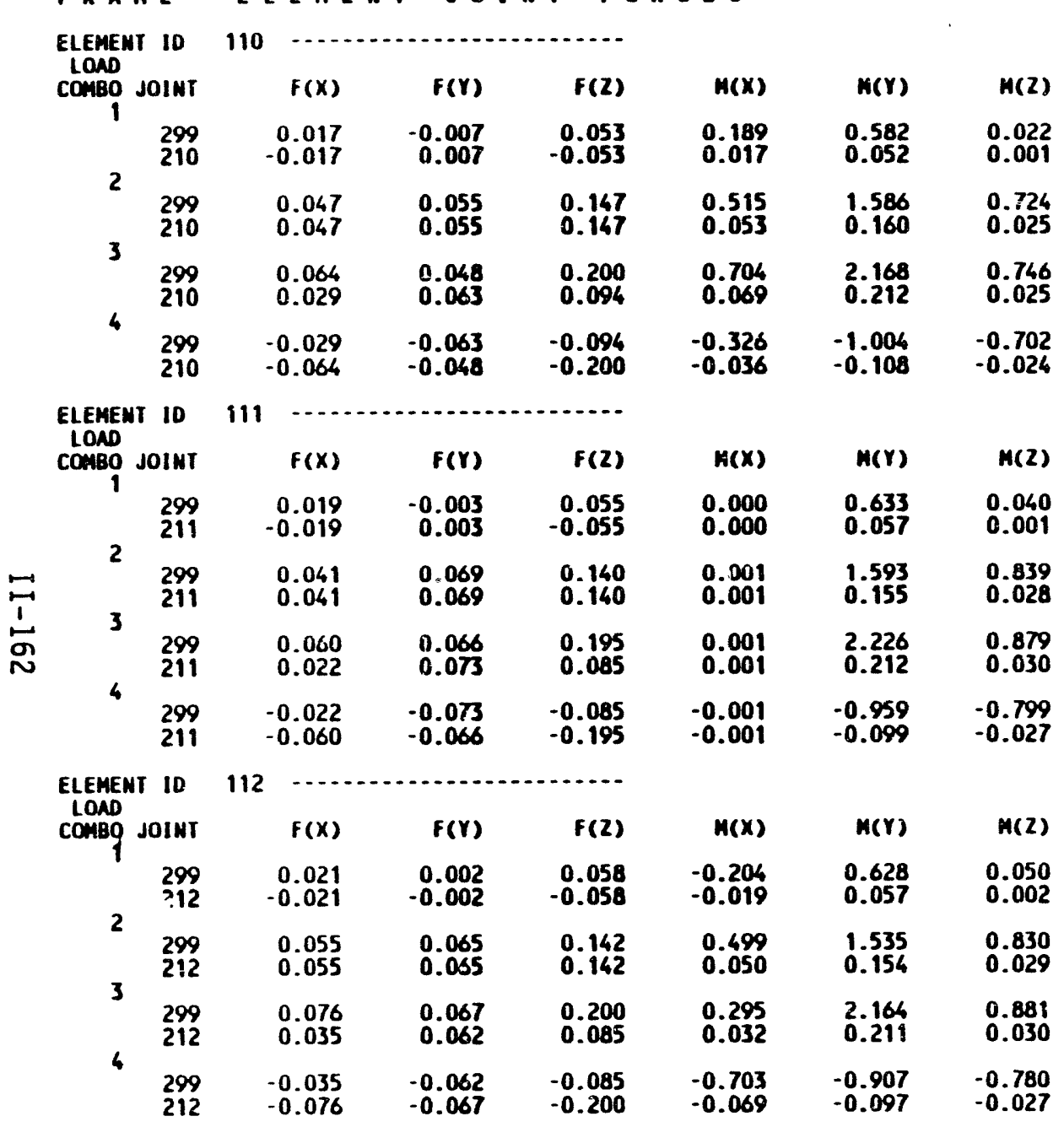

HHC/MPF CAMERA ASSEMBLY MODEL PROGRAM:SAP90/FILE:CH-A-03.FEF

FRAE ELENENT JOINT FORCES

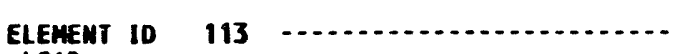

\begin{tabular}{|c|c|c|c|c|c|c|c|}
\hline CONBO & OINT & $F(x)$ & $F(Y)$ & $f(z)$ & $M(x)$ & $M(Y)$ & $M(z)$ \\
\hline & $\begin{array}{l}299 \\
213\end{array}$ & $\begin{array}{r}0.020 \\
-0.020\end{array}$ & $\begin{array}{r}0.011 \\
-0.011\end{array}$ & $\begin{array}{r}0.060 \\
-0.060\end{array}$ & $\begin{array}{l}-0.405 \\
-0.037\end{array}$ & $\begin{array}{l}0.557 \\
0.051\end{array}$ & $\begin{array}{l}0.042 \\
0.001\end{array}$ \\
\hline & $\begin{array}{l}299 \\
213\end{array}$ & $\begin{array}{l}0.067 \\
0.067\end{array}$ & $\begin{array}{l}0.052 \\
0.052\end{array}$ & $\begin{array}{l}0.149 \\
0.149\end{array}$ & $\begin{array}{l}0.987 \\
0.105\end{array}$ & $\begin{array}{l}1.359 \\
0.144\end{array}$ & $\begin{array}{l}0.654 \\
0.022\end{array}$ \\
\hline & $\begin{array}{l}299 \\
213\end{array}$ & $\begin{array}{l}0.088 \\
0.047\end{array}$ & $\begin{array}{l}0.063 \\
0.042\end{array}$ & $\begin{array}{l}0.209 \\
0.088\end{array}$ & $\begin{array}{l}0.582 \\
0.068\end{array}$ & $\begin{array}{l}1.917 \\
0.196\end{array}$ & $\begin{array}{l}0.696 \\
0.023\end{array}$ \\
\hline & 299 & $\begin{array}{l}-0.047 \\
-0.088\end{array}$ & $\begin{array}{l}-0.042 \\
-0.063\end{array}$ & $\begin{array}{l}-0.088 \\
-0.209\end{array}$ & $\begin{array}{l}-1.392 \\
-0.143\end{array}$ & $\begin{array}{l}-0.802 \\
-0.093\end{array}$ & $\begin{array}{l}-0.612 \\
-0.020\end{array}$ \\
\hline
\end{tabular}

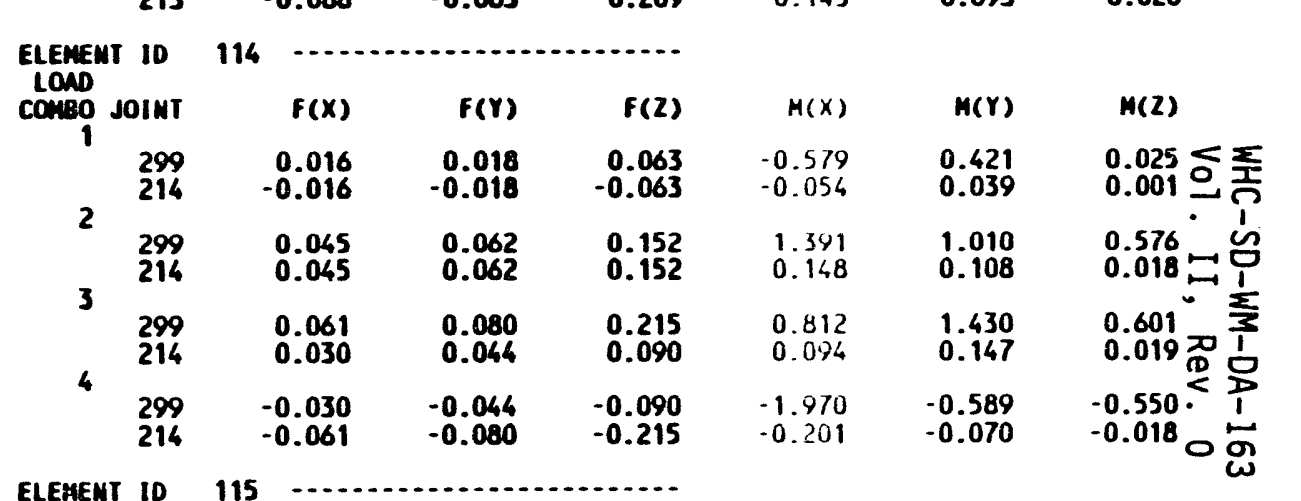

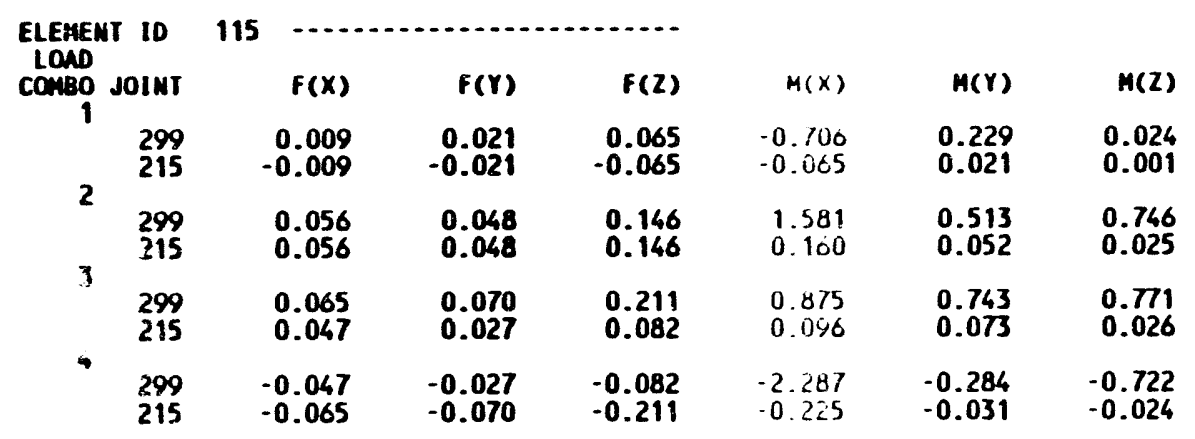


PROGRAM:SAP9O/FILE:CM-A-03. FEF

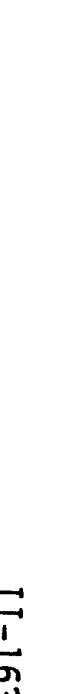

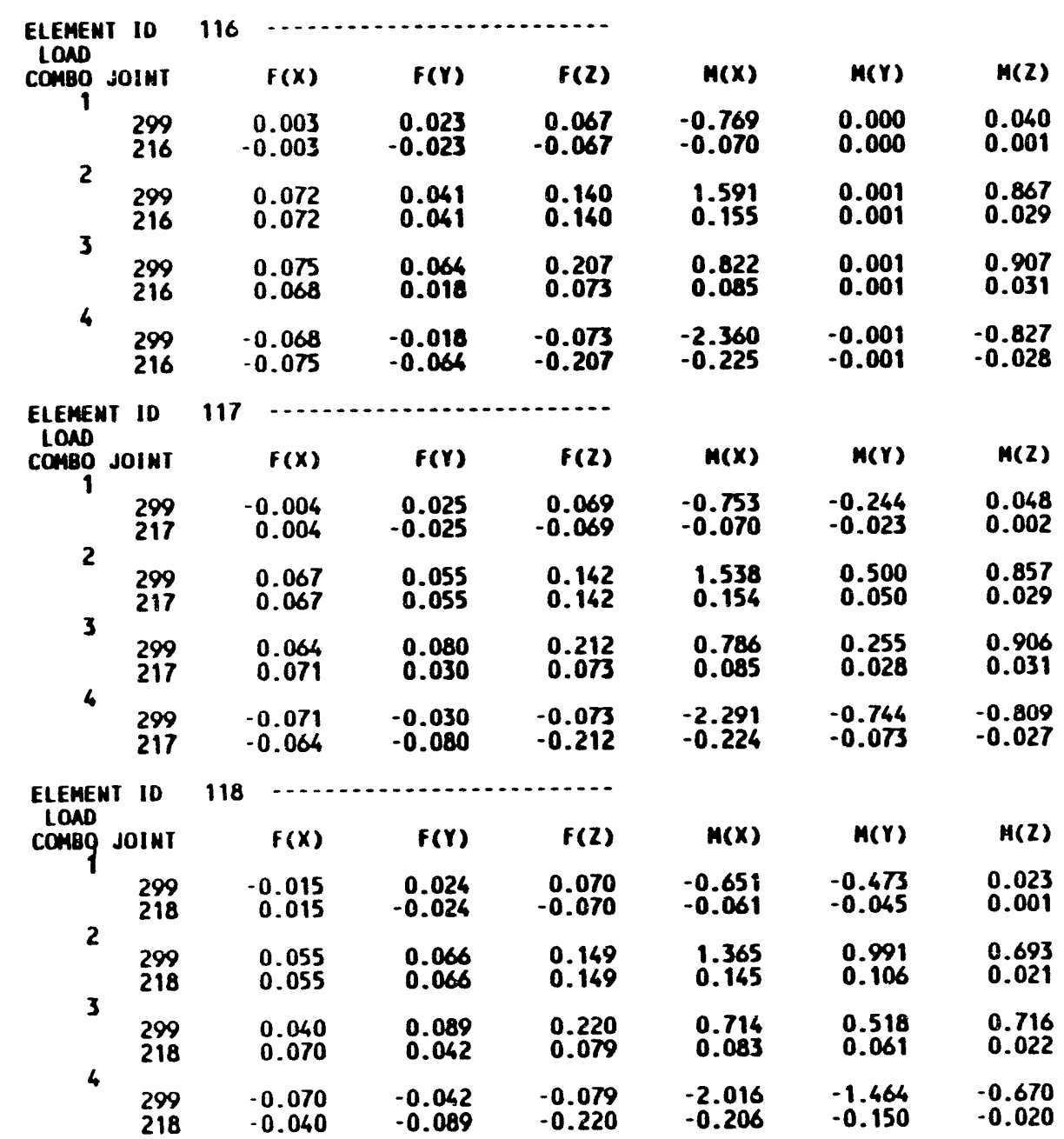

PAGE 7 PROGAM:SAP90/FILE:CH-A-03.FEF
PRA

FRME ELENENT JOINT FORCES

ELEMEMT ID 119

\begin{tabular}{|c|c|c|c|c|c|c|c|}
\hline & OINT & $f(x)$ & $F(r)$ & $f(z)$ & $H(x)$ & $M(Y)$ & $M(2)$ \\
\hline & $\begin{array}{l}299 \\
219\end{array}$ & $\begin{array}{r}-0.024 \\
0.024\end{array}$ & $\begin{array}{r}0.015 \\
-0.015\end{array}$ & $\begin{array}{r}0.070 \\
-0.070\end{array}$ & $\begin{array}{l}-0.473 \\
-0.045\end{array}$ & $\begin{array}{l}-0.651 \\
-0.061\end{array}$ & $\begin{array}{l}-0.023 \\
-0.001\end{array}$ \\
\hline & $\begin{array}{l}299 \\
219\end{array}$ & $\begin{array}{l}0.061 \\
0.061\end{array}$ & $\begin{array}{l}0.048 \\
0.048\end{array}$ & $\begin{array}{l}0.153 \\
0.153\end{array}$ & $\begin{array}{l}1.014 \\
0.109\end{array}$ & $\begin{array}{l}1.397 \\
0.149\end{array}$ & $\begin{array}{l}0.590 \\
0.018\end{array}$ \\
\hline & $\begin{array}{l}299 \\
219\end{array}$ & $\begin{array}{l}0.038 \\
0.085\end{array}$ & $\begin{array}{l}0.063 \\
0.033\end{array}$ & $\begin{array}{l}0.223 \\
0.082\end{array}$ & $\begin{array}{l}0.561 \\
0.064\end{array}$ & $\begin{array}{l}0.746 \\
0.087\end{array}$ & $\begin{array}{l}0.567 \\
0.017\end{array}$ \\
\hline & $\begin{array}{l}299 \\
219\end{array}$ & $\begin{array}{l}-0.085 \\
-0.038\end{array}$ & $\begin{array}{l}-0.033 \\
-0.063\end{array}$ & $\begin{array}{l}-0.082 \\
-0.223\end{array}$ & $\begin{array}{l}-1,487 \\
-0.154\end{array}$ & $\begin{array}{l}-2.048 \\
-0.210\end{array}$ & $\begin{array}{l}-0.613 \\
-0.018\end{array}$ \\
\hline
\end{tabular}

\begin{tabular}{|c|c|c|c|c|c|c|c|}
\hline $\begin{array}{l}\text { Lown } \\
\text { conso }\end{array}$ & JOINT & $f(x)$ & $f(Y)$ & $f(z)$ & $H(x)$ & $M(Y)$ & $M(z)$ \\
\hline & $\begin{array}{l}299 \\
220\end{array}$ & $\begin{array}{r}-0.025 \\
0.025\end{array}$ & $\begin{array}{r}0.004 \\
-0.004\end{array}$ & $\begin{array}{r}0.069 \\
-0.069\end{array}$ & $\begin{array}{l}-0.245 \\
-0.023\end{array}$ & $\begin{array}{l}-0.753 \\
-0.070\end{array}$ & $\begin{array}{l}-0.048 \\
-0.002\end{array}$ \\
\hline 2 & $\begin{array}{l}299 \\
220\end{array}$ & $\begin{array}{l}0.047 \\
0.047\end{array}$ & $\begin{array}{l}0.056 \\
0.056\end{array}$ & $\begin{array}{l}0.147 \\
0.147\end{array}$ & $\begin{array}{l}0.516 \\
0.053\end{array}$ & $\begin{array}{l}1.589 \\
0.161\end{array}$ & $\begin{array}{l}0.745 \\
0.025\end{array}$ \\
\hline 3 & $\begin{array}{l}299 \\
220\end{array}$ & $\begin{array}{l}0.022 \\
0.071\end{array}$ & $\begin{array}{l}0.060 \\
0.052\end{array}$ & $\begin{array}{l}0.216 \\
0.078\end{array}$ & $\begin{array}{l}0.271 \\
0.030\end{array}$ & $\begin{array}{l}0.836 \\
0.091\end{array}$ & $\begin{array}{l}0.697 \\
0.023\end{array}$ \\
\hline 8 & $\begin{array}{l}299 \\
220\end{array}$ & $\begin{array}{l}-0.071 \\
-0.022\end{array}$ & $\begin{array}{l}-0.052 \\
-0.060\end{array}$ & $\begin{array}{l}-0.078 \\
-0.216\end{array}$ & $\begin{array}{l}-0.761 \\
-0.075\end{array}$ & $\begin{array}{l}-2.342 \\
-0.230\end{array}$ & $\begin{array}{l}-0.793 \\
-0.027\end{array}$ \\
\hline
\end{tabular}




\section{UNOLLWSULAAVIS}

WnL-JU-WIH-UA-IOS

Vol. II, Rev. 0

Sheet No. 73.1

Job \#:66481-01 Job: WHC/MPF CAMERA ASSEMBLY ANALYSIS

By ilie Date b/s/a4

Client: WHC Subject: SAP90 ANALYSIS DOCUMENTATION

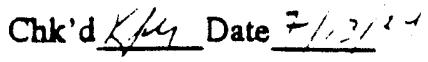

\subsection{Check Weld Connections}

Hand calculations for checking weld connections are provided in this section. 


$$
m / x s \tau=\left(2 / x^{2} \times s=x+0\right. \text { ᄀr }
$$

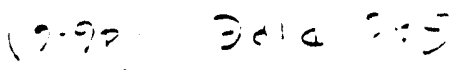

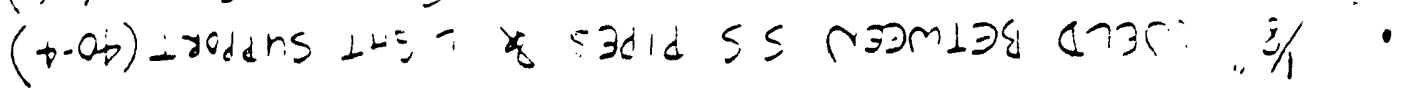

$$
\begin{aligned}
& m / x S L \varepsilon=(91 / \varepsilon \times 2) \times S 2 x+0: \neg \forall \perp \partial H \quad \exists S \forall \varepsilon \\
& \text { ( }
\end{aligned}
$$

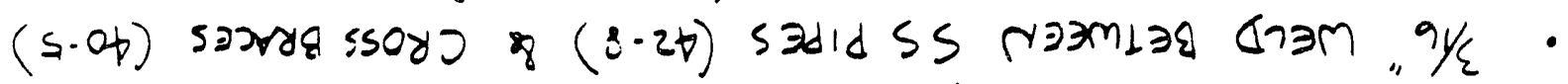

$$
m / x a L 81=(9 / \varepsilon) \times s \tau x+0.7 \gamma \perp \partial W \quad \ni s+\varepsilon
$$

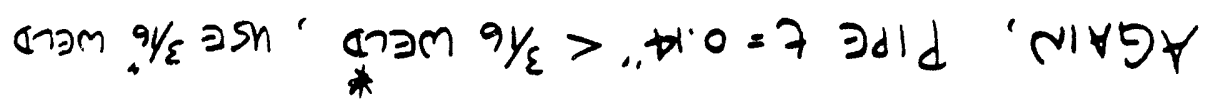

$(z-0)$ ) 3rาd wo1108 \&

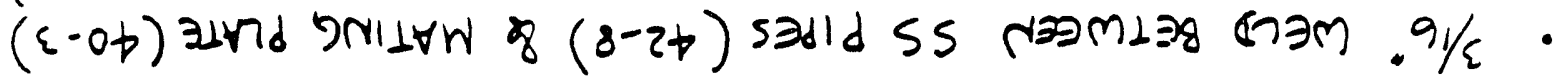

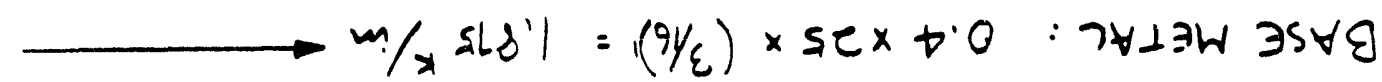

$$
\text { * }
$$

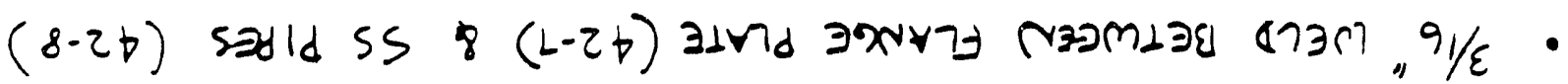

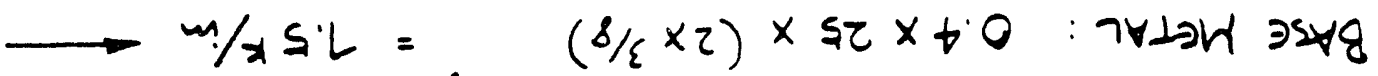

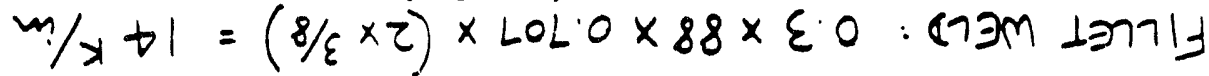

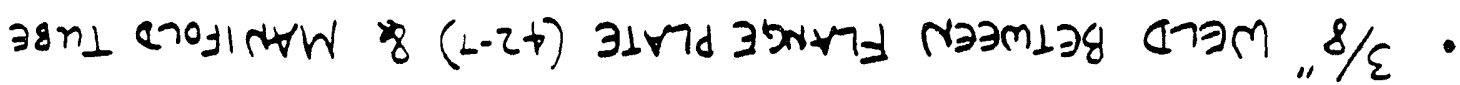

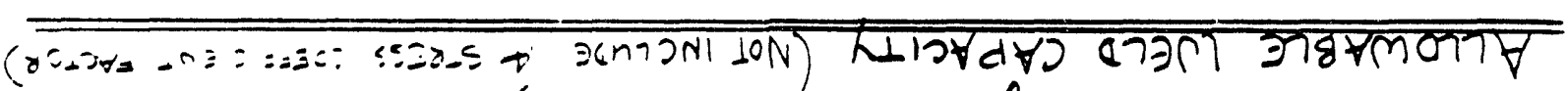

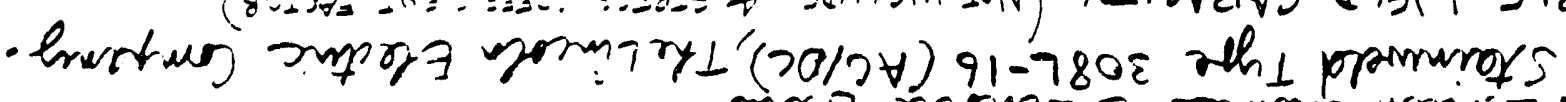

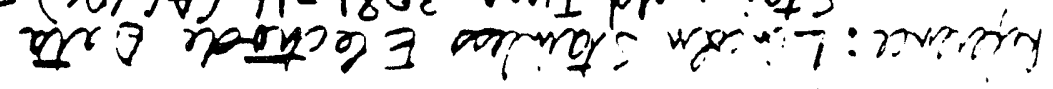

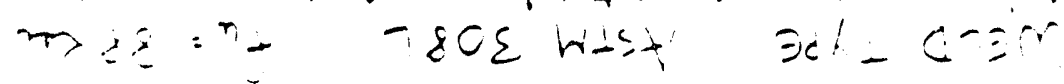

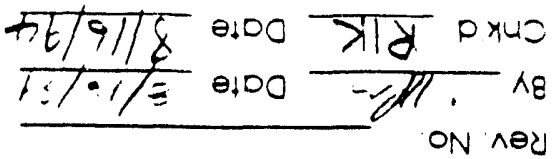

ON JOSOS

$\tau \varepsilon L$ ON IəəU⿻

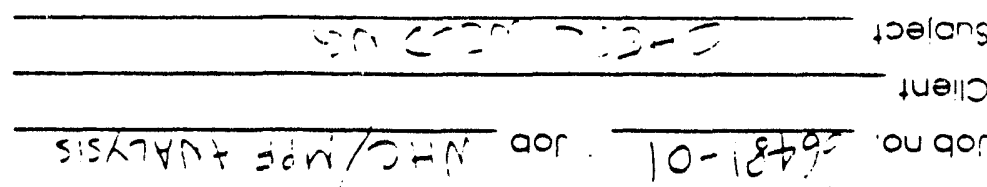

sdeəu!bug 'seje!ooss 8 ounla * uyor/sun $0 \cdot \wedge$ ' II ' $10 \wedge$ 'ONI 'szuezInsuos sun E9I- $\forall O-W M-O S-J H M$ 
URS Consultants, ING. Vol. II, Rev. 0 URS/John A. Blume \& Associates, Engineers job no. $481-01$ Job WHC/MPF ANALYSLS Client Subject

Sheet No. $1,3,3$

Calc. No.

Rev. No.

By Pu Date $\frac{1 / 28 / 94}{8 / 3 / 94}$ Cnk'd RIK Date $8 / 3 / 94$

\section{CALCULATION:- UEUP PROPERTIES LW, Sw}

Flange plate $(42.7)$ \& MANIFOLd Tube (45.5)

$$
\begin{aligned}
& L_{\omega}=\pi D=22 \pi=69.12^{\prime \prime} \\
& S_{\omega}=\pi r^{2}=\pi\left(\frac{22}{2}\right)^{2}=380 \mathrm{~m}^{3} / \mathrm{m}
\end{aligned}
$$

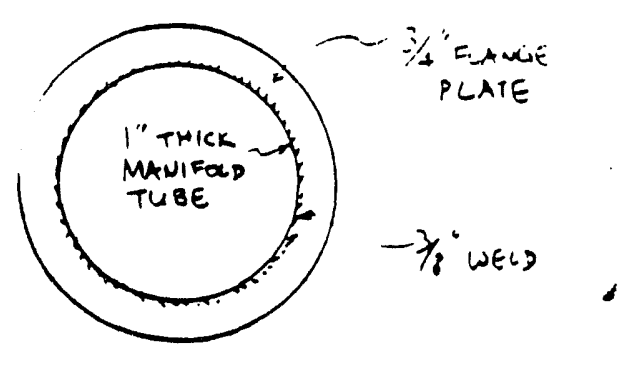

Flange PLATE $(42-7)$ \& SSPIPE (42.8)

$$
\begin{aligned}
& L_{\omega}=\pi D=1.66 \pi=5.22^{\prime \prime} \\
& S_{\omega}=\pi r^{3}=\pi\left(\frac{1.66}{2}\right)^{2}=2.164 \mathrm{~m}^{3} / \mathrm{m}
\end{aligned}
$$

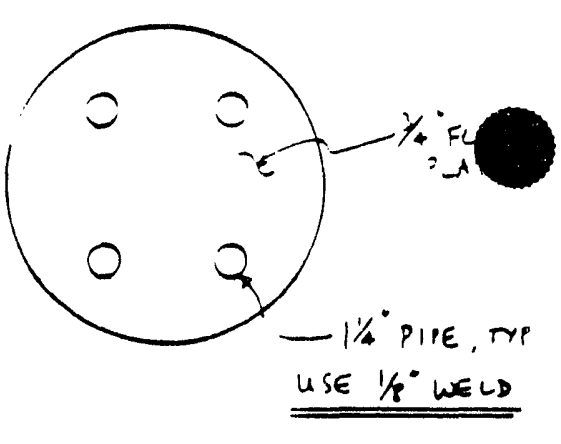

SS PIPE (42.8) \& MATING PLATE (40.3) BOTTOM PLATE $(40-2)$

$$
\begin{aligned}
& L_{\omega}=\pi D=1.66 \pi=5.22^{\prime \prime} \\
& S_{\omega}=\pi r^{2}=\pi\left(\frac{1.66}{2}\right)^{2}=2164 \mathrm{in}^{3} / \mathrm{m}
\end{aligned}
$$

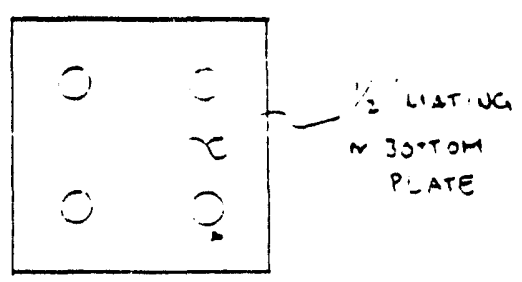

i, 
WHC-jD-WM-DA-163

URS Consultants, INC.

- URS/John A. Blume \& Associates, Engineers

Job no. $6481-01$ Job WHC/MPF ANALYSIS

Client

Subject
SheetNo. 7.34

Calc. No.

Rev. No.

By Pue Date $\frac{-128 / 9 t}{8 / 3 / 944}$

Cnk'd RIK Date $8 / 3 / 94$

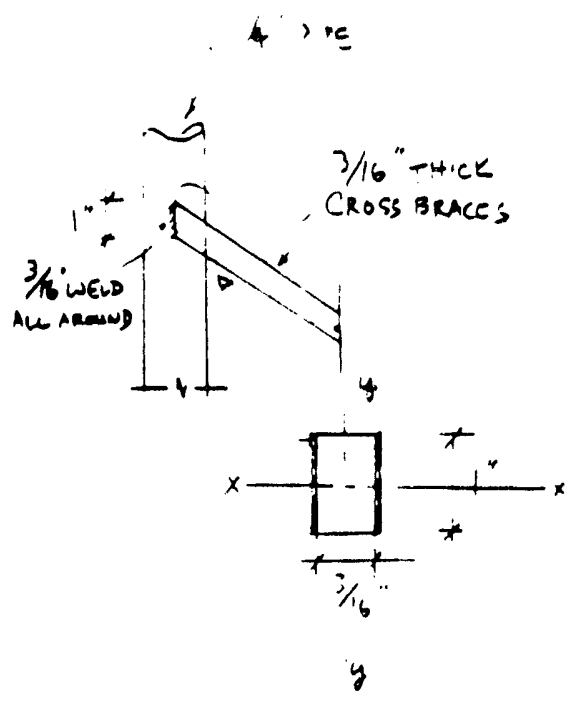

-ight SUPPOR-
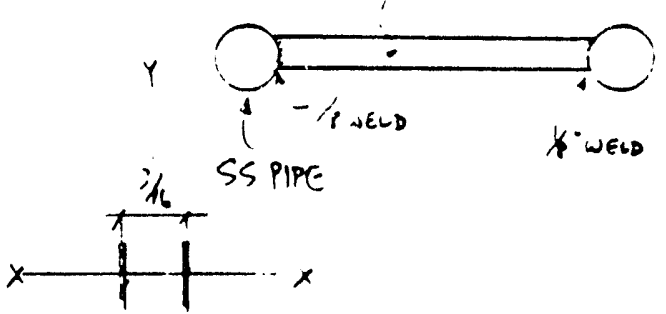

Y

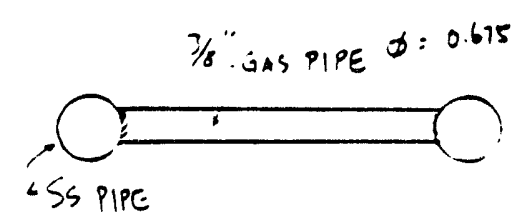

II-167 
URS Consultants, INC. $\mathrm{V}_{0} 1$. II, Rev. 0 URS/John A. Blume \& Associates, Engineers

Job no. $66+8$ i-ol Joo iHC/MPE ANAiysis

Client

Subject

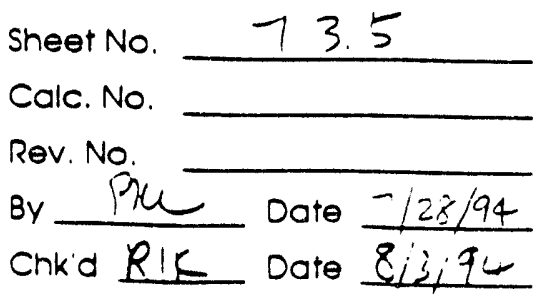

Eivge Plate ix Manifold Tube

$$
\begin{aligned}
& S_{12}(M A X)=0481 \mathrm{ke} ; \text {;EE ONOTS } \\
& S_{12}(\text { MAX })=0.0755 \mathrm{Ner}, 73.19 \rightarrow 7: 26
\end{aligned}
$$
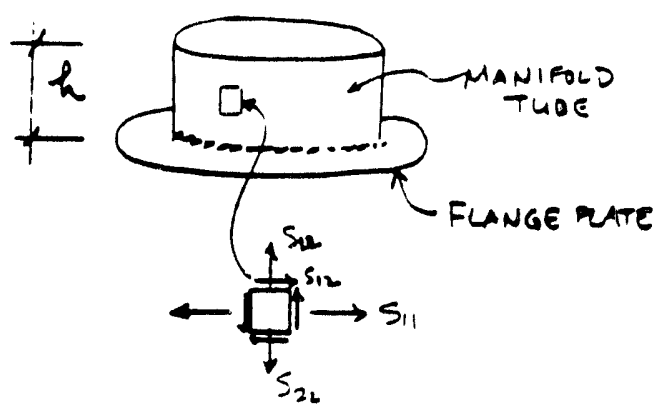

TENSION FORCE PER UNIT LENGTH

$$
0.481(t)(1)=0.481 \mathrm{~K} / \mathrm{m}
$$

SHEAR FORCE PER UNIT LENGTH

$$
0.0755(t)(i)=0.0755 \mathrm{k} / \mathrm{a}
$$

$$
\begin{aligned}
& \text { Should } \\
& \text { be trubled } \\
& \text { becen } t=2 \text {. }
\end{aligned}
$$$$
\text { was ind - rient }
$$

\begin{tabular}{|c|c|c|c|c|c|c|}
\hline & \multirow{3}{*}{$\begin{array}{l}\text { BEAM } \\
\text { ELEMENT } \\
\text { NUMBERS }\end{array}$} & \multicolumn{2}{|c|}{$M A X}$. & \multicolumn{3}{|c|}{ MAX. } \\
\hline & & $1-2$ & plane & & $1-3 p$ & ANE \\
\hline & & SHEAR & MoMNT & AXIAL & SHEAR & MOMENT \\
\hline Flange plate/ss pipes & $201 \rightarrow 204$ & 0.059 & 0.829 & 1.126 & 0038 & 0.831 \\
\hline MatING PLATE/SS PIPES & $233 \rightarrow 236$ & 0.019 & 0.258 & 0.748 & 0.019 & 0259 \\
\hline BOTTOMPLATE/SSPIPES & $265 \rightarrow 268$ & 0.043 & 0.687 & 0.333 & 0.040 & 3770 \\
\hline CROSS BRACES/SSPIES & $401 \rightarrow 420$ & 0.050 & 0.350 & 0.116 & 0.001 & 3008 \\
\hline LIGHTSUPPORT/... .. & $501 \rightarrow 503$ & 0.003 & 0.027 & 0.006 & 2017 & 0112 \\
\hline Z/8 GASPIPES/. & $504 \rightarrow 505$ & 0.002 & 0.007 & 0.029 & 0.010 & 0.048 \\
\hline
\end{tabular}

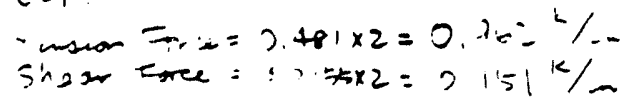

\section{BEAM ELEMENT FORCES}

$$
\text { (SEE Pg } 73.7 \rightarrow 7.3 .18 \text { ) }
$$

From SAP9O. F3F file the enveloped element forces are TABULATED AS FOLLOWS: 
WHC/MPF CAMERA ASSEMBLY

WELD STRESS RATIOS
PROJECT NO: FILENAME:

\section{1-01 \\ WELDSTR.WQ1}

PREPARED BY: Pro

CHECKED EY: RIK
DATE: $7 / 28 / 94$

DATE: $8 / 3 / 94$

$t w=\operatorname{SORT}\left[(F x / L w)^{\wedge} 2+(F y / L w)^{\wedge} 2+(F z / L w+M x x / S w x x+M y y / S w y y)^{\wedge} 2\right]$

\begin{tabular}{|c|c|c|c|c|c|c|c|c|c|c|c|c|c|c|}
\hline CONNECTION & $F_{x}$ & Fy & $\mathbf{F z}$ & Mxx & Myy & $M(s r s s)$ & $M x x+M y$ & Lw & $\operatorname{ses} x x$ & Swyy & $\mathrm{Sw}(\min )$ & iw & $\mathbf{F w}^{*}$ & $\begin{array}{r}\text { Stress } \\
\text { Ratio } \\
\text { S.R. }\end{array}$ \\
\hline Flange Plate / Manitold Tube & 0.151 & - & 0.962 & -. & -. & -- & -- & - & -. & - &.- & 0974 & 10.500 & 0.093 \\
\hline Flange Plate / SS Pipes & 0.059 & 0.058 & 1.126 & 0.829 & 0.831 & 1.174 & & 5.220 & 2.164 & 2.164 & 2.164 & $0 / 58$ & 2.625 & 0.289 \\
\hline Mating Plate / SS Pipes & 0.019 & 0.019 & 0.748 & 0.258 & 0.259 & 0.366 & & 5.220 & 2.164 & 2.164 & 2.164 & 0312 & 2.625 & 0.119 \\
\hline Bottom Plate / SS Pipes & 0.043 & 0.040 & 0.333 & 0.687 & 0.770 & 1.032 & & 5.220 & 2.164 & 2.164 & 2.164 & $0: 4 i$ & 2.625 & 0.206 \\
\hline Cross Braces / SS Pipes & 0.050 & 0.001 & 0.116 & 0.350 & 0.008 & & 0.358 & 2.000 & 0.333 & 0.188 & 0.188 & $1 \cdot 908$ & 5.250 & 0.375 \\
\hline Light Suppon / SS Pipes & 0.003 & 0.017 & 0.006 & 0.027 & 0.112 & & 0.139 & 2.000 & 0.333 & 0.188 & 0.188 & $6 / 50$ & 3.500 & 0.214 \\
\hline 3/8" Gas Pipes / SS Pipes & 0.002 & 0.010 & 0.029 & 0.007 & 0.048 & 0.049 & & 2.120 & 0.358 & 0.358 & 0.358 & $0: 49$ & 3.500 & 0.043 \\
\hline
\end{tabular}

* Fw $=1.4$ * Allowable weld capacity for Extreme Load case (DL + $/$ EQ) 
WHC-SD-WM-DA-163

Vol. II, Rev. 0

This Page Intentionally Left Blank 
UHC/MPF CAMERA ASSEMBLY MODEL JO94: 66458-01 RUM: CM-A-04

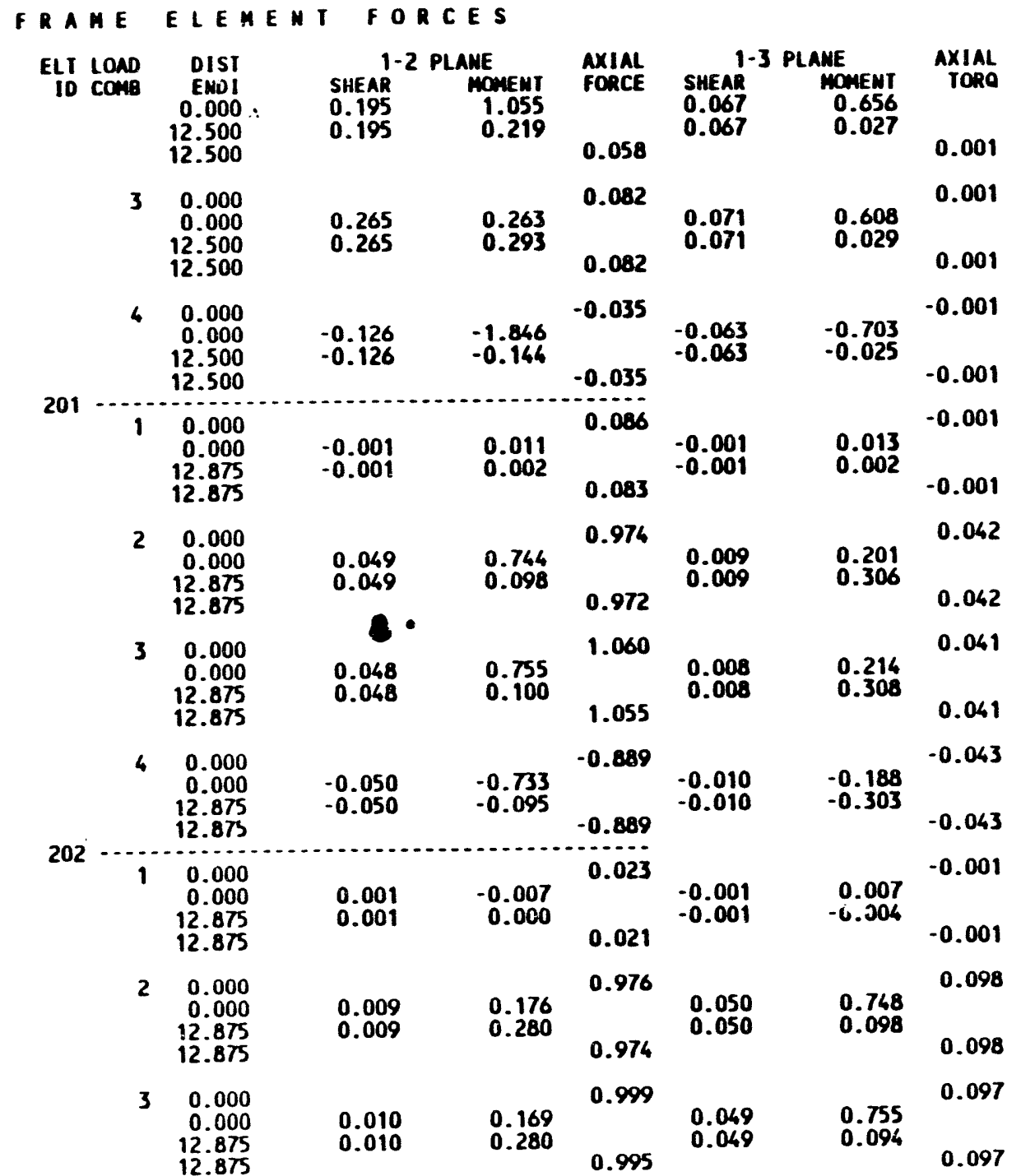

PROGRAM: SAP90/FILE:CM-A-04.F3F FRA AE ELEMEN T FORCES

ELI LOND OIST 1-2 PLAME AXIAL 1 -3 PLAME AXIAL ID COMB EMDI SHEAR MONEMT FORCE SHEAR MOA MOMENT AXIAL

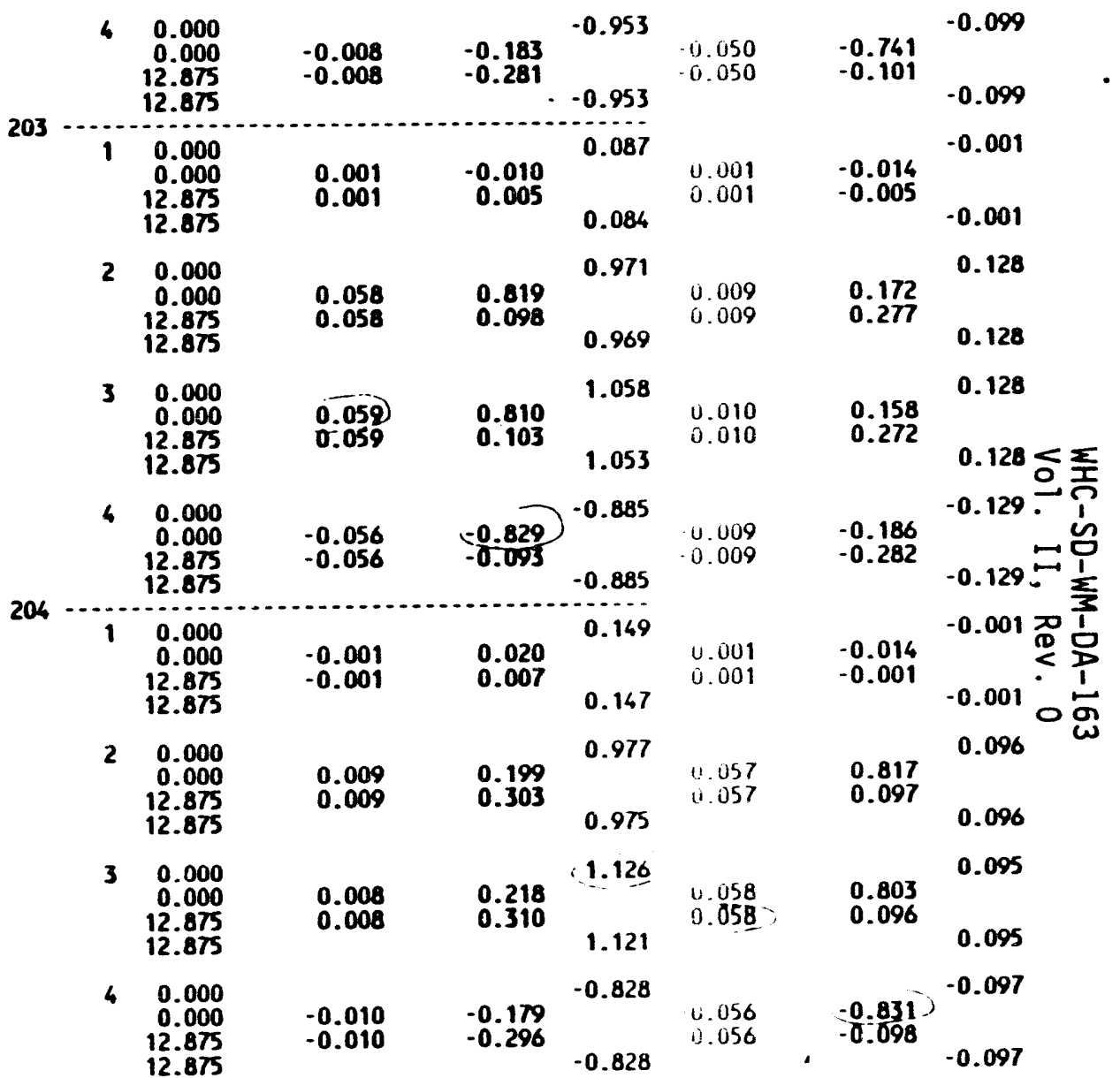

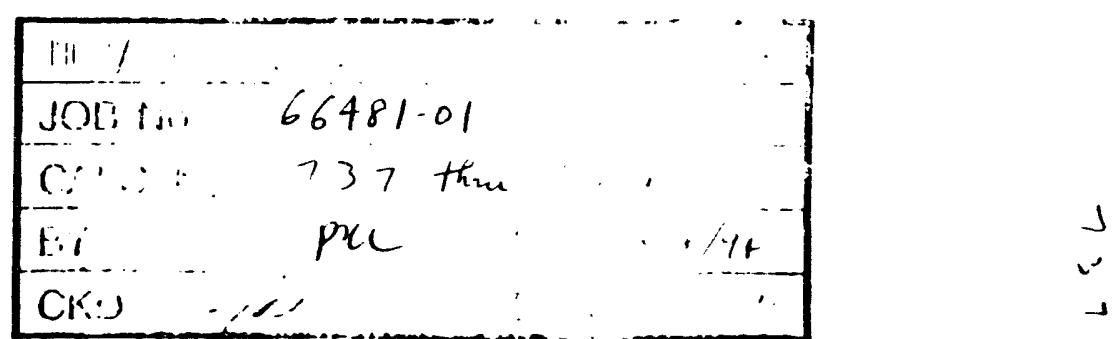




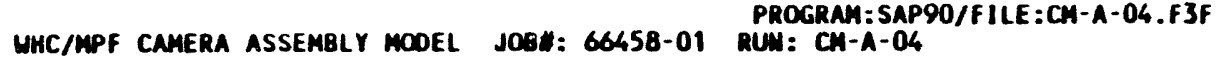

\begin{tabular}{|c|c|c|c|c|c|c|c|c|}
\hline ELI & $\begin{array}{l}\operatorname{Land} \\
\operatorname{con} 8\end{array}$ & $\begin{array}{l}\text { DISI } \\
\text { ENDI }\end{array}$ & SHEAR & $\begin{array}{l}\text { PLANE } \\
\text { MOMENT }\end{array}$ & $\begin{array}{l}\text { AXIAL } \\
\text { FOACE }\end{array}$ & SHEAR & $\begin{array}{l}\text { PLAME } \\
\text { MOMENT }\end{array}$ & $\begin{array}{r}\text { AXIAL } \\
\text { TORO }\end{array}$ \\
\hline & 4 & $\begin{array}{r}0.000 \\
0.000 \\
13.893 \\
13.893\end{array}$ & $\begin{array}{l}-0.030 \\
-0.030\end{array}$ & $\begin{array}{l}-0.108 \\
-0.528\end{array}$ & $\begin{array}{l}-0.605 \\
-0.605\end{array}$ & $\begin{array}{l}-0.014 \\
-0.014\end{array}$ & $\begin{array}{l}-0.126 \\
-0.075\end{array}$ & $\begin{array}{l}-0.086 \\
-0.086\end{array}$ \\
\hline & 1 & $\begin{array}{r}0.000 \\
0.000 \\
13.893 \\
13.893\end{array}$ & $\begin{array}{l}0.000 \\
0.000\end{array}$ & $\begin{array}{l}0.002 \\
0.002\end{array}$ & $\begin{array}{l}0.132 \\
0.130\end{array}$ & $\begin{array}{l}0.000 \\
0.000 \\
.\end{array}$ & $\begin{array}{r}-0.002 \\
0.000\end{array}$ & $\begin{array}{l}-0.001 \\
-0.001\end{array}$ \\
\hline & 2 & $\begin{array}{r}0.000 \\
0.000 \\
13.893 \\
13.893\end{array}$ & $\begin{array}{l}0.015 \\
0.015\end{array}$ & $\begin{array}{l}0.137 \\
0.079\end{array}$ & $\begin{array}{l}0.680 \\
0.678\end{array}$ & $\begin{array}{l}0.031 \\
0.031\end{array}$ & $\begin{array}{l}0.108 \\
0.532\end{array}$ & $\begin{array}{l}0.091 \\
0.091\end{array}$ \\
\hline & 3 & $\begin{array}{r}0.000 \\
0.000 \\
13.893 \\
13.893\end{array}$ & $\begin{array}{l}0.015 \\
0.015\end{array}$ & $\begin{array}{l}0.139 \\
0.080\end{array}$ & $\begin{array}{l}0.812 \\
0.807\end{array}$ & $\begin{array}{l}0.031 \\
0.031\end{array}$ & $\begin{array}{l}0.107 \\
0.532\end{array}$ & $\begin{array}{l}0.090 \\
0.090\end{array}$ \\
\hline & 4 & $\begin{array}{r}0.090 \\
0.000 \\
13.893 \\
13.893\end{array}$ & $\begin{array}{l}-0.015 \\
-0.015\end{array}$ & $\begin{array}{l}-0.136 \\
-0.07\end{array}$ & $\begin{array}{l}-0.548 \\
-0.548\end{array}$ & $\begin{array}{l}-0.031 \\
-0.031\end{array}$ & $\begin{array}{l}-0.110 \\
-0.5332\end{array}$ & $\begin{array}{l}-0.091 \\
-0.091\end{array}$ \\
\hline & 1 & $\begin{array}{r}0.000 \\
0.000 \\
10.107 \\
10.107\end{array}$ & $\begin{array}{l}0.000 \\
0.000\end{array}$ & $\begin{array}{l}0.002 \\
0.002\end{array}$ & $\begin{array}{l}0.065 \\
0.064\end{array}$ & $\begin{array}{l}0.000 \\
0.000\end{array}$ & $\begin{array}{l}-0.002 \\
-0.002\end{array}$ & $\begin{array}{l}-0.001 \\
-0.001\end{array}$ \\
\hline · & 2 & $\begin{array}{r}0.000 \\
0.000 \\
10.107 \\
10.107\end{array}$ & $\begin{array}{l}0.017 \\
0.017\end{array}$ & $\begin{array}{l}0.229 \\
0.067\end{array}$ & $\begin{array}{l}0.629 \\
0.627\end{array}$ & $\begin{array}{l}0.014 \\
0.014\end{array}$ & $\begin{array}{l}0.075 \\
0.202\end{array}$ & $\begin{array}{l}0.105 \\
0.105\end{array}$ \\
\hline & 3 & $\begin{array}{r}0.000 \\
0.000 \\
10.107 \\
10.107\end{array}$ & $\begin{array}{l}0.017 \\
0.017\end{array}$ & $\begin{array}{l}0.231 \\
0.069\end{array}$ & $\begin{array}{l}0.694 \\
0.691\end{array}$ & $\begin{array}{l}0.014 \\
0.014\end{array}$ & $\begin{array}{l}0.072 \\
0.200\end{array}$ & $\begin{array}{l}0.105 \\
0.105\end{array}$ \\
\hline & 4 & $\begin{array}{r}0.000 \\
0.000 \\
10.107 \\
10.107\end{array}$ & $\begin{array}{l}-0.017 \\
-0.017\end{array}$ & $\begin{array}{l}-0.227 \\
-0.066\end{array}$ & $\begin{array}{l}-0.563 \\
-0.563\end{array}$ & $\begin{array}{l}-0.014 \\
-0.014\end{array}$ & $\begin{array}{l}-0.077 \\
-0.204\end{array}$ & $\begin{array}{l}-0.106 \\
-0.106\end{array}$ \\
\hline & 1 & $\begin{array}{l}0.000 \\
0.000\end{array}$ & 0.000 & 0.002 & 0.002 & 0.000 & -0.002 & -0.001 \\
\hline
\end{tabular}

PROCHAM:SAP90/FILE:CM-A-04.F3F

FRAME. ELEME T FORCES

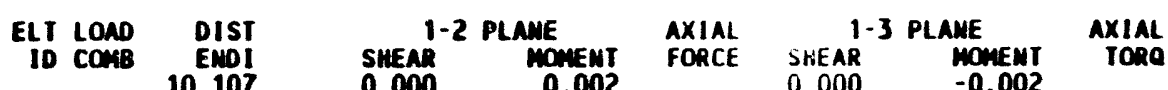
$0.002 \quad 0.0000 .000 \quad-0.002$

$\begin{array}{lllll}2 & 0.000 & 0.617 & 0.102\end{array}$ $\begin{array}{rlllll}0.000 & 0.012 & 0.079 & & 0.016 & 0.224 \\ 10.107 & 0.012 & 0.191 & & 0.016 & 0.077 \\ 10.107 & & 0.616 & & \end{array}$

$\begin{array}{lllll}30.000 & 0.620 & 0.102\end{array}$

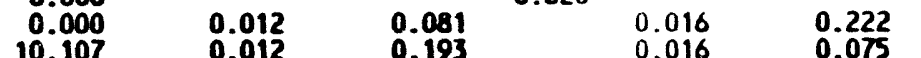
$\begin{array}{lllllll}10.107 & 0.012 & 0.193 & 0.616 & 0.016 & 0.075 & 0.102\end{array}$

\begin{tabular}{|c|c|c|c|c|c|c|c|}
\hline 4 & $\begin{array}{r}0.000 \\
0.000 \\
10.107 \\
10.107\end{array}$ & $\begin{array}{l}-0.012 \\
-0.012\end{array}$ & $\begin{array}{l}-9.077 \\
-0.189\end{array}$ & $\begin{array}{l}-0.615 \\
-0.615\end{array}$ & $\begin{array}{r}-0.016 \\
-0.016\end{array}$ & $\begin{array}{l}-0.226 \\
-0.078\end{array}$ & $\begin{array}{l}-0.103 \\
-0.103\end{array}$ \\
\hline 1 & $\begin{array}{l}0.000 \\
0.000\end{array}$ & 0.000 & 0.002 & 0.066 & 0.000 & -0.001 & -0.001 \\
\hline
\end{tabular}

$\begin{array}{rrrrrrr}0.000 & 0.000 & 0.002 & 0.066 & 0.000 & -0.001 & -0.001 \\ 10.107 & 0.000 & 0.001 & & 0.000 & -0.002 & \\ 10.107 & & & 0.064 & & & -0.001\end{array}$

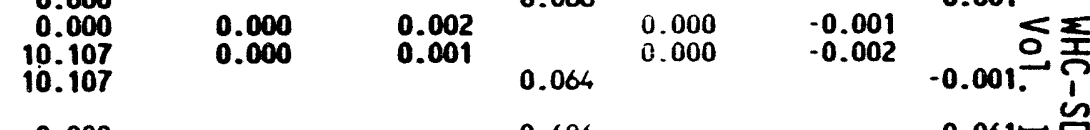

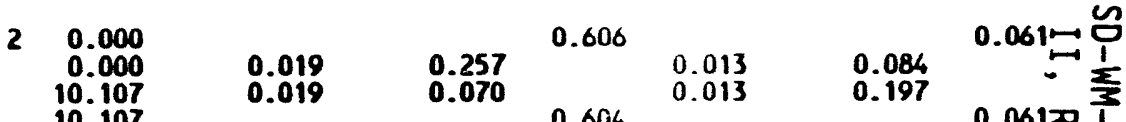

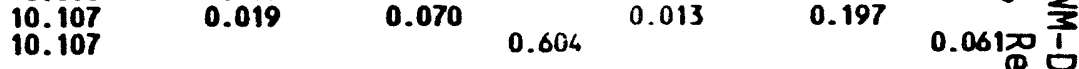

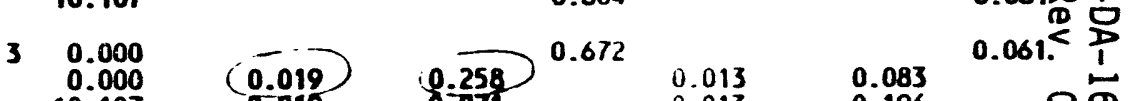

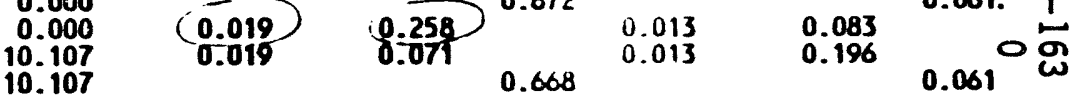

$4 \quad \begin{array}{lllllll}0.000 & -0.019 & -0.255 & -0.540 & 0.013 & -0.086 & -0.062\end{array}$ $\begin{array}{rrrrr}0.000 & -0.019 & -0.255 & 0.013 & -0.086 \\ 10.107 & -0.019 & -0.069 & 0.013 & -0.199\end{array}$

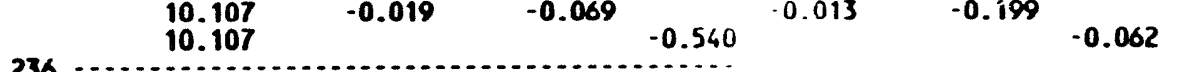
0.000
0.000 10.107
10.107

20.000 0.000
10.107 10.107
10.107

0.000

0.001

129 $\begin{array}{ll}0.000 & 0.001\end{array}$

0.127

0.000

$-0.002$ $-0.001$ $\begin{array}{ll}0.002 & -0.001\end{array}$ 0.014
0.014 0.077
0.205

0.620

0.019

0.257
0.072 0.070 0.070 0.070 

UHC/APF CAMERA ASSEMBLY MODEL J09": 66458-01 RLW: CH-A-OK

FRAME ELEMENT FORCES

\begin{tabular}{|c|c|c|c|c|c|c|c|}
\hline $\begin{array}{l}\text { ELI LOAD } \\
\text { ID CONB }\end{array}$ & $\begin{array}{r}\text { DIST } \\
\text { ENDI } \\
0.000 \\
10.107 \\
10.107\end{array}$ & $\begin{array}{l}1- \\
\text { SHEAR } \\
0.016 \\
0.014\end{array}$ & $\begin{array}{l}\text { AME } \\
\text { MOMENT } \\
0.079 \\
0.206\end{array}$ & $\begin{array}{l}\underset{\text { AXIAL }}{\text { FORCE }} \\
0.765\end{array}$ & $\begin{array}{l}1- \\
\text { SHEAR } \\
0.019 \\
0.019\end{array}$ & $\begin{array}{l}\text { PLAME } \\
\text { MOMENT } \\
0.255 \\
0.070\end{array}$ & $\begin{array}{l}\underset{\text { NXIAL }}{\text { TORO }} \\
0.070\end{array}$ \\
\hline & $\begin{array}{r}0.000 \\
0.000 \\
10.107 \\
10.107\end{array}$ & $\begin{array}{l}-0.014 \\
-0.014\end{array}$ & $\begin{array}{l}-0.076 \\
-0.206\end{array}$ & $\begin{array}{l}-0.491 \\
-0.491\end{array}$ & $\begin{array}{l}-0.019 \\
-0.019\end{array}$ & $\begin{array}{l}-0.259 \\
-0.074\end{array}$ & \\
\hline
\end{tabular}


HHC/MPF CAMERA ASSEMBLY MODEL JOBU: 66458-01 PROGRAM:SAP90/FILE:CH-A-04.F3

\section{FRAME ELENENT FORCES}

\begin{tabular}{|c|c|c|c|c|c|c|c|c|}
\hline -ELI I & $\begin{array}{l}\operatorname{LOnd} \\
\operatorname{cons}\end{array}$ & $\begin{array}{r}\text { DISI } \\
\text { ENDI } \\
16.892 \\
16.892\end{array}$ & $\begin{array}{l}\text { SHEAR } \\
\text { SH- } \\
0.000\end{array}$ & $\begin{array}{l}\text { PLAME } \\
\text { MONENT } \\
0.002\end{array}$ & $\begin{array}{l}\text { AXIAL } \\
\text { FORCE } \\
0.038\end{array}$ & $\begin{array}{l}\text { SHEAR }^{1-3} \\
0.000\end{array}$ & $\begin{array}{c}\text { PLAME } \\
\text { MOMENT } \\
-0.001\end{array}$ & $\begin{array}{r}\text { AXIAL } \\
\text { TORQ } \\
0.000\end{array}$ \\
\hline & 2 & $\begin{array}{r}0.000 \\
0.000 \\
16.892 \\
16.892\end{array}$ & $\begin{array}{l}0.014 \\
0.014\end{array}$ & $\begin{array}{l}0.272 \\
0.487\end{array}$ & $\begin{array}{l}0.221 \\
0.218\end{array}$ & $\begin{array}{l}0.021 \\
0.021\end{array}$ & $\begin{array}{l}0.309 \\
0.667\end{array}$ & $\begin{array}{l}0.073 \\
0.073\end{array}$ \\
\hline & 3 & $\begin{array}{r}0.000 \\
0.000 \\
16.892 \\
16.892\end{array}$ & $\begin{array}{l}0.014 \\
0.014\end{array}$ & $\begin{array}{l}0.273 \\
0.489\end{array}$ & $\begin{array}{l}0.262 \\
0.256\end{array}$ & $\begin{array}{l}0.021 \\
0.021\end{array}$ & $\begin{array}{l}0.306 \\
0.665\end{array}$ & $\begin{array}{l}0.073 \\
0.073\end{array}$ \\
\hline & 4 & $\begin{array}{r}0.000 \\
0.000 \\
16.892 \\
16.892\end{array}$ & $\begin{array}{l}-0.014 \\
-0.016\end{array}$ & $\begin{array}{l}-0.271 \\
-0.485\end{array}$ & $\begin{array}{l}-0.180 \\
-0.180\end{array}$ & $\begin{array}{l}-0.021 \\
-0.021\end{array}$ & $\begin{array}{l}-0.311 \\
-0.668\end{array}$ & $\begin{array}{l}-0.073 \\
-0.073\end{array}$ \\
\hline & 1 & $\begin{array}{r}0.000 \\
0.000 \\
16.892 \\
16.892\end{array}$ & $\begin{array}{l}0.000 \\
0.000\end{array}$ & $\begin{array}{l}0.002 \\
0.002\end{array}$ & $\begin{array}{l}0.106 \\
0.103\end{array}$ & $\begin{array}{l}0.000 \\
0.000\end{array}$ & $\begin{array}{l}-0.002 \\
-0.002\end{array}$ & $\begin{array}{l}0.000 \\
0.000\end{array}$ \\
\hline & 2 & $\begin{array}{r}0.000 \\
0.000 \\
16.892 \\
16.892\end{array}$ & $\begin{array}{l}0.021 \\
0.021\end{array}$ & $\begin{array}{l}0.376 \\
0.733\end{array}$ & $\begin{array}{l}0.241 \\
0.238\end{array}$ & $\begin{array}{l}0.009 \\
0.009\end{array}$ & $\begin{array}{l}0.286 \\
0.433\end{array}$ & $\begin{array}{l}0.087 \\
0.087\end{array}$ \\
\hline & 3 & $\begin{array}{r}0.000 \\
0.000 \\
16.892 \\
16.892\end{array}$ & $\begin{array}{l}0.021 \\
0.021\end{array}$ & $\begin{array}{l}0.378 \\
0.735\end{array}$ & $\begin{array}{l}0.347 \\
0.341\end{array}$ & $\begin{array}{l}0.009 \\
0.009\end{array}$ & $\begin{array}{l}0.284 \\
0.431\end{array}$ & $\begin{array}{l}0.086 \\
0.086\end{array}$ \\
\hline . & 4 & $\begin{array}{r}0.000 \\
0.000 \\
16.892 \\
16.892\end{array}$ & $\begin{array}{l}-0.021 \\
-0.021\end{array}$ & $\begin{array}{l}-0.375 \\
-0.731\end{array}$ & $\begin{array}{l}-0.135 \\
-0.135\end{array}$ & $\begin{array}{l}-0.009 \\
-0.009\end{array}$ & $\begin{array}{l}-0.288 \\
-0.435\end{array}$ & $\begin{array}{l}-0.087 \\
-0.087\end{array}$ \\
\hline & 1 & $\begin{array}{l}0.000 \\
0.000 \\
2.250 \\
2.250\end{array}$ & $\begin{array}{l}0.000 \\
0.000\end{array}$ & $\begin{array}{l}0.001 \\
0.001\end{array}$ & $\begin{array}{l}0.038 \\
0.037\end{array}$ & $\begin{array}{l}0.000 \\
0.000\end{array}$ & $\begin{array}{l}-0.002 \\
-0.002\end{array}$ & $\begin{array}{l}0.000 \\
0.000\end{array}$ \\
\hline & 2 & $\begin{array}{l}0.000 \\
0.000 \\
2.250 \\
2.250\end{array}$ & $\begin{array}{l}0.008 \\
0.008\end{array}$ & $\begin{array}{l}0.412 \\
0.431\end{array}$ & $\begin{array}{l}0.209 \\
0.209\end{array}$ & $\begin{array}{l}0.023 \\
0.023\end{array}$ & $\begin{array}{r}0.712 \\
0.770 \\
\end{array}$ & $\begin{array}{l}0.078 \\
0.078\end{array}$ \\
\hline & 3 & 0.000 & & & 0.247 & & & 0.079 \\
\hline
\end{tabular}

UHC/MPF CAMERA ASSEMQIY MODEL J08*: 66458-01 PROCIKAM:SAP90/FILE:CH-A-04.F3F

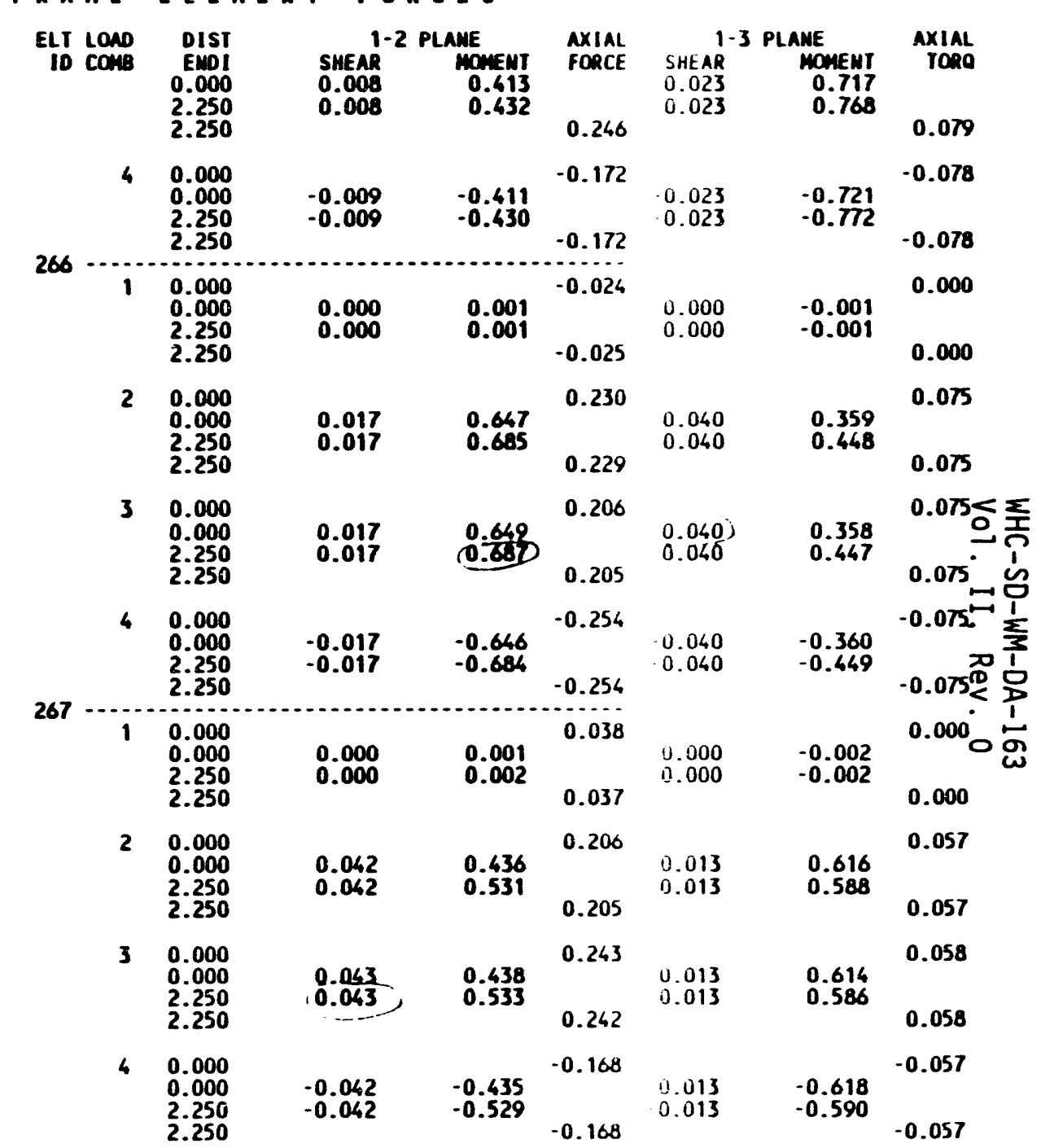


WHC/MPF CAMERA ASSEMBLY MOOEL JOQN: 66458-01 PROGRAM:SAP90/FILE:CH-A-04.F3F

\begin{tabular}{|c|c|c|c|c|c|c|c|}
\hline \multirow{5}{*}{$\begin{array}{r}\text { ELI } \\
10 \\
268\end{array}$} & $\begin{array}{l}\text { DISI } \\
\text { EMDI }\end{array}$ & $\begin{array}{c}1 \\
\text { SHEAR }\end{array}$ & AME & $\underset{\text { FORCE }}{\operatorname{axIAL}}$ & SHEAR & PLAME & $\begin{array}{r}\text { AXIAL } \\
\text { TORO }\end{array}$ \\
\hline & $\begin{array}{l}0.000 \\
0.000 \\
2.250 \\
2.250\end{array}$ & $\begin{array}{l}0.000 \\
0.000\end{array}$ & $\begin{array}{l}0.003 \\
0.002\end{array}$ & $\begin{array}{l}0.103 \\
0.102\end{array}$ & $\begin{array}{l}0.000 \\
0.000\end{array}$ & $\begin{array}{l}-0.002 \\
-0.002\end{array}$ & $\begin{array}{l}0.000 \\
0.000\end{array}$ \\
\hline & $\begin{array}{l}0.000 \\
0.000 \\
2.250 \\
2.250\end{array}$ & $\begin{array}{l}0.008 \\
0.008\end{array}$ & $\begin{array}{l}0.678 \\
0.660\end{array}$ & $\begin{array}{l}0.230 \\
0.230\end{array}$ & $\begin{array}{l}0.009 \\
0.009\end{array}$ & $\begin{array}{l}0.434 \\
0.454\end{array}$ & $\begin{array}{l}0.078 \\
0.078\end{array}$ \\
\hline & $\begin{array}{l}0.000 \\
0.000 \\
2.250 \\
2.250\end{array}$ & $\begin{array}{l}0.008 \\
0.008\end{array}$ & $\begin{array}{l}0.681 \\
0.662\end{array}$ & $\begin{array}{l}0.333 \\
0.332\end{array}$ & $\begin{array}{l}0.009 \\
0.009\end{array}$ & $\begin{array}{l}0.432 \\
0.453\end{array}$ & $\begin{array}{l}0.078 \\
0.078\end{array}$ \\
\hline & $\begin{array}{l}0.000 \\
0.000 \\
2.250 \\
2.250\end{array}$ & $\begin{array}{l}-0.009 \\
-0.009\end{array}$ & $\begin{array}{l}-0.676 \\
-0.658\end{array}$ & $\begin{array}{l}-0.128 \\
-0.128\end{array}$ & $\begin{array}{l}-0.009 \\
-0.009\end{array}$ & $\begin{array}{l}-0.436 \\
-0.456\end{array}$ & $\begin{array}{l}-0.078 \\
-0.078\end{array}$ \\
\hline
\end{tabular}

जั

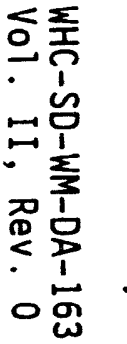


UHC/MPF CAMERA ASSE'RBLY MOOEL JOBH: 66458-01 RROGRAM:SAP90/FILE:CM-A-04.F3F

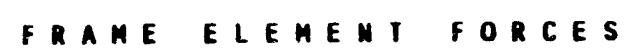

\begin{tabular}{|c|c|c|c|c|c|c|c|c|}
\hline \multirow[t]{2}{*}{ ELT } & WD & $\begin{array}{l}\text { DIST } \\
\text { ENDI } \\
0.941\end{array}$ & SHEAR & $\begin{array}{l}\text { AME } \\
\text { MONENT }\end{array}$ & $\begin{array}{l}\text { AXIAL } \\
\text { FORCE } \\
0.027\end{array}$ & $\begin{array}{r}1 \\
\text { SHEAR }\end{array}$ & $\begin{array}{l}\text { PLAME } \\
\text { MOMENT }\end{array}$ & $\begin{array}{c}\text { AXIAL } \\
\text { TORO } \\
0.158\end{array}$ \\
\hline & 4 & $\begin{array}{l}0.000 \\
0.000 \\
0.941 \\
0.941\end{array}$ & $\begin{array}{l}-0.088 \\
-0.088\end{array}$ & $\begin{array}{l}-0.210 \\
-0.127\end{array}$ & $\begin{array}{l}-0.027 \\
-0.027\end{array}$ & $\begin{array}{l}-0.034 \\
-0.034\end{array}$ & $\begin{array}{l}-0.033 \\
-0.006\end{array}$ & $\begin{array}{l}-0.159 \\
-0.159\end{array}$ \\
\hline & 1 & $\begin{array}{l}0.000 \\
0.000 \\
0.94 \\
0.94\end{array}$ & $\begin{array}{l}0.000 \\
0.000\end{array}$ & $\begin{array}{l}0.000 \\
0.000\end{array}$ & $\begin{array}{l}0.000 \\
0.000\end{array}$ & $\begin{array}{l}0.000 \\
0.000\end{array}$ & $\begin{array}{l}0.000 \\
0.000\end{array}$ & $\begin{array}{l}0.000 \\
0.000\end{array}$ \\
\hline & 2 & $\begin{array}{l}0.000 \\
0.000 \\
0.941 \\
0.941\end{array}$ & $\begin{array}{l}0.089 \\
0.089\end{array}$ & $\begin{array}{l}0.206 \\
0.123\end{array}$ & $\begin{array}{l}0.028 \\
0.028\end{array}$ & $\begin{array}{l}0.035 \\
0.035\end{array}$ & $\begin{array}{l}0.032 \\
0.003\end{array}$ & $\begin{array}{l}0.155 \\
0.155\end{array}$ \\
\hline & 3 & $\begin{array}{l}0.000 \\
0.000 \\
0.941 \\
0.941\end{array}$ & $\begin{array}{l}0.089 \\
0.089\end{array}$ & $\begin{array}{l}0.205 \\
0.122\end{array}$ & $\begin{array}{l}0.028 \\
0.028\end{array}$ & $\begin{array}{l}0.035 \\
0.035\end{array}$ & $\begin{array}{l}0.032 \\
0.003\end{array}$ & $\begin{array}{l}0.155 \\
0.155\end{array}$ \\
\hline & 4 & $\begin{array}{l}0.000 \\
0.000 \\
0.941 \\
0.941\end{array}$ & $\begin{array}{l}-0.089 \\
-0.089\end{array}$ & $\begin{array}{l}-0.206 \\
-0.123\end{array}$ & $\begin{array}{l}-0.028 \\
-0.028\end{array}$ & $\begin{array}{l}-0.035 \\
-0.035\end{array}$ & $\begin{array}{l}-0.032 \\
-0.003\end{array}$ & $\begin{array}{l}-0.155 \\
-0.155\end{array}$ \\
\hline 34 & 1 & $\begin{array}{l}0.000 \\
0.000 \\
0.941 \\
0.941\end{array}$ & $\begin{array}{l}0.000 \\
0.000\end{array}$ & $\begin{array}{l}0.000 \\
0.000\end{array}$ & $\begin{array}{l}0.000 \\
0.000\end{array}$ & $\begin{array}{l}0.000 \\
0.000\end{array}$ & $\begin{array}{l}0.000 \\
0.000\end{array}$ & $\begin{array}{l}0.000 \\
0.000\end{array}$ \\
\hline ' & 2 & $\begin{array}{l}0.000 \\
0.000 \\
0.941 \\
0.941\end{array}$ & $\begin{array}{l}0.101 \\
0.101\end{array}$ & $\begin{array}{l}0.234 \\
0.139\end{array}$ & $\begin{array}{l}0.031 \\
0.031\end{array}$ & $\begin{array}{l}0.040 \\
0.040\end{array}$ & $\begin{array}{l}0.038 \\
0.003\end{array}$ & $\begin{array}{l}0.175 \\
0.175\end{array}$ \\
\hline & 3 & $\begin{array}{l}0.000 \\
0.000 \\
0.941 \\
0.941\end{array}$ & $\begin{array}{l}0.101 \\
0.101\end{array}$ & $\begin{array}{l}0.233 \\
0.139\end{array}$ & $\begin{array}{l}0.031 \\
0.031\end{array}$ & $\begin{array}{l}0.040 \\
0.040\end{array}$ & $\begin{array}{l}0.038 \\
0.003\end{array}$ & $\begin{array}{l}0.174 \\
0.174\end{array}$ \\
\hline & 4 & $\begin{array}{l}0.000 \\
0.000 \\
0.941 \\
0.941\end{array}$ & $\begin{array}{l}-0.100 \\
-0.100\end{array}$ & $\begin{array}{l}-0.234 \\
-0.140\end{array}$ & $\begin{array}{l}-0.031 \\
-0.031\end{array}$ & $\begin{array}{l}-0.039 \\
-0.039\end{array}$ & $\begin{array}{l}-0.038 \\
-0.003\end{array}$ & $\begin{array}{l}-0.175 \\
-0.175\end{array}$ \\
\hline & 1 & 0.000 & & & 0.001 & & & 0.000 \\
\hline
\end{tabular}

UHC/MPF CAMERA ASSEMBLY MOEL PAGE S6

FRAME ELEMENT FORCES

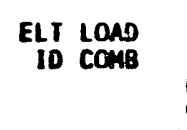

DIST
EMDI
0.000
9.454
14.096
14.096

1-2 PLAMP
SHEAR
0.000
0.000
0.000

20.000

0.000
0.000
14.096

14.096
14.096

0.029
0.030

$3 \quad 0.000$ 0.000 14.096
14.096

MLAE
MOMENT
0.001
0.000
0.000

AXIA
FORC
001

IAL

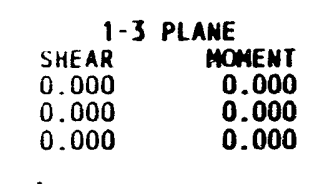

AxIAL

000

0.002

0.000

0.001
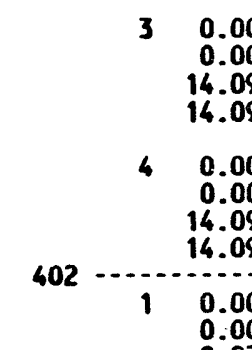
0.000
0.000 14.096
14.096

0.000
0.000 0.000
8.971
14.096

0.02

0.029
0.030

0.210
0.211

0.004

0.08 s

0.001

0.006

0.001

0.001

8.971
14.096

$-0.030$

0.211

0.090

0.001

0.004
0.006

0.001

0.001

$-0.001$

0.004
0.006

-0.001 으ㅇㅗㅗ

0.000

0.000

0.000

0.000

0.000
0.000

0.001

0.001

14.096
0.000

0.000
0.000

0.000
16.096

0.02

0.000
0.000

0.000
0.000

0.001

14.096

0.026
0.027

0.07

0.00

$3 \quad 0.000$ 0.000
14.096 14.096
14.096

0.026

0.001
0.001

0.005

0.005
0.006

0.000

0.001

0.079

0.027

0.187

0.187

0.000

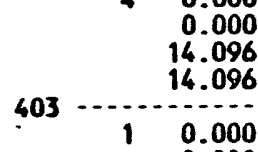

$\begin{array}{rr} & 0.000 \\ & 14.096 \\ 14.096 & 10.00\end{array}$

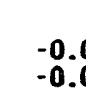

-0.026
-0.026

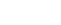

$-0$.

079

0.00

0.001

0.005

0.001

0.001

0.005
0.006

0.001

$-0.001$

$-0.005$

$-0.001$

0.000

0.000
6.374
14.096

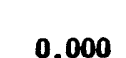

0.186

..........

a.cor

0.000

0.000

0.000

$2 \quad 0.000$

0.000

0.000

0.000
0.000

0.000

0.001 

AOE J08: 66458-01 RUN: CM-A-O4

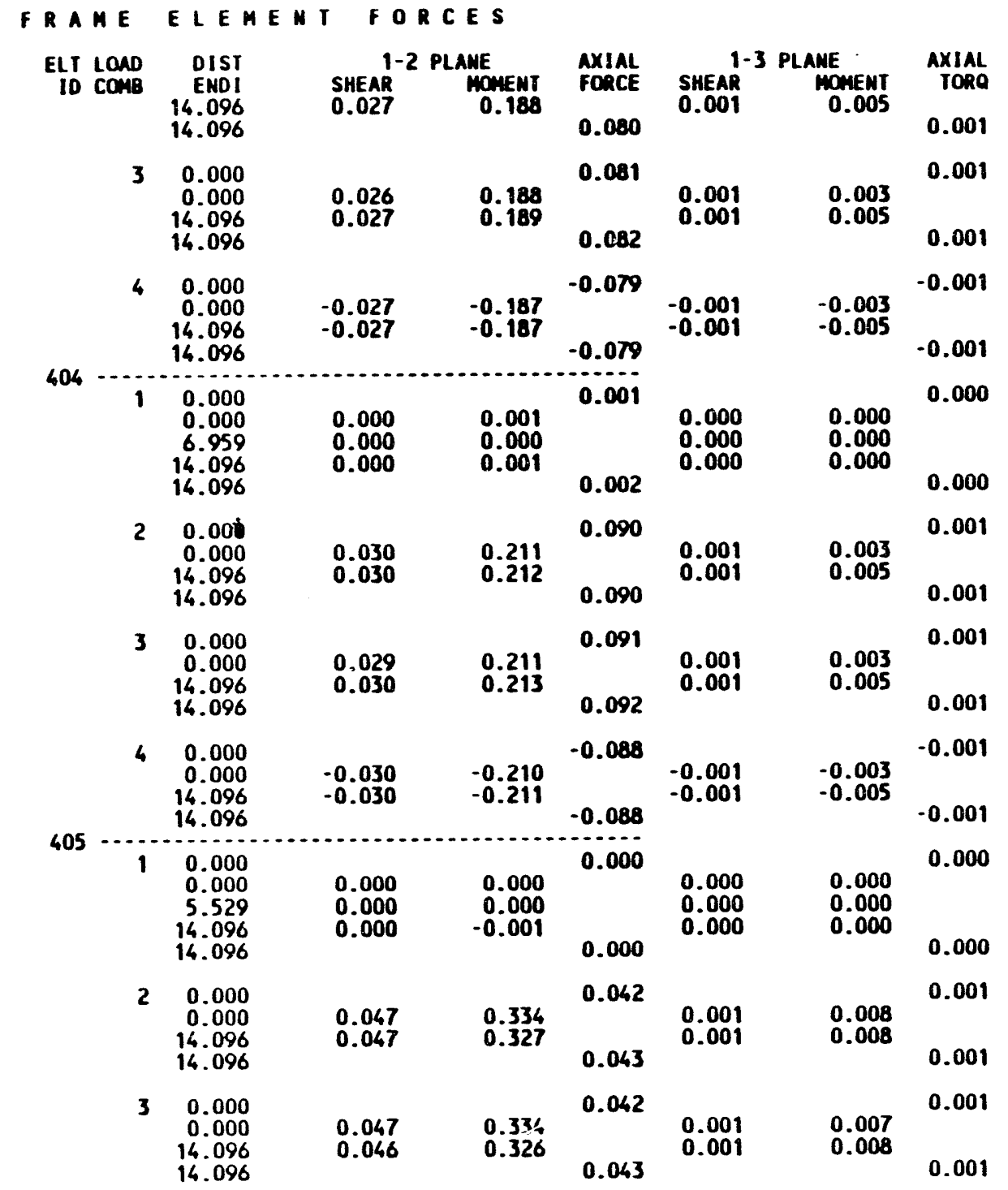

UHC/MPF CAMERA ASSEMBLY MODEL JC8: 66458-01 PROGRAM:SAP90/FILE:CH-A-04.F3F

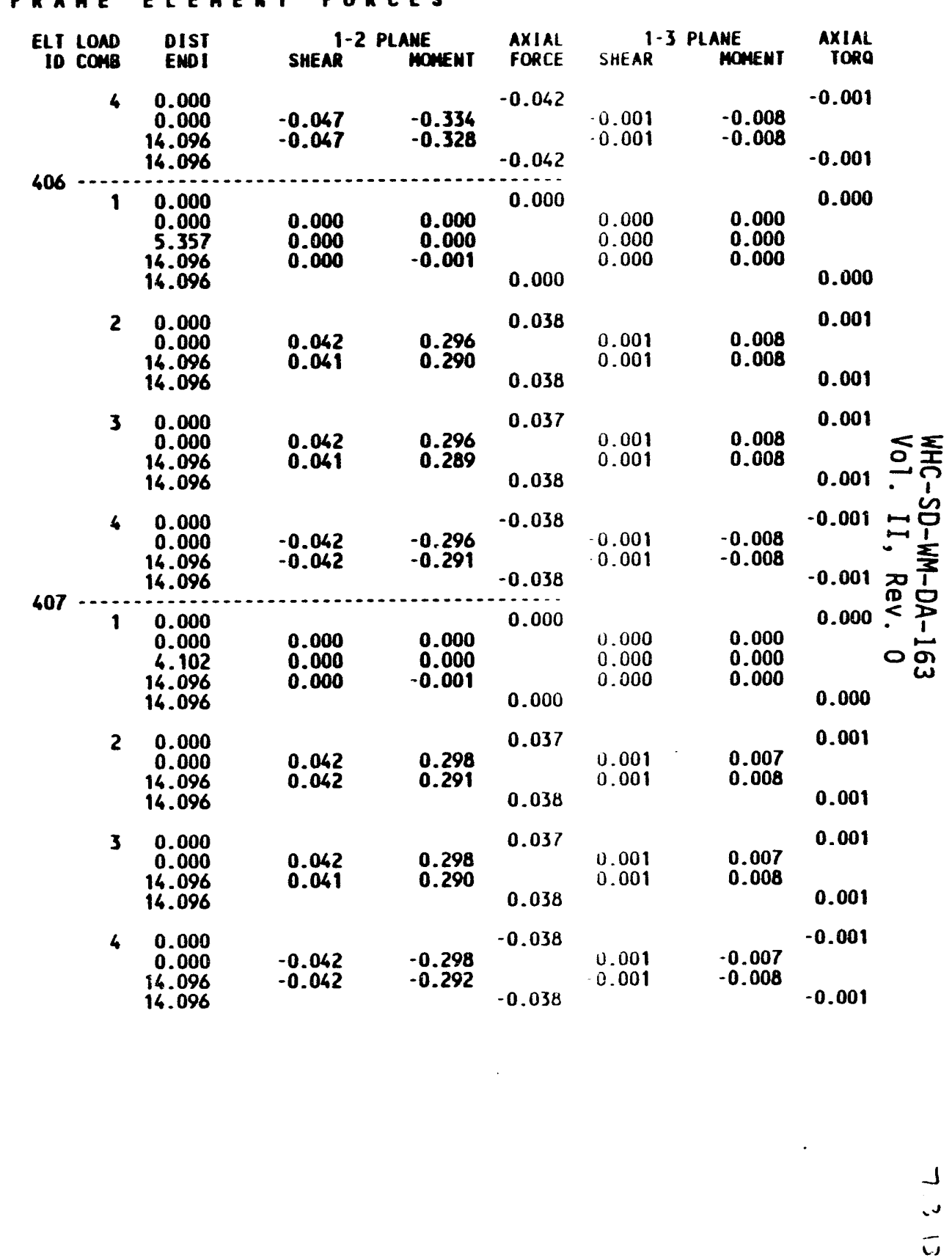


UHC/MPF CAMERA ASSEMBLY MOOEL JOB*: 66458-01 RUM: CH-A-04

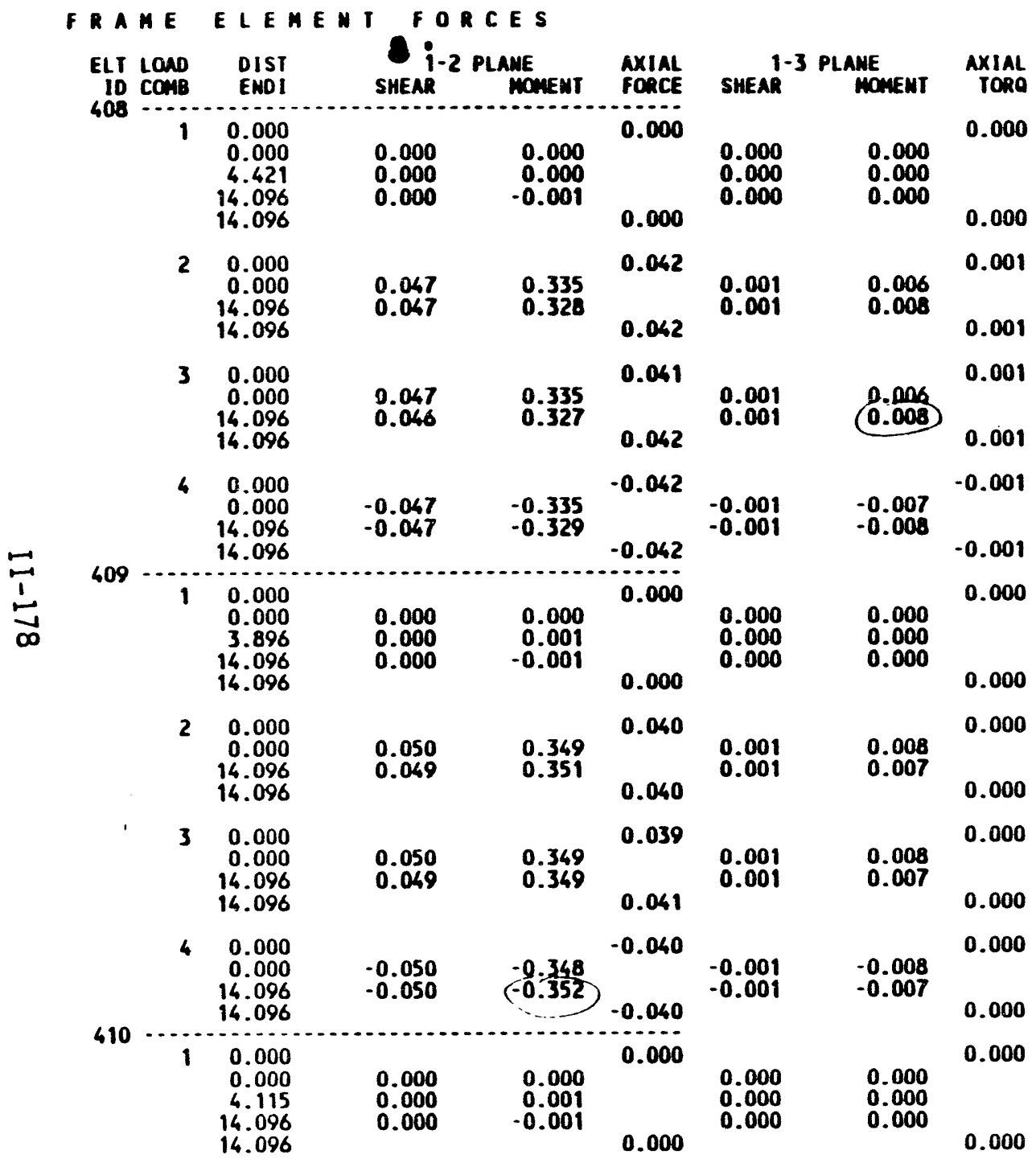

PAGE 58
PROLRAM:SAP90/FILE:CH-A-04.F3F

frane elenent forces

ELI LOAD DIST TI-2 PLAME AXIAL 1-3 PLAME

20.000

0.000
14.096

$0.046 \quad 0.3090 .036$

0.046
0.044

$\mathbf{0} .309$
$\mathbf{0 . 3 1 0}$

0.036

0.001
0.001

0.008

0.000

16.096

0.036
0.035

0.000
0.000 0.000
14.096

0.044
0.043

0.309

0.036

0.001

0.008

0.000

14.096

$4 \quad 0.000$ 0.000
$1 \% .096$
14.096

$-0.036$ 14.096

$-0.044$

-0.309
-0.312

$3 \quad 0.000$ 0.000
14.096 14.096

$4 \quad 0.000$ 0.000
14.096
14.096

0.000

0.000
0.000

0.000

0.036

0.044
0.046

0.310

0.001

.001

0.036

$$
\begin{aligned}
& 0.044 \\
& 0.043
\end{aligned}
$$

0.310
0.309

0.036

0.001

0.037

14.096

$-0.3$

$-0.037$

37

-0.001
.0 .001

0.008
0.008

0.000

0.000

0.000

0.000

0.000

0.000
0.000

0.000 $0.000^{\circ}$

$0.000 \leftrightarrows$

0.008

0.000 备

$0.000^{\circ}$

0.008

0.000

0.000$$
412
$$

$3 \quad 0.000$

$$
0.000
$$$$
0.000
$$$$
0.000
$$
0.000 0.000

0.000
0.000 0.000
0.000 0.000 0.000 0.008
0.008 0.000 0.000 


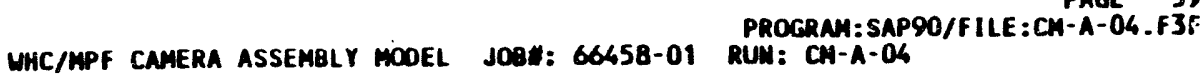

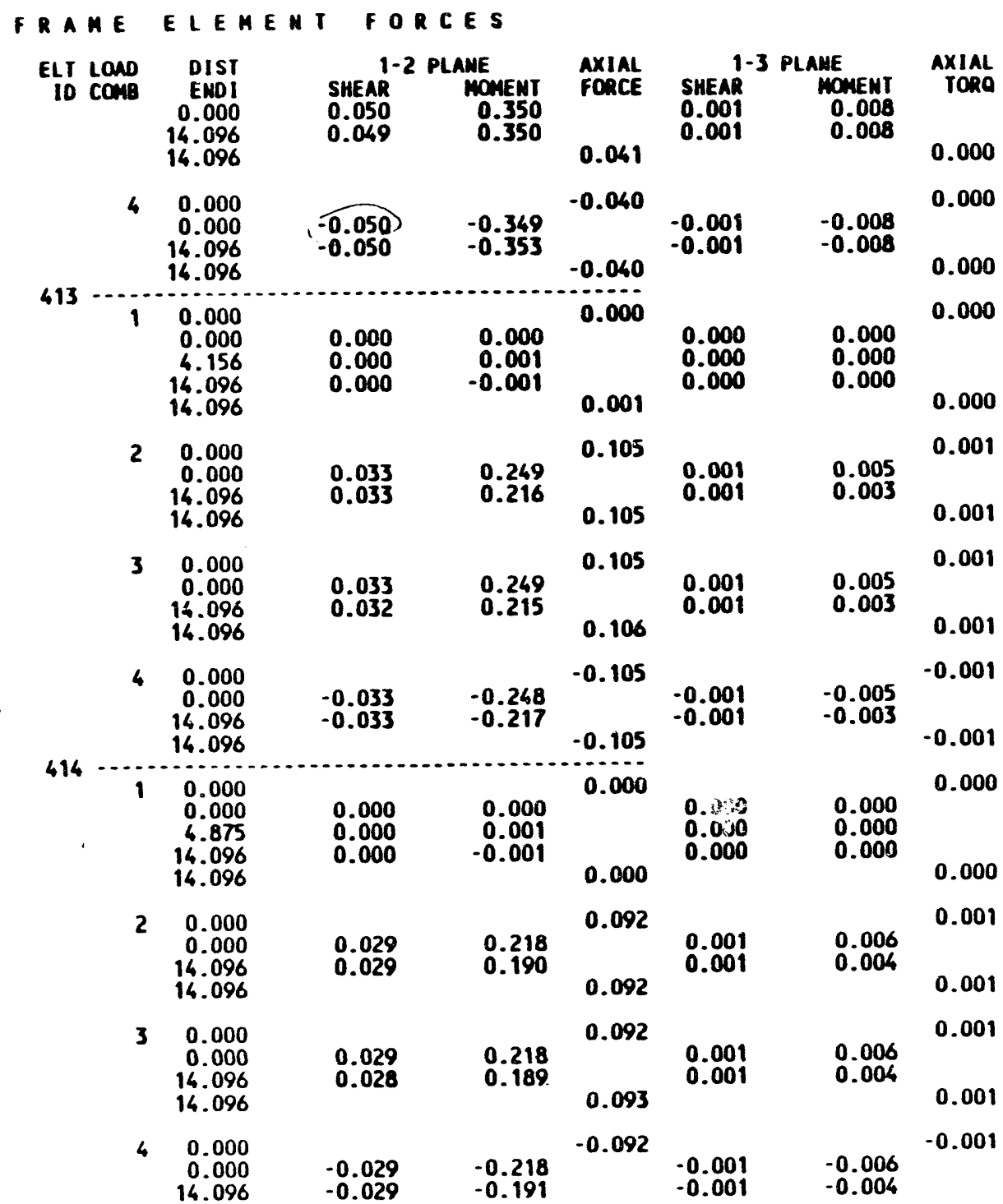

PROGRAM:SAP90/FILE:CM-A-04.F3F UHC/MPF CAMERA ASSEMBLY MOOEL JOBE: 66458-01 RUN: CH-A-O4

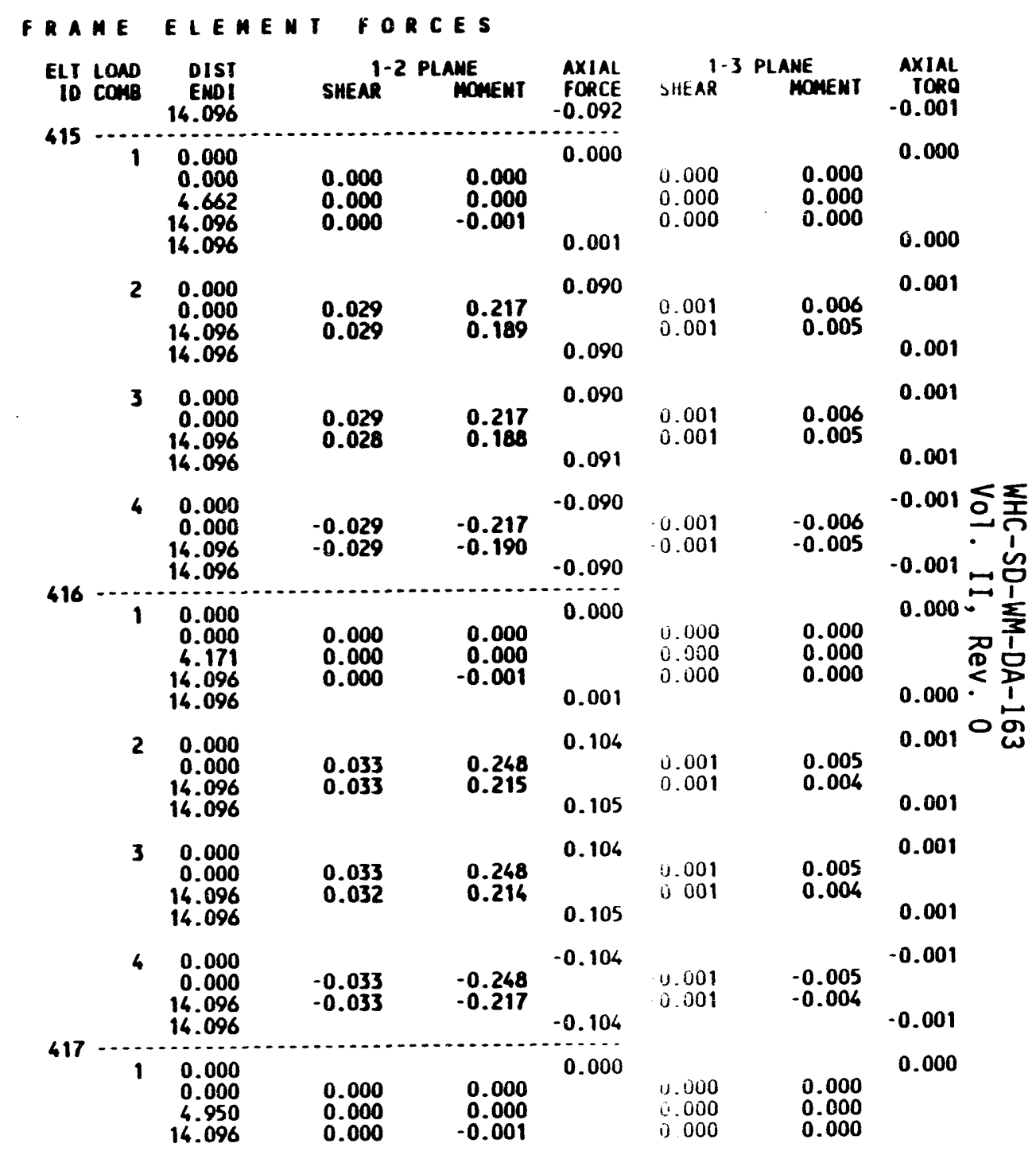


UHCIMPF CAMERA ASSEMBLY MODEL JO9: 66458-01 RLW: CH-A-04

\begin{tabular}{|c|c|c|c|c|c|c|c|}
\hline ELI LOAD & $\begin{array}{r}\text { DISI } \\
\text { ENDI } \\
14.096\end{array}$ & SHEAR & $\begin{array}{l}\text { LAME } \\
\text { MONEMT }\end{array}$ & $\begin{array}{l}\text { AXIAL } \\
\text { FORCE } \\
0.001\end{array}$ & SHEAR & $\begin{array}{l}\text { PLAME } \\
\text { MOMENT }\end{array}$ & $\begin{array}{l}\text { AXIAL } \\
\text { TORO } \\
0.000\end{array}$ \\
\hline 2 & $\begin{array}{r}0.000 \\
0.000 \\
14.096 \\
14.096\end{array}$ & $\begin{array}{l}0.034 \\
0.033\end{array}$ & $\begin{array}{l}0.224 \\
0.251\end{array}$ & $\begin{array}{l}0.114 \\
0.115\end{array}$ & $\begin{array}{l}0.001 \\
0.001\end{array}$ & $\begin{array}{l}0.005 \\
0.007\end{array}$ & $\begin{array}{l}0.001 \\
0.001\end{array}$ \\
\hline 3 & $\begin{array}{r}0.000 \\
0.000 \\
14.096 \\
14.096\end{array}$ & $\begin{array}{l}0.034 \\
0.033\end{array}$ & $\begin{array}{l}0.224 \\
0.250\end{array}$ & $\begin{array}{l}0.115 \\
0.116\end{array}$ & $\left(\begin{array}{l}0.001 \\
0: 001\end{array}\right)$ & $\begin{array}{l}0.005 \\
0.007\end{array}$ & $\begin{array}{l}0.001 \\
0.001\end{array}$ \\
\hline 4 & $\begin{array}{r}0.000 \\
0.000 \\
14.096 \\
14.096\end{array}$ & $\begin{array}{l}-0.034 \\
-0.034\end{array}$ & $\begin{array}{l}-0.224 \\
-0.252\end{array}$ & $\begin{array}{l}-0.116 \\
-0.116\end{array}$ & $\begin{array}{l}-0.001 \\
-0.001\end{array}$ & $\begin{array}{l}-0.005 \\
-0.007\end{array}$ & $\begin{array}{l}-0.001 \\
-0.001\end{array}$ \\
\hline 1 & $\begin{array}{r}0.000 \\
0.000 \\
6.640 \\
14.096 \\
14.096\end{array}$ & $\begin{array}{l}0.000 \\
0.000 \\
0.000\end{array}$ & $\begin{array}{r}0.000 \\
0.000 \\
-0.001\end{array}$ & 0.000 & $\begin{array}{l}0.000 \\
0.000 \\
0.000\end{array}$ & $\begin{array}{l}0.000 \\
0.000 \\
0.000\end{array}$ & 0.000 \\
\hline 2 & $\begin{array}{r}0.000 \\
0.000 \\
14.096 \\
14.096\end{array}$ & $\begin{array}{l}0.031 \\
0.030\end{array}$ & $\begin{array}{l}0.203 \\
0.227\end{array}$ & $\begin{array}{l}0.093 \\
0.094\end{array}$ & $\begin{array}{l}0.001 \\
0.001\end{array}$ & $\begin{array}{l}0.006 \\
0.006\end{array}$ & $\begin{array}{l}0.001 \\
0.001\end{array}$ \\
\hline 3 & $\begin{array}{r}0.000 \\
0.000 \\
14.096 \\
14.096\end{array}$ & $\begin{array}{l}0.031 \\
0.030\end{array}$ & $\begin{array}{l}0.202 \\
0.227\end{array}$ & $\begin{array}{l}0.093 \\
0.094\end{array}$ & $\begin{array}{l}0.001 \\
0.001\end{array}$ & $\begin{array}{l}0.004 \\
0.006\end{array}$ & $\begin{array}{l}0.001 \\
0.001\end{array}$ \\
\hline 4 & $\begin{array}{r}0.000 \\
0.000 \\
14.096 \\
14.096\end{array}$ & $\begin{array}{l}-0.031 \\
-0.031\end{array}$ & $\begin{array}{l}-0.203 \\
-0.228\end{array}$ & $\begin{array}{l}-0.093 \\
-0.093\end{array}$ & $\begin{array}{l}-0.001 \\
-0.001\end{array}$ & $\begin{array}{l}-0.004 \\
-0.006\end{array}$ & $\begin{array}{l}-0.001 \\
-0.001\end{array}$ \\
\hline 1 & $\begin{array}{r}0.000 \\
0.000 \\
6.053 \\
14.096 \\
14.096\end{array}$ & $\begin{array}{l}0.000 \\
0.000 \\
0.000\end{array}$ & $\begin{array}{r}0.000 \\
0.000 \\
-0.001\end{array}$ & 0.000 & $\begin{array}{l}0.000 \\
0.000 \\
0.000\end{array}$ & $\begin{array}{l}0.000 \\
0.000 \\
0.000\end{array}$ & 0.000 \\
\hline 2 & $\begin{array}{r}0.000 \\
0.000 \\
14.096 \\
14.096\end{array}$ & $\begin{array}{l}0.030 \\
0.029\end{array}$ & $\begin{array}{l}0.198 \\
0.221\end{array}$ & $\begin{array}{l}0.094 \\
0.095\end{array}$ & $\begin{array}{l}0.001 \\
0.001\end{array}$ & $\begin{array}{l}0.004 \\
0.006\end{array}$ & $\begin{array}{l}0.001 \\
0.001\end{array}$ \\
\hline
\end{tabular}

MHC/MPF CAMERA ASSEMBLY HODEL JO8: 66458-01 PROGRAM:SAP90/FILE:CH-A-04.F3F

FRAME ELEMENT FúRES

\begin{tabular}{|c|c|c|c|c|c|c|c|c|}
\hline $\begin{array}{l}\text { ELI LOND } \\
\text { ID CONB }\end{array}$ & $\begin{array}{l}\text { DIST } \\
\text { EMDI }\end{array}$ & SHEAR & $\begin{array}{l}\text { ANE } \\
\text { MONENT }\end{array}$ & $\underset{\text { FXIAL }}{\text { FORCE }}$ & SHEAR & $\begin{array}{l}\text { PLAME } \\
\text { MOMENT }\end{array}$ & $\begin{array}{r}\text { AXIALL } \\
\text { TORO }\end{array}$ & \\
\hline 3 & $\begin{array}{r}0.000 \\
0.000 \\
14.096 \\
14.096\end{array}$ & $\begin{array}{l}0.030 \\
0.029\end{array}$ & $\begin{array}{l}0.197 \\
0.220\end{array}$ & $\begin{array}{l}0.094 \\
0.096\end{array}$ & $\begin{array}{l}0.001 \\
0.001\end{array}$ & $\begin{array}{l}0.004 \\
0.006\end{array}$ & $\begin{array}{l}0.001 \\
0.001\end{array}$ & \\
\hline 4 & $\begin{array}{r}0.000 \\
0.000 \\
14.096 \\
14.096\end{array}$ & $\begin{array}{l}-0.030 \\
-0.030\end{array}$ & $\begin{array}{l}-0.198 \\
-0.222\end{array}$ & $\begin{array}{l}-0.095 \\
-0.095\end{array}$ & $\begin{array}{l}-0.001 \\
-0.001\end{array}$ & $\begin{array}{l}-0.004 \\
-0.006\end{array}$ & $\begin{array}{l}-0.001 \\
-0.001\end{array}$ & \\
\hline 1 & $\begin{array}{r}0.000 \\
0.000 \\
5.455 \\
14.096 \\
16.096\end{array}$ & $\begin{array}{l}0.000 \\
0.000 \\
0.000\end{array}$ & $\begin{array}{r}0.000 \\
0.000 \\
-0.001\end{array}$ & $\begin{array}{l}0.000 \\
0.001\end{array}$ & $\begin{array}{l}0.000 \\
0.000 \\
0.000\end{array}$ & $\begin{array}{l}0.000 \\
0.000 \\
0.000\end{array}$ & 0.000 & \\
\hline 2 & $\begin{array}{r}0.000 \\
0.000 \\
14.096 \\
14.096\end{array}$ & $\begin{array}{l}0.034 \\
0.033\end{array}$ & $\begin{array}{l}0.223 \\
0.251\end{array}$ & $\begin{array}{l}0.107 \\
0.107\end{array}$ & $\begin{array}{l}0.001 \\
0.001\end{array}$ & $\begin{array}{l}0.004 \\
0.006\end{array}$ & $\begin{array}{l}0.001 \\
0.001\end{array}$ & 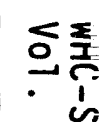 \\
\hline 3 & $\begin{array}{r}0.000 \\
0.000 \\
14.096 \\
14.096\end{array}$ & $\begin{array}{l}0.036 \\
0.033\end{array}$ & $\begin{array}{l}0.223 \\
0.250\end{array}$ & $\begin{array}{l}0.107 \\
0.108\end{array}$ & $\begin{array}{l}0.001 \\
0.001\end{array}$ & $\begin{array}{l}0.004 \\
0.006\end{array}$ & $\begin{array}{l}0.001 \\
0.001\end{array}$ & 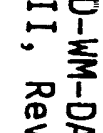 \\
\hline 4 & $\begin{array}{r}0.000 \\
0.000 \\
14.096 \\
14.096\end{array}$ & $\begin{array}{l}-0.034 \\
-0.034\end{array}$ & $\begin{array}{l}-0.224 \\
-0.253\end{array}$ & $\begin{array}{l}-0.107 \\
-0.107\end{array}$ & $\begin{array}{r}-1) .001 \\
-0.001\end{array}$ & $\begin{array}{l}-0.004 \\
-0.006\end{array}$ & $\begin{array}{l}-0.001 \\
-0.001\end{array}$ & o $\stackrel{1}{\sigma}$ \\
\hline
\end{tabular}


UHC/MPF CAMERA ASSEMBLY MOOEL JOB:: 66458-01 RUW: CH-A-04

\section{FRAME ELEMENT FORCES}

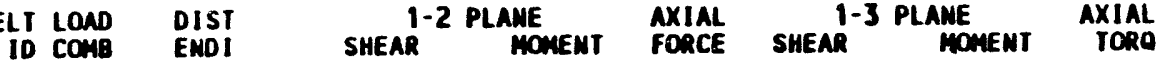

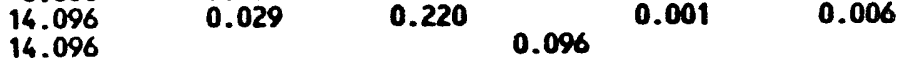

$4 \quad 0.000 \quad 0.030 \quad-0.198-0.095$ $\begin{array}{lllll}0.000 & -0.030 & -0.198 & -0.001 & -0.004\end{array}$

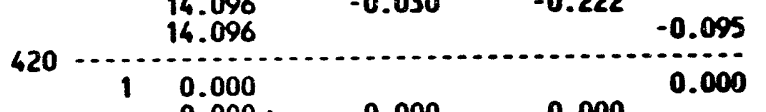

$\begin{array}{rrr}5.455 & 0.000 & 0.000 \\ 14.096 & 0.000 & -0.001\end{array}$

14.096

20.000 0.000
14.096 14.096
14.096

$\stackrel{\square}{a}$

0.001

0.001

$-0.00$

$-0.001$

0.000

$0.000 \quad 0.000$

$0.000 \quad 0.000$

0.107

$\begin{array}{ll}0.034 & 0.223 \\ 0.033 & 0.251\end{array}$

$\begin{array}{ll}0.034 & 0.223 \\ 0.033 & 0.250\end{array}$

0.107

0.00

0.107

0.108

0.001

0.001

0.001
$30.000 \quad 0.094$

$\begin{array}{rrrrrr}0.000 & 0.030 & 0.197 & 0.094 & 0.001 & 0.004 \\ 14.096 & 0.029 & 0.220 & 0.001 & 0.006\end{array}$

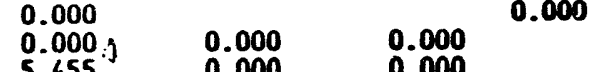

0.000 0.000
16.096 14.096
14.096

$4 \quad 0.000$ 0.000
14.096 14.096
14.096

0.000

\section{LIGHT} C, Ullort

\begin{tabular}{|c|c|c|c|c|}
\hline & $\begin{array}{l}14.753 \\
15.556\end{array}$ & 0.000 & 0.000 & 0.000 \\
\hline 2 & $\begin{array}{r}0.000 \\
0.803 \\
14.753 \\
15.556\end{array}$ & $\begin{array}{l}0.003 \\
0.003\end{array}$ & $\begin{array}{l}0.009 \\
0.027\end{array}$ & $\begin{array}{l}0.006 \\
0.006\end{array}$ \\
\hline 3 & $\begin{array}{l}0.000 \\
0.803\end{array}$ & 0.002 & 0.009 & 0.005 \\
\hline
\end{tabular}
14.753

$4 \quad 0.000$
PAGE 62
CM-A-04.F $3 F$

$-0.107$

$\begin{array}{ll}-0.034 & -0.224 \\ -0.034 & -0.253\end{array}$

$-0.107$

0.107

$-0.001$

$0.001 \quad-0.002$

$\begin{array}{lll}0.004 & \end{array}$

$\begin{array}{ll}0.016 & 0.103 \\ 0.014 & 0.103\end{array}$

0.004

0.00

$\begin{array}{ll}0.016 & 0.102 \\ 0.016 & 0.101\end{array}$

0.005

0.006

$-0.001$

$-0.001$

0.000

0.000

0.004

0.004 $\begin{array}{ll}0.001 & -0.001\end{array}$
UHC/MPF CAMERA ASSEMBLY MODEL JOB.:

FRAME ELEMENT FORCES

ELI LOND DIST TI-2 PLAME MAXIAL $1-3$ PLAME

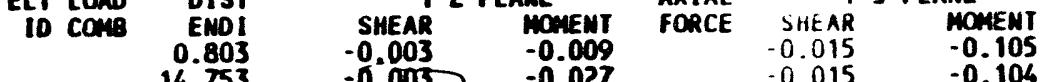

502

$\begin{array}{llll}0.000 & 0.000 & 0.000 & 0.000\end{array}$

$\begin{array}{llllll}0.803 & 0.000 & 0.000 & 0.001 & -0.002 & 0.000\end{array}$

$\begin{array}{rrrrr}7.838 & 0.000 & 0.000 & 0.000 & 0.002 \\ 14.753 & 0.000 & 0.000 & -0.001 & 0.001\end{array}$

0.000

$\begin{array}{llllll}\text { LIGHT } 2 & 0.000 & 0.000 & 0.006 & 0.002\end{array}$

0.017

0.004

$\begin{array}{ll}\text { culpoRT } & 14.753 \\ & 15.556\end{array}$

$0.000 \quad 0.004$

$3 \quad 0.000$

0.803
16.753

16.753
15.556

0.002
0.002

$\begin{array}{llll}0.000 & 0.006 & 0.002 & 0.017 \\ 0.000 & 0.004 & & 0.015\end{array}$

$4 \quad 0.000$

0.004

0.002

0.003

0.000

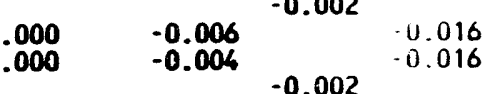

15.556

0.002

0.000

1.000

\begin{tabular}{|rrrr} 
& 0.803 & 0.000 & 0.000 \\
LKGT & 7.814 & 0.000 & 0.000 \\
& 14.753 & 0.000 & 0.000
\end{tabular}

$2 \quad 0.000$

0.803
16.753
15.556

$\begin{array}{ll}0.000 & 0.000\end{array}$

0.001

0.000
0.001

0.003

0.001

0.0

$3 \quad 0.000$

0.803
14.753

$\begin{array}{ll}0.003 & 0.023 \\ & 0.019\end{array}$

0.001

(1) 15.556

$\begin{array}{ll}0.003 & 0.022 \\ 0.003 & 0.019\end{array}$

0.001

$4 \quad 0.000$

0.803
14.753

0.000

$\begin{array}{ll}-0.003 & -0.023 \\ -0.019\end{array}$
0.000

0.000

0.000
504
GAS
PIPE
0.1110 .004

0.004

0.109

0.109

0.004 동포 $1^{-0.006} \cdot$ i $\begin{array}{ll}-0.112 \\ -0.112 & -0.004: 04\end{array}$ 0.000 邓 -0.092
0.001

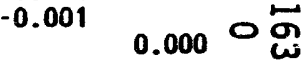

0.003

0.082 0.003 0.003 0.080 0.003 $-0.003$

0.083
-0.083 $-0.003$ 0.000 

UHC/MPF CAMERA ASSEMBLY WODEL JOBN: 66458-01 RUN: CH-A-04

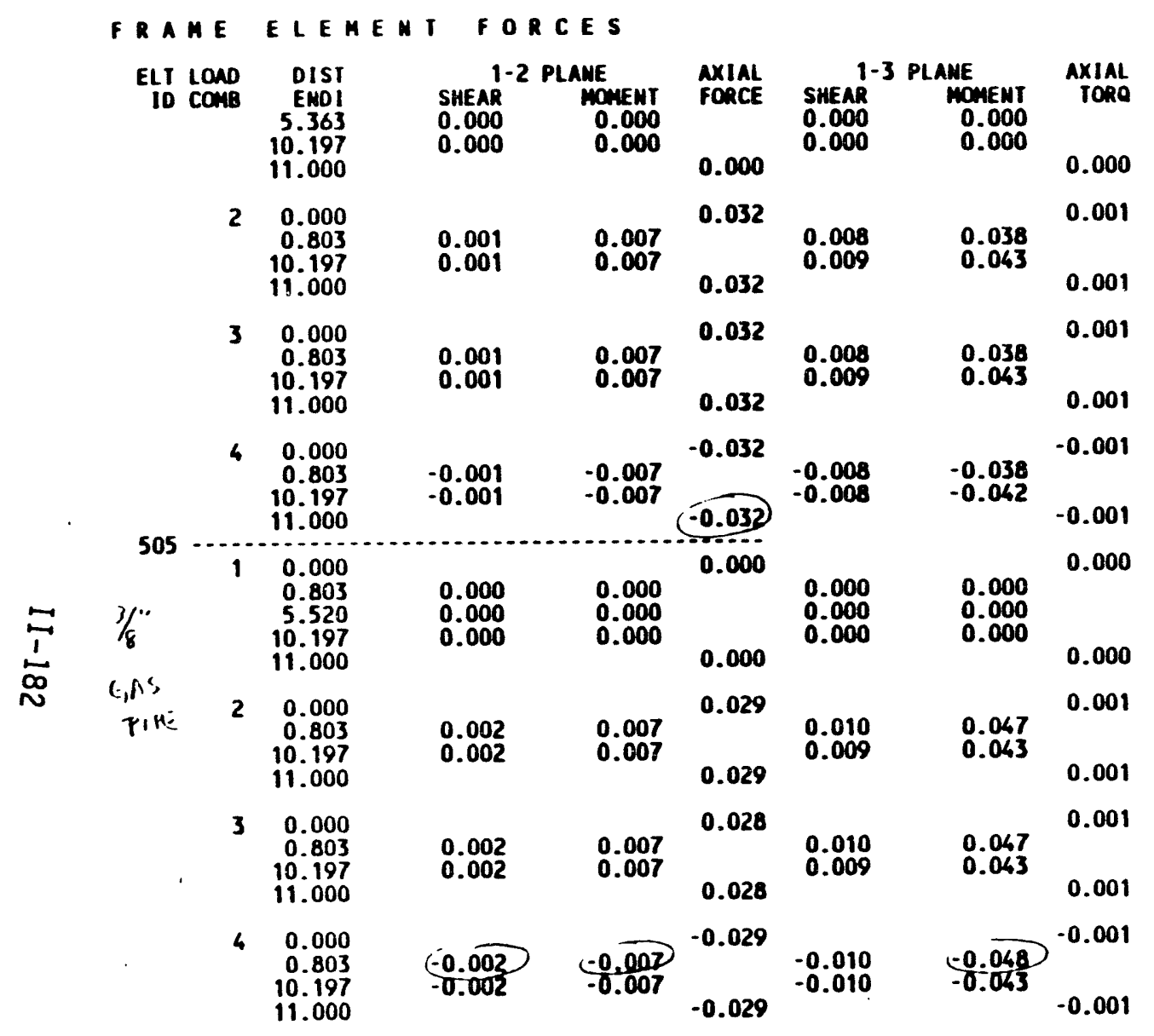


WHC-SD-WM-DA-163
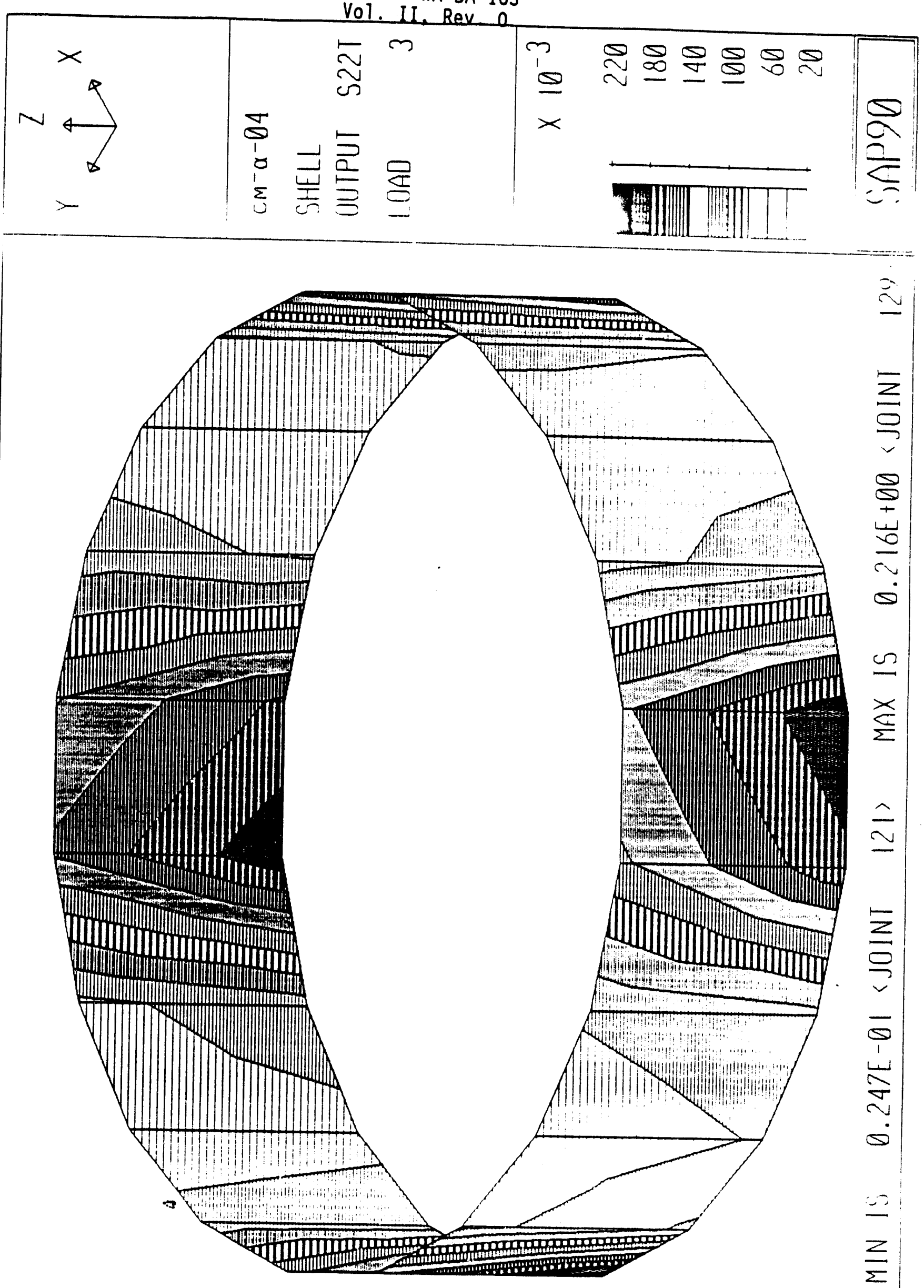

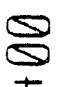

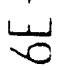

iv

$\Phi$

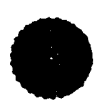

m.m.

m

m.nIm)

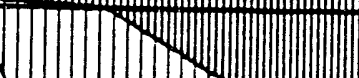

(I)

$1+\ldots$ 


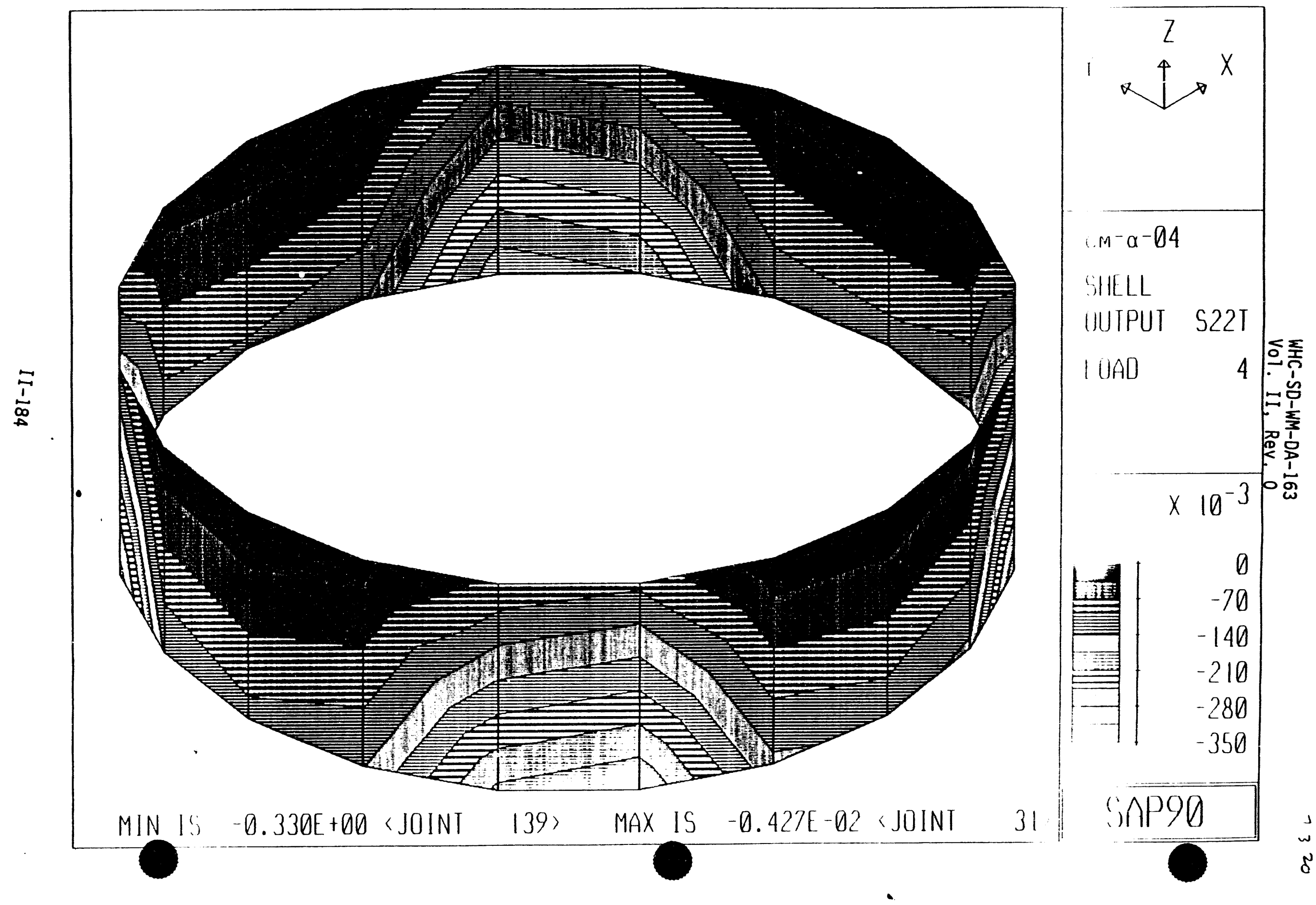




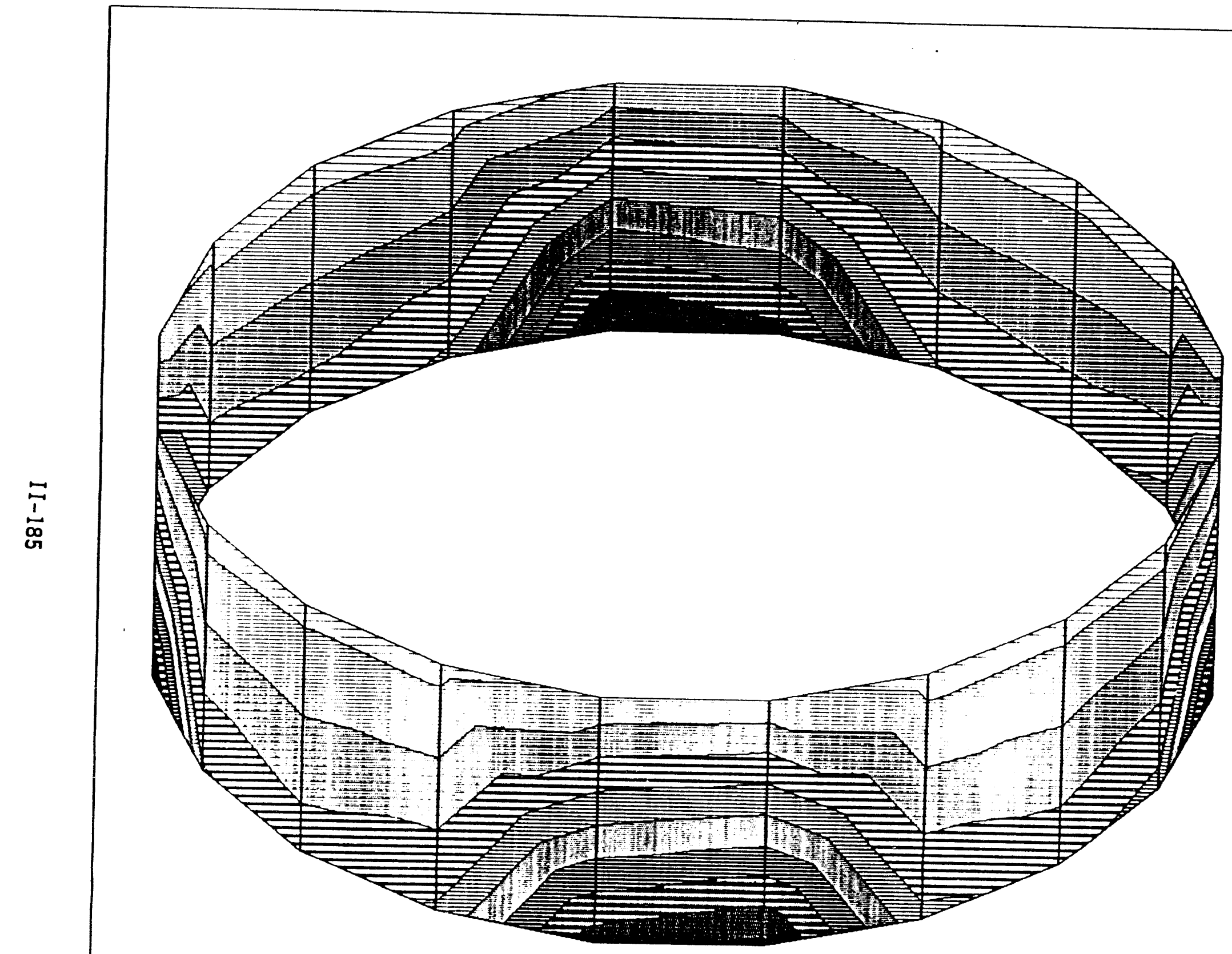

MIN IS $-0.262 E-01<J 0 I N T$ 40> MAX IS.

$0.481 E+00<J O I N T$ 139 


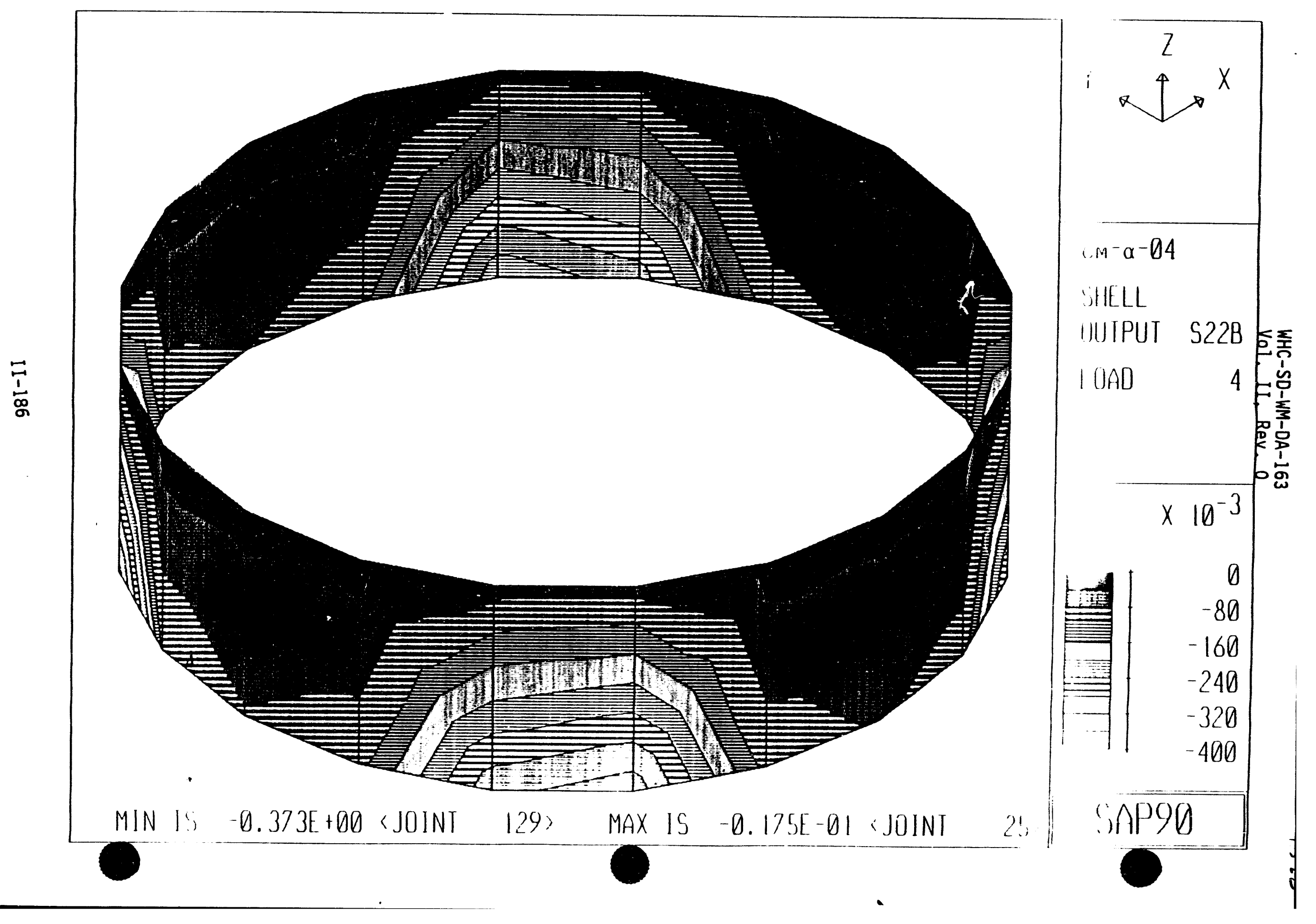


WHC-SD-WM-DA-163

Vol. II, Rev. 0
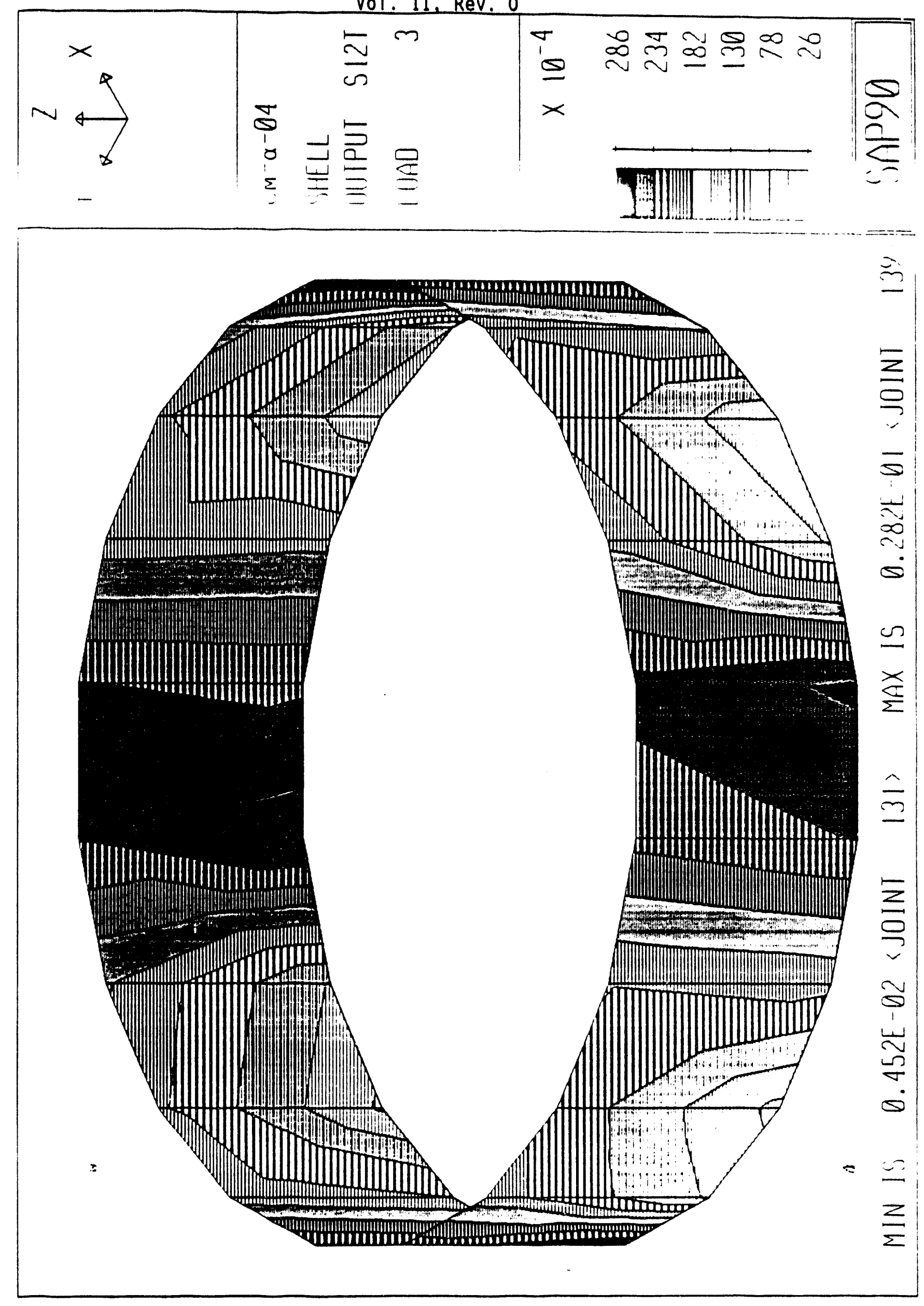


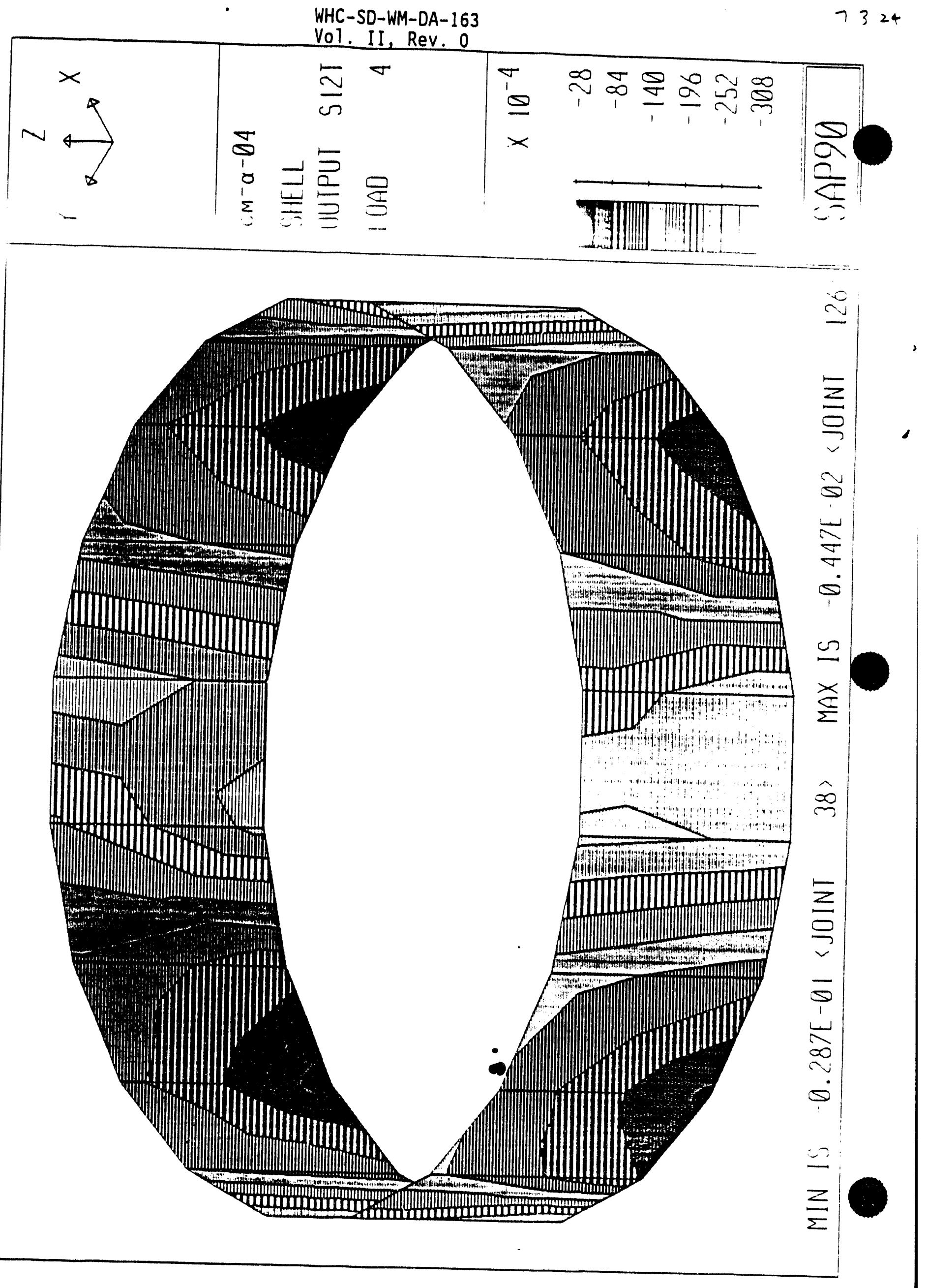


WHC-SD-WM-DA-163

Vol. II, Rev. 0
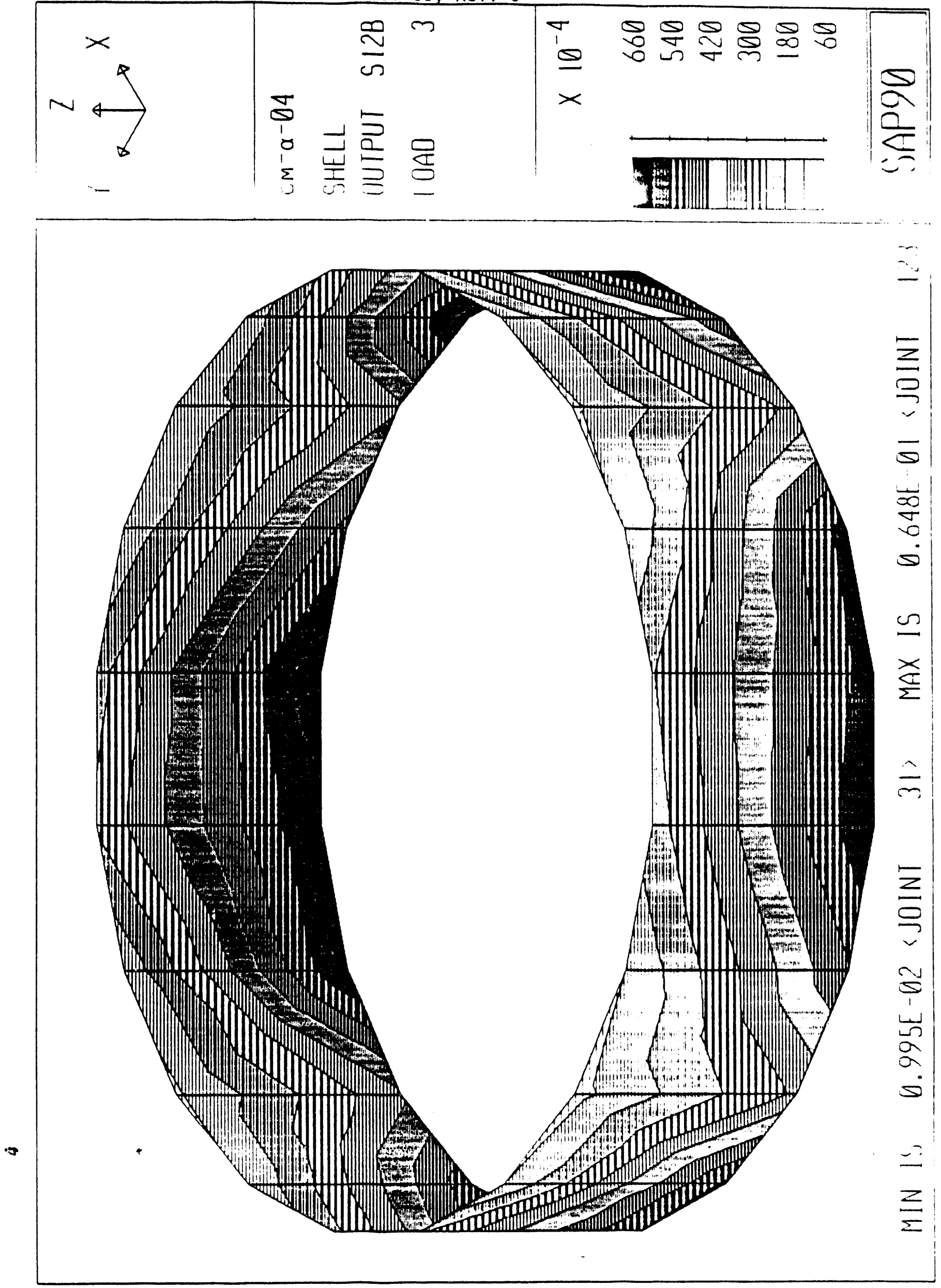


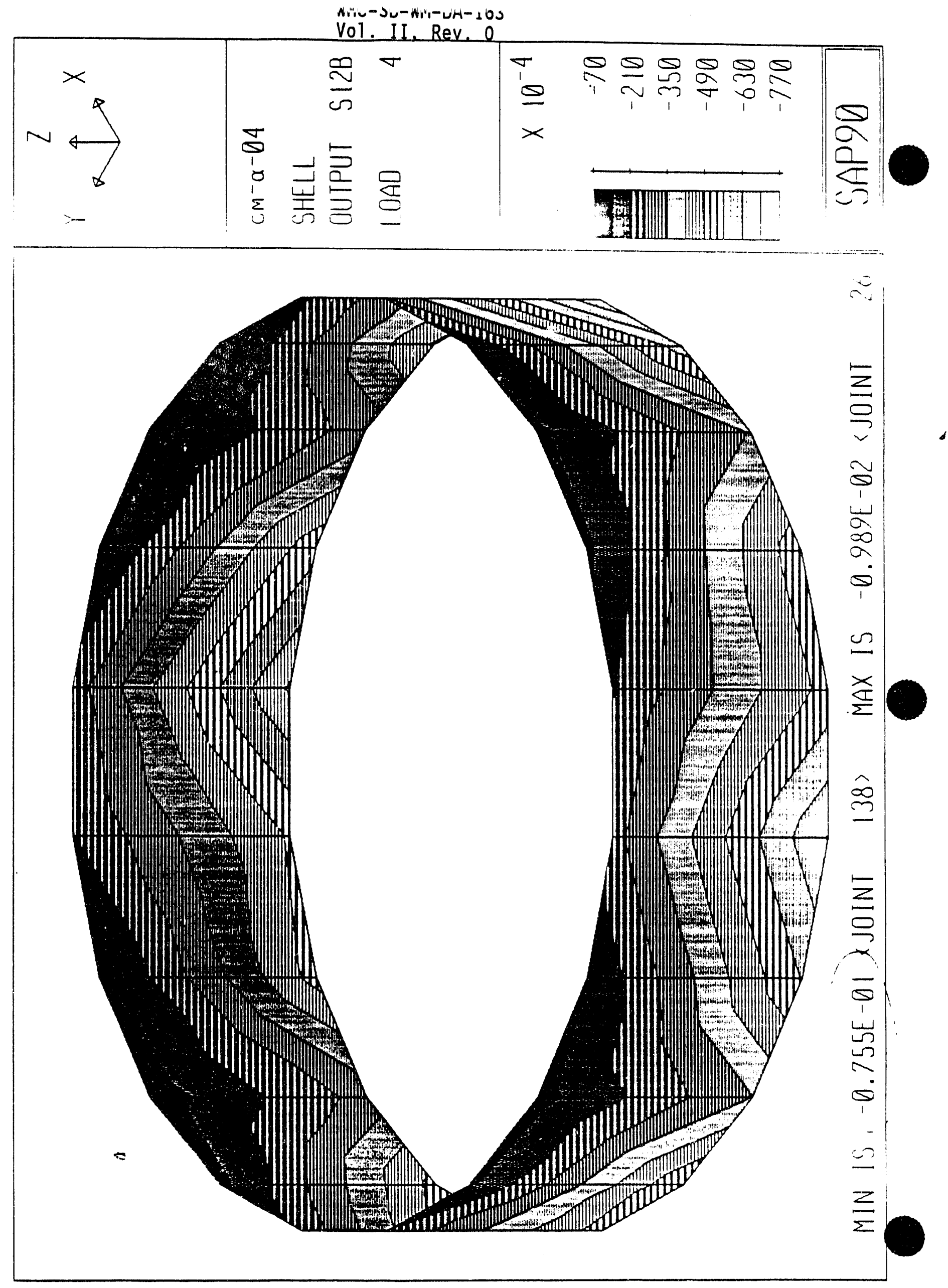




\section{URS CONSULTANTS}

WHC-SD-WM-DA-163

Vol. II, Rev. 0

Job \#:66481-01 Job: WHC/MPF CAMERA ASSEMBLY ANALYSIS

Client: WHC Subject: SAP9O ANALYSIS DOCUMENTATION
Sheet No. \& 0

By PuL Date 6/8/94 Chk'd U/M Date $7 / 1 \geqslant /: y$

\section{TABLE OF CONTENTS}

\section{SECTION CONTENTS}

8.1

8.2
General Discussion

Summary of Displacements
PAGE NO.

б.11

$8.21-82.7$ 


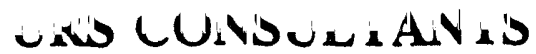

WHC-SD-WM-DA-163

Vol. II, Rev. 0

Job \#:66481-01 Job: WHC/MPF CAMERA ASSEMBLY ANALYSIS

Sheet No. 81.1

Client: WHC Subject: SAP9O ANALYSIS DOCUMENTATION

By PMU Date $7 / 25 / 94$

Chk'd RIK Date 8:3:94

\subsection{General Discussion}

To determine the minimum clearance that needs to be provided $t: 2$ the installed camera to avoid interference with the riser and/or adjacent equipment already installed, results from SAP90 Model $B$ were used. Model $B$ is the same as Model $A$, except, it also included the amplification from the MPF riser stick model.

To be conservative, the center of gravity of the 128 lbs camera was located at the far corner coordinates of the camera geometry specified in the reference drawings (see Attachment A). 


\section{URS CONSULTANTS}

WHC-SD-WM-DA-163

Vol. II, Rev. 0

Sheet No. 3.2 .1

Job \#:66481-01 Job: WHC/MPF CAMERA ASSEMBLY ANALYSIS

By Pu Date $6 / 8 / 94$

Client: WHC Subject: SAP90 ANALYSIS DOCUMENTATION

Chk'd Y/n Date $z, Y$;

8.2 Summary of Displacements

The displacements at the center of gravity of the camera are given below. As for comparison purposes, displacements from both Model $A$ and Model $B$ were extracted from the SAP9O CM-A-04.SOL and CM-B-01.SOL files, respectively.

\section{Direction}

$X$ (horizontal)

$Y$ (horizontal)

$z$ (vertical)
Model A

Disp (inches)

0.3708

0.3747

0.00625
Model B

Disp (inches)

0.377

0.382

0.00732

As shown here, there are only slight differences between the displacements from Model $A$ and Model $B$. The added MPF riser stick model was quite rigid. 
06/02/1994 16:31 Filename: PISP-A

PAGE 121 WHC/MPF CAMERA ASSEMBLY MODEL JOBN: 66458-01 RUW: CH-A-04 JOINT DISPLACEMEMTS

LOND CONBINATION 1 - DISPLACEMENTS "U" AMD ROTATIONS "R"

$\begin{array}{lllllll}2001 & -0.006597 & 0.007191 & -0.001297 & 0.000058 & 0.000059 & 0.000021\end{array}$

LOND COMBINATION 2 - DISPLACEMENTS "UM" AND ROTATIONS MR"

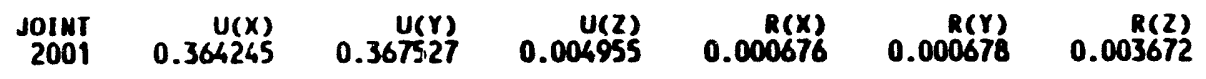

LOAD CONBIMATION 3 - DISPLACEMEMTS WUM AND ROTATIONS "R"

$\begin{array}{rrrrrrr}\text { JolnT } & U(X) & U(Y) & U(z) & R(X) & R(Y) & R(z) \\ 2001 & 0.357649 & 0.374718 & 0.003657 & 0.000734 & 0.000737 & 0.003692\end{array}$

LOAD COMBIMATION 4 - DISPLACEMENTS "UM AMD ROTATIONS WR"

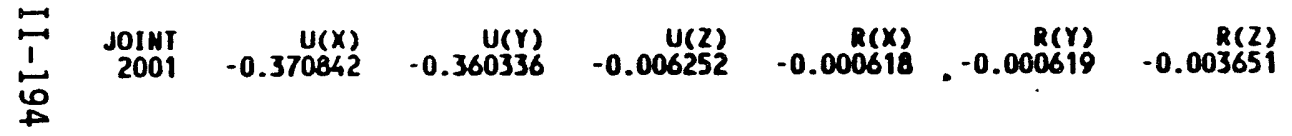

\section{DISPL of CAMERA @ NOW ....}

SAPIO MODEL A (FIXEd at CEnTt, riange plate)

$$
\begin{aligned}
& \frac{\text { ALS DISPL of CAMERA }}{U(X)}=0.3708^{\circ} \\
& U(Y)=0.3747^{\prime \prime} \\
& u(z)=0.00625^{\prime \prime}
\end{aligned}
$$

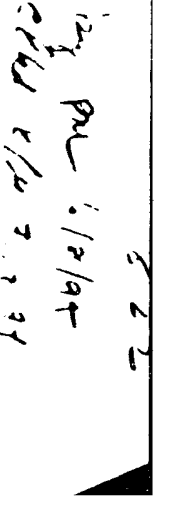




\section{SAP90 MODEL B (INCL RIST). "ENTER of FLANG, PLATE is} UHC/MPF CAMERA ASSEMBLY MODEL JOBN: 66458-01 RUW: CH-B-01

JOINT DISPLACENENTS

LOND COMBIMATION 1 - DISPLACENENTS wU AND ROTATIONS "R" $D L$ $\begin{array}{rrrrrrr}J 01 N T & U(X) & U(Y) & U(Z) & R(X) & R(Y) & R(Z) \\ 299 & -0.1816 E-03 & -0.1867 E-04 & -0.4891 E-03 & 0.5561 E-06 & -0.2816 E-05 & 0.0000 E+00 \\ 2001 & -0.006173 & 0.007293 & -0.001790 & 0.000059 & 0.000056 & 0.000022\end{array}$

LOAD COMBIMATION 2 - DISPLACEMENTS "UN AMD ROTATIONS MR" $E Q$

$\begin{array}{rrrrrrr}J 01 N I & U(x) & U(Y) & U(z) & R(X) & R(Y) & R(z) \\ 299 & 0.001960 & 0.001967 & 0.000167 & 0.000021 & 0.000019 & 0.000001 \\ 2001 & 0.369091 & 0.372765 & 0.005365 & 0.000692 & 0.000691 & 0.003707\end{array}$

LOND COMBImation 3 - Displacenents "u" and rotations mpu $D L+E Q$

\begin{tabular}{|c|c|c|c|}
\hline $\begin{array}{l}\text { JoInT } \\
299\end{array}$ & $\begin{array}{r}U(x) \\
0.001778\end{array}$ & $U(Y)$ & \\
\hline
\end{tabular}

$\begin{array}{rrrrrrr}299 & 0.001778 & 0.001948 & -0.000322 & 0.000021 & 0.000017 & 0.000001 \\ 2001 & 0.362918 & 0.380058 & 0.003575 & 0.000750 & 0.000747 & 0.003729\end{array}$

LOAD COMBIMATION 4 - DISPLACEMENIS "UW AMD ROTAJIONS "R" DL - EQ

$\begin{array}{rrrrrrr}\text { JoINT } & U(x) & U(y) & U(z) & R(x) & R(y) & R(z) \\ 299 & -0.002142 & -0.001986 & -0.000656 & -0.000020 & -0.000022 & -0.000001\end{array}$

$\begin{array}{rrrrrrr}299 & -0.002142 & -0.001986 & -0.000656 & -0.000020 & -0.000022 & -0.000001 \\ 2001 & -0.375263 & -0.365472 & -0.007155 & -0.000633 & -0.000635 & -0.003685\end{array}$ $\frac{\text { ABS DISPL of CAMERA }}{\text { Nude zool }}$

$u(x)=0.369091+0.00617 \%+0.00146$

$=0.377^{\prime \prime}$

$U(Y)=0.372765+0.007243+0001967$

$=0.382^{\prime \prime}$

$u(z)=0.005365+000179+0.000167$

$=0.00732 "$ 


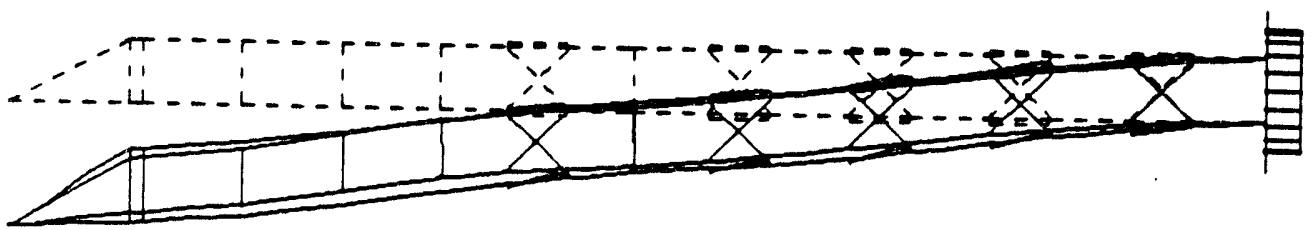

$\begin{array}{ll}5 & 5 \\ 0 & 0 \\ 0 & 0 \\ j & r \\ n & 5 \\ \omega & \end{array}$

$\stackrel{+}{r}$
+
$\stackrel{m}{p}$

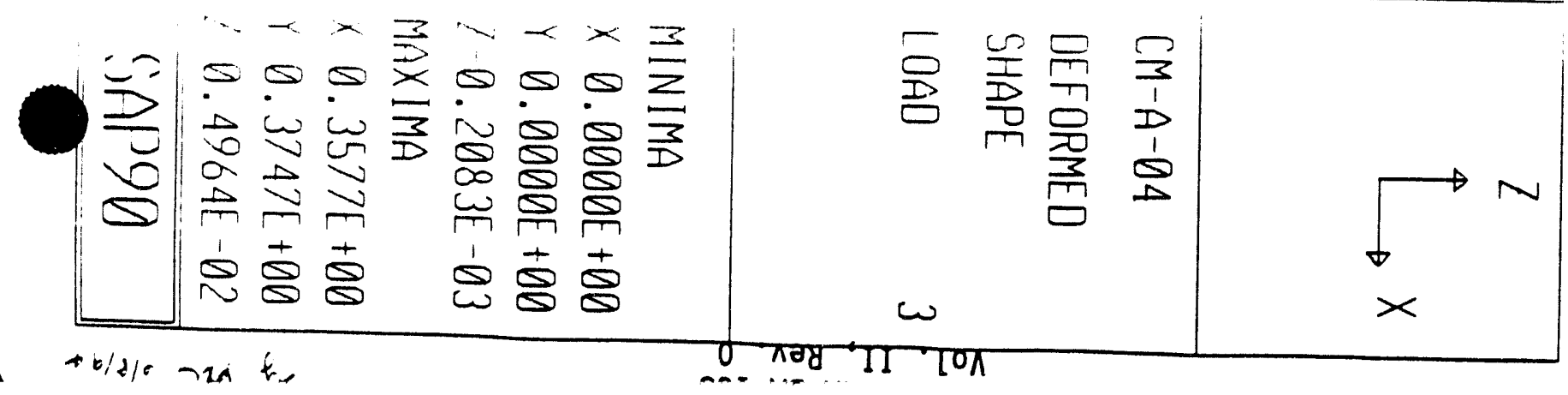


525 WHC-SD-WM-DA-163 ey one 6/5/a4

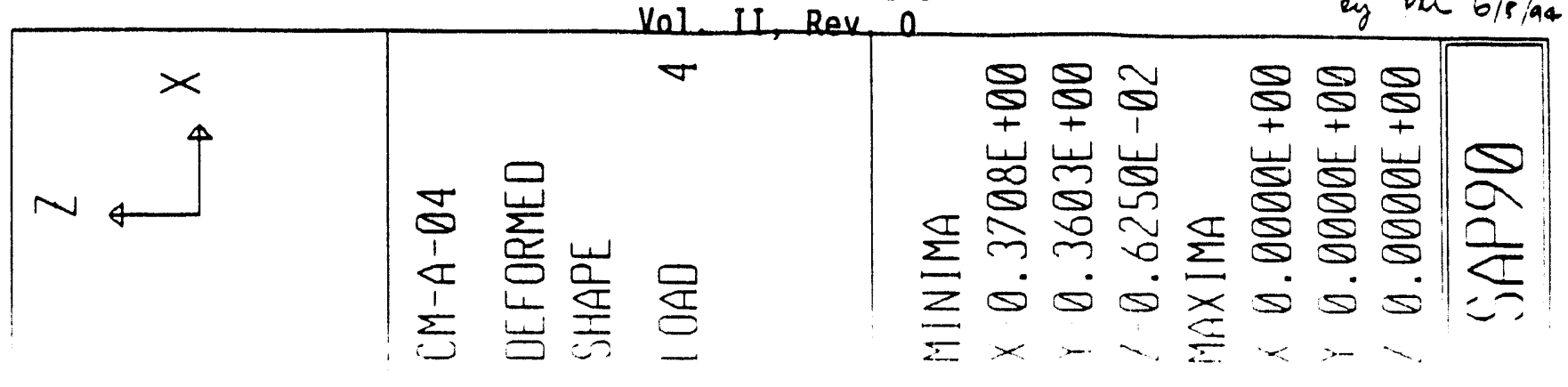

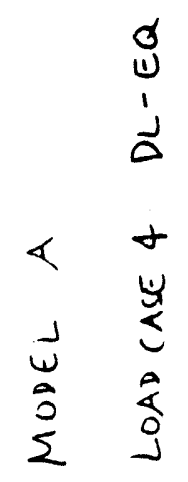

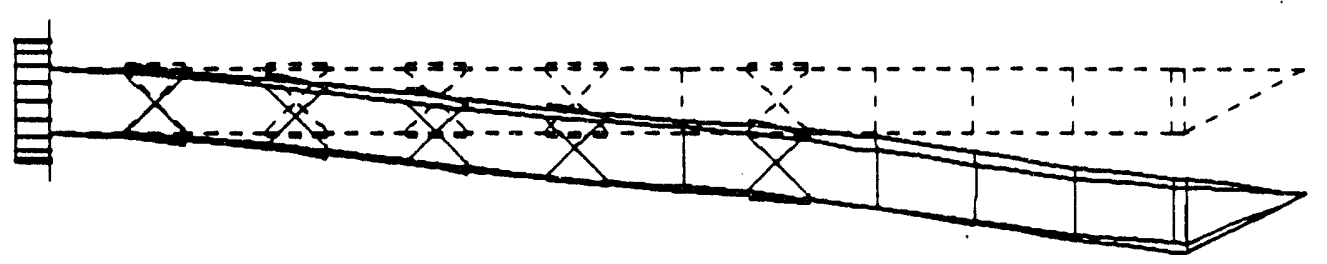


WHC-SD-WM-DA-163

lay lin 6/8/04

Vol. II Rey 0

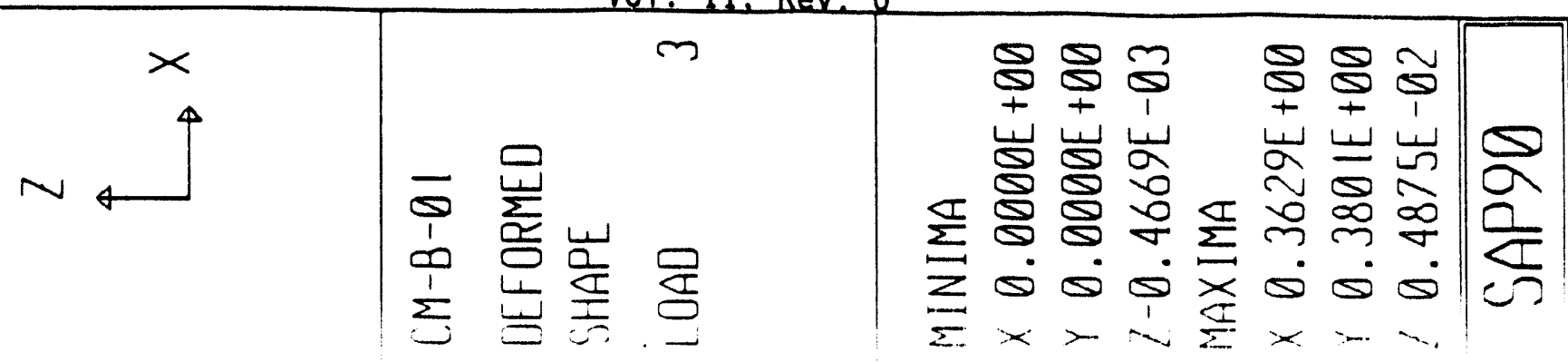

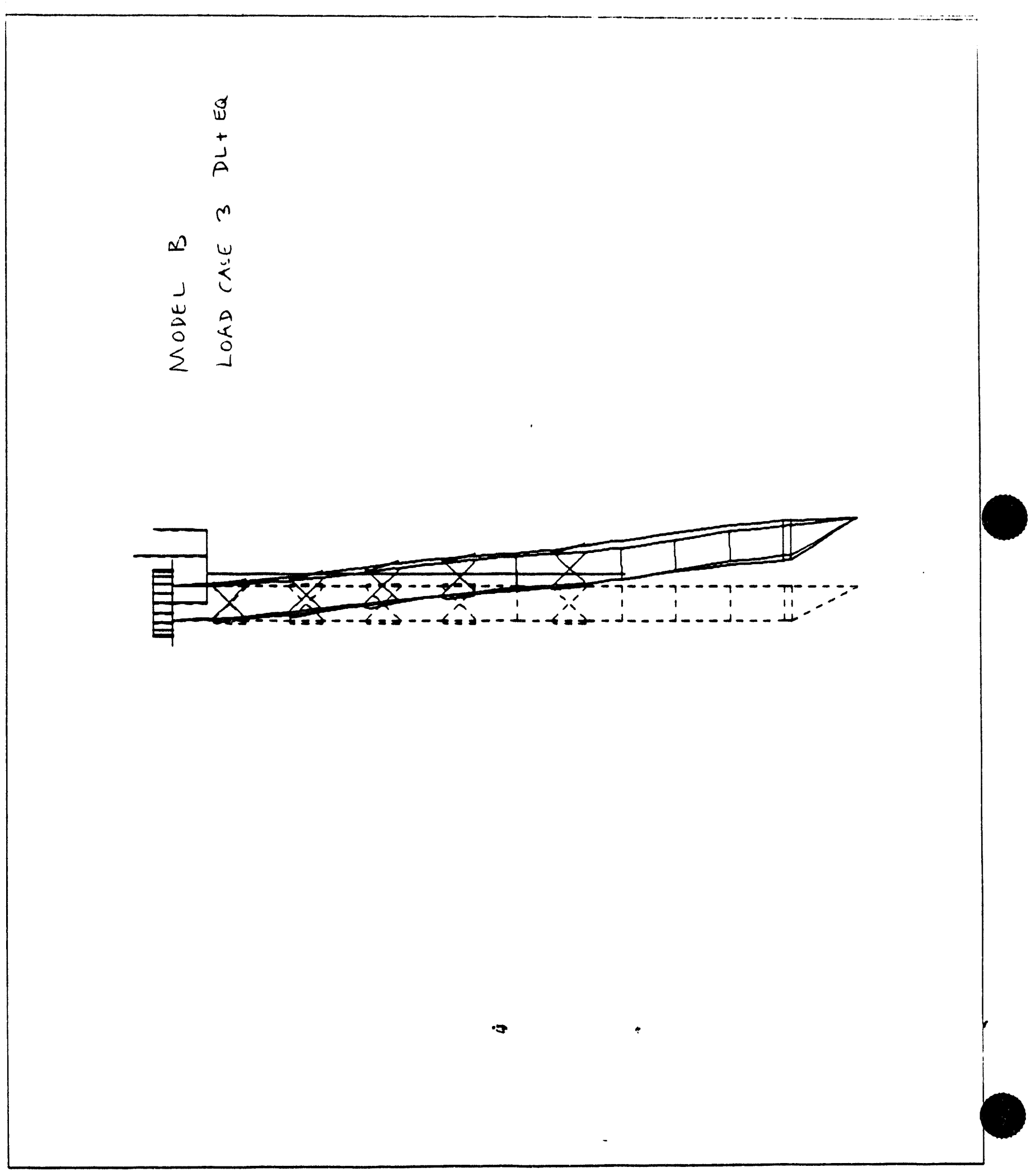




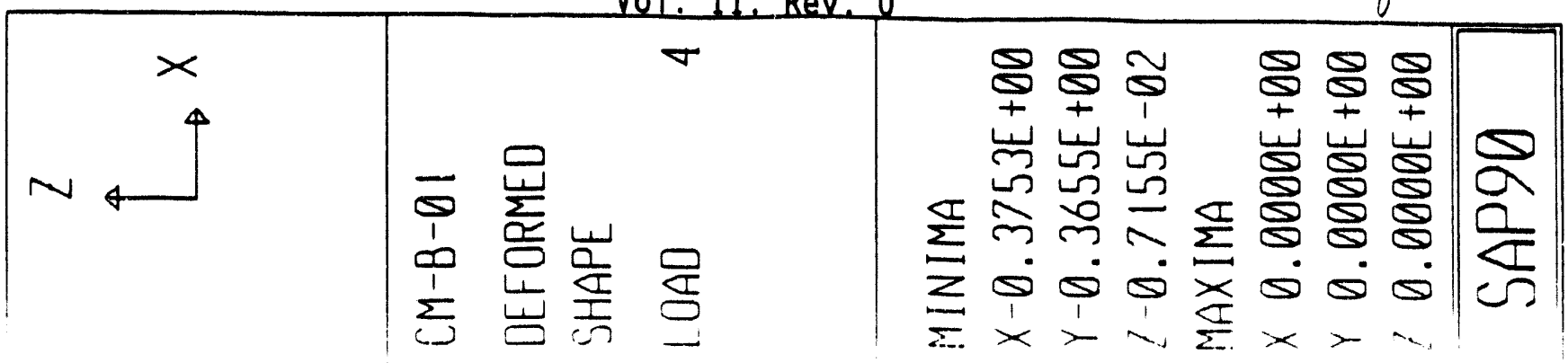
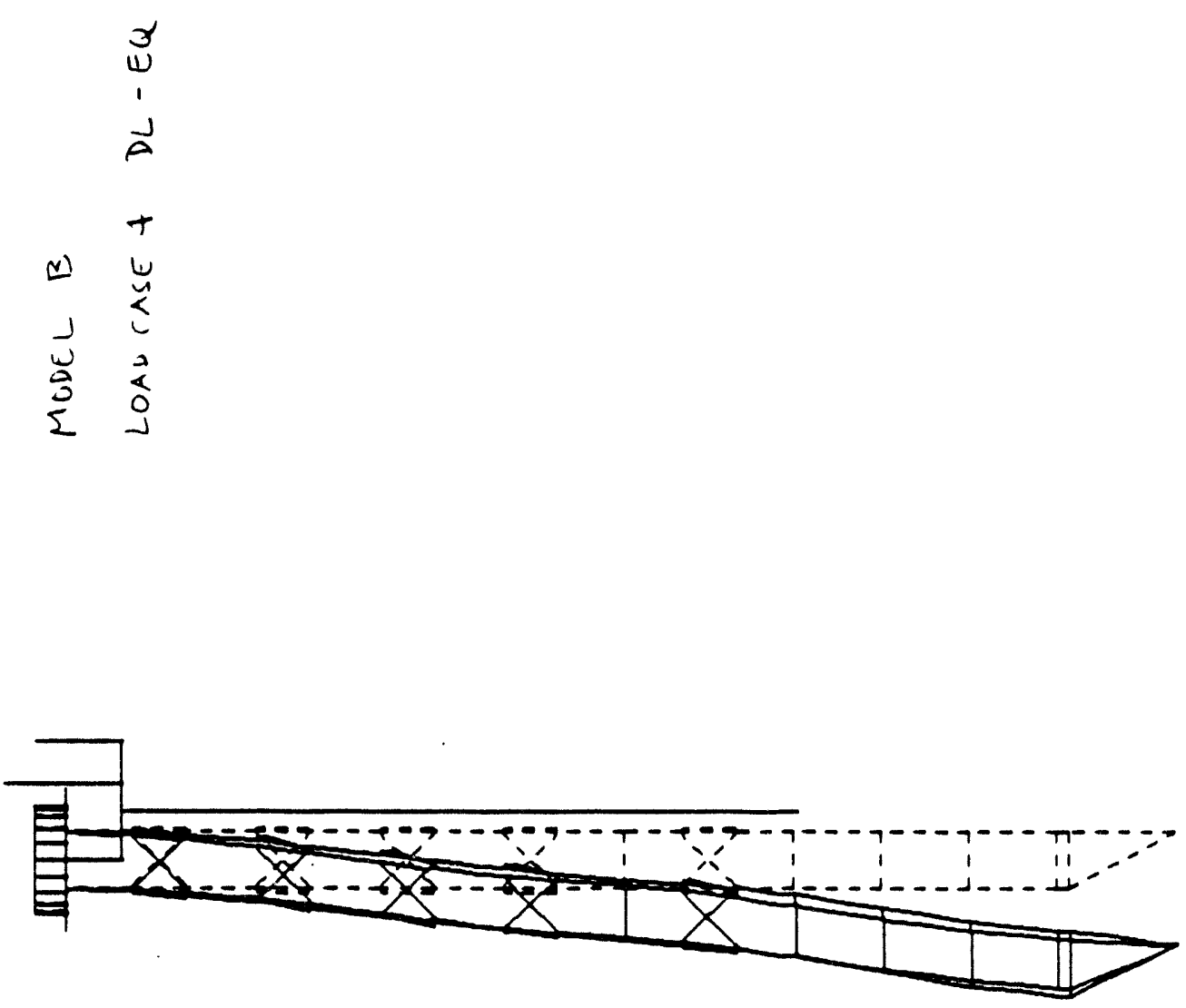
URS CONSULTANTS

WHC-SD-WM-DA-163

Vol. II. Rev. 0

Job \#:66481-01 Job:

WHC/MPF CAMERA ASSEMBLY ANALYSIS

Client: WHC

SAP90 ANALYSIS DOCUMENTATION

Sheet No.

By idf Date 29

Chk'dRIKDate $2 / 3 / 2 x$

\section{SUMMARY}

\section{TABLE OF CONTENTS}

\section{SECTION CONTENTS}

9.1

Summary of stress Ratios

9.2

$9 \cdot 3$
Overall Analysis Conservatism

Recommended Modifications
PAGE NO.

$911 \rightarrow 713$

92

$93.1 \rightarrow 93=$ 
URS CONSULTANTS WHC-SD-WM-DA-163

Job \#:66481-01 Job: WHC/MPF CAMERA ASSEMBLY ANALYSIS

Client: WHC Subject: SAP9O ANALYSIS DOCUMENTATION
Sheet No.

By Pre Date ${ }^{\top}$ ar

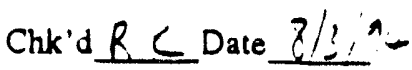

9. 1 Summary of stress Ratios

The stress ratios of each part and lts connections are ziriidit: in Table $9-1$. 
TABLE 9-1: SUMMARY OF STRESS RATIOS

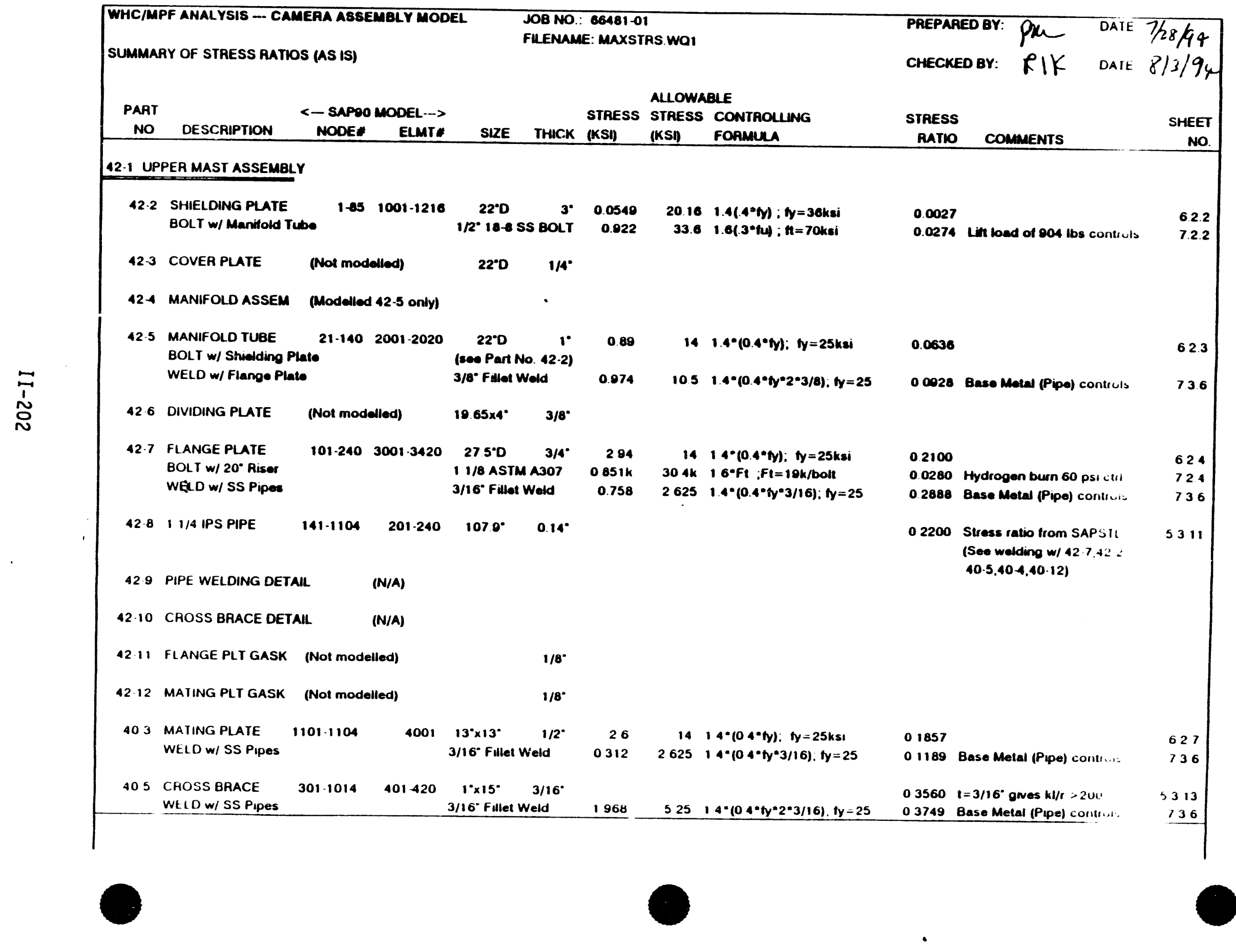


TABLE 9-1: SUMMARY OF STRESS RATIOS (CONTINUED)

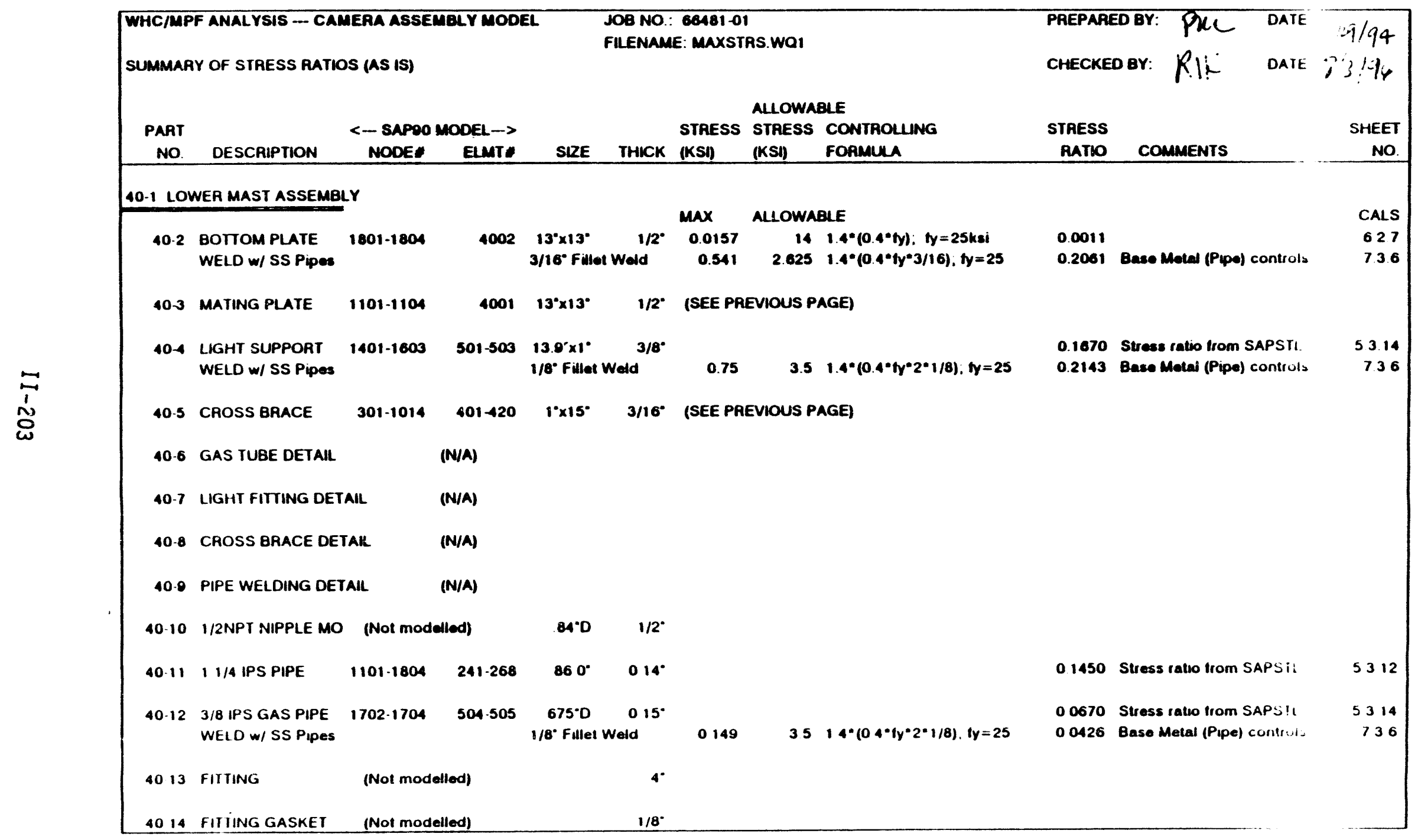




\section{URS CONSULTANTS}

Job $\#: 66481-01$ Job:

WHC/MPF CAMERA ASSEMBLY ANALYSIS

Client: WHC Subject: SAP90 ANALYSIS DOCUMENTATION

WHC-SD-WM-DA-163

Vol. II. Rev. 0
9.2 Overall Analysis Conservatism
Sheet No. $7=$

By Plli- Date Pra/a4

Chk'dRIK Date $8 / 3 / 94$

There are conservatism in this analysis, the main ones are as follows:

1. Response spectra for vertical direction was not scaled to 2/3. $100 \%$ of the Non-safety Class 2 spectrum $10.12 \mathrm{~g}$ ZPA) was used.

2. $2 \%$ damped response spectrum was used in the preliminary analysis. In fact, $5 \%$ damped response spectrum can be used which lower the spectrum by approximately $20 \%$ in the frequency range of .1 to $10 \mathrm{~Hz}$. Note that the first mode frequencies for the entire camera assembly are $3.21 \mathrm{~Hz}$ for Model A and $3.18 \mathrm{~Hz}$ for Model B.

3. For material specified as 300 series SS, 308L specifications was used:

$$
\begin{array}{ll}
\text { Allowable yield stress } & =25 \mathrm{ksi} \\
\text { Tensile strength } & =70 \mathrm{ksi}
\end{array}
$$

4. The center of gravity of the camera roll mechanism (Dwg. 4-11, pg. A.33) is located at the far corner of the unit to create the worst case of eccentricity. 
UTS CONSULTANTS

$$
\begin{aligned}
& \text { WHC-SD-WM-DA-163 } \\
& \text { Vol. II, Rev. } 0
\end{aligned}
$$

Sheet No. 9.3 .1

Job \#:66481-01 Job: WHC/MPF CAMERA ASSEMBLY ANALYSIS

By Pu Date $7 / 29 / 94$

Client: WHC Subject: SAP9O ANALYSIS DOCUMENTATION

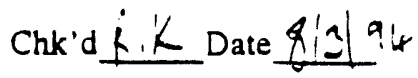

9.3 Recommended Modifications

From the analysis, it is recommended the following be noulined:

The KL/R ratios of the cross braces (40-5) exceed 200 . Four options were under considerations as shown on Figure $9-1$.

It is recommended to incorporate the modifications as shown on Option No. 4.

See attachment e (Pg. e.0.1 $\rightarrow$ E 0.3) For final "as-built" DETAILS OF CROSS. BRACES.

by. Dou $8 / 8 / 94$

CRed. RIK 8/8/94

II -205 
WHC-SD-WM-DA-163

Vol. II, Rev. 0

2f pree $7 / 29 / a+$

$2 k \dot{R}$ RIK $8 / 3 / 9$
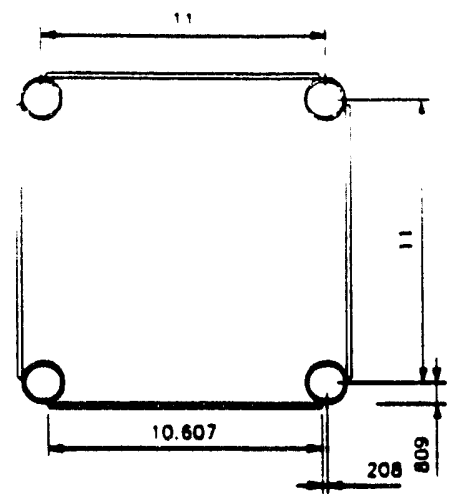

42-10 CROSS BRACE OETAL

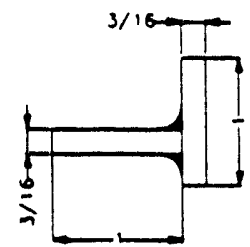

SECTION A-A

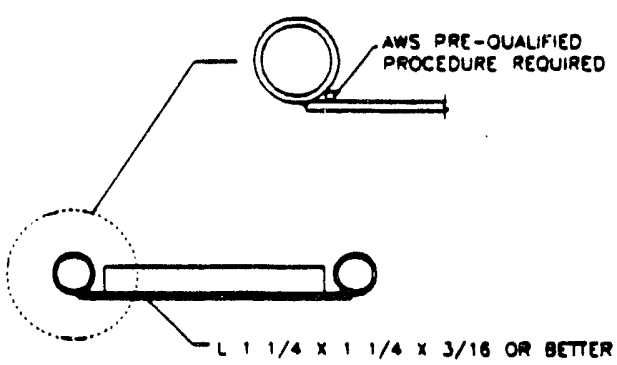

OPTION I USE ANGLE AS SHOWN (LEC IN)

OPTION 2 WELO $3 / 16^{\circ}$ PLATE AS SHOWN
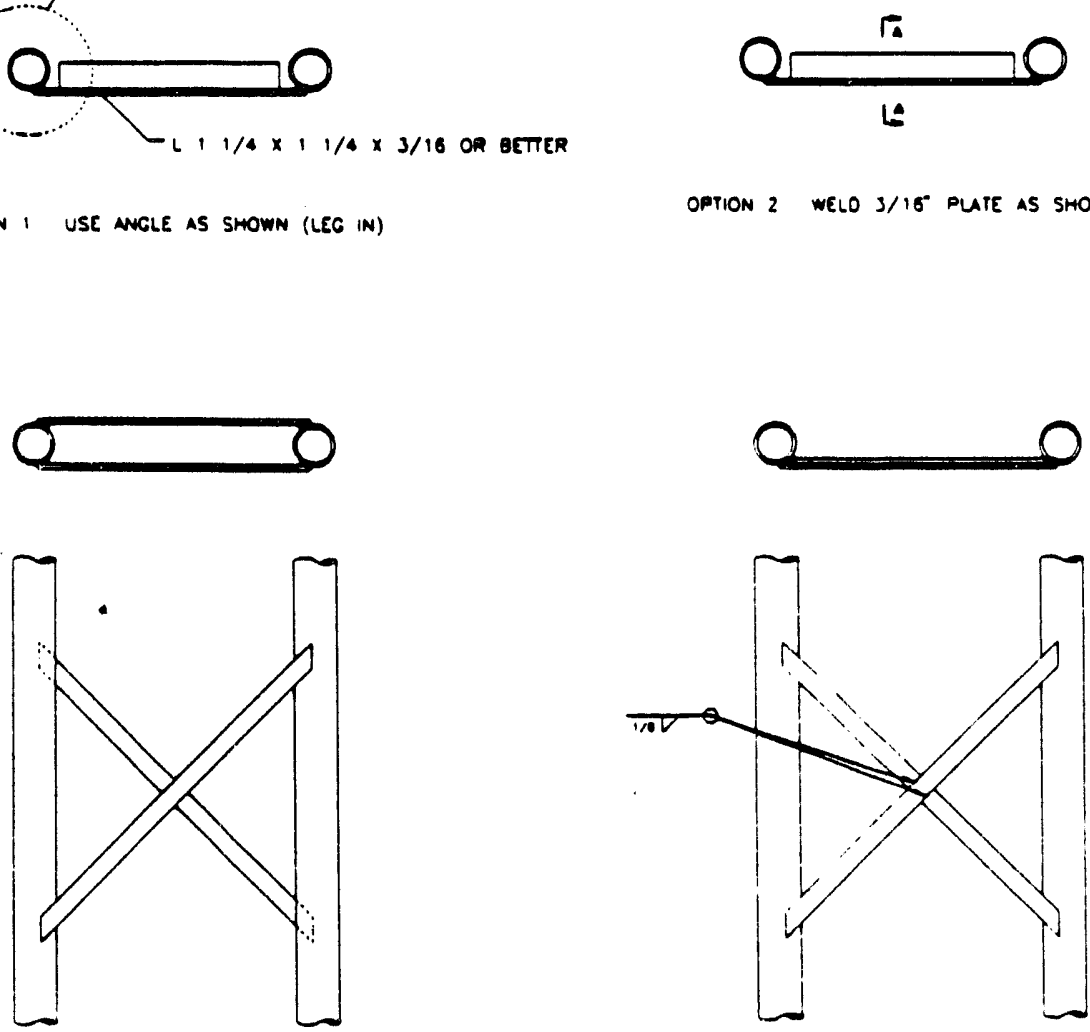

JPIDN : WELO ANOTHER J/16 PLATE INSIOE

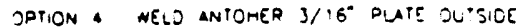

=g. 9-1 CROSS-BRACES MOOHEATOON OPTICIS 
WHC-SD-WM-DA-163

Vol. II. Rev. 0

ATTACHMENT A

: 


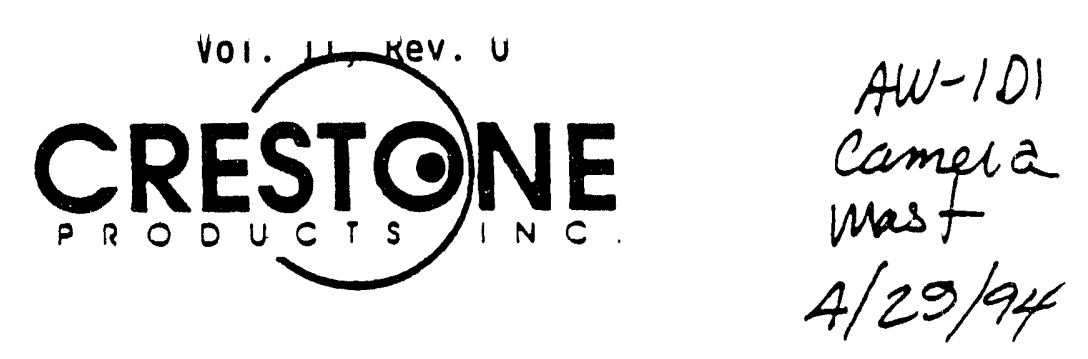

$42 \cdot 1$

$42-2$

$42 \cdot 3$

$42-4$

42.5

$42 \cdot 6$

42.7

42.8

$42-9$

42-10

$42-11$

$42-12$

40-3

$40-5$

UPPER MAST ASSEMBLY

SHIELDING PLATE

COVER PLATE

MANIFOLD ASSEMBLY

MANIFOLD TUBE

DIVIDING PLATE

FLNGE PLATE

1 1/4 IPS UPPER PIPE

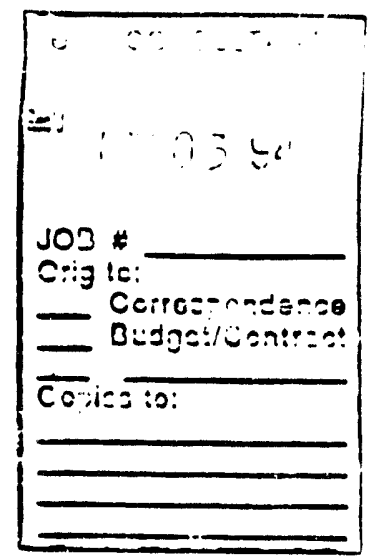

PIPE WELDING DETAIL

CROSS BRACE DETAIL

FLANGE PLATE GASKET

MATING PLATE GASKET

MATING PLATE

CROSS BRACE

OTY PER ASSEMBLY

\section{MAST}

\section{1 \\ 1 \\ 1 \\ 1 \\ 4}

1

1

16

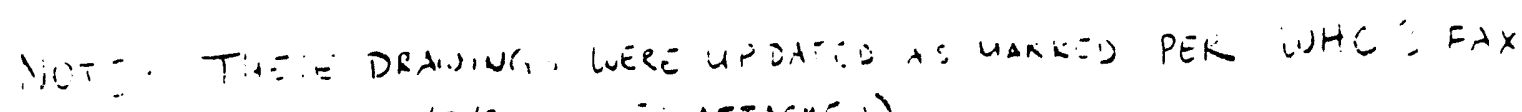

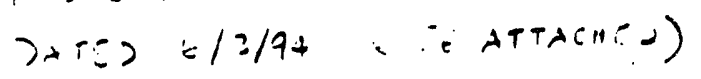


WHC-SD-WM-DA-163

Vol. II, Rev. 0

42-1 UPPER MAST ASSEMBLY WITH SHIELDING PLATE

THREADED STEEL EYEBOLT

$11 / 8-7 \times 23 / 4$ THREAD

12,000 LBS WORKING LDAD

THREE INCH THICK BY 22 INCH DIAMETER CARBON STEEL SHIELDING PLATE $(42-2)$

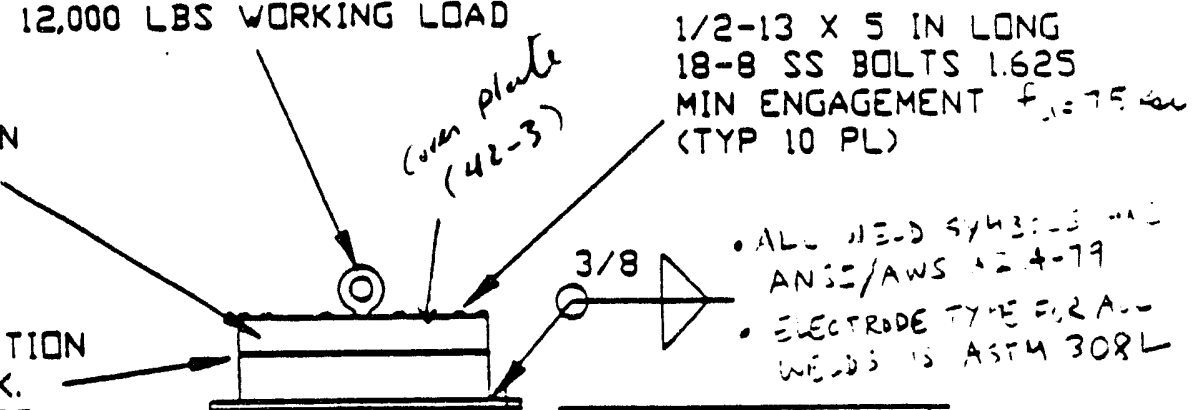

17-4 PH STAINLESS STEEL JUNCTION BOX. 22 IN Q.D., 1.0 WALL THICK. WELDED TO FLANCE PLATE INSIDE AND QUTSIDE LEAK TICHT

FLANCE PLATE 3/4 INCH THICK STAINLESS STEEL MATES TD ISO\# 20 INCH IPS RISER FLANGE $(4 i-7)$

$11 / 4$ SCHEDULE 40304 STAINLESS STEEL PIPE 1.66 Q.D. $X$ 1.38 I.D. (TYP 4 PL)

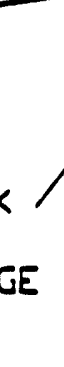

$3 / 16 \times 1$ WELDED CROSS BRACE (TYP 16 PLACES)

BASE PLATE I/Z IN THICK BY 13 IN SQUARE STAINLESS STEEL. SET I 1/4 IPS PIPES INTQ BASE PILDT SOCKETS AND WELD ALL AROUND. MUST BE LEAK TICHT. PIPES PERPENDICULAR TO BASE PLATES. SAME WELDING DETAIL IN TOP FLANCE PLATE.

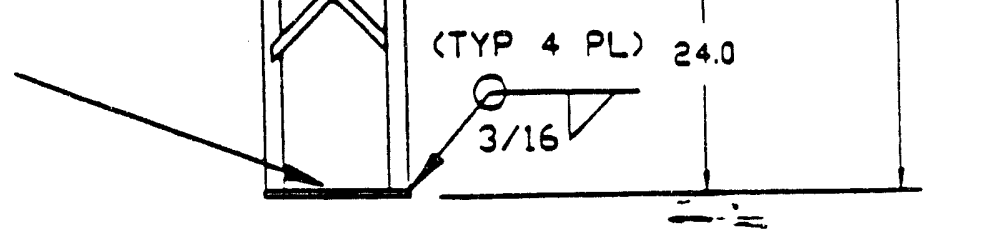

CRESTONE PRODUCTS, INO.

4175 W. QUAIL RIDGE DR.

BOISE, ID 83703

TEL 208-368.7998

FAX 208-368.7999 


\section{2-3 CQVER PLATE}

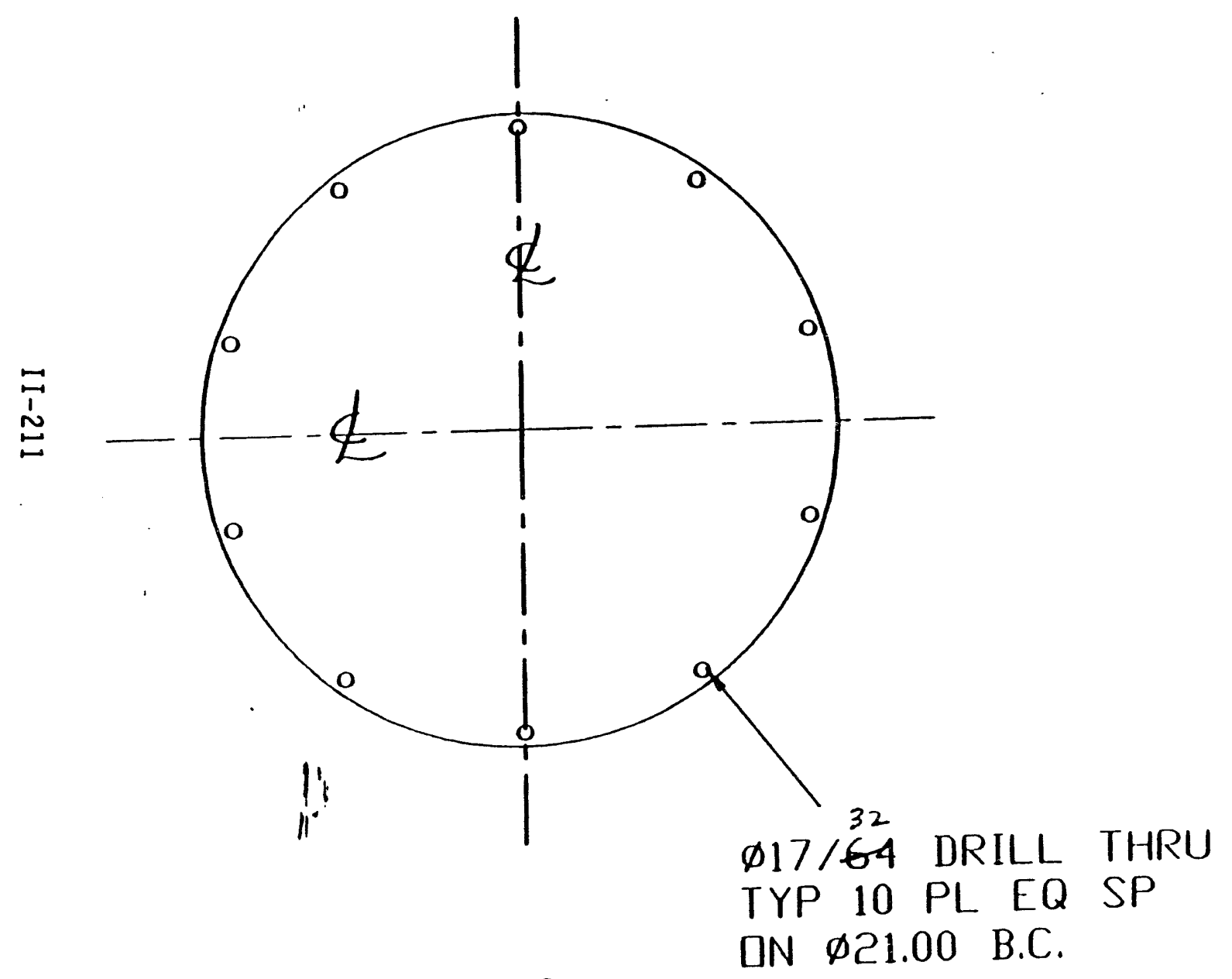

CRESTONE PRODUCTS, NC

4175 W. QUAIL RIDGE DR.

BOISE. ID 83703

TEL 208-368-7998

FAX 208-368.7999
MATL: 300 SERIES $: S$

TOL: $. X X \pm .020 \quad \times \quad 1.050$

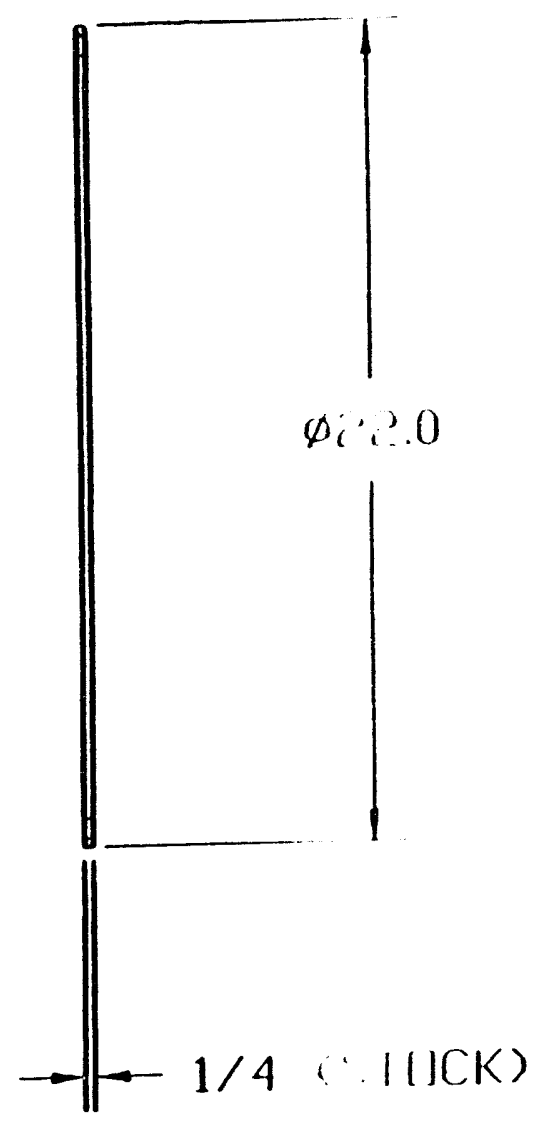


42-4 MANIFQLD ASSEMBLY

CAESTONE PRODUCTS, INC.

$\triangle 175 W$. QUAIL RIDGE DR.

BOISE. ID 83703

TEL 208-368-7998

FAX 208-368.7999
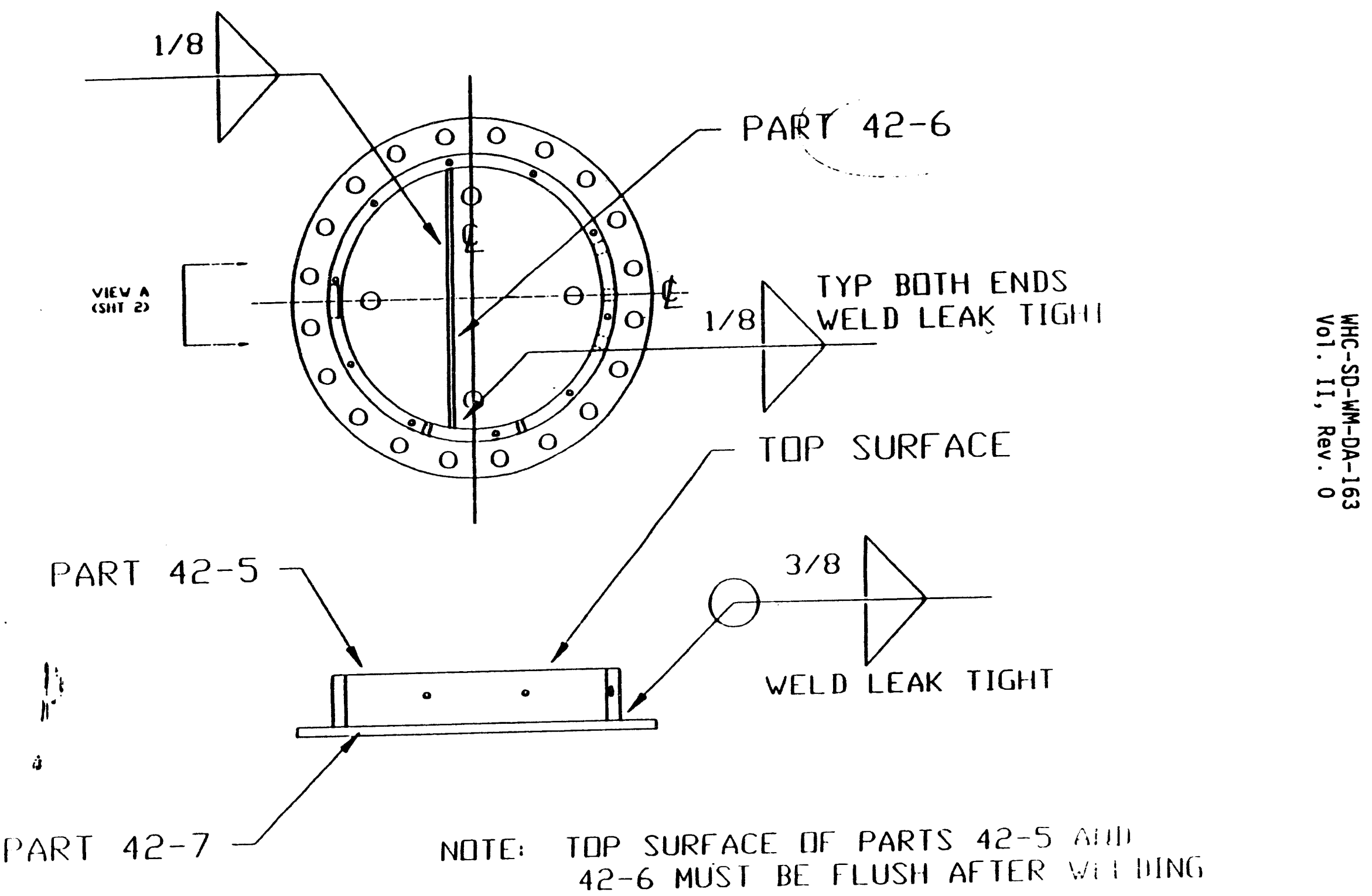


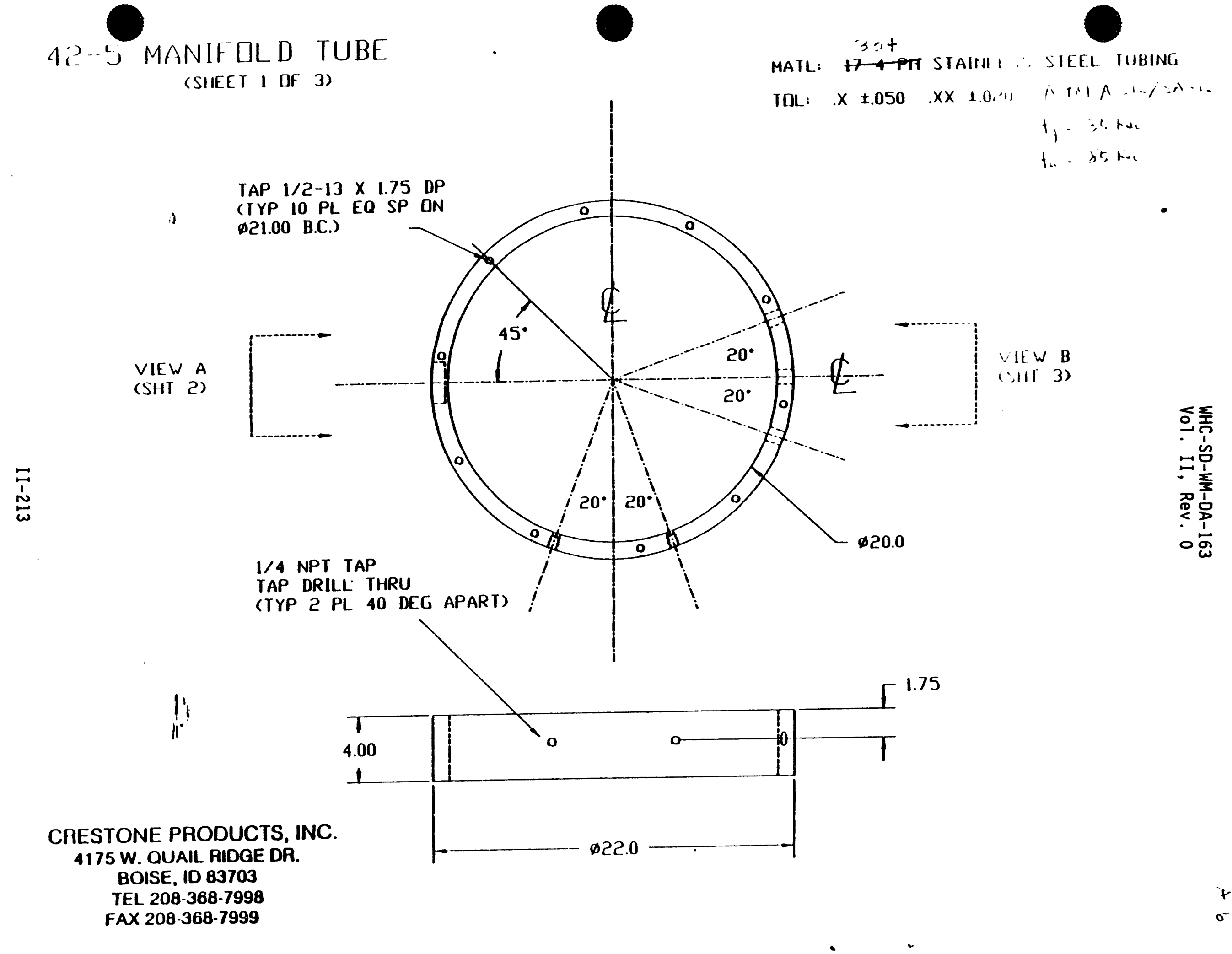


42-5 MANIF OLD TUBE

(SHEET 2 OF 3)
CRESTONE PRODUCTS, INC.

$4175 W$. QUAIL RIDGE DP.

BOISE, ID 83703

TEL 208-368-7998

FAX 208-368-7999

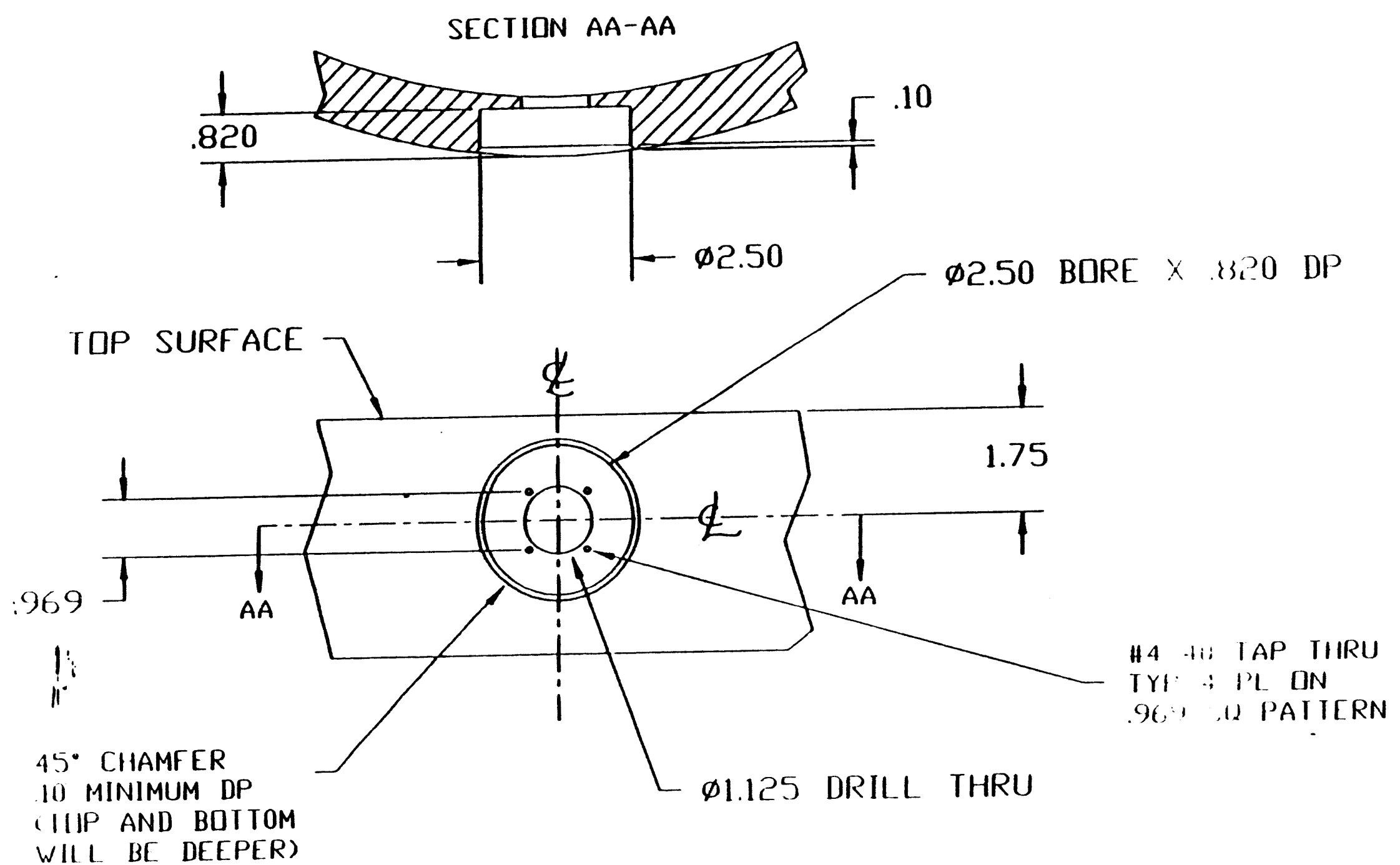


$42-5$ MANIF LL D TUBE
(SHEET 2 OF 3)

\section{LIGHT CONNECTOR MACHINING} (TYP 3 PL 20 DEG APART)

$\underset{ \pm}{ \pm}$
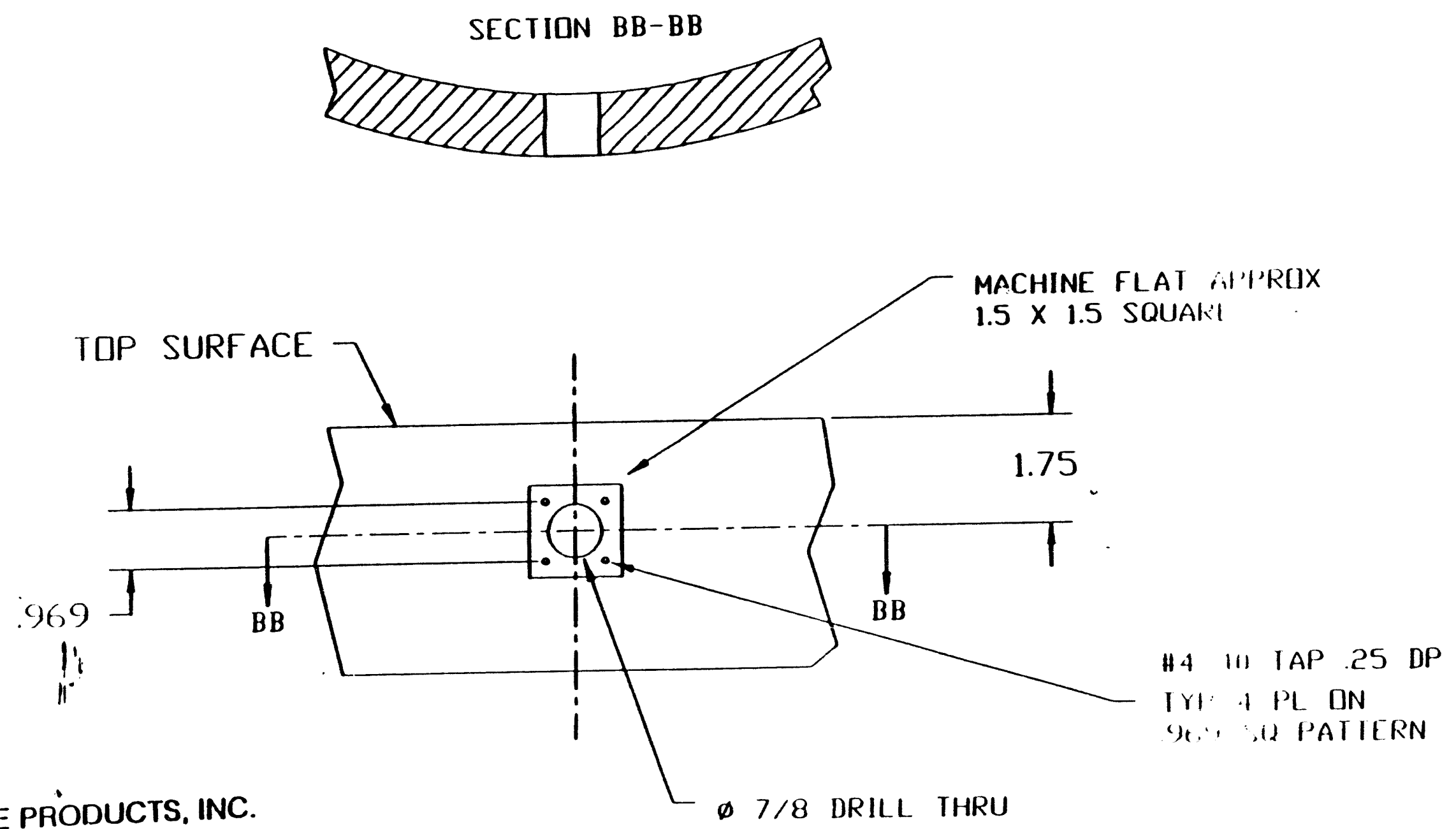
42-6 DIVIDING PLATE

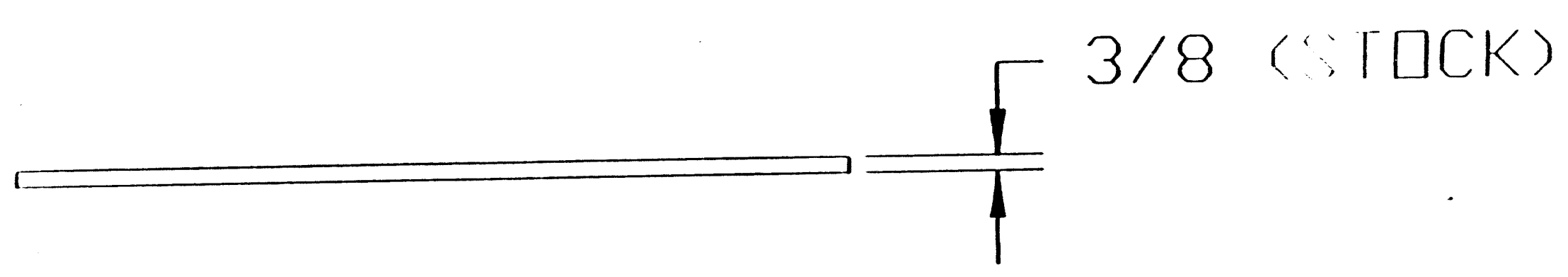

F

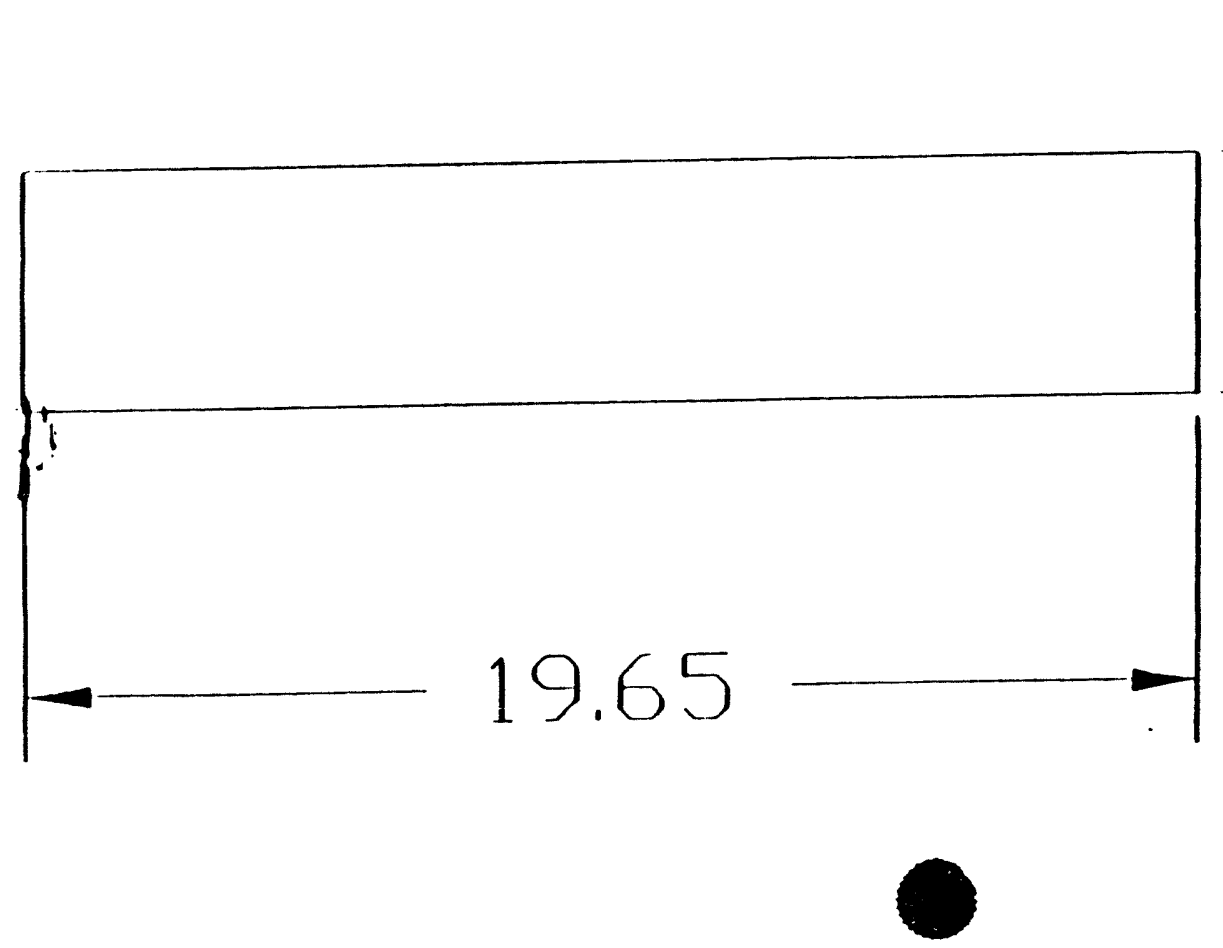

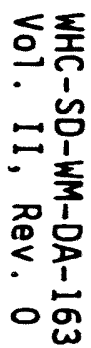

4.00

4175 W. QUAII HIDGE DR.

BOISE. 11 ; $B 3703$

FAX $2003121 / 999$
CRESTONE PHUUUCTS. INC.

TEL 208 ind 7998
MATL: 300 SERII $S S$

TOL: $. X X \pm .020$. \pm .050 
42-7 FLANGE PLATE

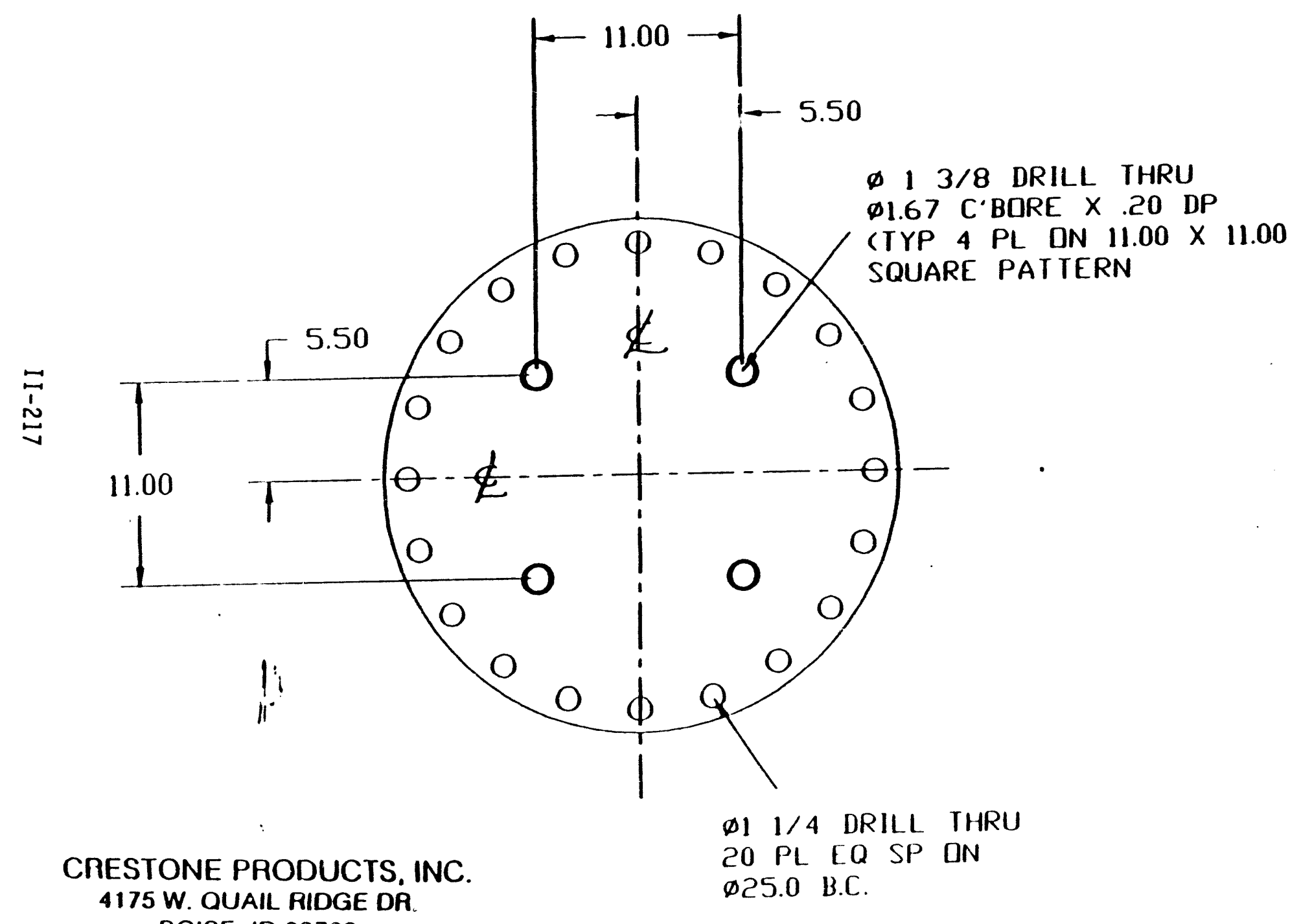

$3 u t$

MATL: 300 SEK!I $S$ SS A.HII ALTO

TOL: $. X X \pm .020 \times \pm .050$ 


\title{
42-8 11/4 IPS UPPER PIPE
}

\author{
MATL: $\quad 1 \quad 1 / 4$ IPS SCH 40 \\ 304 SS PII'L. \\ 1.66 D.D. $\times 1.38$ I.D. \\ TOL: .XX $\pm .020 \quad \times \pm .050$
}

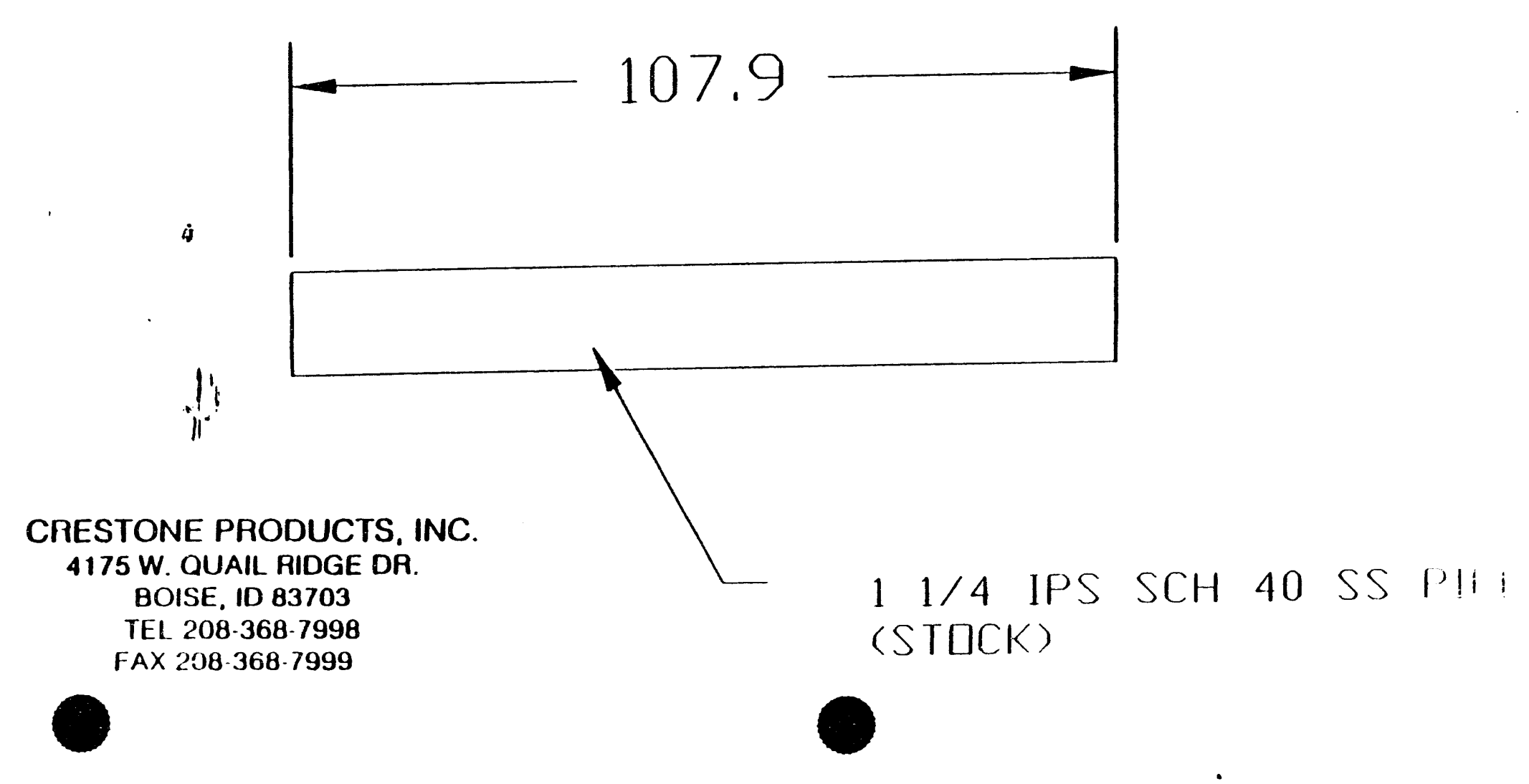

CAESTONE PRODUCTS, INC.

(1D 83703

1 1/4 IPS SCH 40 SS PII:

TEL 208-368-7998

(STOCK) 
CRESTONE PRODUCTS, INC.

\section{2-9 PIPE WELDING DETAIL}

4175 W. QUAIL FIDGE DR.

BOISE. ID 83703

TEL 208-368-7998

FAX 208-368.7999

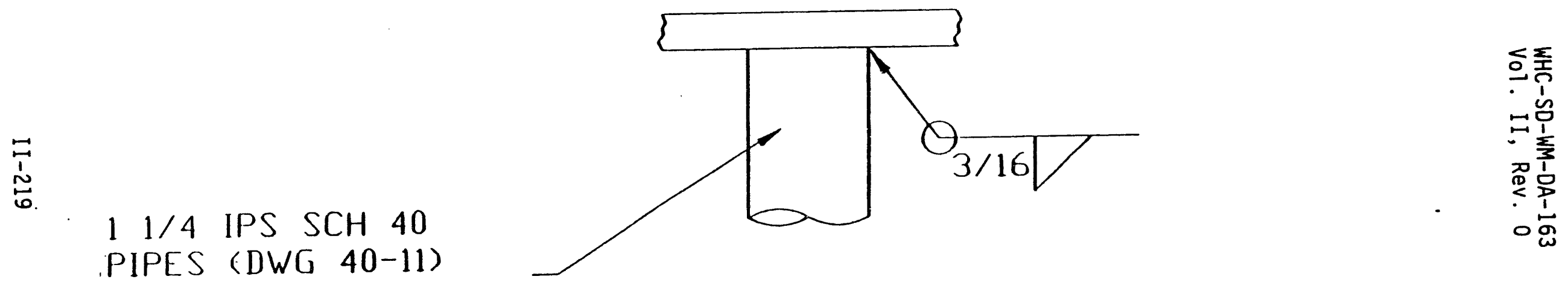

FIT PIPES INTO PILUT SOCKETS IN FLANGE PLATE (4c ' AND MATING PLATE (40-3). TUBES MUST BE PERPENDI(III AR TO PLATES. WELD ALL ARDUND EACH PIPE. MUST BE $1: \mathrm{AK}$ TIGHT. (TYP FOUR PIPES EACH END) 
42-10 CRQSS BRACE DETAIL (D)
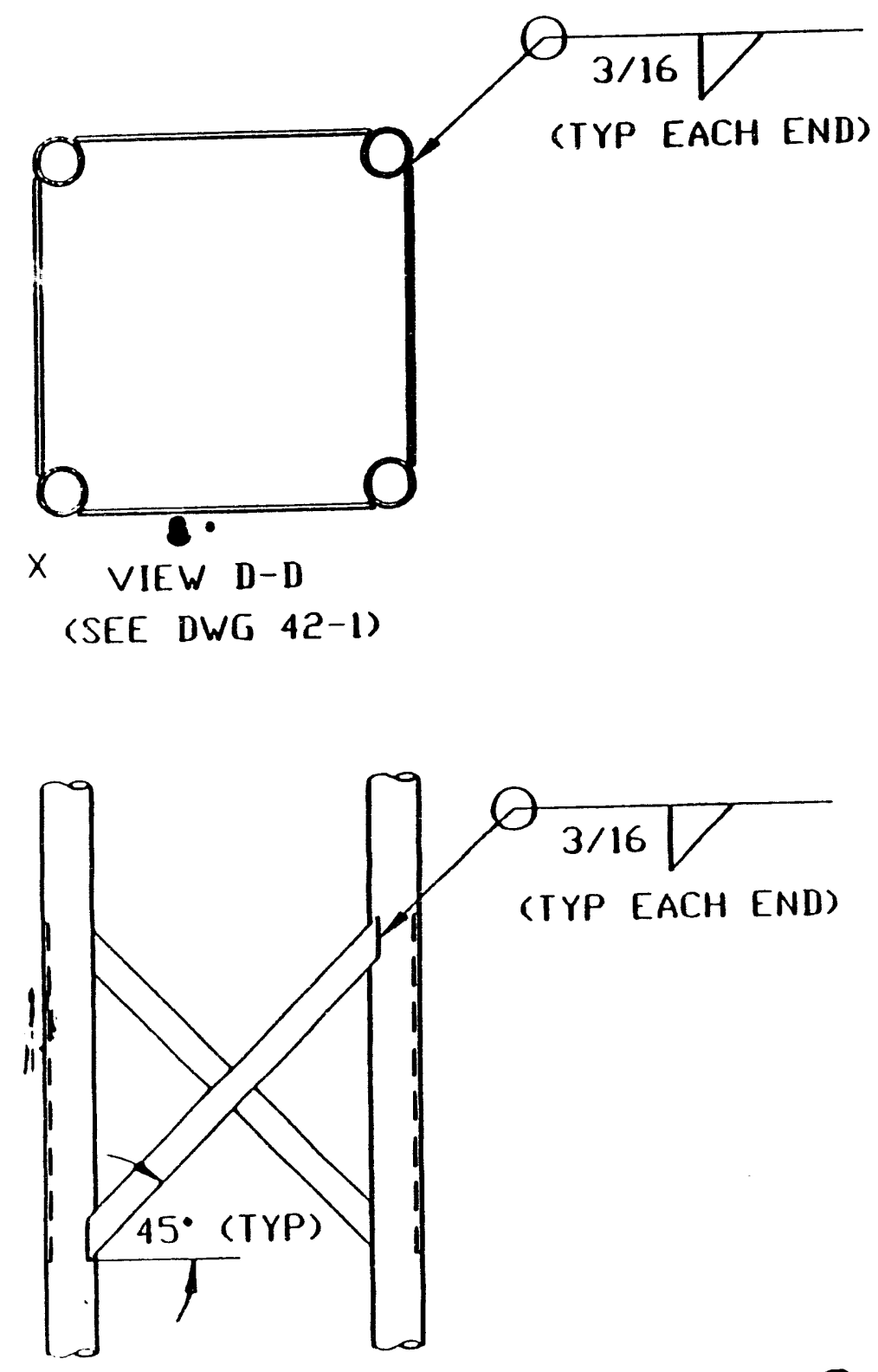

CRESTONE PRODUCTS. INC. $4175 W$. QUAIL RIDGE DR.

BOISE, ID 83703

TEL 208-368-7998

FAX 208-368-7999

$11 / 4$ IPS SCH 40

SS PIPE (SEE DWG 1, - 8)

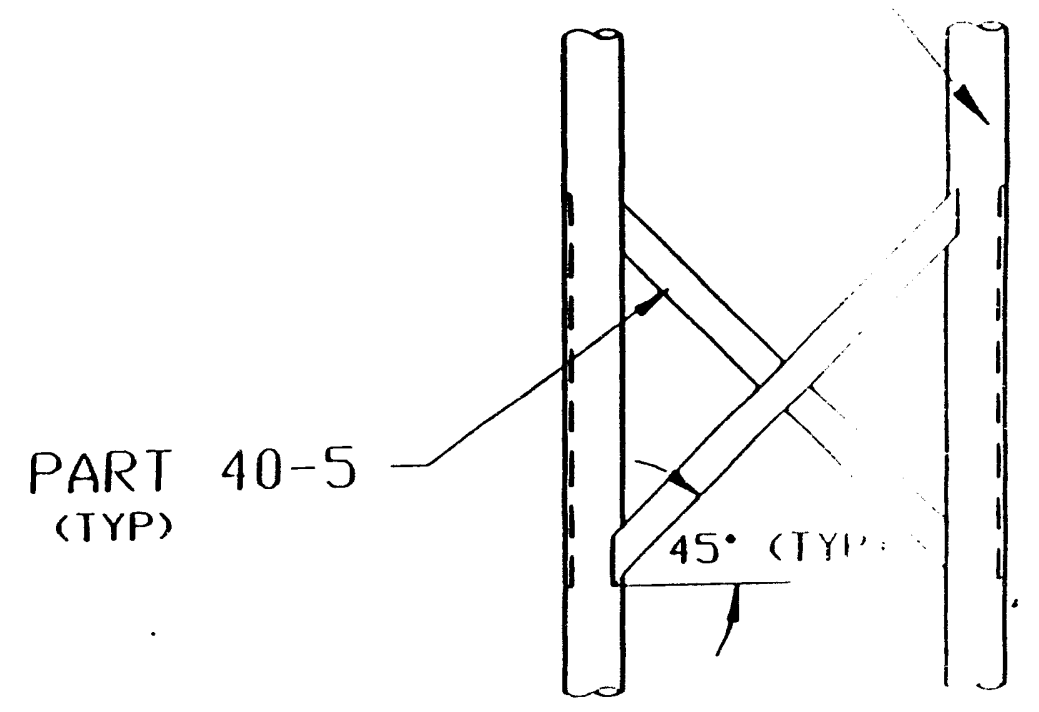

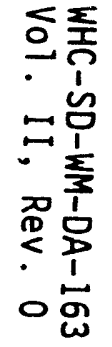

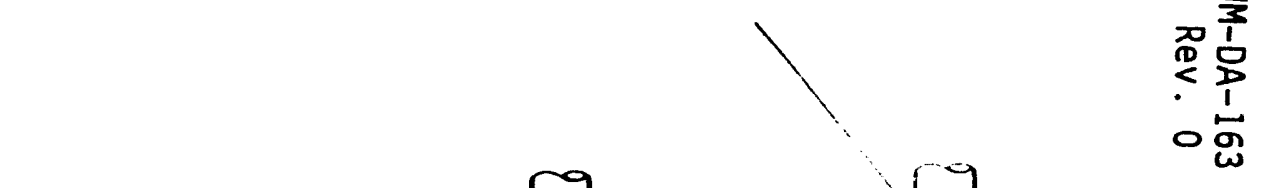




\section{2-11 FLANGE PLATE GASKET}

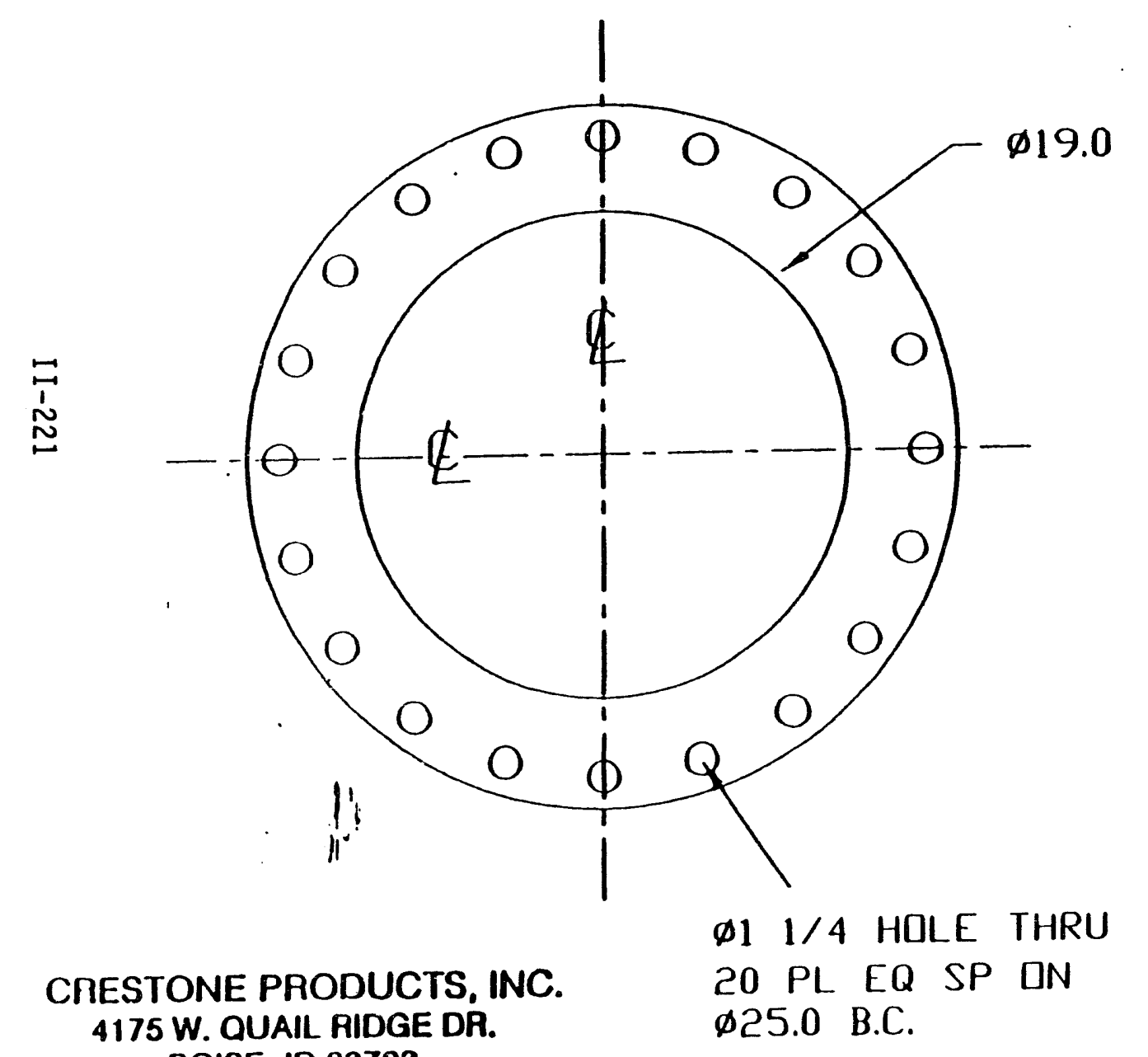

MATL: $1 / 8$ THICK GARLDCK 3000 GASKET MATERIAL (UR EQUIVAL ENT)

TOL: $. X X \pm .020 \quad . X \pm .050$

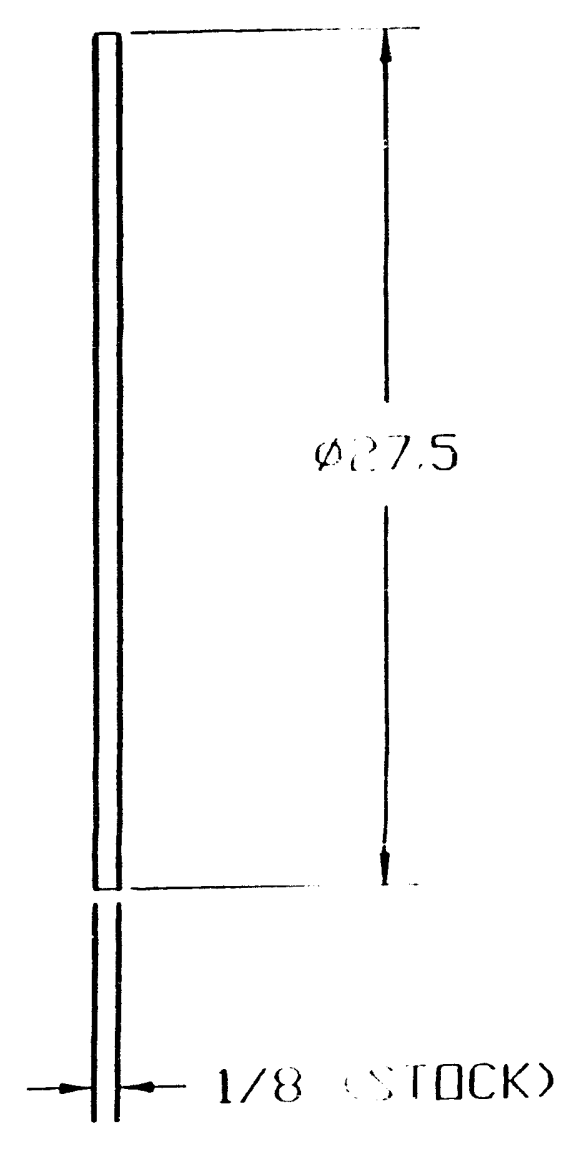


i)

42-12 MATING PLATE GASKET
MATL: $1 / 8$ THICK GARLOCK 31000 GASKET MATERIAL (OR EQUIVAI LNT)

TOL: $. X X \pm .020 \quad \times \pm .050$

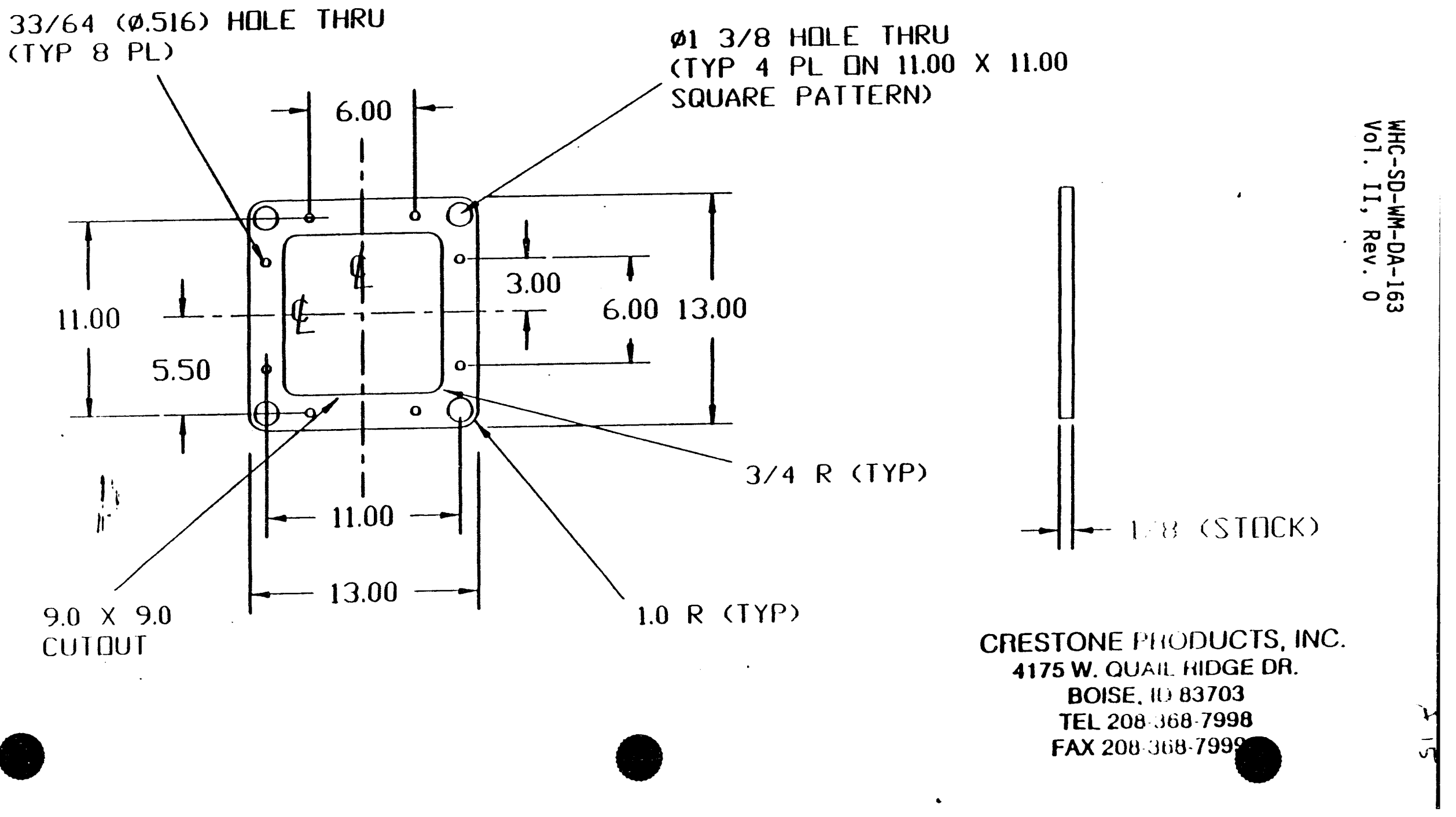


MATL, 1/2 IHICK 300 SHWIIS STAINLESS

TOL, XX $\pm .020 \quad$.XXX $\quad \pm .0111$
$A=T H A<+1$

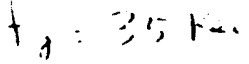

t $\quad 8 \div 1-$

泀
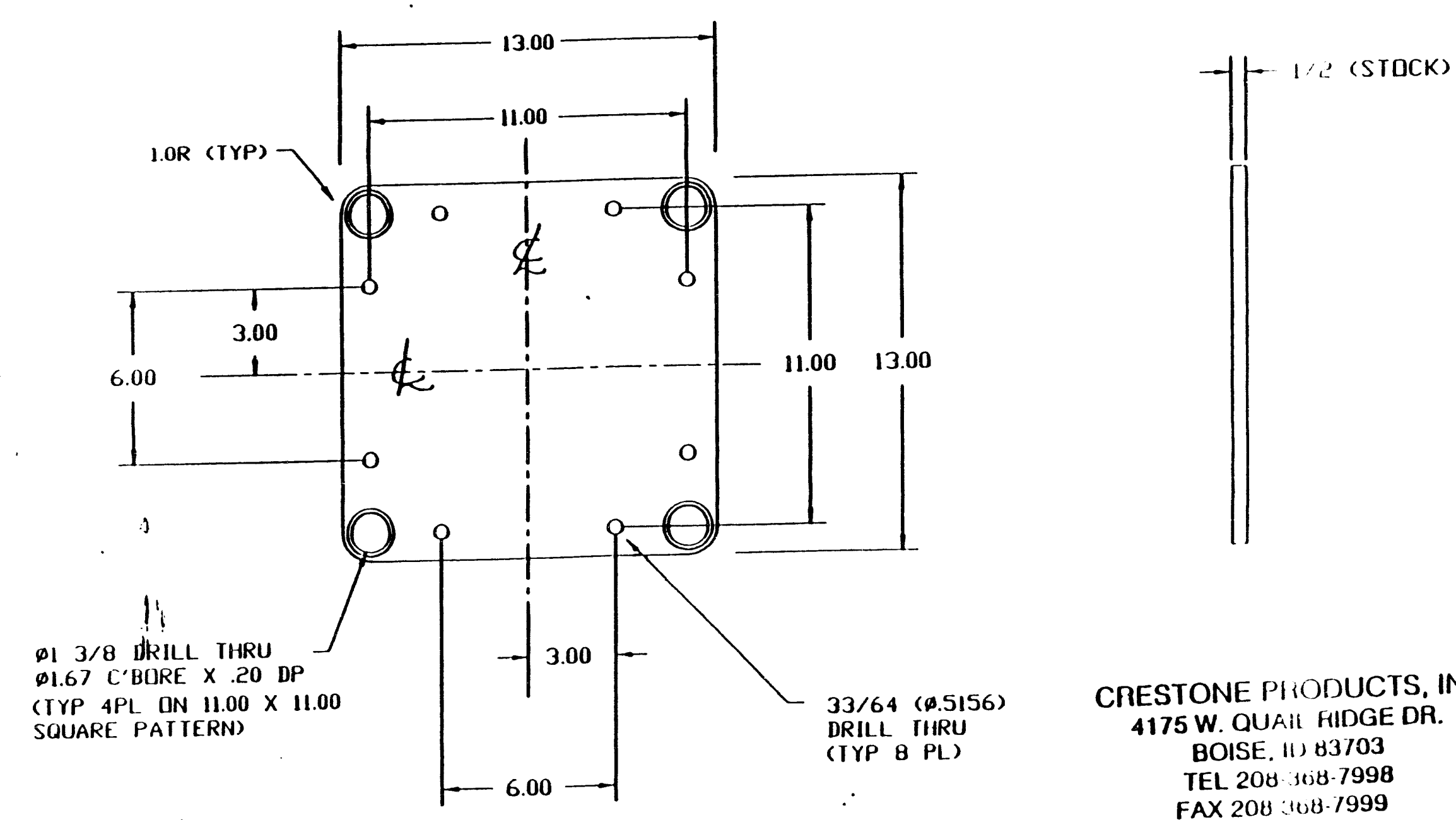

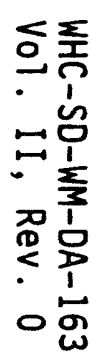

CRESTONE PHODUCTS, INC. 4175 W. QUAII FIDGE DR. BOISE. 11) 83703

TEL 208-368-7998 FAX $208 \div 108 \cdot 7999$ 


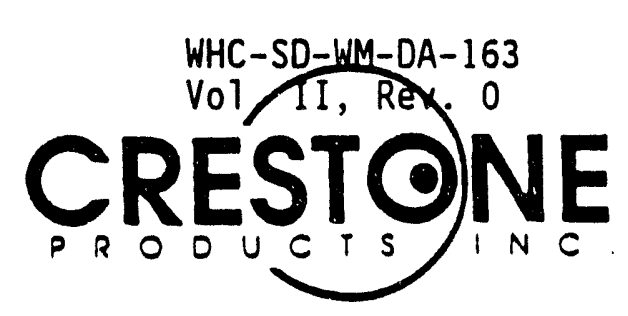

DESCRIPTION

QTY PER ASSEMBLY

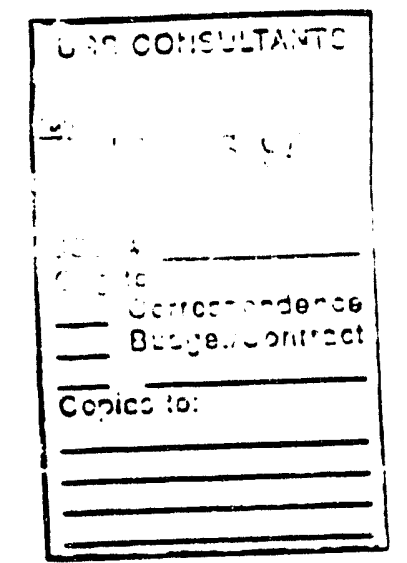

40-1

40-2

40-3

$40-4$

$40-5$

$40-6$

40.7

40.8

$40-9$

40.10

40-11

40.12

40-13

40-14
LOWER MAST ASSEMBLY

BOTTOM PLATE

MATING PLATE

LIGHT SUPPORT

CROSS BRACE

GAS TUBE DETAIL (A)

LIGHT FITTING DETAIL (B)

CROSS BRACE DETAIL (D)

PIPE WELDING DETAIL

1/2 NPT NIPPLE MODS

$11 / 4$ IPS LOWER PIPES

3/8 IPS GAS PIPE

FITTING

FITTING GASKET
1

1

3

4 

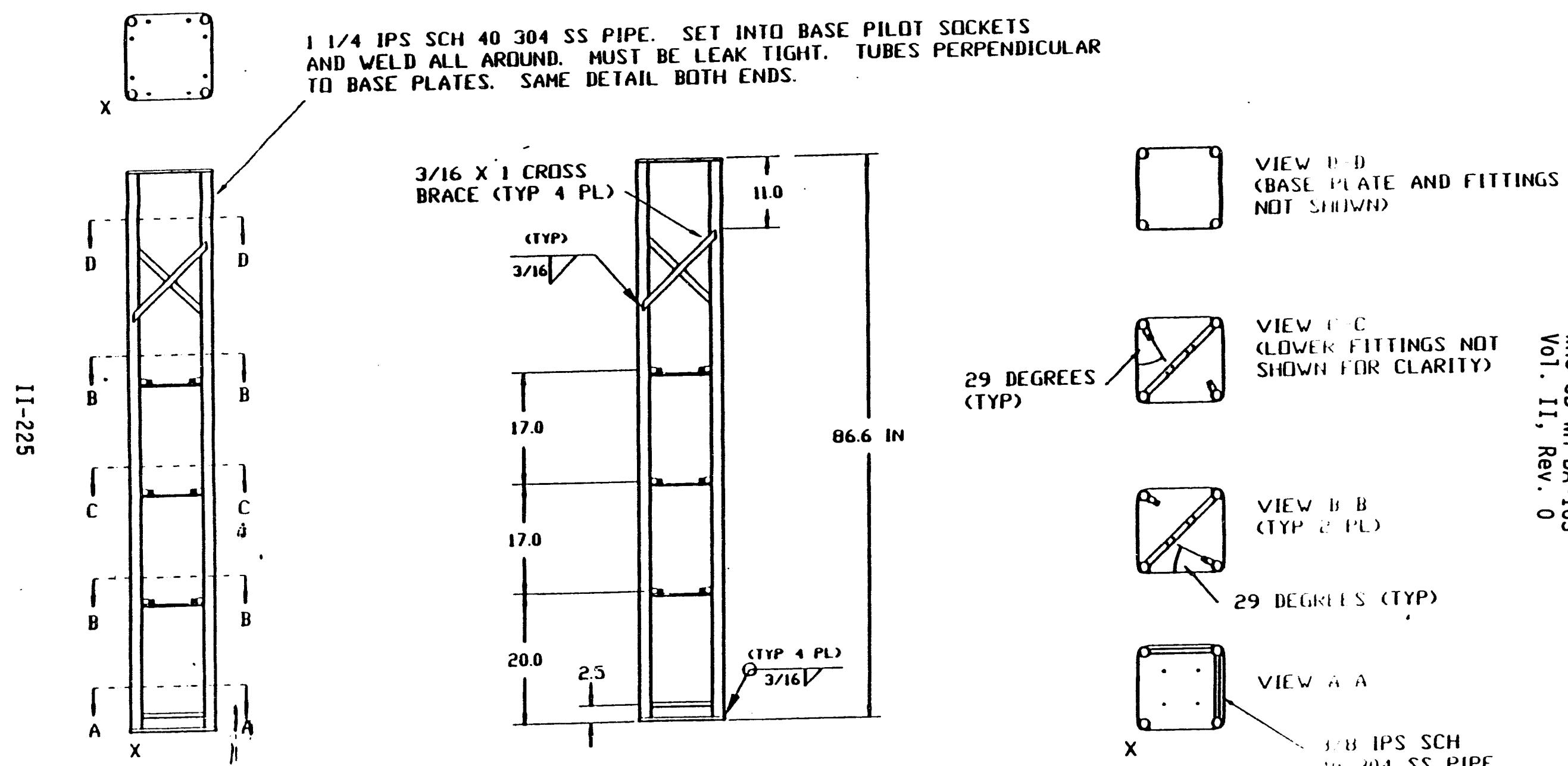

VIEW $\quad \mathrm{C}$

GOWEK FITIINGS NOT SHOWH HOR CLARITY)

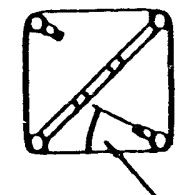

VIEW is B

(TYH $\because \mathrm{PL}$ )

○

29 DEGNEtS (TYP)

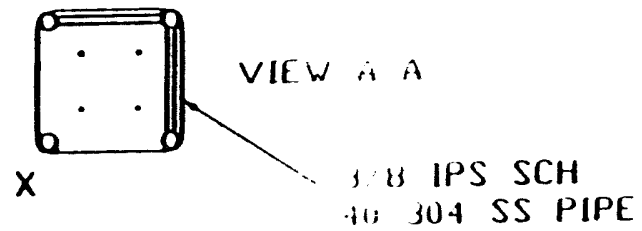

NOTES, (1) $x$ INDICAIES LOCATIONS OF THE PRIMARY MAST TUBE FOR IV AND PQT WIRING AND INPUT NIIROGEN GAS

(2) MEASUREMENTS IO WELDED PIPES ARE FROM BOITOM OF LOWER PLATE TO CENTER L.INES OF CROSS PIPES AND BETWEEN CENTERS OF CROSS PIPES 
MATL, I/2 IHICK 300 SERII: $I$ IAINLESS

IOL, .XX $\quad$.020 .XXX 1.010

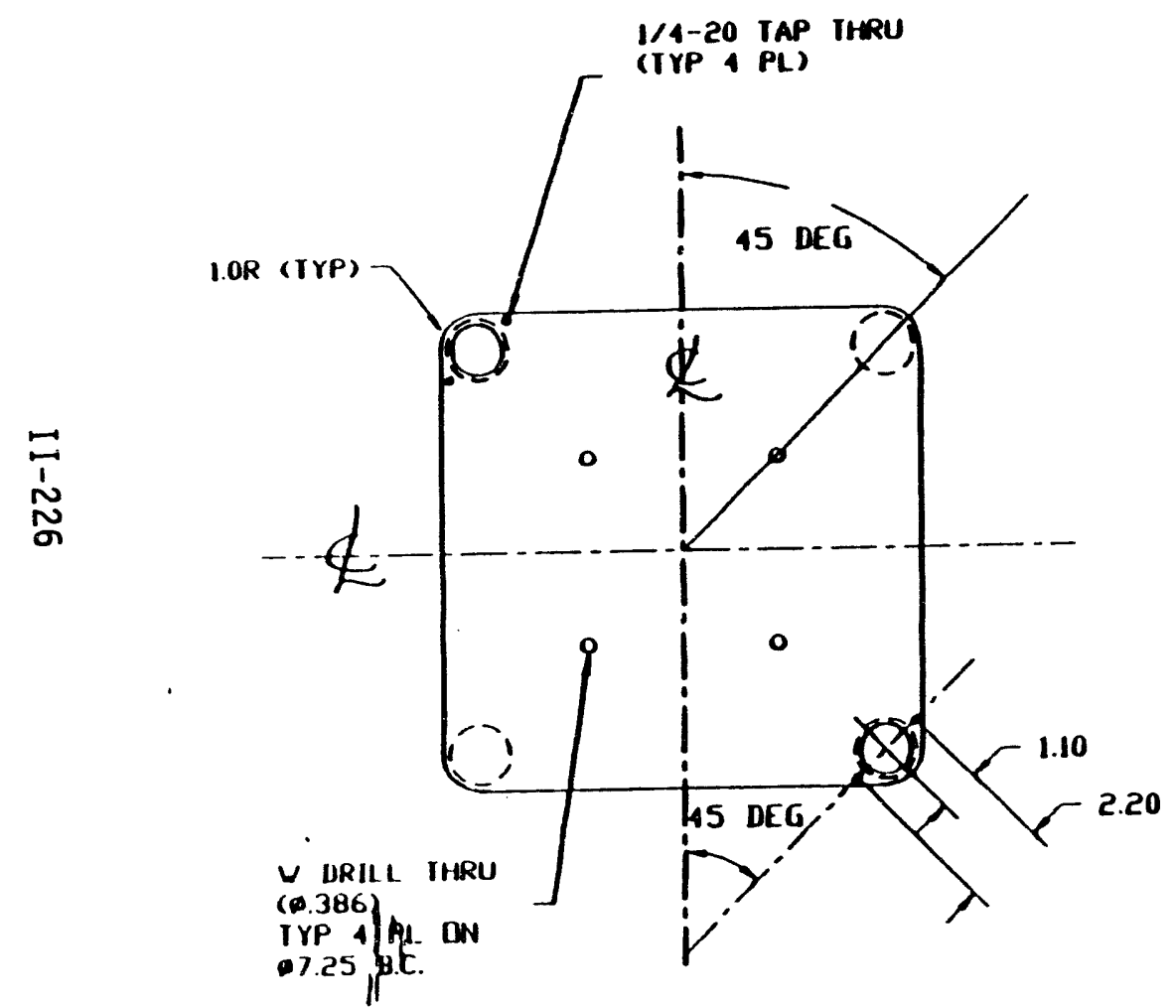

-
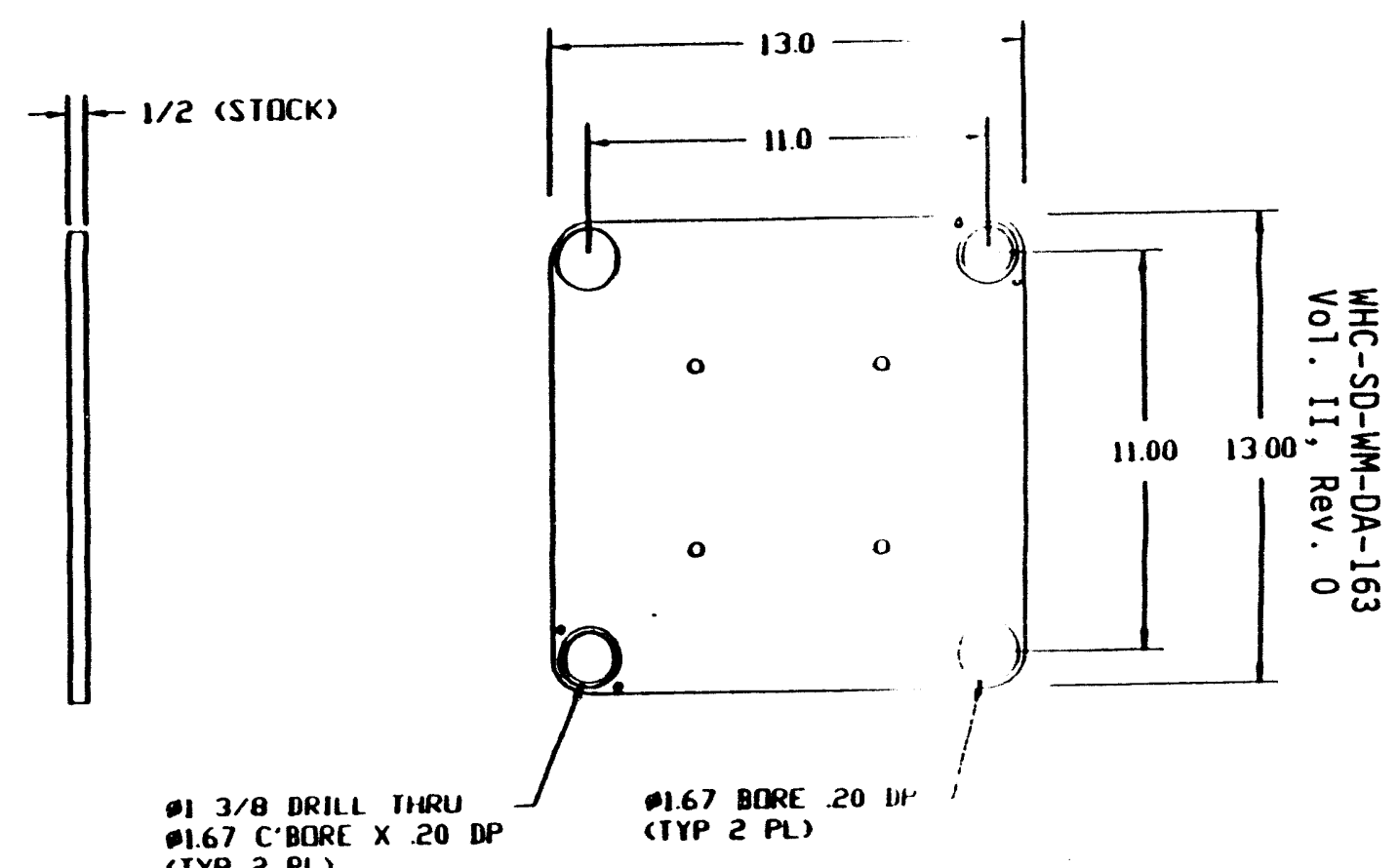

1.67 C'BORE $\times .20$

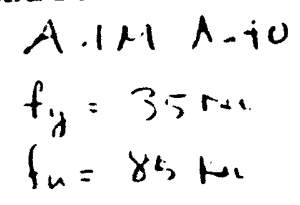
(IYP 2 PL)
CRESTONE PRODUCTS, INC.

1175 W. QUAIL RIIDGE DR.

BOISE, ID 83/03

TEL 208-368-7998

FAX 208-368-7.399 
MATL, 1/2 THICK 300 SINII; STAINIESS

TOL, .XX $\pm .020 \quad . \times \times X \pm 11111$

$11 / 1 \hat{A}<+0$

$f_{y}=351-$.

$f_{\text {. }}=6 i ; 1$.
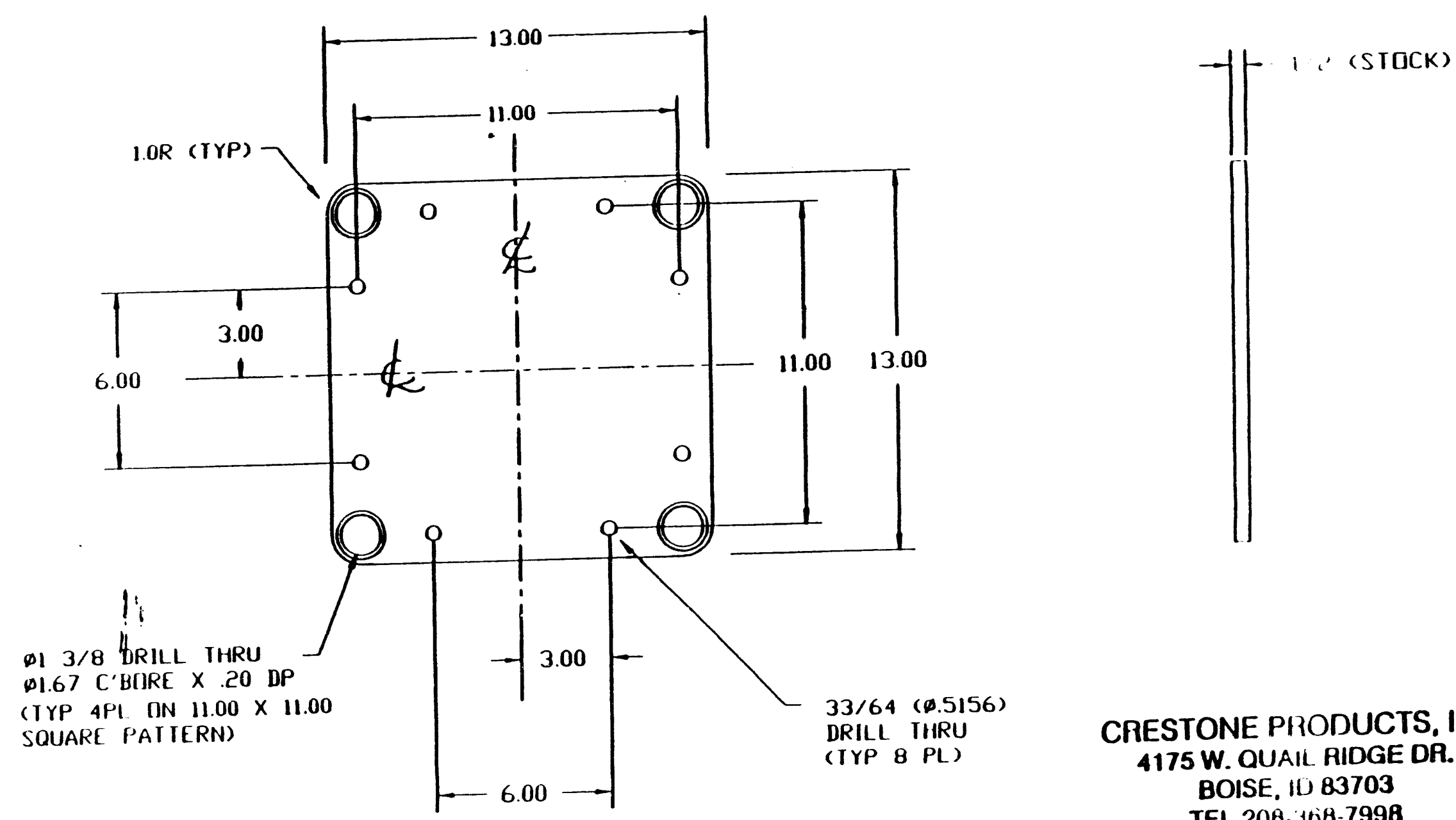

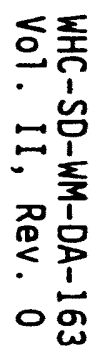

CRESTONE PRODUCTS, INC.

4175 W. QUAIL RIDGE DR.

BOISE, IO 83703

TEL 208-3168-7998

FAX 208 . $168-7999$ 


\section{0-4 LIGHT SUPPQRT}

MATL: $3 / 8 \times 1300$ SERIF $\triangle \because \because$ FAT

TOL, .XX $\pm .020 \quad . X X X \quad \pm .010$

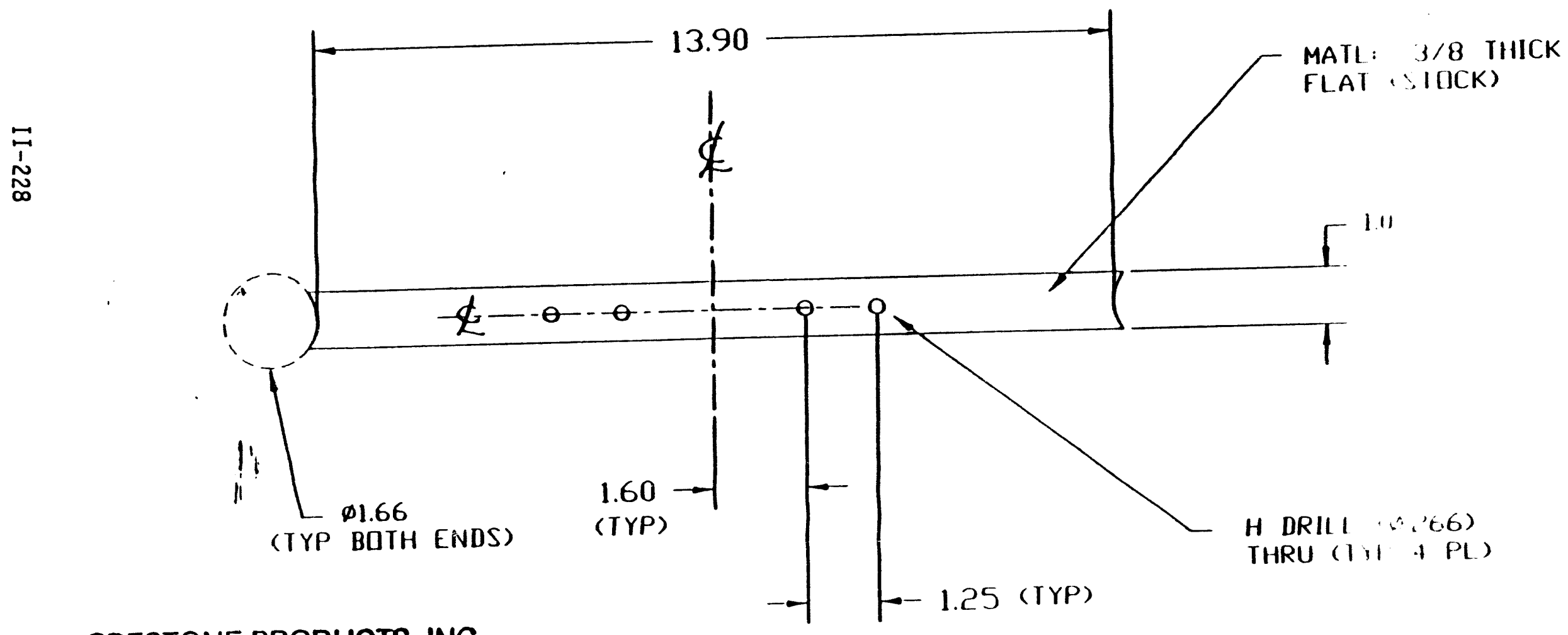

CAESTONE PRODUCTS, INC.

4175 W. QUAIL RIDGE DR.

BOISE. ID 83703

TEL 208-368-7998

AX 208-368-7999 


\section{0-5 CRISS BRACE}

MATL: $3 / 16 \times 1300$ SIHIIS SS FLAT

TOL, .XX $\pm .020 \quad . X \times X \quad \pm .1111$

1

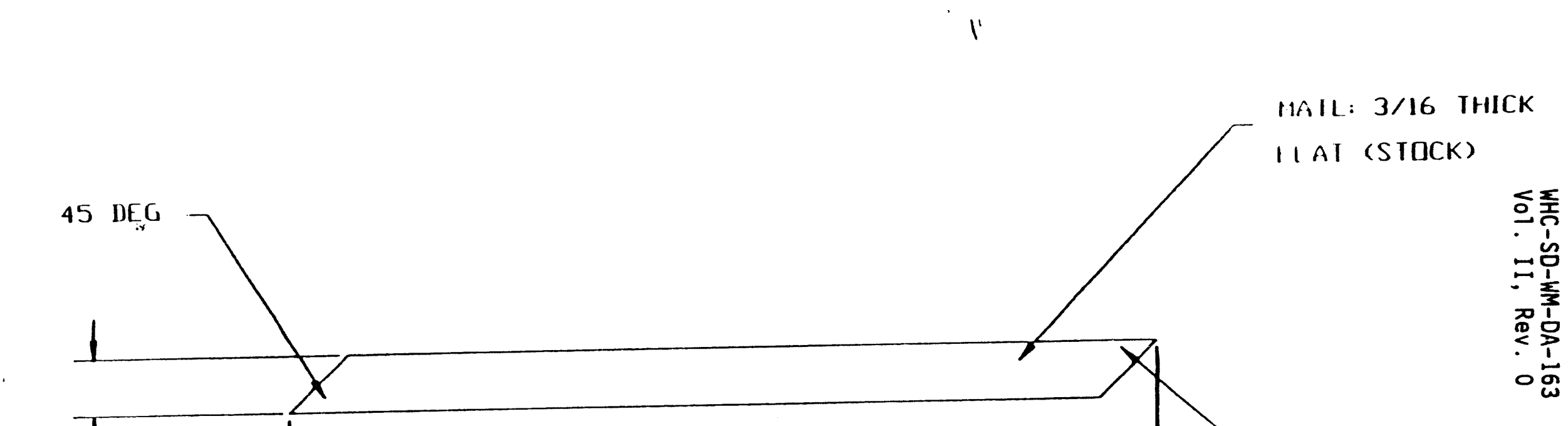

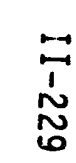

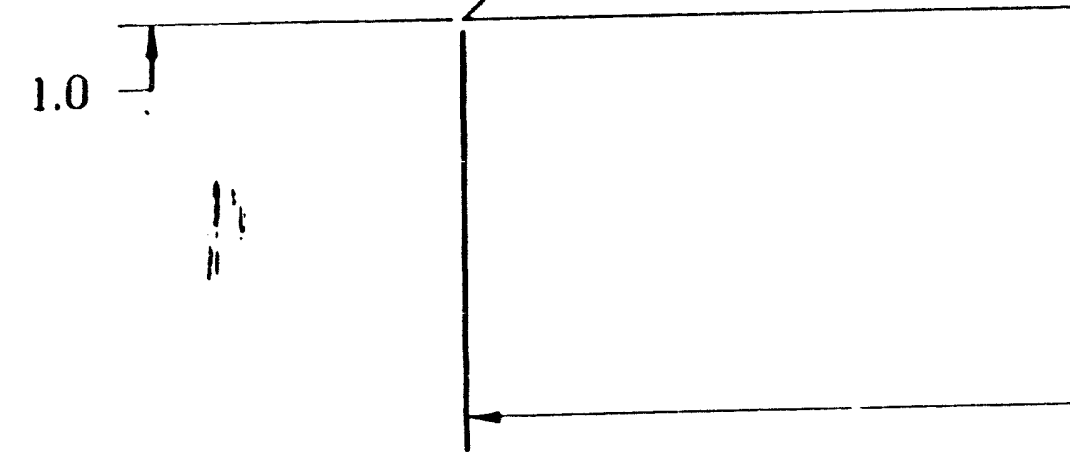

15.0 
CRESTONE PRODUCTS, INC.

4175 W. QUAIL HIDGE DR.

BOISE. II) 83703

TEL 208-308-7998

FAX 2083 (its 7999

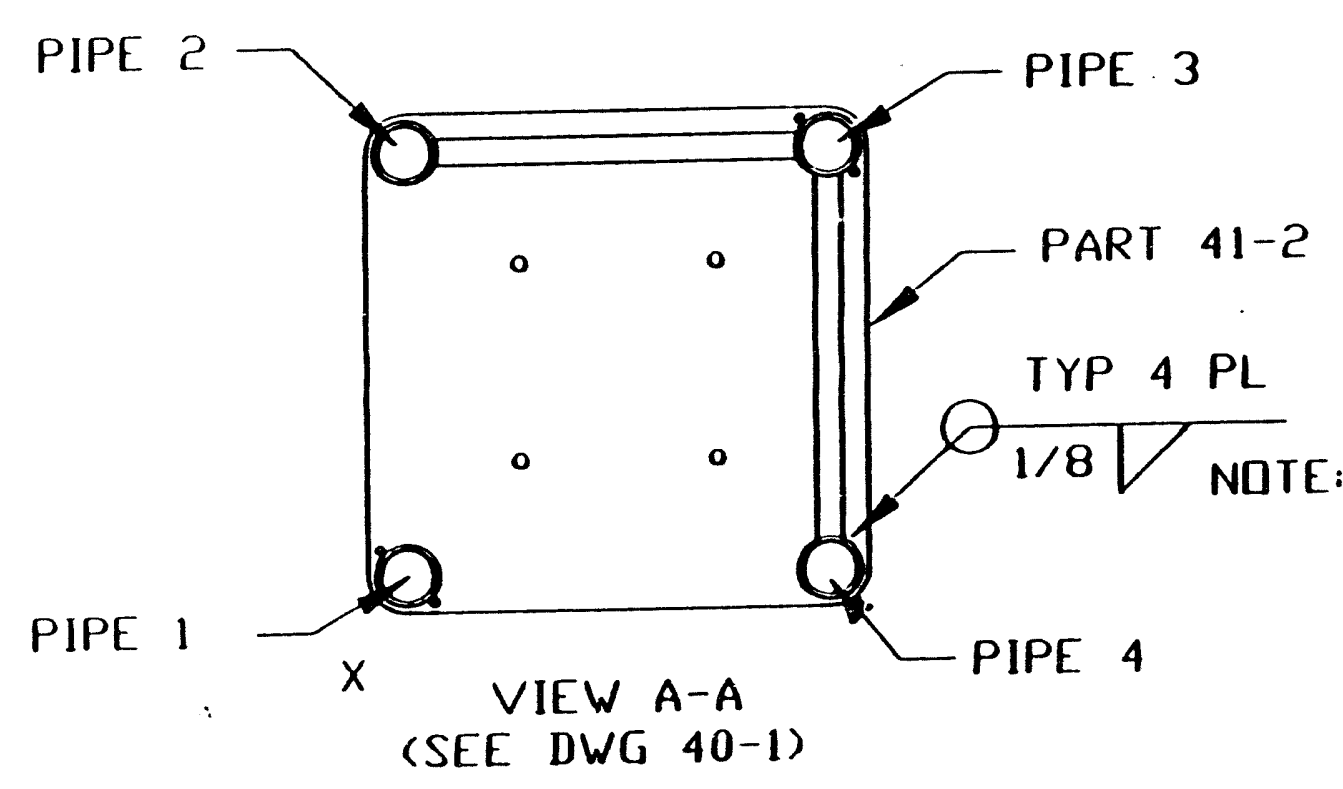

PART $40-2$

li!

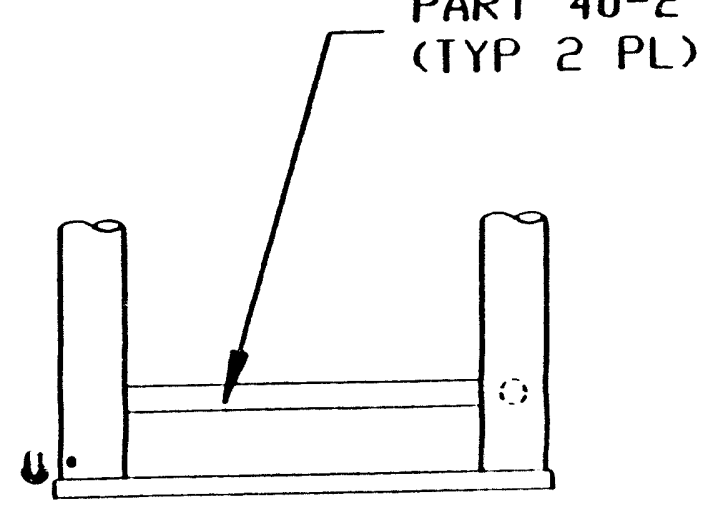

NDTE: CENTER PIPES QVF: HOLES IN $11 / 4$ PIIIS

AND WELD IN POSIIIIIN

WELDS MUST BE

LEAK TIGHT
$11 / 4$ IPS SCH 40

SS PIPE (SEE DWG 411 11)

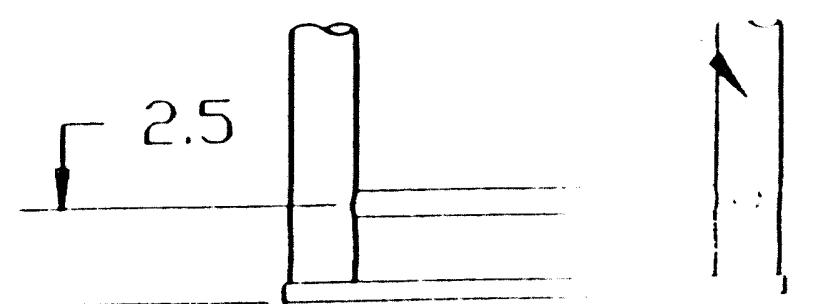


40-7 LIGHT FITTING DETAIL (B)

CRESTONE PRODUCTS, W.

4175 W. OUAIL IIIDGE DR.

BOISE. ID 43703

TEL 208-36U 7998

FAX 208-368. 7999

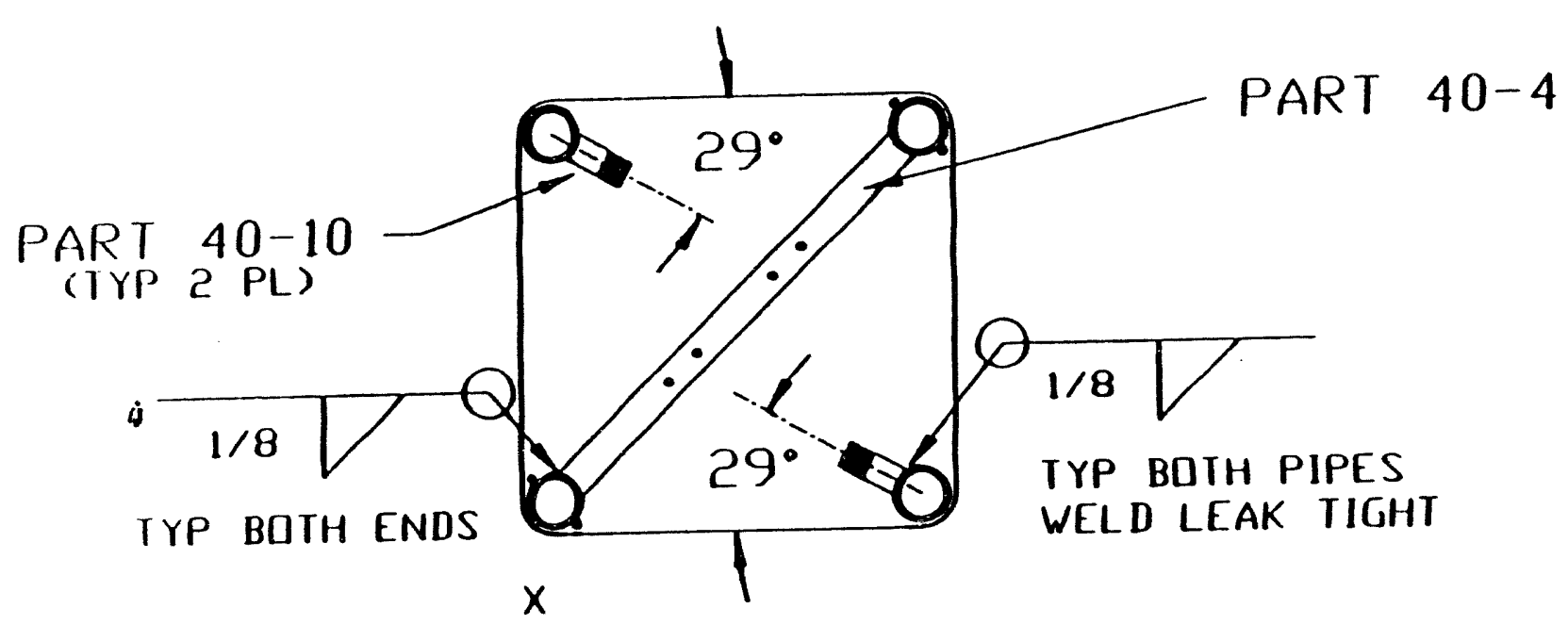

$$
\begin{aligned}
& \text { VIEW B-B } \\
& \text { (SEE DWG 40-1) }
\end{aligned}
$$

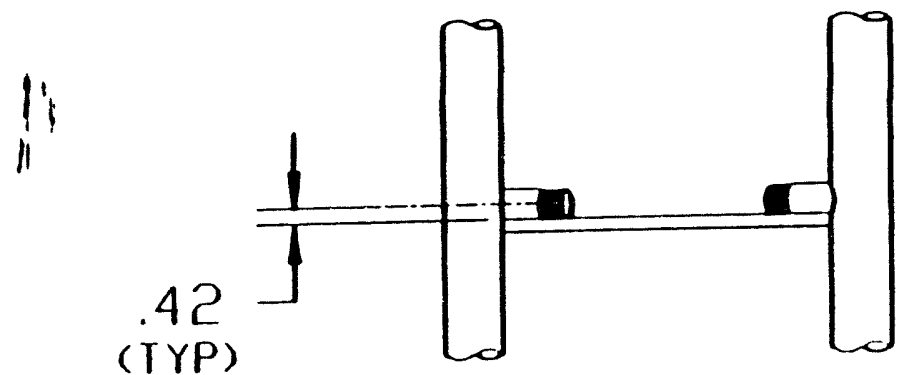

DIMENSION FROM IOP OI LIGHT SUPPORT (40-4) TO CENTERLINE OF STUB PIPES $(40-10)$
1 1/4 IPS SC11 10

SS PIPE (SET iW 40-11)

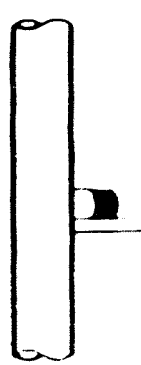


40-8 CRDSS BRACE DETAIL (D)

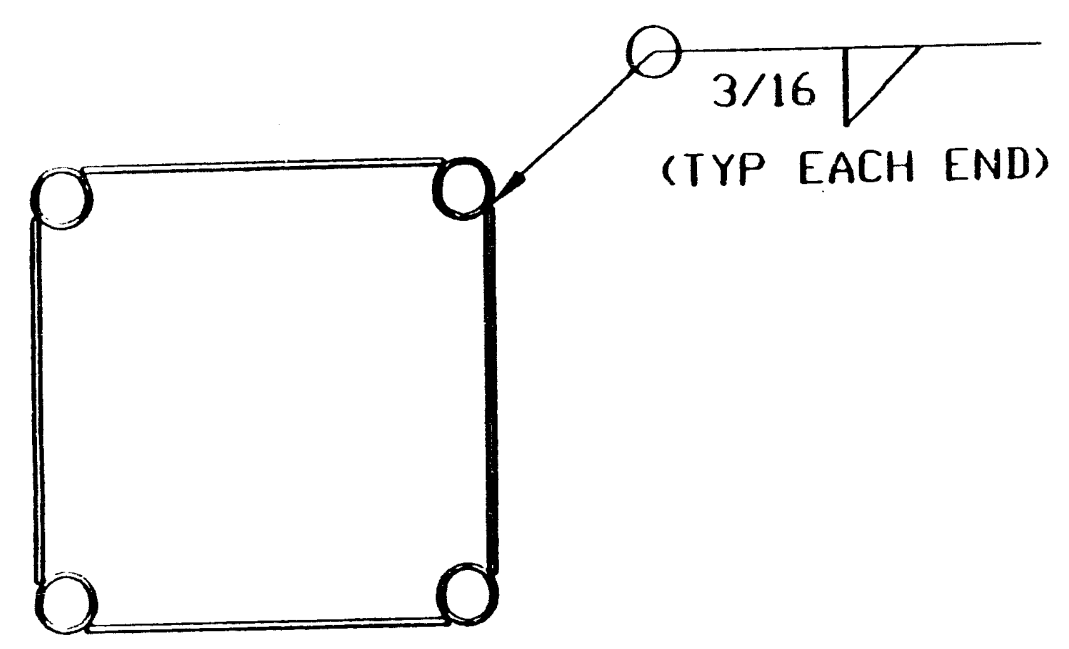

$\stackrel{N}{\omega}$

$$
\text { i(SEE DWG 40-1) }
$$

$11 / 4$ IPS SCH 111

SS PIPE (SEE IWW 40-11)
$4175 \mathrm{~W}$. QUAIL RIDGE DR.
CRESTONE PRODUCTS,

BOISE, ID 83703

TEL 208.368-7998

FAX $2013368-7999$

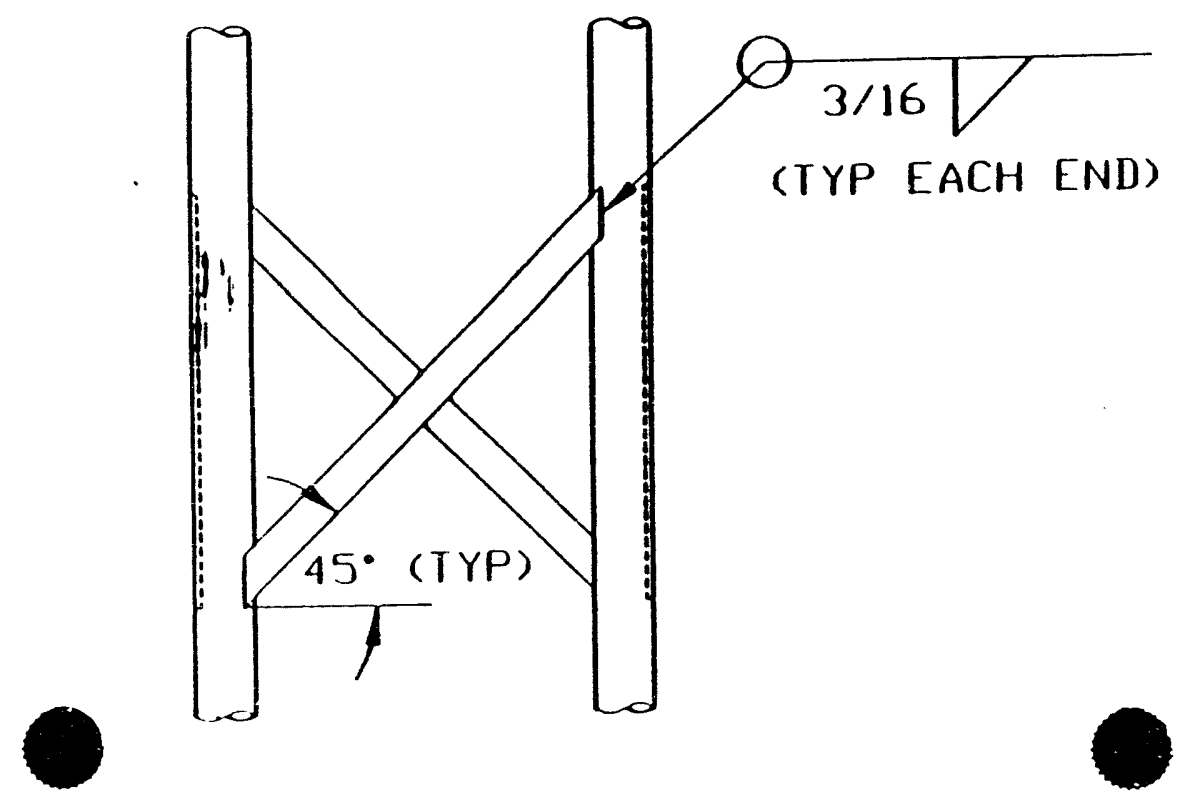

PART $40-5$ (TYP)

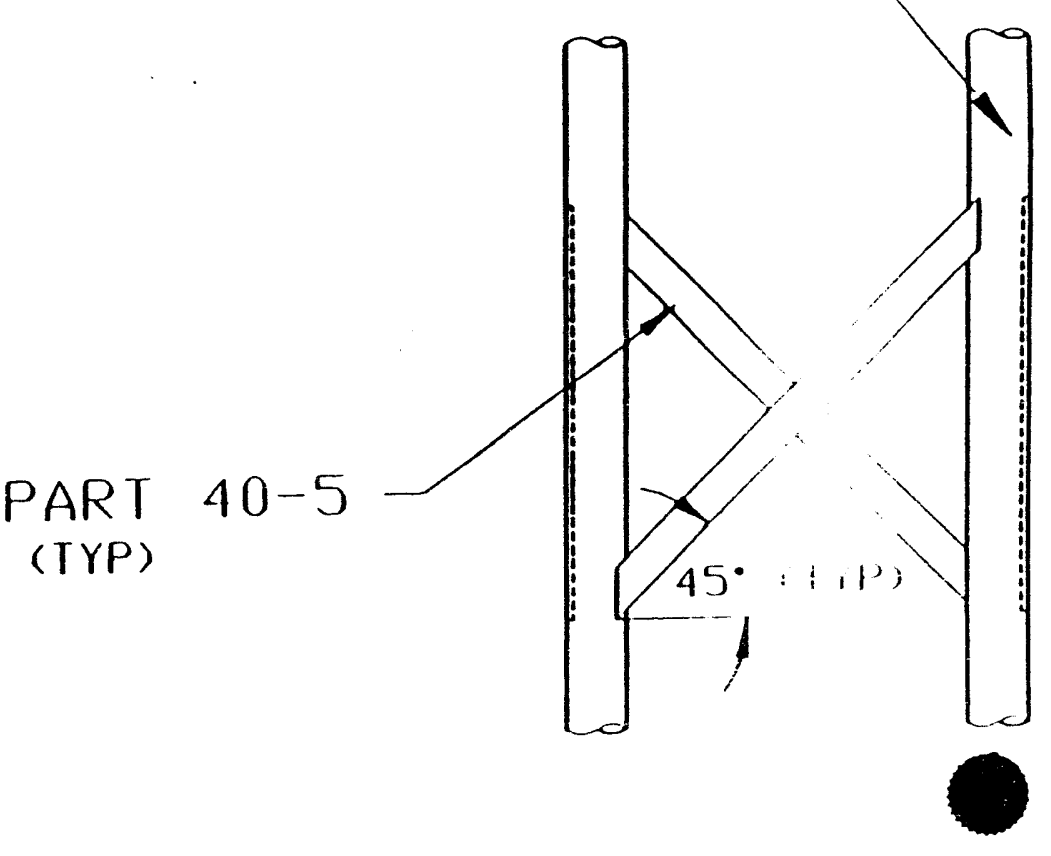


WHC-SD-WM-DA-163

\section{ín.}

능용요용

는

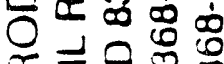

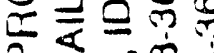

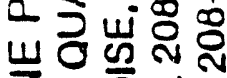

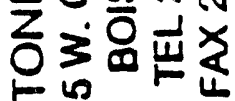

的

峞
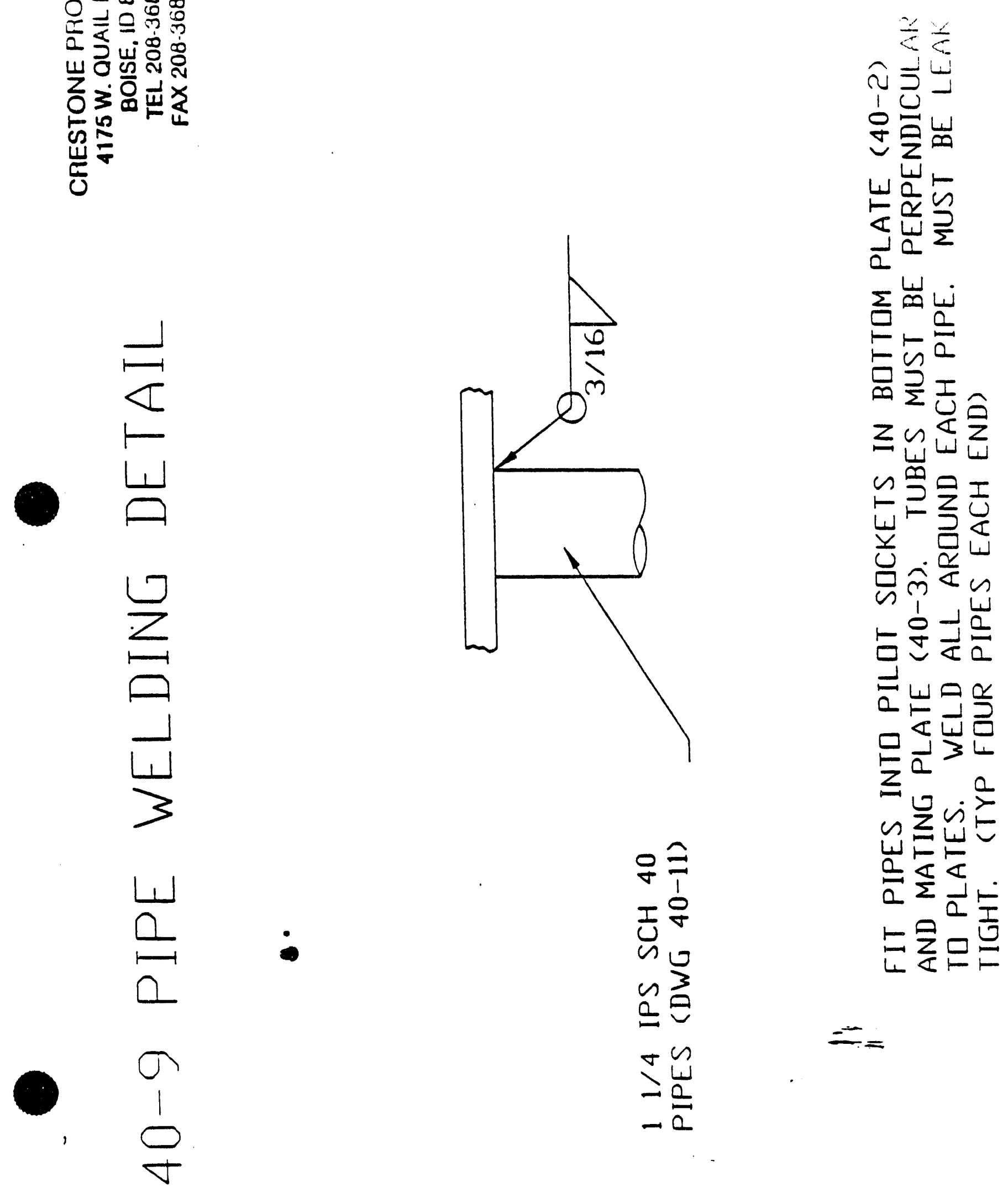

$\pm \frac{1}{6}$

Vol. II, Rev. 0 


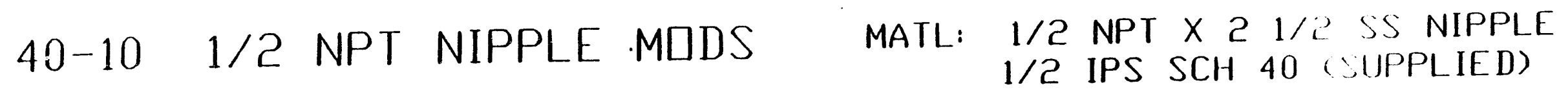

TQL: $. X X \pm .020 \quad . X X X \quad 1.010$

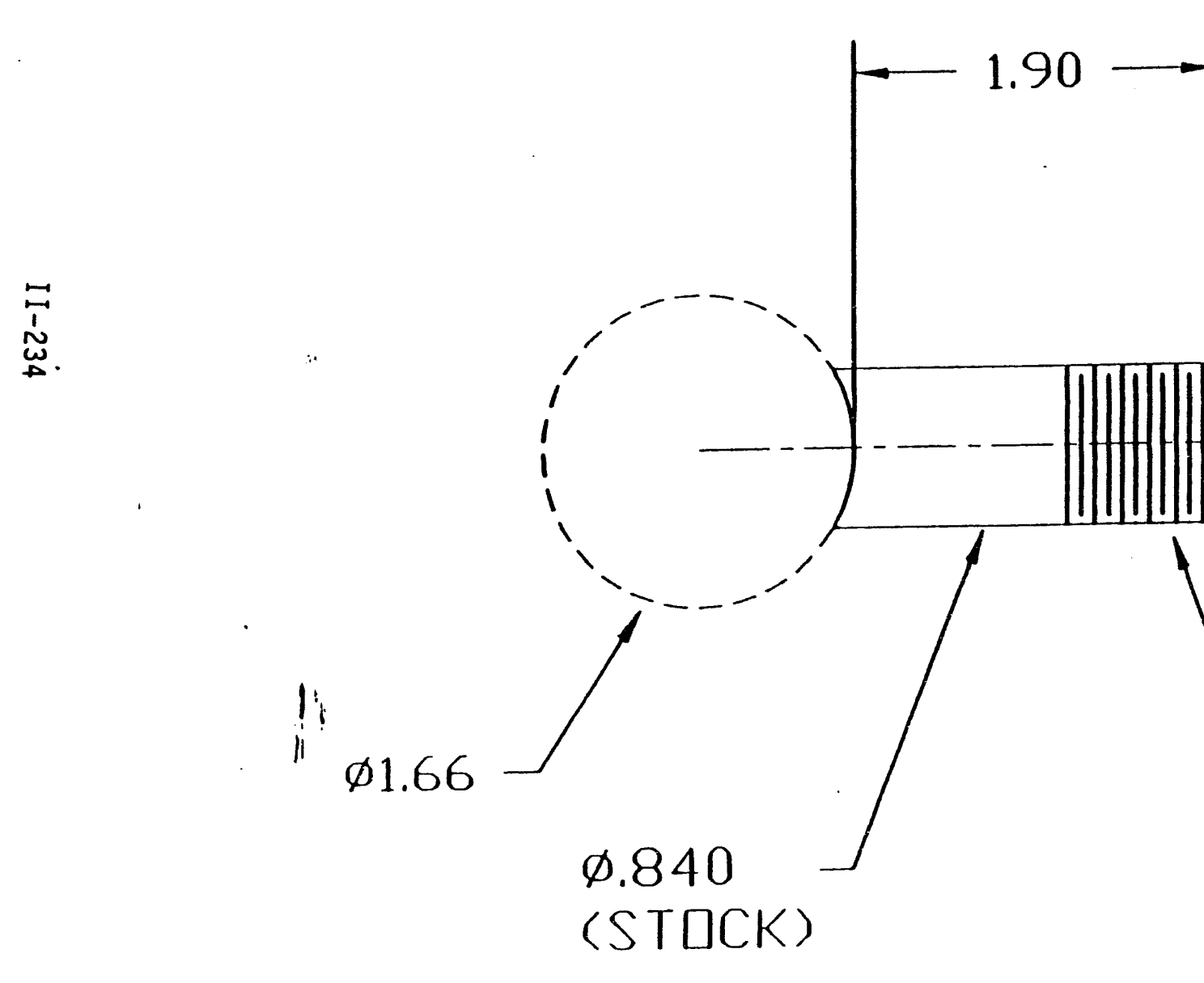

CRESTONE PRODUCTS, INC. 4175 W. QUAIL RIDGE DR.

BOISE. ID 83703

TEL 208-368-7998

FAX 208 368-7999

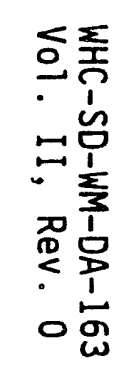

STANDARD

$1 / 2$ NPT THRT ADS (STOCK)

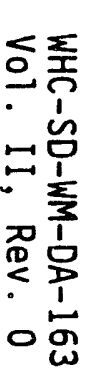




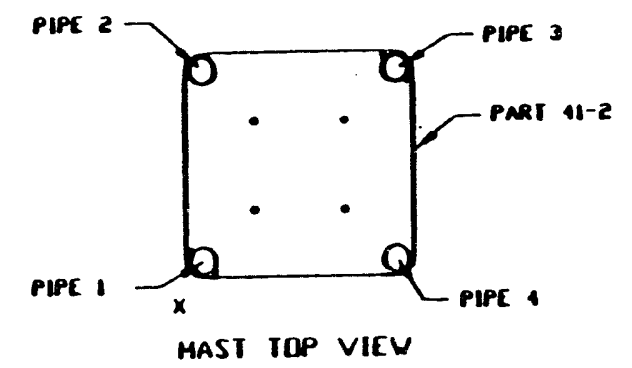
CRESTONE PRODUCTS, INC. $4175 W$. QUAIL. FIDGE DR.
BOISE, 1103703
TEL 208-368-7998
FAX 208-368-7999

$\underset{\omega}{\tilde{\omega}}$

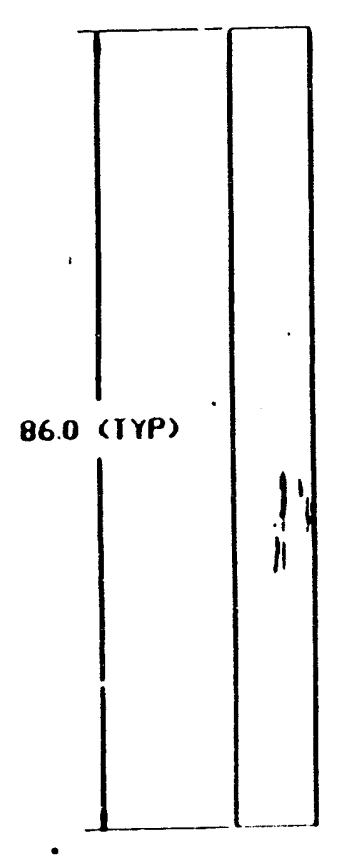

PIPE ।

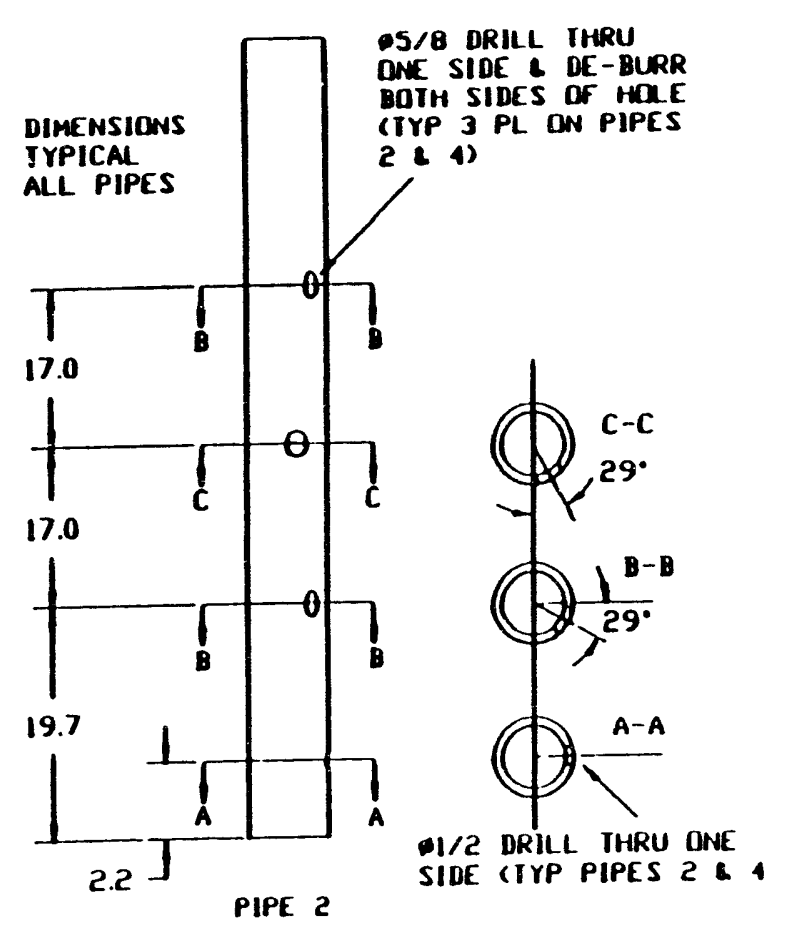

SIDE CIYP PIPES 2 L

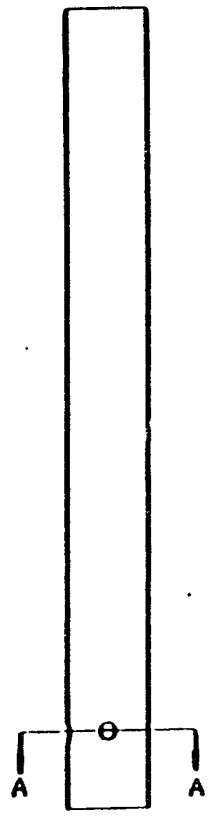

PIPE 3 SIDE (IYP 2 PL 90. APART)

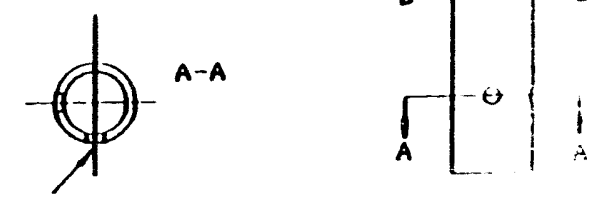

PIPE

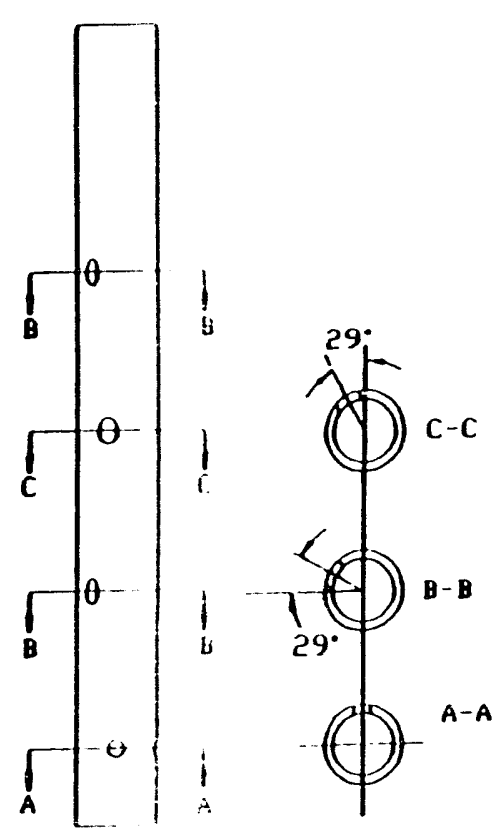

$p$ 

40-12 3/8 IPS GAS PIPE
$\begin{array}{ll}\text { MATL: } & 3 / 8 \text { IPS SCll } 40 \\ 304 & \text { SS PIPE }\end{array}$
TOL: $. X X \pm .020 \quad . X X X \quad \pm .010$

\begin{abstract}
NQTE: MACHINE BQTH ENDS TQ FIT CONTQUR
UF $\$ 1.66$ MAST PIPES
\end{abstract}

i)

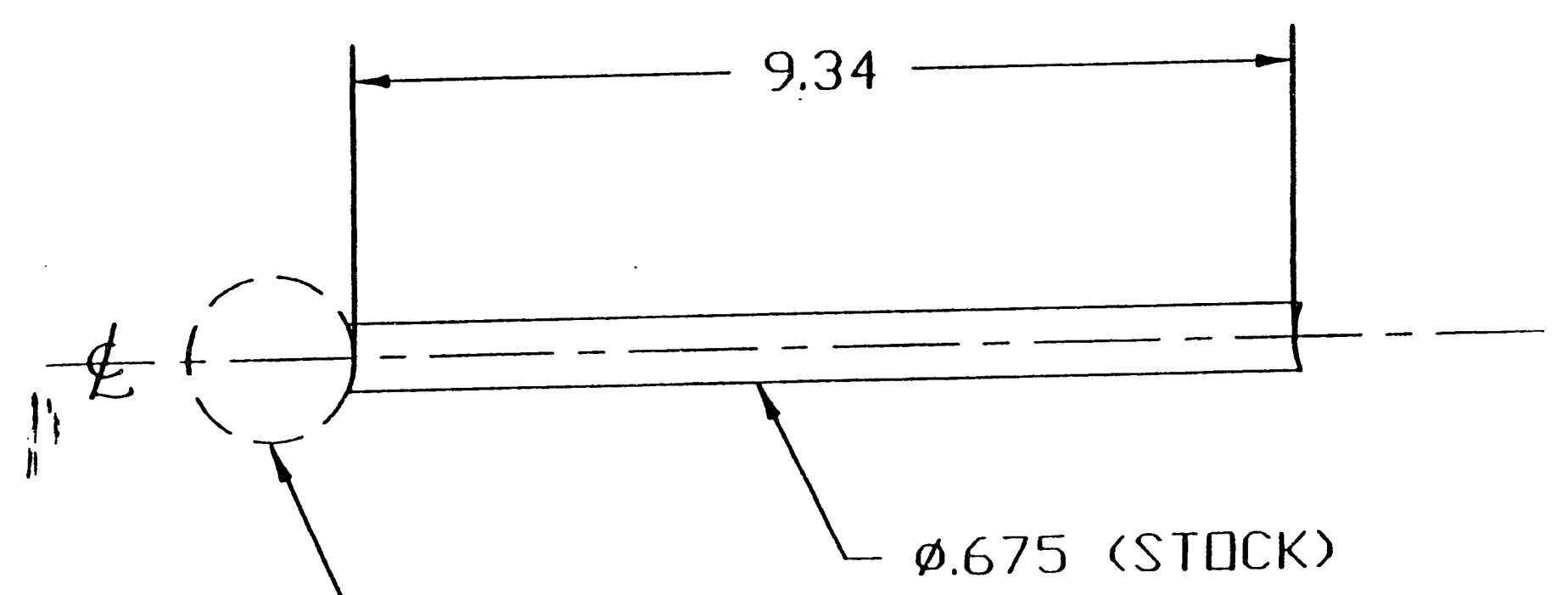

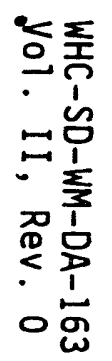

占

$\varnothing 1.66$ (TYP BDTH ENDS)

CNESTONE I HODUCTS, INC. 4175 W. OL; A.IL RIDGE DR. 


\section{0-13 FITTING}

MATL, 300 SERIES STAIIHISS STEEL

TOL, .XX $\quad \pm .020$
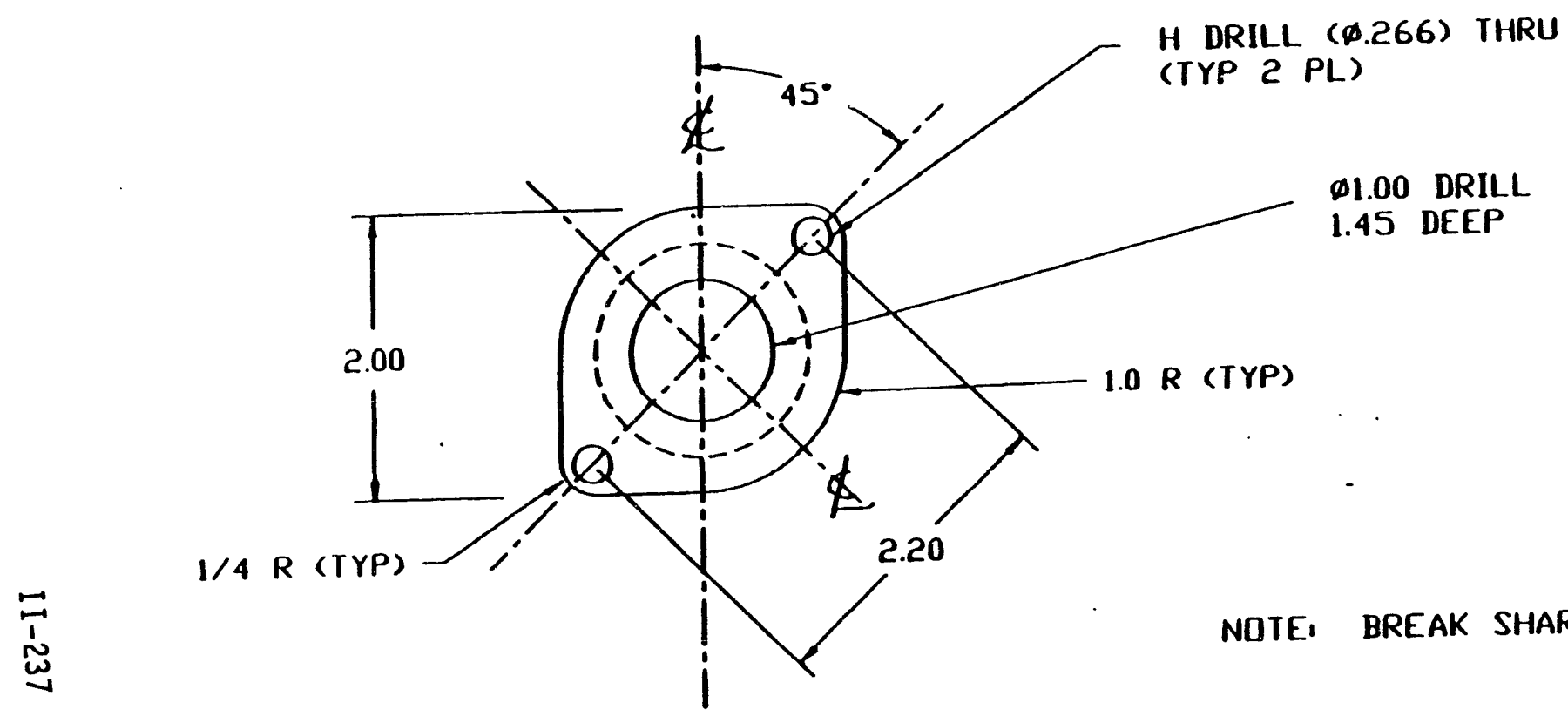

CRESTONE PRODUCTS, INC. 4175 W. QUAIL RIDGE DR. BOISE. ID 83703

TEL 208-368-7998

FAX 208-368-7999

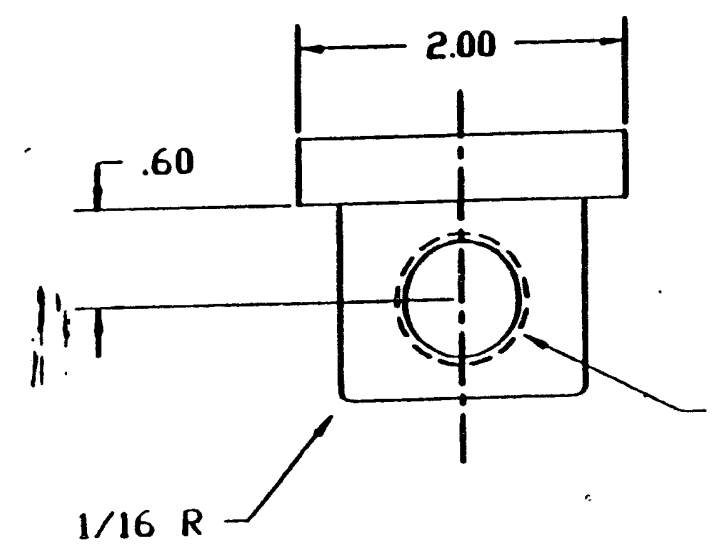

1/2 NPT TAP

THRU DNE SIDE
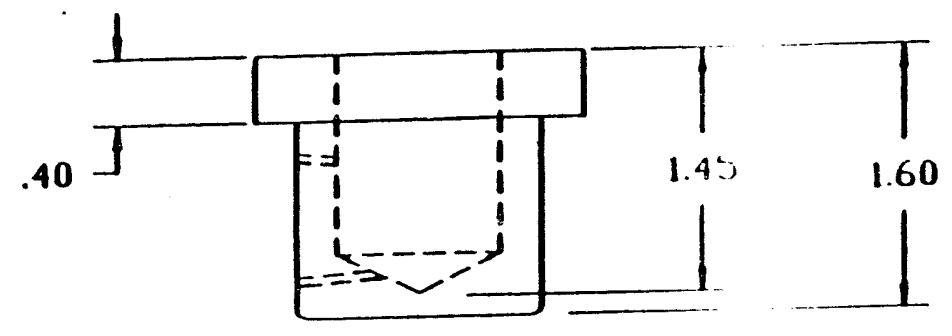

$\mid-\infty 1.50-1$ 


\section{MATERIAL (UR EQUIVAI ENT) \\ TDL: $\quad . X X \quad \pm .020$}

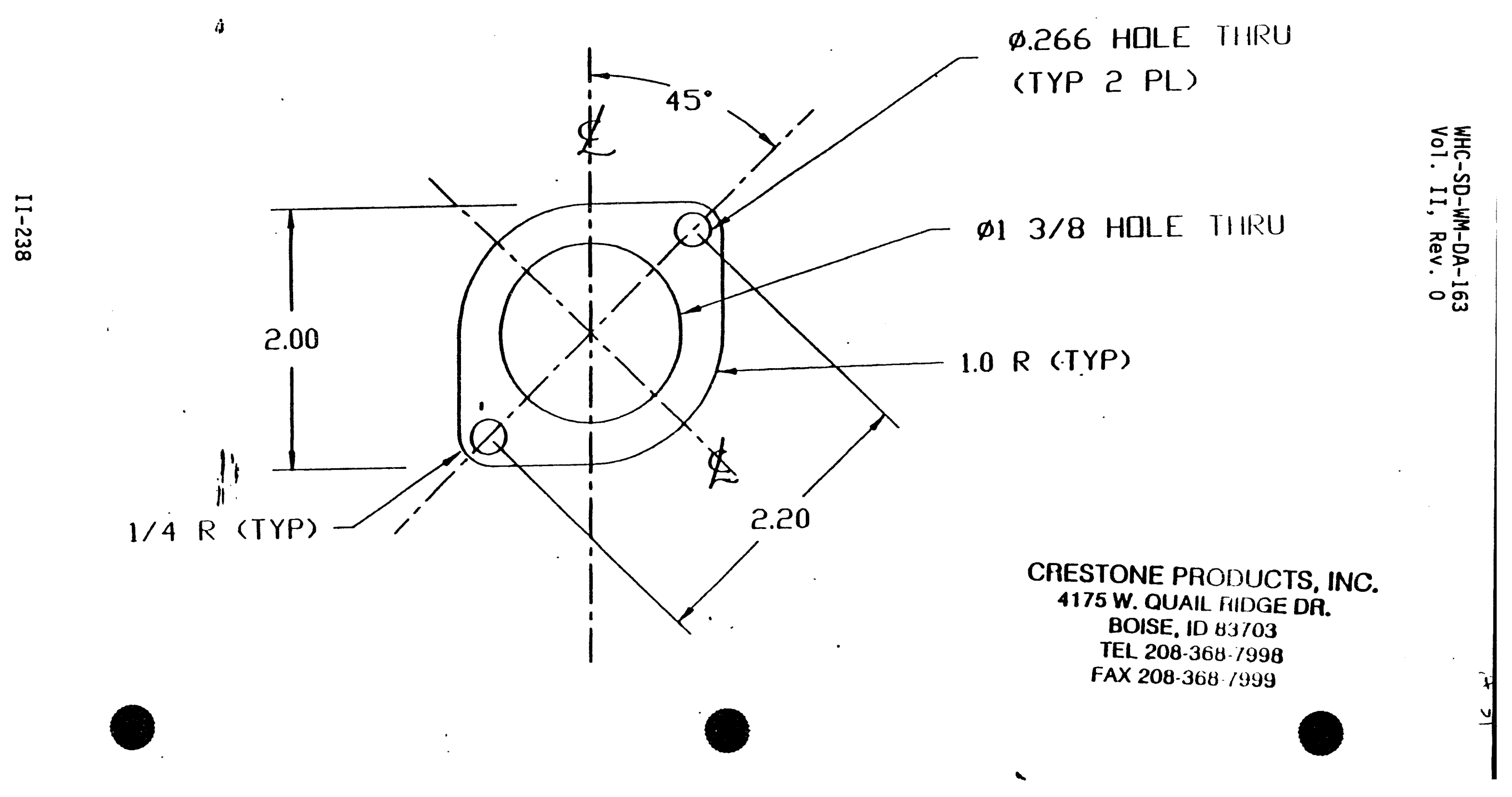




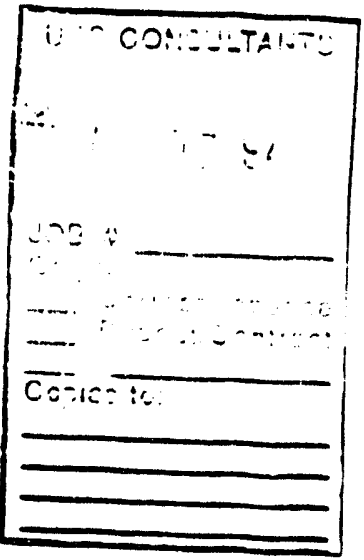

$\begin{array}{ll}41.1 & \text { ROLL ASSEMBLY } \\ 41.2 & \text { BEARING PLATE \#1 } \\ 41.3 & \text { BEARING PLATE \#2 } \\ 41.4 & \text { SIDE PLATE } \\ 41.5 & \text { END PIECE } \\ 41.6 & \text { CAMERA GEAR SPACER } \\ 41.7 & \text { P\&T BACK RING } \\ 41.8 & \text { P\&T OUTPUT SPACER } \\ 41.9 & \text { P\&T SPACER *2 } \\ 41.10 & \text { OELRIN RING } \\ 41.11 & \text { SPROCKET }\end{array}$



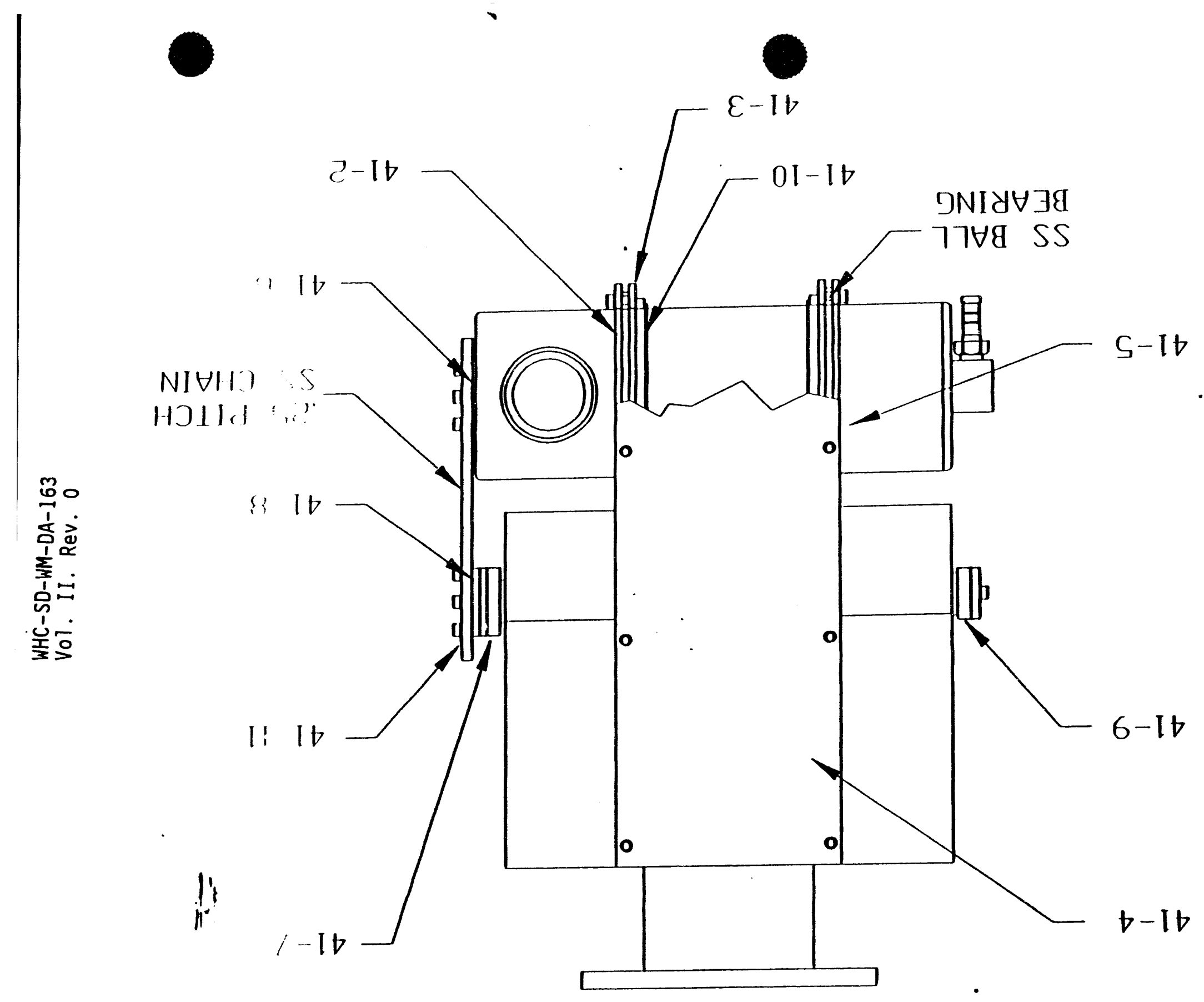

\begin{tabular}{l}
0 \\
\multirow{1}{1}{} \\
1 \\
$\square$
\end{tabular}

666L-89E-80Z XVJ

866L-89E-80Z $7 \exists 1$

cOLEB ol ' $\exists S 108$

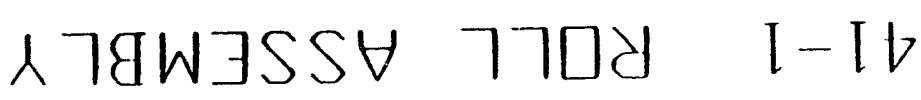
'ONI 'SIONOOHd JNOLS 


\section{1-2 BEARING PLATE \#1}

MATL: ALUM $1,061-T 6$

TOL: $\quad X X \pm .0 i^{\prime \prime} \quad X X X \pm .010$

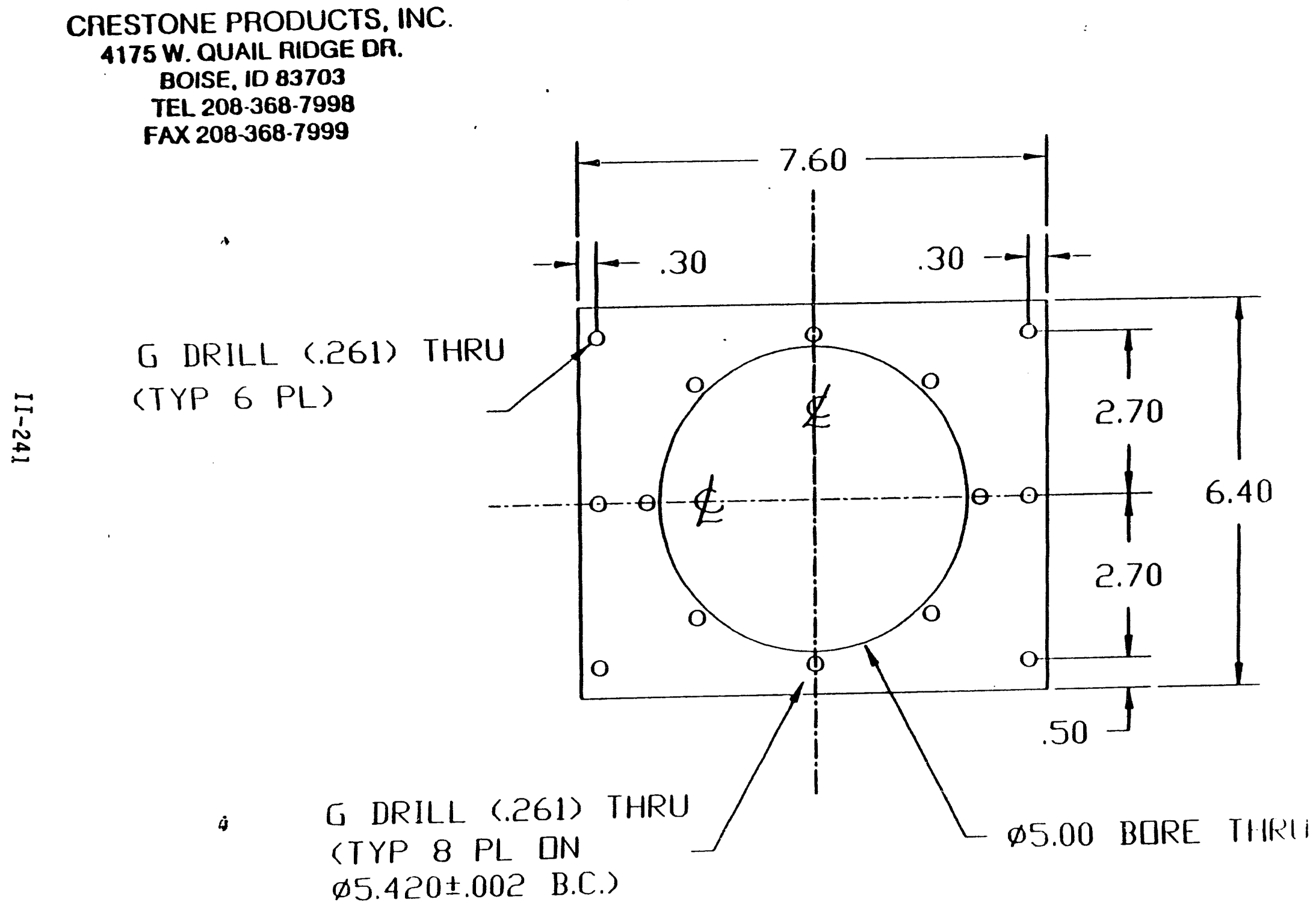

i.

208-368-7998

AX 208-368.7999

G DRILL (.261) THRU 


\section{1-3 BEARING PLATE \#2 \\ MATL: ALUM $1.061-T 6$ \\ TOL: .XX $\pm .11 ' 11 \quad . X X X \pm .010$}

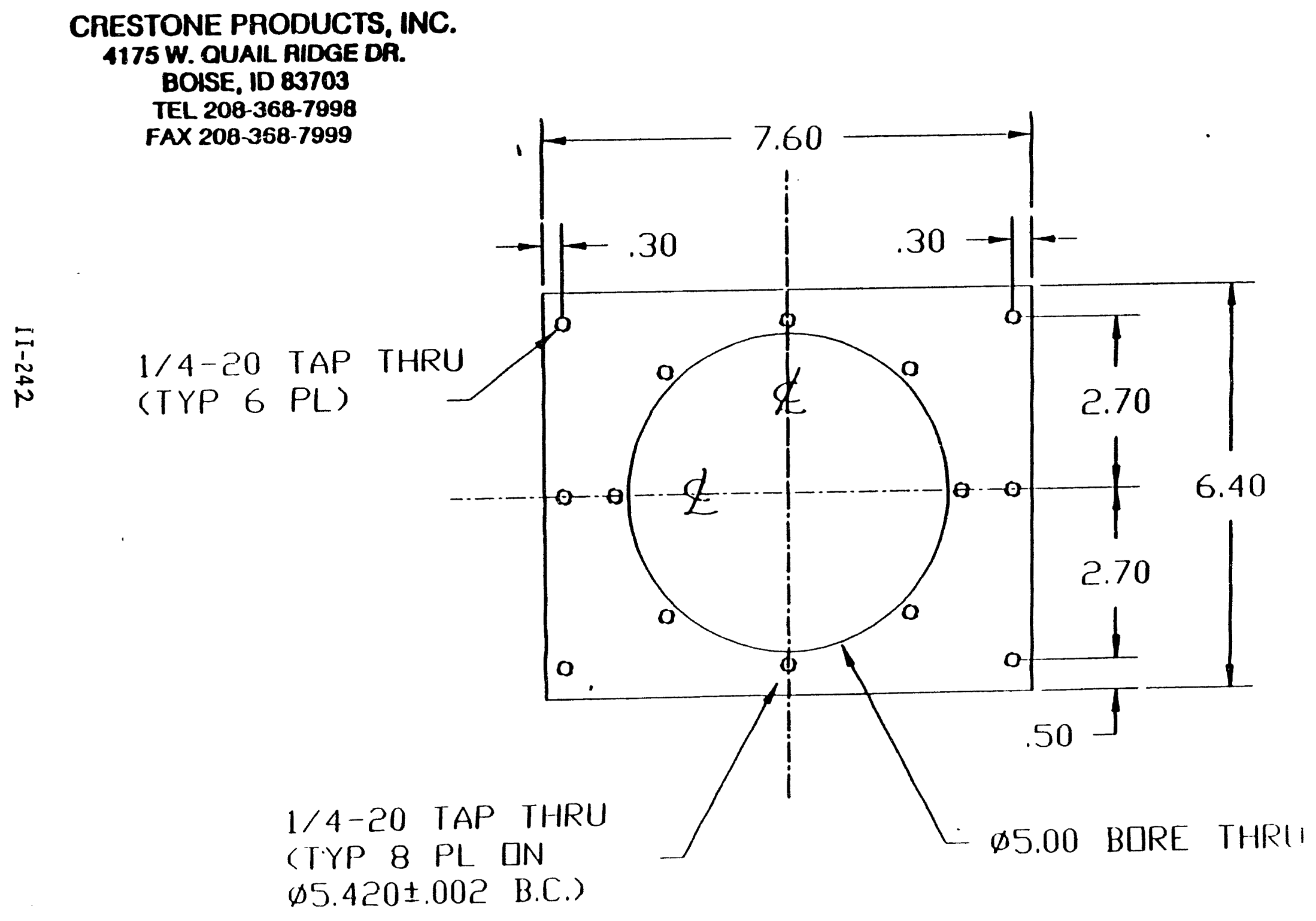

il

TEL 208-368-7998

FAX 208-368-7999

1/4-20 TAP THRU

(T.YP 8 PL DN

$-11-3 / 16$

1/4-20 TAP THRU

$\emptyset 5.420 \pm .002$ B.C.) 


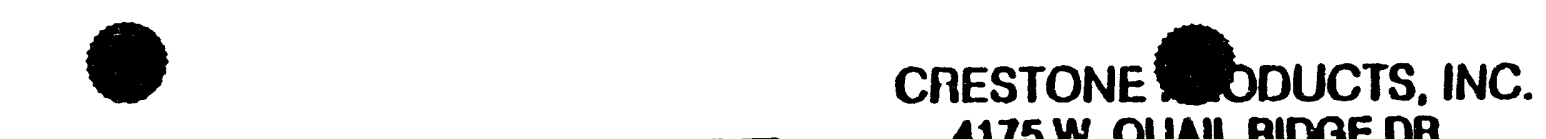

41-4 SIDE PLATE
4175 W. QUAIL RIDGE DR.

BOISE, 1083703

TEL 208-368-7998

FAX 208-368-7999
MATL: ALUM 601.1 16

TOL: $. X X \pm .020 \quad \times X X \pm .010$

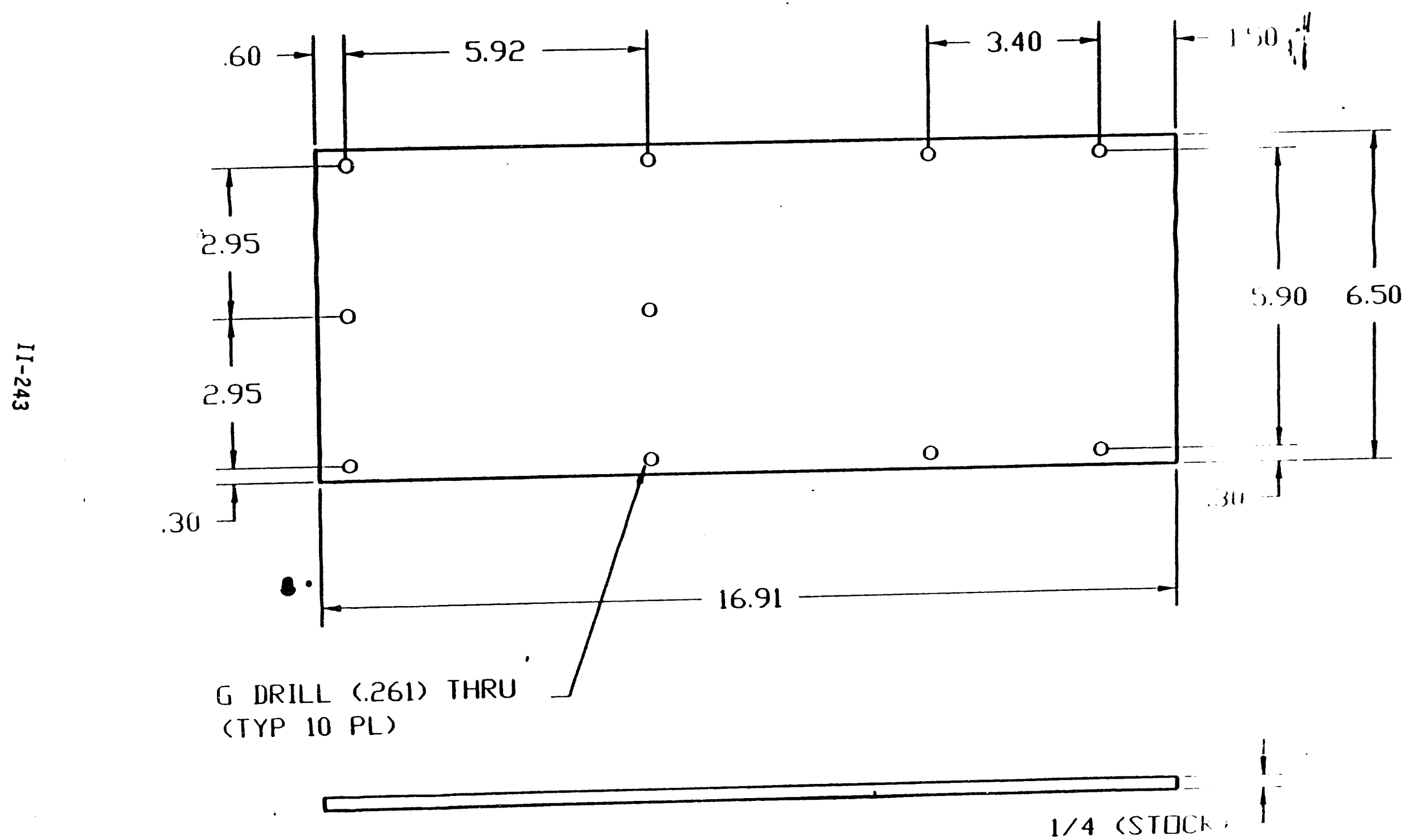

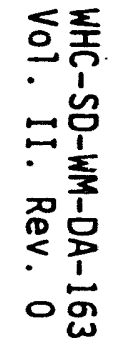


WHC-SD-WM-DA-163
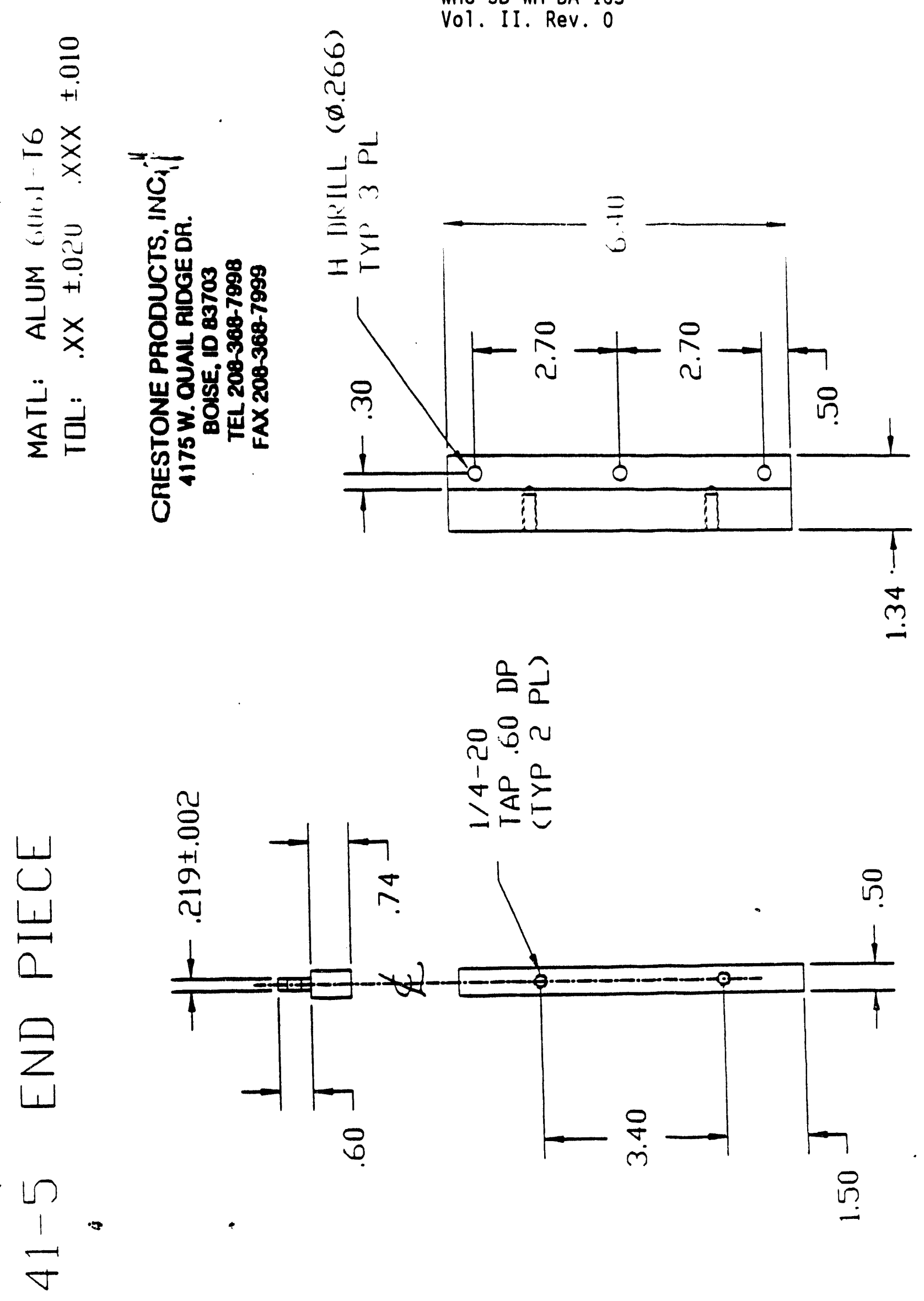


\section{1-6 CAMERA GEAR SPACER}

MATL: $\quad$ ALUM $1.1161-T 6$

TOL: .XX $\pm .020 \quad . X X X \pm .010$
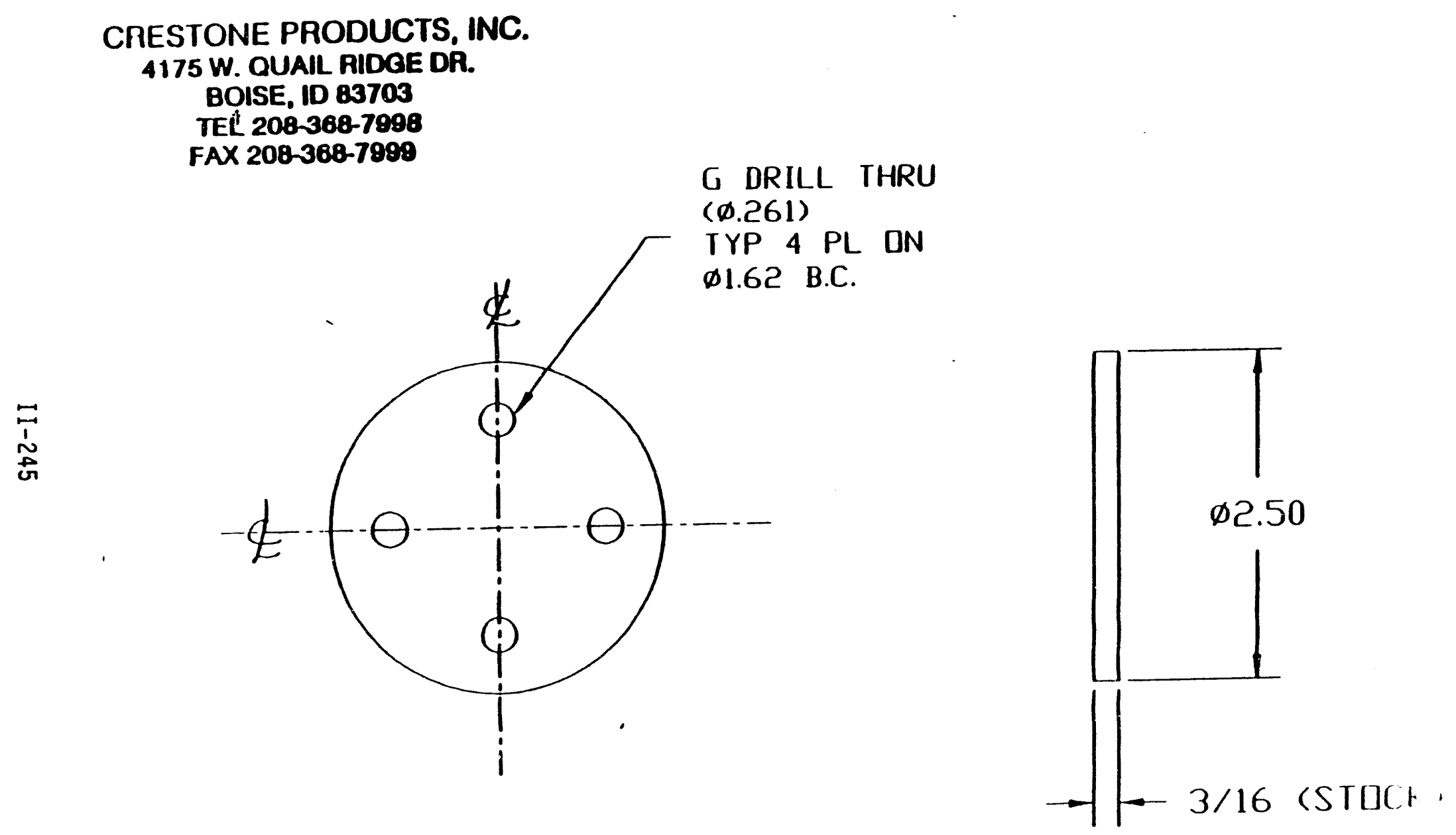

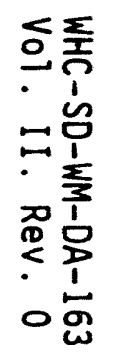



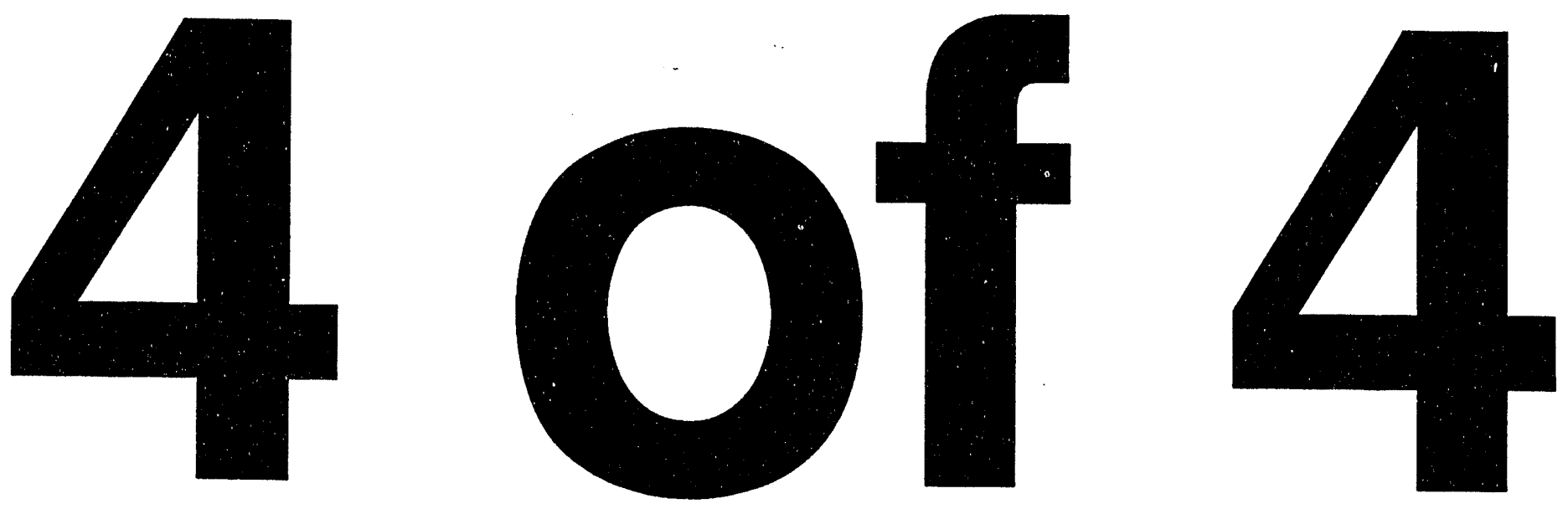


\section{1-7 P\&T BACK RING}

MATL: ALUM $0,061-T 6$

TOL: $\left.. X X \pm .11^{\prime}\right) \quad . X X X \quad \pm .011$

CRESTONE PRODUCTS, INC.

$4175 \mathrm{~W}$. QUAIL RIDGE DR.

BOISE, ID 83703

TEL 208-368-7998

FAX 208-368-7998
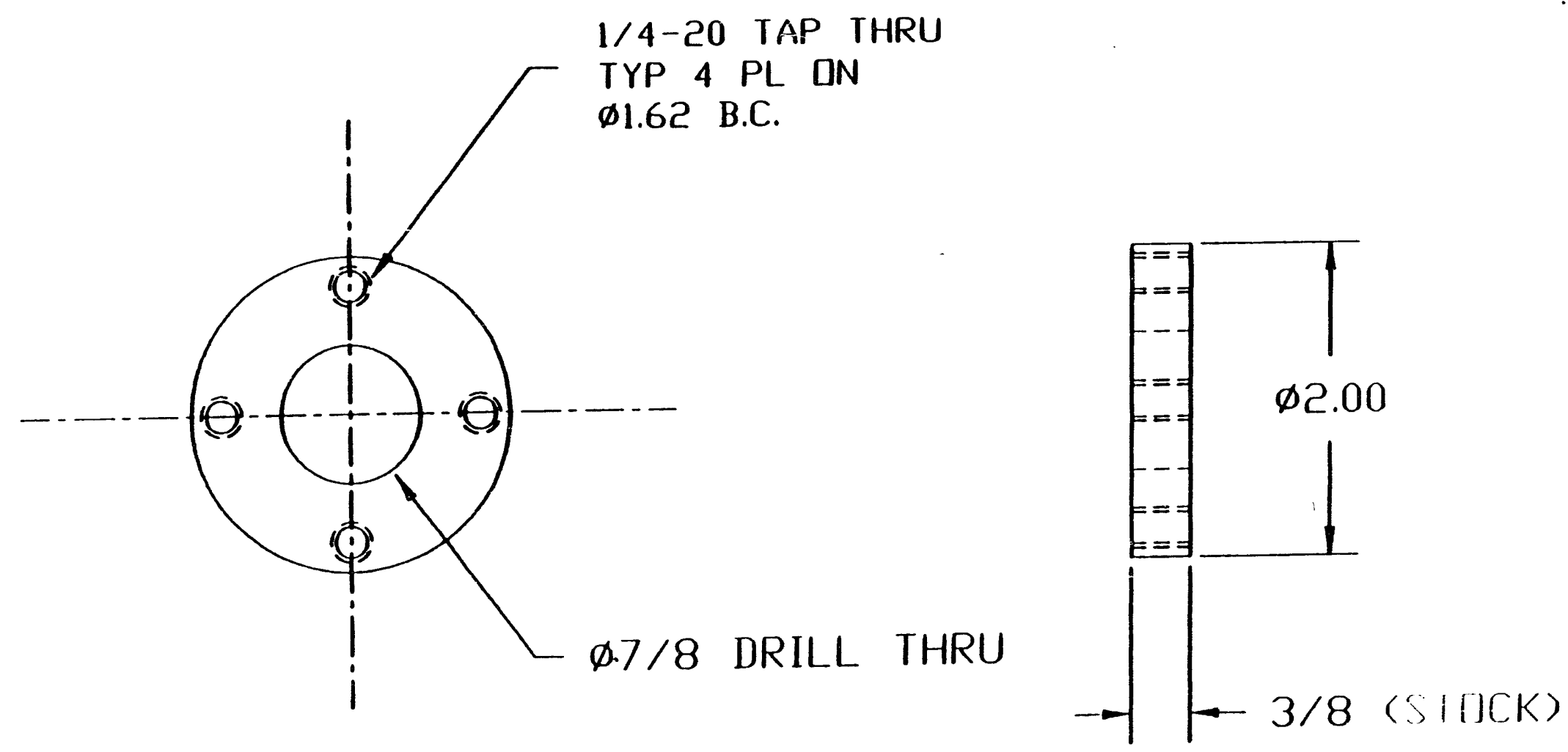

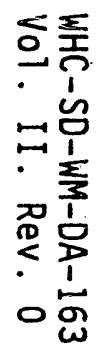


CRESTONE PRODUCTS, INC. 4175 W. QUAIL RIDGE DF.

BOISE, ID 83703

TEL 208-368-7898

FAX 208-368-7999

ڤ્⿱

G DRILL THRU $(\emptyset .261)$
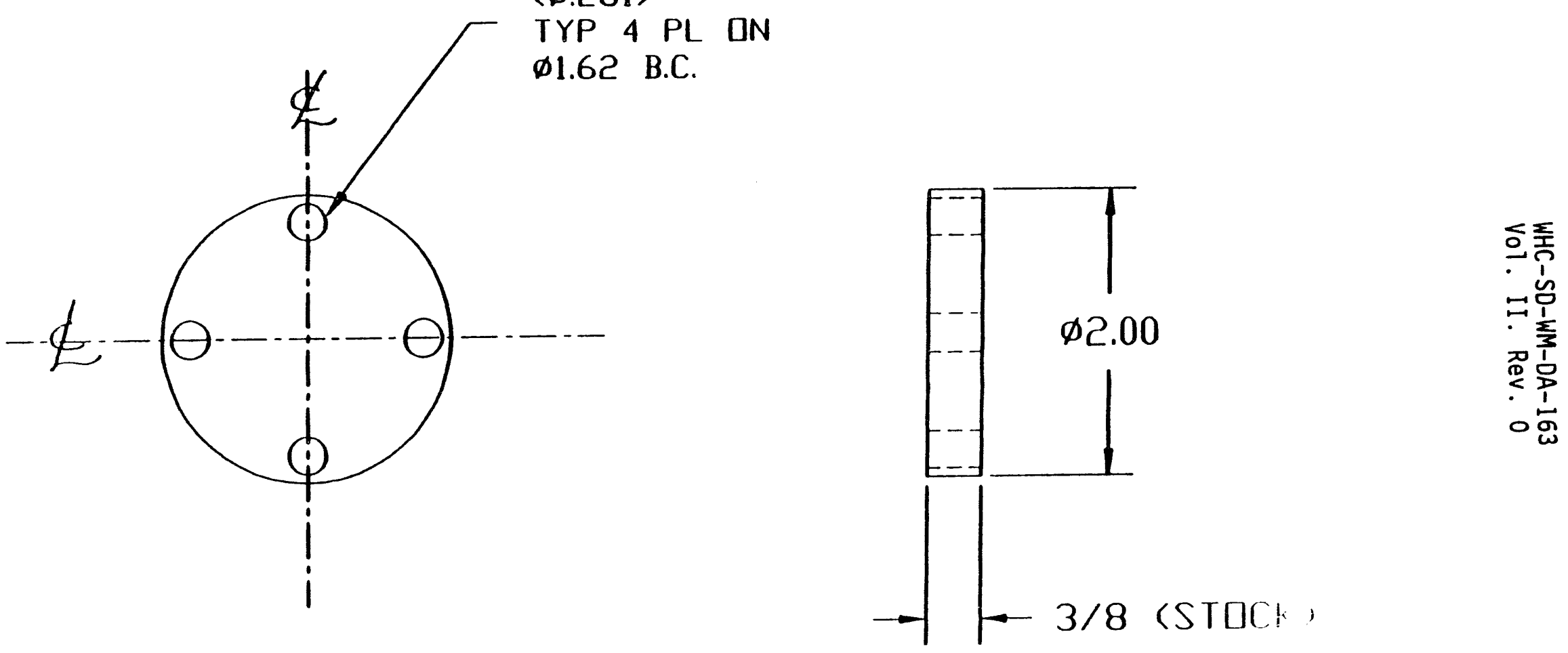


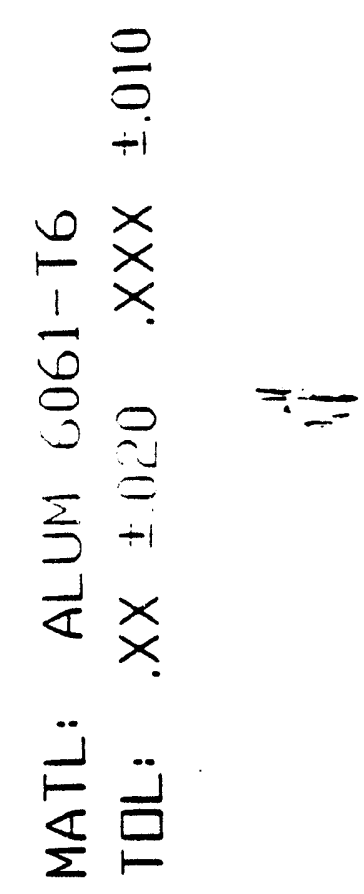

$\sim$

\#

$\frac{\alpha}{4} \frac{1}{4}$

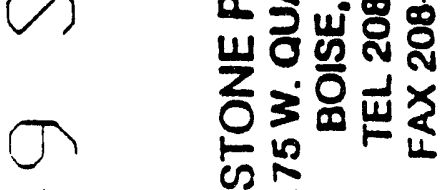

$\frac{1}{\sigma}$

jo

ตั

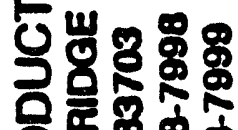

웅혀

둥응

䐆

峞

WHC-SD-WM-DA-163

Vol. II. Rev. 0

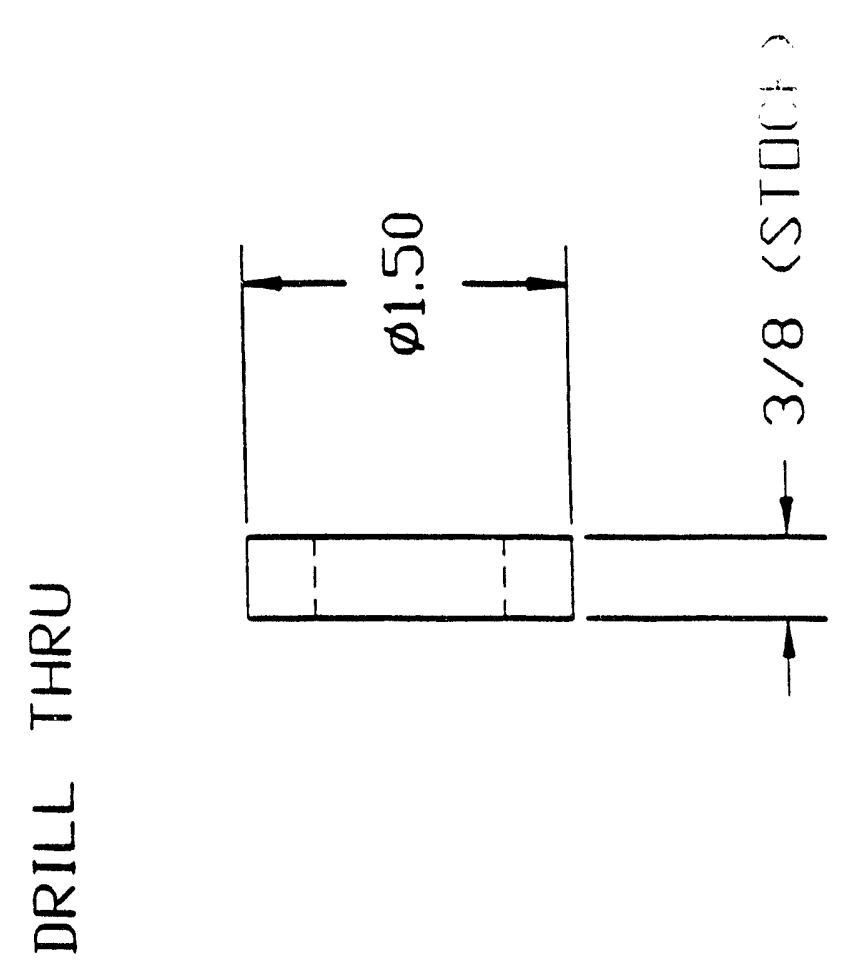

$\frac{1}{2}$

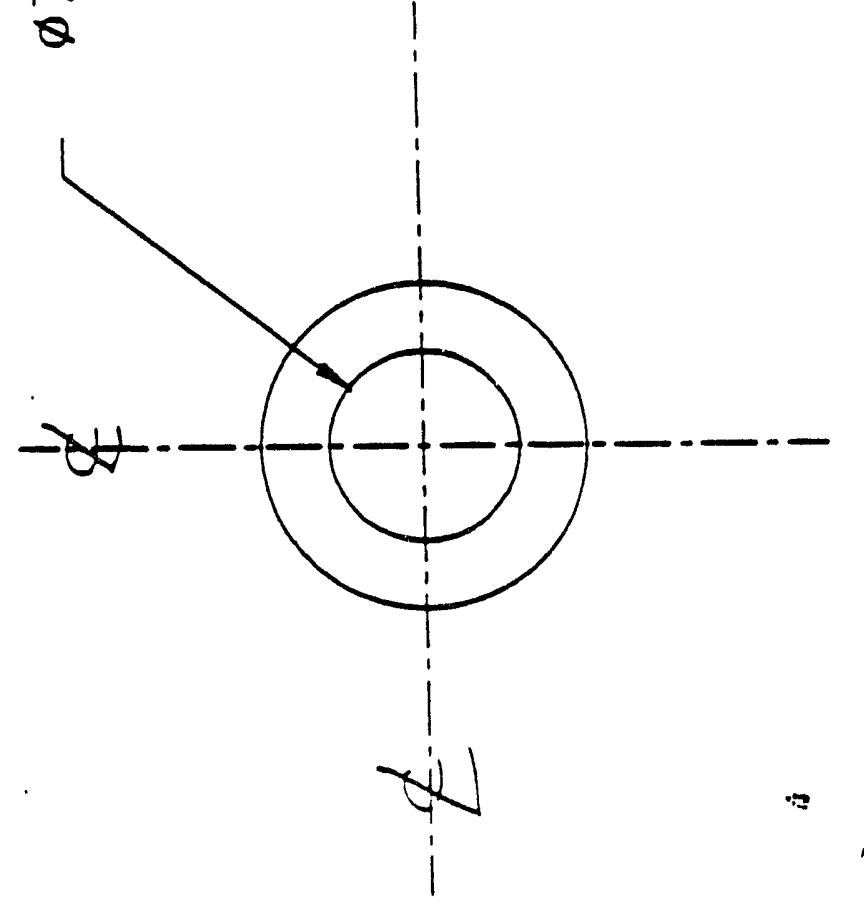




\section{1-10 DELRIN RING}

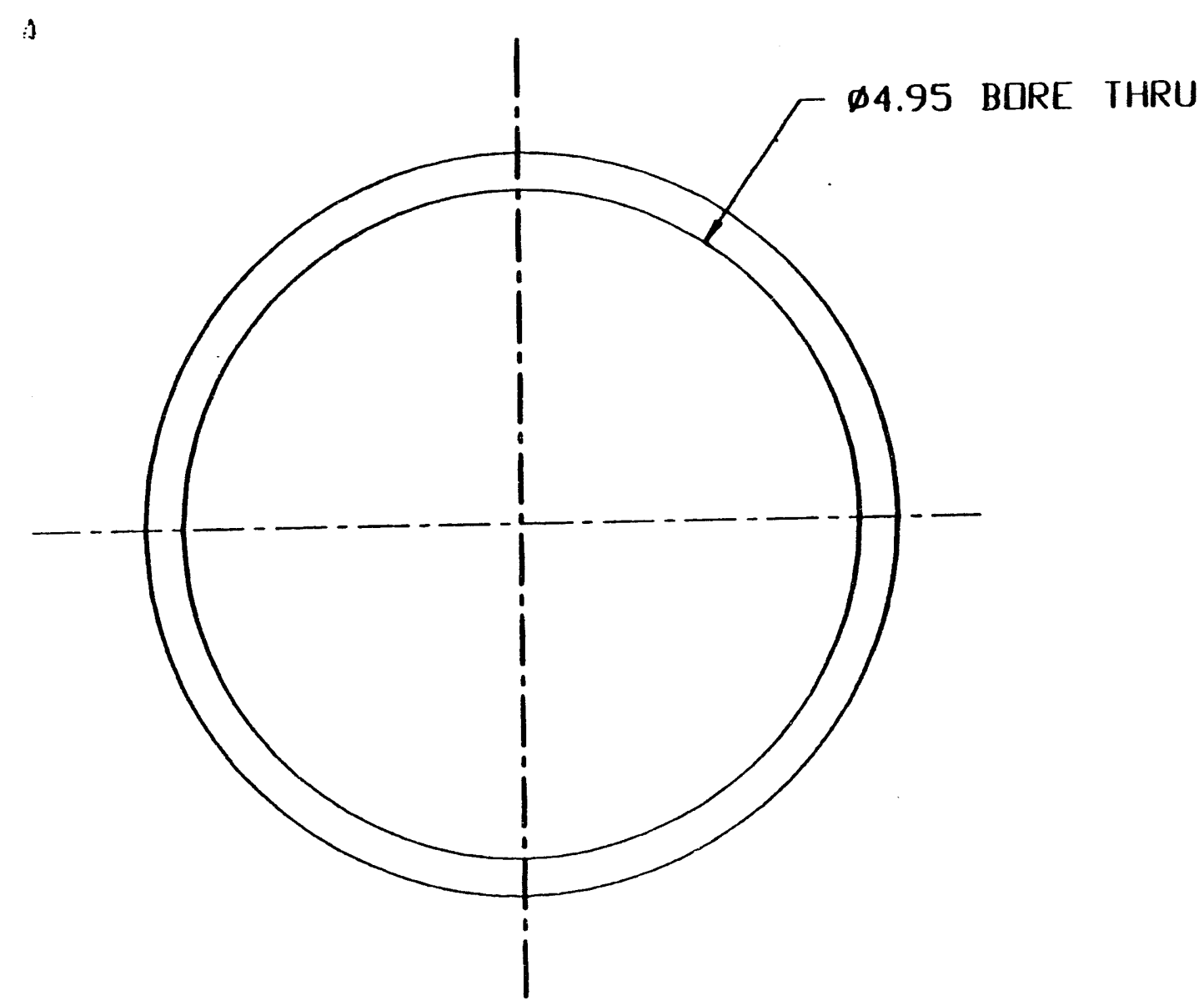

CRESTONE PRODUCTS, INC.

$\triangle 175 W$. QUAIL RIDGE DR.

$$
\begin{aligned}
& \text { BOISE, ID } 83703 \\
& \text { TEL 208-368-7998 } \\
& \text { FAX 208-368-7999 }
\end{aligned}
$$

MAIL, 1/4 HIILK BLACK DELRIN

TOL: .XX 1.0?O .XXX \pm .010

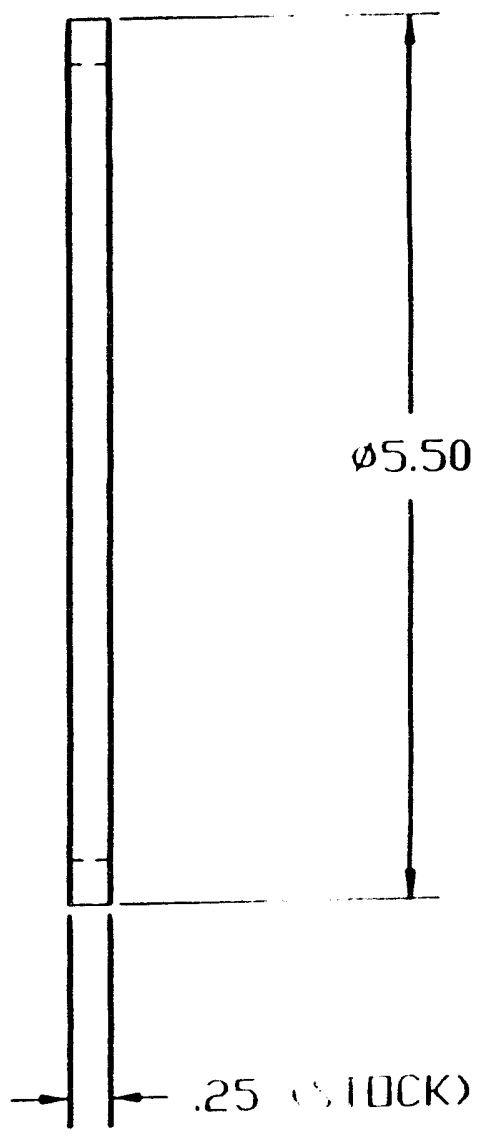


WHC-SD-WM-DA-163

Vol. II. Rev. 0

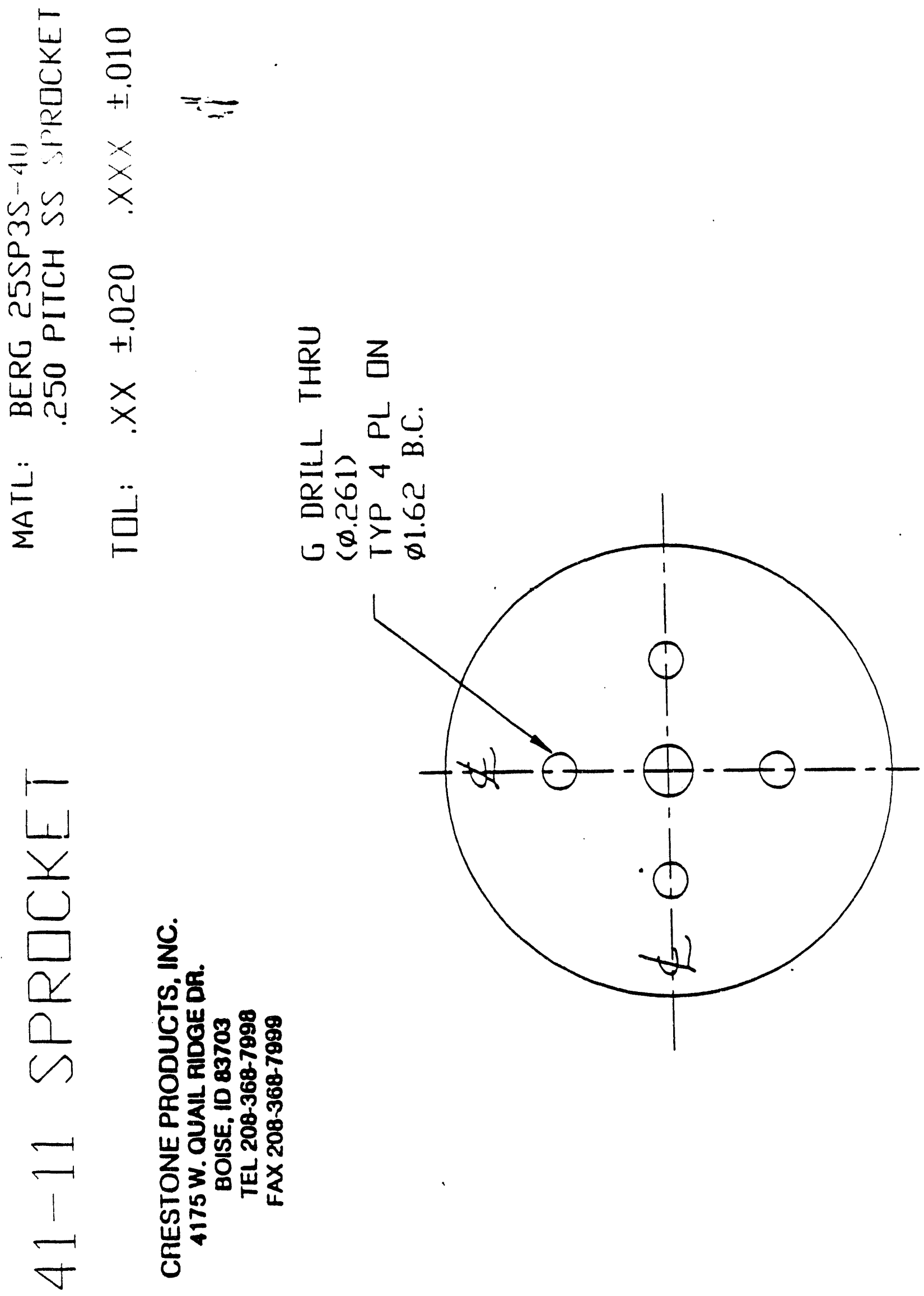




$$
\begin{aligned}
& \text { WHC-SD-WM-DA-163 } \\
& \text { Vol. II. Rev. } 0
\end{aligned}
$$

FAX MESSAGE

$$
\begin{aligned}
& \text { WESTINGHOUSE HANFORD COMPANY } \\
& \text { MECHANICAL ENGINEERING } \\
& \text { PO BOX 1970 MSIN HE-70 } \\
& \text { RICHLAND WA } 99352
\end{aligned}
$$

$4001 / 197$

Date: $6 / 3 / 94$

Number of Pages including this one

4

To: Barron Mosnwoms

Fax Number. $(415) 398-1904$

Telephone:
From: J ann SiREHCUW

Fax Number.

Telephone: (509) $376-5009$

Message:

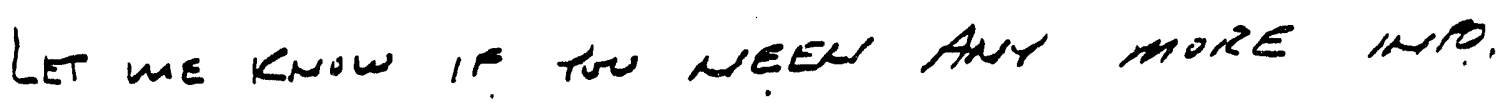

Therese, Some

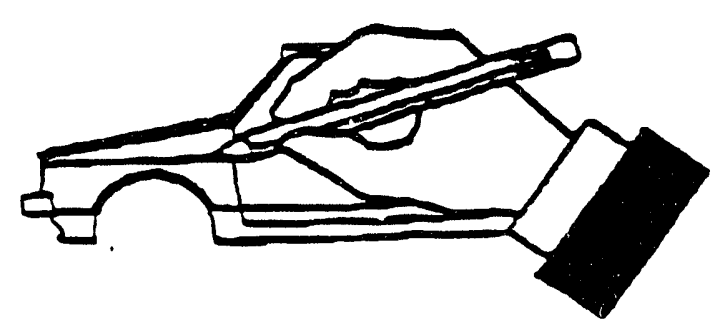

$=$

We're Making Sure!

CC: BM, DKL, FILE $\underbrace{43-66481-01}_{\text {org }}$

II -251 


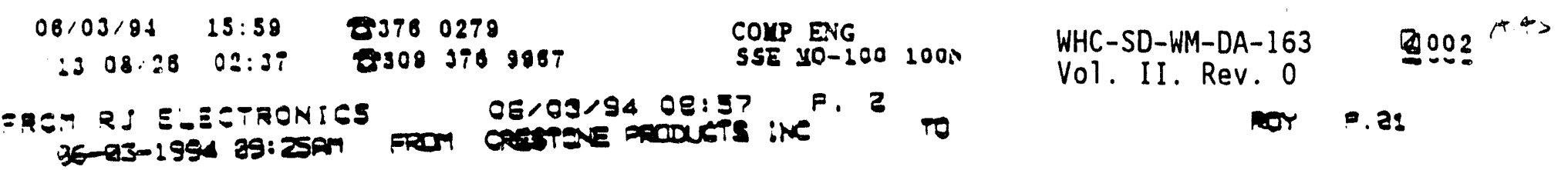

CRESTONE

PaOUETS ING.
4175 W. Qued Rudoo Dr.. Bolad, 1089703

TH (203) 382-7983 312 (203) 333-7999

\section{FAX COVER SHEET}

Po:Bov

Tal No.:

Company:Awh Elnamator

Dato: Jun 3.1994

Number of poges inchellng thls sheor:?

Prom:_ruen

Hoy:

Hore is Informaton regarding equedons on the uppar mases syoum for 241 -AW. 101:

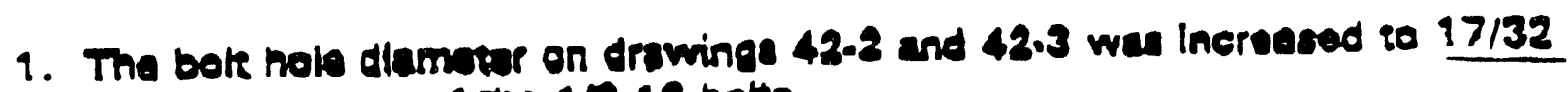
(.538) for Gloaranes of the 1/2.13 botts.

2. The muturial for the manifold tube (drawling 42.0 ) has been ohanged from 17.4 PH sealinlasestool to 304 sealinless seod.

3. The wold ormbols cre aceording to Amortean Nedlond Stundard ANSWAWS A2. 4.79

4. The abouede trie for all molde is ASTM 30th.

5. Maturial for the shdeding plate is struerural eapoon sted ASTM A.38 with a yleld strength of 38,000 ail and tensile strength of 58,000-80,000 psi.

8. Matarial for the manlfold iube is 304 tainless sted ASTM g-312/5A-312. 304 s9 has a yidd strength of epproximateh 35,000 psl and tensite strenget of approximataly 85,000 pol.

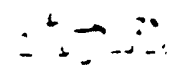

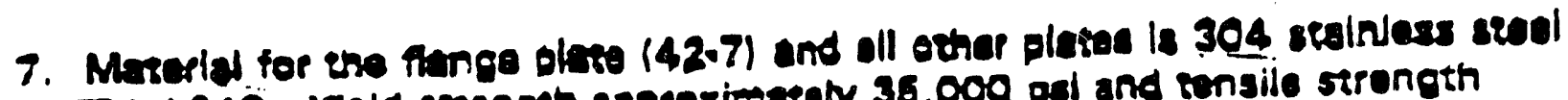
ASTM A24Q Yld strenght approximarely 36,000 pol and rensile strongth appreximativ 88.009 pol. 


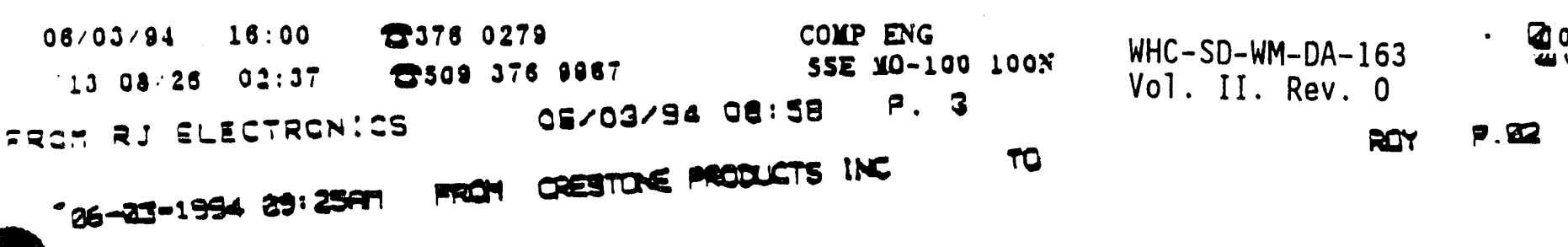

8. Th $1 / 2-13 \times 5$ inch boles usod to retain the ohiolding plase are 18.8 sealniass stool wien an folnimum isnjils seponget of 73,000 pai lorderad from MeMaster Carr).

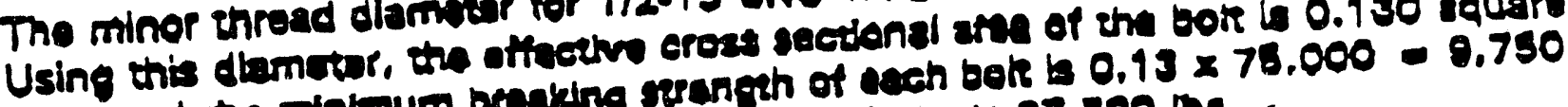

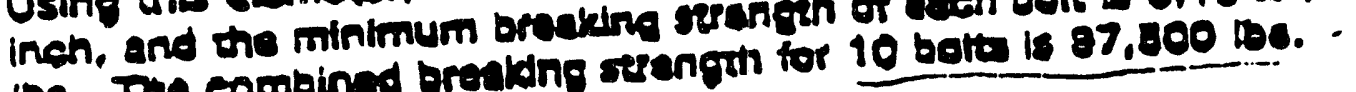

1BS. The combinad brealang seph

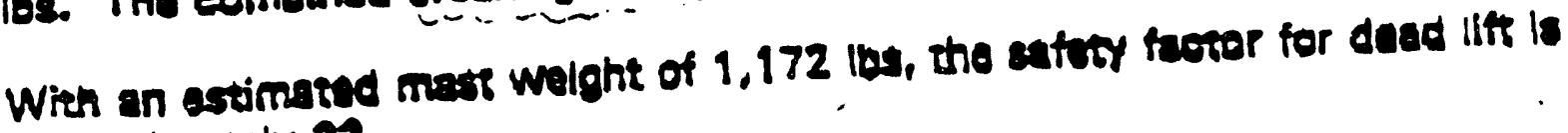
approximately 83.

Bese rogurds.

Bruce 


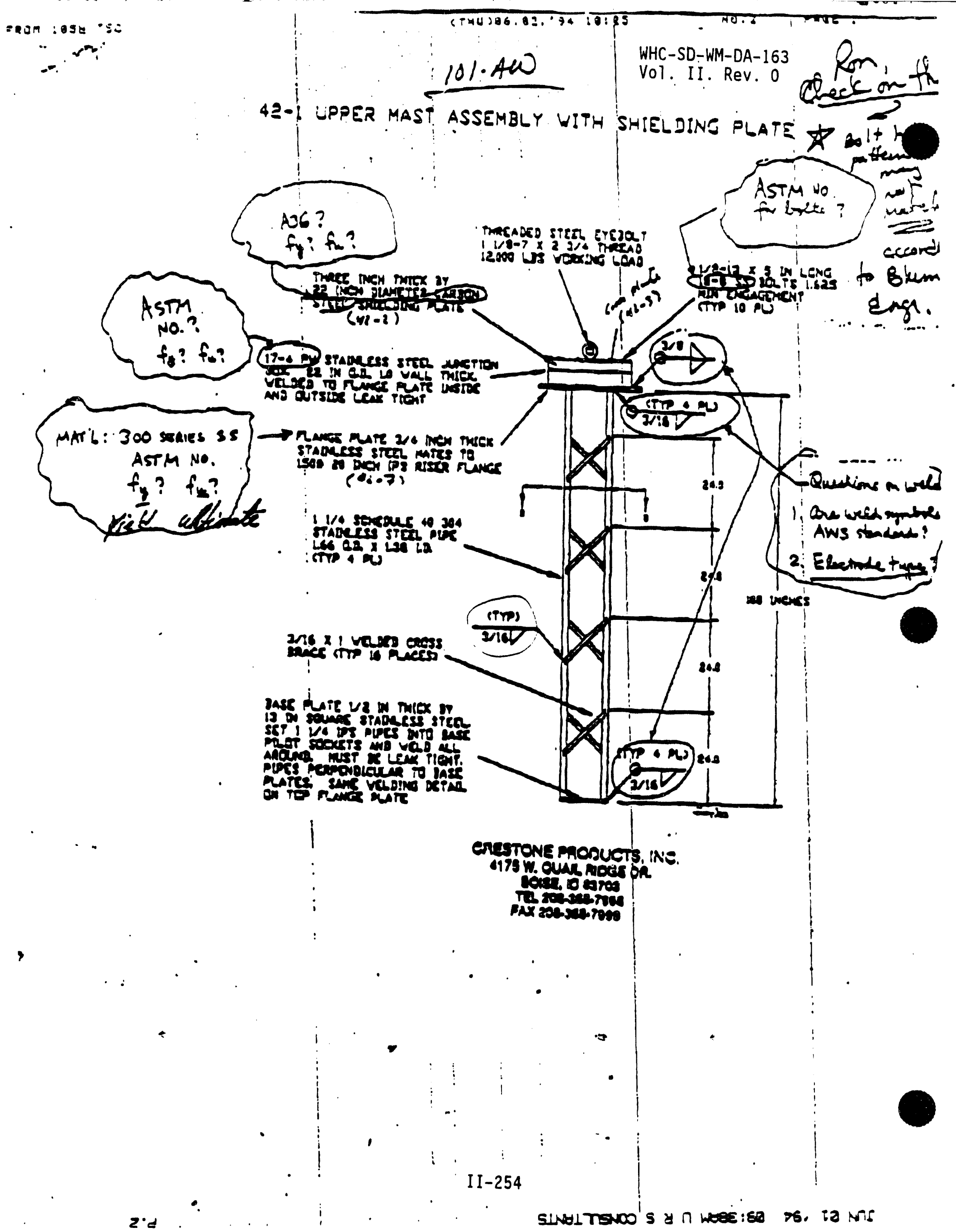


WHC-SD-WM-DA-163

Vol. II. Rev. 0

\section{ATTACHMENT B}


WHC-SD-WM-DA-163

Vol. II, Rev. 0

This Page Intentionally Left Blank

(Attachment B Not Used)

$\dot{0}$ 
WHC-SD-WM-DA-163

Vol. II, Rev. 0

ATTACHMENT C 


\section{URS Consultants URS/John A. Blume \& Associates, Engineers \\ WHC-SD-WM-DA-163}

Job no. $66458-01$ Job WHC/MPF ANALYSis

Client

Subject AlLONABLE STRESSES
Sheet No.

Colc. No.

Rev. No.

By PrL Date $\frac{5 / 31 / 94}{7 / 13 / 4}$

Cnk'd He

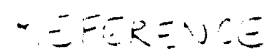

DEZIGN =AzRICATION $\times$ ERECTION", AMERICAN UT unal BTHDARD.

ANSI/AISC N690.1984

\section{ALOUABLE STRESSES}

STRUCTURAL STEEL (SECT Q1.5.1)

TENSION. $\quad F_{t}=0.6 f_{y}$ on gross area

$F_{t}=0.5 f_{u} \quad$ on effective net area

$F_{t}=0.45 f_{y} \quad$ for pin-connected members

SHEAR: $\quad F_{v}=0.40 f_{y}$

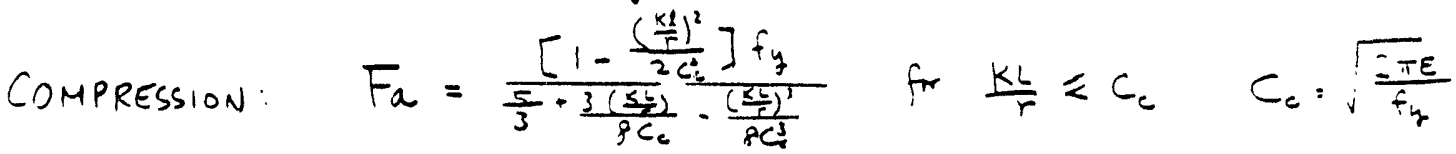

$F_{a}=\frac{12 \pi^{2} E}{23\left(\frac{K L}{T}\right)^{2}} \quad$ ir $\frac{K L}{r}>c_{c}$

BENDING: $\quad F_{b}=0.66 f_{y}<0.7 f_{u}$ COMPACT SECTION

$F_{b}=0.6 f_{y}<0.7 f_{u}$ non-compact section

$F_{b}=0.75 f_{y}$ Comeact, DENDing in minde Axi:

STAINLESS STEEL (SECT Q/59)

$$
\begin{array}{rlrl}
\text { COMPRESSIOU: } & F_{a}=\frac{f_{y}}{2.15}-\left(\frac{\frac{f_{y}}{2.15}-6}{120}\right) \frac{k l}{r} & \text { fr } \frac{k l}{r} \leqslant 120 \\
F_{a}=12-\frac{1}{20}\left(\frac{k l}{r}\right) & \text { fr } \frac{k l}{r}>120
\end{array}
$$

$=$

ENSION, SHEAR, BENDING sme as above for structural steel 
URS Consultants WHC-SD-WM-DA-163 URS/John A. Blume \& Associates, Engineers Job no. $=6458$-0l Job WHC/MPF ANALYSIS Client

Subject LOAD IOMBINATIENS

Sheet No. Cálc. No. Rev. No.

By $\frac{\text { Phe Date } \frac{1}{6 / 1 / 94}}{\text { Chk'd } 1 / 1 .}$

-OAD SOMBIUATIOU a STRESS - MIT COEFF TABLE QIS71)
NORMAL
$1.0 \times D L$
$D L=$ DEAD LOAD
EXTREME
$1.6(D L \pm E Q)$
$E Q=$ SEISMIC LOAD
1.4 (DL $\pm E Q$ ) for SHEAR \& BOLTS 
WHC-SD-WM-DA-163

Vol. II, Rev. 0

ATTACHMENT D 
WHC-SD-WM-DA-163

Vol. II, Rev. 0

May 25,1994

Kevin $M$. Jones

Westinghouse Hanford Company

P.0. Box 1970 MSIN H5-56

Richland, Washington 99352

Bahram Mosaddad

URS Blume and Associates

100 California St., Suite 500

San Francisco, California $94111-4529$

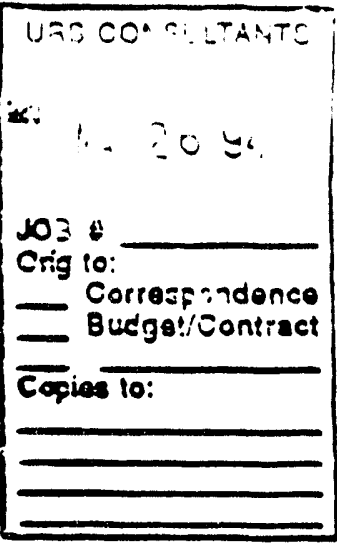

Mr. Bahram:

This package contains the information requested by URS Blume for the mulitport flange and video camera analyses. The comments that follow address specific questions and the following 1 ist describes the information included in this package.

Sincerely,

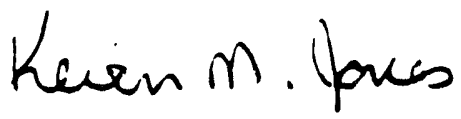

Kevin M. Jones

Engineer -- Component Stress Analysis 
WHC-SD-WM-DA-163

Comments:

Vo1. II, Rev. 0

1. The material called out for the risers on tanks 241 AWl01 and -SYl01 are given on drawings $H-2-70420$ and $H-2-37792$, respectively. The material for the riser is ASTM A134 A283 Grade A Carbon Steel. The specification is clear on drawing H-2-70420 for AW101. Although it is unclear on S101, it is the same material and grade.

2. The length and, or elevations for the 42 in piser on fiwlol will nave :a be determined. As per drawing H-2-70396 only the top elevation is specified. For SYlOl the length is specified on drawing $\mathrm{H}-2-37776$ and the elevation of the top of the riser is specified on drawing $\mathrm{H}-2-72213$ which is included in this package. Drawings H-2-70396 and -37776 were previously provided.

3. The estimated weight of the Multiport flange will be provided ASAP.

4. The weights for the different camera components are as follows:

The weight of just the camera and the pan and tilt which are bolted to the bottom of the camera mast assembly is estimated at 128 lof.

The weight of the camera structural assembly is estimated at 1099 lbf.

The weight of the entire video camera to be placed in tank 241 AW101 is 1227 ibf.

5. In response to the facsimile from Peter Lum asking for the size and description of the part labeled 40-2 on sheet 40-6 of the drawings for the lower mast of the camera assembly, the part should be labeled $40-12$ which is gas piping and is clearly described on sheet 40-12.

6. For the multiport flange both 304 and $304 \mathrm{~L}$ stainless steel are called out. The lower yield strength of should be used.

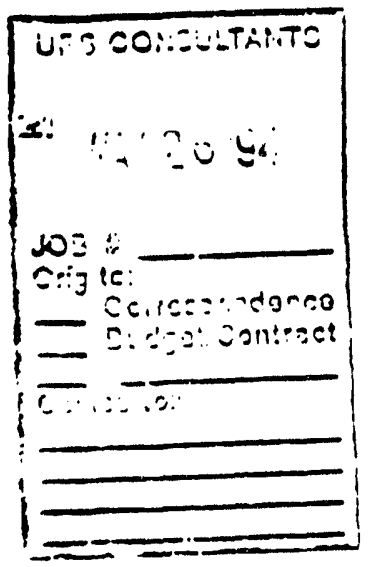




\section{Item $\quad$ Item Description}

Vol. II, Rev. 0

1 .

2.

3.

4.

5.

6.

7.

8.

9.
Tank 241SY101 and -AW101 tank section drawings showing tank dome information (Drawings $\mathrm{H}-2-37706,-37707,-37708,-37772,-70307$, $-70308,-70309,-70394)$.

Orawing $\mathrm{H}-2-37778$.

An information sheet from the manufacturer giving the strengths : the weld studs. The construction specification sent earlier gives the type of anchor bolts (H4L).

A copy of the weld specification HS-VS-0013.

Drawing $\mathrm{H}-2-72213$.

Pressure time history information for the 1.25 MEB Hydrogen burn event.

7. Displacement time history information for the 1.25 MEB Hydrogen burn event.

Seismic time histories. (On $31 / 2$ in. diskette)

The current Multiport flange drawings.

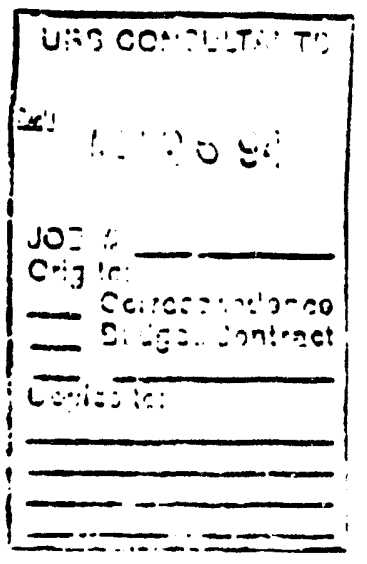


WHC-SD-WM-DA-163

Vol. II, Rev. 0

ATTACHMENT E 
URS Consultants

URS/John A. Blume \& Associates, Engineers

WHC-SD-WM-DA-163

Vol. II, Rev. 0

Job \#: 66481-01 Job: $\quad$ Structural Analysis for MPF Riser Assembly

Client: WHC

Subject: $1.25^{\circ} \mathrm{MEB}$ Hydrogen Burn Analysis

Sheet No. $=.1$

By

Chk'd RIK Date $8 / 16 / 9$ -

The following table contains a list of the "As-Built" drawings for the Crestone Camera These drawings were recelved by URS on $8 / 1 / 94$

\begin{tabular}{|c|c|c|c|c|}
\hline \# & $\begin{array}{c}\text { Drawing/Part } \\
\text { Number }\end{array}$ & Drawing Name & $\begin{array}{l}\text { Drawing } \\
\text { Revision }\end{array}$ & $\begin{array}{c}\text { Revision } \\
\text { Date }\end{array}$ \\
\hline 1 & 404142 & 241-AW-101 TV System & -- & $6 / 18 / 94$ \\
\hline 2 & $42 \mathrm{U1}$ & Upper Mast & - & $6 / 18 / 94$ \\
\hline 3 & $42 \cup 2$ & Upper Mast & - & $6 / 18 / 94$ \\
\hline 4 & $42 \cup 3$ & Upper Mast & - & $6 / 18 / 94$ \\
\hline 5 & $42 \cup 4$ & Upper Mast & -- & $6 / 18 / 94$ \\
\hline 6 & $42 \cup 5$ & Upper Mast & -- & $6 / 18 / 94$ \\
\hline 7 & $42 \cup 6$ & Upper Mast & - & $6 / 18 / 94$ \\
\hline 8 & $42 \cup 7$ & Upper Mast & - & $6 / 18 / 94$ \\
\hline 9 & $42 \cup 8$ & Upper Mast & - & $6 / 18 / 94$ \\
\hline 10 & $42 \cup 9$ & Upper Mast & - & $6 / 27 / 94$ \\
\hline 11 & $40 L 1$ & Lower Mast & - & $6 / 18 / 94$ \\
\hline 12 & $40 L 2$ & Lower Mast & -- & $6 / 18 / 94$ \\
\hline 13 & $40 L 3$ & Lower Mast & - & $6 / 18 / 94$ \\
\hline 14 & $40 L 4$ & Lower Mast & - & $6 / 18 / 94$ \\
\hline 15 & $40 L 5$ & Lower Mast & - & $6 / 18 / 94$ \\
\hline 16 & 40L6 & Lower Mast & - & $6 / 18 / 94$ \\
\hline 17 & $41 R 1$ & Roll Mechanism & - & $6 / 18 / 94$ \\
\hline 18 & $41 R 2$ & Roll Mechanism & - & $6 / 18 / 94$ \\
\hline 19 & 41R3 & Roll Mechanism & - & $6 / 18 / 94$ \\
\hline 20 & 41R4 & Roll Mechanism & -- & $6 / 18 / 94$ \\
\hline 21 & $41 R 5$ & Roll Mechanism & -- & $6 / 18 / 94$ \\
\hline
\end{tabular}


URS Consultants, INC. Vol. II, Rev. 0

\section{URS/John A. Blume \& Associates, Engineers}

jobno. 66481-01 Job NHC/MPF ANALYSIS

Client

Subject EVALUATIONS of CHANGE;
Sheet No.

Calc. No.

Rev. No.

Cyk'd RIK Date $\frac{2 / 3 / 94}{8 / 3 / 94}$
E. -

\begin{tabular}{|c|c|c|c|c|}
\hline NO & $\begin{array}{l}\therefore \text { - unive } \\
\text { PATER No }\end{array}$ & $\begin{array}{l}+5-3,11 T \\
\text { PAGE NO. }\end{array}$ & DESCRIPT ON of CHANGES & INPACT EUALUATIONS \\
\hline 1 & A. 2 & $E .2$ & $\begin{array}{l}\text { MAT'L Of JUNCTION BOX } \\
\text { (MANIFOLD TUBE) CHANGED } \\
\text { FROM } 17.4 \text { PHSS TO } 304 \text { SS }\end{array}$ & $\begin{array}{l}f_{y}=25, f_{t}=70 \mathrm{kni} \text { wers } \\
\text { USED; no IMPACT }\end{array}$ \\
\hline 2 & A. 2 & E. 2 & CROSS-BRACES ARE ADDED & $\begin{array}{l}\text { THIS CHANGE SHOULD LOWER } \\
\text { THE STRESSES ET WILL BE } \\
\text { BETTER STRUCTURALLY. }\end{array}$ \\
\hline 3 & $A_{3}$ & $E \dot{3}$ & $\begin{array}{l}\not 17 / 64 \text { CHANGED TO } \\
\not 17 / 32 \text { DRILL HOLES }\end{array}$ & $\begin{array}{l}\text { IT WAS AN ERROR BEING } \\
\text { CORRECTED. OK }\end{array}$ \\
\hline 4 & A.5 & E.4 & $\begin{array}{l}\text { "WELD LEAK TIGHT" IS } \\
\text { ADDED TO } 1 / 8 \text { WELD }\end{array}$ & OK \\
\hline 5 & A. 6 & E. 5 & $\begin{array}{l}\text { - } 20^{\circ} \text { Changes to } 18^{\circ} \\
\text { - 1/4 NPT } \\
\text { - TYP } 2 \text { R } 40^{\circ} \text { APART TO } 36^{\circ} \text { APNeT } \\
\text { - MAT } 17-4 \text { SS TO } 304 \mathrm{SS}\end{array}$ & $\begin{array}{l}\text { No IMPACT IN } \\
\text { STRUCTURAL ANALYSIS }\end{array}$ \\
\hline 6 & A.10 & $E .6$ & $\begin{array}{l}\text { ORIENTATION Of THE } \\
4 \text { SS PIES HAS BEEN CHANGED }\end{array}$ & NO IMPACT \\
\hline 7 & A. 13 & E. 7 & $\begin{array}{l}\text { DETAILS of CROSS BRACES } \\
\text { - ADDED WELD @ CENTER of BRACES } \\
\text { - REMOVE ALL AROUND WELD SYMBOLL } \\
\text { BETWEEN BRACE } 3 \text { PIPE }\end{array}$ & 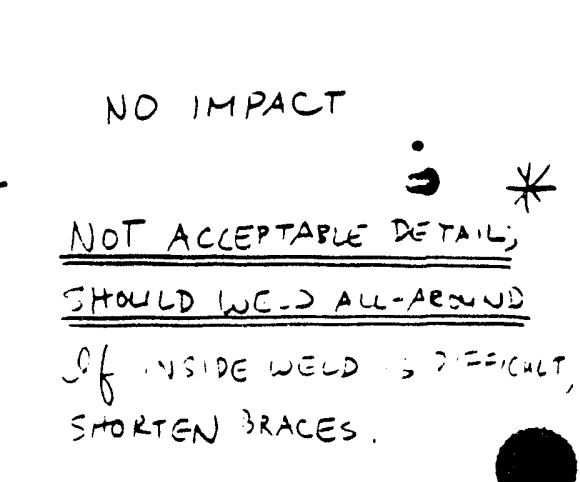 \\
\hline
\end{tabular}

- ADDEDWELD@CENTER of braces

- Remove all Alound WELd SYMBOL Between brace 3 PIPE 


\section{FAX MESSAGE}

FROM: MECHANICAL ENGINEERING

MAILSTOP H5-TO

WESTINGHOUSE HANFORD COMPANY

P.O. BOX 1970

RICHLANO, WA 99352

DATE: $8 / 8 / 94$

TO: RABIEH KHOURT

FAX NUMBER: (415) 398-MOl

TELEPHONE:
Number of Pages Including This Ono FROM: JoHN STREHLOW FAX NUMBER:

TELEPHONE: (509) $376-5009$

MESSAGE:

I have inspected the Aw-101 camera AsSEmbly ANO FOUMO THAT THE QROSS BRACE WELOS WERE PERFORMED AS REQUIRED IN THE FOLWWUMh IMSTRUCTIOU MEMO TO THE MANUFACTURER. THE VenDer PrAWIMLS WILL BE WPOATED TO REFLECT THLS AS-BUILT COMFIGURAIION.

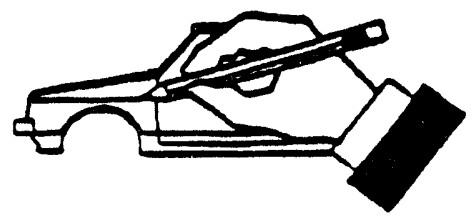

We're Making Sure!

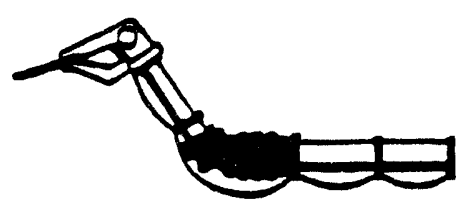

$\rightarrow$ 
DON'T SAY IT - Write It!

TO: Ron Harding
DATE: June 9, 1994

FROM: Ed Kohlman

Telephone: $6-3696$

SUBJECT: Additional bracing required on AW-101 camera structure

According to feedback from Blun (analysts group doing seismic and structural work on the camera), an additional brace or T-section welded over the existing brace is required for it to meet code. We request that an additional brace be added at each location as shown on the sketch below.
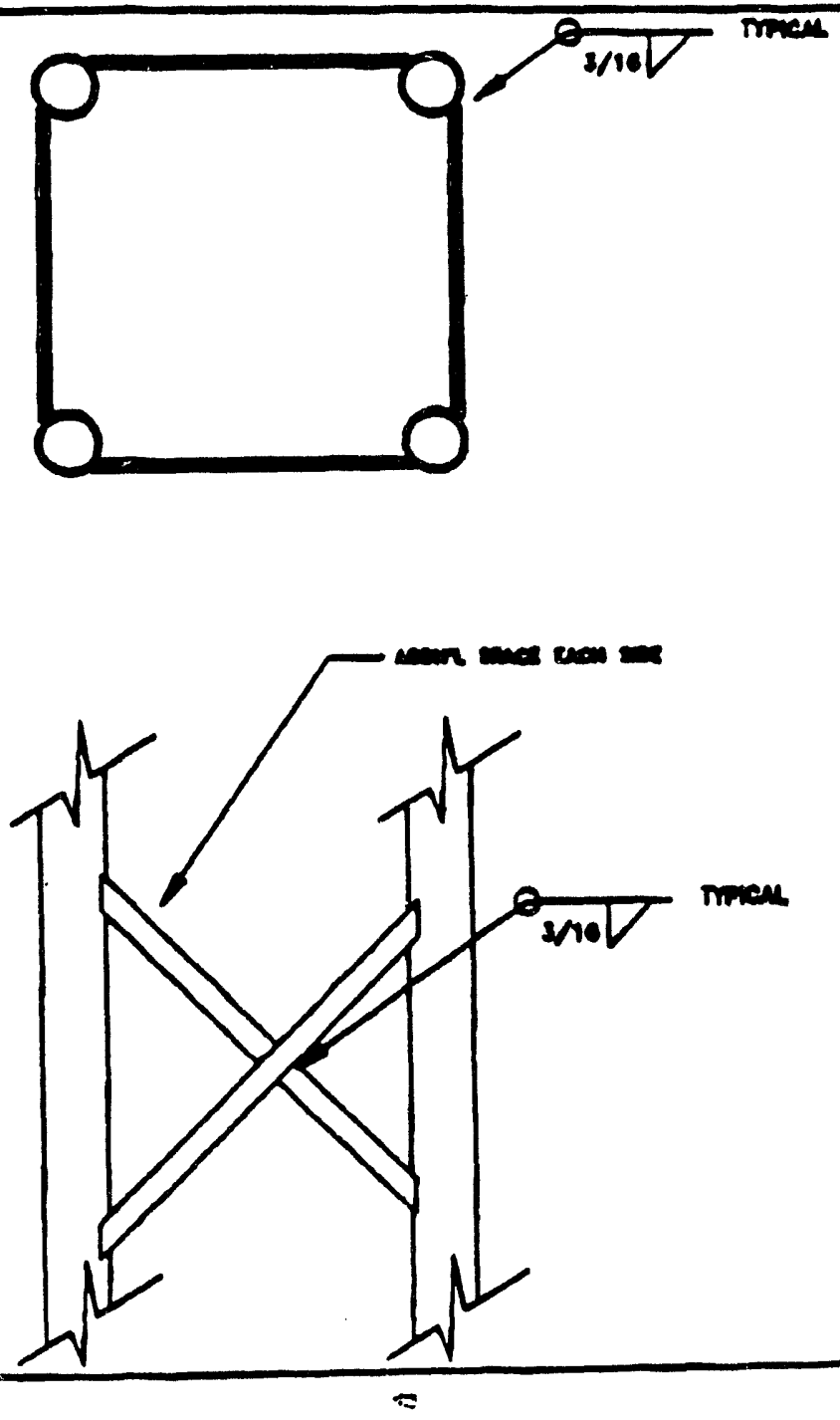
WHC-SD-WM-D.A-163

Vol. II, Rev. 0

Attachment I to Volume II

Resolution of Independent Reviaw Comments

0 


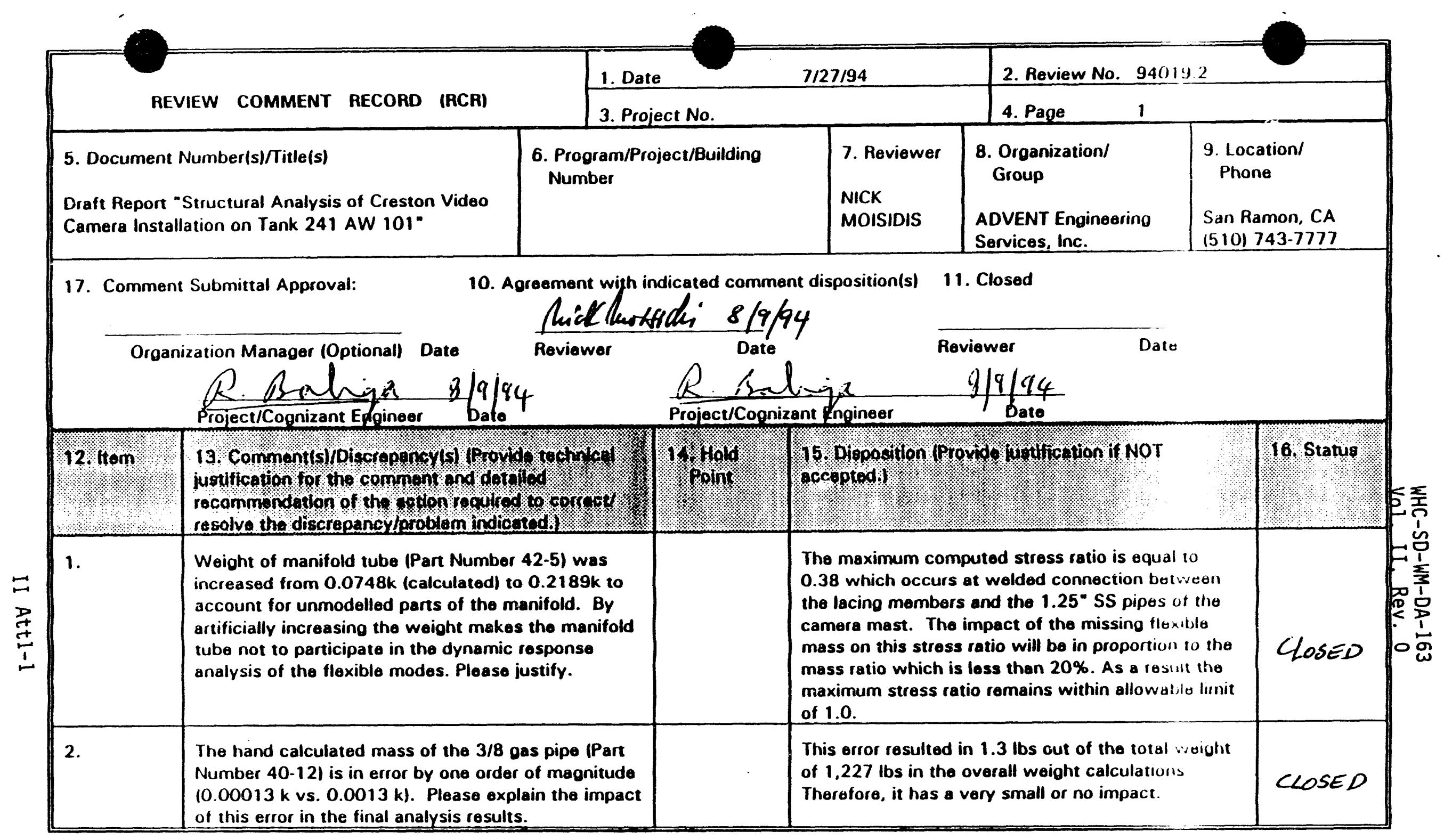




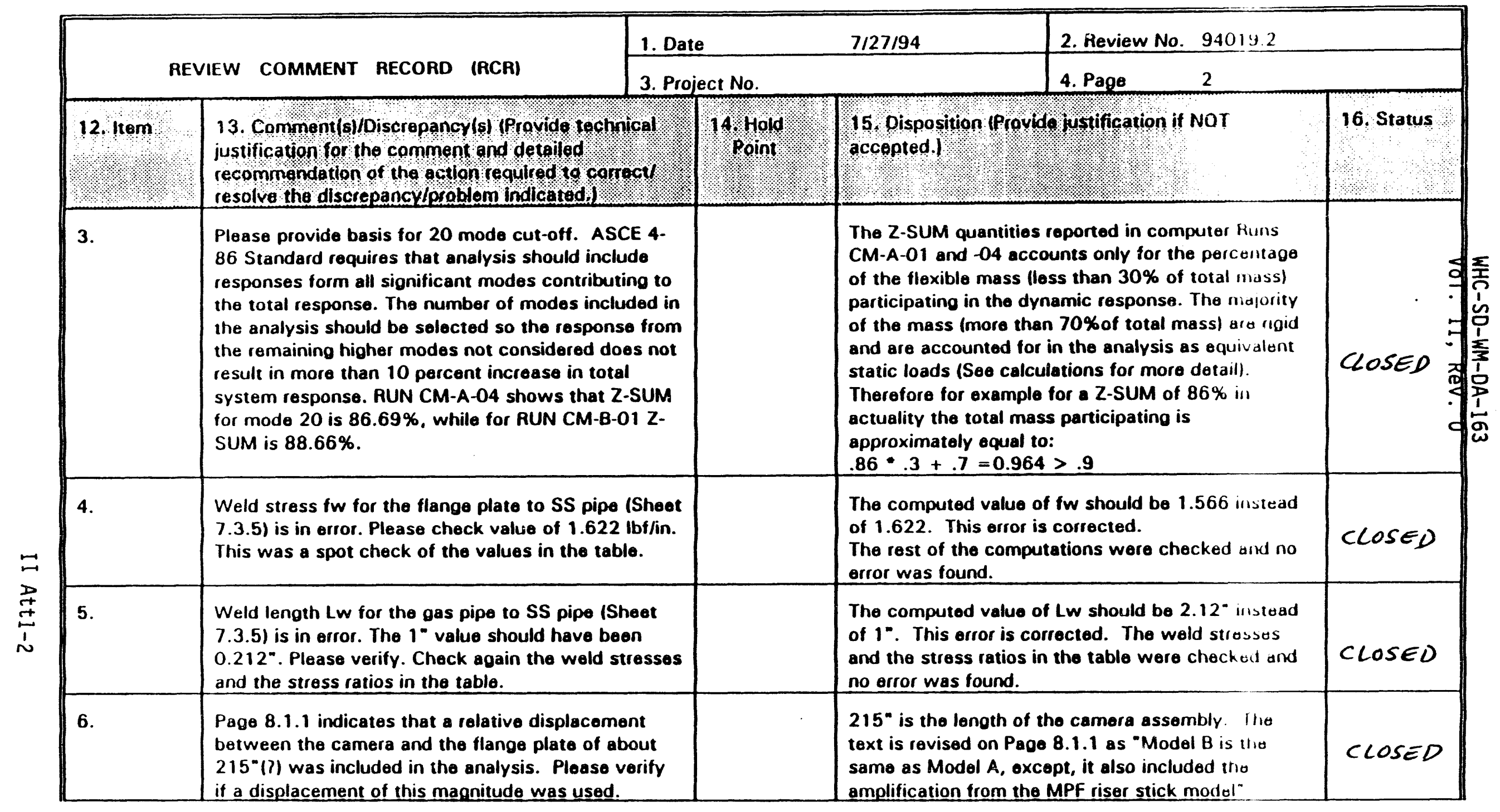
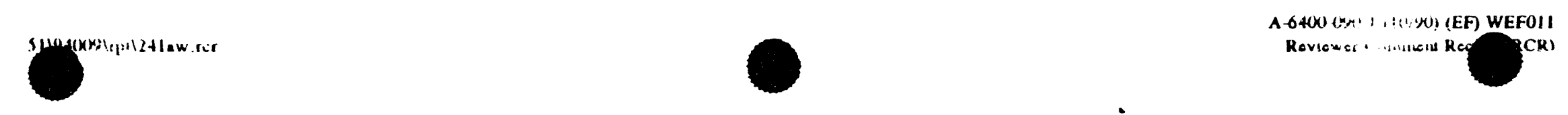


\begin{tabular}{|c|c|c|c|c|}
\hline 12. liem & $\begin{array}{l}\text { 13. Comment(s)/Disciepancy(s) (Provide technical } \\
\text { justification tor the cenmert and detalied } \\
\text { recommendation of the action required to conrect) } \\
\text { resolve the discrepancy/problem indicated.) }\end{array}$ & 14. Hold & 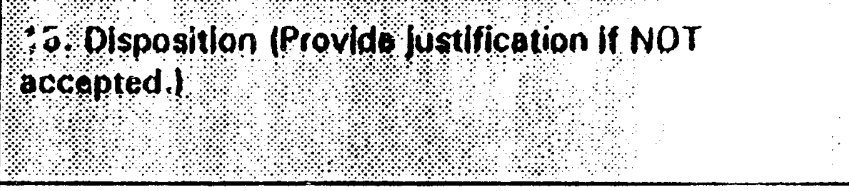 & 16. Status \\
\hline 7. & $\begin{array}{l}\text { Provide basis for the } 30 \mathrm{~Hz} \text { natural frequency for } \\
\text { the camera unit specified on Sheet } 9.1 .1 \text {. This } \\
\text { frequency is the first mode frequency of the } \\
\text { structure including the camera unit. }\end{array}$ & & $\begin{array}{l}\text { The text is revised as "Note that the first mode } \\
\text { frequencies for the entire camera assembly are } 3.21 \\
\mathrm{~Hz} \text { for Model A and } 3.18 \mathrm{~Hz} \text { for Model B. }\end{array}$ & CCOSED \\
\hline 8. & $\begin{array}{l}\text { Is Figure } 1 \text { of Section } 9 \text { the one providing the } \\
\text { proposed revised configuration of the bracing? If } \\
\text { not, replace with the proper figure and describe } \\
\text { figure and reference in the conclusion section. }\end{array}$ & & $\begin{array}{l}\text { Section } 9.3 \text { and Figure } 9-1 \text { are added. Section } 9.3 \\
\text { describes the recommended modifications in more } \\
\text { detail. }\end{array}$ & $C \operatorname{CoS} E D$ \\
\hline
\end{tabular}




\begin{tabular}{|c|c|c|c|c|c|}
\hline \multirow{2}{*}{\multicolumn{2}{|c|}{ REVIEW COMMENT RECORD (RCA) }} & 1. Date & $2 / 27 / 94$ & \multicolumn{2}{|l|}{ 2. Review No. 940192} \\
\hline & & 3. Project No. & & 4. Page 3 & \multirow[b]{2}{*}{ 16. Status } \\
\hline 12. Itam & 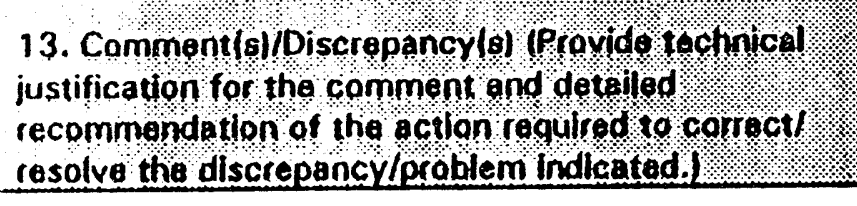 & 14. Hold & $\begin{array}{l}15.0 \text { Disp } \\
\text { accepted }\end{array}$ & Uustification if nor & \\
\hline 9. & $\begin{array}{l}\text { Where are references } 1 \text { through } 5 \text { and } 10 \text { used in } \\
\text { the report? Are they needed? If so, indicate in the } \\
\text { text of the report. }\end{array}$ & & $\begin{array}{l}\text { Referenc } \\
\text { indicated }\end{array}$ & $\begin{array}{l}5 \text { and } 10 \text { are now used and } \\
\text { the final report. }\end{array}$ & CLOSED \\
\hline 10. & $\begin{array}{l}\text { In the second paragraph of page } 6 \text { of the report, } \\
\text { describe Table } 2-2 \text { which summarizes the results of } \\
\text { frequency analysis. Provide reasons why stopped at } \\
20 t h \text { mode and justify that contribution from higher } \\
\text { modes is not significant to the total system } \\
\text { response. }\end{array}$ & & $\begin{array}{l}\text { Text is e } \\
\text { modes. }\end{array}$ & $\begin{array}{l}\text { final report to justify } 20 \\
\text { the Final report. }\end{array}$ & $C \operatorname{Cos} E D$ \\
\hline 11. & $\begin{array}{l}\text { On page } 6 \text { of the report, the 3rd paragraph indicates } \\
\text { that Table 2-2, rather than Table 2-3, summarizes } \\
\text { the computed stress ratios. Please revise. }\end{array}$ & & Text is re & & CLOSED \\
\hline 12. & $\begin{array}{l}\text { On page } 6 \text { of the report, at the last paragraph, } \\
\text { provide reference for the maximum lateral } \\
\text { displacement of } 0.6^{*} \text { at the location of the camera. } \\
\text { Also illustrate this displacement on Figure } 2-1 \text { of the } \\
\text { report, either as a detail to the model or provide a } \\
\text { mode shape. }\end{array}$ & & $\begin{array}{l}\text { A new Fi } \\
\text { shape of }\end{array}$ & $\begin{array}{l}\text { Ided to depict the deflected } \\
\text { nbly in the Final report }\end{array}$ & CLOSED \\
\hline 13. & $\begin{array}{l}\text { Describe Figure } 1.4 \text { in the report. Indicate that this } \\
\text { Figure illustrates the proposed modification for the } \\
\text { structure bracing. }\end{array}$ & & $\begin{array}{l}\text { The desc } \\
\text { bracings } \\
\text { report. }\end{array}$ & $\begin{array}{l}\text { proposed modification for the } \\
\text { Figure } 1-4 \text { is added to } 4110\end{array}$ & C COSED \\
\hline 14. & $\begin{array}{l}\text { Third paragraph on page } 6 \text { should read: "Table 2-3 } \\
\text { summarizes ...for different ...structure. As shown } \\
\text { in Table 2-3,.. for components of camera ..with the } \\
\text { largest ...support (part No.40-4) respectively." }\end{array}$ & & $\begin{array}{l}\text { Revised } \\
\text { Section }\end{array}$ & $\begin{array}{l}\text { ded. See expanded vorsion in } \\
\text { ort. }\end{array}$ & CLOSED \\
\hline 15 . & $\begin{array}{l}\text { On page } 1.1 \text { paragraph } 4 \text { and page } 1.2 \text { section } 1.3 \\
\text { paragraph } 3 \text {, replace "pounding" with "interference" }\end{array}$ & & Revised & ded. & $C \operatorname{COSED}$ \\
\hline
\end{tabular}

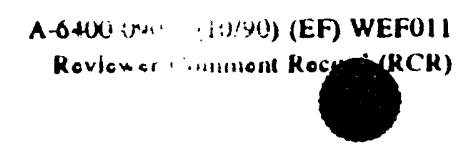




\begin{tabular}{|c|c|c|c|c|c|c|c|}
\hline \multirow{2}{*}{\multicolumn{2}{|c|}{ REVIEW COMMENT RECORD (RCA) }} & \multicolumn{2}{|c|}{ 1. Date } & $7 / 27 / 94$ & \multicolumn{3}{|c|}{ 2. Review No. 94019.2} \\
\hline & & \multicolumn{2}{|c|}{ 3. Project No. } & & \multicolumn{2}{|l|}{ 4. Pago } & \\
\hline 12. & 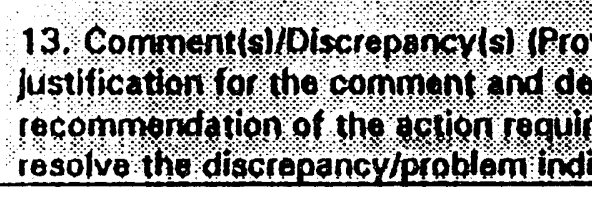 & (201: & : $14:$ Hold: $:=$ & \multicolumn{3}{|c|}{ 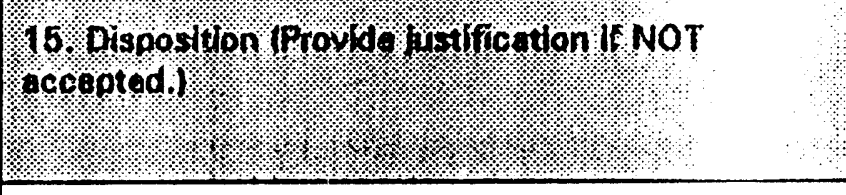 } & 16. Status \\
\hline 16. & $\begin{array}{l}\text { On page } 2.1 .1 \text { the last sentence of I } \\
\text { Restraints should read: "This approa } \\
\text { subjected to loads ... remain station }\end{array}$ & $\mathrm{n}$ it is & & \multicolumn{3}{|c|}{ Revised as recommended. } & C Cosed \\
\hline 17. & $\begin{array}{l}\text { On page } 2.1 .2 \text { first paragraph should } \\
\text { the drawings..., the description of p } \\
\text { tabulated in Table...." } \\
\text { The second paragraph should read: } \\
\text { fixed... and shares the same node w } \\
\text { riser." }\end{array}$ & sing & & \multicolumn{3}{|c|}{ Revised as recommendad. } & $C \cos E D$ \\
\hline 18. & On page 2.1.3 give table number an & & & \multicolumn{3}{|c|}{$\begin{array}{l}\text { It is re-formatted as "Table 2-1: Description of } \\
\text { parts". Also, the same for "Table 9-1: Summary of } \\
\text { Stress Ratios". }\end{array}$} & CLOSED \\
\hline 19. & $\begin{array}{l}\text { On page 2.2.1, paragraph } 3 \text { should o } \\
\text { entire camera assembly is given as } 1 \\
\text { adjustment factors... }\end{array}$ & io the & & \multicolumn{3}{|c|}{ Revised as recommended. } & CCOSED \\
\hline 20. & $\begin{array}{l}\text { On page 2.4.1, paragraph } 2 \text { should } \\
\text { material property parameter, } M N=N\end{array}$ & $\ldots$. & & \multicolumn{3}{|c|}{ Revised as recommended. } & CLOSED \\
\hline 21. & $\begin{array}{l}\text { On page } 3.1 .1 \text {, paragraph } 3 \text { should } \\
\text { the spectrum can be scaled by } 2 / 3 \mathrm{ff} \\
\text { direction, however, for conservatism } \\
\text { at } 100 \% \text { in all three directions." }\end{array}$ & that & & \multicolumn{3}{|c|}{ Text is revised. Seo page 3.1.1 for details. } & CLOSED \\
\hline
\end{tabular}




\begin{tabular}{|c|c|c|c|c|c|}
\hline \multirow{2}{*}{\multicolumn{2}{|c|}{ REVIEW COMMENT RECORD (RCR) }} & 1. Date & $7 / 27 / 94$ & \multicolumn{2}{|l|}{ 2. Review No. 94019.2} \\
\hline & & 3. Project No. & & 4. Page 5 & \multirow[b]{2}{*}{$\begin{array}{l}16, \text { status } \\
\text { (4), }\end{array}$} \\
\hline 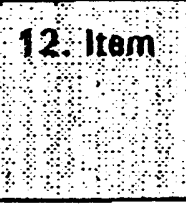 & 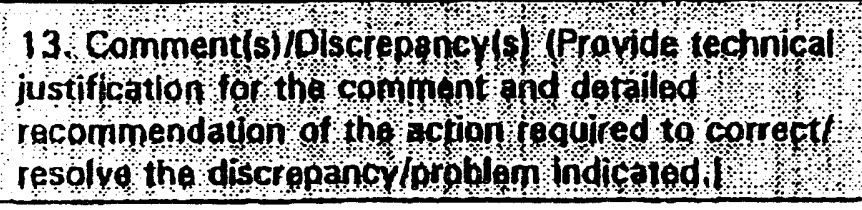 & $\begin{array}{l}14 . \mathrm{Hold} \\
\text { poing }\end{array}$ & 15 oispo & de justification if NaT & \\
\hline 22. & $\begin{array}{l}\text { On page 3.3.1, paragraph } 3 \text { should be reworded } \\
\text { for clarity. }\end{array}$ & & \multicolumn{2}{|c|}{ Reworded as recommended. } & CCOSED \\
\hline 23. & $\begin{array}{l}\text { On page 4.1.1, paragraph } 1 \text { and the paragraph } \\
\text { describing the history of computer runs must be } \\
\text { reworded for clarity. }\end{array}$ & & \multicolumn{2}{|c|}{ Page 4.1.1 is revised for clarity. } & $C \cos =0$ \\
\hline 24. & On page 7.1.1 fix typo: "deal" instead of "dead". & & \multicolumn{2}{|c|}{ Fixed as recommended. } & $c \cos =0$ \\
\hline 25. & $\begin{array}{l}\text { On page } 8.1 .1 \text { replace "pounding" with } \\
\text { "intererence". }\end{array}$ & & \multicolumn{2}{|c|}{ Replaced as recommended. } & $C \cos \theta 0$ \\
\hline 26. & $\begin{array}{l}\text { On page 9.1.1 text needs to be reworded for } \\
\text { clarity. }\end{array}$ & & \multicolumn{2}{|c|}{$\begin{array}{l}\text { Section } 9.1 \text { and } 9.2 \text { are revised for clarity. Also, } \\
\text { Section } 9.3 \text { is added. }\end{array}$} & CCOSED \\
\hline & & & & & C COSED \\
\hline 27. & $\begin{array}{l}\text { An error was found in SAP9O model that the } \\
\text { thickness of the manifold tube was input as } 2^{*} \\
\text { instead of } 1^{*} \text {. }\end{array}$ & & \multicolumn{2}{|c|}{$\begin{array}{l}\text { Element stress and the stress ratios are revised } \\
\text { and it had insignificant or no impact to the } \\
\text { analysis because the stress ratios were very low. }\end{array}$} & $C \cos E D$ \\
\hline 28. & $\begin{array}{l}\text { The thickness of the SS pipes is } 0.14 \text {, less than } \\
3 / 16^{*} \text { the size of the wold. }\end{array}$ & & \multicolumn{2}{|c|}{$\begin{array}{l}\text { With proper care per AWS specifications, the } \\
3 / 16^{\prime \prime} \text { weld can be used without damaging the SS } \\
\text { pipes. }\end{array}$} & $C \cos E D$ \\
\hline & & & & & \\
\hline & & & & & \\
\hline & & & & & \\
\hline & & & & & \\
\hline
\end{tabular}



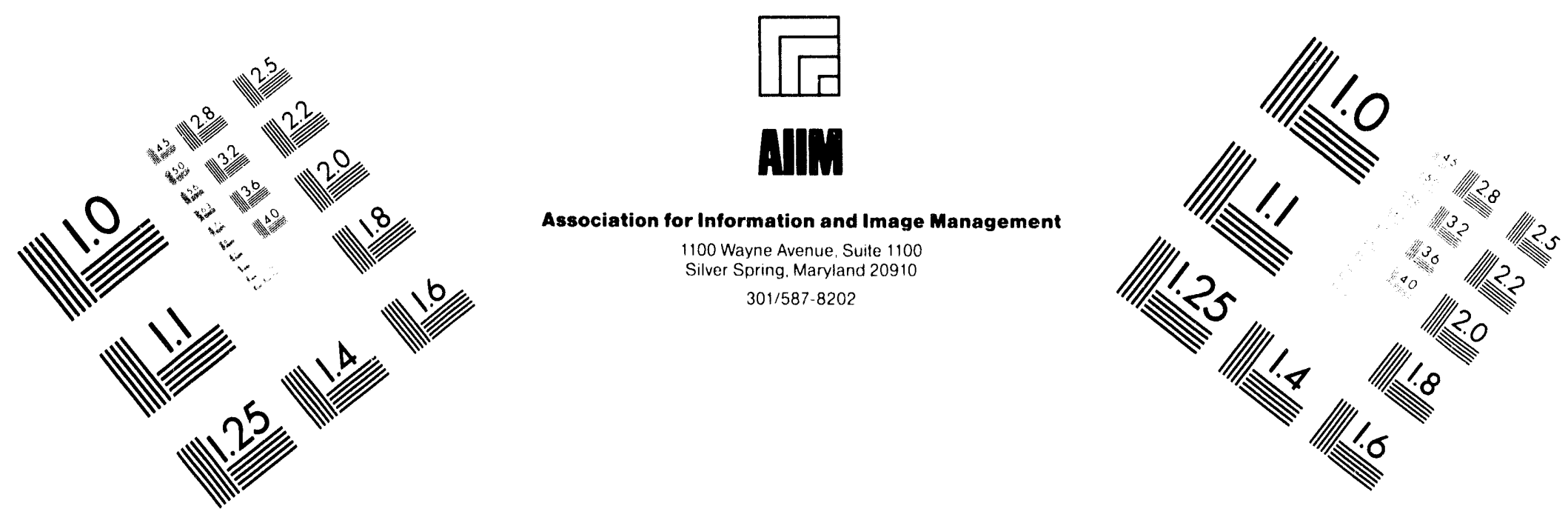

\section{Centimeter}

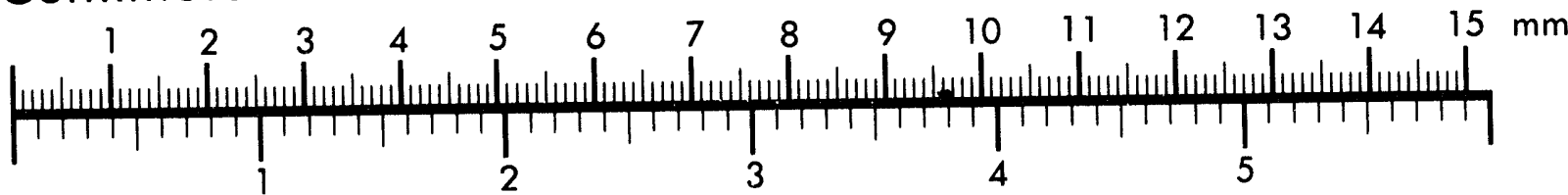
Inches
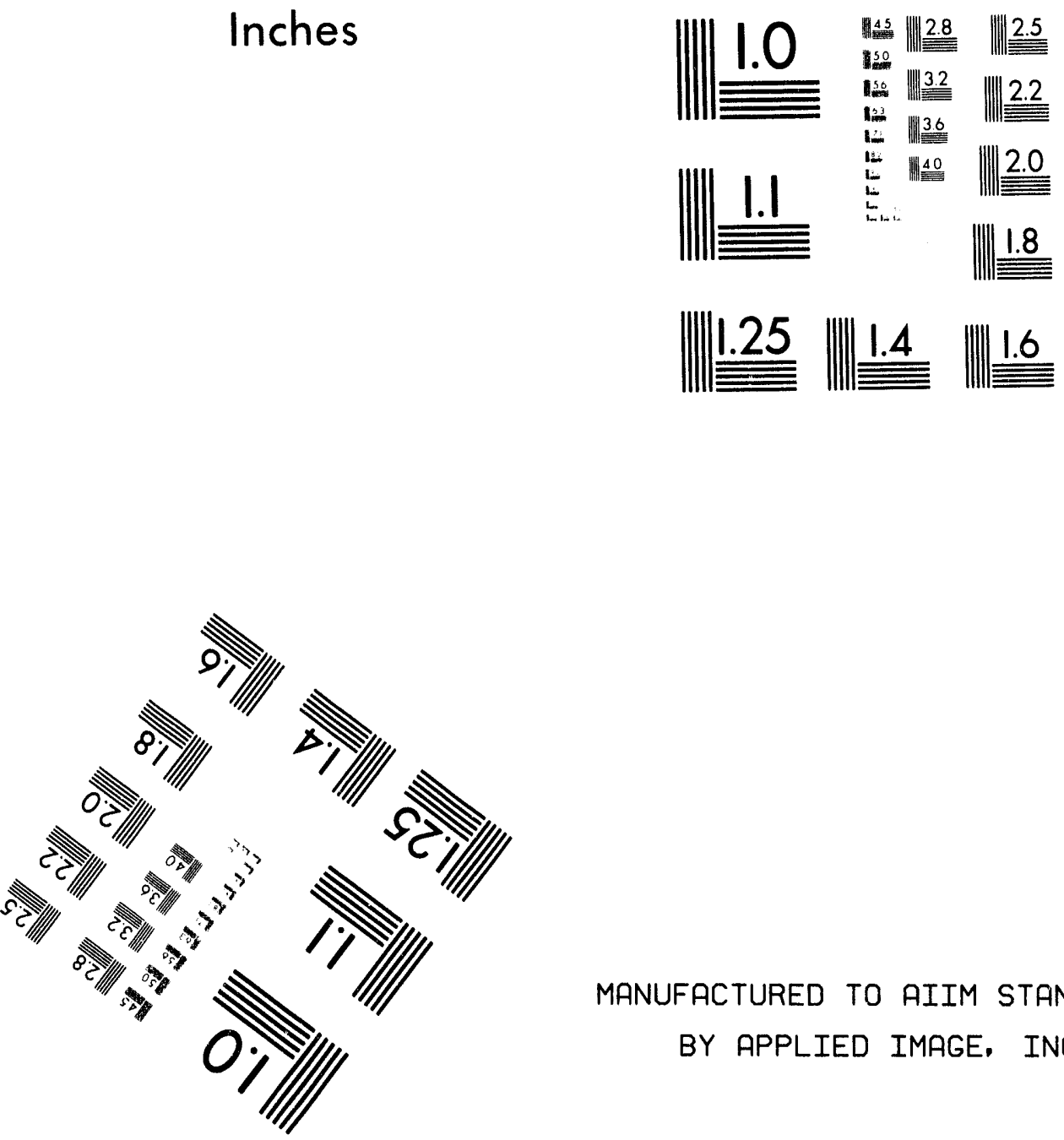

MANUFACTURED TO AIIM STANDARDS

BY APPLIED IMAGE, INC.

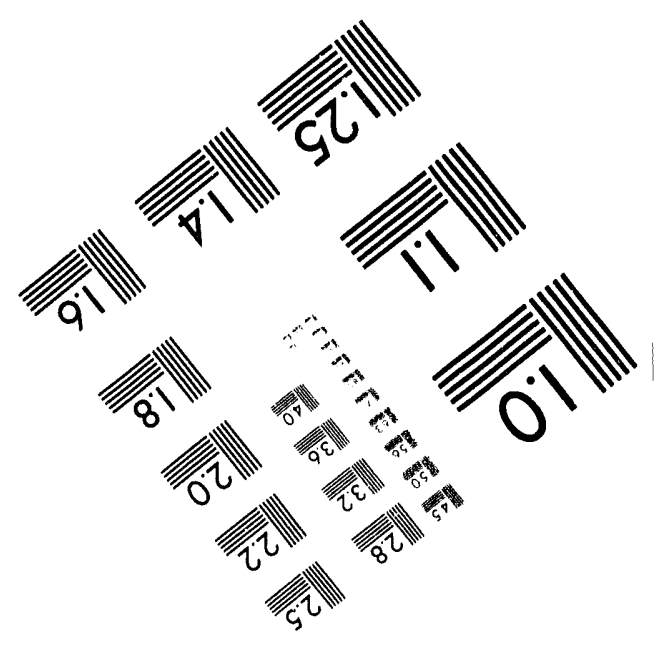




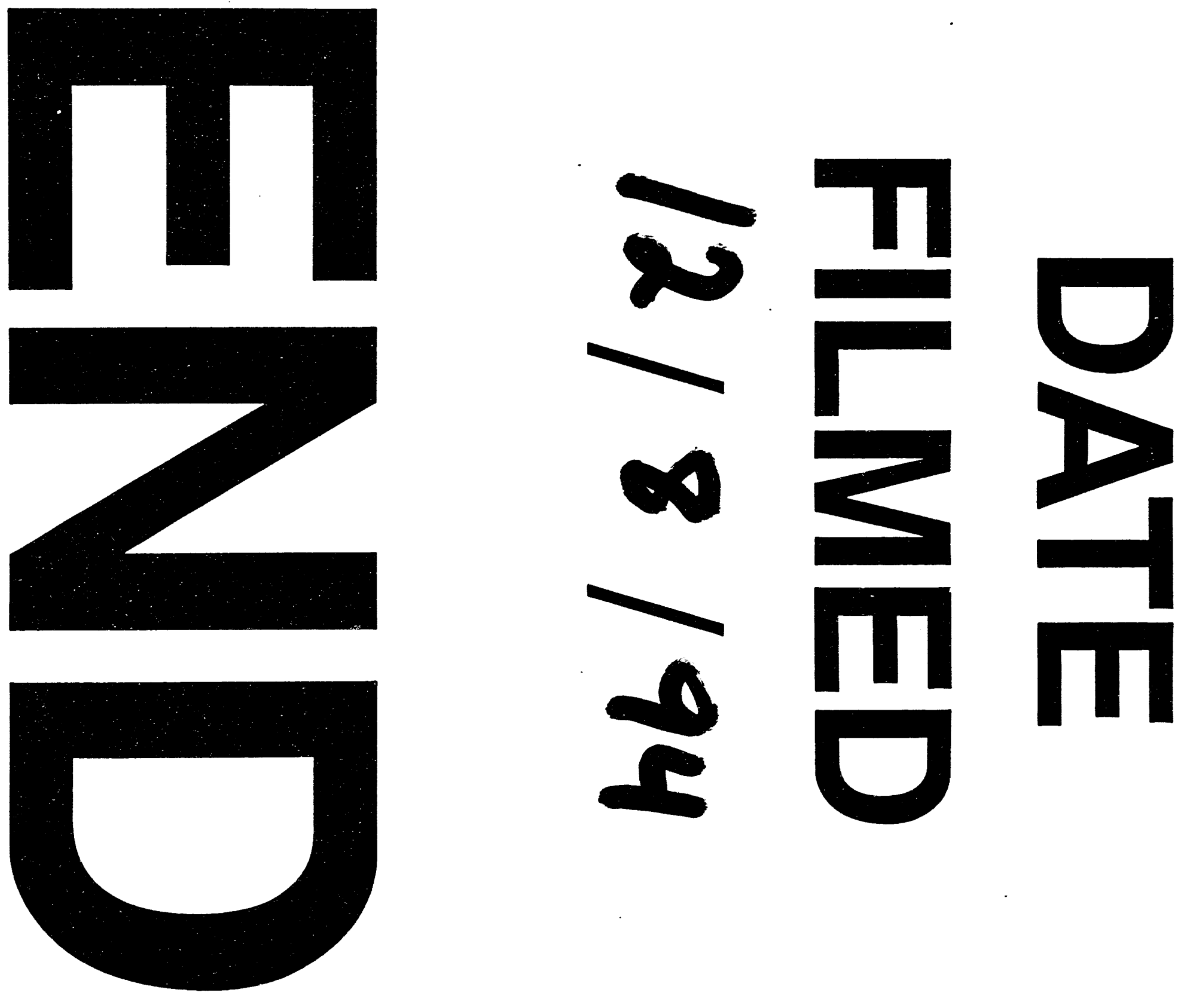